\title{
FINAL REPORT FOR THE FLOW EXCURSION FOLLOW-ON TESTING (U)
}

\author{
by C. A. Nash
}

Westinghouse Savannah River Company

Savannah River Site

Aiken, South Carolina 29808

Other Authors:

T. W. Walters

(WSRC)

Babcock \& Wilcox

This paper was prepared in connection with work done under Contract No. DE-AC09-89SR18035 with the U. S. Department of Energy. By acceptance of this paper, the publisher and/or recipient acknowledges the U. S. Government's right to retain a nonexclusive, royalty-free license in and to any copyright covering this paper, along with the right to reproduce and to authorize others to reproduce all or part of the copyrighted paper. 


\section{DISCLAIMER}

This report was prepared as an account of work sponsored by an agency of the United States .Government. Neither the United States Government nor any agency thereof, nor any of their employees, makes any warranty, express or implied, or assumes any legal liability or responsibility for the accuracy, completeness, or usefulness of any information, apparatus. product, or process disclosed, or represents that its use would not infringe privately owned rights. Reference herein to any specific commercial product, process, or service by trade name, trademark, manufacturer, or otherwise does not necessarily constitute or imply its endorsement, recommendation, or favoring by the United States Government or any agency thereof. The views and opinions of authors expressed herein do not necessarily state or reflect those of the United States Government or any agency thereof.

This report has been reproduced directly from the best available copy.

Available to DOE and DOE contractors firom the Office of Scientific and Technical Information, P.O. Box 62, Oak Ridge, TN 37831; prices available from (615) 576-8401, FTS 626-8401.

Available to the public from the National Technical Information Service, U.S. Department of Commerce, 5285 Port Royal Rd., Springfield, VA 22161. 
To

C. A. NASH - WESTINGHOUSE SAVANNAH RIVER COMPANY

rom

T. W. WALTERS - NUCLEAR STEAM SYSTEMS \& COMPONENTS SECTION, ARC

\begin{tabular}{ll|c}
\hline -ust. & WESTINGHOUSE SAVANNAH RIVER COMPANY & $\begin{array}{l}\text { File No. } \\
\text { RDD:93:44260-102-006:01 }\end{array}$ \\
\hline ubj. & $\begin{array}{l}\text { FINAL REPORT FOR THE FLOW EXCURSION FOLLOW-ON } \\
\text { TESTING }\end{array}$ & $\begin{array}{l}\text { Date } \\
\text { AUGUST 5, 1992 }\end{array}$ \\
\hline
\end{tabular}

This letter to cover one customer and one subject only

\section{SUMMARY}

The purpose of the Mark 22 Flow Excursion Follow-On testing was to investigate the theory that approximately $15 \%$ of the flow bypassed the primary flow channels in previous testing, whereas the design called for only a 3\% bypass. The results of the follow-on tests clearly confirmed this theory. The testing was performed in two phases. During the first phase, characterization tests performed during the earlier test program were repeated.

DOES NOT CONTAIN

UNCLASSIFIED CONTROLLEO

NUCLEAR INFORMATION

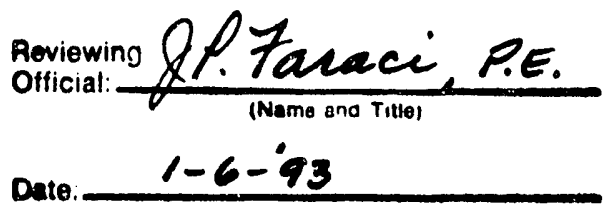


The data were in good agreement with earlier tests, thereby establishing a common baseline. The model fuel assembly was then partially disassembled, inspected, and fitted with plugs to prevent flow through the bypass channels. The inspection showed a larger gap size in the outer target extension than previously estimated. This area is suspected of being the primary leak path that permitted the bypass in earlier tests. The characterization tests were repeated once again, and results compared to the baseline. All pressure drop and flow data clearly indicated that the fuel assembly with bypass channel plugs installed had an increased flow through the primary flow channels relative to the total supplied flow. Analysis indicates the increased flow was approximately $15 \%$. The flow and pressure data were provided to WSRC. The data can be used to assess the impact of the bypass flow on previous test results.

Prepared By:

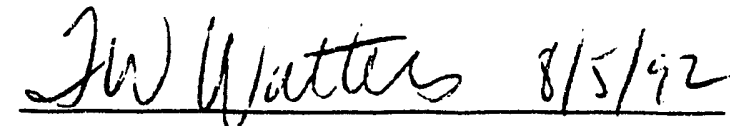

Trey W. Walters, Project Engineer

Reviewed By:

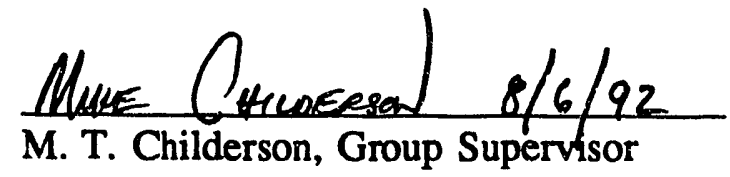

Approved By:

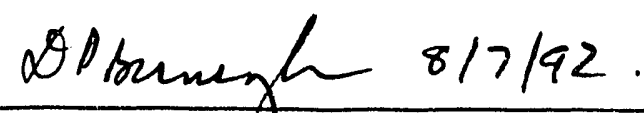

D. P. Birminghah, Section Manager Nuclear Steam Systems \& Components

L

Attachment

Keywords: Savannah River, Mark 22 fuel assembly, downflow, pressure drop, plugging, flow excursion 


\section{INTRODUCTION}

The Flow Excursion tests were performed in 1989 and documented in the Reference 1 final report. The test program investigated the behavior of a Mark 22 nuclear fuel assembly model under various simulated accident conditions. The model was an electrically heated fuel assembly mockup, which simulated the heating profile in an actual Mark 22 nuclear fuel assembly. Details on the design of the model fuel assembly are given in Reference 1.

An independent, subsequent test program used the spare fuel assembly from the Flow Excursion testing (Reference 2). The spare fuel assembly was of the same design as the former, however it was tested with coolant flowing in the upward direction, rather than the downward direction as in the Flow Excursion test.

Because the two fuel assemblies were of the same design, it would be expected that the frictional pressure drop should be similar at similar flow rates. However, the data from the Upflow and Downflow Test Programs indicated a significant difference, with the Flow Excursion (downflow) fuel assembly appearing to have a lower frictional pressure drop. It was theorized that the Flow Excursion fuel assembly had a leakage that permitted $15 \%$ of the coolant supply to bypass the heated, primary channels. Other data was also offered in support of this theory (Reference 3).

At this time, it was suspected that differences in fabrication of the outer target extension were primarily responsible for the different bypass characteristics. This part consists of two halves cut from a single machined part. The process of cutting the two halves results in some loss of material, which further results in a gap between the two halves when installed. However, the spare fuel assembly used for NPR upflow had one of the halves destroyed during the fabrication process. The replacement half was cut to achieve a better fit with the existing half, which greatly reduced the gap size on the spare assembly. The larger gaps in the downflow outer target extension provided a large leak path for the primary flow.

Further analysis was performed which made use of the pitot tube measurements installed in the three primary flow channels. The dynamic pressure measured by the pitots was converted to an approximate flow rate, and compared against the venturi flowmeter flow rates. Good agreement (within 1\%) was demonstrated for the NPR upflow test data, but an approximate 15\% discrepancy existed in the Flow Excursion downflow data (Reference 4).

A decision was made to go back to the Flow Excursion test loop and to physically plug the bypass channels, and repeat the unheated pressure drop characterization tests. If the theory was correct, then the pressure data should change in a manner consistent with a $15 \%$ increase in flow through the primary channels. 


\section{BASELINE TESTING}

In order to establish a common reference point, a pressure drop test was run on June 3, 1992. This test followed the Reference 5 technical procedure. This procedure characterizes the pressure drop behavior of the fuel assembly. The June 3rd test was then compared to characterization test results from the 1989 Flow Excursion tests to confirm that the fuel assembly characteristics have not changed.

The Flow Excursion downflow test fuel assembly measurements are shown schematically in Figures 1-9, taken from Reference 1, Volume 2. Miost of the temperature measurements shown in these figures were not activated for the unheated follow-on tests and can thus be ignored.

The Flow Excursion loop has redundant flow rate measurements: a turbine flowmeter and three venturi flowmeters in parallel. The turbine meter (WSTM01 in gpm/WSTM20 in lbm/hr) and the sum of the three venturis (IPVN20 in lbm/hr) differed by $1-3 \%$ during the follow-on tests. This disagreement was also observed during 1989 tests. After the baseline test it was concluded that the flow rate as measured by the turbine flowmeter was less reliable for the following reasons:

1) Disagreement between the venturi flow rate and the turbine meter flow rate ranged from 1 to $3 \%$, with the turbine meter reading higher. The pressure data was repeatable when plotted against the venturi measured flow rate, while it was not repeatable when plotted against the turbine meter flow rate.

2) The same calibrations of the venturi flowmeters were used for both tests, while the turbine meter used different calibrations.

3) The turbine meter also read higher by 2 to $3 \%$ on the previous Flow Excursion tests.

All results presented in this report are plotted against the venturi based flow rate converted to gpm.

Test 827_25 was the final pressure drop characterization test performed in 1989. It is documented in Reference 1. The baseline follow-on test (827_31) was performed on June 3, 1992. Each of these tests followed the Reference 5 technical procedure, which specified data acquisition at 8 flow rate plateaus, with 2 repeat tests for a total of 10 tests. 
Comparisons between the two tests are made in Figures 10-14. The pressure drop data is shown in Figures 10-12. No significant differences are apparent. Figure 13 compares the pitot data in the channels, while Figure 14 shows the flow splits as determined from the pitot data. It appears in Figures 13 and 14 that the flow splits are slightly different, but this is not considered significant. The data in numerical form are given in Table 1. The frictional pressure drop is given in Table 2.

It was concluded that a successful baseline had been established, and so disassembly for inspection and plug installation was commenced.

\section{PLUGGED BYPASS TESTING}

The test with the plugged bypass was performed in two parts. Test 827_32 addressed the first 9 flow rate plateaus. An equipment problem in the facility flow supply necessitated terminating Test 827_32 and performing the tenth flow rate plateau in Test 827_33. These results are combined together and reported numerically in Table 3. The frictional pressure drop is given in Table 4.

A deviation from the Reference 5 technical procedure was made in Test 827_32. The deviation involved skipping over the first high flow rate plateau and postponing it to the end. This decision was made because it appeared that the pitot pressure measurement C2DP02 would over-range at the higher flow rates. This pitot was a 10 psi range unit. Since this was a critical measurement, it was decided to alter the test procedure in order to obtain good data at all lower flow rate plateaus before risking over-ranging C2DP02. When the high flow rate plateau was reached, C2DP02 did over-range at times, but its averaged value was $10.0 \mathrm{psi}$. In addition, measurement BFAP02 was set to NOREAD for the plugged bypass tests (Table 3). This was done because the outer channel (channel 1) plug installation at the bottom fitting was greatly simplified by covering the BFAP02 pressure tap. The BFAP02 tap was not used for any other pressure measurements (Figure 9), and was not considered of primary importance in the test.

The data for Tests $827 \_32$ and 33 are plotted against the 827 _31 baseline test in Figures 15-20. It is clear from the figures that the pressure drop increased significantly in the primary flow channels, as expected. Figure 15 shows the overall assembly pressure drops. The increased pressure drop is apparent. Figure 16 shows the individual channel pressure drops, while Figure 17 shows pressure drops across various parts of the loop. Again, all pressure drops which include the channels show an increase.

The pitot data, which reflect the quantity of flow in the channels, increased in all channels as shown in Figure 18. The channel frictional pressure drops also increased in Figure 19. The flow split, shown in Figure 20, did change slightly. 
It is of interest to plot the results of the plugged tests $\left(827 \_32,33\right)$ against those of Test 827 31 with the assumption of $15 \%$ increased flow. With $15 \%$ increased flow through all the channels, it would be expected that the channel frictional pressure drops would increase by the square of the increased flow. That is, the predicted frictional pressure drop for the plugged test should increase by a factor of $1.15^{2}=1.32$.

By applying this factor to the 827_31 baseline test frictional pressure drops, the other relevant pressure measurements can be back-calculated. The results presented in Figures 21-25 are obtained. The overall pressure drop shown in Figure 21 (IPAP01 - RTAP01), was in very good agreement with the prediction. Examination of the channel pressure drops, Figure 22, shows that channel 4 exceeded the prediction, while channel 2 fell below. Channel 3 was well predicted. These results are zonsistent with the altered flow split discussed previously.

The results shown in Figures 21-25 show that the plugged bypass test results increased in a manner consistent with the $15 \%$ bypass theory. calibrations.

Included in Appendices A and B are copies of the test log book and the instrument

\section{PITOT DATA ANALYSIS}

The Reference 4 analysis technique was applied to the follow-on data in order to give further insight into the results. As discussed previously, the analysis computes channel flow rates from the pitot data, and compares the sum of the three channels to IPVN20, the flow rate as determined by the venturis.

Figures 26 and 27 show the results for the 827 31 baseline test. These figures indicate that a 14-15\% discrepancy existed between the pitots and the venturis. This agrees with the $15 \%$ bypass theory. Figures $28-31$ show results for the 827_32, 33 plugged bypass tests. The agreement between pitots and venturis was within $1 \%$.

\section{DOWNFLOW/UPFLOW COMPARISON}

A comparison of the frictional pressure drops in the Flow Excursion fuel assembly and the Reference 2 upflow fuel assembly can be made because they are of the same design. Frictional pressure drop data are plotted in Figure 32. Comparisons of individual channel pressure drops are complicated by flow split differences and quadrant to quadrant differences resulting from imperfect concentricity of the cylindrical channels. 
A better comparison would be the overall pressure drop over the assembly, but this is complicated by differences in the inlet and outlet geometries of the two assemblies. The individual channel pressure drops are thus the best indicators available. For completeness, a plot of the overall pressure drop in the tests is shown in Figure 33.

\section{INSPECTION AND PLUG INSTALLATION}

The inspection involved partially disassembling the model fuel assembly. At the top fitting (see Figure 1), the top extension spool was raised about 8 inches (Figure 34). The outer target top extension was photographed and videotaped while in place. Unfortunately, the photographs of this part were of poor quality and are not shown. A sketch of this part is shown in Figure 35. The gaps on each split line of the outer target extension were suspected of being a primary source of potential leakage (Reference 4). In Reference 4, the best estimate for the width of these gaps was 0.020 to 0.030 inches. However, the inspection showed the gaps to be 0.078 (North side) and 0.050 inches (South side), thus providing a larger path for the leakage. All other parts, including teflon gaskets, were in place and intact. No other bypass paths were visible.

The analysis summarized in Reference 4 is detailed in Reference 6 . The Reference 6 analysis was modified in light of the inspection results. The results indicate that about $80 \%$ of the bypass flow can now be accounted for. No further work along these lines was pursued.

Bypass flow through the fuel assembly was possible through both channels 1 and 5. To simulate the operation of an actual Mark 22 fuel assembly, a 3-4\% bypass was designed to flow through these channels. In order to eliminate flow through the split line gap and from other sources reaching the outer bypass channel (channel 1) at the top of the channel length, a teflon gasket was placed over the orificed flange connecting the assembly inlet flow to channel 1 (Figure 36). To eliminate flow through the center channel (channel 5), the orifice screw at the top of the channel length was removed and replaced with a solid screw.

To ensure complete bypass blockage, the outlets of channels 1 and 5 were also plugged. New parts were fabricated to perform this task. The inner channel (\#5) was plugged with a metal plug, with a machined groove included to hold a rubber $\mathrm{O}$-ring. This part is shown at the right of Figure 37, along with the bottom fitting insert. The plug is shown installed in Figure 38.

The outer channel (\#1) seal included a base ring with 4 extension bars that hold a seal support ring in place (Figure 39). The seal support ring had a rubber seal glued to the top surface (Figure 40, to the left of the center channel plug). This surface, when installed, blocks flow from exiting the outer channel. This part can also be seen installed in Figure 38. 41.

The bottom fitting insert, with plugs in place and ready for installation, is shown in Figure 


\section{CONCLUSIONS}

Test results for the Flow Excursion Follow-On Program showed that approximately 15\% of the flow to the fuel assembly mockup bypassed the heated channels during the original (Reference 1) Flow Excursion test program. These results confirm the $15 \%$ bypass theory discussed in References 3 and 4. Based on inspections, it is suspected that most of the bypass flow was the result of a larger than designed split line in the downflow fuel assembly outer target extension. However, identification of the specific site for the bypass flow (Channel 1 vs. 5) was not within the scope of this test.

\section{REFERENCES}

1. "Flow Excursion Experiments With a Savannah River Mark 22 Fuel Assembly Mockup," B\&W Alliance Research Center Report RDD:90:4427-13-01-01:01, February, 1990.

2. "New Production Reactor Flow Instability Experiments With Coolant Upflow," B\&W Alliance Research Center Report RDD:92:4024-0101:01, April, 1992.

3. "Possible Flow Leakage for B\&W Downflow Test," Letter from J. E. Halverson, WSRC, to M. T. Childerson and J. E. Blake, B\&W ARC, August 2, 1991.

4. "Flow Excursion Test Leakage Question, NPR-566," Letter from M. T. Childerson and J. E. Blake, B\&W ARC, to J. E. Halverson, WSRC, November 5, 1991.

5. "SRP Characterization test - Fuel Assembly Pressure Drop at SteadyState," B\&W Alliance Research Center Technical Procedure ARC-TY827, Revision 2, December 13, 1989.

6. "Blake's Estimate on Leakage," B\&W Alliance Research Center Memorandum, SRP-1366, November 20, 1991. 
Table 1. Average and 2-sigma Data for Follow-on Flow Excursion Test 827_31

\begin{tabular}{|c|c|c|c|c|c|c|c|c|c|c|c|c|c|}
\hline $\begin{array}{r}\text { IPVN20 } \\
\text { GPM } \\
\end{array}$ & $\begin{array}{r}\text { (PSIA) } \\
\text { BPAP1 } \\
\end{array}$ & $\begin{array}{r}\text { (PSIA) } \\
\text { BPAPI? } \\
\end{array}$ & $\begin{array}{r}\text { (PSID) } \\
\text { BDPPI } \\
\end{array}$ & $\begin{array}{r}\text { (PSID) } \\
\text { BDDP2 } \\
\end{array}$ & $\begin{array}{r}\text { (F) } \\
\text { BRTTL1 } \\
\end{array}$ & $\begin{array}{r}\text { (PSIA) } \\
\text { C2AP1 } \\
\end{array}$ & $\begin{array}{r}\text { (PSIA) } \\
\text { C2APY } \\
\end{array}$ & $\begin{array}{r}\text { (PSLA) } \\
\text { C2APQ3 } \\
\end{array}$ & $\begin{array}{r}\text { (PSIA) } \\
\text { CAAPEA } \\
\end{array}$ & $\begin{array}{r}\text { (PSIA) } \\
\text { CAAPIS } \\
\end{array}$ & $\begin{array}{r}\text { (PSLA) } \\
\text { C2APS } \\
\end{array}$ & $\begin{array}{r}\text { (PSIA) } \\
\text { C2APQ7 } \\
\end{array}$ & $\begin{array}{r}\text { (PSIA) } \\
\text { CZAPOB } \\
\end{array}$ \\
\hline \multirow[t]{2}{*}{184.0} & 33.29 & 3.24 & -1.454 & -1.659 & 77.91 & 33.2 & 33.22 & 33.81 & 33.7 & 34.33 & 34.32 & 32.06 & 32.17 \\
\hline & 0.34 & 0.39 & 0.19 & 0.15 & 0.56 & 0.36 & 0.37 & 0.35 & 0.35 & 0.34 & 0.35 & 0.37 & 0.38 \\
\hline \multirow[t]{2}{*}{218.2} & 35.51 & 35.41 & -2.022 & -2.358 & 82.12 & 36.96 & 36.97 & 37.35 & 37.21 & 37.63 & 37.61 & 35.92 & 36.12 \\
\hline & 0.78 & 0.75 & 0.25 & 0.24 & 0.12 & 1 & 1 & 0.98 & 1 & 0.95 & 0.97 & 1 & 1.1 \\
\hline \multirow[t]{2}{*}{254.0} & 38.19 & 38.02 & -3.001 & -3.209 & 79.78 & 41.39 & 41.4 & 41.52 & 41.34 & 41.51 & 415 & 40.46 & 40.7 \\
\hline & 0.62 & 0.62 & 0.26 & 0.27 & 0.39 & 0.78 & 0.79 & 0.75 & 0.75 & 0.72 & 0.74 & 0.81 & 0.81 \\
\hline \multirow[t]{2}{*}{281.4} & 40.37 & 40.14 & -3.616 & -3.921 & 82.23 & 45.18 & 45.18 & 45.09 & 44.89 & 44.84 & 44.8 & 44.37 & 44.7 \\
\hline & 1.2 & 1.1 & 0.42 & 0.39 & 0.27 & 1.7 & 1.7 & 1.6 & 1.7 & 1.5 & 1.5 & 1.8 & 1.8 \\
\hline \multirow[t]{2}{*}{313.6} & 43.75 & 43.41 & 4.585 & 4.837 & 78.67 & 50.54 & 50.54 & 50.18 & 49.97 & 49.62 & 49.57 & 49.91 & 50.27 \\
\hline & 0.52 & 0.58 & 0.37 & 0.4 & 0.3 & 0.83 & 0.79 & 0.74 & 0.8 & 0.7 & 0.71 & 0.86 & 0.85 \\
\hline \multirow[t]{2}{*}{327.0} & 45.09 & 44.75 & -5.15 & -5.268 & 81.42 & 52.74 & 52.74 & 52.25 & 52.01 & 51.55 & 51.51 & 52.14 & 52.51 \\
\hline & 0.55 & 0.7 & 0.4 & 0.41 & 0.27 & 0.69 & 0.7 & 0.67 & 0.67 & 0.65 & 0.65 & 0.72 & 0.71 \\
\hline \multirow[t]{2}{*}{338.4} & 46.72 & 46.38 & -5.411 & -5.711 & 77.52 & 55.31 & 55.26 & 54.66 & 54.43 & 53.83 & 53.76 & 54.8 & 55.2 \\
\hline & 0.68 & 0.74 & 0.45 & 0.46 & 0.14 & 0.93 & 0.95 & 0.89 & 0.89 & 0.85 & 0.87 & 0.94 & 0.98 \\
\hline \multirow[t]{2}{*}{341.4} & 47.01 & 46.63 & .5 .659 & -5.813 & 81.76 & 55.69 & 55.60 & 55.06 & 54.79 & 54.19 & 54.15 & 55.14 & 55.55 \\
\hline & 0.67 & 0.73 & 0.45 & 0.39 & 0.16 & 0.85 & 0.83 & 0.85 & 0.86 & 0.82 & 0.78 & 0.85 & 0.85 \\
\hline \multirow[t]{2}{*}{355.4} & 48.63 & 48.16 & -5.888 & -6.214 & 75.62 & 58.44 & 58.39 & 57.62 & 57.36 & 56.58 & 56.53 & 58.03 & 58.5 \\
\hline & 1 & 1.1 & 0.45 & 0.55 & 0.2 & 0.89 & 0.89 & 0.91 & 0.9 & 0.92 & 0.92 & 0.88 & 0.89 \\
\hline \multirow[t]{2}{*}{366.8} & 49.96 & 49.5 & -6.427 & -6.652 & 82.31 & 60.51 & 60.46 & 59.63 & 59.32 & 58.48 & 58.41 & 60.12 & 60.59 \\
\hline & 0.91 & 0.99 & 0.42 & 0.54 & 0.14 & 0.93 & 0.98 & 0.9 & 0.92 & 0.89 & 0.93 & 0.95 & 0.98 \\
\hline
\end{tabular}

$\begin{array}{llllllllllllll}\text { IPYN20 } & \text { (PSLA) } & \text { (PSIA) } & \text { (PSID) } & \text { (PSID) } & \text { (PSID) } & \text { (PSIA) } & \text { (PSIA) } & \text { (PSIA) } & \text { (PSID) } & \text { (PSID) } & \text { (PSIA) } & \text { (PSIA) } & \text { (PSIA) }\end{array}$

\begin{tabular}{|c|c|c|c|c|c|c|c|c|c|c|c|c|c|}
\hline GPM & C2APOS & C2AP10 & C2PPO1 & C2DPe2 & C2DPE3 & CXAPII & CaAP & C3APQ3 & C3DPO1 & C3DP02 & CAAPII & CAAPE & CAAPOz \\
\hline \multirow[t]{2}{*}{184.0} & 33.99 & 34.1 & 2.01 & 1.173 & 1.989 & 33.4 & 33.52 & 33.41 & 0.2854 & 1.054 & 32.14 & 33.3 & 33.48 \\
\hline & 0.34 & 0.37 & 0.21 & 0.12 & 0.14 & 0.38 & 0.34 & 0.35 & 0.26 & 0.027 & 0.37 & 0.37 & 0.34 \\
\hline \multirow[t]{2}{*}{218.2} & 36.67 & 36.78 & 0.856 & 1.686 & 0.7078 & 37.69 & 35.98 & 35.86 & -1.608 & 1.482 & 36.04 & 35.62 & 35.87 \\
\hline & 0.86 & 0.86 & 0.28 & 0.31 & 0.27 & 1.2 & 0.81 & 0.8 & 0.44 & 0.084 & 1.1 & 0.78 & 0.79 \\
\hline \multirow[t]{2}{*}{254.0} & 39.91 & 39.97 & -0.3889 & 2215 & -0.6735 & 42.82 & 38.95 & 38.83 & -3.723 & 1.988 & 40.52 & 38.44 & 38.73 \\
\hline & 0.7 & 0.66 & 0.32 & 0.24 & 0.32 & 0.88 & 0.64 & 0.66 & 0.41 & 0.067 & 0.81 & 0.63 & 0.63 \\
\hline \multirow[t]{2}{*}{281.4} & 42.61 & 42.65 & -1.611 & 2.794 & .2 & 47.13 & 41.4 & 41.29 & -5.593 & 2.439 & 44.43 & 40.74 & 41.06 \\
\hline & 1.4 & 1.4 & 0.49 & 0.28 & 0.45 & 1.9 & 1.3 & 1.3 & 0.7 & 0.16 & 1.7 & 1.2 & 1.2 \\
\hline \multirow[t]{2}{*}{313.6} & 46.64 & 46.63 & -3.102 & 3.432 & -3.626 & 53.24 & 45.12 & 44.99 & -8.044 & 3.024 & 49.75 & 44.25 & 44.63 \\
\hline & 0.62 & 0.58 & 0.46 & 0.3 & 0.4 & 0.94 & 0.57 & 0.58 & 0.49 & 0.09 & 0.86 & 0.55 & 0.55 \\
\hline \multirow[t]{2}{*}{327.0} & 48.23 & 48.24 & -3.754 & 3.695 & -4.252 & 55.7 & 46.59 & 46.46 & -9.009 & 3.265 & 52.07 & 45.68 & 46.04 \\
\hline & 0.6 & 0.6 & 0.39 & 0.27 & 0.32 & 0.74 & 0.57 & 0.59 & 0.38 & 0.049 & 0.7 & 0.57 & 0.56 \\
\hline \multirow[t]{2}{*}{338.4} & 50.18 & 50.11 & -4.46 & 3.99 & -5.066 & 58.56 & 48.34 & 48.23 & -10.12 & 3.529 & 54.64 & 47.34 & 47.75 \\
\hline & 0.8 & 0.76 & 0.47 & 0.21 & 0.48 & 1.1 & 0.72 & 0.74 & 0.57 & 0.093 & 0.95 & 0.69 & 0.7 \\
\hline \multirow[t]{2}{*}{341.4} & 50.45 & 50.46 & -4.52 & 4.048 & -5.082 & 59 & 48.68 & 48.52 & -10.26 & 3.562 & 55.05 & 47.66 & 48.04 \\
\hline & 0.74 & 0.72 & 0.39 & 0.34 & 0.3 & 0.9 & 0.71 & 0.69 & 0.4 & 0.052 & 0.84 & 0.71 & 0.69 \\
\hline \multirow[t]{2}{*}{355.4} & 52.44 & 52.39 & -5.452 & 4.327 & -6.08 & 62.14 & 50.44 & 50.3 & -11.64 & 3.866 & 57.8 & 49.33 & 49.76 \\
\hline & 0.96 & 0.99 & 0.46 & 0.42 & 0.39 & 0.85 & 0.98 & 0.98 & 0.44 & 0.038 & 0.9 & 1.1 & 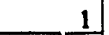 \\
\hline \multirow[t]{2}{*}{366.8} & 54 & 53.99 & -5.978 & 4.655 & -6.614 & 64.42 & 51.92 & 51.77 & -12.48 & 4.098 & 59.93 & 50.74 & $51 . \overline{14}$ \\
\hline & 0.96 & 0.94 & 0.45 & 0.41 & 0.45 & 0.98 & 0.93 & 0.95 & 0.6 & 0.083 & 0.99 & 0.97 & 0.93 \\
\hline
\end{tabular}

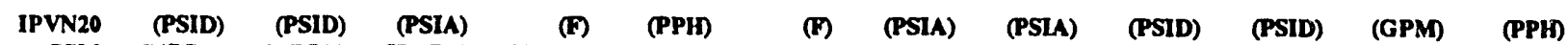

\begin{tabular}{|c|c|c|c|c|c|c|c|c|c|c|c|c|}
\hline GPM & CADPOI & CADPQ2 & IPAPE & IPRTOI & IPVN20 & MSTCR & BTAPOI & TFAPOI & TrDPO1 & TPDP2 & WSTMM & WSTM2? \\
\hline \multirow[t]{2}{*}{184.0} & 1.051 & 1.687 & 33.85 & 77.83 & 91990 & 76.49 & 28.36 & 32.99 & 0.8397 & -0.6657 & 187.6 & 93610 \\
\hline & 0.18 & 0.042 & 0.41 & 0.64 & 1300 & 0.14 & 0.36 & 0.38 & 0.084 & 0.32 & 7.5 & 3700 \\
\hline \multirow[t]{2}{*}{218.2} & 0.5481 & 2.468 & 39.34 & 81.94 & 109100 & 76.52 & 28.36 & 37.35 & -1.945 & -3.969 & 211.7 & 105600 \\
\hline & 0.34 & 0.14 & 1.4 & 0.11 & 3200 & 0.16 & 0.45 & 1.2 & 0.22 & 0.65 & 7.4 & 3700 \\
\hline \multirow[t]{2}{*}{254.0} & .2 .158 & 3.231 & 45.86 & 79.59 & 127000 & 76.39 & 28.2 & 42.53 & .3 .302 & -7.762 & 255.5 & 127500 \\
\hline & 0.31 & 0.11 & 1 & 0.38 & 2400 & 0.16 & 0.37 & 0.89 & 0.18 & 0.59 & 5.9 & 3000 \\
\hline \multirow[t]{2}{*}{281.4} & .3 .795 & 4.075 & 51.43 & 82.05 & $140700:$ & 76.47 & 28.02 & 46.89 & -4.48 & -11.15 & 286.4 & 142900 \\
\hline & 0.53 & 0.26 & 2.4 & 0.21 & 4800 & 0.14 & 0.36 & 1.9 & 0.44 & 1.2 & 16 & 7900 \\
\hline \multirow[t]{2}{*}{313.6} & -5.617 & 4.975 & 59.1 & 78.46 & 156800 & 75.6 & 28.3 & 53.15 & .5 .972 & -15.45 & 316.2 & 157900 \\
\hline & 0.45 & 0.15 & 1.2 & 0.24 & 2600 & 0.14 & 0.23 & 0.93 & 0.29 & 0.92 & 9.1 & 4600 \\
\hline \multirow[t]{2}{*}{327.0} & -6.476 & 5.404 & 62.28 & 81.19 & 163500 & 76.55 & 28.06 & 55.56 & 6.661 & -17.26 & 333.4 & 166400 \\
\hline & 0.4 & 0.081 & 0.91 & 0.24 & 1700 & 0.15 & 0.3 & 0.75 & 0.2 & 0.62 & 6.6 & 3300 \\
\hline \multirow[t]{2}{*}{338.4} & .7 .402 & 5.863 & 65.79 & 77.35 & 169200 & 75.86 & 28.5 & 58.54 & -7.273 & -19.22 & 344.2 & 171900 \\
\hline & 0.53 & 0.16 & 1.3 & 0.13 & 2700 & 0.24 & 0.39 & 1.1 & 0.29 & 0.83 & 15 & 7300 \\
\hline \multirow[t]{2}{*}{341.4} & -7.445 & 5.938 & 66.41 & 81.52 & 170700 & 76.67 & 28.4 & 58.9 & -7.457 & -19.5 & 345 & 172200 \\
\hline & 0.38 & 0.085 & 1 & 0.14 & 1500 & 0.16 & 0.43 & 0.89 & 0.19 & 0.6 & 5.6 & 2800 \\
\hline \multirow[t]{2}{*}{355.4} & .8 .55 & 6.428 & 70.35 & 75.42 & 177700 & 76.37 & 28.52 & 62.14 & .8 .209 & -21.86 & 368 & 183800 \\
\hline & 0.42 & 0.067 & 0.79 & 0.19 & 1500 & 0.16 & 1.2 & 0.85 & 0.19 & 0.64 & 7.6 & 3800 \\
\hline \multirow[t]{2}{*}{366.8} & -9.263 & 6.878 & 73.34 & 82.04 & 183400 & 76.46 & 28.41 & 64.39 & -8.877 & -23.47 & 371.2 & 185200 \\
\hline & 0.48 & 0.15 & 1.1 & 0.13 & 2000 & 0.16 & 0.95 & 0.98 & 0.27 & 0.78 & 6.5 & 3200 \\
\hline
\end{tabular}


Table 2. Frictional Pressure Drop Data Across Fuel Assembly Test 827_31

\begin{tabular}{|c|c|c|c|c|c|c|c|}
\hline IPVN20 & (PSID) & (PSID) & (PSID) & (PSID) & (PSID) & (PSTD) & $\begin{array}{l}\text { (PSID) } \\
\text { TFDPO2* }\end{array}$ \\
\hline GPM & C2DPO1* & C2DP03* & C3DPO1* & CADPO1* & TFDP01* & TFDP02* & -TFDP01* \\
\hline 184.0 & -3.27 & -3.35 & -5.05 & -4.29 & -2.69 & -8.64 & -5.95 \\
\hline 218.2 & -4.48 & -4.63 & -6.94 & -5.88 & -3.79 & -11.94 & -8.15 \\
\hline 254.0 & -5.73 & -6.01 & -9.06 & -7.49 & -5.15 & -15.73 & -10.59 \\
\hline 281.4 & -6.95 & -7.34 & -10.93 & -9.13 & -6.33 & -19.12 & -12.80 \\
\hline 313.6 & -8.44 & -8.96 & -13.38 & -10.95 & -7.82 & -23.42 & -15.60 \\
\hline 327.0 & -9.09 & -9.59 & -14.35 & -11.81 & -8.51 & -25.23 & -16.72 \\
\hline 338.4 & -9.80 & -10.40 & -15.46 & -12.74 & -9.12 & -27.19 & -18.07 \\
\hline 341.4 & -9.86 & -10.42 & -15.60 & -12.78 & -9.30 & -27.47 & -18.17 \\
\hline 355.4 & -10.79 & $\cdot 11.42$ & -16.98 & -13.89 & -10.05 & -29.83 & -19.78 \\
\hline 366.8 & -11.31 & -11.95 & -17.82 & -14.60 & -10.72 & -31.44 & -20.72 \\
\hline $\begin{array}{l}\text { DPfric } \\
\text { where: }\end{array}$ & $\begin{array}{l}\text { Density = 6) } \\
\text { H (C2DP01) } \\
\text { H (C2DP03) } \\
\text { H (C3DP01) } \\
\text { H (C42DP0 } \\
\text { H (TFDP01) } \\
\text { H (TFDP02) }\end{array}$ & $\begin{array}{l}221 \mathrm{lbm} / \mathrm{ft} 3 \\
=12.35 \mathrm{ft} \\
=12.35 \mathrm{ft} \\
=12.35 \mathrm{ft} \\
=12.35 \mathrm{ft} \\
=4.271 \mathrm{ft} \\
=18.448 \mathrm{ft}\end{array}$ & & & & 144 & \\
\hline
\end{tabular}


Table 3. Average and 2-sigma Data for Follow-on Flow Excursion Test 827_32,33

\begin{tabular}{|c|c|c|c|c|c|c|c|c|c|c|c|c|c|}
\hline $\begin{array}{r}\text { IPVN20 } \\
\text { GPM }\end{array}$ & $\begin{array}{r}\text { (PSUA) } \\
\text { BAAPOL }\end{array}$ & $\begin{array}{r}\text { (PSLA) } \\
\text { BRAPO? }\end{array}$ & $\begin{array}{r}\text { (PSID) } \\
\text { BDPPI }\end{array}$ & $\begin{array}{r}\text { (PSID) } \\
\text { BPDP2 }\end{array}$ & $\begin{array}{r}\text { (P) } \\
\text { BRRTO1 }\end{array}$ & $\begin{array}{r}\text { (PSLA) } \\
\text { C2APQ1 }\end{array}$ & $\begin{array}{r}\text { (PSIA) } \\
\text { C2APE2 }\end{array}$ & $\begin{array}{r}\text { (PSIA) } \\
\text { C2APQ3 } \\
\end{array}$ & $\begin{array}{r}\text { (PSLA) } \\
\text { CAPA }\end{array}$ & $\begin{array}{r}\text { (PSIA) } \\
\text { C2APES }\end{array}$ & $\begin{array}{r}\text { (PSLA) } \\
\text { C2APCS }\end{array}$ & $\begin{array}{r}\text { (PSLA) } \\
\text { C2AP1 }\end{array}$ & $\begin{array}{r}\text { (PSIA) } \\
\text { C2APQ8 } \\
\end{array}$ \\
\hline \multirow[t]{2}{*}{185.3} & 32.85 & 0 & -1.402 & -1.65 & 76.29 & 34.82 & 34.84 & 35.39 & 35.24 & 35.85 & 35.82 & 33.81 & 34.01 \\
\hline & 0.14 & 0 & 0.14 & 0.13 & 0.26 & 0.12 & 0.13 & 0.11 & 0.12 & 0.11 & 0.13 & 0.13 & 0.13 \\
\hline \multirow[t]{2}{*}{220.4} & 34.97 & 0 & -2.081 & -2.319 & 76.9 & 39.26 & 39.28 & 39.55 & 39.4 & 39.73 & 39.69 & 38.47 & 38.79 \\
\hline & 0.22 & 0 & 0.19 & 0.18 & 0.12 & 0.21 & 0.22 & 0.2 & 0.22 & 0.2 & 0.22 & 0.21 & 0.22 \\
\hline \multirow[t]{2}{*}{254.4} & 375 & 0 & -2.941 & -3.051 & 77.04 & 44.33 & 44.33 & 44.4 & 44.19 & 44.32 & 44.24 & 43.66 & 44.05 \\
\hline & 0.26 & 0 & 0.23 & 0.2 & 0.14 & 0.25 & 0.27 & 0.23 & 0.25 & 0.23 & 0.26 & 0.26 & 0.25 \\
\hline \multirow[t]{2}{*}{281.0} & 39.88 & 0 & -3.591 & -3.723 & 76.99 & 49 & 49.02 & 48.81 & 48.58 & 48.45 & 48.35 & 48.58 & 49.1 \\
\hline & 0.34 & 0 & 0.27 & 0.28 & 0.11 & 0.34 & 0.35 & 0.33 & 0.36 & 0.33 & 0.35 & 0.35 & 0.35 \\
\hline \multirow[t]{2}{*}{313.3} & 42.87 & 0 & 4.557 & 4.679 & 76.96 & 55 & 55.01 & 54.49 & 54.22 & 53.77 & 53.65 & 54.87 & 55.54 \\
\hline & 0.38 & 0 & 0.35 & 0.35 & 0.16 & 0.43 & 0.45 & 0.41 & 0.46 & 0.41 & 0.42 & 0.44 & 0.44 \\
\hline \multirow[t]{2}{*}{326.8} & 44 & 0 & -5.019 & -4.982 & 77.23 & 57.44 & 57.44 & 56.85 & 56.57 & 56.04 & 55.9 & 57.29 & 57.97 \\
\hline & 0.32 & 0 & 0.41 & 0.33 & 0.1 & 0.36 & 0.38 & 0.31 & 0.4 & 0.3 & 0.36 & 0.39 & 0.39 \\
\hline \multirow[t]{2}{*}{340.3} & 45.37 & 0 & .5 .455 & -5.42 & 77.15 & 60.23 & 60.24 & 59.49 & 59.19 & 58.51 & 58.36 & 60.22 & 60.99 \\
\hline & 0.37 & 0 & 0.4 & 0.38 & 0.21 & 0.4 & 0.43 & 0.38 & 0.45 & 0.37 & 0.41 & 0.4 & 0.43 \\
\hline \multirow[t]{2}{*}{344.1} & 45.99 & 0 & -5.556 & -5.635 & 76.9 & 61.23 & 6122 & 60.41 & 60.06 & 59.32 & 59.18 & 61.37 & 62.19 \\
\hline & 0.44 & 0 & 0.4 & 0.34 & 0.11 & 0.45 & 0.48 & 0.43 & 0.48 & 0.41 & 0.46 & 0.47 & 0.47 \\
\hline \multirow[t]{2}{*}{356.3} & 47.19 & 0 & -6.039 & -6.03 & 76.61 & 63.74 & 63.74 & 62.77 & 62.43 & 61.51 & 61.38 & 64.03 & 64.94 \\
\hline & 0.91 & 0 & 0.35 & 0.44 & 0.2 & 0.76 & 0.81 & $0 . \pi$ & 0.77 & 0.78 & 0.8 & 0.78 & 0.77 \\
\hline \multirow[t]{2}{*}{364.2} & 48.18 & 0 & -6.28 & -6.307 & 77.68 & 65.71 & 65.69 & 64.71 & 64.36 & 63.4 & 63.27 & 65.99 & 66.88 \\
\hline & 1.2 & 0 & 0.51 & 0.49 & 0.32 & 1.2 & 1.2 & 1.1 & 1.2 & 1.2 & 1.2 & 1.1 & 1.2 \\
\hline
\end{tabular}

\begin{tabular}{|c|c|c|c|c|c|c|c|c|c|c|c|c|c|}
\hline $\begin{array}{r}\text { IPVN20 } \\
\text { GPM }\end{array}$ & $\begin{array}{r}\text { (PSIA) } \\
\text { C2APCS }\end{array}$ & $\begin{array}{r}\text { (PSIA) } \\
\text { C2APIO }\end{array}$ & $\begin{array}{r}\text { (PSID) } \\
\text { CIDPOI }\end{array}$ & $\begin{array}{r}\text { (PSID) } \\
\text { C2DPO2 }\end{array}$ & $\begin{array}{r}\text { (PSID) } \\
\text { C2DPO3 }\end{array}$ & $\begin{array}{r}\text { (PSLA) } \\
\text { C3APY1 }\end{array}$ & $\begin{array}{r}\text { (PSIA) } \\
\text { C3API? }\end{array}$ & $\begin{array}{r}\text { (PSLA) } \\
\text { CXAPE3 }\end{array}$ & $\begin{array}{c}\text { (PSID) } \\
\text { CSDPO1 }\end{array}$ & $\begin{array}{r}\text { (PSID) } \\
\text { C3DPQ2 }\end{array}$ & $\begin{array}{r}\text { (PSTA) } \\
\text { CAAPOI }\end{array}$ & $\begin{array}{r}\text { (PSIA) } \\
\text { C4APO2 }\end{array}$ & $\begin{array}{r}\text { (PSIA) } \\
\text { CAAPO3 }\end{array}$ \\
\hline \multirow[t]{2}{*}{185.3} & 34.87 & 35.08 & 1.363 & 1.607 & 1.134 & 34.83 & 33.23 & 33.25 & -1.257 & 1.433 & 33.54 & $33.0 !$ & 33.34 \\
\hline & 0.14 & 0.15 & 0.14 & 0.013 & 0.21 & 0.14 & 0.14 & 0.14 & 0.17 & 0.012 & 0.13 & 0.16 & 0.13 \\
\hline \multirow[t]{2}{*}{220.4} & 37.95 & 38.13 & -0.2217 & 2.172 & -0.6284 & 39.8 & 35.64 & 35.69 & -3.793 & 2042 & 37.96 & 35.27 & 35.64 \\
\hline & 0.22 & 0.23 & 0.16 & 0.011 & 0.25 & 0.22 & 0.21 & 0.22 & 0.21 & 0.014 & 0.21 & 0.25 & 0.21 \\
\hline \multirow[t]{2}{*}{254.4} & 41.66 & 41.83 & -1.685 & 3.072 & -2.185 & 45.38 & 38.45 & 38.65 & -6.427 & 2.68 & 43 & 37.94 & 38.4 \\
\hline & 0.28 & 0.28 & 0.22 & 0.021 & 0.33 & 0.28 & 0.26 & 0.27 & 0.24 & 0.024 & 0.27 & 0.31 & 0.26 \\
\hline \multirow[t]{2}{*}{281.0} & 45 & 45.14 & -3.257 & 3.519 & -3.945 & 50.58 & 41.11 & 41.36 & -8.907 & 3.291 & 47.62 & 40.48 & 40.94 \\
\hline & 0.36 & 0.36 & 0.25 & 0.024 & 0.39 & 0.36 & 0.35 & 0.34 & 0.31 & 0.022 & 0.35 & 0.41 & 0.33 \\
\hline \multirow[t]{2}{*}{313.3} & 49.3 & 49.43 & -5.246 & 4.312 & -6.086 & 57.28 & 44.52 & 44.82 & .12 .16 & 4.112 & 53.6 & 43.64 & 44.17 \\
\hline & 0.46 & 0.44 & 0.26 & 0.036 & 0.45 & 0.45 & 0.39 & 0.43 & 0.33 & 0.032 & 0.44 & 0.49 & 0.39 \\
\hline \multirow[t]{2}{*}{326.8} & 51.09 & 51.24 & -5.872 & 4.885 & -6.674 & 59.85 & 45.69 & 46.18 & -13.38 & 4.41 & 55.9 & 44.83 & 45.4 \\
\hline & 0.34 & 0.35 & 0.32 & 0.11 & 0.46 & 0.42 & 0.31 & 0.32 & 0.38 & 0.034 & 0.37 & 0.4 & 0.3 \\
\hline \multirow[t]{2}{*}{340.3} & 53.08 & 53.19 & -6.821 & 5.15 & -7.763 & 62.95 & 47.25 & 47.77 & -14.91 & 4.789 & 58.66 & 46.33 & 46.89 \\
\hline & 0.41 & 0.38 & 0.31 & 0.035 & 0.46 & 0.44 & 0.37 & 0.39 & 0.39 & 0.031 & 0.4 & 0.42 & 0.35 \\
\hline \multirow[t]{2}{*}{344.1} & 53.79 & 53.89 & $\cdot 7.239$ & 5.153 & -8.326 & 64.16 & 48.01 & 48.43 & -15.44 & 4.943 & 59.75 & 46.93 & 47.52 \\
\hline & 0.48 & 0.46 & 0.33 & 0.047 & 0.48 & 0.49 & 0.43 & 0.47 & 0.4 & 0.04 & 0.47 & 0.53 & 0.42 \\
\hline \multirow[t]{2}{*}{356.3} & 55.58 & 55.67 & -8.123 & 5.654 & -9.263 & 66.99 & 49.39 & 49.82 & -16.89 & 5.307 & 62.24 & 48.2 & 48.78 \\
\hline & 0.85 & 0.84 & 0.42 & 0.061 & 0.56 & 0.79 & 0.88 & 0.88 & 0.56 & 0.054 & 0.79 & 0.92 & 0.89 \\
\hline \multirow[t]{2}{*}{364.2} & 56.93 & 57.17 & -8.636 & 6.182 & -9.748 & 69.03 & 50.52 & 50.8 & -17.85 & 5.48 & 64.08 & 49.34 & 49.97 \\
\hline & 1.2 & 1.2 & 0.45 & 0.12 & 0.58 & 1.1 & 1.2 & 1.3 & 0.58 & 0.075 & 1.2 & 1.3 & 1.2 \\
\hline
\end{tabular}

\begin{tabular}{|c|c|c|c|c|c|c|c|c|c|c|c|c|}
\hline $\begin{array}{r}\text { IPVN20 } \\
\text { GPM } \\
\end{array}$ & $\begin{array}{r}\text { (PSID) } \\
\text { CADPD1 } \\
\end{array}$ & $\begin{array}{r}\text { (PSID) } \\
\text { CADPQ? } \\
\end{array}$ & $\begin{array}{r}\text { (PSUA) } \\
\text { IPAPOI } \\
\end{array}$ & $\begin{array}{r}\text { (F) } \\
\text { IPRTI } \\
\end{array}$ & $\begin{array}{r}\text { (PPH) } \\
\text { mVN20 } \\
\end{array}$ & $\begin{array}{r}\text { (F) } \\
\text { MSTCe? } \\
\end{array}$ & $\begin{array}{r}\text { (PSLA) } \\
\text { RTAPQ1 } \\
\end{array}$ & $\begin{array}{r}\text { (PSLA) } \\
\text { TFAPO1 } \\
\end{array}$ & $\begin{array}{r}\text { (PSID) } \\
\text { TPDP1 } \\
\end{array}$ & $\begin{array}{r}\text { (PSID) } \\
\text { TPPPO2 } \\
\end{array}$ & $\begin{array}{r}\text { (GPM) } \\
\text { WSTMO1 } \\
\end{array}$ & $\begin{array}{r}\text { (PPH) } \\
\text { WSTM2 } \\
\end{array}$ \\
\hline \multirow[t]{2}{*}{185.3} & -0.5227 & 2.559 & 35.71 & 76.16 & 92540 & 64.42 & 28.3 & 34.78 & -0.8985 & -2.825 & 185.3 & 92520 \\
\hline & 0.17 & 0.021 & 0.14 & 0.22 & 660 & 0.15 & 0.12 & 0.13 & 0.04 & 0.2 & 4.7 & 2300 \\
\hline \multirow[t]{2}{*}{220.4} & -2.706 & 3.654 & 42.06 & 76.71 & 110100 & 64.98 & 282 & 39.97 & -2.035 & -7.098 & 213.4 & 106600 \\
\hline & 0.24 & 0.025 & 0.22 & 0.13 & 680 & 0.23 & 0.16 & 0.21 & 0.051 & 0.26 & 5.6 & 2800 \\
\hline \multirow[t]{2}{*}{254.4} & -5.041 & 4.837 & 49.13 & 76.82 & 127000 & 64.47 & 28.28 & 45.75 & -3.323 & -11.58 & 258.2 & 128900 \\
\hline & 0.26 & 0.039 & 0.27 & 0.14 & 750 & 0.15 & 0.21 & 0.26 & 0.068 & 0.36 & 7.4 & 3700 \\
\hline \multirow[t]{2}{*}{281.0} & -7.158 & 5.936 & 55.72 & 76.83 & 140300 & 64.86 & 28.4 & 51.18 & -4.474 & -15.79 & 287.8 & 143700 \\
\hline & 0.29 & 0.032 & 0.37 & 0.1 & 790 & 0.19 & 0.28 & 0.35 & 0.094 & 0.33 & 11 & 5400 \\
\hline \multirow[t]{2}{*}{313.3} & -9.961 & 7.389 & 64.2 & 76.73 & 156500 & 65.04 & 28.36 & 58.15 & -5.996 & -21.29 & 319.7 & 159700 \\
\hline & 0.43 & 0.054 & 0.47 & 0.15 & 1100 & 0.17 & 0.33 & 0.43 & 0.11 & 0.46 & 11 & 5500 \\
\hline \multirow[t]{2}{*}{326.8} & -11.06 & 7.985 & 67.58 & 77.01 & 163200 & 64.65 & 28.27 & 60.82 & -6.707 & -23.51 & 334.6 & 167100 \\
\hline & 0.42 & 0.046 & 0.44 & 0.21 & 1100 & 0.17 & 0.17 & 0.39 & 0.11 & 0.54 & 9.2 & 4600 \\
\hline \multirow[t]{2}{*}{340.3} & -12.31 & 8.661 & 71.52 & 76.91 & 170000 & 64.72 & 28.21 & 64.06 & .7 .413 & -26.1 & 347.9 & 173800 \\
\hline & 0.42 & 0.056 & 0.51 & 0.17 & 1000 & 0.15 & 0.2 & 0.44 & 0.14 & 0.55 & 9.6 & 4800 \\
\hline \multirow[t]{2}{*}{344.1} & -12.32 & 8.912 & 72.98 & 76.64 & 171800 & 65.1 & 28.31 & 65.33 & -7.61 & -26.94 & 356.7 & 178100 \\
\hline & -0.47 & 0.071 & 0.52 & 0.11 & 960 & 0.14 & 0.36 & 0.47 & 0.14 & 0.56 & 4.7 & 2300 \\
\hline \multirow[t]{2}{*}{356.3} & .14 .03 & 9.582 & 76.64 & 76.37 & 178000 & 65.24 & 28.14 & 68.28 & -8.311 & -29.4 & 366.7 & 183200 \\
\hline & 0.53 & 0.12 & 0.75 & 0.19 & 1600 & 0.16 & 1 & 0.77 & 0.18 & 0.72 & 8.3 & 4100 \\
\hline \multirow[t]{2}{*}{364.2} & .14 .73 & 9.96 & 79.14 & 77.45 & 181800 & 67.72 & 28.34 & 70.32 & -8.756 & -30.94 & 376.6 & 188000 \\
\hline & 0.67 & C.14 & 1.1 & 0.29 & 1700 & 0.16 & 1.4 & 1.1 & $\therefore 0.21$ & 0.83 & 6 & 3000 \\
\hline
\end{tabular}


Table 4. Frictional Pressure Drop Data Across Fuel Assembly Test 827_32,33

\begin{tabular}{|c|c|c|c|c|c|c|c|}
\hline IPVN20 & (PSID) & (PSID) & (PSID) & (PSID) & (PSID) & (PSID) & $\begin{array}{r}\text { (PSID) } \\
\text { TFDP02* }\end{array}$ \\
\hline GPM & C2DP01* & C2DPO3* & C3DP01* & C4DP01* & TFDPO1* & TFDP02 & -TFDP01 \\
\hline 185.3 & -3.97 & -4.20 & -6.59 & -5.86 & -2.74 & -10.80 & -8.05 \\
\hline 220.4 & -5.56 & -5.96 & -9.13 & -8.04 & -3.88 & -15.07 & -11.19 \\
\hline 254.4 & -7.02 & -7.52 & -11.76 & -10.38 & -5.17 & -19.55 & -14.38 \\
\hline 281.0 & -8.59 & -9.28 & -14.24 & -12.49 & -6.32 & -23.76 & -17.44 \\
\hline 313.3 & -10.58 & -11.42 & -17.50 & -15.30 & -7.84 & -29.26 & -21.42 \\
\hline 326.8 & -11.21 & -12.01 & -18.72 & -16.40 & -8.55 & -31.48 & -22.93 \\
\hline 340.3 & -12.16 & -13.10 & -20.25 & -17.65 & -9.26 & -34.07 & -24.81 \\
\hline 344.1 & -12.58 & -13.66 & -20.78 & -18.16 & -9.46 & -34.91 & -25.4 \\
\hline 356.3 & -13.46 & -14.60 & -22.23 & -19.37 & -10.16 & -37.37 & -27 \\
\hline 364.2 & -13.97 & -15.08 & -23.19 & -20.07 & -10.60 & -38.91 & -28. \\
\hline $\begin{array}{l}\text { DPfric } \\
\text { where }\end{array}$ & $\begin{array}{l}\mathrm{n}[\mathrm{psid}]=\mathrm{DP} \\
\text { Density }=62 \\
\mathrm{H}(\mathrm{C} 2 \mathrm{DPO1}) \\
\mathrm{H}(\mathrm{C} 2 \mathrm{DPO3}) \\
\mathrm{H}(\mathrm{C} \text { (CPO1) } \\
\mathrm{H}(\mathrm{C} 4 \mathrm{DPO1}) \\
\mathrm{H}(\mathrm{TFDP01}) \\
\mathrm{H}(\mathrm{TFDPO2})\end{array}$ & $\begin{array}{l}\text { measured[p } \\
22 \mathrm{lbm} / \mathrm{ft} 3 \\
=12.35 \mathrm{ft} \\
=12.35 \mathrm{ft} \\
=12.35 \mathrm{ft} \\
=12.35 \mathrm{ft} \\
=4.271 \mathrm{ft} \\
=18.448 \mathrm{ft}\end{array}$ & (De & $n / f(3$ & to-ta & & \\
\hline
\end{tabular}




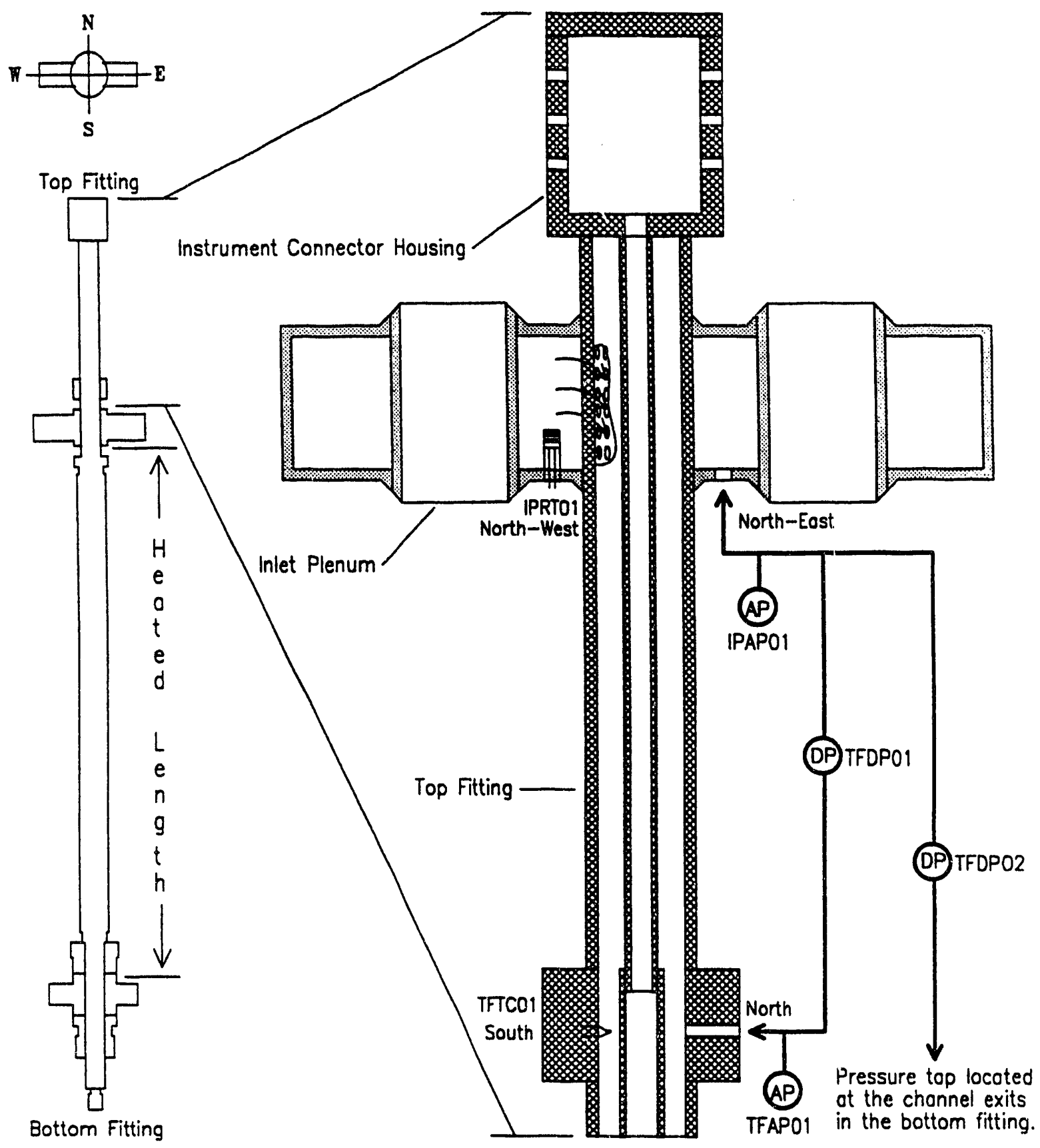

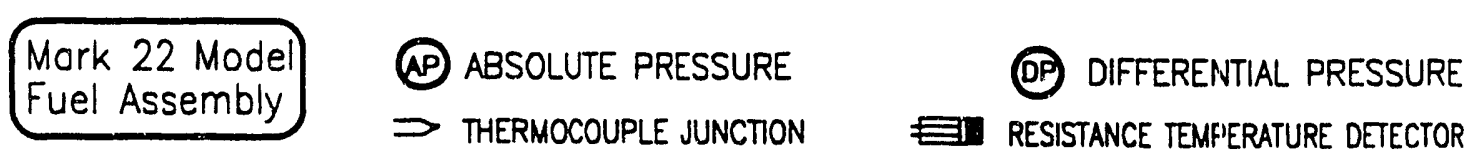

Figure 1. Fuel Assembly Top Fitting Instruments 


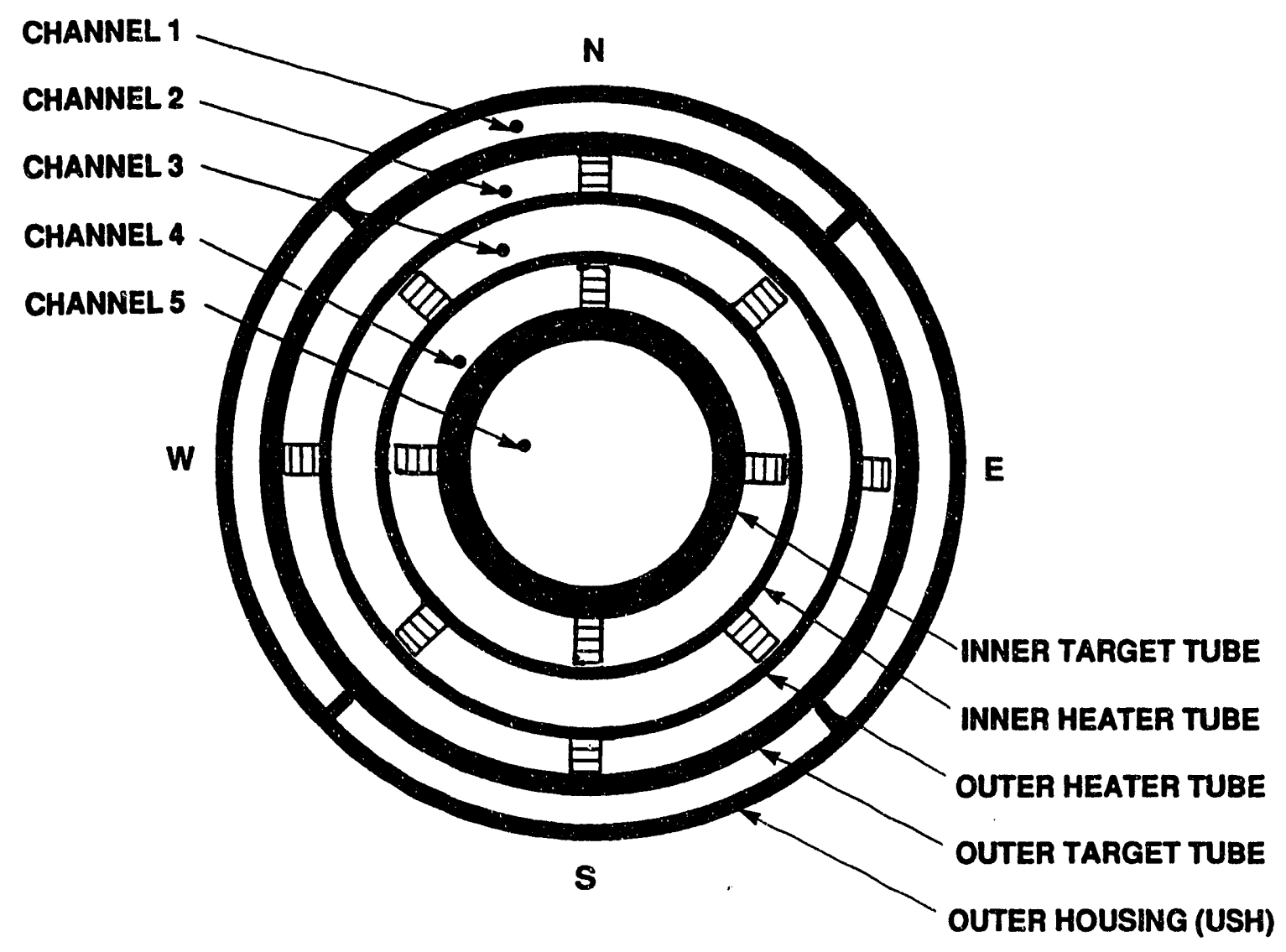

Figure 2. Cross-Section View of Model Fuel Assembly 


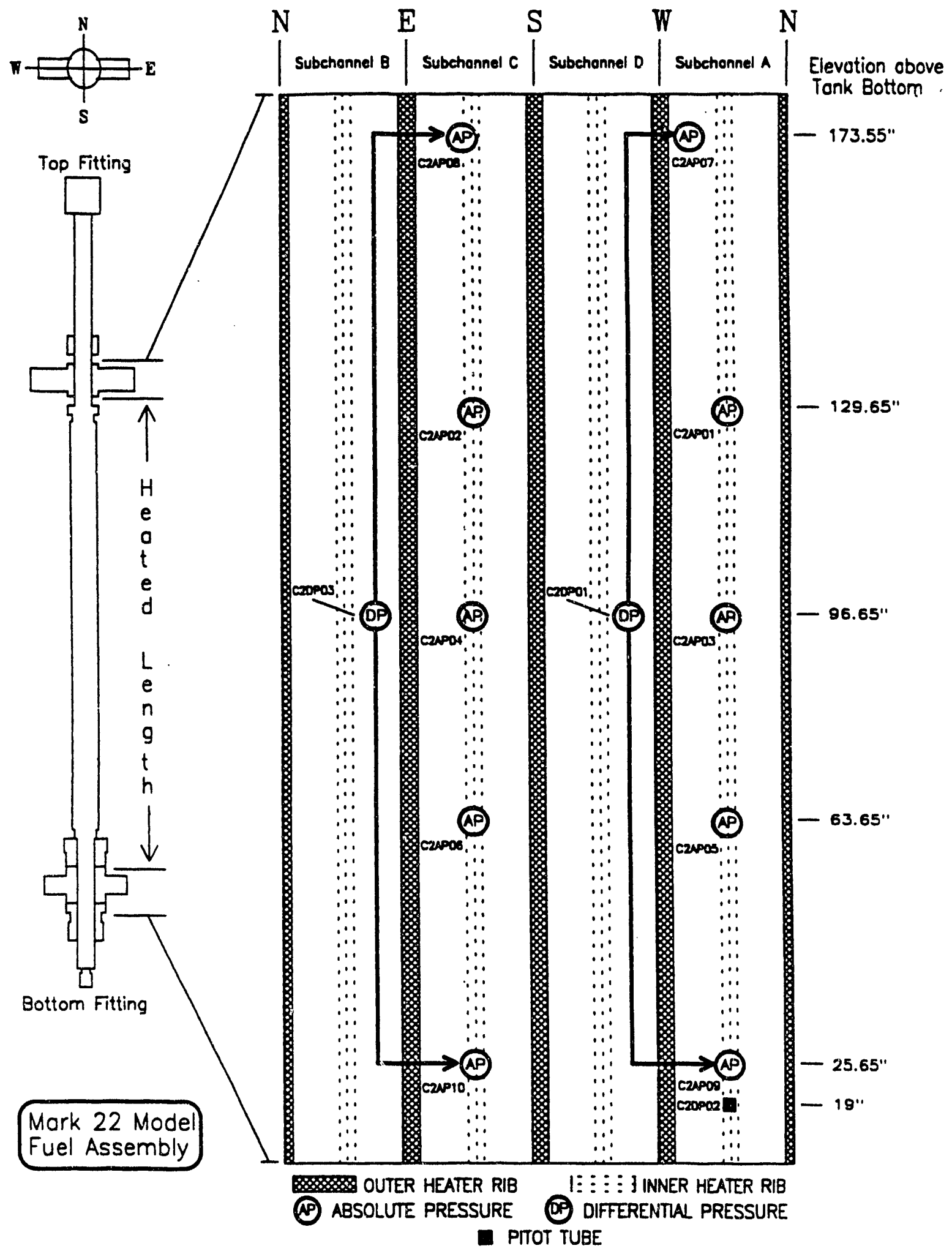

Figure 3. Fuel Assembly Channel 2 Pressure and Differentlal Pressure Taps 


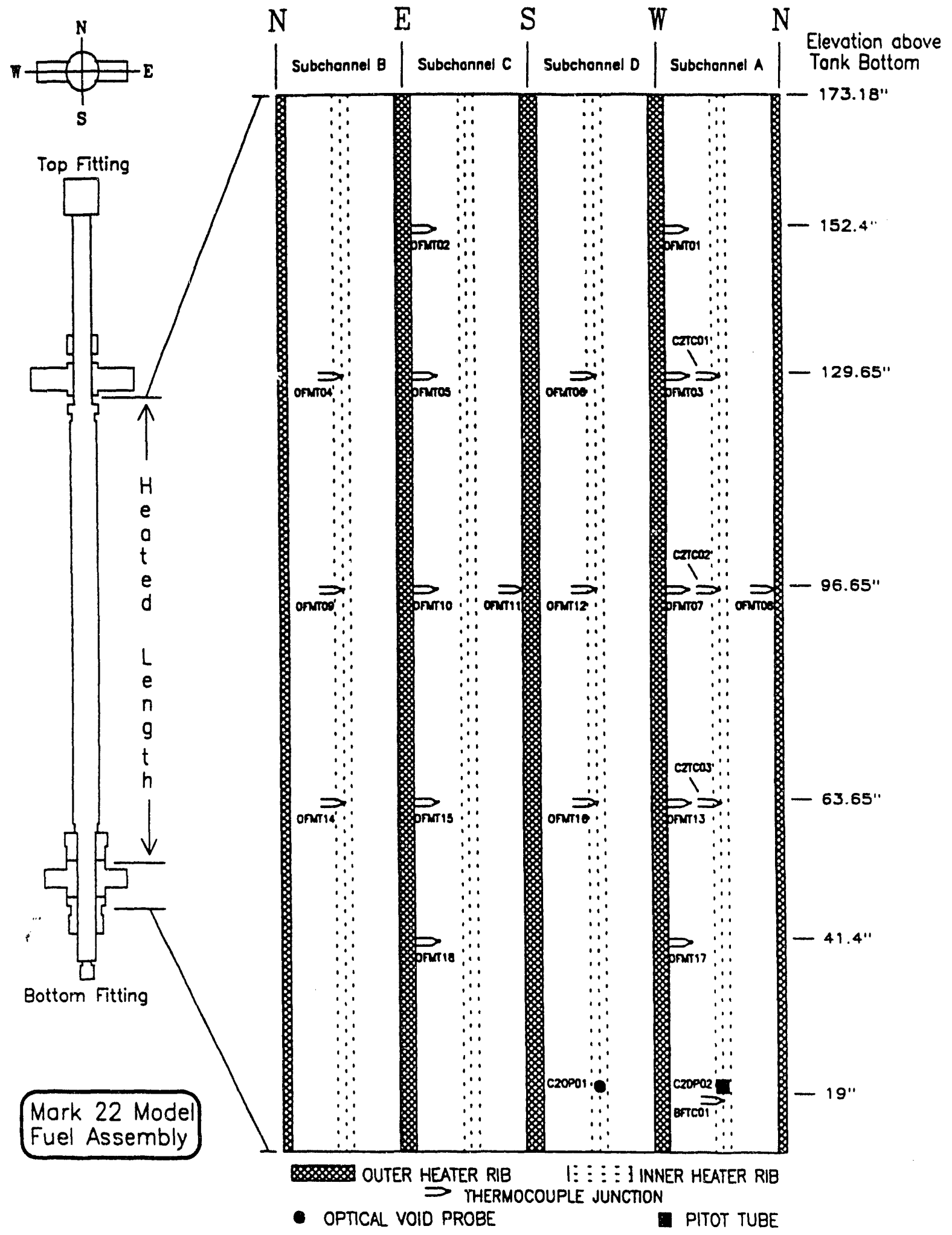

Figure 4. Fuel Assembly Channel 2 and Outer Heater Tube Instruments 


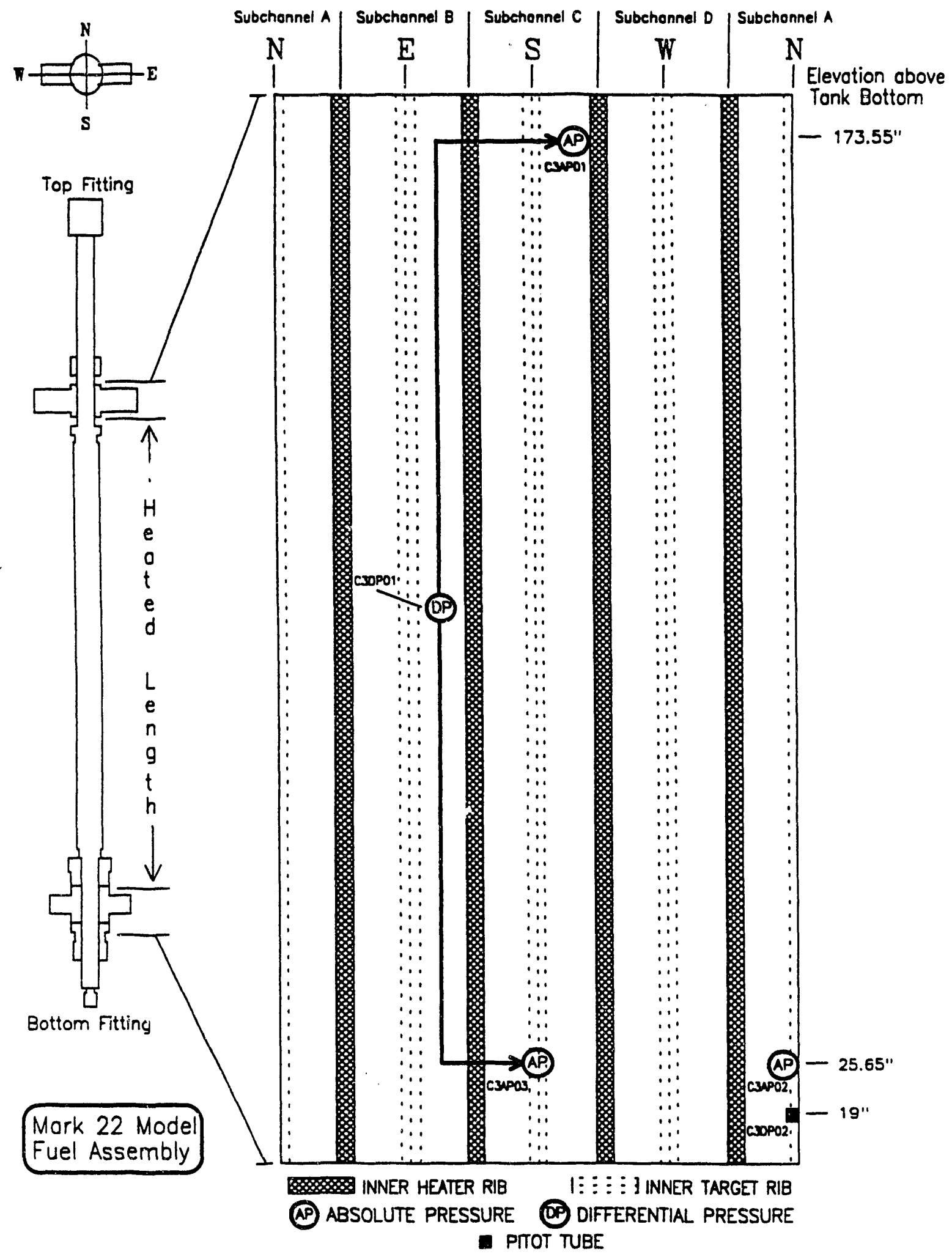

Figure 5. Fuel Assembly Channel 3 Pressure and Differential Pressure Taps 


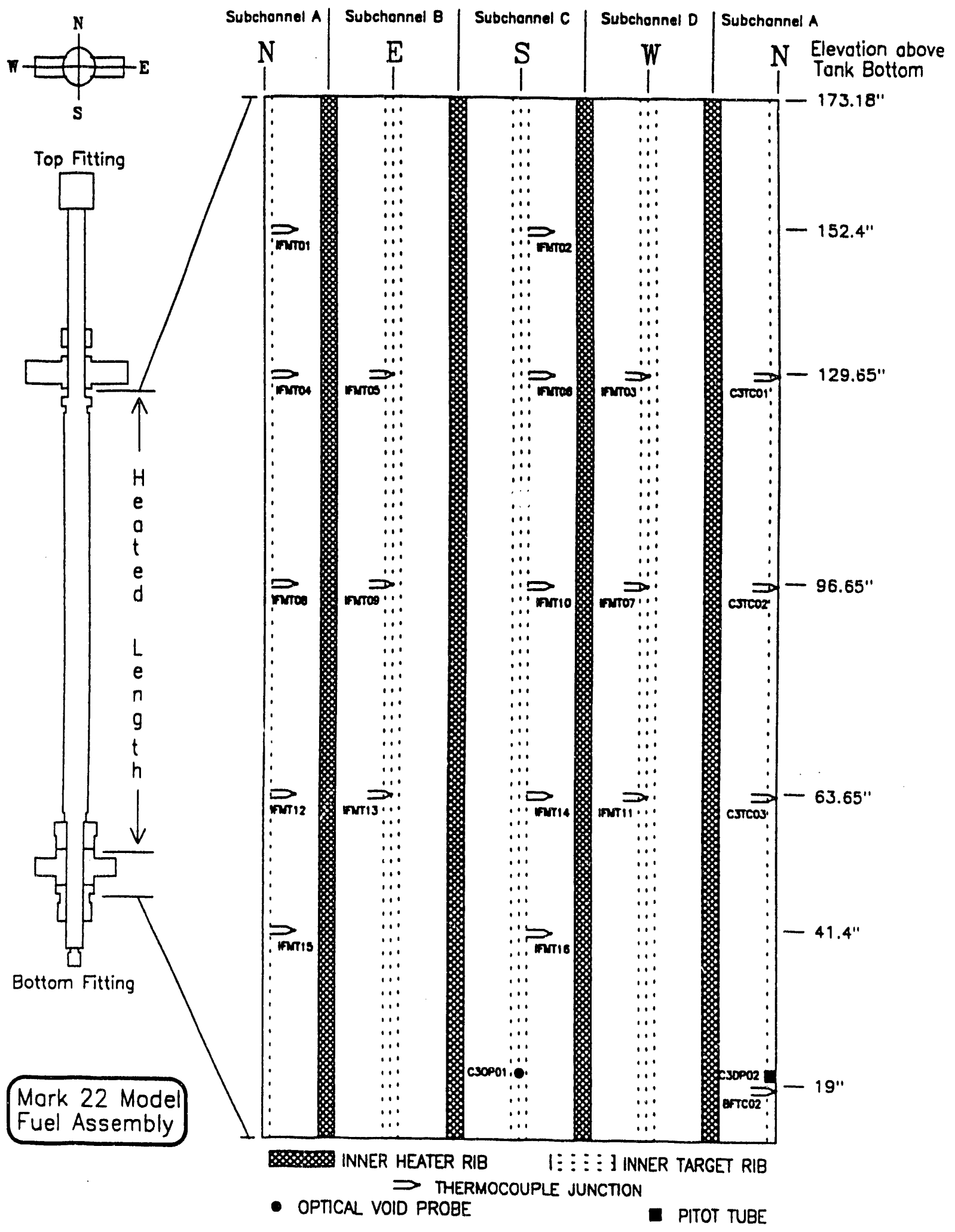

Figure 6. Fuel Assembly Channel 3 and Inner Heater Tube Instruments 


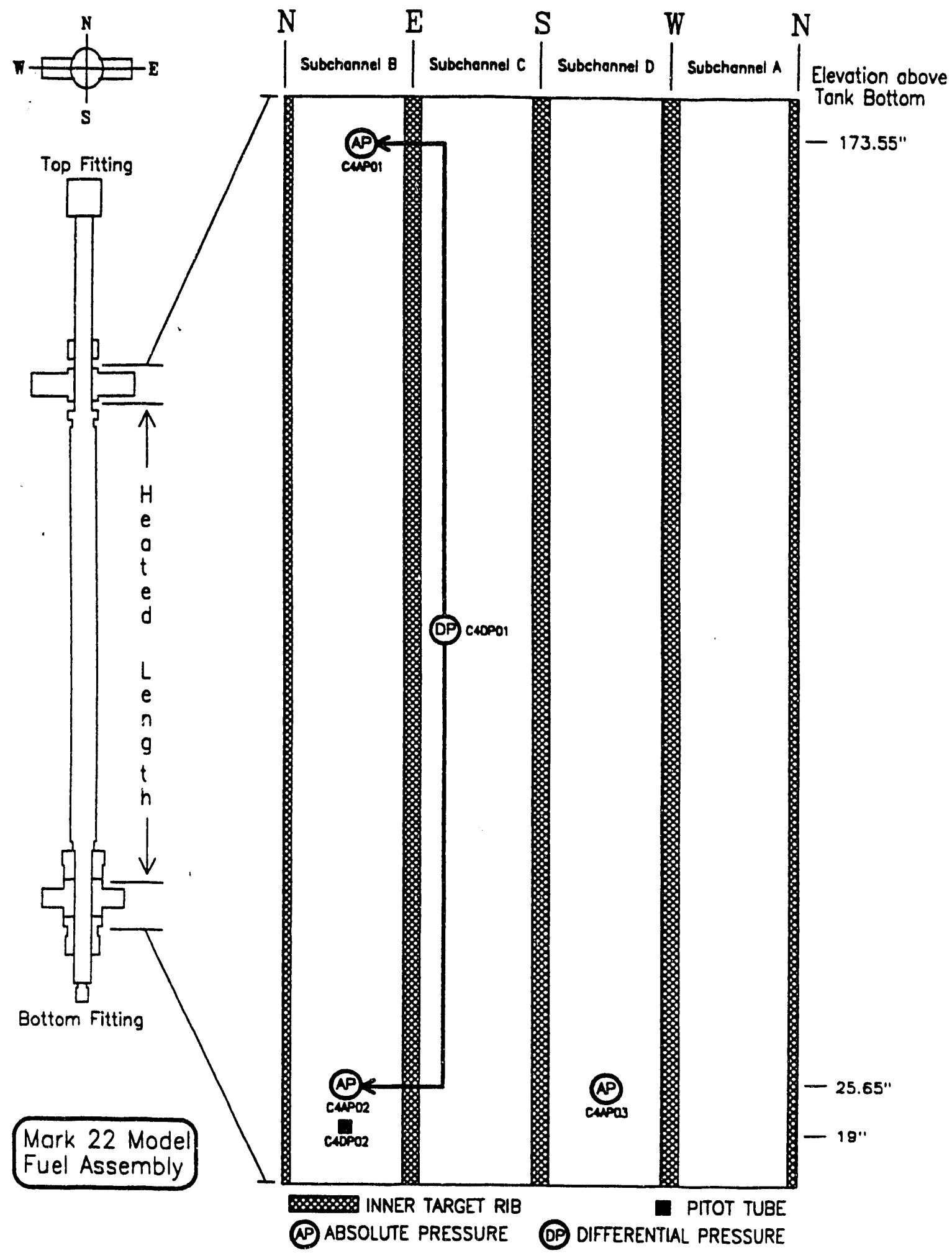

Figure 7. Fuel Assembly Channel 4 Pressure and Differentlal Pressure Taps 


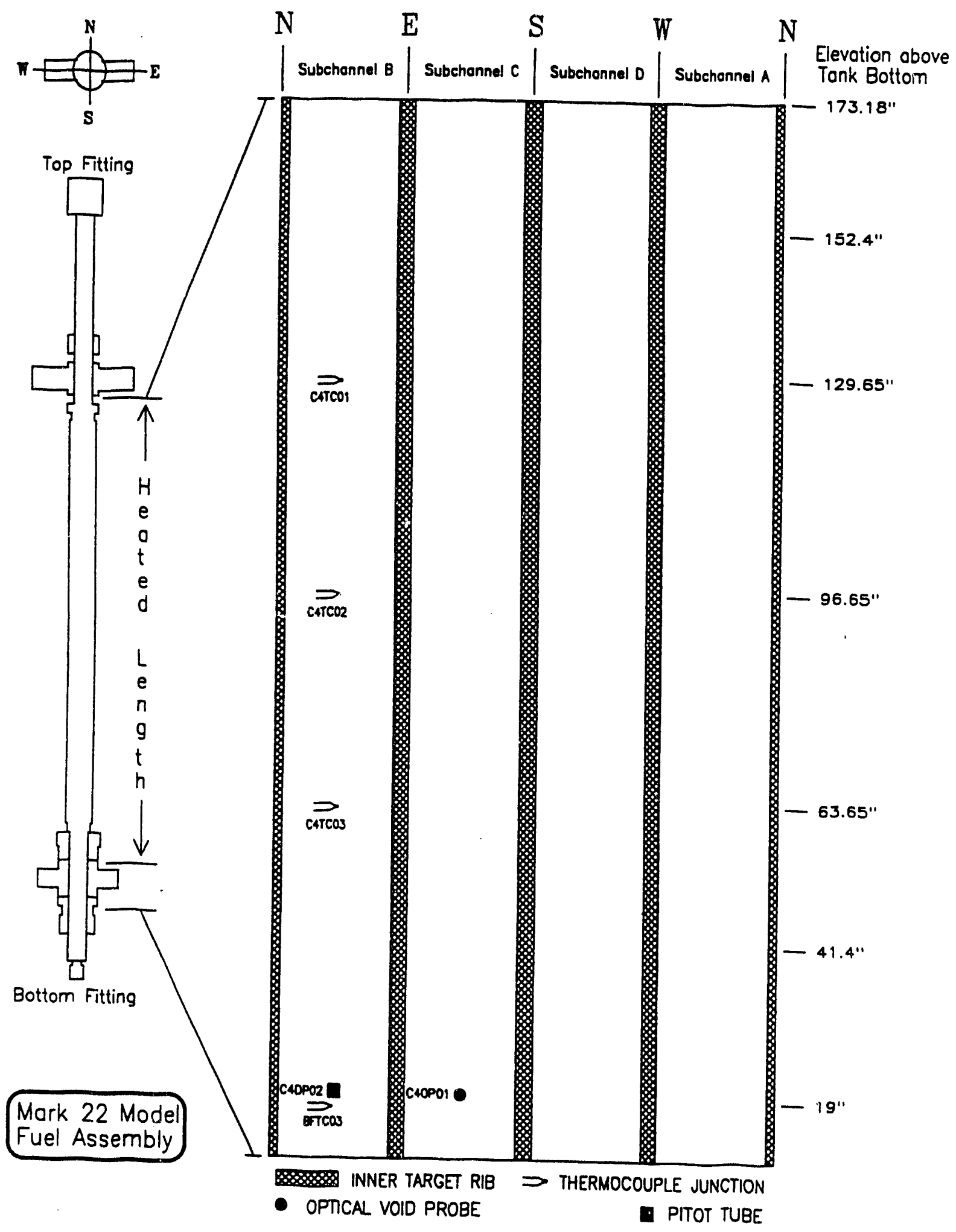

Figure 8. Fuel Assembly Channel 4 Instruments 


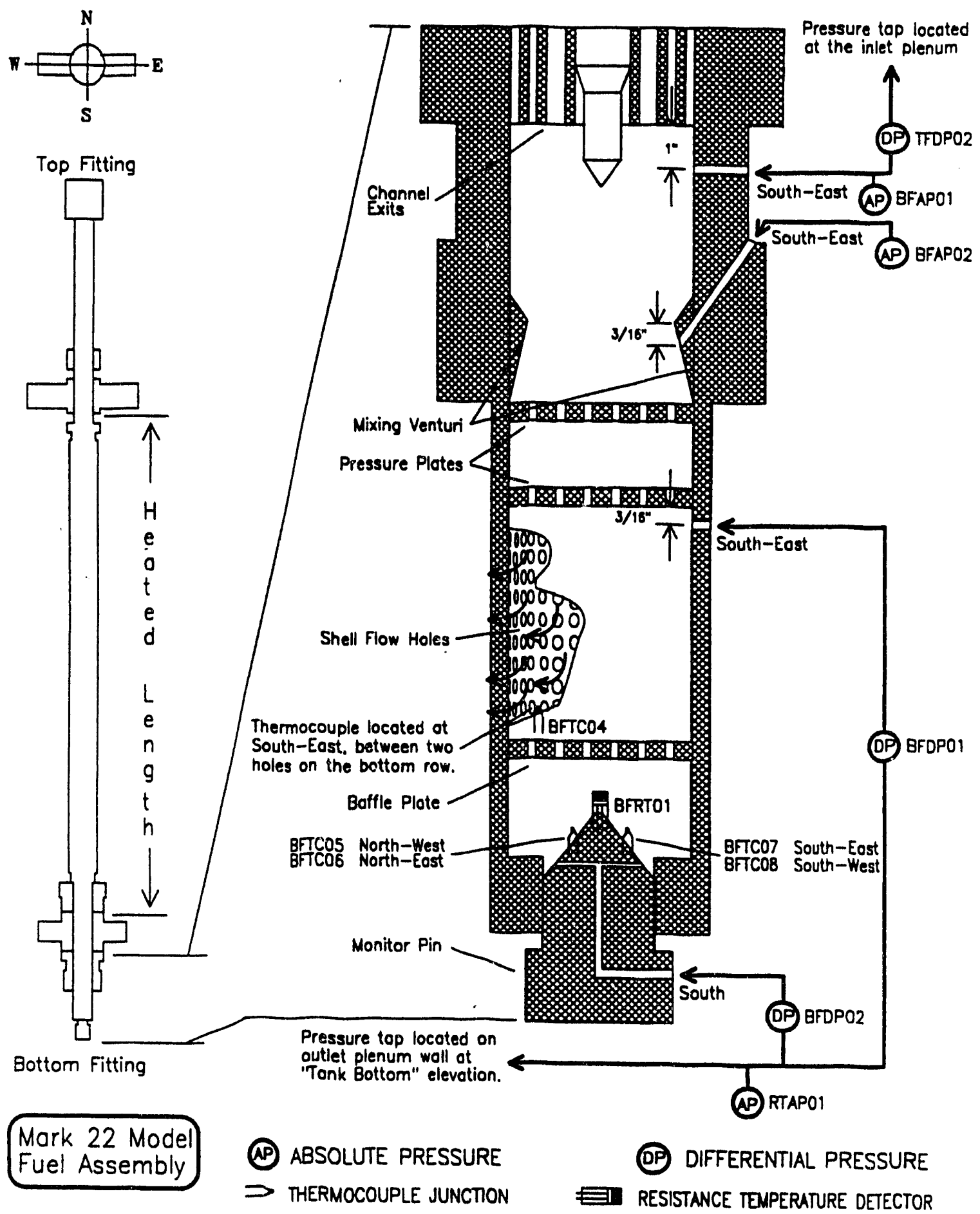

Figure 9. Fuel Assembly Bottom Fitting Instruments 


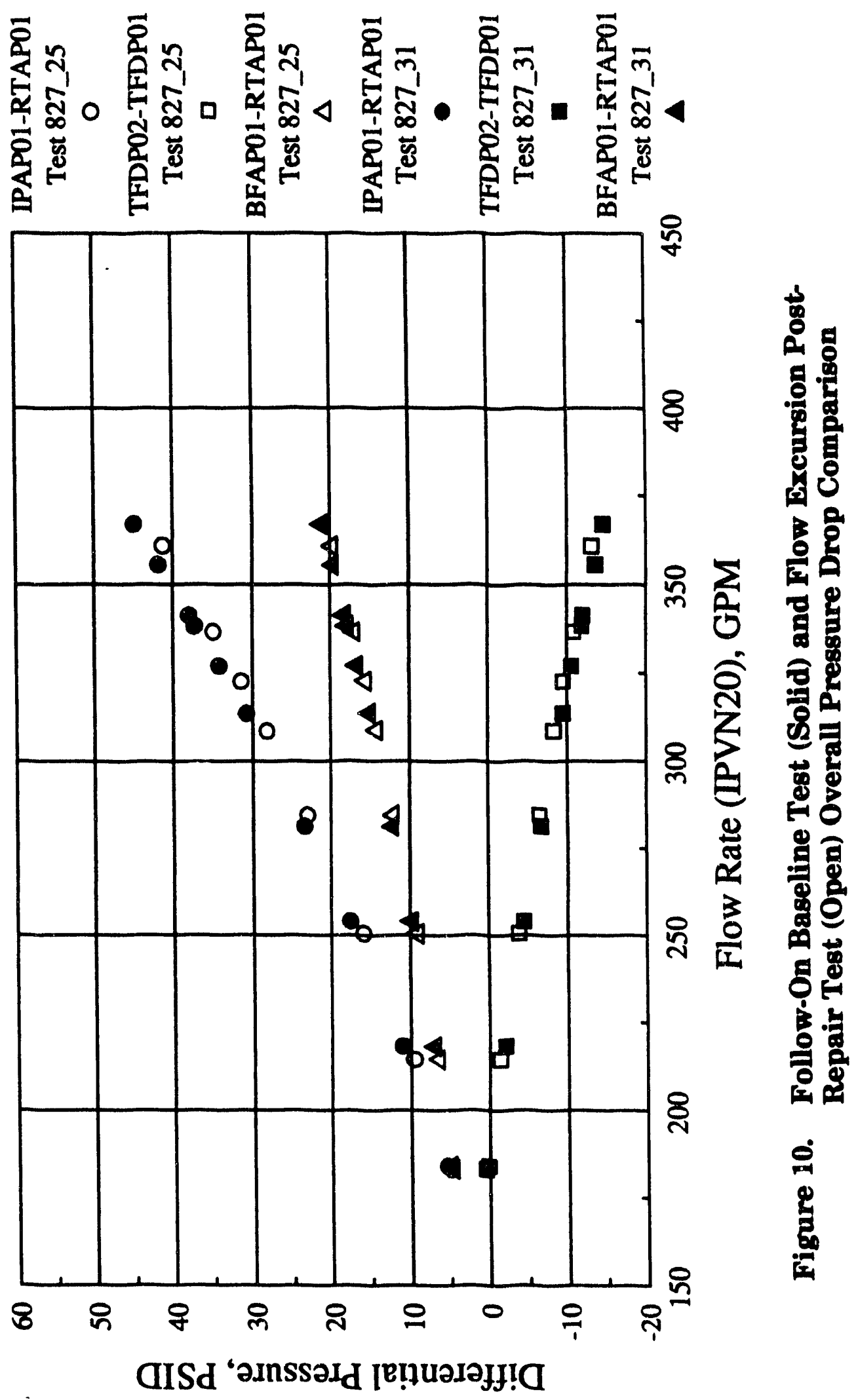




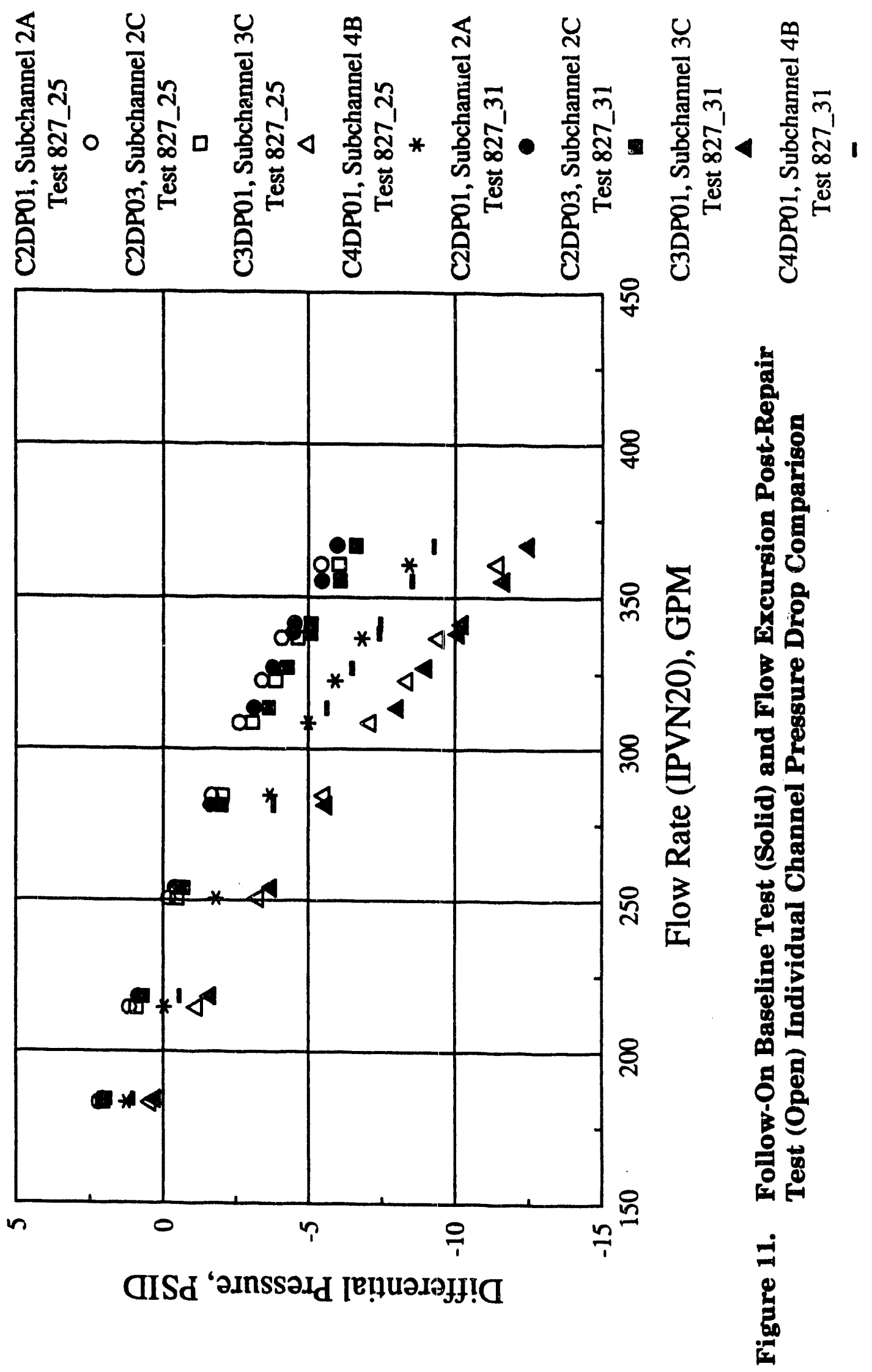




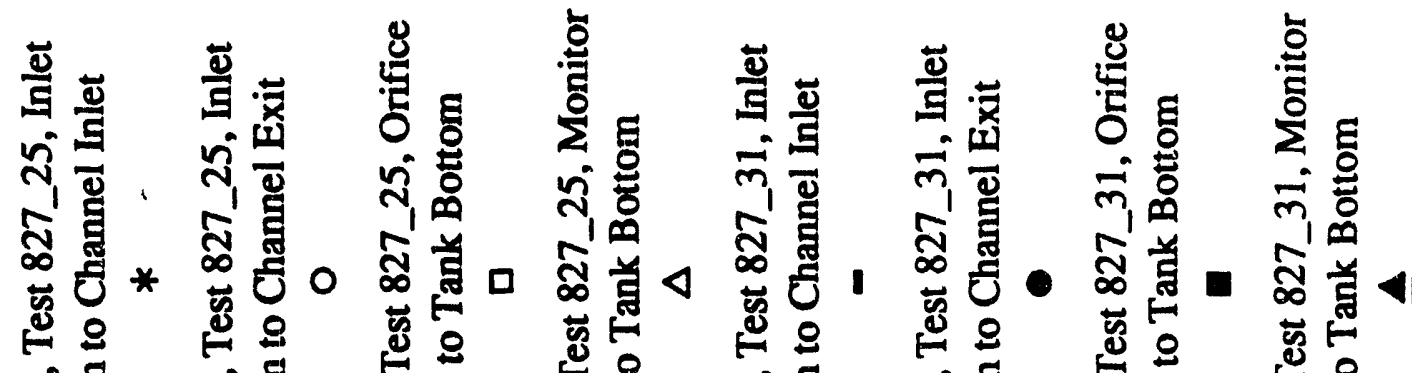

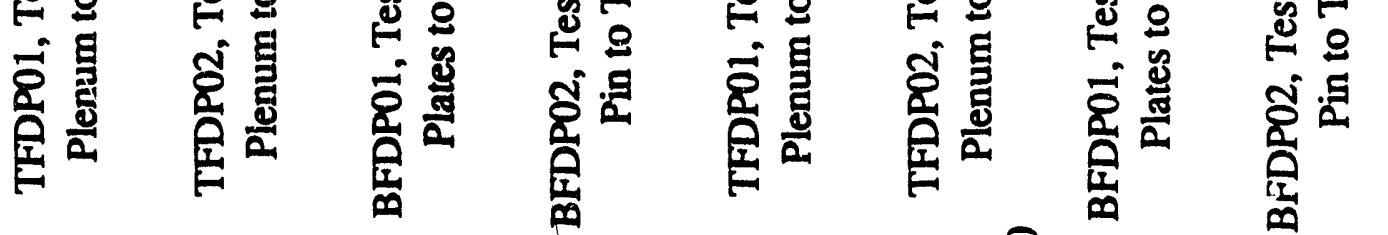

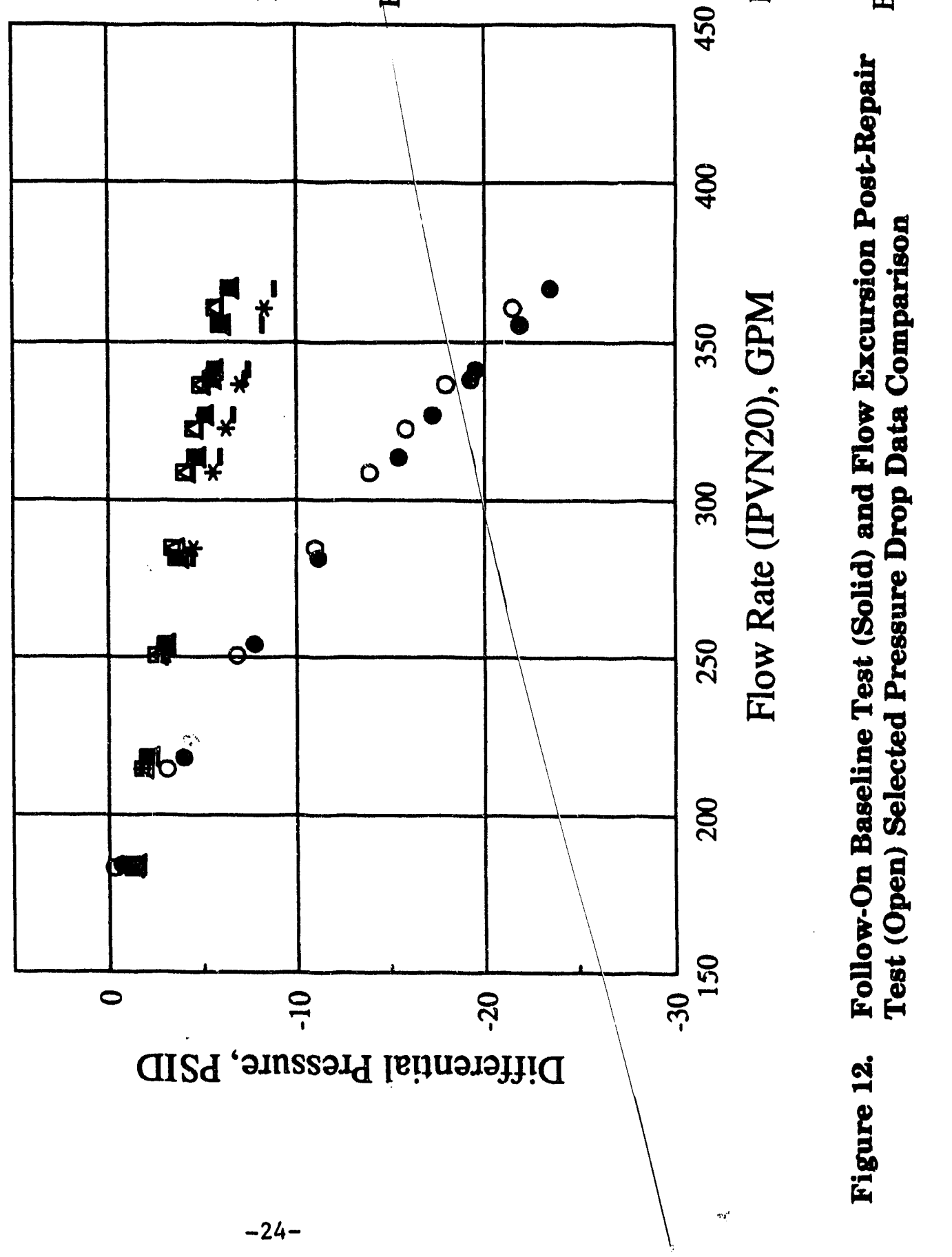




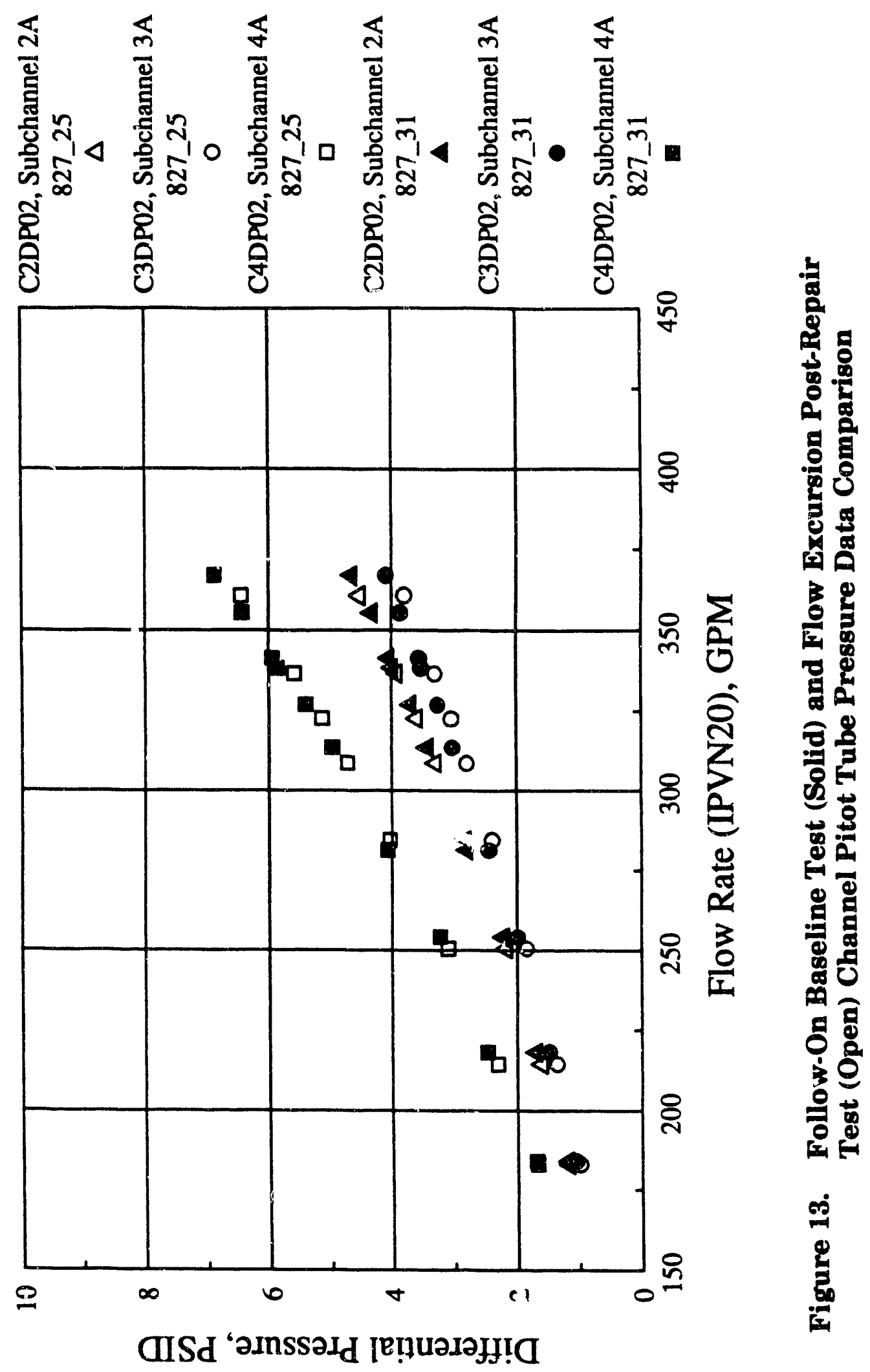




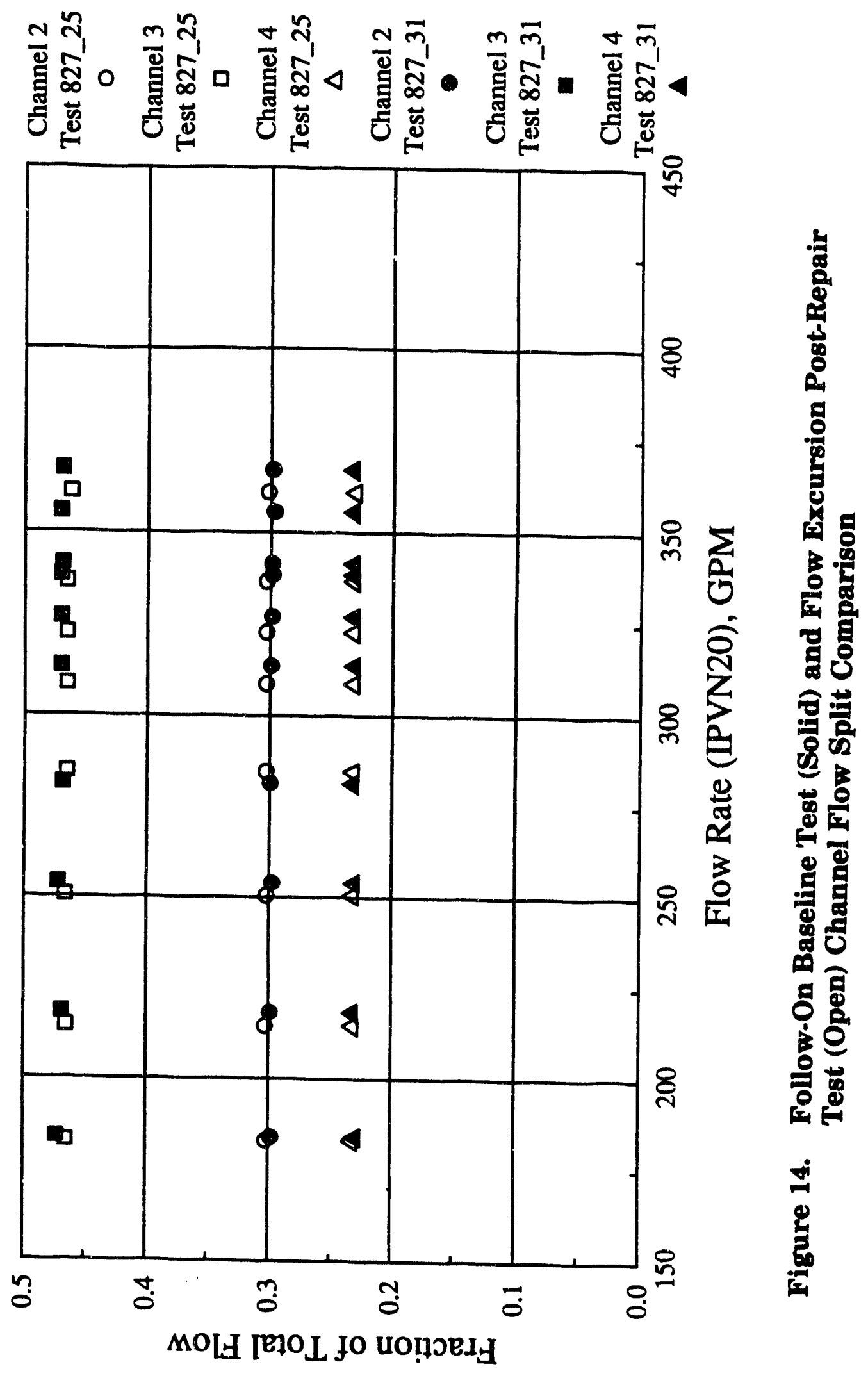




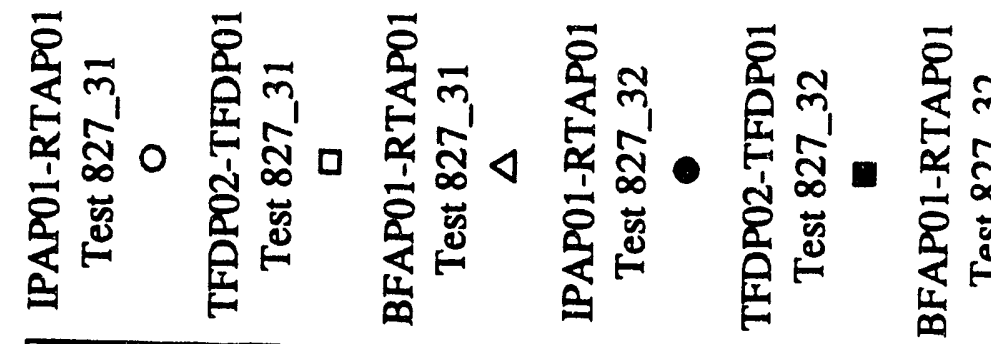

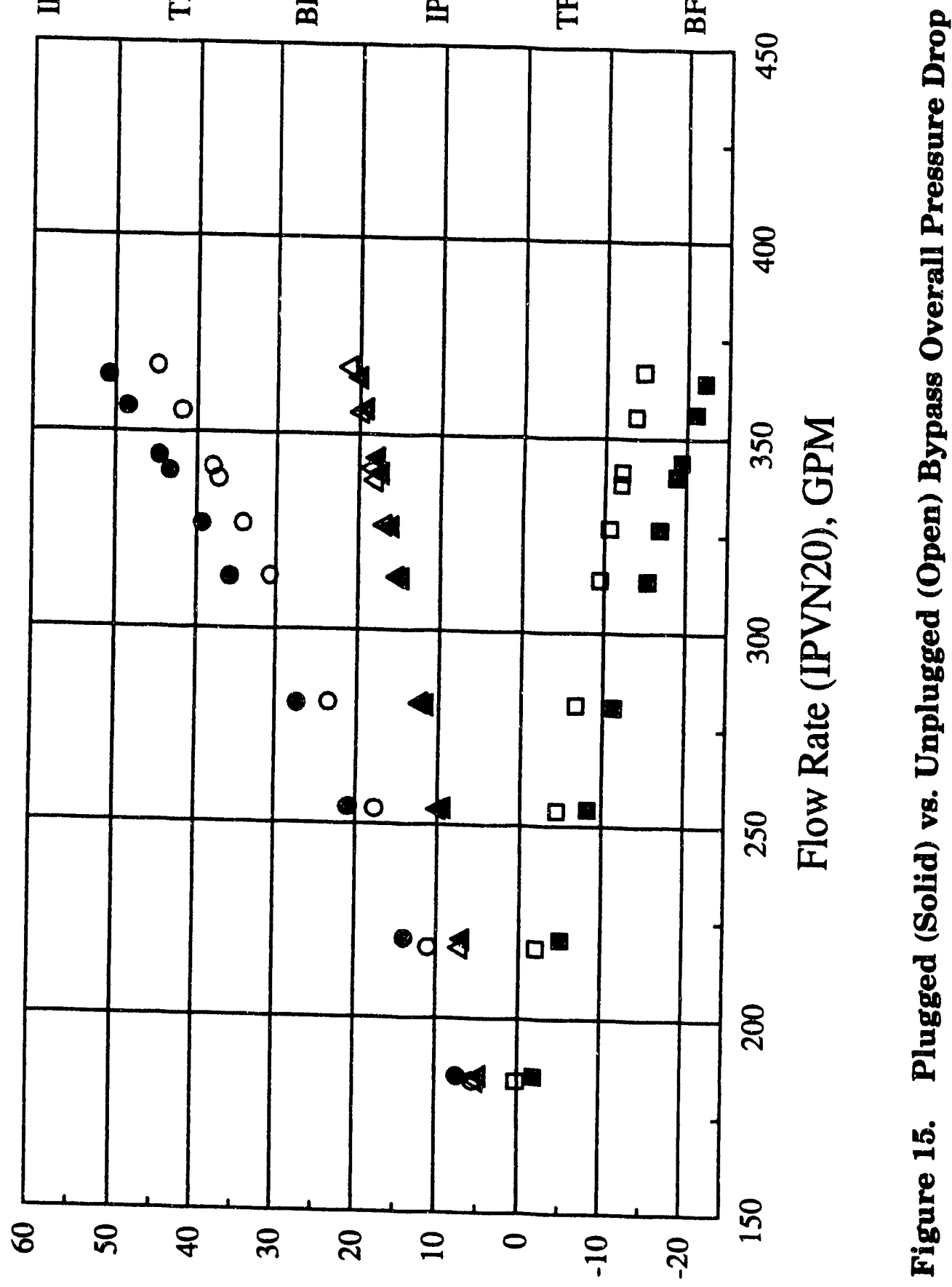

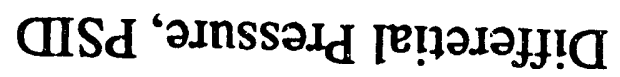




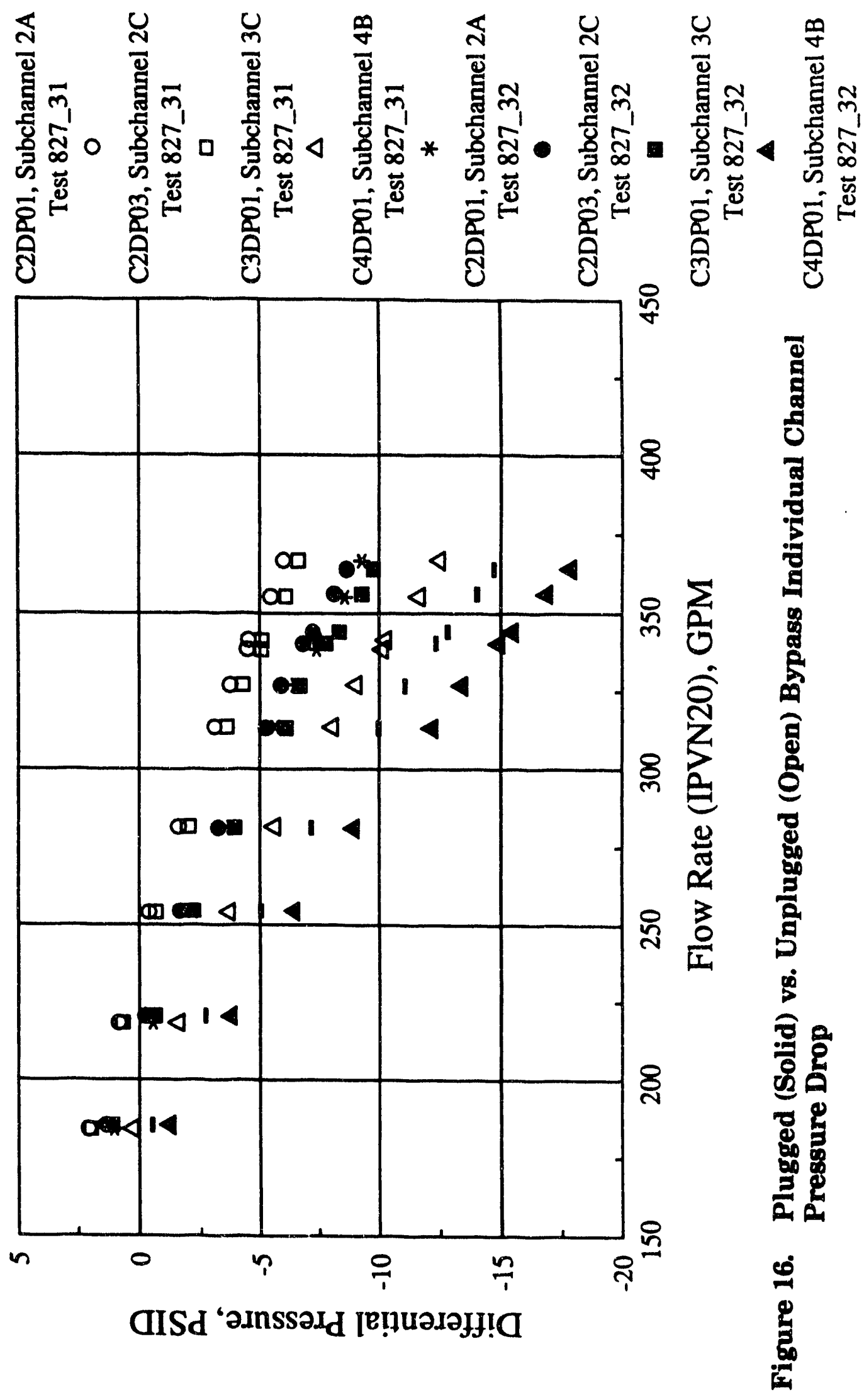



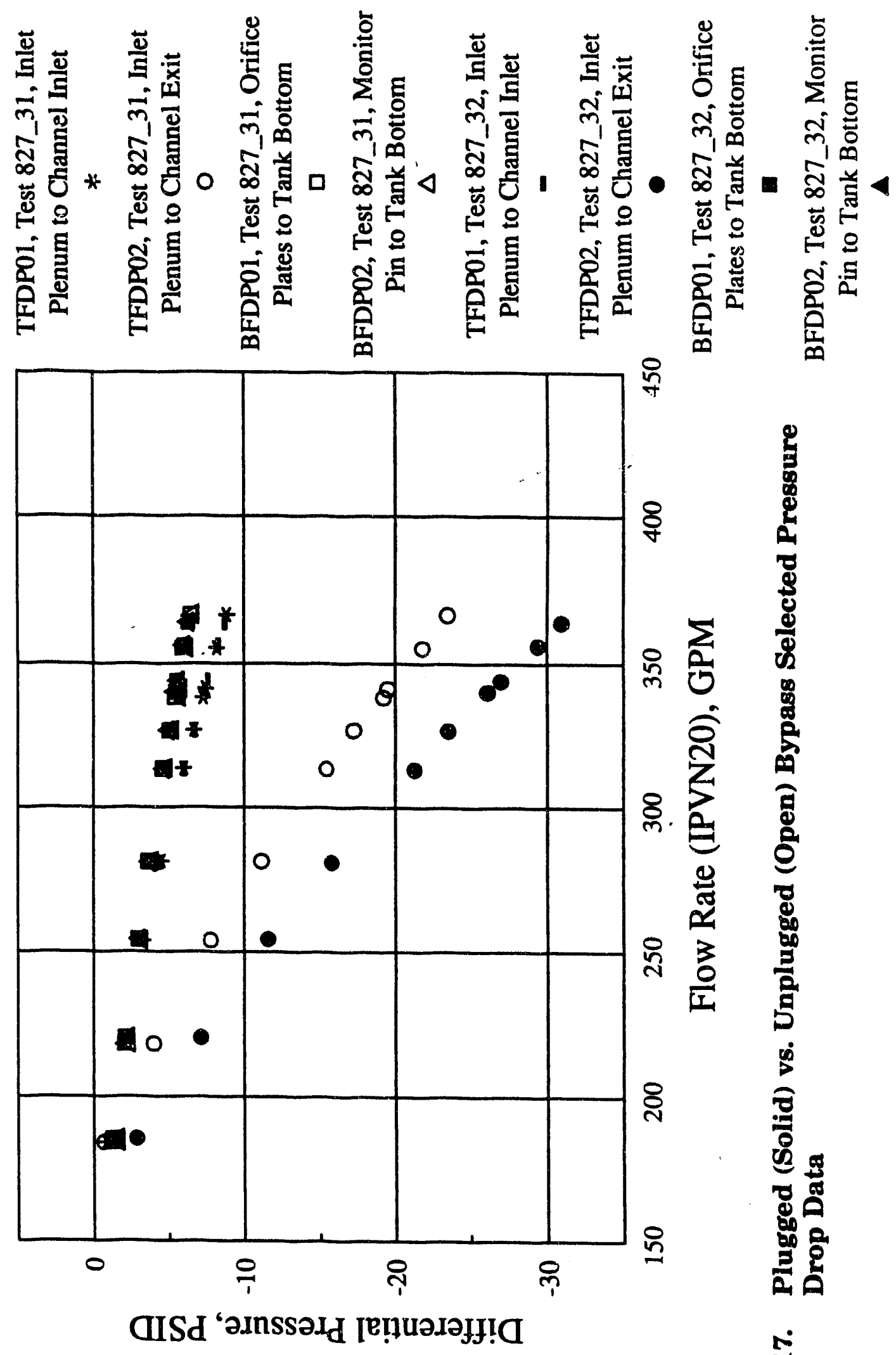


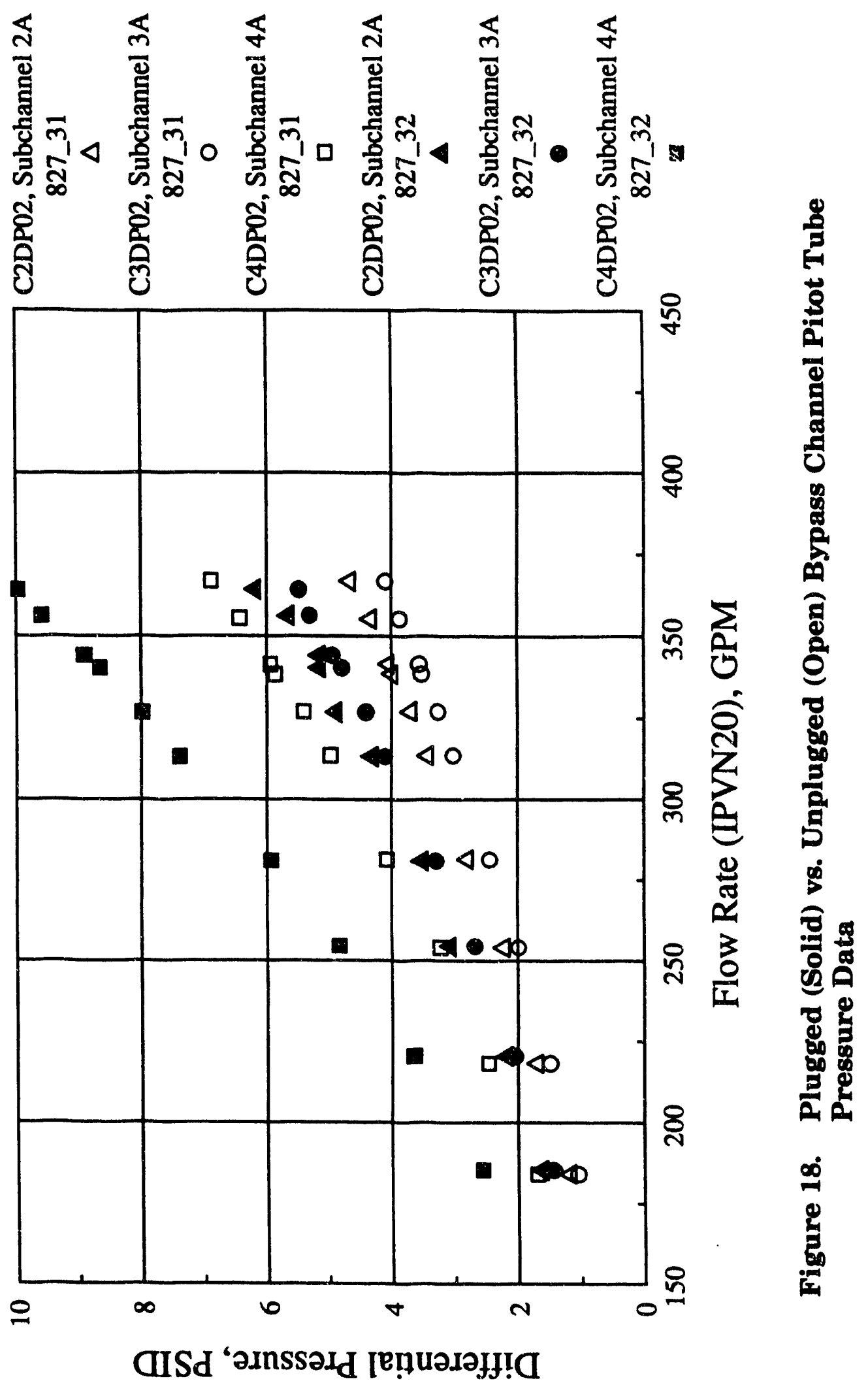




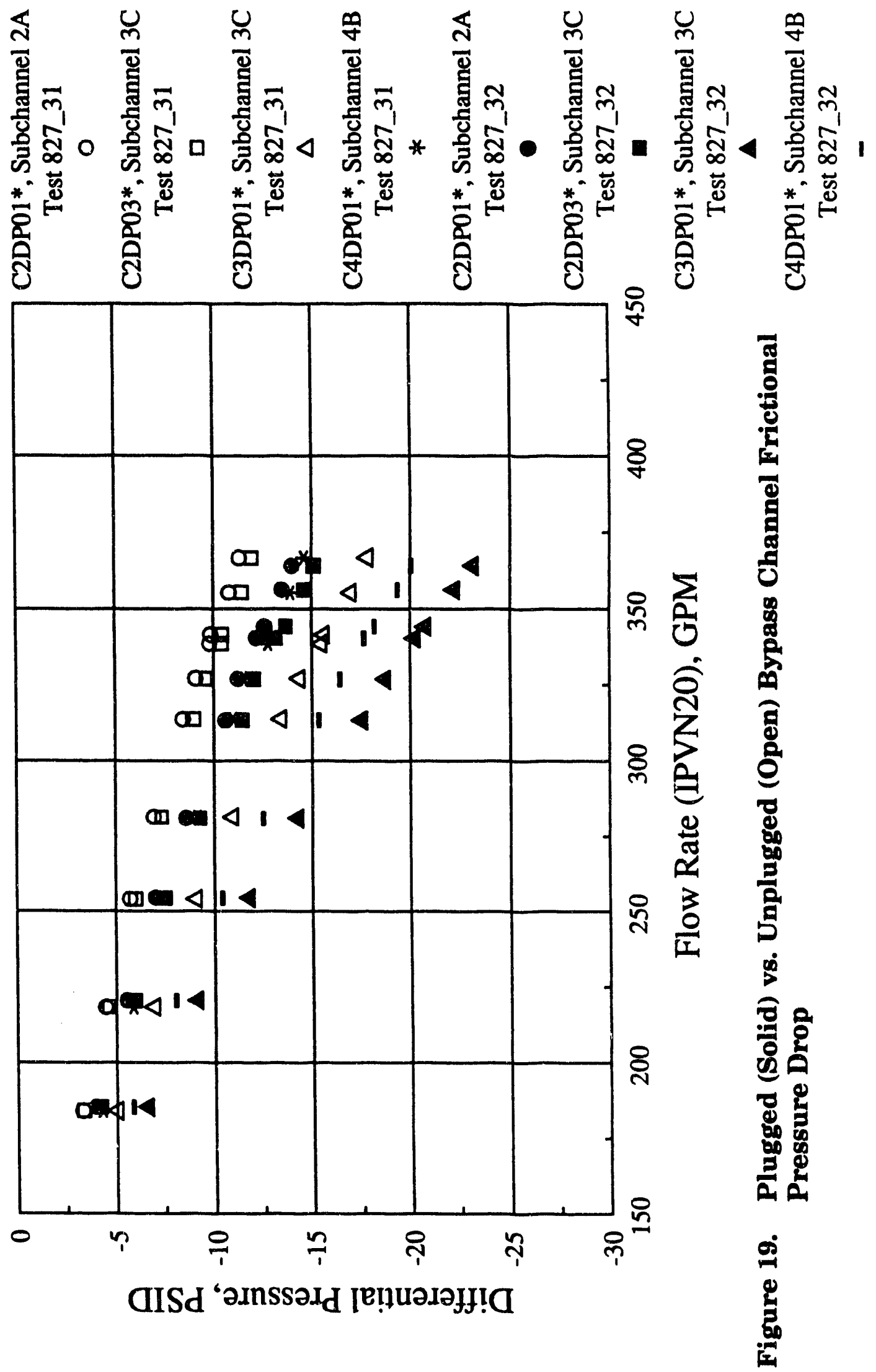




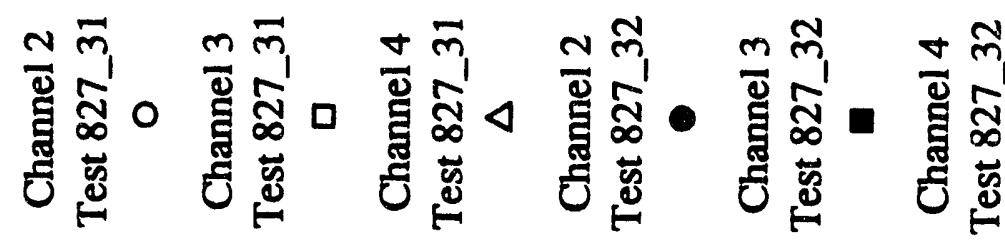

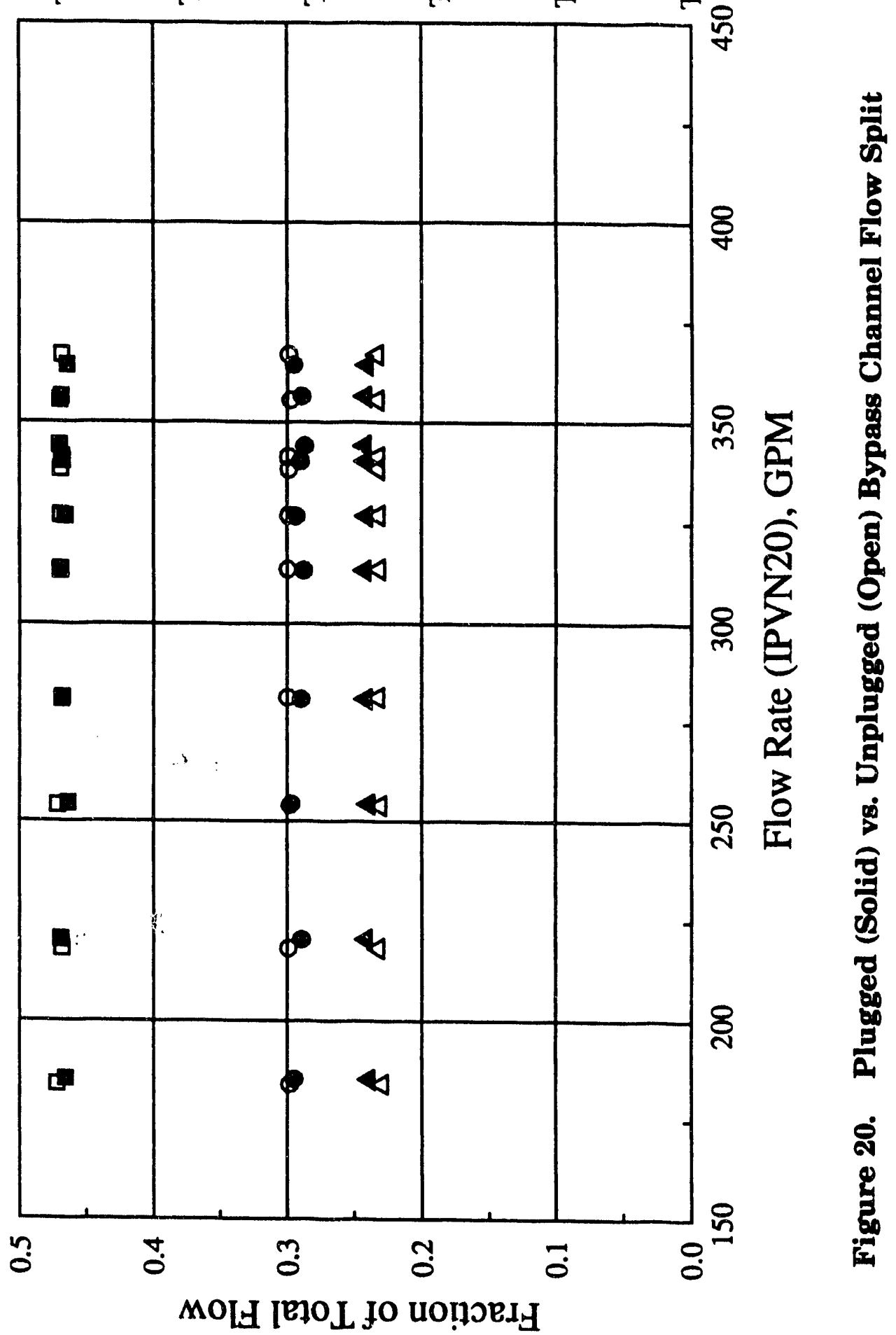



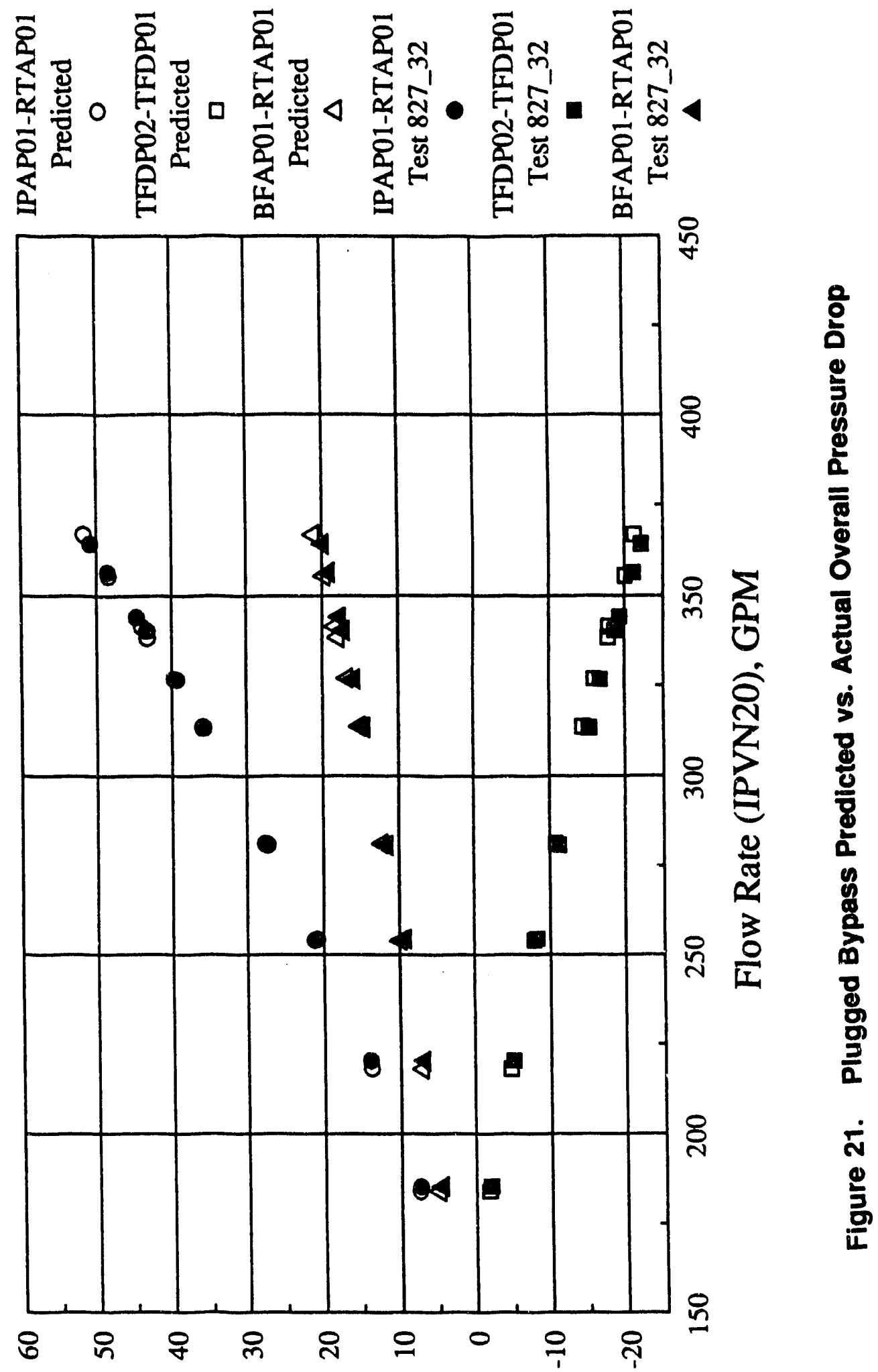

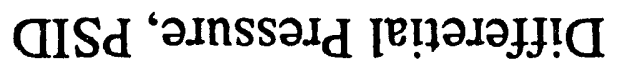




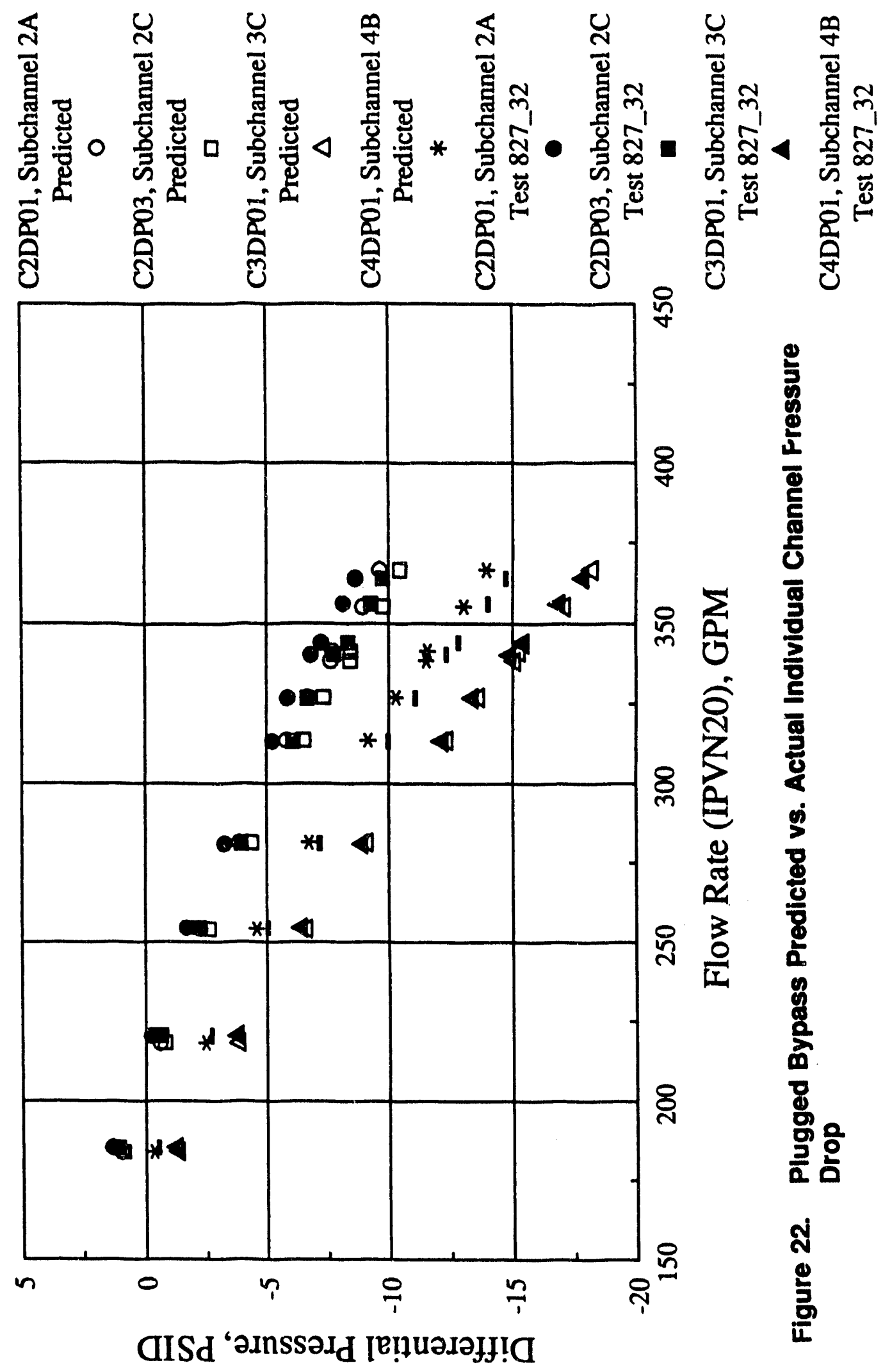




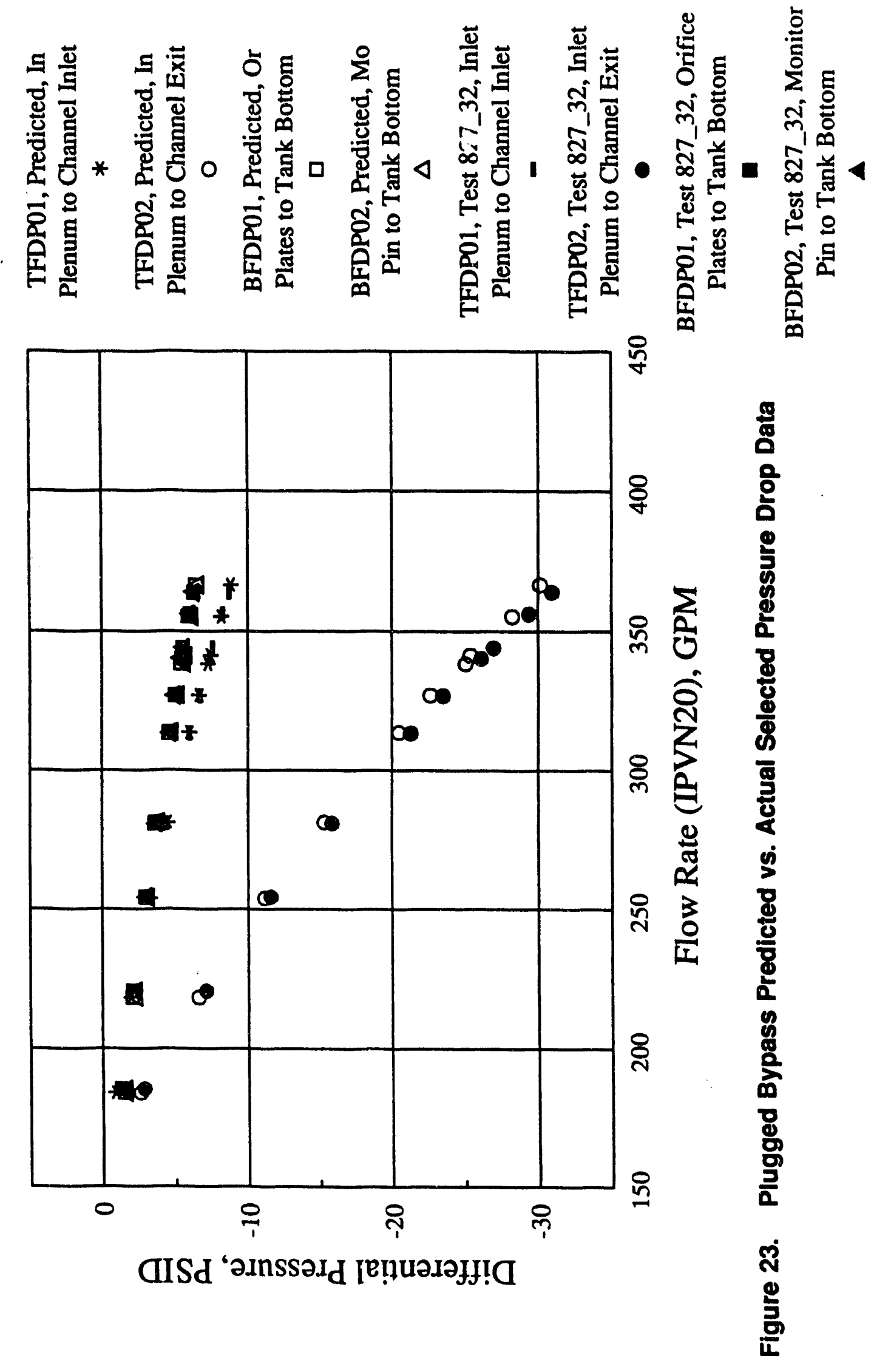




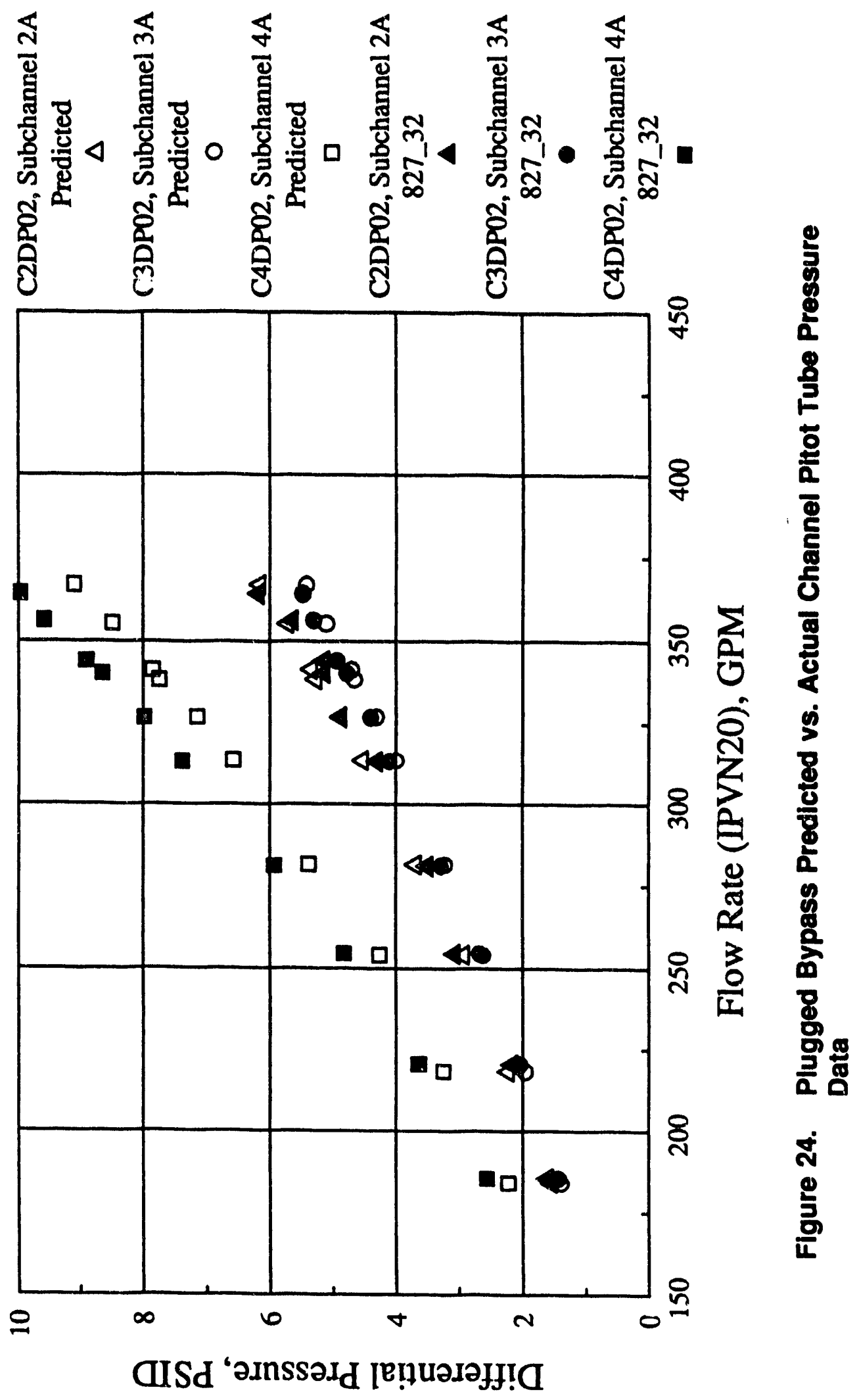




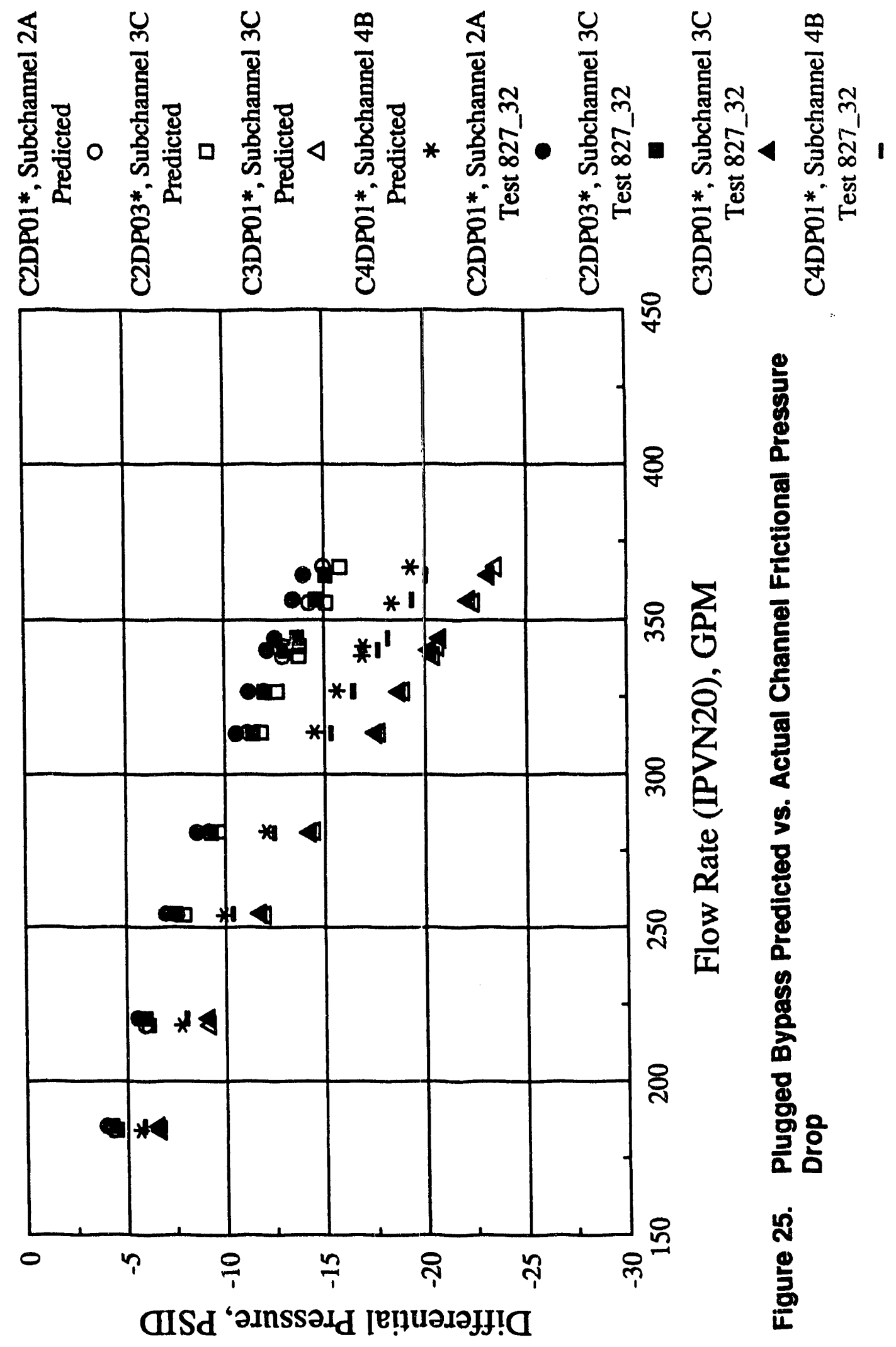




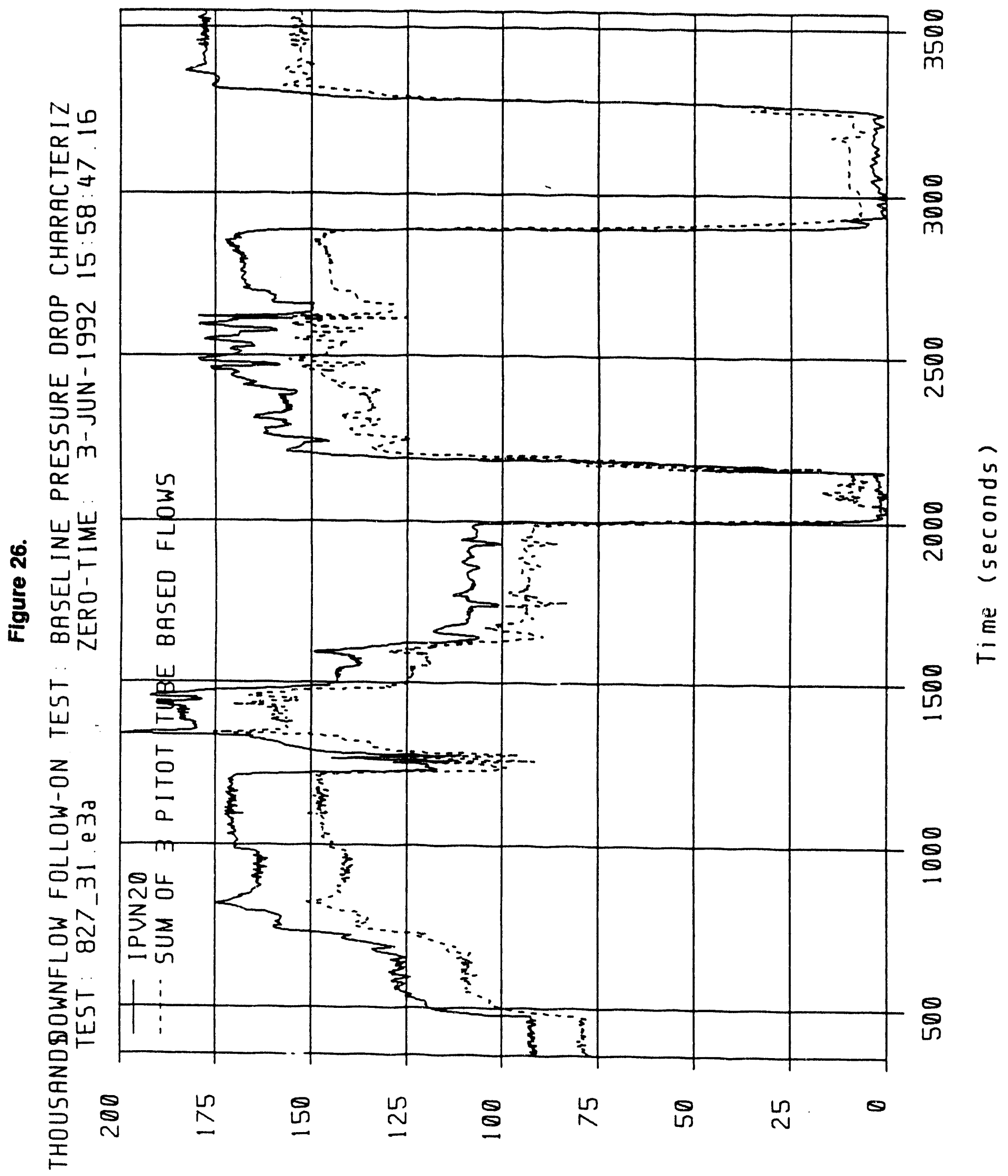




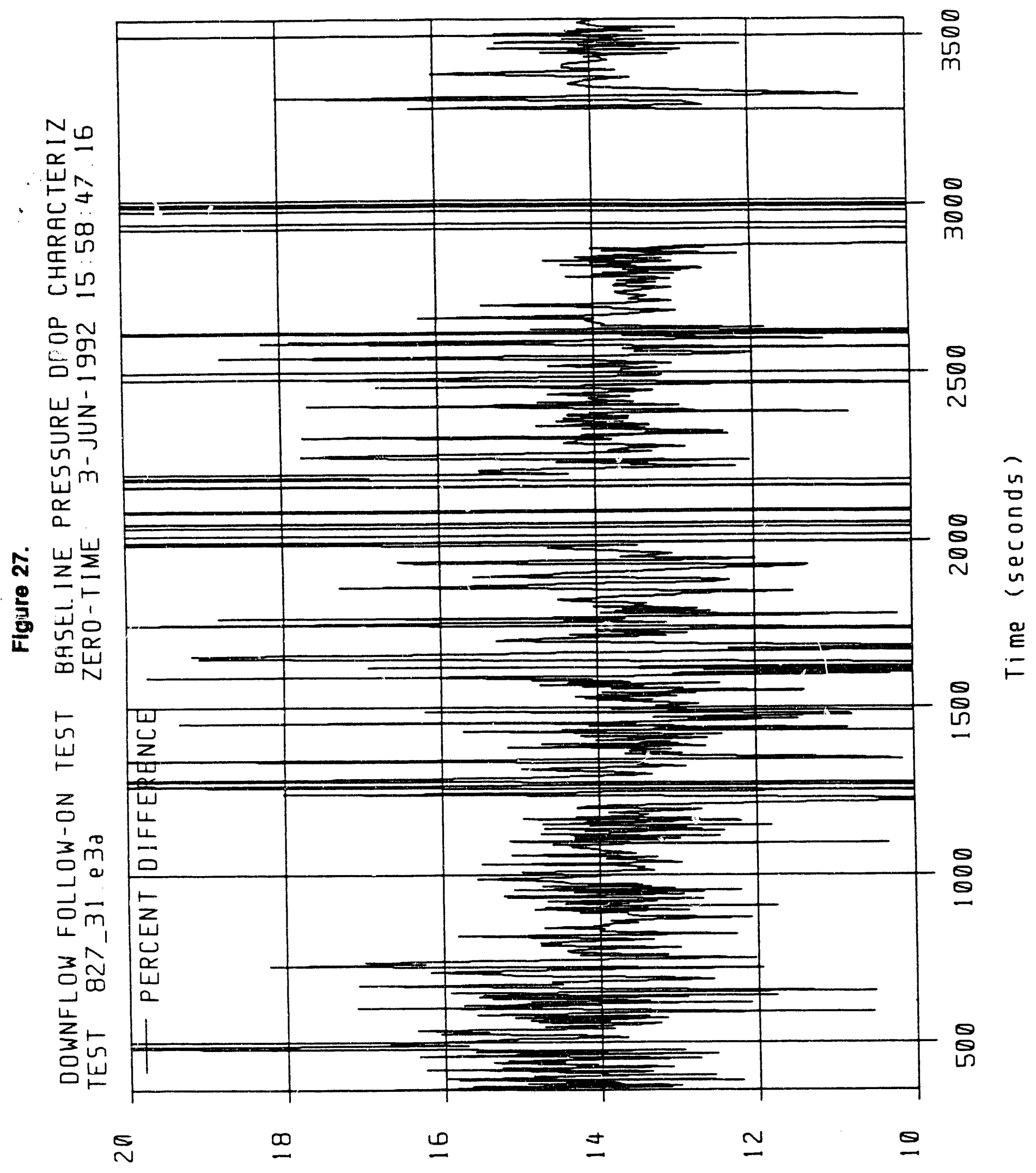

느๘山Zト 


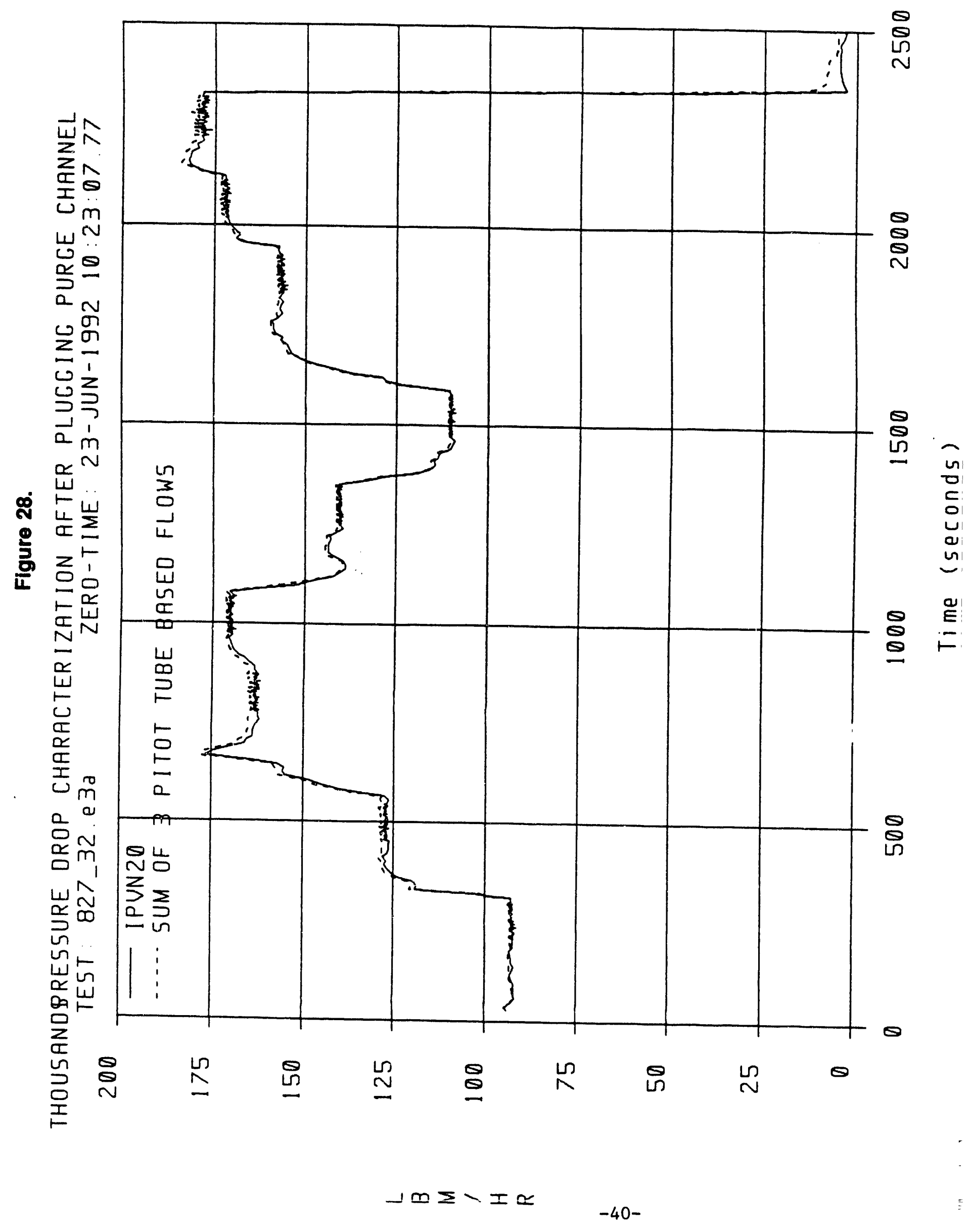




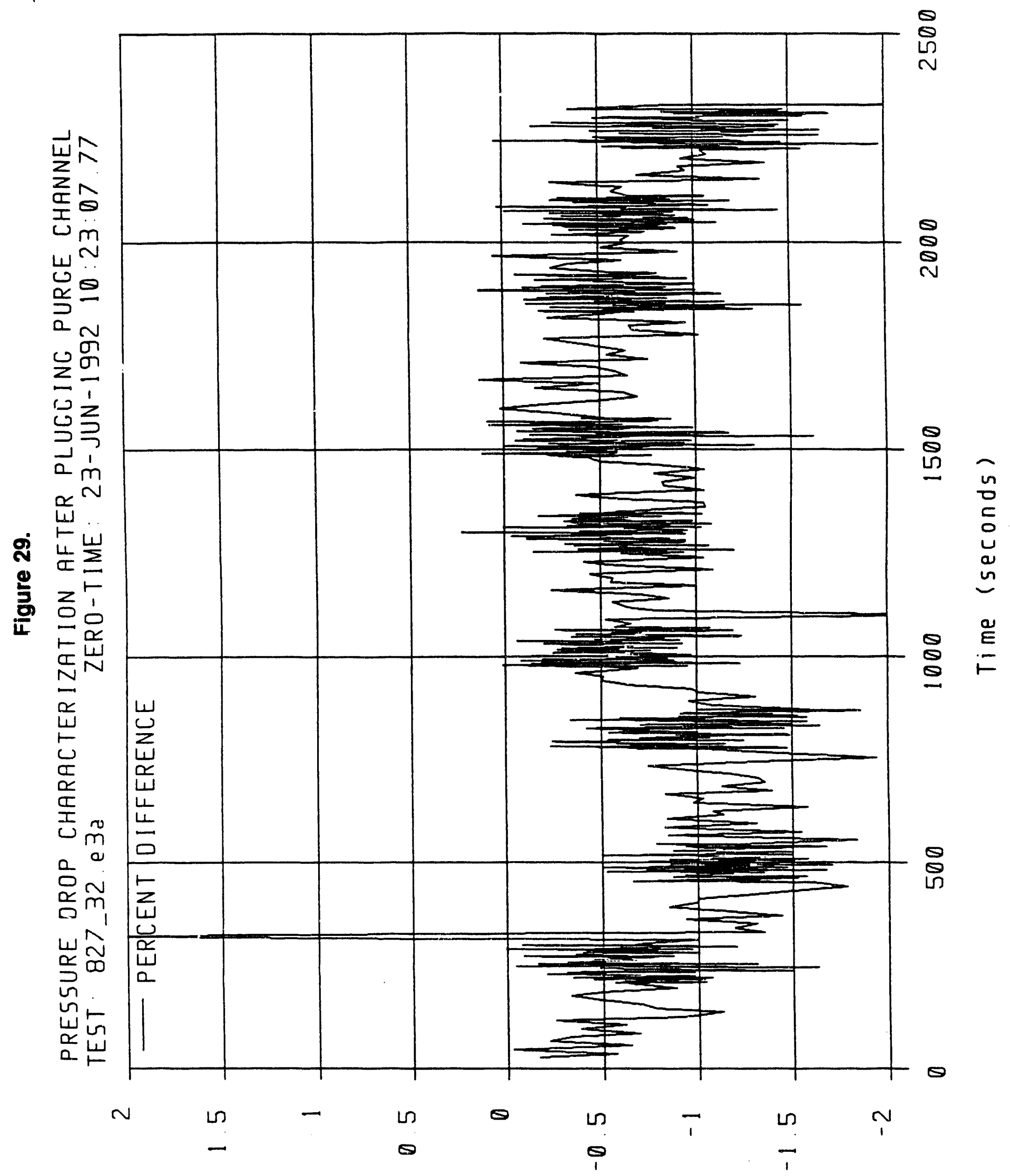

ロயェU山スト 


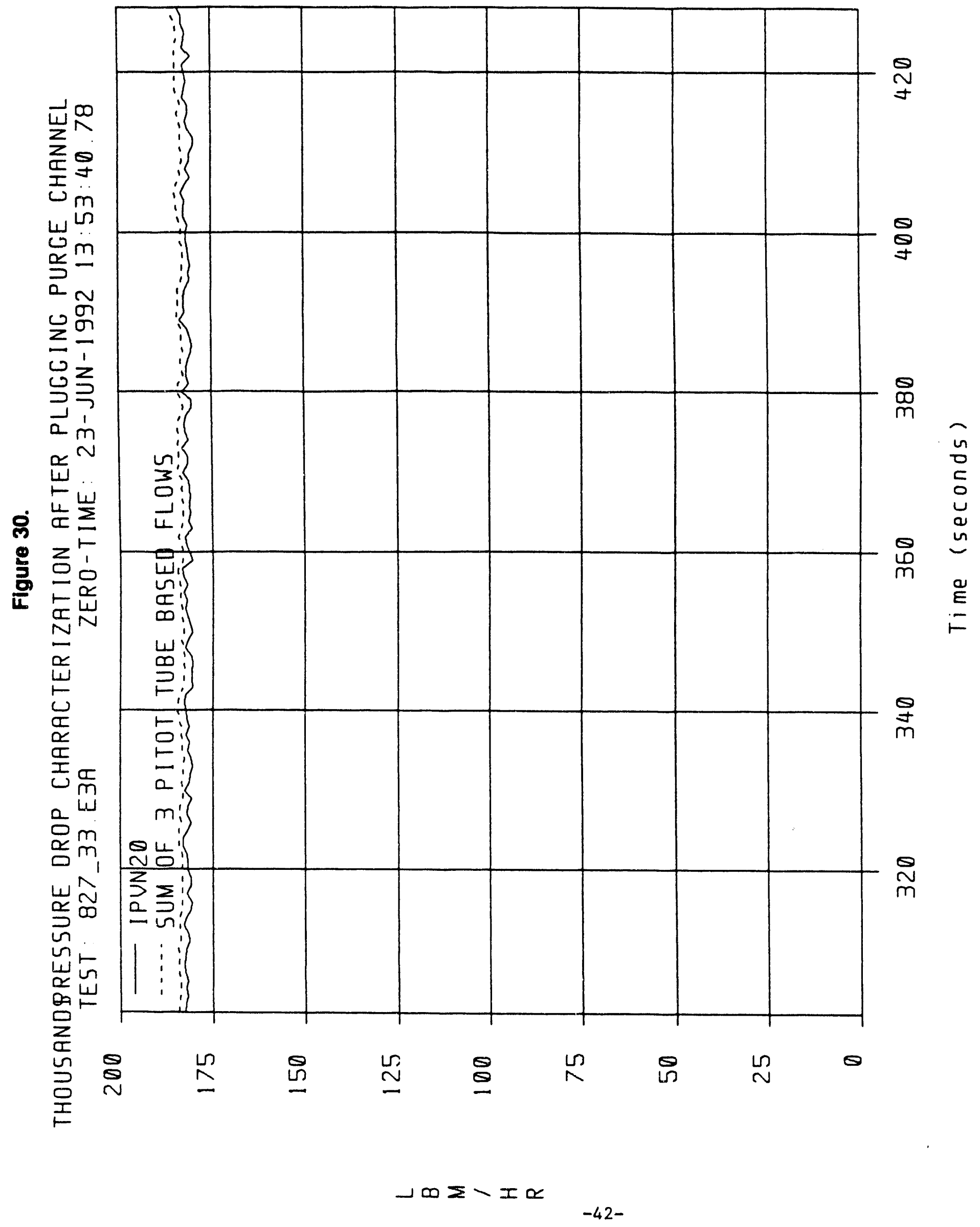




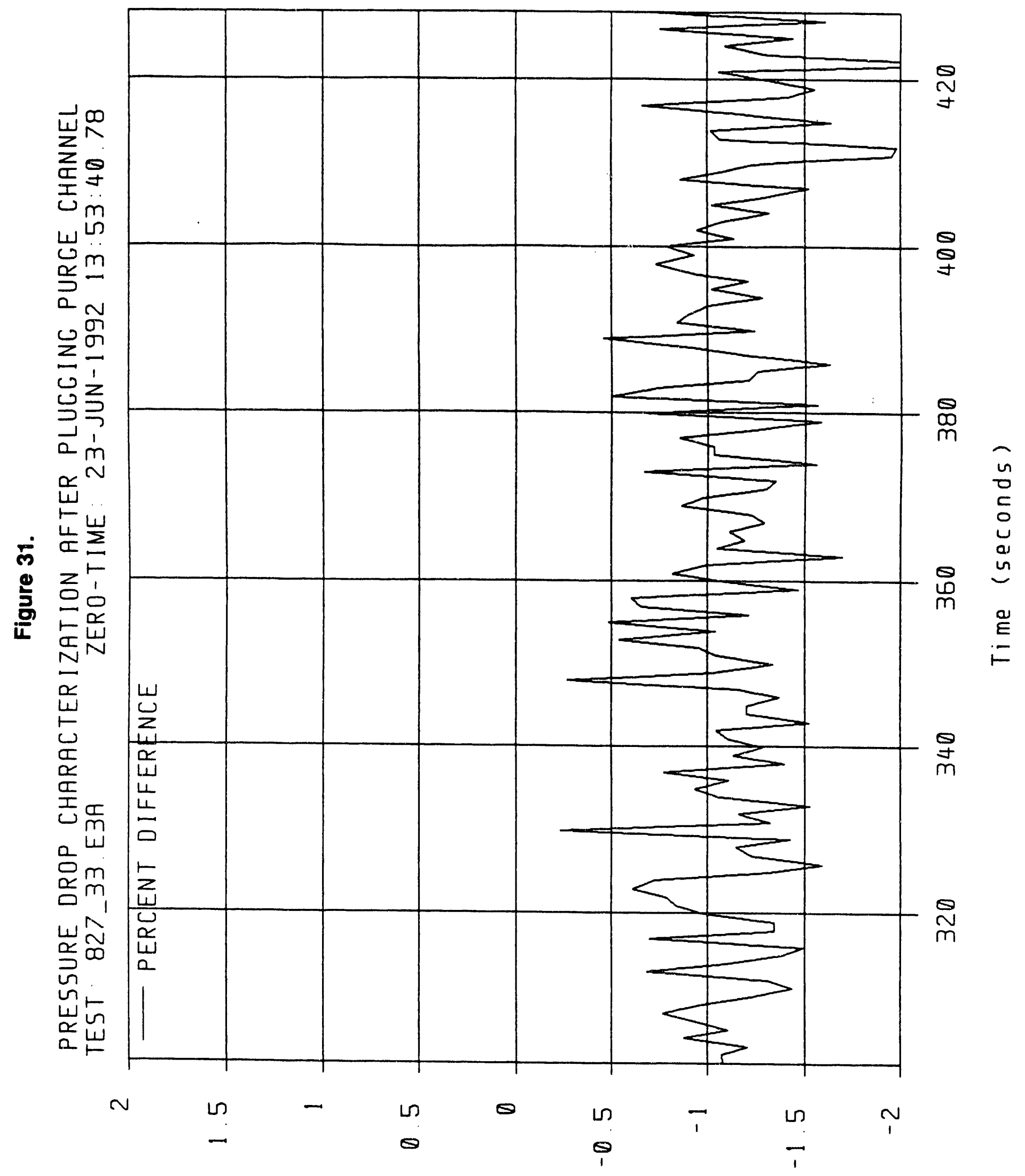

ㄴ $\propto \circlearrowleft \omega Z \vdash$ 


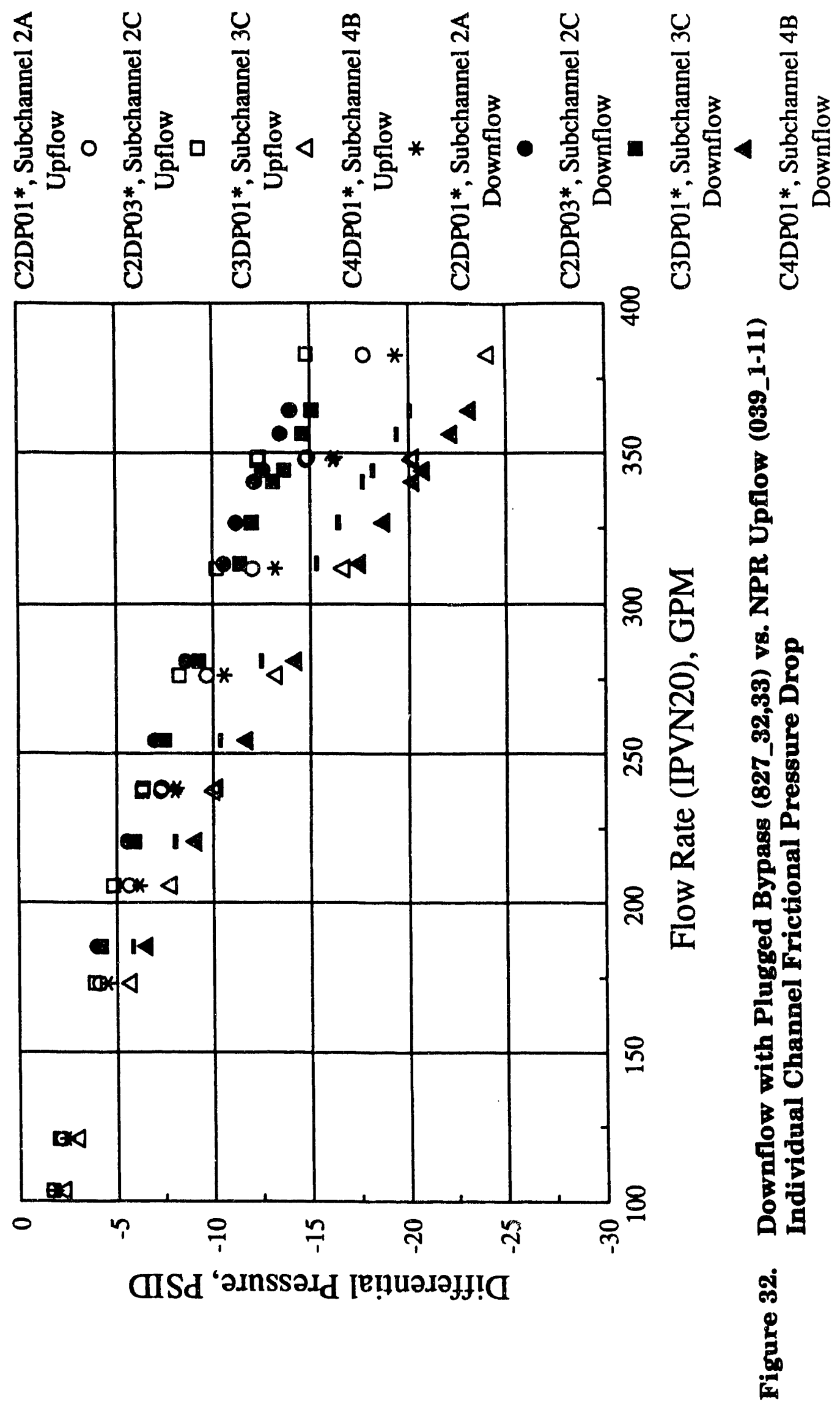




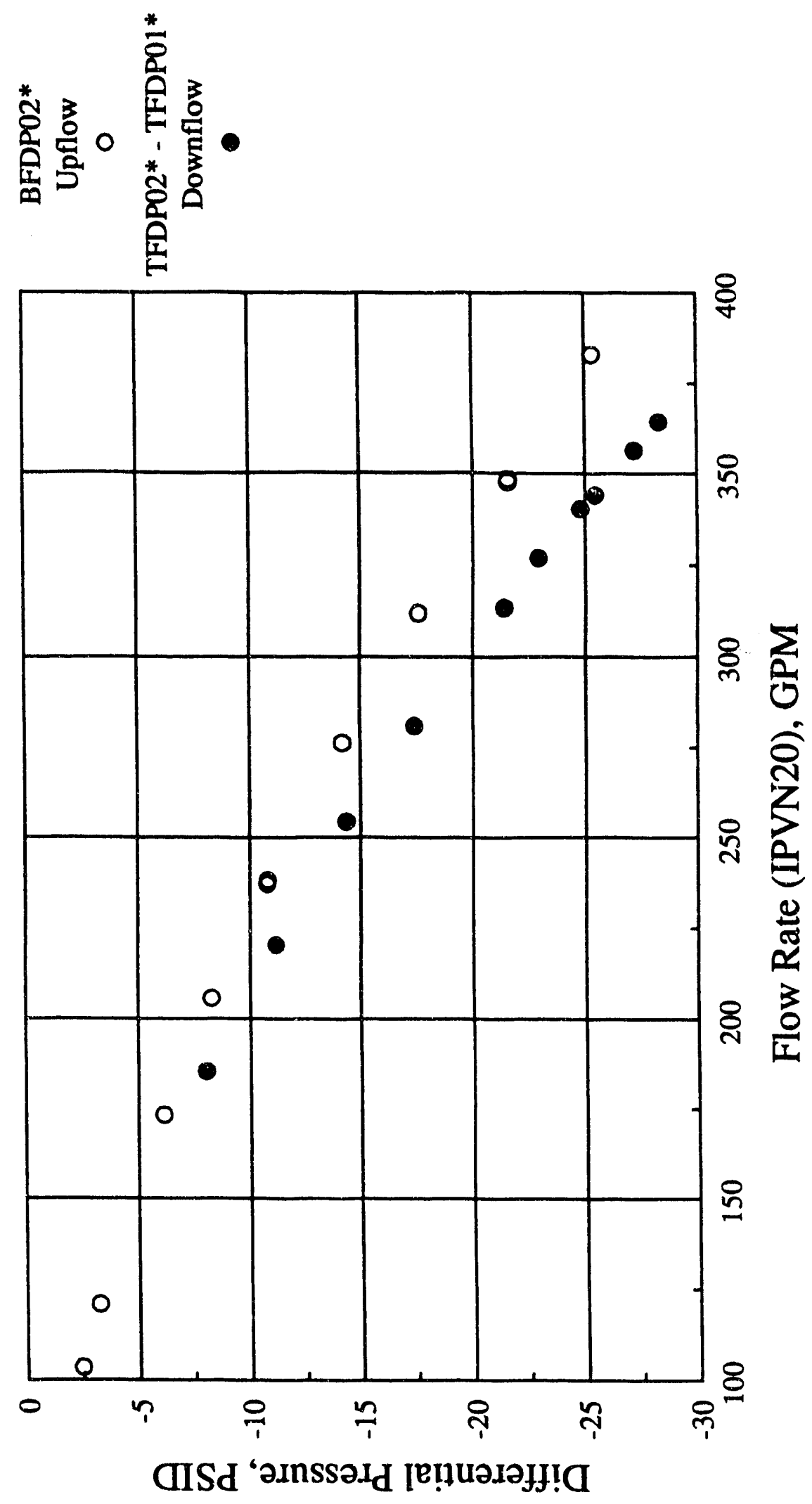

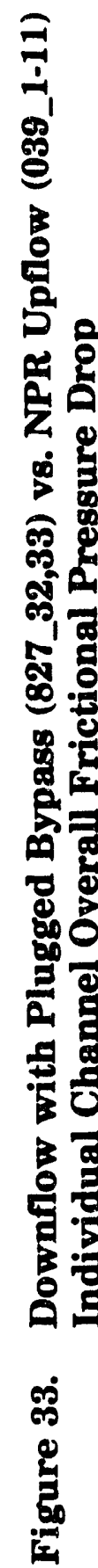




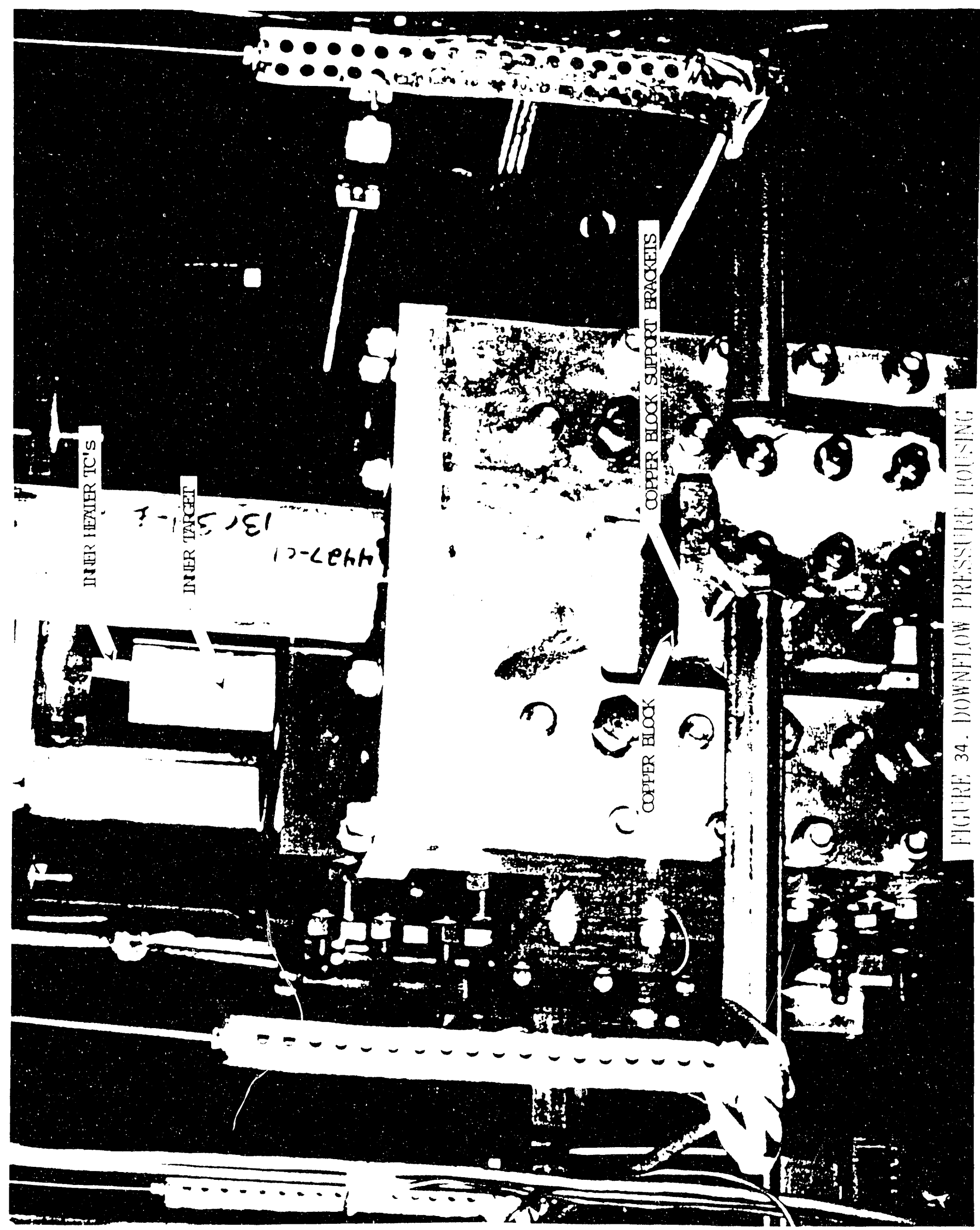




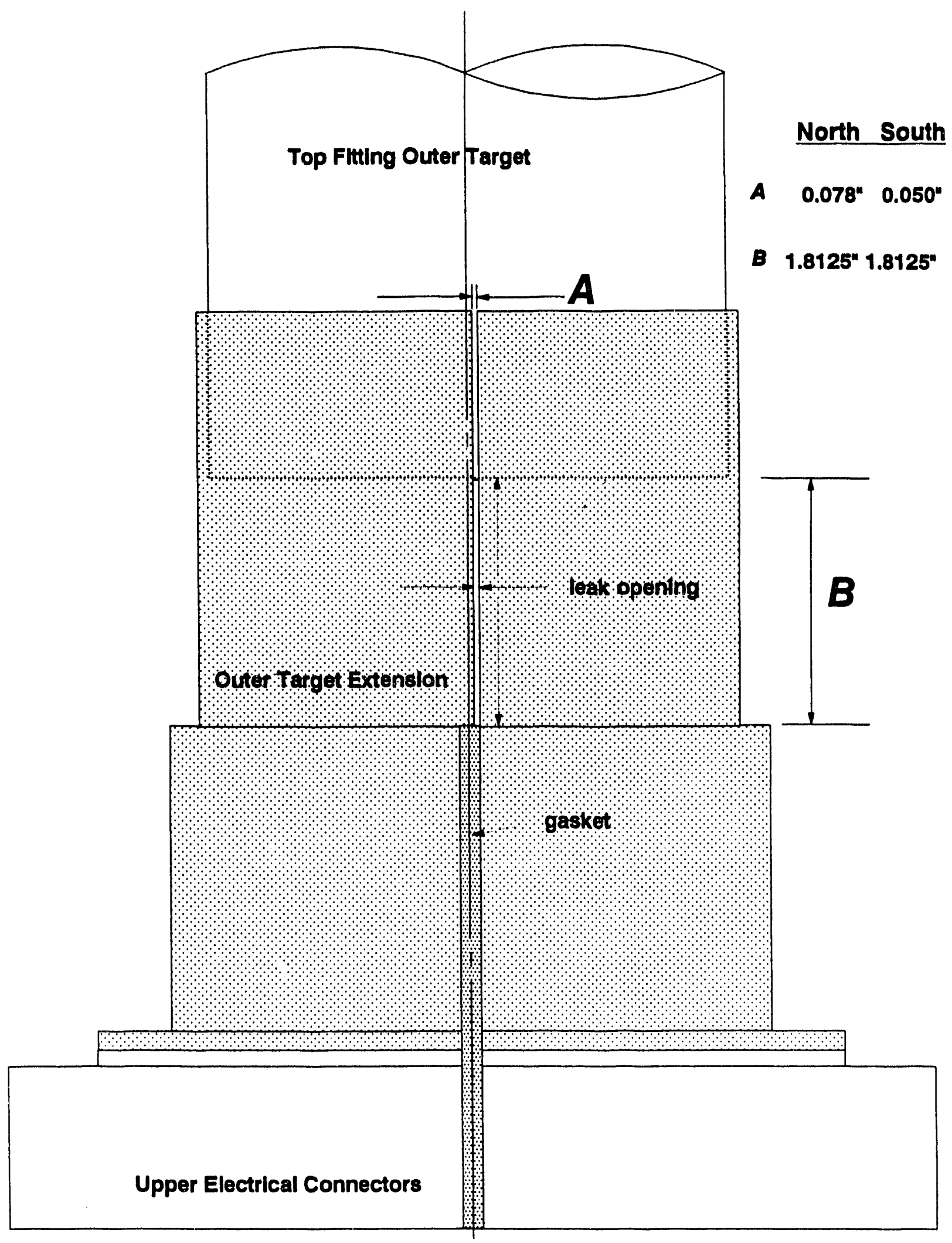

Figure 35. Primary Leakage Path at Outer Target Extension 


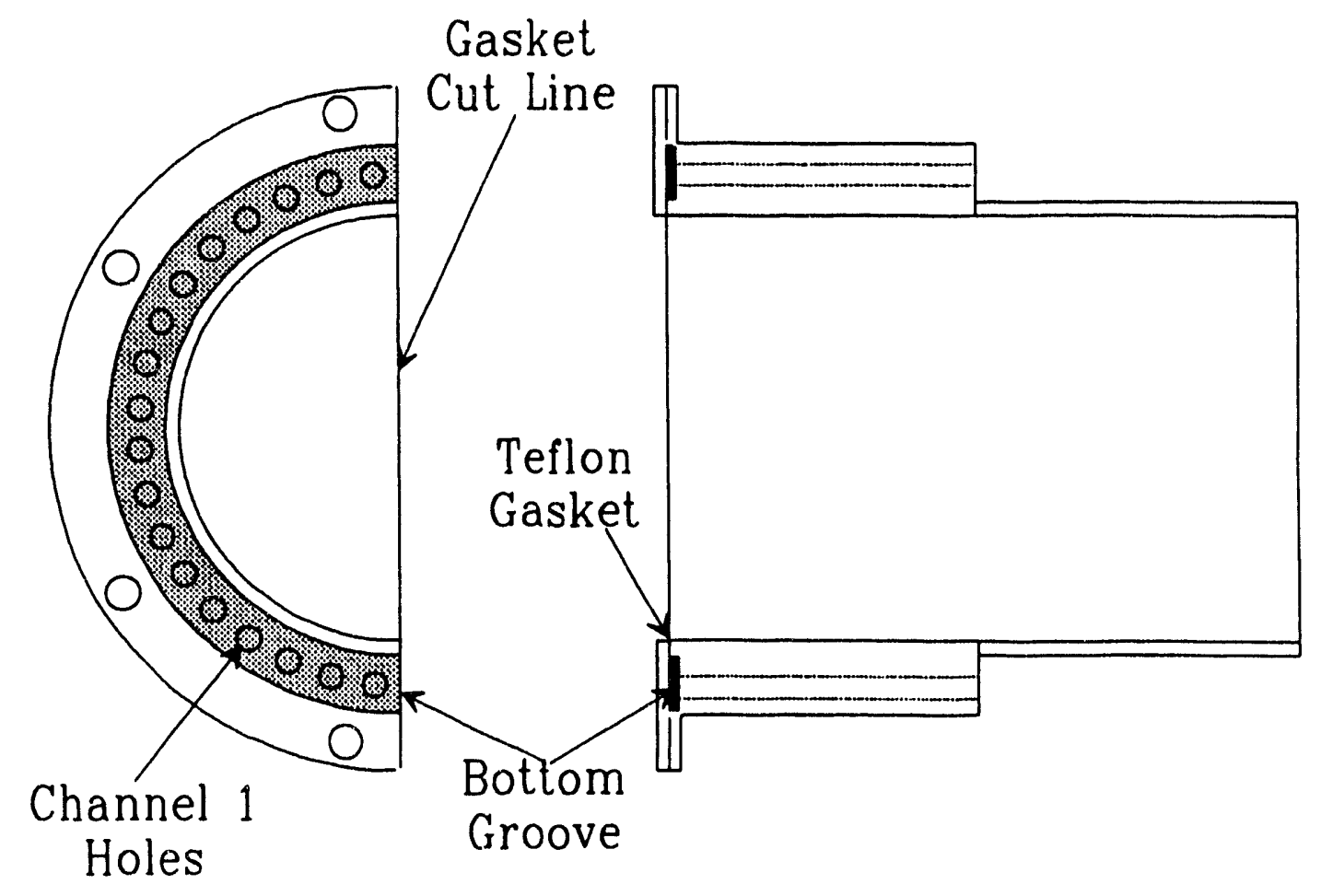

Figure 36. Teflon Gasket Location in Outer Target Extension 


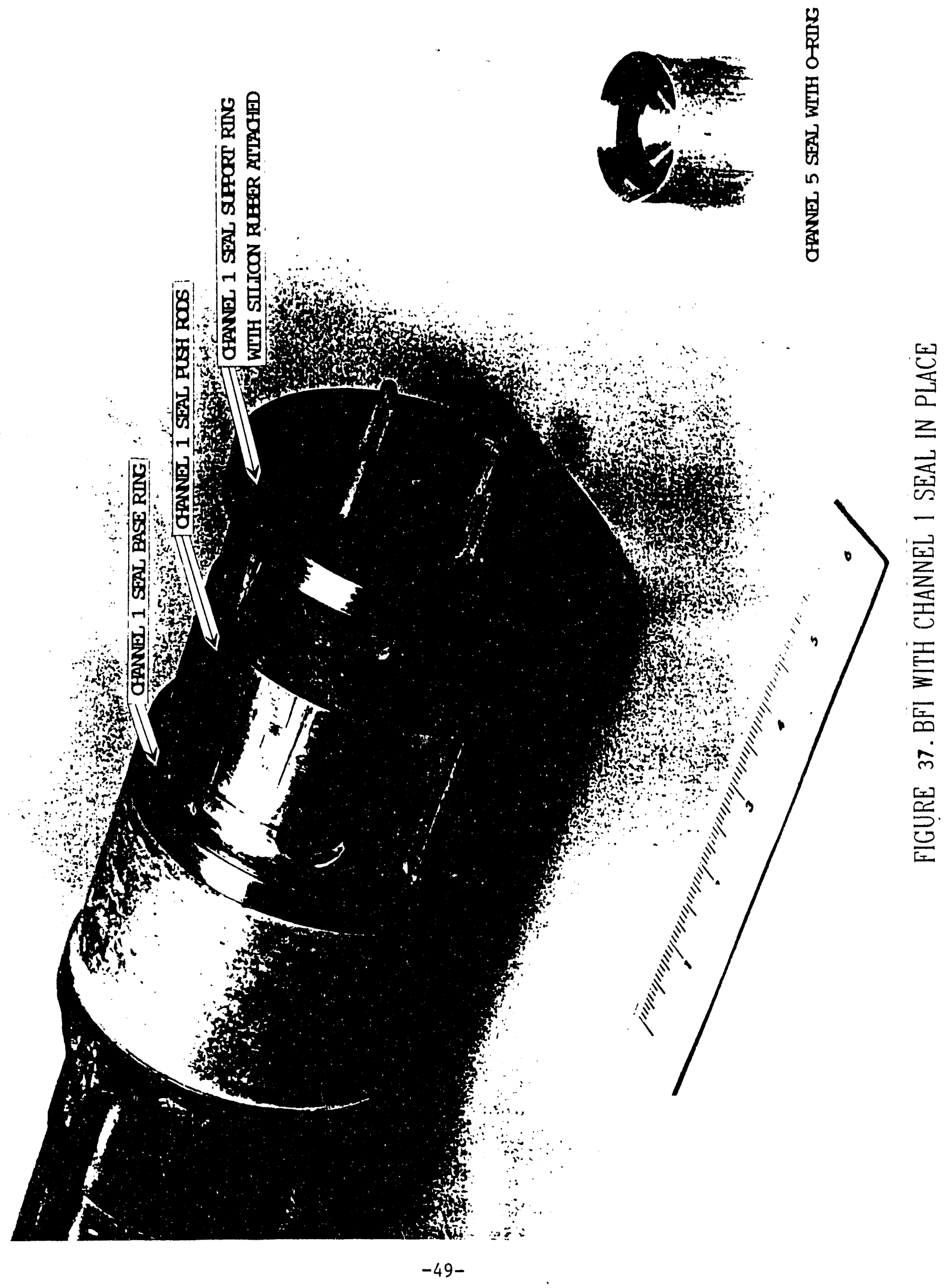




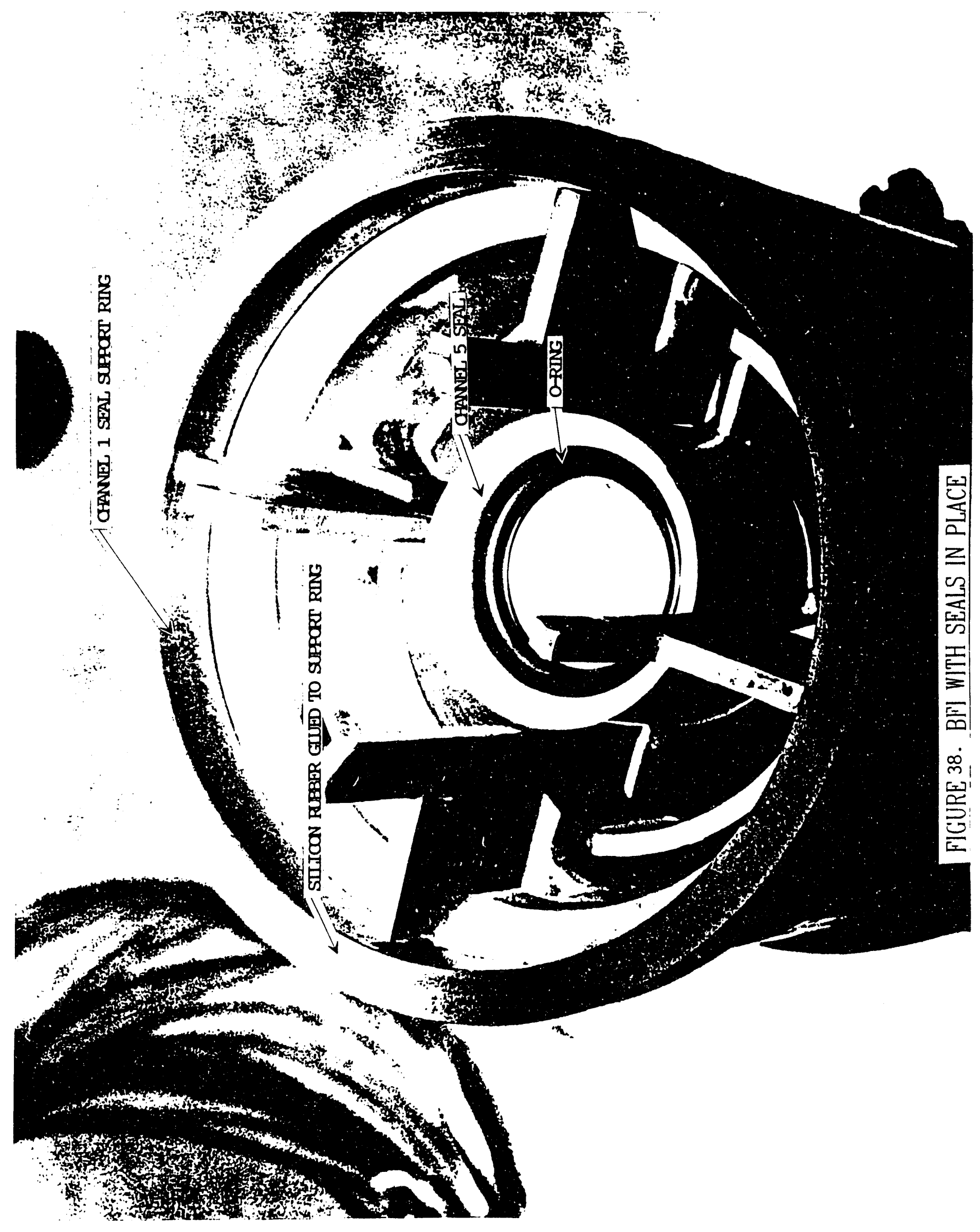




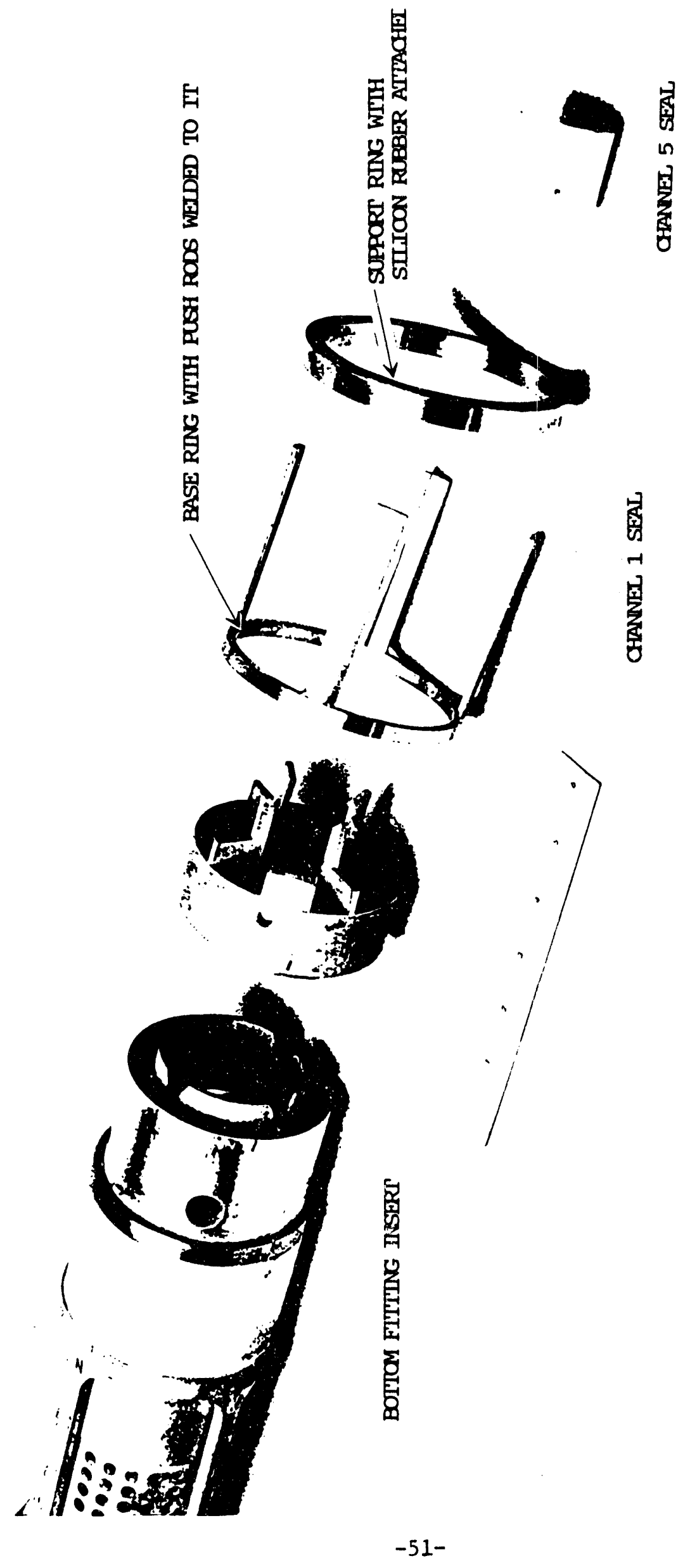

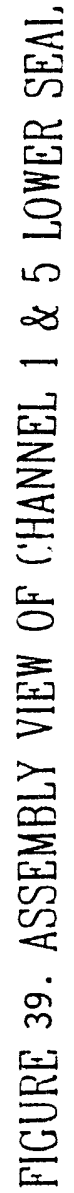




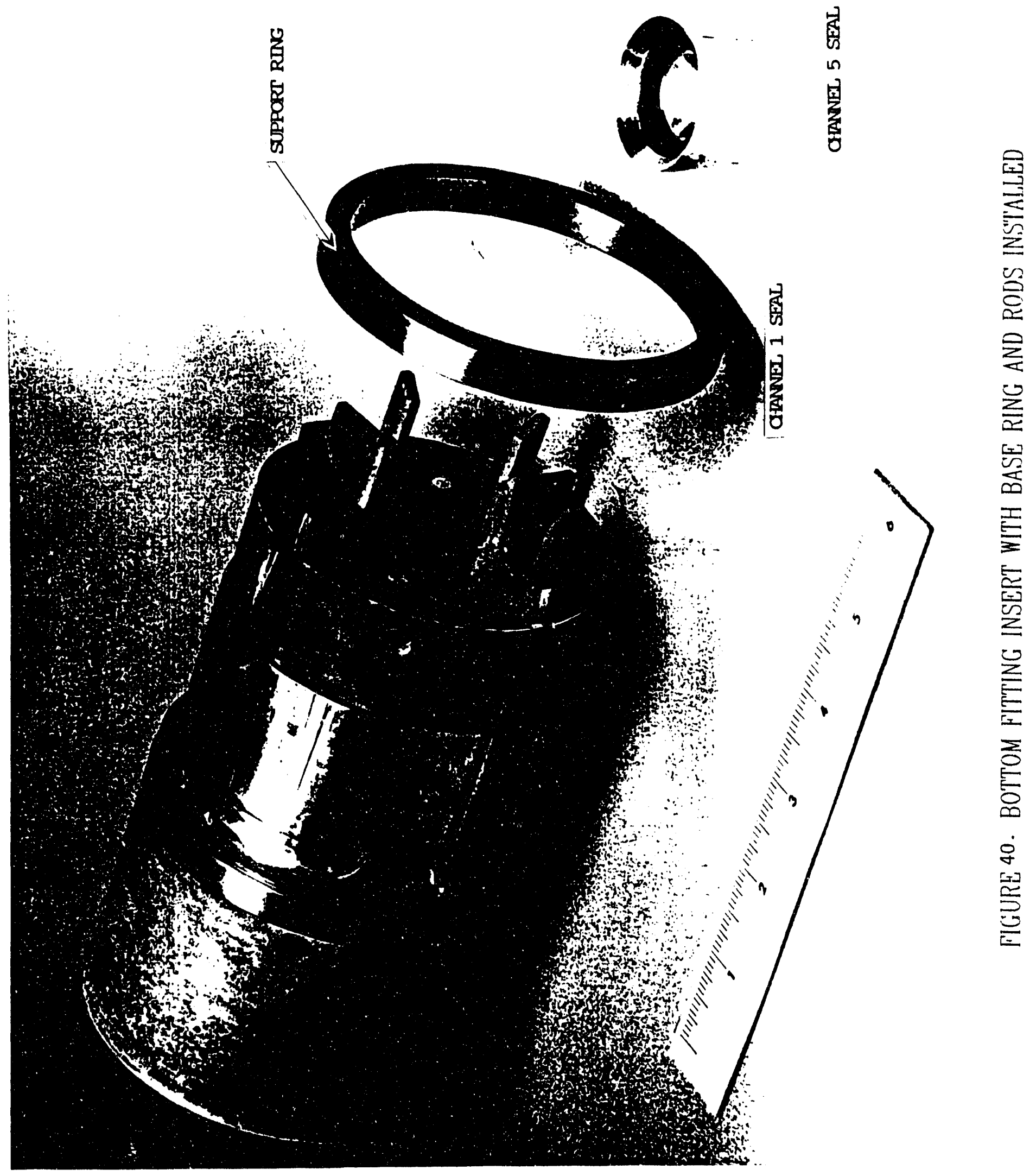




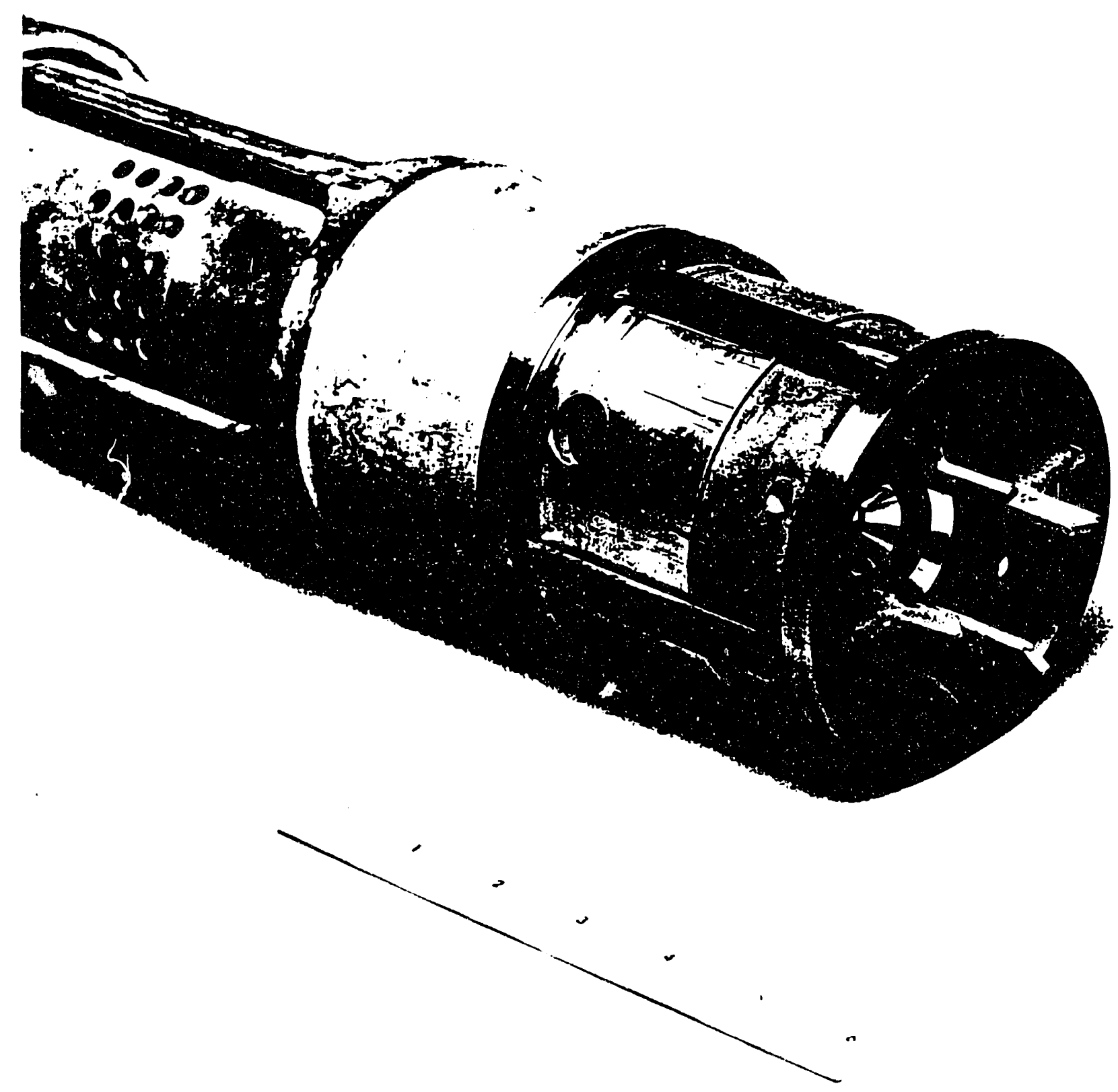

FIGURE 41. BOTTOM FITTING INSERT WITH SEALS INSTALLED 


\section{APPENDIX A}

Logbook Records from Flow Excurison Follow-on Testing 
LE

Book No.

om Page No.

May -5, 1992

Preparing the dounfluw hop for follow ion presence drop tets.

i/5/92- turned $90^{\circ}$ elbow $180^{\circ}$ from de hod pump ard connect it $t$ the dounflow piping

- removed the up flow piping to th holding tank and connected th downer exit piping $t$ it

- removed th blank in the 4000 com return hin....

- replaced th gashes or each venture and-tigitined them in place.

- reinstalled the turbine meter after laving it actihad.

- installed the fallowing TC's in the i sespecterie

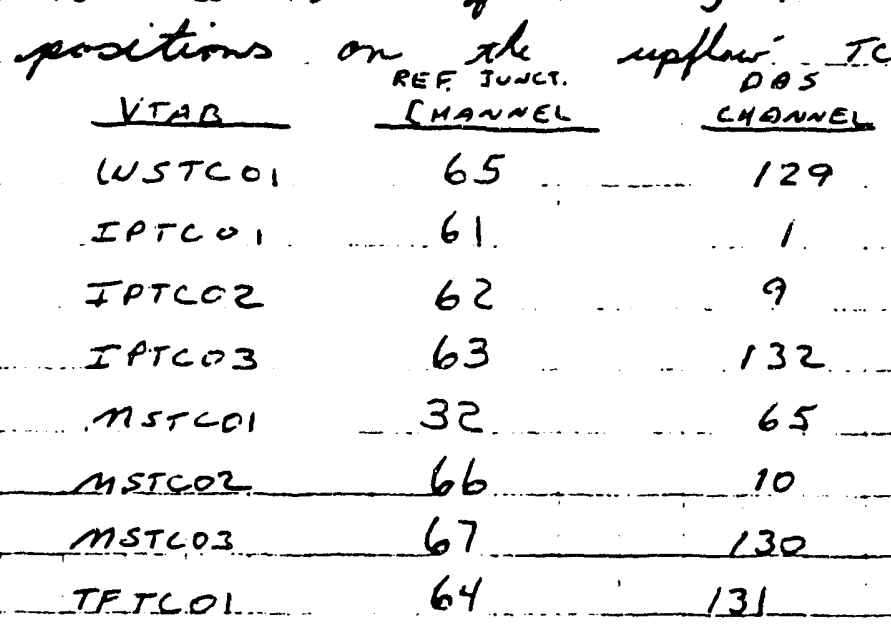

5/7/92 - Calibration all of th AP'S and DP's on the dounfliow top putters each in a Calibration states.

$5 / 13 / 92$ - Debugging the diminflew loop found wscvos flow control value not operating properly having instruments look. at it.

Spent

To Page No

Witnessed \& Understood by me,

Date

Invented by

Date

Recorded by $A-1$ 
Book No.

TLE

$$
5 / 27 / 92
$$

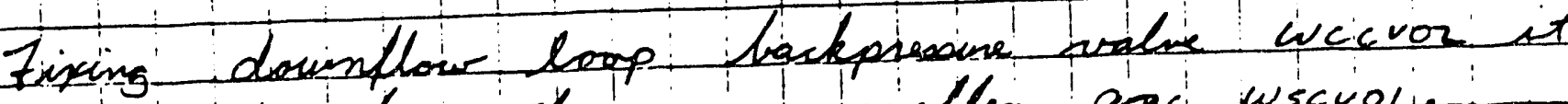

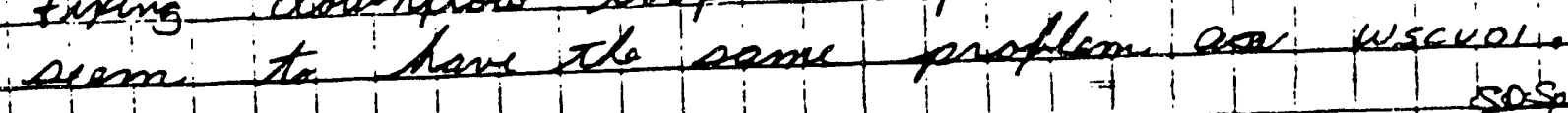

$$
5 / 28 / 92
$$
sosong

Replaced hundle inmer heater conintes useight asom and - applied the weight semored the tessim ban inad to hald it in place inhile inotellive the durang teat:- section.

Athing the hundh with de high puits wath sapply

$$
5 / 2 9 \longdiv { 9 2 }
$$
sapual

- Backfillure to hoop DP's with hish punity wats.

- forward filling th loop AP's

14:0s - Checking zers on AP's 2 op's $\therefore$ all AP's. poud

- all OP's prosed except CrOPOZ, C3DPO1,. CHDPOZ having inotreuments cleck thease owt

15:02 - 4000 gpm lomp teet pimp ion

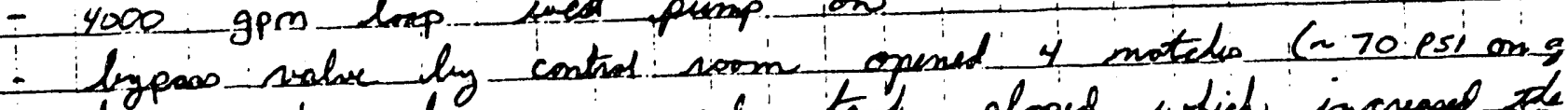
- hyposs value $l_{y} 12,000$ gal tank clowed which increane the

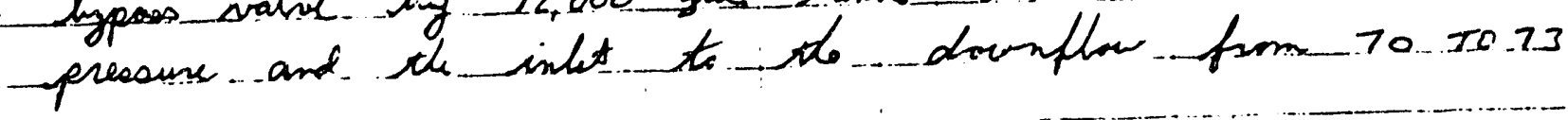
15izo - filling th inlet sovenoi using the 4000 gpm pumpo Q about $100 \mathrm{gpmi}$ bypaso presane $=68$ paig 4000 horp is 1750 gpm.

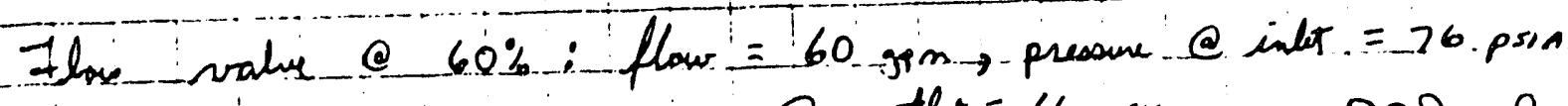

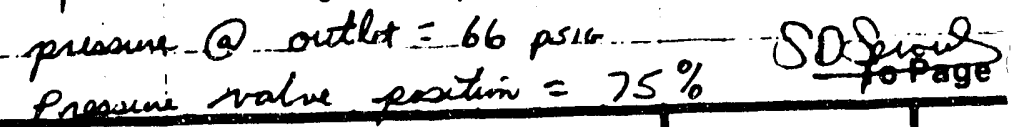

Witnessed 8 Understood by me,

Date

Invented by $4 \cdot 02$ Daie

Recorded by 
Book No.

.LE

$$
5 / 29 / 92
$$

- 4000 - gpm lop lypas set-up in alo s motsh thon claed pocitim unite de flowiet at $1250 \mathrm{sem}$

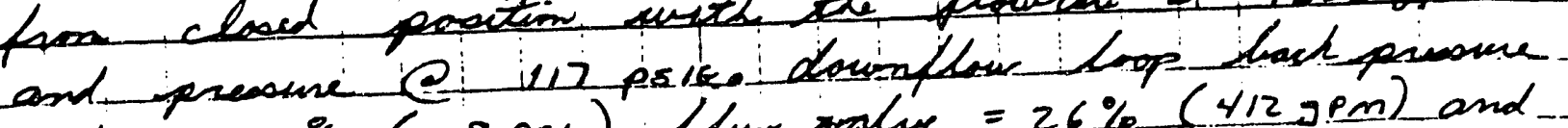
value $=35 \%(2.8 \mathrm{ps})$ fluw walue $=26 \%(4 / 2 \mathrm{JPm})$ and inlot presome $=82$ psin

-.. 16:28 - 7uel assimbly joolated

$16: 29$ - 4000 spm pump off

fune 1,1992

Paver in the plass wos shet doun dis paet wecker whid at antrmatically opens the backprosen value go to the holding taxk aloo the inder flum and quect o which drained the loop.

08:00 - filling the sosth 12,000 gal tanh is preperation to fill the drumflow loop.

- computer wers went down with th power outage without a contraled shectoown which wiped out the data hase seinstallens the vitalle and all de constants for each N tal.

$15: 05$ - Tumed on 4000 opm hop weat pump.

- about 70 psi at the lypros en th. 4000 happ.

- hyposs valive in the 411 motes fim closed

- fiting the dounflaw loop including the inlet seservios, upper plemum (inbt), buende and outbet plemem to backpesane value.

15:16-Syption is now full, venting off all high point verts sage To Page A

\begin{tabular}{|l|l|l|l|l|l|}
\hline Witnessed \& Understooo by me, & Date & Invented by \\
\hline Recorded by
\end{tabular}


From Page No.

Gione $1,-1992$ (cint)

$15: 18-$ isolating

Lomiflow: loop.

$15: 20-4000$ spos

- high penis wote sipply on to dawnflow loop.

to kep . it. wata salid.

fune 2,1992

Sospron

07:00 - instrament checking out Pp' CrPPOR, IPPPO4, IPopo6 they didint pas. zeroschat.

- Ligh purity water acpply line sprang a leak fizing with a new ligne.

- perfarmins continuits clecks on all instuments being used on this that.

\begin{tabular}{|l|l|}
\hline $\begin{array}{l}\text { Instrument } \\
\text { Group }\end{array}$ & Method \\
\hline Thermocouple & Unplug the thermocouple at its conneceor. \\
\hline
\end{tabular}

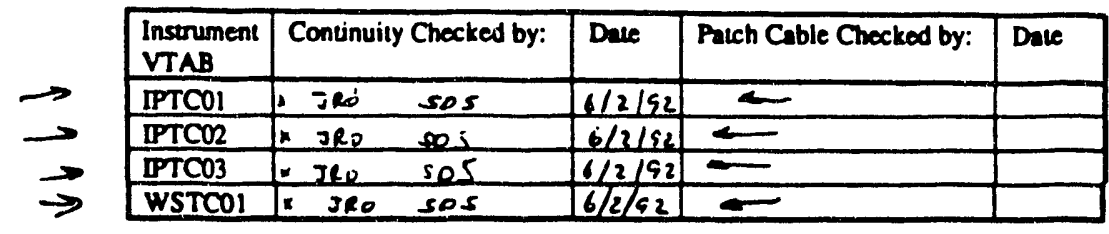

\begin{tabular}{|l|l|}
\hline $\begin{array}{l}\text { Instrument } \\
\text { Group }\end{array}$ & Method \\
\hline RTD's & Disconnoct either the whice or preen gingl wire3 ss the RTD connector block. \\
\hline
\end{tabular}

\begin{tabular}{|c|c|c|c|c|}
\hline $\begin{array}{l}\text { Instrument } \\
\text { VTAB }\end{array}$ & Continuity Checked by: & Dave & Patch Cable Chocked by: & Dale \\
\hline BFRTOI & Say $/$ sent $^{2}$ & $6 / 2 \sqrt{90}$ & Jay & $6 / 262$ \\
\hline opatpl- & Jay $/$ secer & $6 / 2 / 2$ & $I 4$ & $6 / 2 / 52$ \\
\hline TFRTOI & $\sim / A$ & & & \\
\hline WCRTOI & $M / A$ & & & \\
\hline
\end{tabular}

$(\cdots+\cdots$

$+\cdot$

\begin{tabular}{|l|l|}
\hline Date & Invented by 9.4 \\
\hline Recorded by
\end{tabular}

To Page $\mathbf{r}$ 
=rom Page No.

1 fene 2 f $1992(\operatorname{con} t)$

1 -Continuity check continuel.

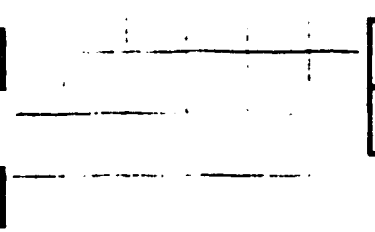

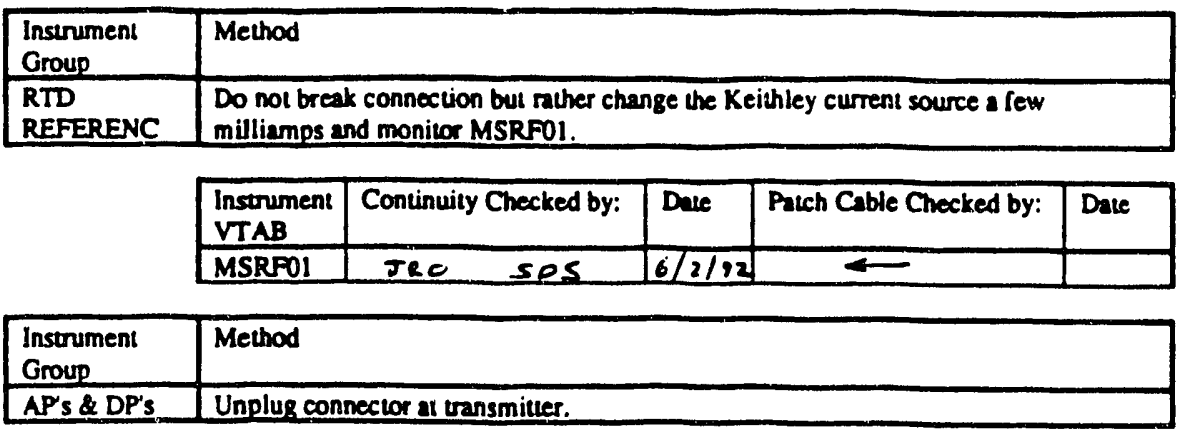

\begin{tabular}{|c|c|c|c|c|c|}
\hline $\begin{array}{l}\text { Instrument } \\
\text { VTAB }\end{array}$ & Continuity C & hecked by: & Dave & Patch Cable Chocked by: & Dale \\
\hline BFAP01 & $x$ JRO & sos & $6 / 2 / 92$ & $=$ & \\
\hline BFDPOI & $=$ & & & 7 & \\
\hline BFDPO2 & $x$ & & & & \\
\hline \multicolumn{6}{|c|}{ 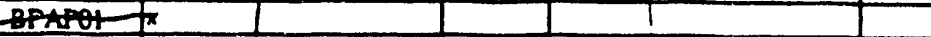 } \\
\hline \multicolumn{6}{|c|}{\begin{tabular}{|l|l|} 
B_AP02 & \\
\end{tabular}} \\
\hline \multirow{2}{*}{\multicolumn{6}{|c|}{\begin{tabular}{|l|l} 
C2APO1 \\
C2APO2
\end{tabular}}} \\
\hline & & & & & \\
\hline \multicolumn{6}{|c|}{ C2APO3 } \\
\hline \multicolumn{6}{|l|}{ C2APOA } \\
\hline \multirow{2}{*}{\multicolumn{6}{|c|}{\begin{tabular}{l|l} 
C2APO5 & $x$ \\
C2APO6
\end{tabular}}} \\
\hline & & & $!$ & & \\
\hline \multicolumn{6}{|l|}{ C2AP07 } \\
\hline \multirow{2}{*}{\multicolumn{6}{|c|}{\begin{tabular}{l|l} 
C2APOB & $x$ \\
C2APOQ
\end{tabular}}} \\
\hline & & & & & \\
\hline \multicolumn{6}{|l|}{ C2AP10 } \\
\hline \multicolumn{6}{|l|}{ C2DPOI } \\
\hline C2DPO2 & 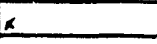 & & & & \\
\hline C2DPO3 & $E$ & & & & \\
\hline \multicolumn{6}{|c|}{ C3APO1 } \\
\hline \multicolumn{6}{|c|}{$\begin{array}{l}\text { C3APO2 } \\
\text { C3APO3 }\end{array}$} \\
\hline \multicolumn{6}{|c|}{\begin{tabular}{l|l} 
C3APO3 & $x$ \\
\end{tabular}} \\
\hline \multirow{2}{*}{\multicolumn{6}{|c|}{\begin{tabular}{l|l} 
C3DPO1 & $x$ \\
C3DPO2
\end{tabular}}} \\
\hline & & & & & \\
\hline \multicolumn{6}{|c|}{ CAAPOI ${ }_{x}$} \\
\hline \multicolumn{6}{|c|}{ CAAPO2 $x$} \\
\hline \multicolumn{6}{|c|}{ CAAPO3 3} \\
\hline \multirow{2}{*}{\multicolumn{6}{|c|}{$\begin{array}{l}\text { CADPO1 } \\
\text { CADPO2 }\end{array}$}} \\
\hline & & & & & \\
\hline \multicolumn{6}{|l|}{ FCAPOI } \\
\hline \multicolumn{6}{|c|}{\begin{tabular}{l|l}
$\mathrm{FCDPOI}$ & $N_{A}$ \\
\end{tabular}} \\
\hline & $N A$ & & & & \\
\hline \multicolumn{6}{|c|}{ FCDPO3 we } \\
\hline \multicolumn{6}{|c|}{ FCDPO4 NA } \\
\hline HWAPOI & Ne JRO & sos & $6 / 2 / 72$ & $=$ & \\
\hline
\end{tabular}

1

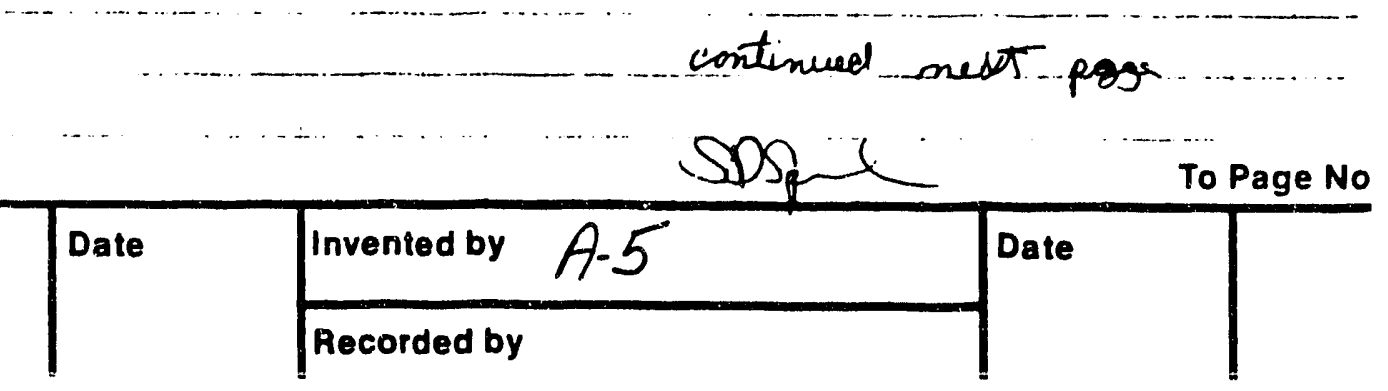

\begin{tabular}{|l|l|l|l|}
\hline Witnessed \& Understood by me, & Date & $\begin{array}{l}\text { Invented by } A-5 \\
\text { Recorded by }\end{array}$ & Date
\end{tabular}


BOOK NO.

From Page No. -

fine $2,1992(\mathrm{cont})$

Conteneisty checks continued:

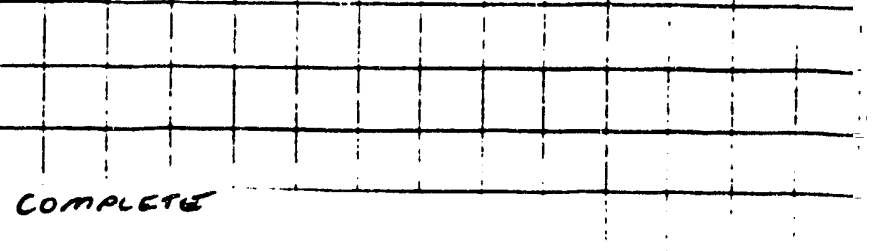

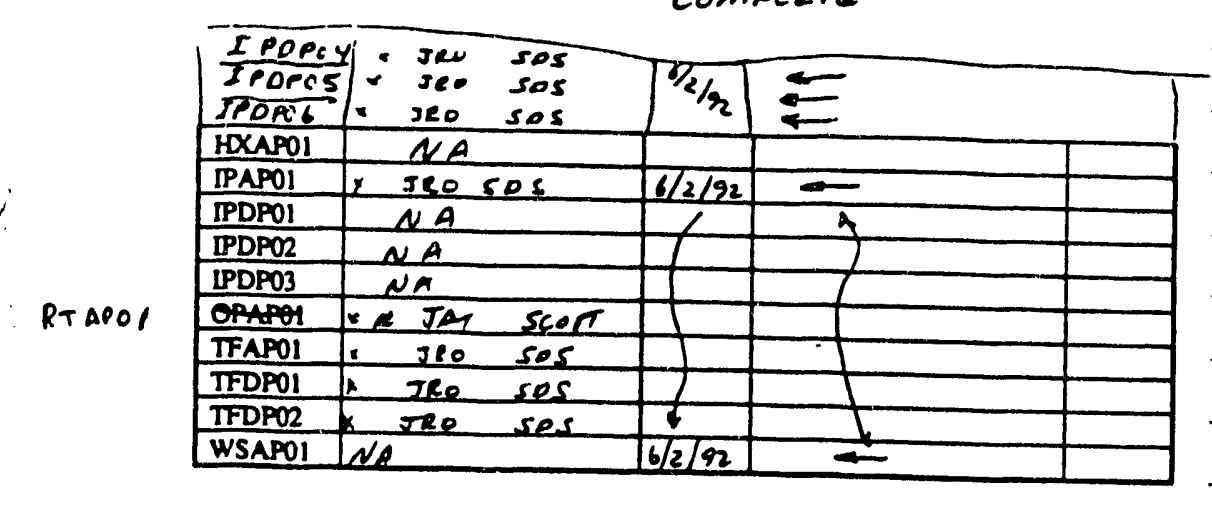

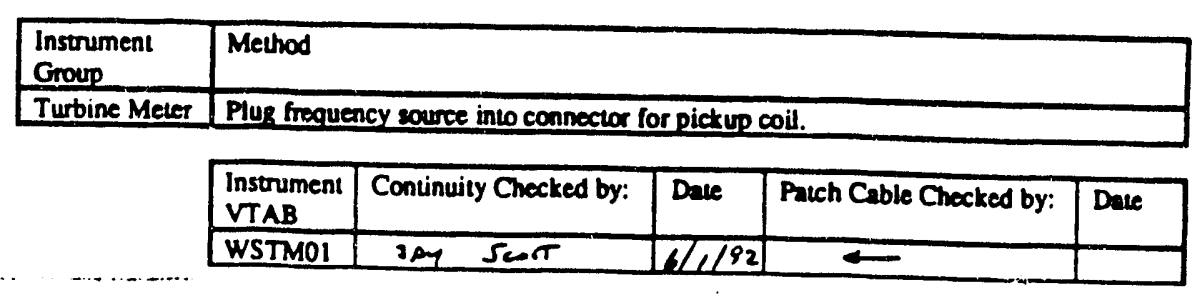

fine 3,1992

07:00 - filling 13,000 gal. tanks (NoRTh and south).

071.5 - Termed on che west 4000 cen pump wat. up to $\sim 80$ of

11:45 - Pump off (4000), wats temperature = 81.0

-11:45 - instruments now "checking out cropor.., IPDPou, IPpopt.

1515 - Starting 4000 sp m (west) pump.

- 15:20- Venting High point vent on piping leading into in lat reese - loop supply (have) isolated..

\begin{tabular}{|l|l|l|l|}
\hline Witnessed \& Understood by me, & Date & Invented by $A-6$ & Date
\end{tabular}


E

bun inv.

in Page No.-

fume $3,19.92 !($ cont $)$

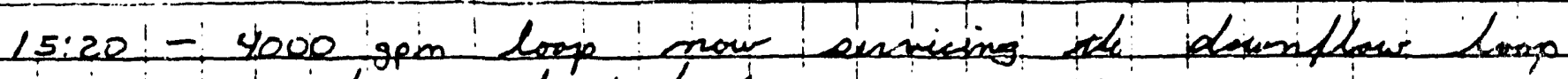

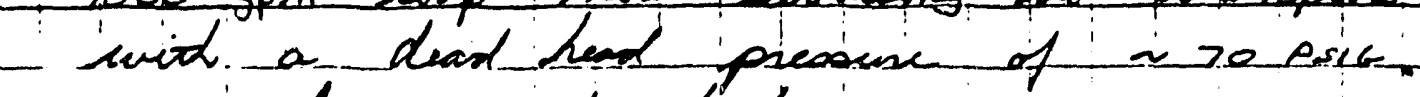

- 4000 hoop supply tank e 12.9 FT

- 4000 loop-enpply irate 72 of

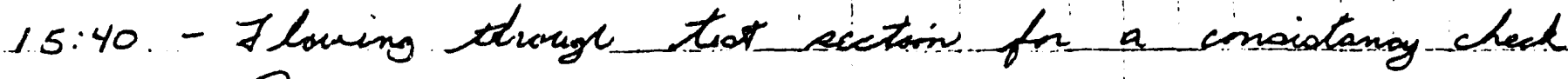
........200 ppm.

$$
\begin{aligned}
& \text { 15:49:30 - Stativin Run } \& 27-31 \text { supply temp = 810 } \\
& 16: 03-\text { flow }=185 \mathrm{gpm} @ 80^{\circ} \mathrm{F} \\
& 16.08-\text { flow = } 257.5 \mathrm{pm} @ 80^{\circ} \mathrm{F} \\
& 16: 15-\text { flow }=330 \mathrm{gPm} @ 80^{\circ} \mathrm{F} \\
& 16: 18 \text { - flow }=344 \operatorname{spm} 81.5 \% \\
& 16: 23 \text { - flow }=368 \mathrm{gpm} \text { Q } 81 \% \\
& 1625-\text { flow }=289^{-9 p m} 82^{\circ} \mathrm{F} \\
& 16: 29=\text { flow }=212 \mathrm{spm} \text { e } 82^{\circ} \mathrm{F}
\end{aligned}
$$

$16: 33$ - Stopped flowing though tot section termprisaily to

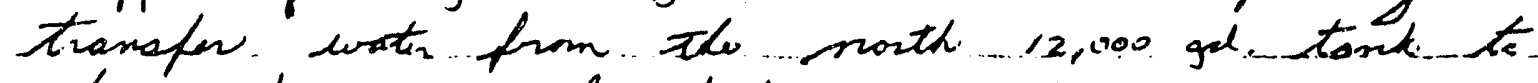
the souter 1,2,000 sol tank.

$16: 38-f l o w=315 \mathrm{gpm}$ @ $70^{\circ} \mathrm{F}$

$16: 46-$ flow $=345 \mathrm{gm}$ (c) 79 of

(continued neat page)

\begin{tabular}{|l|l|l|l|l|}
\hline Witnessed \& Understood by me, & Date & Invented by A-7 & Date & Recorded by
\end{tabular}


rrojeci IVU. Book No.

E

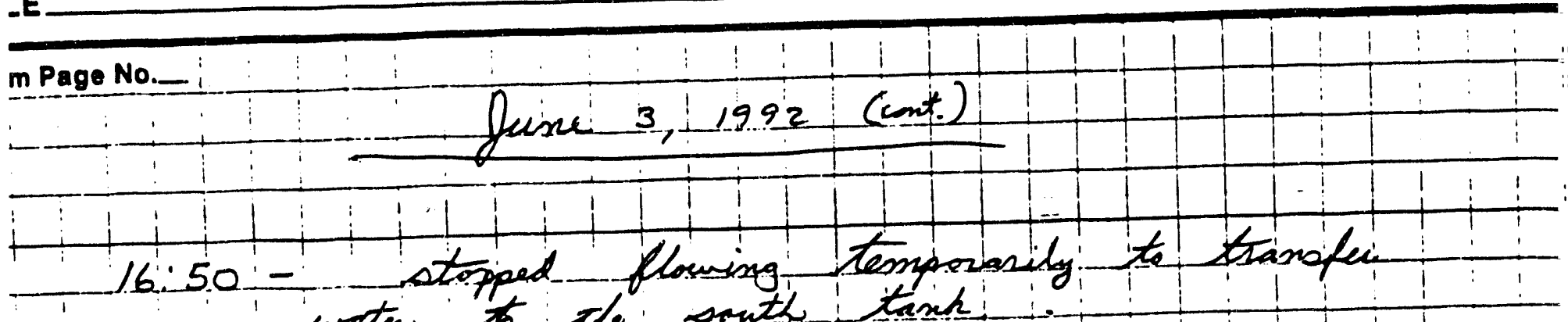
water to de sonth tran

$16: 57-$ flow $=368$ gpm $@ 75.5$ of

$1.2 .01=$ stopped flow though fued nowemilly 17:0.2 - stopfed … yo0 -gpm! permp

- taking ..... zenos

- Troper out of upe plighty ty leopor woirs

- sunning - past toot checks fos sum 827_31.

- hower supply uata an duomflow hosp !

fune 4,1992

Sppone

Analgzing date to detimine if the presouse drop acroos the fuel asembly onatch that from hofies

15:10 - draining the inlet sesernion and finel desanily in preposation for the ingection of the fuel aserme

- apened sesevior drain and vent.

- opened coutlet plemum drain and vent.

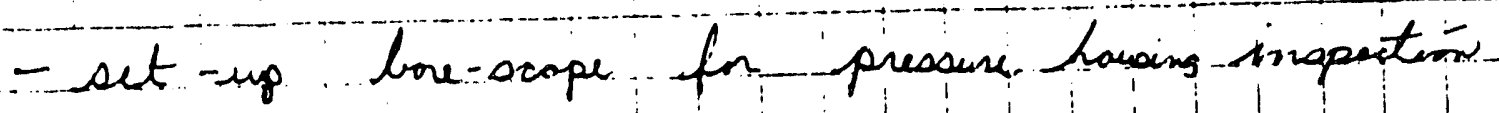

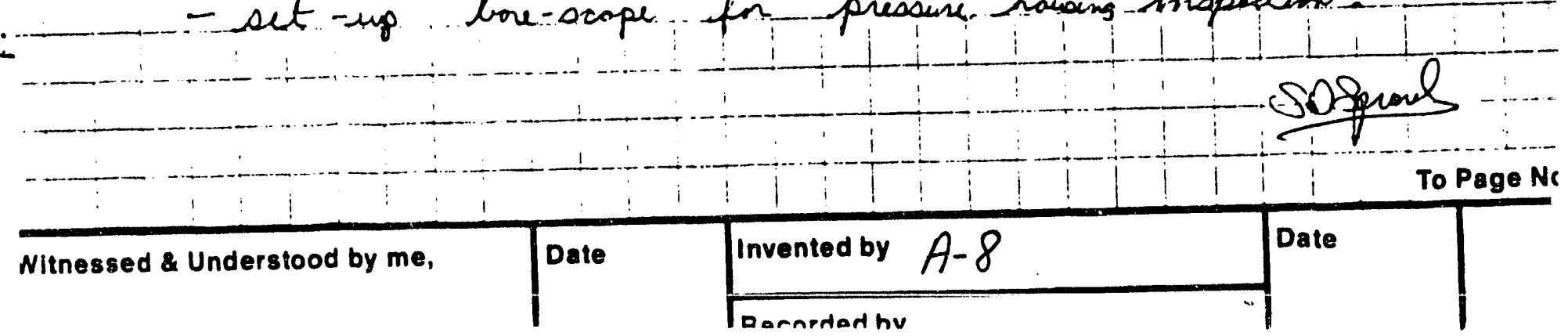


Book No.

fese -1992

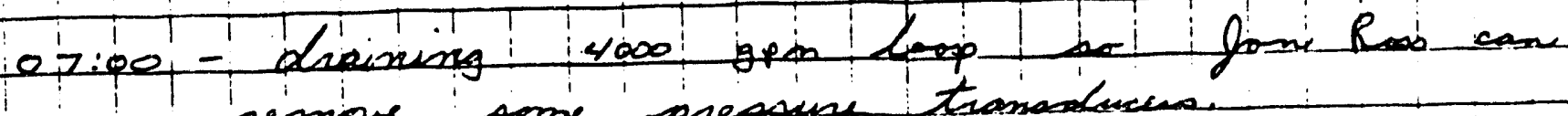

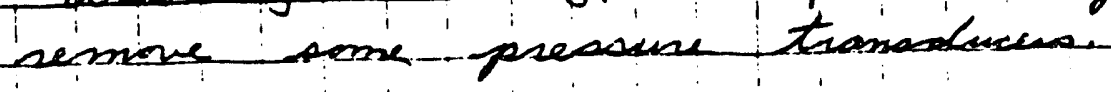

- copened the bettom of thentest phenem

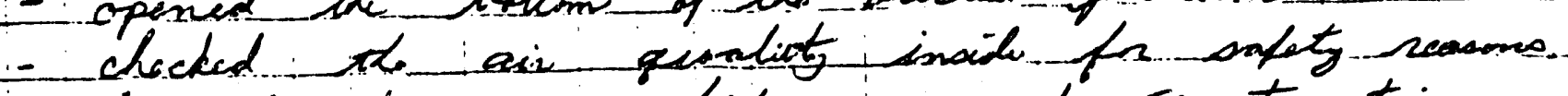

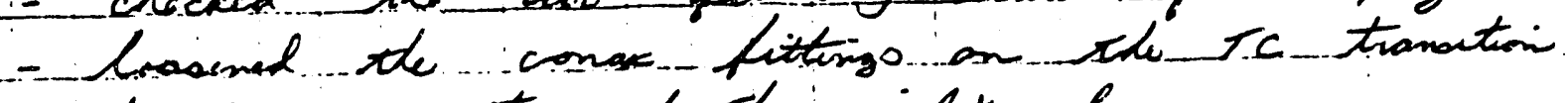
lowe on top of th inlet plenum.

- liftid te top extesision opol up 18 " fading th TC. wises though while doing eo.

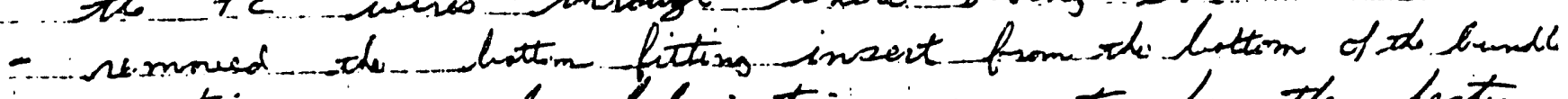
- encetion pevonnel fabicating suppents fo the hester.

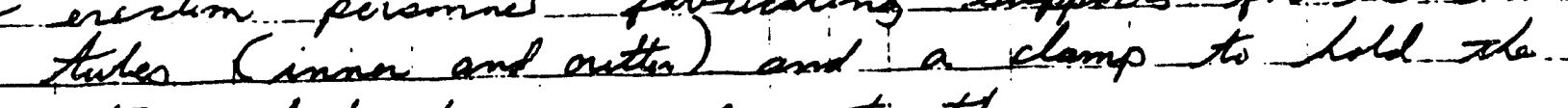
twe elestricd connector togetheo.

- pesformed a visual inspection in the top of the pressese hruaing; sothing secmo te be out of -plare or -lase. (sce vider).

Gine 6, 1992

$07: 00$ - preparing pressure houting for disasiombly: - suppentiong hesters (sinnes esd actor). from alme

- nemoued ... crunto weight casm and weights

- clampes brth heats (coppen) Whatis togother to hep them from separatisin: (see sketch noit page)

- suppostid tho heate hlaho brace from the besmo above.

- semored all of the conas packing glando from the TC boul on de top sinseriad that all of th thannocouplos would

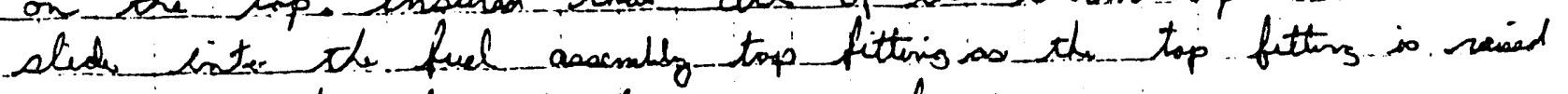
te expose th top of -to preaure housing:

Sa Sringl ToPage No.

Witnessed \& Understood by me,

Date

\begin{tabular}{|l|l|}
\hline Invented by $A-9$ & Date \\
\hline Recorded by &
\end{tabular}


TLE

Book No.

om Page No. -

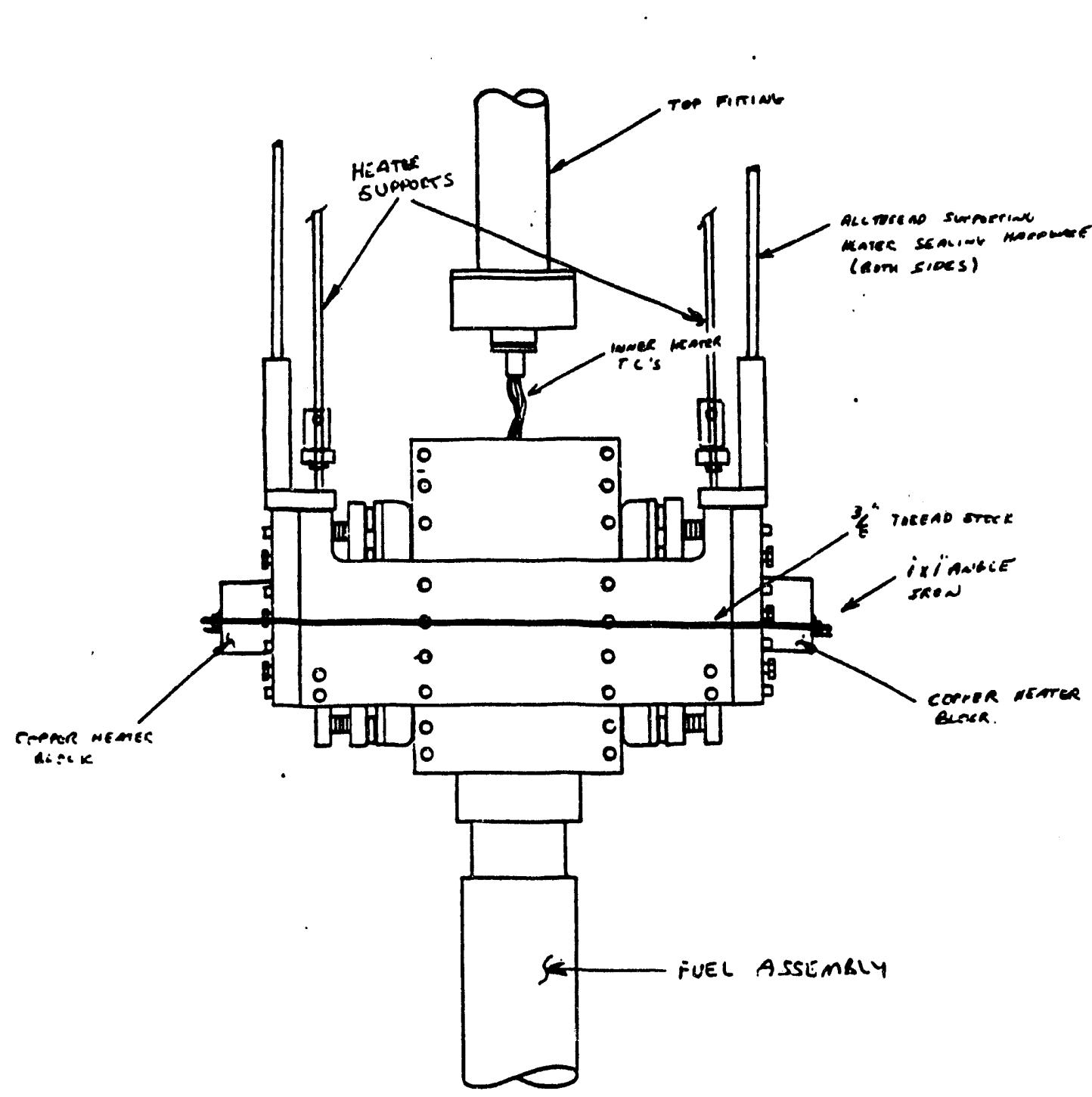

func $81992(c \sin )$
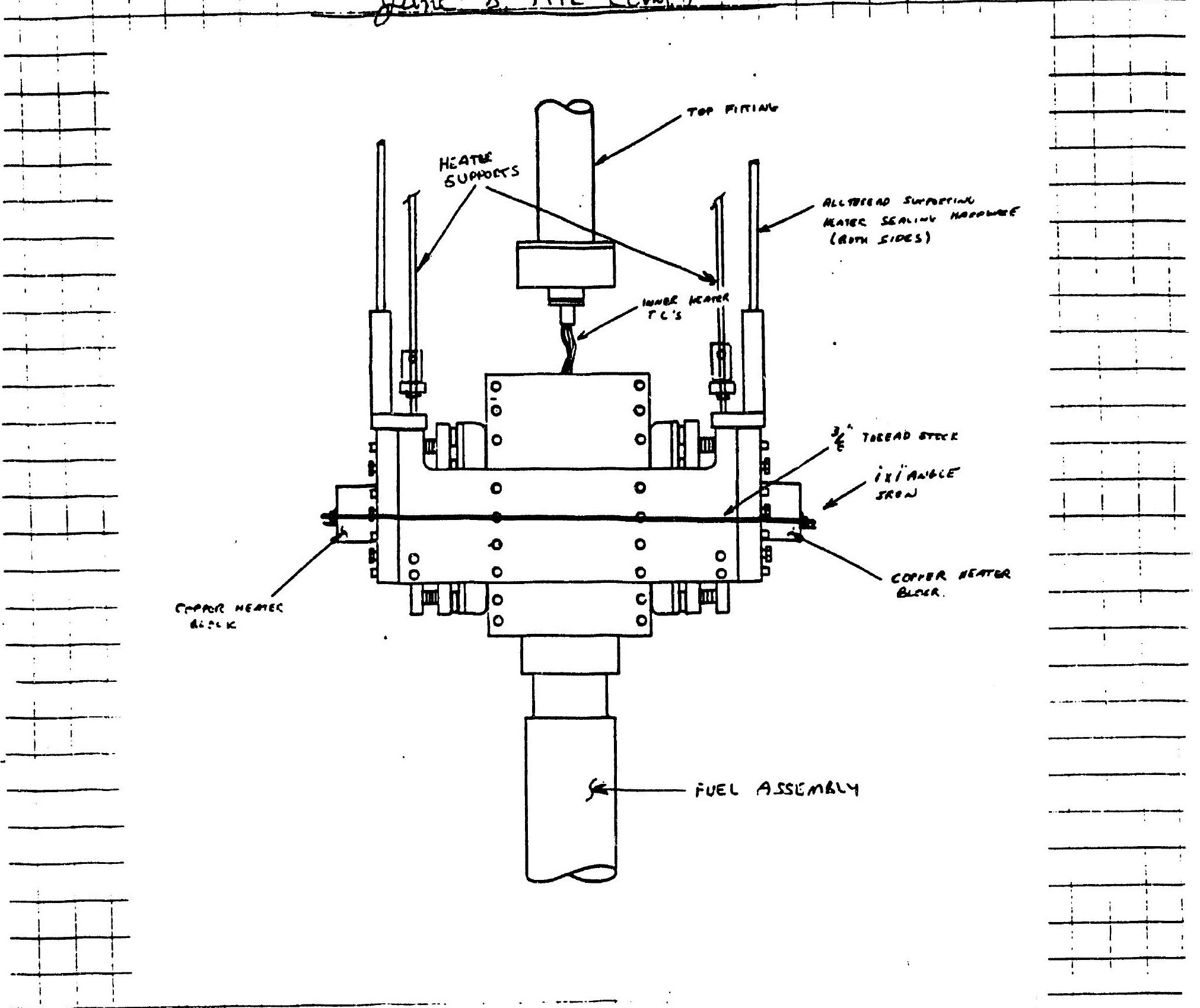

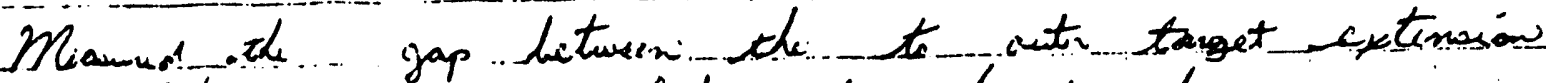

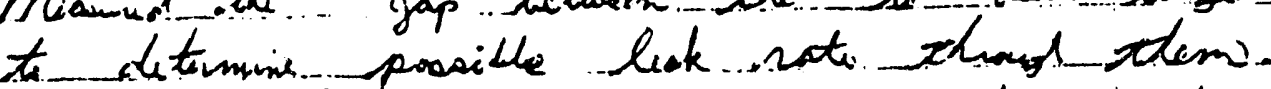

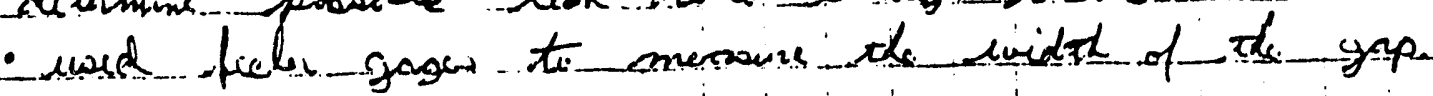
see dhemins onstit-poge 
Book No.

LE

in Page No.-

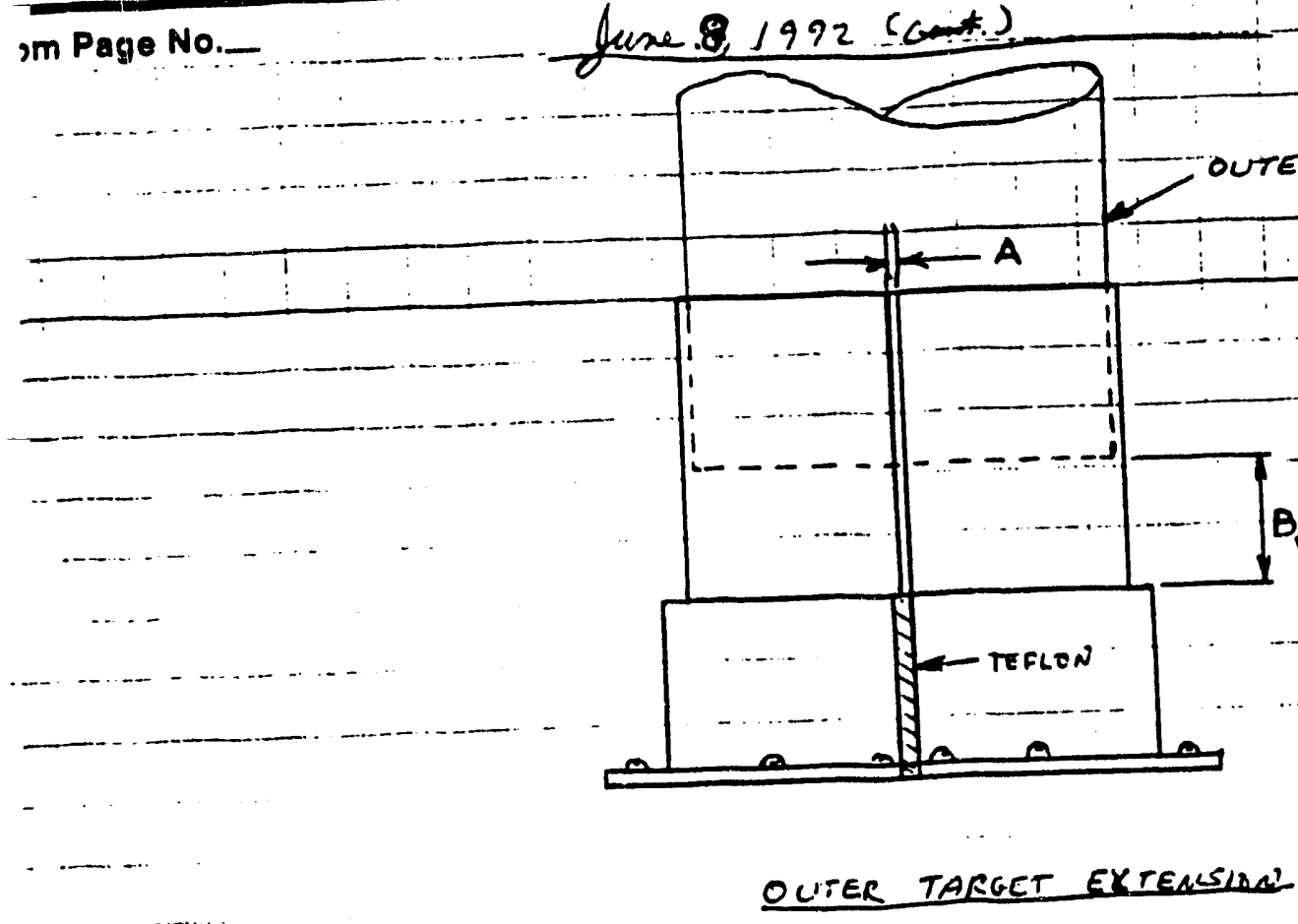

DIMENSION " $B$ " above IS ACquired by

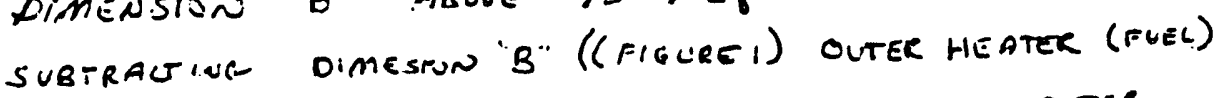
EXTENSION PRIM TSP FITTING FLANGE. FR EM OUTER TARGET EXTEUSIUA TO PRESSURE HOMING SURFACE WERE TA TIMING FLANGE IS CONNECTED. THE OUTER FUEL EXTENSION SEALS THE Gal. , over the distance it is inserted, with TEFLON BAND

- finisher remiviar the pressure housing components PND REMOVEO THE IARGET EXTENSIONhahea.

- Prepared ide two hales of the outer tongerextanaion for

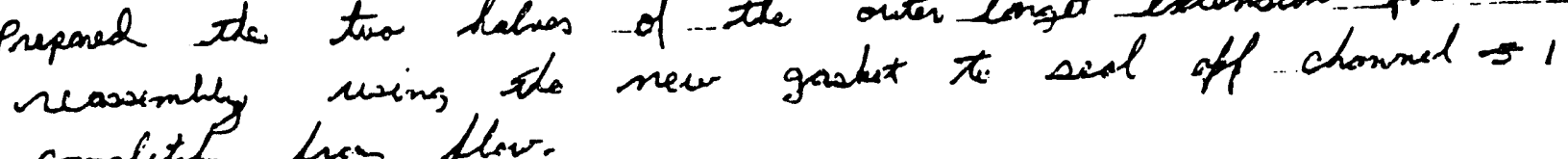
complete from flow.

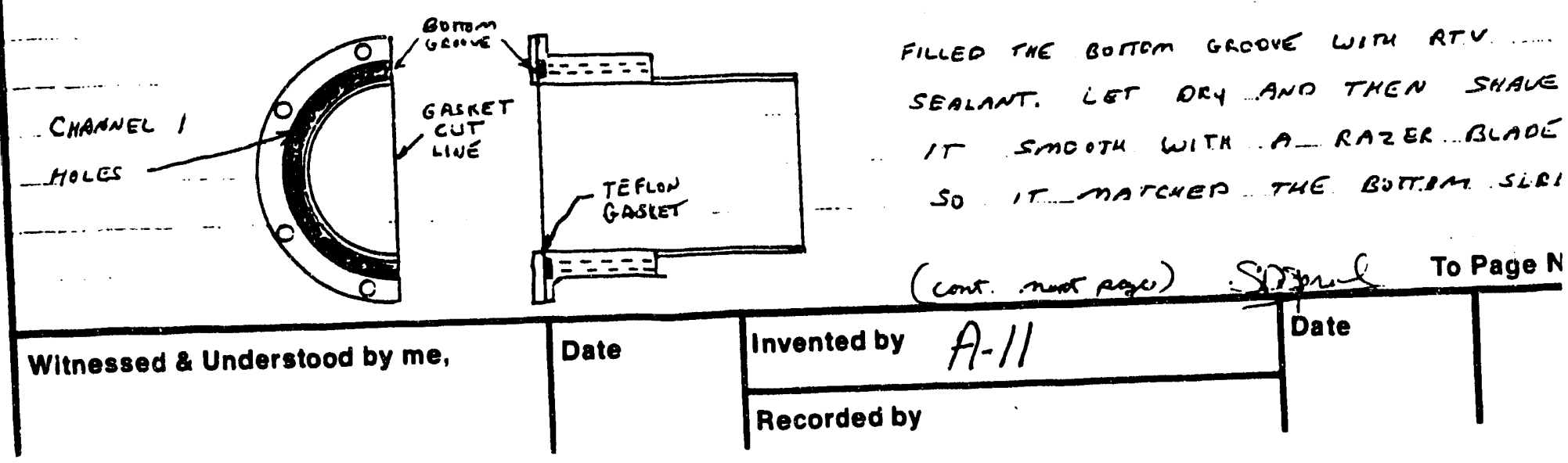


From Page No.-

fune 9.1992

a new teflow gakes inow aned to conea

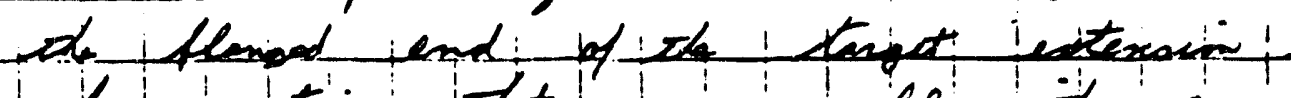

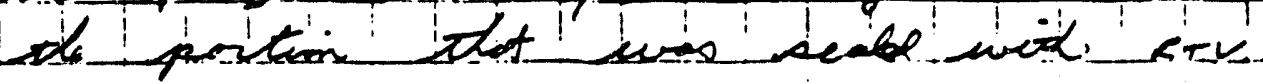

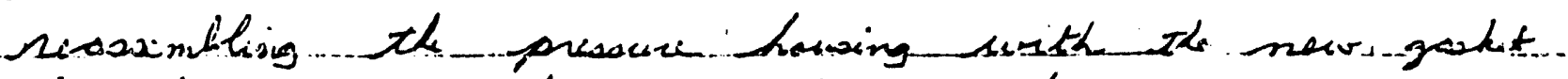

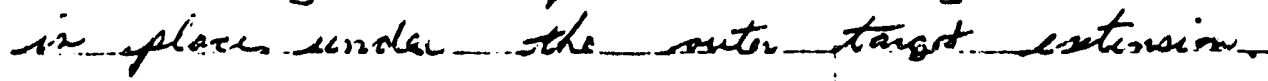

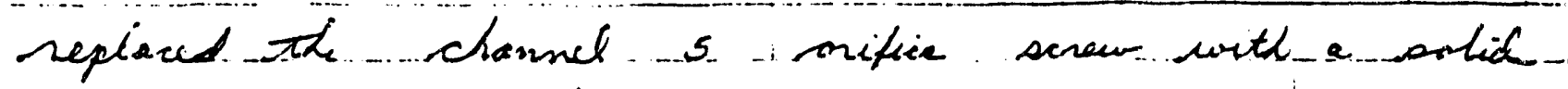
screw. see ... videos.

Gene 10,1992

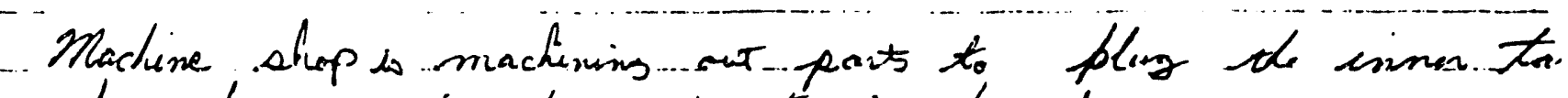

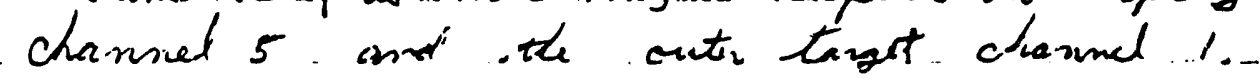

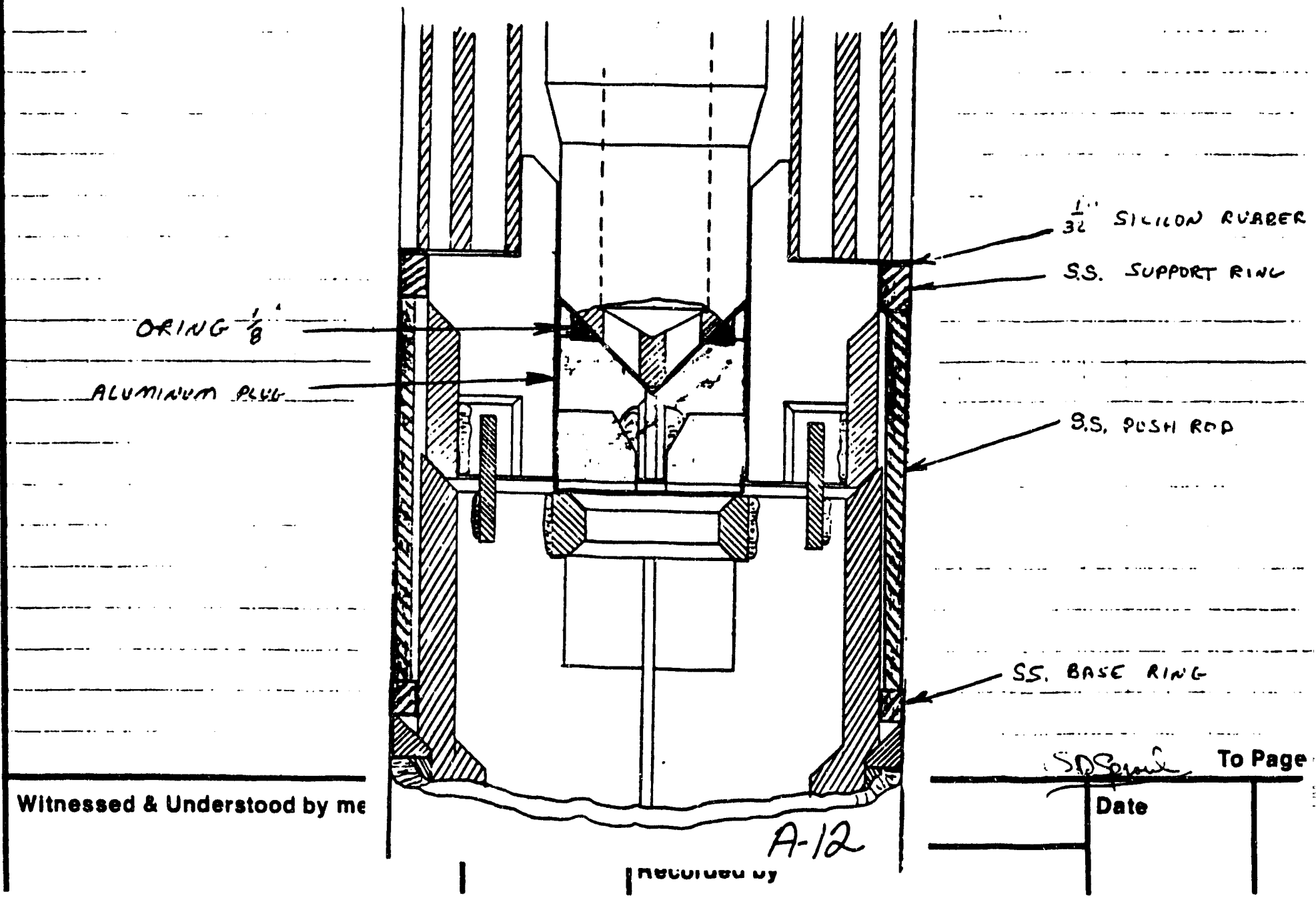


1LE

From Page No.

Oune 10,1992

The seabs aud it Lidente

the inme tanget and the

Iouta tarot an idesignél

to senl with the Bottom

Fetiono enout in it.

propes proition ap againot.

the uppes sinuside faxe of

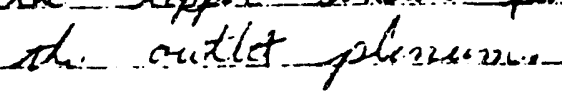
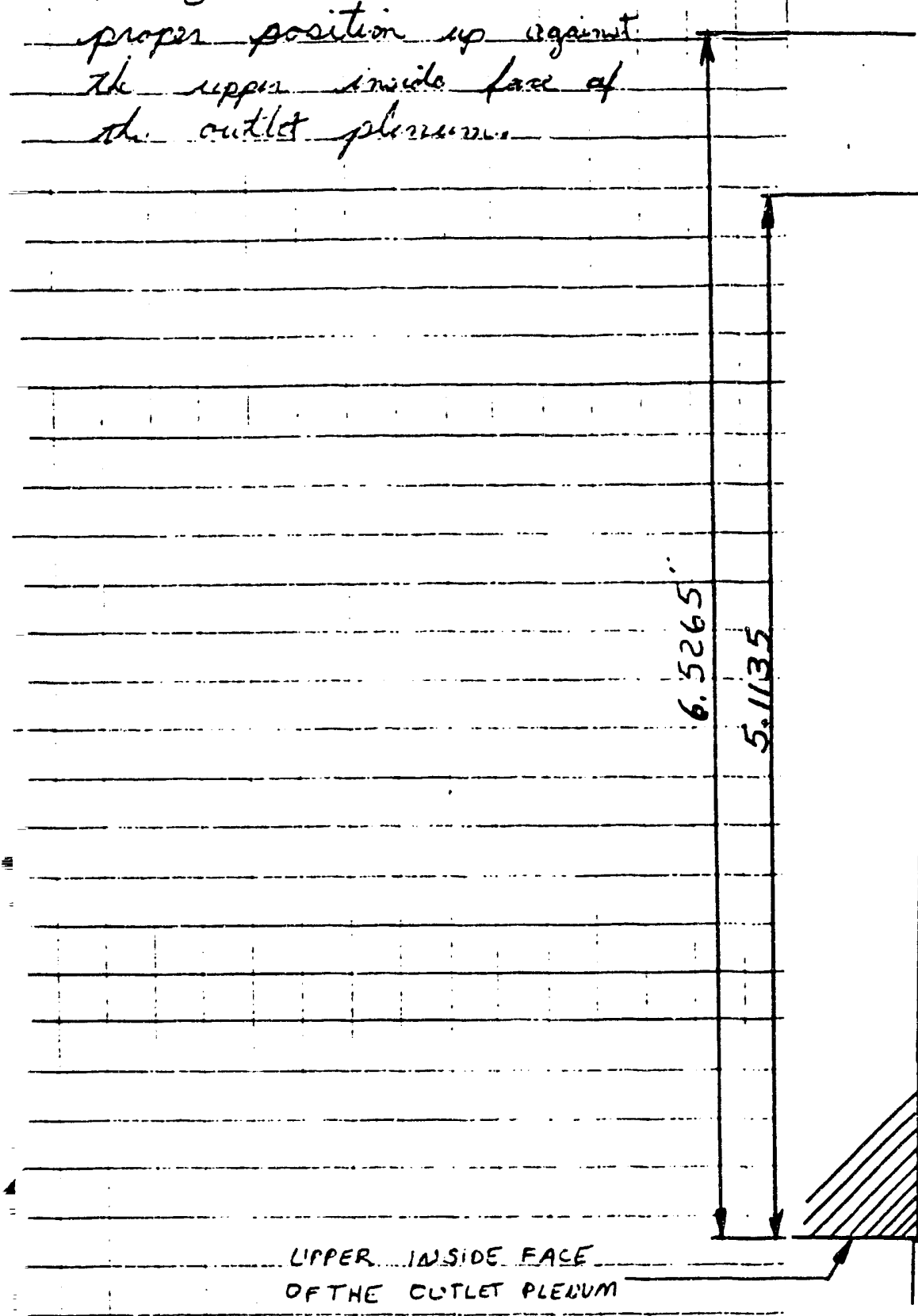
Book No.

TLE

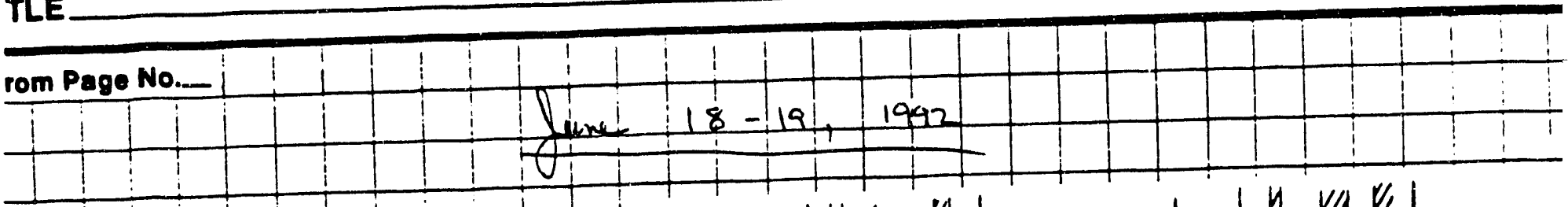

Some of the dismentions cudid to plose the scial 'wirch piopen com = previon.

The chansal is phes enas machinesed binges than ineinded so that uben put in plice it would show- the same oveisige diminaria: at the uppen indelfece of the oul plemum.

Thio ans done with ost th 0 -sing in plane to insene a netal to conetu context the dimersion of gsop hetereesi ds sepper inaid farc of the autlet flemern, (UI F O.O.P), and th SEI proitiming amfese hip is I them marhemed off of de botton of the jelug indis

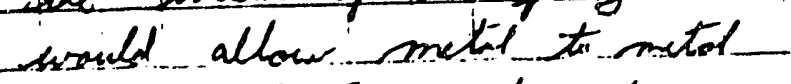
contact hetween to plug and

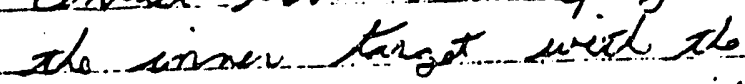
a EI in it proper fesitiv. With the o sing adiled afters ds anill allim it to be

- compresid $\sim 1 / 32$ of a inc
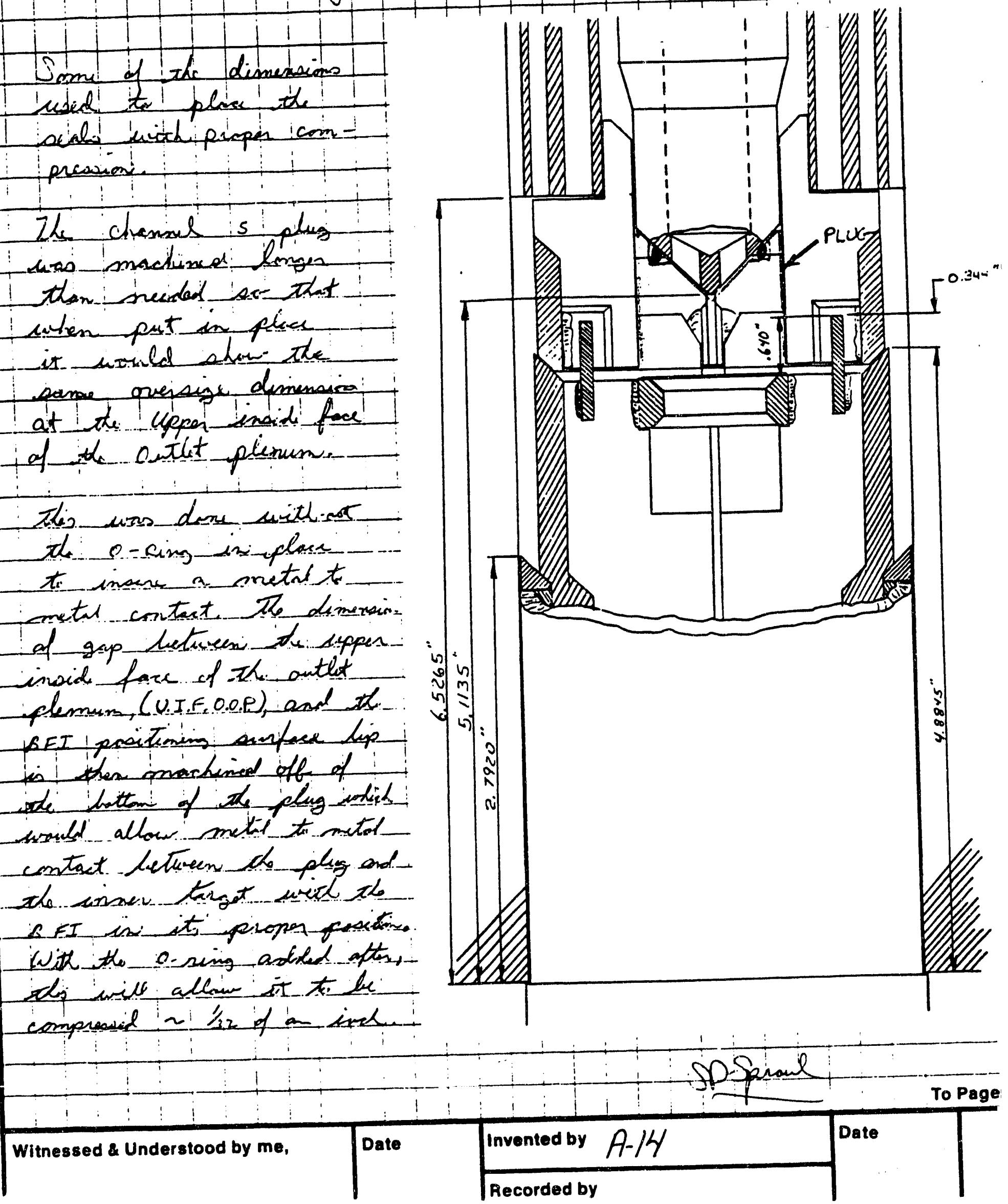
TLE

rom Page No._:

func $18+19,1992+(0,29)$

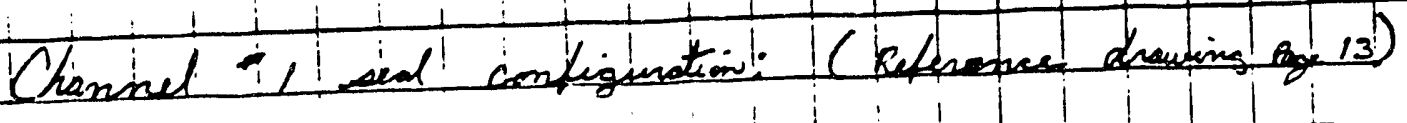

Channal 1 seal consinto i $\frac{1}{32}$ RED SICICNE of a 132 ... sultur sud _ SUBSER SEAL

materiot glued to a suppost sing. Tha is to tor heid in place by de pirctinarion has

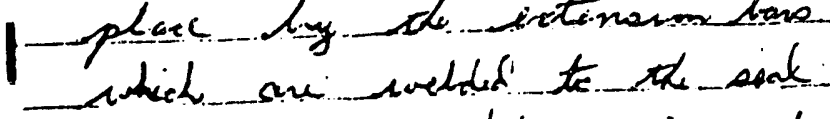
bave sing. 'Whih sizing this. 1 sual aszombly the Channal

5 plig is semoned th hup form

SEAL SUMCE RING

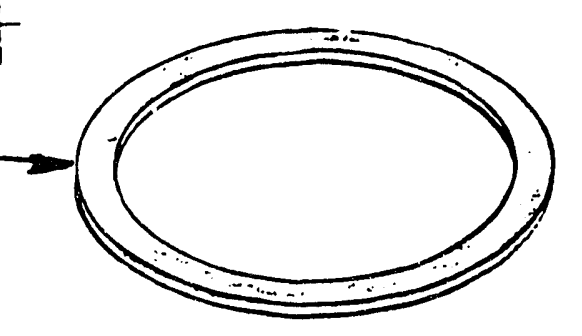

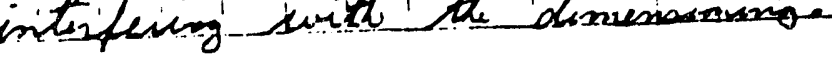

The sual asomity placis ons

Th B.F.I. and insented int

the outht plome

The gap is then mianecred

betwein the uppir inside.

fare of de cutict plonum

and the B.FI. positionsing

surface hp. This onesuremuxt minix: $164 "$ is do aromoned from $x$ th sippen isske of ats

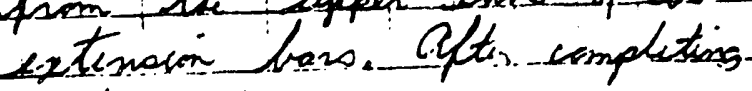

EXTENSION GARS

Thi. the gap menounerment

SEAL BASE RING

stould he in \$14"ushizh will

he compresed with $t h$ BEI

in place wire the holding hed5,

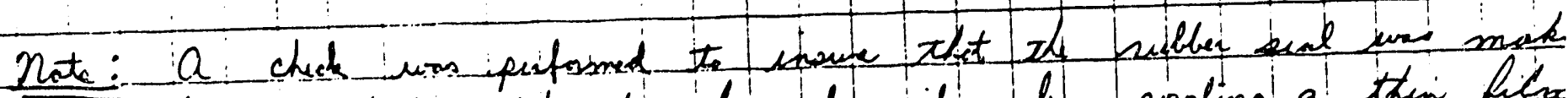
full contart syid th clanos cilo by appling a then filix

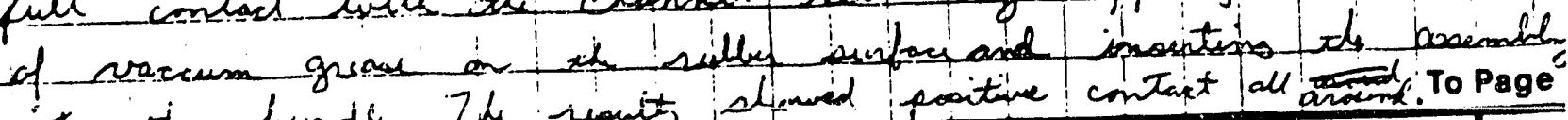

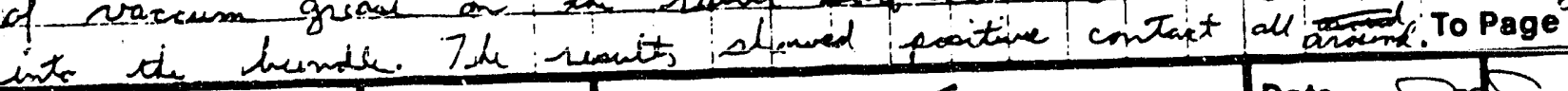

A- 15

Date
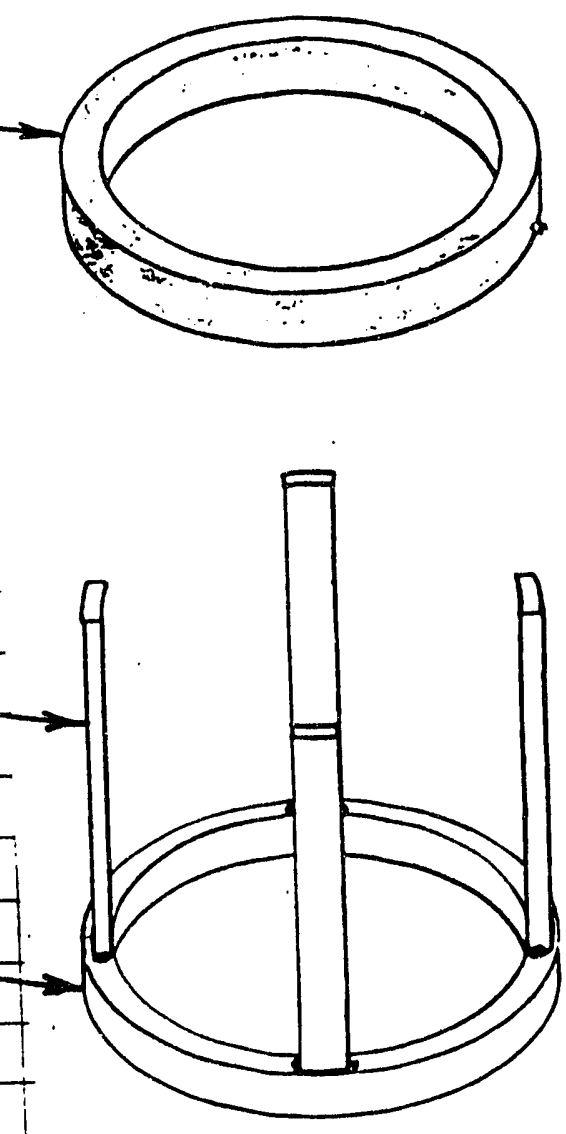
Book No.

TLE

om Page No. - ;

1 furm $18-19.992$ cont.

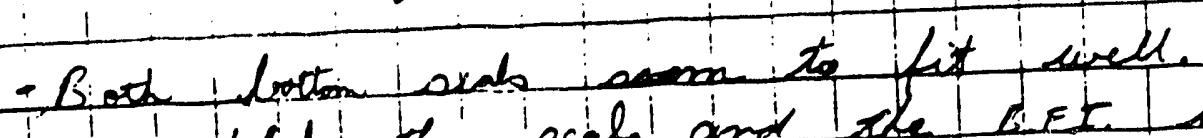

-assembled the soble and the art int the outlit pliemura.

- seconnested the prewerse taps inade tho plemeno

- closer up the plimerm and atarted fithing th

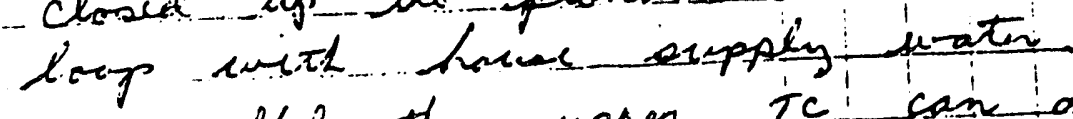

- secosimbere tho upper ic cain on th top of de upper (inker) plenumanis

- suplacert the crnax fittrios wivith th same past. that were semisweds.

Soserom

func 22,1992

08:00 - Repinaed c.20roz 0.10 rsio thamomitto with a spaw due ...t. its slusgin openation.

$$
\begin{aligned}
& \text { CLO } \mathrm{g} / \mathrm{w} S \mathrm{SN}=880 \leq 19 \\
& \text { NEW } \mathrm{B} / \mathrm{WN}=880522
\end{aligned}
$$

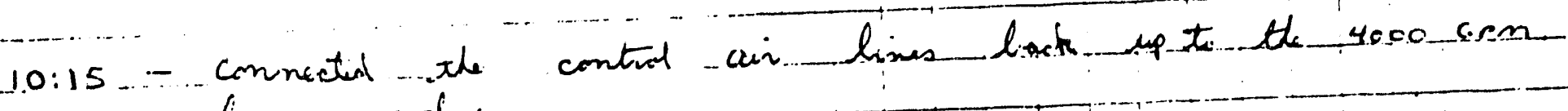
lorp .... value.

- 4000 cen (weat) pump on

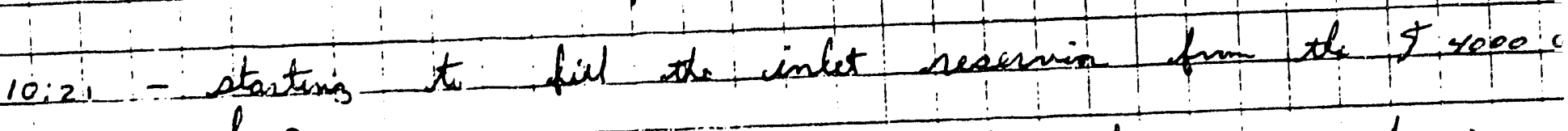

loop.

- varvia almas full maticed that $x d$ privine housing ahe top of the hirdle is leaking move than nowno $10: 42-4000$ - 69 M PUMP OEF

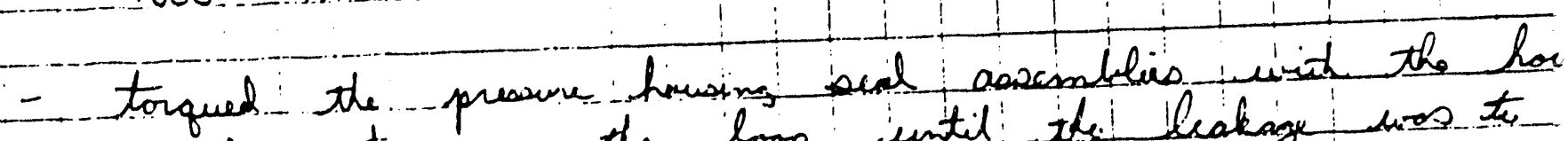
supply unate on the loop suntil th leakag wos th minnimum.

Witnessed $\&$ Understood by me,

Date

sonsprant

To Pag

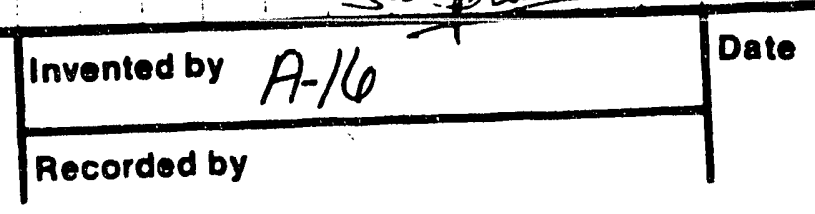


rom Page No. -

funse $22,1992\left(K_{\text {cont }}\right)$

11233 - 4000 afen ureat pump ion

nesionis is baw fill ventiong iff the inlet plemasosa.

11:50 - Pump off

- backfilling all of the pp's.

- fusare filling all of to AP's

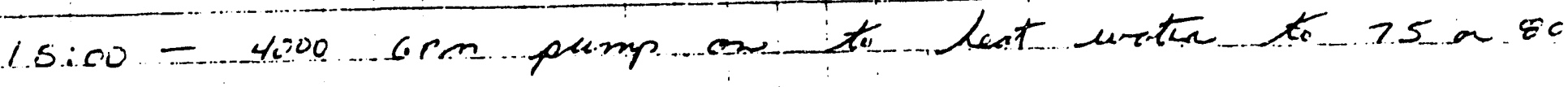

16.40 - wate temp is at 79 of decided to rew teat in the minining - wint 4ine gfm - qump-off.

Snsperal

Cun $\frac{\operatorname{func} 23+1992}{82252}$

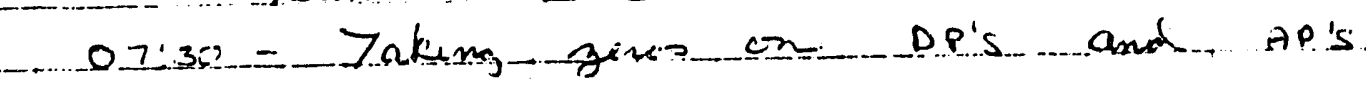

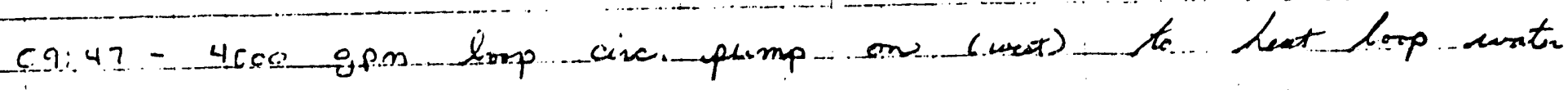

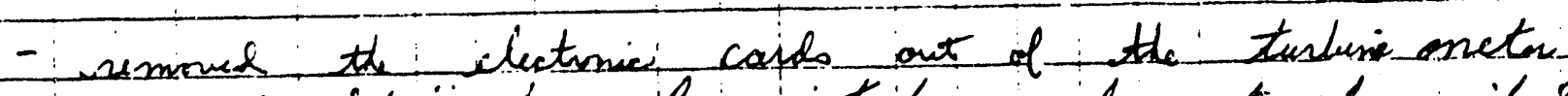

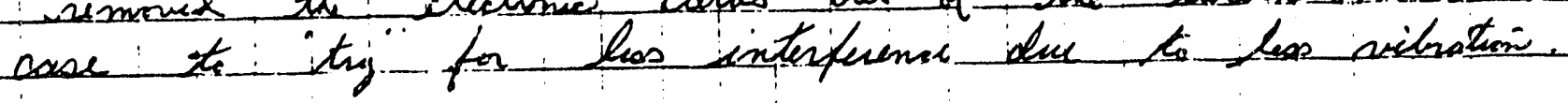

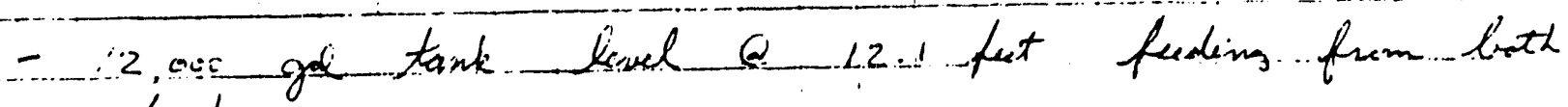
tanks.

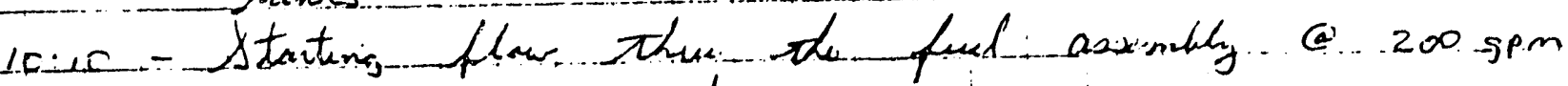

- surmining Coivis chask

10.15 - Stoppil flow than ful arsembly

10.22 - Statat flow again fir tot sumo...

\begin{tabular}{|l|l|l|l|}
\hline Witnessed \& Understood by me, & Date & Invented by A-17 & Date
\end{tabular}




\section{From Page No.}

\section{$\operatorname{com}=82732$}

10:27- betting Lip fing sum e 182 igem 28.5 pas de RTA $10.31+2$ ind dun 2156 gen

$10: 37+3$ ed surn@ @ $333 \mathrm{gpm}$ - sixply tanth levele moth $=8 f t$ esuth $=9$ ft

$10 \cdot 10-4 \div$ sun (A) 344 gPe

$1045-52$ sun Q 286 sfm - hop inlet tompantus = $77^{\circ} \mathrm{F}$ e ICerai

$10 \cdot 99-1: 16$ sun a 218 grm $18: 55-716$ sune e $316-50 n$ $16: 52-8$ th $\operatorname{sen}$ C $345 \operatorname{srn}$

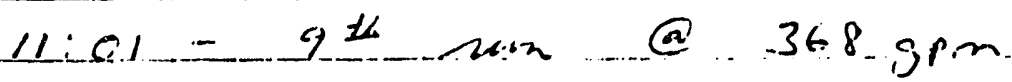
$=10^{\prime \prime}$ sum e $e^{-371}$ spen the wet pump tripped off $11: 15$ - tewnef 4000 spm nest pump hack on e. tupped

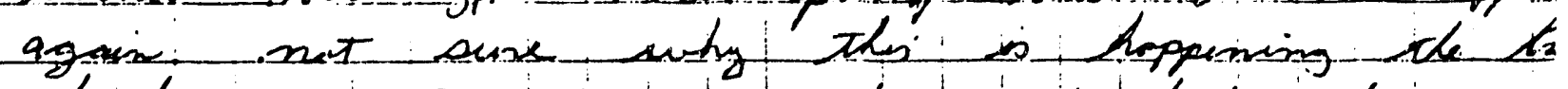

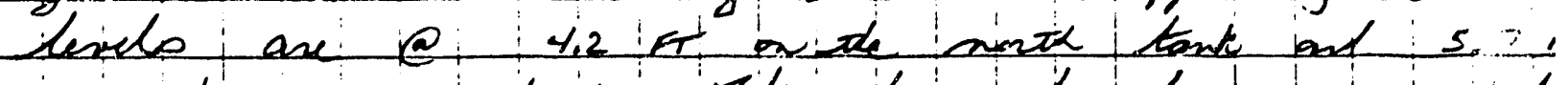

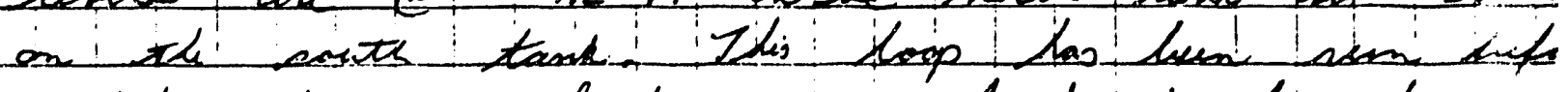

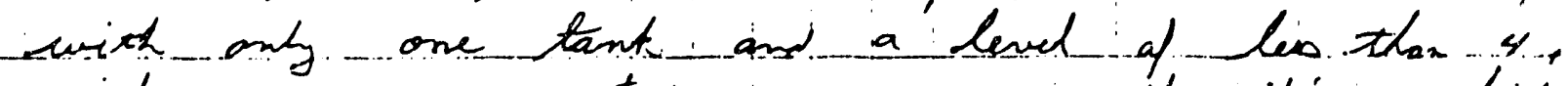
without a pump trip. I not ane if its a high tup or a high temp trip.

letting th purs ect oves lund so it can cond (if that the ponhlem) aldo filling th smute tank.

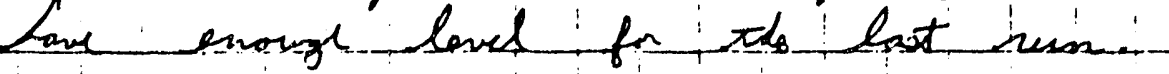

\begin{tabular}{|l|} 
Invented by $A-18$ \\
\hline Recorded by
\end{tabular}


From Page No._.

fun $23,1992(\sin x)$

$12.45=$ South tank loved in now at 9.5 fut

- Turned the west 4000 ppm pump ox to hent up the ito to is -po of ?

$13: 51$ - wat ir temperature es the tank is now 77 of

- tank lived is... Q 10 z pat

13:55- Starting flew then to fat resemble

13:59- taking dit for tho last sum e $371 \mathrm{gsm}$ Kun $827-3.3$

14:01 - Stopped dat save for sun $-827-33$

14:03 - Wert 4000 gem pimp off

14:10 - taking pert teat zines on the DP's and Ap's

- Morning scant frugrom

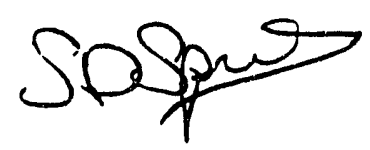

To Page Ni

\begin{tabular}{|l|l|l|l|}
\hline Witnessed \& Understood by me, & Date & Invented by $A-19$ & Date \\
\cline { 3 - 4 } & & Recorded by &
\end{tabular}




\section{APPENDIX B}

Instrument Records Flow Excurison Follow-on Testing 
1.

RD-45A Rev. $11-28-91$

RESEARCH C DEVELOPMENT DIVISION INSTRUMENT SERVICE LOG

\begin{tabular}{|c|c|c|}
\hline INSTRUMENT STATUS & 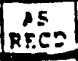 & $\begin{array}{l}\text { AS } \\
E T S \\
\end{array}$ \\
\hline Certified & & \\
\hline Calibrated & & \\
\hline For Ind. Only & $\checkmark$ & \\
\hline standardize & & \\
\hline certified s stand. & & \\
\hline 5 callbrated stand. & & \\
\hline , other & & \\
\hline
\end{tabular}

INSTRUMENT CONDITION

AS RECEIVED (Certified Equipment only)

Meets Manufacturer's Tolerance $N$

Operates within previously defined limits $N$ Deviates from Mfg or previously defined limits $Y$ Inoperative (Describe below) $Y$

AS LEFT

Limited (Describe below)

Meets Manufacturer's Toleranceps other (Describe Below)
Babcock \& Wilcox

a MeDermotr company

B\&W Ser. No. 0880121

SENSOTEC

TJE/708-10

TRANSMITR, PRES

190882

4427-01-020

PROPERTY NO.

ORDER NO.

$\begin{array}{lc}\text { SECTION } & 46 \\ \text { IOCATION } & 115 \\ \text { SCHEDULED (Y-N) } & -\not 4 y \\ \text { RECAL INT.(MOS.) } & 12\end{array}$

TECH. PROCEDURE A0015

Babcock Wilcox RED Division certifies that the performance of this instrument was verified using standards whose accuracies are traceable to the National Institute of standards and Technology, National Institute of a natural physical constant, a ratio type of calibration technique, or to a consensus standard.

\section{STANDARDS USED}

Manufacturer

Model

Item

BCW Serial No.

7 SHCROFT 0 -100PSI PRESS, STD, 1. FluKE $8840 A$ $D M m$

\begin{tabular}{l|l|l|l|l|l|l}
0 & 7 & 0 & 0 & 1 & 2 & 3 \\
0 & 8 & 6 & 0 & 4 & 0 & 4
\end{tabular}

Vendor Cert/Cal/Repair Repair Parts

TOTAL MATERIAL COST

OTHER EOUIPMENT USED

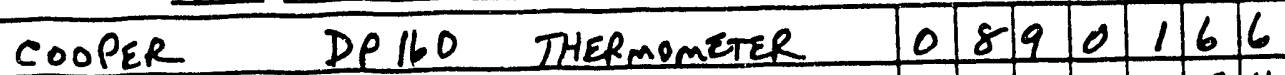
COMPAR PORTARLE COMPVTER

\begin{tabular}{lllllllll}
\hline & & & & & & & \\
\hline & & & & & & & \\
\hline & & & & & & & \\
\hline & & & & & & & \\
\hline
\end{tabular}

TOTAL LABOR HOURS TOTO!1.0 Requested By S, SPROUL Charge Number H4160-102-002 Work Order No. 0296

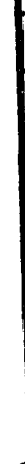

Service Notes $0-100$ PSIG

SEE ATTACHED PATA SHEET,

LIMITED TO DATA PROVIDED, DOES NOT MERT 4:1 RaTTO SPECS. CALIBRATES AS A UNIT WITH 0880123 AMPL. ITRANLDUCER 
AS RECEIVED

TRANSMITTER:

$$
\text { - }
$$

PRESS SOURCE

$$
\begin{aligned}
& \text { ASHCFIOFT } \text { DIGIGAUGE } \\
& \text { O-1OO FSI } \text { FS } \\
& \text { B\&W SER. NO. } 0700123 \\
& \text { TOLERANCE: } 0.05 \%
\end{aligned}
$$

READOUT DEV:
AS

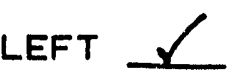

Ambient Temperature (F) 75

Facility I. D. Name

BFAFO1

\author{
B\&W SER. NO. O860404 \\ TOLERANCE: $0.015 \%$
}

\begin{abstract}
VALUE OF CURFENT SENSING FESISTOF USED FOF TEST DATA VALUE OF .CURFENT SENSING RESISTOF USED FOF' CALIBFATION

FESISTOF MEASUFING ACCURACY

14.996 OHMS 14.996 OHMS N.A.
\end{abstract}

FOWEF: SUFF'LY VOLTAGE

TOTAL SEFIIES RESISTANCE

AF'FLIIED F'RESSUFE TFANSMITTEF F'SI FFESS SOUFICE FSI

$\begin{array}{rr}0.000 & 0.000 \\ 10.000 & 10.000 \\ 20.000 & 20.000 \\ 30.000 & 30.000 \\ 40.000 & 40.000 \\ 50.000 & 50.000 \\ 60.000 & 60.000 \\ 70.000 & 70.000 \\ 80.000 & 80.000 \\ 90.000 & 90.000 \\ 100.000 & 100.000 \\ 90.000 & 90.000 \\ 80.000 & 80.000 \\ 70.000 & 70.000 \\ 60.000 & 60.000 \\ 50.000 & 50.000 \\ 40.000 & 40.000 \\ 30.000 & 30.000 \\ 20.000 & 20.000 \\ 10.000 & 10.000 \\ 0.000 & 0.000\end{array}$

\begin{tabular}{cccc}
24 & \multicolumn{1}{c}{ N.A. } \\
14.996 & CALIEFIATION ACCUFACY & $0.17 \%$ \\
& MELIMITED** & \\
IDEAL & MEASUFED & EFFIOF \% \% & OUT \\
OUTFUT SIG & OUTFUT SIG & FULL SCALE & OF \\
MILLIVOLTS & MILLIVOLTS & & TOLEFANCE
\end{tabular}

59.984
83.978
107.971
131.965
155.958
179.952
203.946
227.939
251.935
275.926
299.920
275.926
251.935
227.939
203.946
179.952
155.958
131.965
107.971
83.978
59.984

$0.01 \%$

$0.00 \%$

$0.00 \%$

$0.00 \%$

$0.01 \%$

$0.02 \%$

$0.02 \%$

$0.05 \%$

$0.05 \%$

$0.05 \%$

$0.06 \%$

$0.02 \%$

$-0.01 \%$

$-0.02 \%$

$-0.06 \%$

$-0.06 \%$

$-0.05 \%$

$-0.06 \%$

$-0.04 \%$

$-0.01 \%$

$0.00 \%$ 
CURVEFIT OUTPUT FOR VTAB bfap01

VOLTAGE(V) OBS. PRESS. CALC. PRESS. DIFF. (PSIA) \& DIFFERENCE
DATE: 20-MAX-92

TIME: $16: 55: 19$

(PSIA)

(PSIA)

$\begin{array}{cccccc}1 & 0.1899954 \mathrm{E}-04 & 0.0000000 \mathrm{E}+00 & 0.7316241 \mathrm{E}-02 & -0.7316241 \mathrm{E}-02 & 0.0000000 \mathrm{E}+00 \\ 2 & 0.239900 \mathrm{E}-01 & 10.00000 & 10.01128 & -0.1128313 \mathrm{E}-01 & -0.1128313 \\ 3 & 0.4798100 \mathrm{E}-01 & 20.00000 & 20.01926 & -0.1926320 \mathrm{E}-01 & -0.9631601 \mathrm{E}-01 \\ 4 & 0.7197100 \mathrm{E}-01 & 30.00000 & 30.02249 & -0.2249103 \mathrm{E}-01 & -0.7497010 \mathrm{E}-01 \\ 5 & 0.9599300 \mathrm{E}-01 & 40.00000 & 40.03472 & -0.3471838 \mathrm{E}-01 & -0.8679596 \mathrm{E}-01 \\ 6 & 0.1200050 & 50.00000 & 50.03843 & -0.3843394 \mathrm{E}-01 & -0.7686789 \mathrm{E}-01 \\ 7 & 0.1439920 & 60.00000 & 60.02740 & -0.273980 \mathrm{E}-01 & -0.4566334 \mathrm{E}-01 \\ 8 & 0.1680600 & 70.00000 & 70.04574 & -0.4573720 \mathrm{E}-01 & -0.6533886 \mathrm{E}-01 \\ 9 & 0.1920520 & 80.00000 & 80.02810 & -0.2809871 \mathrm{E}-01 & -0.3512338 \mathrm{E}-01 \\ 10 & 0.2160620 & 90.00000 & 90.01361 & -0.1360888 \mathrm{E}-01 & -0.1512098 \mathrm{E}-01 \\ 11 & 0.2400750 & 100.0000 & 99.95602 & 0.3976312 \mathrm{E}-02 & 0.3976312 \mathrm{E}-02 \\ 12 & 0.2159920 & 90.00000 & 89.98450 & 0.1549708 \mathrm{E}-01 & 0.1721898 \mathrm{E}-01 \\ 13 & 0.1919270 & 80.00000 & 79.97610 & 0.2389886 \mathrm{E}-01 & 0.2987358 \mathrm{E}-01 \\ 14 & 0.1678890 & 70.00000 & 69.97457 & 0.2542640 \mathrm{E}-01 & 0.3632343 \mathrm{E}-01 \\ 15 & 0.1438040 & 60.00000 & 59.94913 & 0.5087444 \mathrm{E}-01 & 0.8479073 \mathrm{E}-01 \\ 16 & 0.1198280 & 50.00000 & 49.96471 & 0.3529070 \mathrm{E}-01 & 0.7058141 \mathrm{E}-01 \\ 17 & 0.9584700 \mathrm{E}-01 & 40.00000 & 39.97388 & 0.2612042 \mathrm{E}-01 & 0.6530106 \mathrm{E}-01 \\ 18 & 0.7183600 \mathrm{E}-01 & 30.00000 & 29.96621 & 0.3378845 \mathrm{E}-01 & 0.1126282 \\ 19 & 0.4788500 \mathrm{E}-01 & 20.00000 & 19.97921 & 0.2077510 \mathrm{E}-01 & 0.1038755 \\ 20 & 0.2395300 \mathrm{E}-01 & 10.00000 & 9.995845 & 0.4154973 \mathrm{E}-02 & 0.4154972 \mathrm{E}-01 \\ 21 & -0.1900046 \mathrm{E}-04 & 0.0000000 \mathrm{E}+00 & -0.8545972 \mathrm{E}-02 & 0.8545972 \mathrm{E}-02 & 0.0000000 \mathrm{E}+00\end{array}$

SGREE OF POLYNOMIAL 2

COEFFICIENTS

$-0.61467204 \mathrm{E}-03$

$0.41742666 \mathrm{E}+03-0.37661537 \mathrm{E}+01$

ESS $=0.1521 \mathrm{E}-01 \quad \begin{aligned} \mathrm{SD} & =0.2907 \mathrm{E}-01 \\ 2 * S D & =0.5814 \mathrm{E}-01\end{aligned}$

$\mathrm{R2}=$

$0.999999 E+00$ 
$\checkmark$

RD-45A ReV. 11-18-91

\begin{tabular}{|c|c|c|}
\hline INSTRUMENT STATUS & \begin{tabular}{|l|l|}
$A S$ \\
RE.CS \\
\end{tabular} & $\begin{array}{ll}\text { AS } \\
\text { LETT }\end{array}$ \\
\hline 1. Certified & & \\
\hline Calibrated & & \\
\hline 3 For Ind. Only & $\checkmark$ & \\
\hline 4 Standardize & & \\
\hline 5 certified stand. & & \\
\hline 6 Calibrated stand. & & \\
\hline 9 other & & \\
\hline
\end{tabular}

INSTRUMENT CONDITION

AS RECEIVED (Certified Equipment only)

Meets Manufacturer's Tolerance $N$

Operates within previously defined limits $N$ Deviates from Mfg or previously defined limits $Y$ Inoperative (Describe below) $y$

AS LEFT

Limited (Describe below)

Meets Manufacturer's Tolerance other (Describe Below)
Babcock \& Wilcox

- McDermott company

B\&W Ser. No. 0880542

MANUFACTURER

MODEL

ITEM

MANUF. SER. NO.

PROPERTY NO.

ORDER NO.

SECTION

IOCATION

46

SCHEDULED $(Y-N)$

RECAL INT.(MOS.)

115

- $y$

12

TECH. PROCEDURE A0015

Babcock \& Wilcox RCD Division certifies that the performance of this instrument was verified using standards whose accuracies are traceable to the

National Institute of Standards and Technology, an accepted value of a natural physical constant. a ratio type of calibration technique, or to a consensus standard.

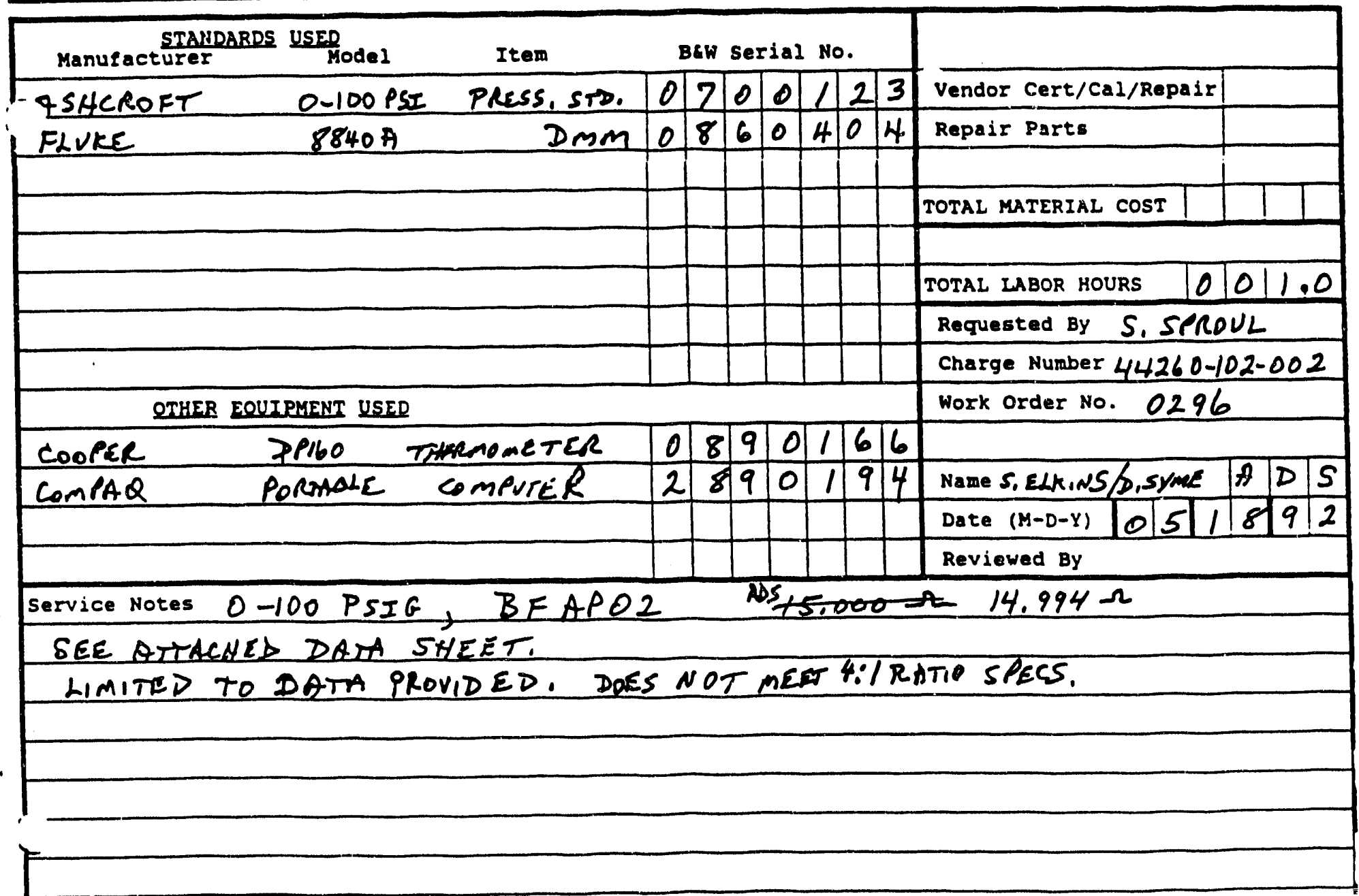


AS RECEIVED AS LEFT
FIANSMITTER:

'RESS SOURICE
EADOUT DEV:

\section{SENSOTEC TJE 100 FSI}

TOLEFIANCE: $0.10 \%$

$$
\begin{aligned}
& \text { ASHCROFT } \text { DIGIGAUGE } \\
& 0-100 \text { FSI } \\
& \text { E\&W SER. NO. } 0700123 \\
& \text { TOLERANCE: } 0.05 \%
\end{aligned}
$$

\section{FLUKE 8840A \\ B\&W Ser. NO. 0860404}

TOLEFANCE: $0.015 \%$
B\&W S.N. O880542

DATE: 18-MaY-92

FROCEDURE : ARC-TF-015-0B

FROGRAM: TOO15_0B.03A

NAME : S, ELKINS\$. Sÿme

Ambient Temperature (F) 72

Facility I. D. Name

BFAF'OZ
ALUE OF CURFENT SENSING RESISTOF USED FOR TEST DATA 'ALIJE OF "CUFFENT SENSING RESISTOR USED FOR CALIBRATION OWEF SUFFLYY VOLTAGE UTAL SEFIES FIESISTANCE

AFF'LIED F'RESSUFE FIANSMITTEF: PSI FFEESS SOURICE

0.000
10.000
20.000
30.000
40.000
50.000
60.000
70.000
80.000
90.000
100.000
90.000
80.000
70.000
60.000
50.000
40.000
30.000
20.000
10.000
0.000
FESISTOF MEASUFIING ACCURACY F'SI

$$
24
$$$$
14.994
$$

IDEAL

\section{DUTFUT SIG} MILLIVOLTS

59.976

0.000

10.000

20.000

30.000

40.000

50.000

60.000

70.000

80.000

90.000

100.000

90.000

80.000

70.000

60.000

50.000

40.000

30.000

20.000

10.000

0.000
83.966

107.957

131.947

155.938

179.928

205.918

227.909

251.899

275.890

259.880

275.890

251.899

227.909

203.918

179.928

155.938

131.947

107.957

83.966

59.976

\section{MILLIVOLTS}

107.948

131.921

155.956

179.946

205.934

228.000

251.977

276.025

300.042

275.955

251.911

227.891

203.792

179.777

155.802

131.792

107.857

83.951

60.012
CALIBFATION ACCUFACY

**LIMITED**

MEASURED EFIROR \%

OUTFUT SIG FLLL SCALE MILLIVOLTS

14.994 OHMS

14.994 OHMS

N.A.

60.005
83.953

$0.01 \%$

$-0.01 \%$

$0.00 \%$

$-0.01 \%$

$0.01 \%$

$0.01 \%$

$0.01 \%$

$0.04 \%$

$0.03 \%$

$0.06 \%$

$0.07 \%$

$0.03 \%$

$0.00 \%$

$-0.01 \%$

$-0.05 \%$

$-0.06 \%$

$-0.06 \%$

$-0.06 \%$

$-0.04 \%$

$-0.01 \%$

$0.02 \%$
$0.17 \%$

OUT

DF

TOLEFIANCE

$$
\Sigma=60.009
$$


CURVEFIT OUTPUT FOR VTAB bEAPO2 $\checkmark$

VOLTAGE (V) OBS. PRESS.
CAIC. PRESS. DIFF. (PSIA)

* DIFFERENCE
(PSIA)

$\begin{array}{ll}1 & -0.3998960 \mathrm{E}-05 \\ 2 & 0.2394400 \mathrm{E}-01 \\ 3 & 0.4793900 \mathrm{E}-01 \\ 4 & 0.7191200 \mathrm{E}-01 \\ 5 & 0.9594700 \mathrm{E}-01 \\ 6 & 0.1199370 \\ 7 & 0.1439250 \\ 8 & 0.1679910 \\ 9 & 0.1919680 \\ 10 & 0.2160160 \\ 11 & 0.2400330 \\ 22 & 0.2159460 \\ 13 & 0.1919020 \\ 14 & 0.1678820 \\ 15 & 0.1437830 \\ 16 & 0.1197680 \\ 17 & 0.9579300 \mathrm{E}-01 \\ 18 & 0.7178300 \mathrm{E}-01 \\ 19 & 0.4784800 \mathrm{E}-01 \\ 1 & 0.2394200 \mathrm{E}-01 \\ \angle 1 & 0.3001040 \mathrm{E}-05\end{array}$

$\begin{array}{lll}0.0000000 \mathrm{E}+00 & 0.2042064 \mathrm{E}-02 \\ 10.00000 & 10.00228 & \\ 20.00000 & 20.01672 & \\ 30.00000 & 30.01656 & \\ 40.00000 & 40.03684 & \\ 50.00000 & 50.03292 & \\ 60.00000 & 60.02276 & \\ 70.00000 & 70.03963 & \\ 80.00000 & 80.01403 & \\ 90.00000 & 90.01253 & \\ 100.0000 & 99.99270 & \\ 90.00000 & 89.98343 & \\ 80.00000 & 79.98658 & \\ 70.00000 & 69.99427 & \\ 60.00000 & 59.96364 & \\ 50.00000 & 49.96252 & \\ 40.00000 & 39.97265 & \\ 30.00000 & 29.96277 & \\ 20.00000 & 19.97875 & \\ 10.00000 & 10.00144 & \\ 0.0000000 \mathrm{E}+00 & 0.4965922 \mathrm{E}-02\end{array}$

$0.0000000 E+00$

10.00000

30.00000

40.00000

50.00000

60.00000

80.00000

90.00000

100.0000

90.00000

80.00000

70.00000

60.00000

40.00000

30.00000

10.00000

$0.0000000 E+00$

(PSIA)

DATE: 20-MAY-92

TIME: $16: 56: 14$

\section{DEGREE OF POLYNOMIAL 2}

COEFFICIENTS

$0.37124058 \mathrm{E}-02 \quad 0.41769390 \mathrm{E}+03-0.47093516 \mathrm{E}+01$

ESS $=0.1133 \mathrm{E}-01 \quad \begin{aligned} \mathrm{SD} & =0.2508 \mathrm{E}-01 \\ 2 * \mathrm{SD} & =0.5017 \mathrm{E}-01\end{aligned} \quad \mathrm{R} 2=0.999999 \mathrm{E}+00$


RE. +5A Rev. $11-18-91$

RESEARCH \& DEVELOPMENT DIVISION INSTRUMENT SERVICE LOG

\begin{tabular}{|l|l|l|}
\hline INSTRUMENT STATUS & RÉCO & AS \\
\hline 1 Certified & \\
\hline Calibrated & & \\
\hline 3 For Ind. OnlY & & \\
\hline 4 Standardize & & \\
\hline 5 Certified \& Stand. & & \\
\hline 6 Calibrated \& Stand. & & \\
\hline 9 other & & \\
\hline
\end{tabular}

INSTRUMENT CONDITION

AS RECEIVED (Certified Equipment only)

Meets Manufacturex's Tolerance $N$

operates within previously defined limits $\mathrm{N}$ Deviates from Mfg or previously defined limits $Y$

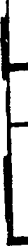
Inoperative (Describe below) $Y$ AS LEFT Limited (Describe below) Meets Manufacturer's Tolerance other (Describe Below)

\section{Babcóck \&' Wilcox \\ a McDermost company}

B\&W Ser. No. 0880540

\section{MANUFACTURER MODEL ITEM} MANUF . SER. NO. PROPERTY NO. ORDER NO.

SECTION

LOCATION SCHEDULED ( $Y-N)$ RECAL INT. (MOS.)

\section{SENSOTEC}

TJE/127810

TRANSMITR, PRES

188767

4427-01-102

\section{TECH. PROCEDURE A0015}

Babcock Wilcox R\&D Division certifies that the performance of this instrument was verified using standards whose accuracies are traceable to the National Institute of Standards and Technology, an accepted value of a natural physical constant. a ratio type of calibration technique, or to a consensus standard.

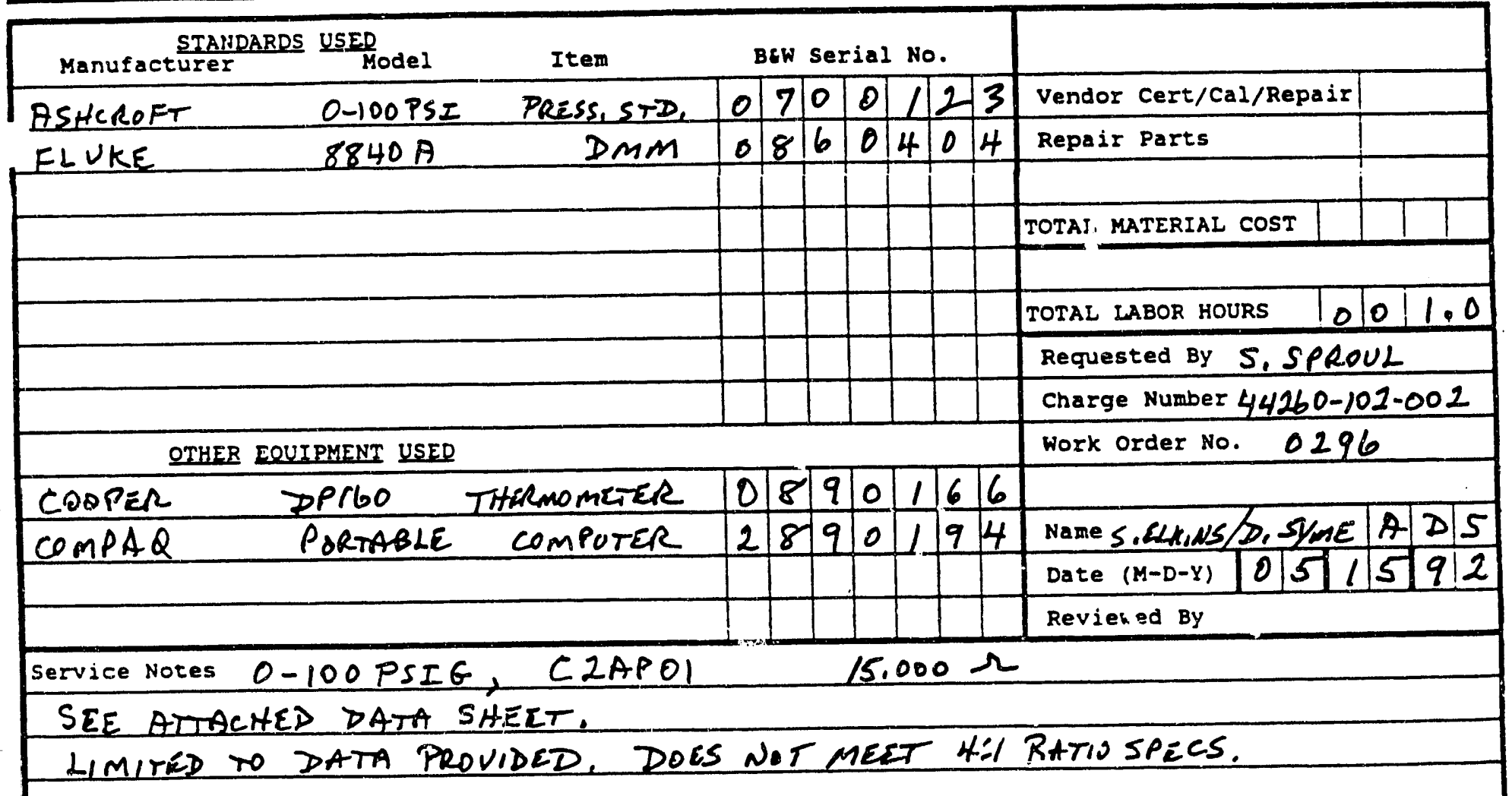


AS FECEIVED

$\begin{array}{cr}\text { TRANSMITTEF: } & \text { SENSOTEC TJE } \\ 100 \text { FSI } & \\ & \text { TOLEFANCE: } 0.10 \% \\ \text { FRESS SOURCE } & \text { ASHCFOFT DIGIGAUGE } \\ & 0-100 \text { FSI } \\ & \text { E\&W SER. NO. } 0700123 \\ & \text { TOLEFINCE: } 0.05 \% \\ \text { FEADOUT DEV: } & \text { FLUKE } 8840 \mathrm{~A} \\ & \text { E\&W SER. NO: } 0860404 \\ & \text { TOLEFANCE: } 0.015 \%\end{array}$

AS LEFT

B\&W S.N. 0880540 DATE: $15-M a \ddot{y}-92$

F'FOCEDURE : AFIC-TP-O15-08

FIROGRAM: TOO15_OB.OSA

NAME : S,ELKINS/D.SYME

Ambient Temperature (F) 70

Facility I. D. Name CZAFO1

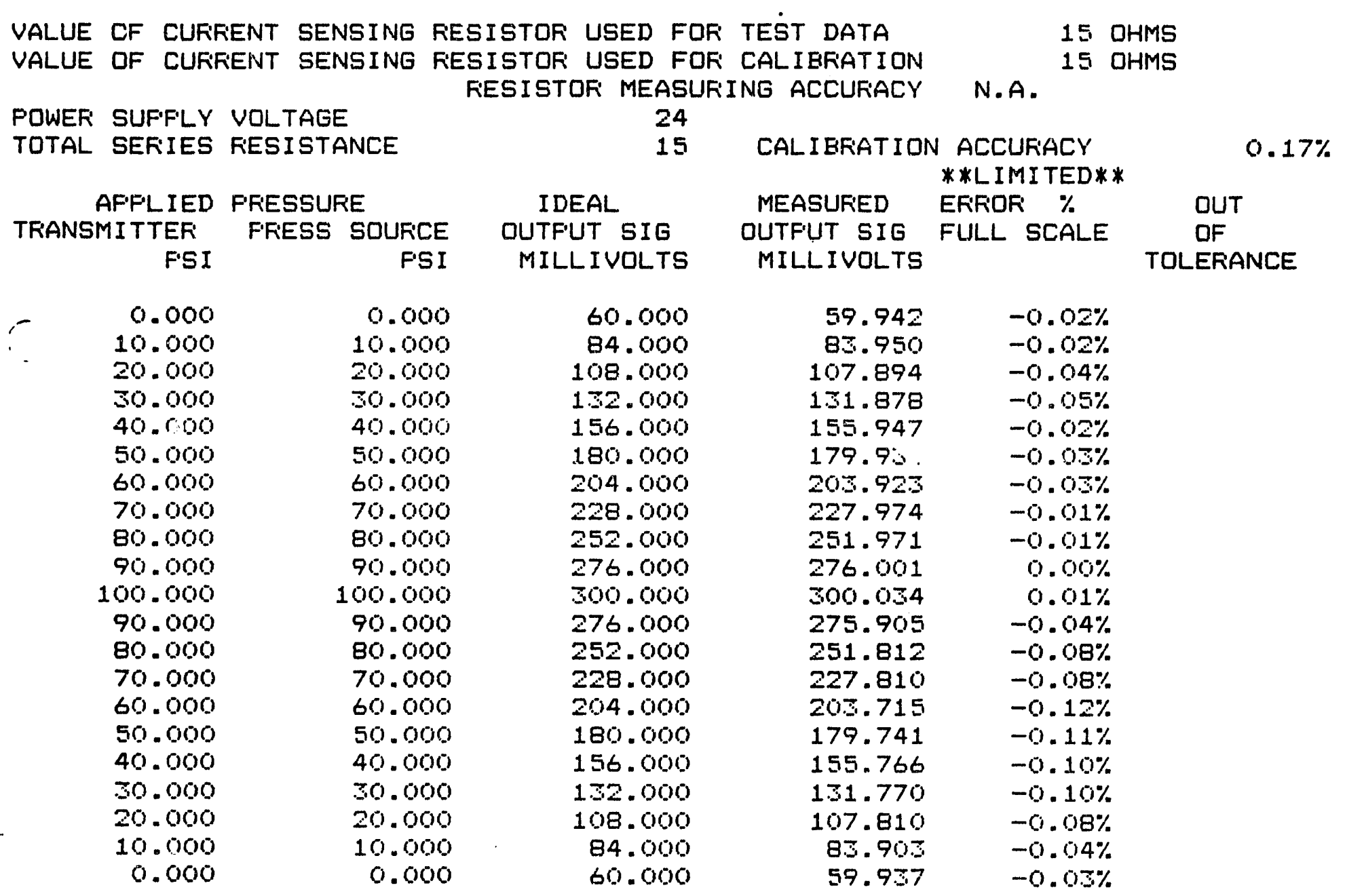


CURVEFIT OUTPUT FOR VTAB C2APOI

DATE: 20-MAY-92

TIME: $16: 38: 36$

VOLTAGE(V) OBS. PRESS. CALC. PRESS. DIFF. (PSIA) \& DIFFERENCE

(PSIA)

(PSIA)

$\begin{array}{rlllll}1 & 0.2000867 \mathrm{E}-05 & 0.0000000 \mathrm{E}+00 & -0.3401342 \mathrm{E}-02 & 0.3401342 \mathrm{E}-02 & 0.0000000 \mathrm{E}+00 \\ 2 & 0.2401000 \mathrm{E}-01 & 10.00000 & 10.02011 & -0.2011020 \mathrm{E}-01 & -0.2011020 \\ 3 & 0.4795400 \mathrm{E}-01 & 20.00000 & 20.01179 & -0.1179454 \mathrm{E}-01 & -0.5897270 \mathrm{E}-01 \\ 4 & 0.7193800 \mathrm{E}-01 & 30.00000 & 30.01506 & -0.1505792 \mathrm{E}-01 & -0.5019306 \mathrm{E}-01 \\ 5 & 0.9600700 \mathrm{E}-01 & 40.00000 & 40.04863 & -0.4862894 \mathrm{E}-01 & -0.1215723 \\ 6 & 0.1199940 & 50.00000 & 50.04289 & -0.4288985 \mathrm{E}-01 & -0.8577971 \mathrm{E}-01 \\ 7 & 0.1439830 & 60.00000 & 60.03287 & -0.3286517 \mathrm{E}-01 & -0.5477529 \mathrm{E}-01 \\ 8 & 0.1680340 & 70.00000 & 70.04352 & -0.4352078 \mathrm{E}-01 & -0.6217255 \mathrm{E}-01 \\ 9 & 0.1920310 & 80.00000 & 80.02657 & -0.2657189 \mathrm{E}-01 & -0.3321486 \mathrm{E}-01 \\ 10 & 0.2160610 & 90.00000 & 90.01822 & -0.1821829 \mathrm{E}-01 & -0.2024255 \mathrm{E}-01 \\ 1.1 & 0.2400940 & 100.0000 & 100.0060 & -0.5974508 \mathrm{E}-02 & -0.5974508 \mathrm{E}-02 \\ .2 & 0.2159650 & 90.00000 & 89.97831 & 0.2168818 \mathrm{E}-01 & 0.2409797 \mathrm{E}-01 \\ 23 & 0.1918720 & 80.00000 & 79.96044 & 0.3955724 \mathrm{E}-01 & 0.4944655 \mathrm{E}-01 \\ 14 & 0.1678700 & 70.00000 & 69.97528 & 0.2472288 \mathrm{E}-01 & 0.3531840 \mathrm{E}-01 \\ .5 & 0.1437750 & 60.00000 & 59.94627 & 0.5373231 \mathrm{E}-01 & 0.8955386 \mathrm{E}-01 \\ .6 & 0.1198010 & 50.00000 & 49.96250 & 0.3750379 \mathrm{E}-01 & 0.7500759 \mathrm{E}-01 \\ 17 & 0.9582600 \mathrm{E}-01 & 40.00000 & 39.97320 & 0.2680475 \mathrm{E}-01 & 0.6701188 \mathrm{E}-01 \\ 28 & 0.7183000 \mathrm{E}-01 & 30.00000 & 29.97002 & 0.2997533 \mathrm{E}-01 & 0.9991776 \mathrm{E}-01 \\ 19 & 0.4787000 \mathrm{E}-01 & 20.00000 & 19.97675 & 0.2324923 \mathrm{E}-01 & 0.1162462 \\ & 0.2396300 \mathrm{E}-01 & 10.00000 & 10.00049 & -0.4923732 \mathrm{E}-03 & -0.4923732 \mathrm{E}-02 \\ 21 & -0.2999133 \mathrm{E}-05 & 0.0000000 \mathrm{E}+00 & -0.5489412 \mathrm{E}-02 & 0.5489412 \mathrm{E}-02 & 0.0000000 \mathrm{E}+00\end{array}$

JEGREE OF POLYNOMIAL 2

OOEFFICIENTS

$-0.42369320 \mathrm{E}-02 \quad 0.41761394 \mathrm{E}+03-0.44477541 \mathrm{E}+01$

ISS $=0.1816 \mathrm{E}-01 \quad \mathrm{SD}=0.3176 \mathrm{E}-01 \quad \mathrm{R2}=0.999999 \mathrm{E}+00$

$2 * S D=0.6353 \mathrm{E}-01$ 
RD-ASA Rev. $12-18-91$

\begin{tabular}{|l|l|l|}
\hline INSTRUMENT STATUS & PF́CD & ALS \\
\hline 1 Certified & & \\
\hline Calibrated & & $\checkmark$ \\
\hline 3 For Ind. Only & $\checkmark$ & \\
\hline 4 standardize & & \\
\hline 5 certified \& stand. & & \\
\hline 6 calibrated s stand. & & \\
\hline 9 other & & \\
\hline
\end{tabular}

INSTRUMENT CONDITION

AS RECEIVED (Certified Equipment Only)

Meets Manufacturer's Tolerance $N$

operates within previously defined limits $N$

Deviates from Mfg or previously defined limits $Y$

Inoperative (Describe below) $y$

AS LEFT

Limited (Describe below)

Meets Manufacturer's Tolerance

other (Describe Below)
บレオ่レン

\section{Babcock \& Wilcox}

acDermott company

B\&W Ser. No. 0880550

MANUFACTURER SENSOTEC

MODEL

ITEM

TJE/127810

MANUF. SER. NO.

TRANSMITR, PRES:

201308

PROPERTY NO. 4427-01-112

ORDER NO.

$\begin{array}{lr}\text { SECTION } & 46 \\ \text { LOCATION } & 115\end{array}$

$\operatorname{SCHEDULED~}(Y-\mathrm{N})$ \#Y

RECAL INT.(MOS.) 12

\section{TECH. PROCEDURE A0015}

Babcock Wilcox RGD Division certifies that the performance of this instrument was verified using standards whose accuracies are traceable to the National Institute of standards and Technology, an accepted value of a natural physical constant, a ratio type of calibration technique, or to a consensus standard.

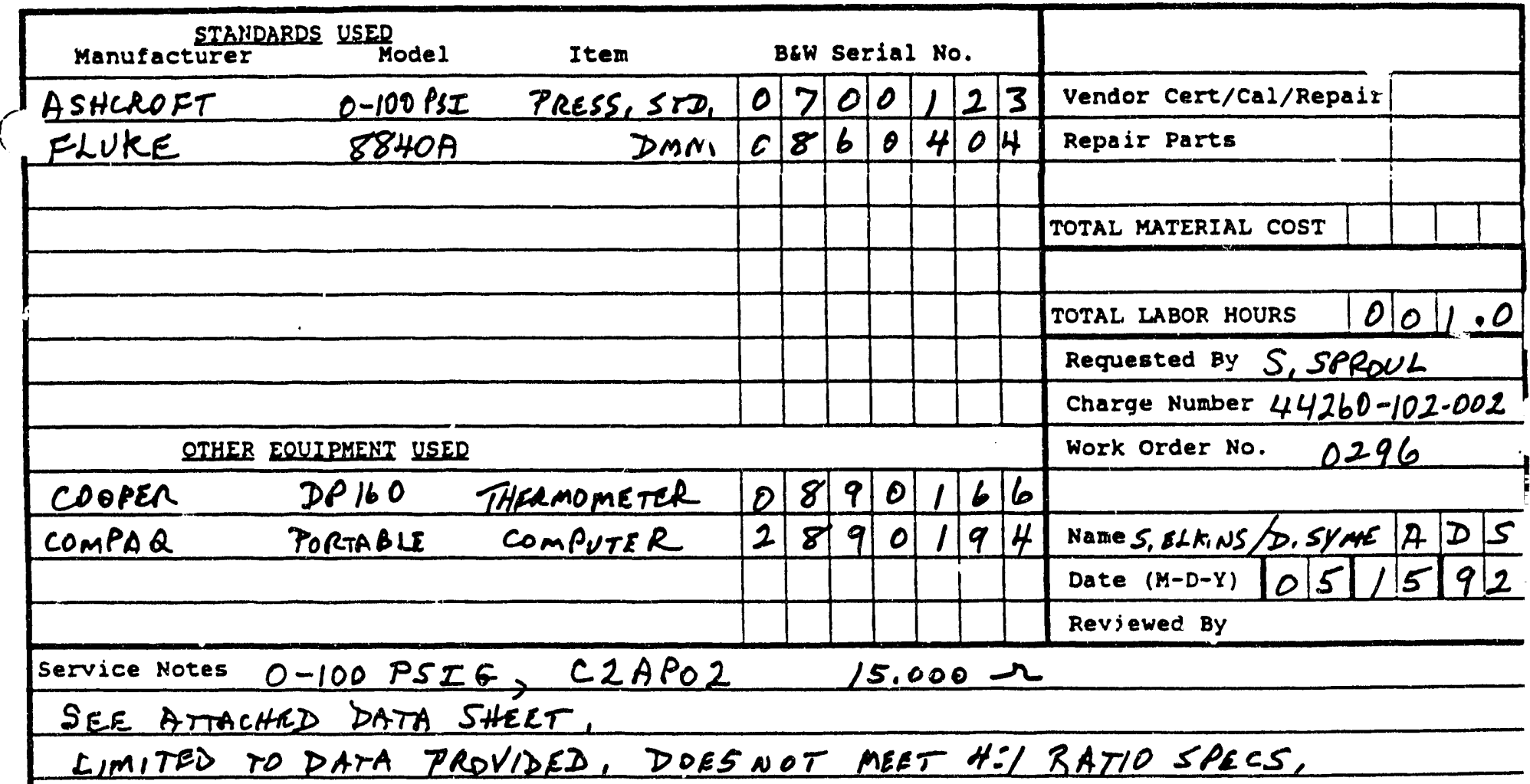


AS RIECEIVED

\begin{tabular}{|c|c|c|}
\hline FIANSMI TTEF: & $\begin{array}{r}\text { SENSOTEC } \\
100 \\
\text { TOLERANCE: }\end{array}$ & $\begin{array}{l}\text { TJE } \\
\text { FSI } \\
0.10 \%\end{array}$ \\
\hline 'RESS SOURECE & $\begin{array}{r}\text { ASHCFOFT } \\
0-100 \\
\text { E\&W SER. NO } \\
\text { TOLEFIANCE: }\end{array}$ & $\begin{array}{l}\text { DIGIGAUGE } \\
\text { FSI } \\
0700125 \\
0.05 \%\end{array}$ \\
\hline ADOUT DEV: & $\begin{array}{l}\text { FLUKE } \\
\text { E\&W SER. NO: } \\
\text { TOLEFANCE: }\end{array}$ & $\begin{array}{l}8840 A \\
0860404 \\
0.015 \%\end{array}$ \\
\hline
\end{tabular}

AS LEFT

E\&W S.N. 0880550 .. DATE: 15-May-92 F'FOCEDURE : ARC-TF-015-08 FFIOGFIAM: TOO15_08.03A NAME: S,ELK,NS/D,SYME

Ambient Temperature (F) 70

Facility I. D. Name CZAFOZ

-ALUE OF CUFFENT SENSING FESISTOF USED FOF TEST DATA IALUE OF CUFFENT SENSING RESISTOF' USED FOR CALIEFATION FIESISTOF: MEASUFIING ACCUFIACY

BWEE SUEFIES YOLTISTANCE

AF'FLIED F'FESSURE I RAANSMITTEF: F'SI F'RESS SOURCE PSI

$\begin{array}{rr}0.000 & 0.000 \\ 10.000 & 10.000 \\ 20.000 & 20.000 \\ 30.000 & 30.000 \\ 40.000 & 40.000 \\ 50.000 & 50.000 \\ 60.000 & 60.000 \\ 70.000 & 70.000 \\ 80.000 & 80.000 \\ 90.000 & 90.000 \\ 100.000 & 100.000 \\ 90.000 & 90.000 \\ 80.000 & 80.000 \\ 70.000 & 70.000 \\ 60.000 & 60.000 \\ 50.000 & 50.000 \\ 40.000 & 40.000 \\ 30.000 & 30.000 \\ 20.000 & 20.000 \\ 10.000 & 10.000 \\ 0.000 & 0.000\end{array}$

$$
14.997
$$

I DEAL QUTFUT SIG MILLIVOLTS

59.988
$8 . .98$.
107.978
1.1 .974
155.969
179.964
207.959
227.954
251.950
275.945
299.940
275.945
251.950
227.954
20.959
179.964
155.969
1.1 .974
107.978
85.985
59.988

$\begin{array}{ccc}\text { CALIBFATION ACCUFACY } & \\ \text { **LIMITED** } & \\ \text { MEASUFED } & \text { EFR'RF } \% & \text { OUT } \\ \text { OUTFUT SIG } & \text { FULL SCALE } & \text { OF } \\ \text { MILLIVOLTS } & & \text { TOLEFIANCE }\end{array}$

TOLEFIANCE

\begin{abstract}
14.997 DHMS
14.997 OHMS
\end{abstract}

N.A.

$\begin{array}{rr}59.948 & -0.02 \% \\ 85.955 & -0.02 \% \\ 107.861 & -0.05 \% \\ 131.829 & -0.06 \% \\ 155.884 & -0.04 \% \\ 179.837 & -0.05 \% \\ 205.852 & -0.04 \% \\ 227.907 & -0.02 \% \\ 251.914 & -0.01 \% \\ 275.951 & 0.00 \% \\ 299.970 & 0.01 \% \\ 275.880 & -0.03 \% \\ 251.799 & -0.06 \% \\ 227.764 & -0.08 \% \\ 203.708 & -0.10 \% \\ 179.699 & -0.11 \% \\ 155.721 & -0.10 \% \\ 151.712 & -0.11 \% \\ 107.765 & -0.09 \% \\ 83.858 & -0.05 \% \\ 59.964 & -0.01 \% \\ & \end{array}$


CURVEFIT OUTPUT FOR VTAB C2APO2

VOLTAGE(V) OBS. PRESS. CALC. PRESS. DIFF. (PSIA) \& DIFFERENCE
DATE: 20-MAY-92

TIME: $16: 39: 53$

\begin{tabular}{|c|c|c|c|c|c|}
\hline \multirow{2}{*}{\multicolumn{2}{|c|}{ VOLTAGE $(V)$}} & OBS. PRESS. & CALC. PRESS. & DIFF.(PSIA) & \& DIFFERENCE \\
\hline & & (PSIA) & (PSIA) & & \\
\hline $\begin{array}{r}1 \\
2 \\
3 \\
4 \\
5 \\
6 \\
7 \\
8 \\
9 \\
10 \\
11 \\
12 \\
13 \\
14 \\
15 \\
16 \\
17 \\
18\end{array}$ & $\begin{array}{l}-0.7999255 \mathrm{E}-05 \\
0.2397900 \mathrm{E}-01 \\
0.4790500 \mathrm{E}-01 \\
0.7187300 \mathrm{E}-01 \\
0.9592800 \mathrm{E}-01 \\
0.1198810 \\
0.1438960 \\
0.1679510 \\
0.1919580 \\
0.2159950 \\
0.2400140 \\
0.2159240 \\
0.1918430 \\
0.1678080 \\
0.1437520 \\
0.1197430 \\
0.9576500 \mathrm{E}-01 \\
0.7175700 \mathrm{E}-01 \\
0.4780700 \mathrm{E}-01 \\
0.2390200 \mathrm{E}-01 \\
0.8000745 \mathrm{E}-05\end{array}$ & $\begin{array}{l}0.0000000 \mathrm{E}+00 \\
10.00000 \\
20.00000 \\
30.00000 \\
40.00000 \\
50.00000 \\
60.00000 \\
70.00000 \\
80.00000 \\
90.00000 \\
100.0000 \\
90.00000 \\
80.00000 \\
70.00000 \\
60.00000 \\
50.00000 \\
40.00000 \\
30.00000 \\
20.00000 \\
10.00000 \\
0.0000000 \mathrm{E}+00\end{array}$ & $\begin{array}{l}-0.3350469 \mathrm{E}-03 \\
10.02173 \\
20.01186 \\
30.01307 \\
40.04409 \\
50.02611 \\
60.02750 \\
70.03904 \\
80.02411 \\
90.01518 \\
99.99227 \\
89.98567 \\
79.97630 \\
69.97954 \\
59.96755 \\
49.96862 \\
39.97614 \\
29.96468 \\
19.97095 \\
9.989566 \\
0.6352105 \mathrm{E}-02\end{array}$ & $\begin{array}{r}0.3350469 \mathrm{E}-03 \\
-0.2172712 \mathrm{E}-01 \\
-0.1185764 \mathrm{E}-01 \\
-0.1307112 \mathrm{E}-01 \\
-0.4409268 \mathrm{E}-01 \\
-0.2611472 \mathrm{E}-01 \\
-0.2749775 \mathrm{E}-01 \\
-0.3903826 \mathrm{E}-01 \\
-0.2411445 \mathrm{E}-01 \\
-0.1517565 \mathrm{E}-01 \\
0.7734374 \mathrm{E}-02 \\
0.1432618 \mathrm{E}-01 \\
0.2370131 \mathrm{E}-01 \\
0.2045822 \mathrm{E}-01 \\
0.3245374 \mathrm{E}-01 \\
0.3137605 \mathrm{E}-01 \\
0.2385701 \mathrm{E}-01 \\
0.3531706 \mathrm{E}-01 \\
0.2904843 \mathrm{E}-01 \\
0.1043407 \mathrm{E}-01 \\
-0.6352105 \mathrm{E}-02\end{array}$ & $\begin{array}{l}0.0000000 \mathrm{E}+00 \\
-0.2172712 \\
-0.5928822 \mathrm{E}-01 \\
-0.4357041 \mathrm{E}-01 \\
-0.1102317 \\
-0.5222944 \mathrm{E}-01 \\
-0.4582958 \mathrm{E}-01 \\
-0.5576894 \mathrm{E}-01 \\
-0.3014306 \mathrm{E}-01 \\
-0.1686183 \mathrm{E}-01 \\
0.7734374 \mathrm{E}-02 \\
0.1591798 \mathrm{E}-01 \\
0.2962664 \mathrm{E}-01 \\
0.2922603 \mathrm{E}-01 \\
0.5408957 \mathrm{E}-01 \\
0.6275211 \mathrm{E}-01 \\
0.5964252 \mathrm{E}-01 \\
0.1177235 \\
0.1452421 \\
0.1043407 \\
0.0000000 \mathrm{E}+00\end{array}$ \\
\hline
\end{tabular}

DEGREE OF POLYNOMIAL 2

COEFFICIENTS

$0.30082182 \mathrm{E}-02 \quad 0.41794701 \mathrm{E}+03-0.56221932 \mathrm{E}+01$

ESS $=0.1259 \mathrm{E}-01 \quad \mathrm{SD}=0.2645 \mathrm{E}-01 \quad \mathrm{R2}=0.999999 \mathrm{E}+00$

$2 \star S D=0.5290 \mathrm{E}-01$ 
$R D-45 A$ ReV. $11-18-91$

RESEARCH \& DEVELOPMENT DIVISION INSTRURENT SERVICE IOG

\begin{tabular}{l|l|l|}
\hline INSTRUMENT STATUS & RÉCD & AS \\
\hline 1 Certified & \\
\hline Calibrated & & \\
\hline 3 For Ind. Only & & \\
\hline 4 Standardize & & \\
\hline 5 Certified s stand. & & \\
\hline 6 Calibrated stand. & & \\
\hline 9 other & & \\
\hline
\end{tabular}

INSTRUMENT CONDITION

AS RECEIVED (Certified Equipment Only)

Meets Manufacturer's Tolerance $N$

Operates within previously defined limits $N$ Deviates from Mfg or previously defined limits $Y$ Inoperative (Describe below) $Y$

AS LEFT Limited (Describe below)

Meets Ma ufacturer's Tolerance ther (Describe Below) consensus standard.
Babcock \& Wilcox

a MeDermors company

B\&W Ser. No. 0880543

MANUFACTURER

MODEL

ITEM

MANUF, SER. NO.

PROPERTY NO.

ORDER NO.

SECTION

LOCATION

SCHEDULED ( $Y-N)$

RECAL INT.(MOS.)
SENSOTEC

TJE/ 127810

TRANSMITR, PRES :

189740

4427-01-105

46

115

$-1+y$

12

TECH. PROCEDURE A0015

Babcock Wilcox RED Division certifies that the performance of this instrument was verified using standards whose accuracies are traceable to the National Institute of standards and Technology, an accepted value of a natural physical constant. a ratio type of calibration technique, or to a

\section{STANDARDS USED}

Manutacturer

A SHCROFT FLUKE 1
Item

BEW Serial No.

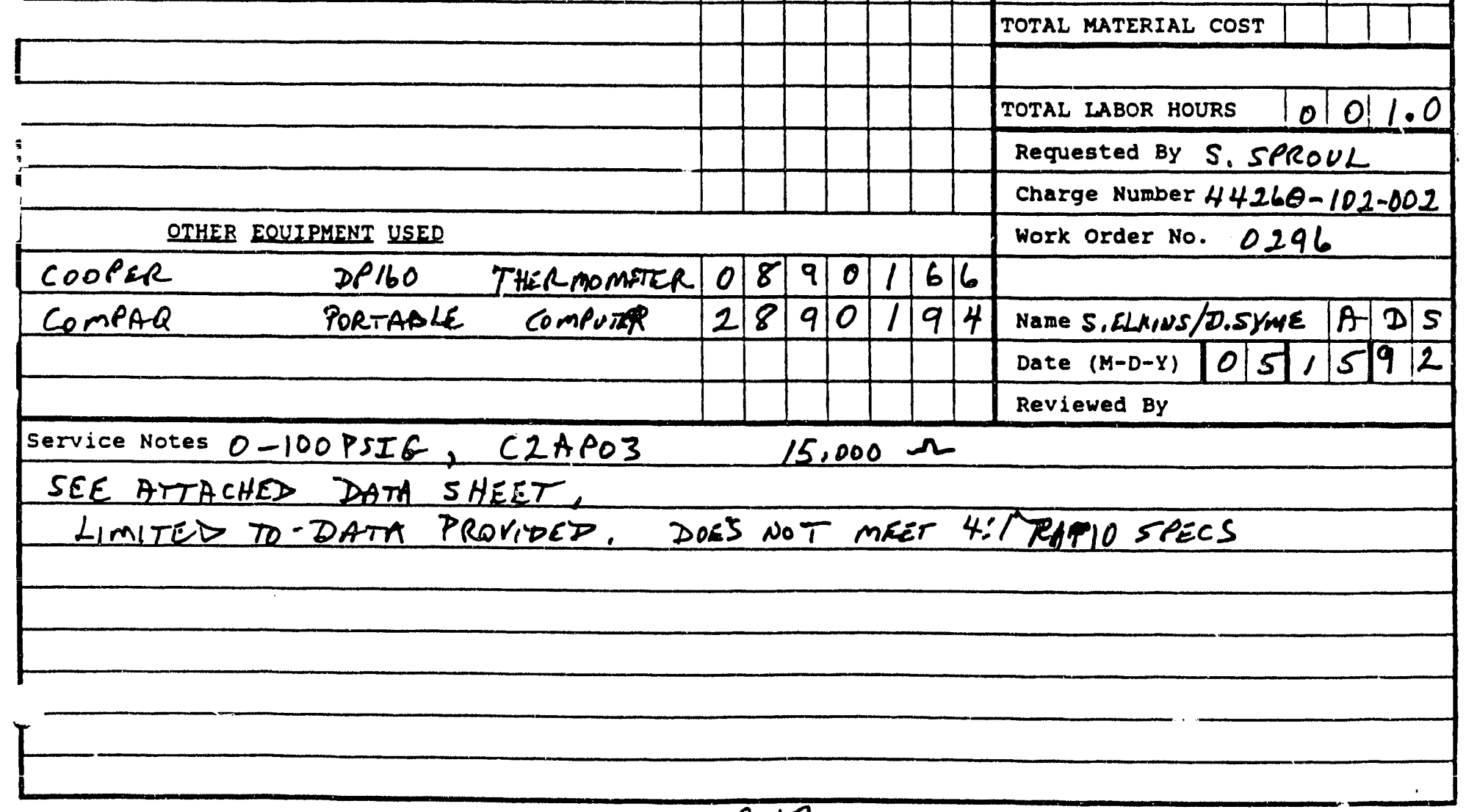


AS FiECEIVED

TRANSMITTER:

F'RESS SOURCE

ASHCFOFT DIGIGAUGE
O-10O FSI
E\&W SER. NO. O700123
TOLEFANCE: $0.05 \%$

FEADOUT DEV:
FLUKE 8840A
B\&W Ser. NO. 0860404

SENSOTEC TJE

100 F'SI

TOLERANCE: $0.10 \%$

TOLEFIANCE: $0.015 \%$
AS

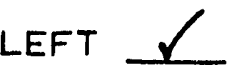

B\&W S.N. 0880543..

DATE: 15-Maÿ-92

F'ROCEDURE : ARC-TP-O15-08

FFIOGFAM: T0015_08.03A

NAME: S,ELkINS LD, SYME

Ambient Temperature ( $F$ ) 70

Facility I. D. Name

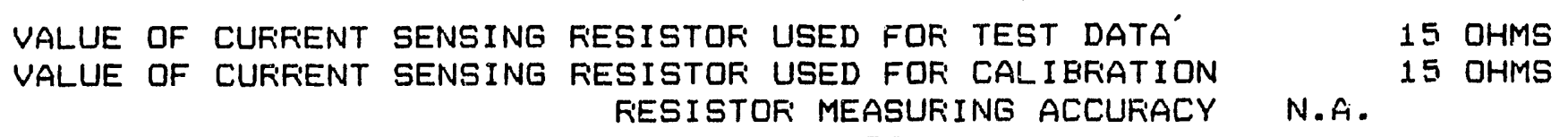

FOWEF SUF'FLY VOLTAGE

TOTAL SE.FIIES FIESISTANCE

AFFLIED F'FESSURE

TFIANSMI TTEF' F'SI

F'RESS SDUFICE F'SI

$\begin{array}{rr}0.000 & 0.000 \\ 10.000 & 10.000 \\ 20.000 & 20.000 \\ 30.000 & 30.000 \\ 40.000 & 40.000 \\ 50.000 & 50.000 \\ 60.000 & 60.000 \\ 70.000 & 70.000 \\ 80.000 & 80.000 \\ 90.000 & 90.000 \\ 100.000 & 100.000 \\ 90.000 & 90.000 \\ 80.000 & 80.000 \\ 70.000 & 70.000 \\ 60.000 & 60.000 \\ 50.000 & 50.000 \\ 40.000 & 40.000 \\ 30.000 & 30.000 \\ 20.000 & 20.000 \\ 10.000 & 10.000 \\ 0.000 & 0.000\end{array}$

24

15

I DEAL OUTFUT SIG MILLIVOLTS

$\begin{array}{cl}\text { CALIEFIATION ACCUFIACY } \\ & \text { **LIMITED** } \\ \text { MEASUFED } & \text { EFFIOF \% } \\ \text { OUTFUT SIG } & \text { FULL SCALE } \\ \text { MILLIVOLTS } & \end{array}$

$0.00 \%$

$-0.02 \%$

$-0.04 \%$

$-0.04 \%$

$-0.02 \%$

$-0.0 .3 \%$

$-0.03 \%$

$-0.01 \%$

$-0.01 \%$

$0.01 \%$

$0.01 \%$

$-0.02 \%$

$-0.06 \%$

$-0.07 \%$

$-0.11 \%$

$-0.10 \%$

$-0.09 \%$

$-0.09 \%$

$-0.06 \%$

$-0.0 .3 \%$

$0.00 \%$
$0.17 \%$

QUT

DF TOLEFIANCE

$$
\bar{x}=59.998
$$


VOLTAGE(V) OBS. PRESS. CALC. PRESS. DIFF.(PSIA) \& DIFFERENCE (PSIA) (PSIA)

\begin{tabular}{llllll}
\hline 1 & $-0.9981780 \mathrm{E}-06$ & $0.0000000 \mathrm{E}+00$ & $0.2553159 \mathrm{E}-02$ & $-0.2553159 \mathrm{E}-02$ & $0.0000000 \mathrm{E}+00$ \\
2 & $0.2395700 \mathrm{E}-01$ & 10.00000 & 10.00869 & $-0.8687259 \mathrm{E}-02$ & $-0.8687259 \mathrm{E}-01$ \\
3 & $0.4791700 \mathrm{E}-01$ & 20.00000 & 20.00994 & $-0.9939915 \mathrm{E}-02$ & $-0.4969957 \mathrm{E}-01$ \\
4 & $0.7189900 \mathrm{E}-01$ & 30.00000 & 30.01465 & $-0.1465083 \mathrm{E}-01$ & $-0.4883608 \mathrm{E}-01$ \\
5 & $0.9595300 \mathrm{E}-01$ & 40.00000 & 40.04365 & $-0.4364508 \mathrm{E}-01$ & -0.1091127 \\
6 & 0.1199270 & 50.00000 & 50.03355 & $-0.3355119 \mathrm{E}-01$ & $-0.6710238 \mathrm{E}-01$ \\
7 & 0.1439290 & 60.00000 & 60.02939 & $-0.2939112 \mathrm{E}-01$ & $-0.4898520 \mathrm{E}-01$ \\
8 & 0.1679870 & 70.00000 & 70.04280 & $-0.427959 \mathrm{E}-01$ & $-0.6113656 \mathrm{E}-01$ \\
9 & 0.1919810 & 80.00000 & 80.02382 & $-0.2382116 \mathrm{E}-01$ & $-0.2977644 \mathrm{E}-01$ \\
0 & 0.2160170 & 90.00000 & 90.01657 & $-0.1656959 \mathrm{E}-01$ & $-0.1841066 \mathrm{E}-01$ \\
1 & 0.2400250 & 100.0000 & 99.99193 & $0.8065981 \mathrm{E}-02$ & $0.8065981 \mathrm{E}-02$ \\
2 & 0.2159440 & 90.00000 & 89.98623 & $0.1377078 \mathrm{E}-01$ & $0.1530087 \mathrm{E}-01$ \\
3 & 0.1918620 & 80.00000 & 79.97433 & $0.2566633 \mathrm{E}-01$ & $0.3208291 \mathrm{E}-01$ \\
14 & 0.1678460 & 70.00000 & 69.98413 & $0.1587454 \mathrm{E}-01$ & $0.2267792 \mathrm{E}-01$ \\
5 & 0.1437470 & 60.00000 & 59.95362 & $0.4638276 \mathrm{E}-01$ & $0.7730460 \mathrm{E}-01$ \\
6 & 0.1197570 & 50.00000 & 49.96273 & $0.3726724 \mathrm{E}-01$ & $0.7453448 \mathrm{E}-01$ \\
17 & $0.9577700 \mathrm{E}-01$ & 40.00000 & 39.97029 & $0.2971484 \mathrm{E}-01$ & $0.7428710 \mathrm{E}-01$ \\
.8 & $0.7179600 \mathrm{E}-01$ & 30.00000 & 29.97169 & $0.2830604 \mathrm{E}-01$ & $0.9435347 \mathrm{E}-01$ \\
.8 & $0.4784900 \mathrm{E}-01$ & 20.00000 & 19.98156 & $0.1843619 \mathrm{E}-01$ & $0.9218092 \mathrm{E}-01$ \\
& $0.2392300 \mathrm{E}-01$ & 10.00000 & 9.994491 & $0.5508898 \mathrm{E}-02$ & $0.5508897 \mathrm{E}-01$ \\
.1 & $0.1001822 \mathrm{E}-05$ & $0.0000000 \mathrm{E}+00$ & $0.3388704 \mathrm{E}-02$ & $-0.3388704 \mathrm{E}-02$ & $0.0000000 \mathrm{E}+00$
\end{tabular}

IEGREE OF POLYNOMIAL 2

TOEFFICIENTS

$0.29701706 E-02 \quad 0.41777243 E+03-0.49792479 E+01$

TSS $=0.1373 \mathrm{E}-01 \quad \mathrm{SD}=0.2762 \mathrm{E}-01 \quad \mathrm{R2}=0.999999 \mathrm{E}+00$

$\begin{aligned} S D & =0.2762 E-01 \\ 2 * S D & =0.5525 E-01\end{aligned} \quad R 2=0.999999 E+00$ 
RD-45A Rev. $11-18-91$

RESEARCH \& DEVELOPMENT DIVISION INSTRURENT SERVICE IOG

\begin{tabular}{|l|l|l|}
\hline \multicolumn{1}{|c|}{ INSTRUMENT STATUS } & RISCE & LES \\
\hline 1 Certified & & \\
\hline Calibrated & & $\checkmark$ \\
\hline 3 For Ind. Only & & \\
\hline 4 standardize & & \\
\hline 5 Certified \& stand. & & \\
\hline 6 Calibrated s stand. & & \\
\hline 9 other & & \\
\hline
\end{tabular}

INSTRUMENT CONDITION

AS RECEIVED (Certified Equipment only)

Meets Manufacturer's Tolerance $N$

Operates within previously defined limits $N$ Deviates from Mfg or previously defined limits $Y$ Inoperative (Describe below) $\mathbf{y}$

AS LEFT

Limited (Describe below)

Meets Manufacturer's Tolerance other (Describe Below)

\section{C2Nio}

Babcock \& Wilcox

- McDermoti company

B\&W Ser. No. 0880544

MANUFACTURER

MODEL

SENSOTEC

ITEM

TJE/127810

MANUF . SER. NO.

TRANSMITR, PRES

PROPERTY NO.

ORDER NO.

4427-01-106

$\begin{array}{lc}\text { SECTION } & 46 \\ \text { LOCATION } & 115 \\ \text { SCHEDULED (Y-N) } & -1-Y \\ \text { RECAL INT.(MOS.) } & 12\end{array}$

TECH. PROCEDURE A0015

Babcock Wilcox RED Division certifies that the performance of this instrument was verified using standards whose accuracies are traceable to the National Institute of Standards and Technology, an accepted value of a natural physical constant. a ratio type of calibration technique, or to a consensus standard.

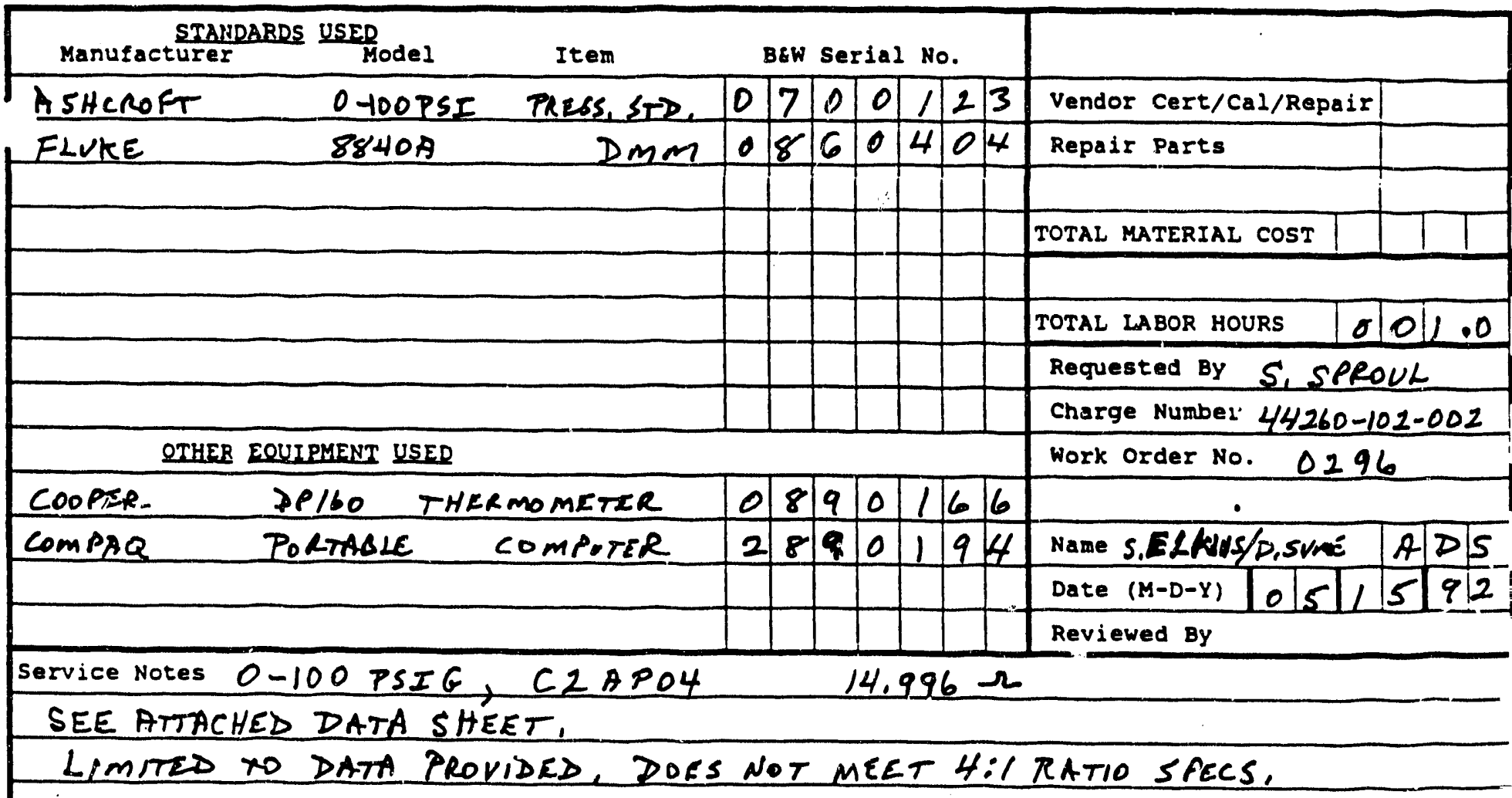


AS REEEIVED

\begin{tabular}{|c|c|c|}
\hline FIANSMITTEF: & $\begin{array}{r}\text { SENSOTEC } \\
100 \\
\text { TOLEFIANCE: }\end{array}$ & $\begin{array}{l}\text { TJE } \\
\text { FSI } \\
0.10 \%\end{array}$ \\
\hline 'RESS SOUFiCE & $\begin{array}{r}\text { ASHCROFT } \\
0-100 \\
\text { B\&W SER. NO } \\
\text { TOLEFIANCE: }\end{array}$ & $\begin{array}{l}\text { DIGIGAUGE } \\
\text { FSI } \\
0700123 \\
0.05 \%\end{array}$ \\
\hline EADOUT DEV: & $\begin{array}{l}\text { FLUKE } \\
\text { E\&W SER. NO. } \\
\text { TOLERANCE: }\end{array}$ & $\begin{array}{l}8840 A \\
0860404 \\
0.015 \%\end{array}$ \\
\hline
\end{tabular}

AS LEFT

E\&W S.N. 0880544 DATE: $\quad 15-M a y-92$ FRIOCEDUFE : AFIC-TF-O15-OB FFIOGFIAM: TOO15_0B.0SA NAME: S,ELKINS/7, SYME

Ambient Temperature (F) 70
Facility I. D. Name C2AFO4
ALUE OF CUFFENT SENSING FESISTOF USED FOF TEST DATA 'ALUE OF CURFENT SENSING FESISTOF' USED FOF' CALIBRATION JWER SUFFLY VOLTAGE OTAL SEFIIES FIESISTANCE FIESISTOF MEASUFIING ACCUFIACY 24

AFFLIED F'FEESSURE I RANSMITTEF: F'SI F'R'ESS SOURICE F'SI

$$
\begin{array}{r}
0.000 \\
10.000 \\
20.000 \\
30.000 \\
40.000 \\
50.000 \\
60.000 \\
70.000 \\
80.000 \\
90.000 \\
100.000 \\
90.000 \\
80.000 \\
70.000 \\
60.000 \\
50.000 \\
40.000 \\
30.000 \\
20.000 \\
10.000 \\
0.000
\end{array}
$$

$$
\begin{array}{r}
0.000 \\
10.000 \\
20.000 \\
30.000 \\
40.000 \\
50.000 \\
60.000 \\
70.000 \\
80.000 \\
90.000 \\
100.000 \\
90.000 \\
80.000 \\
70.000 \\
60.000 \\
50.000 \\
40.000 \\
30.000 \\
20.000 \\
10.000 \\
0.000
\end{array}
$$

24
14.996
IDEAL
OUTFUT SIG
MILLIVOLTS
59.984
85.978
107.971
131.965
155.958
179.952
203.946
227.939
251.933
275.926
299.920
275.926
251.933
227.939
203.946
179.952
155.958
131.965
107.971
85.978
59.984

14.996 OHMS 14.996 OHMS N.A.

\section{CAL I EFFAT ION}

MEASURED

OUTFUT SIG MILLIVOLTS

**LIMITED**

EFFIOF: \%

FULL SCALE
$0.17 \%$

DUT

QF

$\begin{array}{rr}59.969 & -0.01 \% \\ 84.009 & 0.01 \% \\ 108.061 & 0.04 \% \\ 132.083 & 0.05 \% \\ 156.092 & 0.06 \% \\ 180.064 & 0.05 \% \\ 204.012 & 0.03 \% \\ 228.027 & 0.04 \% \\ 251.982 & 0.02 \% \\ 275.928 & 0.00 \% \\ 299.852 & -0.03 \% \\ 275.890 & -0.02 \% \\ 251.880 & -0.02 \% \\ 227.938 & 0.00 \% \\ 205.911 & -0.01 \% \\ 179.952 & 0.00 \% \\ 155.972 & 0.01 \% \\ 1.1 .945 & -0.01 \% \\ 107.941 & -0.01 \% \\ 83.956 & -0.01 \% \\ 59.858 & -0.04 \% \\ & \end{array}$


CURVEFIT OUTPUT FOR VTAB C2AP04/

VOLTAGE (V) OBS. PRESS. CALC. PRESS. DIFF. (PSIA)
DATE: 20-MAY-92

TIME: $16: 42: 00$

(PSIA)

(PSIA)

$\begin{array}{rlll}1 & 0.3499858 \mathrm{E}-04 & 0.0000000 \mathrm{E}+00 & 0.1371127 \mathrm{E}-01 \\ 2 & 0.2407500 \mathrm{E}-01 & 10.00000 & 10.01301 \\ 3 & 0.4812700 \mathrm{E}-01 & 20.00000 & 20.02368 \\ 4 & 0.7214900 \mathrm{E}-01 & 30.00000 & 30.02755 \\ 5 & 0.9615800 \mathrm{E}-01 & 40.00000 & 40.03100 \\ 6 & 0.1201300 & 50.00000 & 50.02331 \\ 7 & 0.1440780 & 60.00000 & 60.00922 \\ 8 & 0.1680930 & 70.00000 & 70.02598 \\ 9 & 0.1920480 & 80.00000 & 80.01995 \\ 10 & 0.2159940 & 90.00000 & 90.01170 \\ 11 & 0.2399180 & 100.0000 & 99.99514 \\ 12 & 0.2159560 & 90.00000 & 89.99585 \\ 13 & 0.1919460 & 80.00000 & 79.97739 \\ 14 & 0.1680040 & 70.00000 & 69.98886 \\ 15 & 0.1439770 & 60.00000 & 59.96710 \\ 16 & 0.1200180 & 50.00000 & 49.97662 \\ 17 & 0.9603800 \mathrm{E}-01 & 40.00000 & 39.98099 \\ 18 & 0.7201100 \mathrm{E}-01 & 30.00000 & 29.97007 \\ 19 & 0.4800700 \mathrm{E}-01 & 20.00000 & 19.97372 \\ 1 & 0.2402200 \mathrm{E}-01 & 10.00000 & 9.990957 \\ 21 & -0.3600142 \mathrm{E}-04 & 0.0000000 \mathrm{E}+00 & -0.1581064 \mathrm{E}-01\end{array}$

$-0.1371127 \mathrm{E}-01$

$-0.1300936 \mathrm{E}-01$

$-0.2368287 \mathrm{E}-01$

$-0.2755379 \mathrm{E}-01$

$-0.3099773 \mathrm{E}-01$

$-0.2331322 \mathrm{E}-01$

$-0.9218788 \mathrm{E}-02$

$-0.2598305 \mathrm{E}-01$

$-0.1995026 \mathrm{E}-01$

$-0.1170438 \mathrm{E}-01$

$0.4863528 \mathrm{E}-02$

$0.4152432 \mathrm{E}-02$

$0.2260765 \mathrm{E}-01$

$0.1114381 \mathrm{E}-01$

$0.3290307 E-01$

$0.2338096 \mathrm{E}-01$

$0.1901200 \mathrm{E}-01$

$0.2993070 \mathrm{E}-01$

$0.2627699 E-01$

$0.9042949 E-02$

$0.1581064 \mathrm{E}-01$
DIFFERENCE

DEGREE OF POLYNOMIAL 3

COEFFICIENTS

$-0.84120217 E-03 \quad 0.41580149 E+03 \quad 0.61177070 E+01-0.82856319 E+01$

ESS $=0.9062 \mathrm{E}-02 \quad \begin{aligned} \mathrm{SD} & =0.2309 \mathrm{E}-01 \\ 2 * \mathrm{SD} & =0.4618 \mathrm{E}-01\end{aligned} \quad \mathrm{R} 2=0.100000 \mathrm{E}+01$ 
ID-45A Rev. $12-18-91$

\begin{tabular}{|c|c|c|}
\hline INSTRUMENT STATUS & 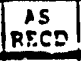 & LES \\
\hline Certified & & \\
\hline Calibrated & & $\checkmark$ \\
\hline For Ind. only & $\checkmark$ & \\
\hline Standardize & & \\
\hline Certified \& stand. & & \\
\hline Calibrated stand. & & \\
\hline Other & & \\
\hline
\end{tabular}

RESEARCH DEVELOPMENT DIVISION INSTRURENT SERVICE LOG

\begin{tabular}{|c|c|}
\hline Certification & \\
\hline Caldbration & \\
\hline Maintenance & \\
\hline Repair & \\
\hline Vendor cert. & \\
\hline Vendor calib. & \\
\hline Vendor Repair & \\
\hline other & \\
\hline
\end{tabular}

AS RECEIVED (Certified Equipment only)

Meets Manufacturer's Tolerance $N$

operates within previously defined limits $\mathrm{N}$

leviates from Mfg or previously defined limits $Y$

Inoperative (Describe below) $Y$

AS LEFT

Iimited (Describe below)

Meets Manufacturer's Tolerance

other (Describe Below)
Babcock \& Wilcox $\checkmark$

a MrDermolt company

B\&W Ser. No. 0880545

MANUFACTURER

MODEL

ITEM

MANUF. SER. NO.

PROPERTY NO.

ORDER NO.

SECTION

46

IOCATION

SCHEDULED ( $Y-N)$

115

IN $Y$

RECAL INT. (MOS.)
SENSOTEC

TJE/127810

TRANSMITR, PRES

194491

4427-01-107

\section{TECH. PROCEDURE A0015}

Babcock Wilcox RED Division certifies that the performance of this instrument was verified using standards whose accuracies are traceable to the National Institute of standards and Technology, an accepted value of a natural physical constant. a ratio type of calibration techinique, or to a consensus standard.

STAIDARDS USED

Manufacturer

ASHCROFT

ELVKE
Model

Item
Bew Serial No.

0 -10OPSI PRESS, STD
Dmm

0 \begin{tabular}{l|l|l|l|l|l|}
\hline & 0 & 0 & 12 & \\
\hline
\end{tabular} $8840 \mathrm{~A}$

\begin{tabular}{l|l|l|l|l|l}
8 & 6 & 0 & 4 & 0 \\
\hline
\end{tabular}

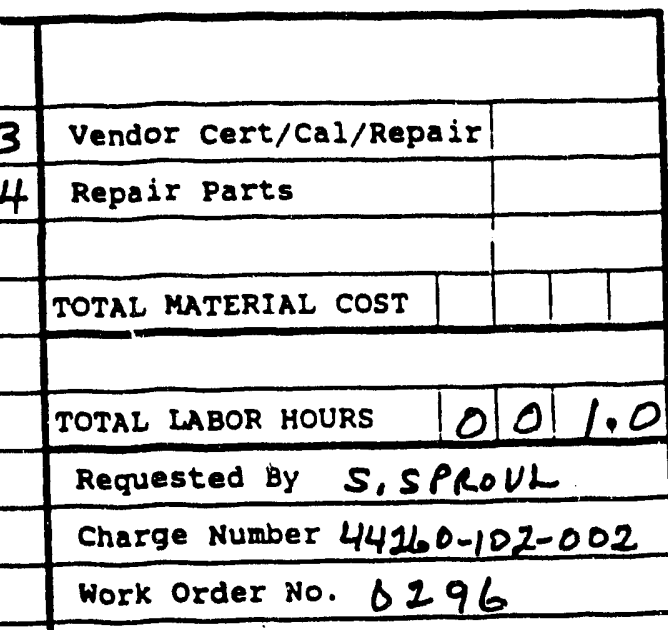

OTHER BOUIPMENT USED

\begin{tabular}{lllllll} 
& & & & & \\
\hline & & & & & \\
\hline & & & & & & \\
\hline & & & & &
\end{tabular}

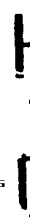

\begin{tabular}{l} 
OTHER EOUIPMENT USED \\
\begin{tabular}{lll|l|l|l|l|l|l|}
\hline COOPER & DP160 THERMOMETER & 0 & 8 & 9 & 0 & 1 & 6 & 6 \\
\hline COMPAR & PORABLE COMPUTER & 2 & 8 & 9 & 0 & 1 & 9 & 4
\end{tabular} \\
\hline
\end{tabular}
Work Order No. 6296

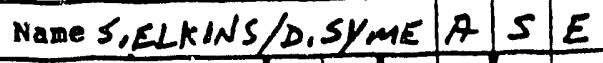

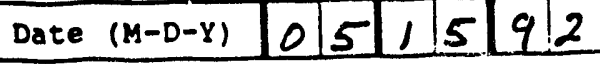

Reviewed By

Service Notes $0-100$ PSIG, CZAPO5
SEE ATTACHED DATA SHEET.

$14.998 \Omega$

LIMITED TO PATA PRO VIDED, DOES NOT MEET 4.1 RATIO SPECS, 
AS FIECEIVED

TRANSMITTER:

F'RESS SOUFICE

ASHCROFT DIGIGAUGE
O-100 FSI
E\&W SER. NO. 0700123

FEADOUT DEV:
TOLEFANCE : $0.05 \%$

SENSOTEC TJE 100 FSI

TOLERANCE: $0.10 \%$

\section{FLUKE $8840 A$}

E\&W SEr. NO. OB60404

TOLERANCE: $0.015 \%$
AS LEFT

E\&W S.N. 0880545

DATE: 15-May-92

FROCEDURE : AFIC-TF-O15-OB

FFIOGRAM: TOO15_08.03A

NAME: S,ELkINS/D, SYME

Ambient Temperature (F) 70

Facility I. D. Name

\section{C2AFOS}

\begin{abstract}
VALUE OF CUFIRENT SENSING FESISTOR USED FOF TEST DATA VALUE OF CUFFENT SENSING RESISTOF USED FOF CALIERATION

FEESISTOF MEASUFIING ACCUFIACY

14.998 DHMS 14.998 OHMS
\end{abstract}

F'OWEF' SUF'FLY VOLTAGE TOTAL SEFIES RIESISTANCE

APFLLIED FFESSURE TFIANSMITTER FFESS SOUFICE F'SI

$\begin{array}{rr}0.000 & 0.000 \\ 10.000 & 10.000 \\ 20.000 & 20.000 \\ 30.000 & 50.000 \\ 40.000 & 40.000 \\ 50.000 & 50.000 \\ 60.000 & 60.000 \\ 70.000 & 70.000 \\ 80.000 & 80.000 \\ 90.000 & 90.000 \\ 100.000 & 100.000 \\ 90.000 & 90.000 \\ 80.000 & 80.000 \\ 70.000 & 70.000 \\ 60.000 & 60.000 \\ 50.000 & 50.000 \\ 40.000 & 40.000 \\ 30.000 & 50.000 \\ 20.000 & 20.000 \\ 10.000 & 10.000 \\ 0.000 & 0.000\end{array}$

24

14.998

I DEAL

OUTFUT SIG

MILLIVOLTS

59.992

83.989

107.986

131.982

179.976

203.973

227.970

251.966

275.963

299.960

275.96 .5

251.966

227.970

20.975

179.976

155.979

131.982

107.986

8.989

59.992
155.979
CALIBFIATION ACCUFIACY

**LIMI TED**

MEASURED

OUTFUT SIG

MILLIVOLTS

\section{FULL SCALE}

$0.17 \%$

DUT

OF
$0.00 \%$

$-0.04 \%$

$-0.06 \%$

$-0.06 \%$

$-0.03 \%$

$-0.03 \%$

$-0.0 .5 \%$

$-0.01 \%$

$-0.02 \%$

$-0.01 \%$

$0.00 \%$

$-0.03 \%$

$-0.07 \%$

$-0.08 \%$

$-0.11 \%$

$-0.10 \%$

$-0.10 \%$

$-0.11 \%$

$-0.10 \%$

$-0.07 \%$

$-0.01 \%$
TOLERANCE

$\bar{z}=59.970$ 
CURVEFIT OUTPUT FOR VTAB C2AP05/

VOLTAGE(V) OBS. PRESS. CAIC. PRESS. DIFF. (PSIA) -DIFFERENCE
DATE: 20-MAY -92

TIME: $16: 43: 16$

(PSIA)

(PSIA)

$\begin{array}{rlllll}1 & 0.1100110 \mathrm{E}-04 & 0.0000000 \mathrm{E}+00 & 0.1171033 \mathrm{E}-01 & -0.1171033 \mathrm{E}-01 & 0.0000000 \mathrm{E}+00 \\ 2 & 0.2391900 \mathrm{E}-01 & 10.00000 & 10.00725 & -0.7248122 \mathrm{E}-02 & -0.7248122 \mathrm{E}-01 \\ 3 & 0.4787500 \mathrm{E}-01 & 20.00000 & 20.01012 & -0.1012296 \mathrm{E}-01 & -0.5061482 \mathrm{E}-01 \\ 4 & 0.7187900 \mathrm{E}-01 & 30.00000 & 30.02228 & -0.2227798 \mathrm{E}-01 & -0.7425992 \mathrm{E}-01 \\ 5 & 0.9594900 \mathrm{E}-01 & 40.00000 & 40.05318 & -0.5317893 \mathrm{E}-01 & -0.1329473 \\ 6 & 0.1199400 & 50.00000 & 50.04443 & -0.4443426 \mathrm{E}-01 & -0.8886853 \mathrm{E}-01 \\ 7 & 0.1439280 & 60.00000 & 60.02975 & -0.2975353 \mathrm{E}-01 & -0.4958922 \mathrm{E}-01 \\ 8 & 0.1679850 & 70.00000 & 70.04112 & -0.4112083 \mathrm{E}-01 & -0.5874404 \mathrm{E}-01 \\ 9 & 0.1919510 & 80.00000 & 80.01398 & -0.1397871 \mathrm{E}-01 & -0.1747338 \mathrm{E}-01 \\ 0 & 0.2159710 & 90.00000 & 90.01069 & -0.1069391 \mathrm{E}-01 & -0.1188212 \mathrm{E}-01 \\ 11 & 0.2399870 & 100.0000 & 100.0092 & -0.9159869 \mathrm{E}-02 & -0.9159869 \mathrm{E}-02 \\ 2 & 0.2159100 & 90.00000 & 89.98530 & 0.1469672 \mathrm{E}-01 & 0.1632969 \mathrm{E}-01 \\ 3 & 0.1918370 & 80.00000 & 79.96654 & 0.3346124 \mathrm{E}-01 & 0.4182655 \mathrm{E}-01 \\ 14 & 0.1678120 & 70.00000 & 69.96913 & 0.3086873 \mathrm{E}-01 & 0.4409818 \mathrm{E}-01 \\ 5 & 0.1437470 & 60.00000 & 59.95442 & 0.4557757 \mathrm{E}-01 & 0.7596263 \mathrm{E}-01 \\ 6 & 0.1197640 & 50.00000 & 49.97116 & 0.2884289 \mathrm{E}-01 & 0.5768577 \mathrm{E}-01 \\ 17 & 0.9576600 \mathrm{E}-01 & 40.00000 & 39.97694 & 0.2305618 \mathrm{E}-01 & 0.5764046 \mathrm{E}-01 \\ 18 & 0.7175400 \mathrm{E}-01 & 30.00000 & 29.97016 & 0.2983556 \mathrm{E}-01 & 0.9945185 \mathrm{E}-01 \\ 9 & 0.4777400 \mathrm{E}-01 & 20.00000 & 19.96797 & 0.3202584 \mathrm{E}-01 & 0.1601292 \\ 1 & 0.2386000 \mathrm{E}-01 & 10.00000 & 9.982598 & 0.1740235 \mathrm{E}-01 & 0.1740235 \\ 21 & -0.1199890 \mathrm{E}-04 & 0.0000000 \mathrm{E}+00 & 0.2087656 \mathrm{E}-02 & -0.2087656 \mathrm{E}-02 & 0.0000000 \mathrm{E}+00\end{array}$

EGREE OF POLYNOMIAL 3

COEFFICIENTS

$0.71077205 E-02 \quad 0.41837706 E+03-0.12855802 E+02 \quad 0.24411512 E+02$

ESS $=0.1651 \mathrm{E}-01 \quad \mathrm{SD}=0.3117 \mathrm{E}-01 \quad \mathrm{R} 2=0.999999 \mathrm{E}+00$

$2 * S D=0.6233 \mathrm{E}-01$ 
$-1=-2$

RD-45A Rev. 11-18-91

FUSEARCH \& DEVEIOPAENT DIVISION INSTRUYENI SERVICE LOG

\begin{tabular}{|l|l|l|}
\hline INSTRUMENT STATUS & $\begin{array}{c}\text { AS } \\
\text { RICD }\end{array}$ & \begin{tabular}{c} 
AS \\
\hline 1 Certified
\end{tabular} \\
\hline Calibrated & & \\
\hline 3 For Ind. Only & & $\checkmark$ \\
\hline 4 standardize & & \\
\hline 5 Certified c stand. & & \\
\hline 6 Calibrated s Stand. & & \\
\hline 9 other & & \\
\hline
\end{tabular}

INSTRUMENT CONDITION

AS RECEIVED (Certified Equipment only)

Meets Manufacturer's rolerance $N$

Operates within previously defined limits $N$ Deviates from Mfg or previously defined limits $Y$ Inoperative (Describe below) $y$

AS IEFT

Limited (Describe below)

Meets Manufacturer's Tolerance

Other (Describe Below)
Babcock \& Wilcox

- McDermoti company

B\&W Ser. No. 0880546

MANUFACTURER

MODEL

ITEM

MANUF. SER. NO

PROPERTY NO.

ORDER NO.

SENSOTEC

TJE/127810

TRANSMITR, PRES

194495

4427-01-108

$\begin{array}{lc}\text { SECTION } & 46 \\ \text { IOCATION } & 115 \\ \text { SCHEDULED (Y-N) } & \mathbb{1} \text { Y } \\ \text { RECAL INT.(MOS.) } & 12\end{array}$

TECH. PROCEDURE A0015

Babcock \& Wllox RED Division certifjes that the performance of this instrument was verified using standards whoise accuracies are traceable to the National Institute of Standards and Technology, an accepted vilue of a natural physical constant, a ratio type of calibration technique, or to a consensus standard.

\section{Manufacturer $\frac{\text { STAIIDARDS USED }}{\text { Hodel Item }}$ BEw serial No.}

\begin{tabular}{lll|l|l|l|l|l|l|l|l|}
\hline ASHCROFT O-100PSI PRISS, STD, & 0 & 7 & 0 & 0 & 1 & 2 & 3 & vendor Cert/Cal/Repair
\end{tabular}

FLUKE $8840 \mathrm{~A} \quad \mathrm{DmM}$

\begin{tabular}{l|l|l|l|l|l|l|}
0 & 8 & 6 & 0 & 4 & 0 & 4
\end{tabular}

Repair Parts

TOTAL MATERIAL COST

\begin{tabular}{l|l|l|l|l}
\hline TOTAL IABOR HOURS & 0 & 0 & 1.0 \\
\hline
\end{tabular}

Requested By S, SPRovL

Charge Number 44260-102-002

OTHER EOUTPMENT USED

work order No. 0296

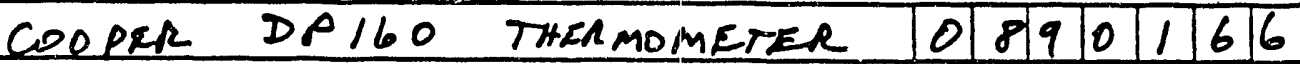

COMPAQ PORABLE COMPUTER 22 899 011194

Name S, ELKINS/D.SYAE $|A| D|S|$

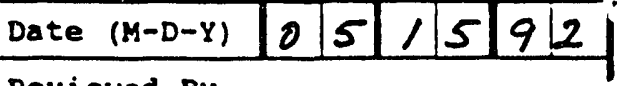

Reviewed By

Service notes $0-100$ PSIG, C2APOG ADS+41998s $14.995 \Omega$

SEE ATTACHED DATA SHEET.

$\angle I M I T E D$ TO DATA PROVIDED, DOES NOT MEET H:I RATO SPECS, 
AS FECE IVED

RAANSMITTER:

FESS SOURICE

ASHCROFT DIGIGAUGE
O-100 FSI
E\&W SER. NO. 0700123

TOLEFIANCE: $0.05 \%$

¿ADOUT DEV:
AS LEFT

B\&W S.N. OB8O546 DATE: 15-May-92

F'ROCEDURE : AFIC-TF-015-08

FFIOGFAM: TOO15_08.03A

NAME: S,ELKWS B.SYONE

Ambient Temperature (F) 70

Facility I. D. Name CZAFO6

E\&:W SER. NO. 0860404

TOLEFIANCE: $0.015 \%$

ALUE OF CURFENT SENSING FESISTOF USED FOR TEST DATA

IALUE OF CUFRENT SENSING FIESISTOF: USED FOR CALIBFATION

JWER SUFF'LY VOLTAGE

OTAL SEFIES FESISTANCE

AF'PLIED F'FESSURE - R̃ANSMITTEF: F'S I

F'FIESS SOURICE

F'S I

0.000

0.000

10.000

20.000

30.000

40.000

50.000

60.000

70.000

80.000

90.000

100.000

90.000

80.000

70.000

60.000

50.000

40.000

30.000

20.000

10.000

0.000
FEESISTOF MEASURIING ACCURACY 24

14.995

IDEAL DUTFUT SIG MILLIVOLTS

59.980

83.972

107.964

151.956

155.948

179.940

20.932

227.924

251.916

275.908

299.900

275.908

251.916

227.924

205.932

179.940

155.948

131.956

107.964

83.972

59.980

\section{CAL IBFAT ION ACCUFACY **LIMITED** MEASUIED OUTFUT SIG FULL SCALE MILLIVOLTS}

$$
14.995 \text { OHMS }
$$$$
14.995 \text { OHMS }
$$

N.A.

$$
\begin{array}{rr}
59.947 & -0.01 \% \\
83.908 & -0.03 \% \\
107.905 & -0.03 \% \\
131.905 & -0.02 \% \\
155.957 & 0.00 \% \\
179.938 & 0.00 \% \\
203.926 & 0.00 \% \\
227.978 & 0.02 \% \\
251.956 & 0.02 \% \\
275.949 & 0.02 \% \\
299.898 & 0.00 \% \\
275.871 & -0.02 \% \\
251.858 & -0.02 \% \\
227.837 & -0.04 \% \\
203.794 & -0.06 \% \\
179.798 & -0.06 \% \\
155.824 & -0.05 \% \\
1.21 .815 & -0.06 \% \\
107.821 & -0.06 \% \\
83.845 & -0.05 \% \\
59.951 & -0.01 \%
\end{array}
$$

OUT
OF
TOLERANCE

$\bar{z}=59.949$ 
CURVEFIT OUTPUT FOR VTAB C2AP06 $J$

VOLTAGE(V) OBS. PRESS. CALC. PRESS. DIFF. (PSIA) \& DIFFERENCE
DATE: 20-MAY-92

TIME: 16:44:21
(PSIA)

$\begin{array}{rl}1 & -0.1999435 \mathrm{E}-05 \\ 2 & 0.2395900 \mathrm{E}-01 \\ 3 & 0.4795400 \mathrm{E}-01 \\ 4 & 0.7195400 \mathrm{E}-01 \\ 5 & 0.9600800 \mathrm{E}-01 \\ 6 & 0.1199890 \\ 7 & 0.1439770 \\ 8 & 0.1680290 \\ 9 & 0.1920070 \\ 10 & 0.2160000 \\ 11 & 0.2399490 \\ 12 & 0.2159220 \\ 13 & 0.1919090 \\ 14 & 0.1678880 \\ 15 & 0.1438450 \\ 16 & 0.1198490 \\ 17 & 0.9587500 \mathrm{E}-01 \\ 18 & 0.7186600 \mathrm{E}-01 \\ 19 & 0.4787200 \mathrm{E}-01 \\ & 0.2389600 \mathrm{E}-01 \\ 21 & 0.2000565 \mathrm{E}-05\end{array}$

$0.0000000 E+00$
10.00000
20.00000
30.00000
40.00000
50.00000
60.00000
70.00000
80.00000
90.00000
100.0000
90.00000
80.00000
70.00000
60.00000
50.00000
40.00000
30.00000
20.00000
10.00000
$0.0000000 E+00$

$0.0000000 \mathrm{E}+00$

$0.3643884 \mathrm{E}-02$

10.00609

20.01453

30.01849

40.04005

50.02796

60.01722

70.03322

80.02015

90.01673

100.0000

89.98422

79.97932

69.97450

59.96225

49.96966

39.98465

29.98182

19.98034

9.979804

$0.5314448 \mathrm{E}-02$
$-0.3643884 \mathrm{E}-02$
$-0.6092025 \mathrm{E}-02$
$-0.1452652 \mathrm{E}-01$
$-0.1849046 \mathrm{E}-01$
$-0.4005150 \mathrm{E}-01$
$-0.2796477 \mathrm{E}-01$
$-0.1722201 \mathrm{E}-01$
$-0.3321841 \mathrm{E}-01$
$-0.2014671 \mathrm{E}-01$
$-0.1672867 \mathrm{E}-01$
$-0.2900127 \mathrm{E}-04$
$0.1577701 \mathrm{E}-01$
$0.2067647 \mathrm{E}-01$
$0.2550184 \mathrm{E}-01$
$0.3774510 \mathrm{E}-01$
$0.3033805 \mathrm{E}-01$
$0.1534951 \mathrm{E}-01$
$0.1818078 \mathrm{E}-01$
$0.1966398 \mathrm{E}-01$
$0.2019567 \mathrm{E}-01$
$-0.5314448 \mathrm{E}-02$ $0.0000000 E+00$ $-0.6092025 E-01$ $-0.7263260 \mathrm{E}-01$

$-0.6163488 E-01$

$-0.1001287$

$-0.5592954 \mathrm{E}-01$

$-0.2870336 \mathrm{E}-01$

$-0.4745487 E-01$

$-0.2518338 E-01$

$-0.1858741 E-01$

$-0.2900127 \mathrm{E}-04$

$0.1753001 E-01$

$0.2584558 \mathrm{E}-01$

$0.3643120 \mathrm{E}-01$

$0.6290850 \mathrm{E}-01$

$0.6067610 \mathrm{E}-01$

$0.3837378 \mathrm{E}-01$

$0.6060260 \mathrm{E}-01$

$0.9831992 E-01$

0.2019567

$0.0000000 \mathrm{E}+00$

\section{DEGREE OF POLYNOMIAL 3}

\section{COEFFICIENTS}

$0.44789299 \mathrm{E}-02$

$0.41764088 E+03-0.85715034 E+01$

$0.20017584 E+02$

$\mathrm{ESS}=0.1014 \mathrm{E}-01$

$$
\begin{aligned}
S D & =0.2442 \mathrm{E}-01 \\
2 \star S D & =0.4884 \mathrm{E}-01
\end{aligned}
$$

$R_{2}=0.999999 E+00$ 
1.

$: D-45 A \quad$ Rev. $11-18-91$

RESEARCH \& DEVELOPMENT DIVISION INSTRUMENT SERVICE LOG

\begin{tabular}{l|l|l|}
\hline INSTRUMENT STATUS & PSES & AS \\
\hline Certified & & \\
\hline Calibrated & & $\checkmark$ \\
\hline For Ind. Only & $\checkmark$ & \\
\hline Standardize & & \\
\hline Certified S Stand. & & \\
\hline Calibrated S Stand. & & \\
\hline Other & & \\
\hline
\end{tabular}

INSTRUMENT CONDITION

AS RECEIVED (Certified Equipment only)

Meets Manufacturer's Tolerance $N$

operates within previously defined limits $N$ leviates from $M f g$ or previously defined limits $Y$ Inoperative (Describe below) $Y$ AS LEFT Limited (Describe below) Meets Manufacturer's Tolerance Other (Describe Below)

\section{(2) 07 \\ Babcock \& Wilcox \\ a MCDermoti company}

B\&W Ser. No. 0880537

MANUFACTURER

MODEL

ITEM

MANUF . SER. NO.

PROPERTY NO.

ORDER NO.

SENSOTEC

TJE/ 127810

TRANSMITR, PRES

188751

4427-01-099

SECTION 46

LOCATION $\quad 115$

SCHEDULED $(Y-N) \quad \# Y$

RECAL INT. (MOS.) 12

\section{TECH. PROCEDURE A0015}

Babcock Wilcox RED Division certifies that the performance of this instrument was verified using standards whose accuracies are traceable to the National Institute of Standards and Technology, an accepted value of a natural physical constant, a ratio type of calibration technique, or to a consensus standard.

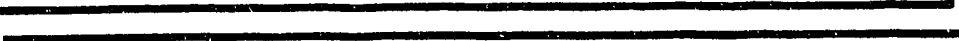

STAUDARDS USED

Manufacturer

Model

Item

BEW Serial No.

7 SHCROFT

O-10OPSI PRESS, STD.

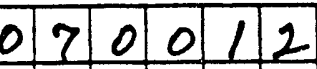

FLUKE $8840 A$ DMM \begin{tabular}{ll|l|llllll}
0 & 8 & 6 & 0 & 4 & 0 & 4 & Repair Parts
\end{tabular}

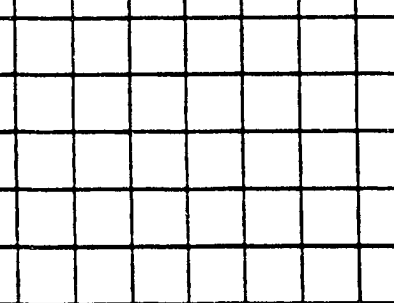

OTHER EOUIPMENT USED

\begin{tabular}{llc|l|l|l|l|l|l|l}
\hline CODPER & DPI6O & THLAMOMETER & 0 & 8 & 9 & 0 & 1 & 6 & 6 \\
\hline COMPAR & PORTABLE & COMPJTER & 2 & 8 & 9 & 0 & 1 & 9 & 4 \\
\hline
\end{tabular}

TOTAL MATERIAL COST

TOTAL LABOR HOURS 001011.0

Requested By S.SAROUL Charge Number 44260-102-002 Work order No. 0296

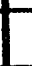

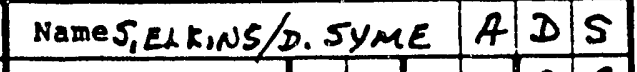
\begin{tabular}{l|l|l|l|l|l|l} 
Date $(M-D-X)$ & 0 & 5 & 1 & 4 & 9 & 2 \\
\hline
\end{tabular}

Reviewed By

ervice Notes 0 -100 PSIG, C2APO7 ADS $14,998 \Omega 14.996 \Omega$

SEE ATTACHED DATA SHEET,

LIMITED TO DATA PROVIDED DOES NOT MEET H:I RATIO SPECS. 
AS FEECEIVED

TFAANSMI TTEF: :

FRESS SOUREE

\section{ASHCFIOFT DIGIGAUGE 0-100 F.SI \\ E\&W Ser. NO. 0700123 \\ TOLEFIANCE : $0.05 \%$}

FEADOUT DEVV:

FLUKE 8840A

E\&W Ser. No. 0860404

TOLEFIANCE: $0.015 \%$
AS LEFT

E\&.W S.N. 08805.87 DATE: 14-May-92

FROCEDUFE : AFIC-TF-015-08

FRIOGFIAM: T0015_08.03A

NAME: S.EKNS/D, SYME

Ambient Temperature (F) 70

Facility I. D. Name

\section{VALUE OF CUFFENT SENSING FESISTOF USED FOF TEST DATA \\ VALUE OF CUFFENT SENSING FESISTOF USED FOF CALIEFIATION FIESISTOF MEASUFING ACCURACY

\author{
14.956 DHMS \\ 14.996 OHMS \\ N.A.
}

FOWEF SUFFLY VOLTAGE

TOTAL SEFIES FESISTANCE

AF'F'LIED F'FIESSURE

TFANSMITTEF: FSI

FRESS SOLFICE

FSI

$\begin{array}{rr}0.000 & 0.000 \\ 10.000 & 10.000 \\ 20.000 & 20.000 \\ 30.000 & 30.000 \\ 40.000 & 40.000 \\ 50.000 & 50.000 \\ 60.000 & 60.000 \\ 70.000 & 70.000 \\ 80.000 & 80.000 \\ 90.000 & 90.000 \\ 100.000 & 100.000 \\ 90.000 & 90.000 \\ 80.000 & 80.000 \\ 70.000 & 70.000 \\ 60.000 & 60.000 \\ 50.000 & 50.000 \\ 40.000 & 40.000 \\ 30.000 & 30.000 \\ 20.000 & 20.000 \\ 10.000 & 10.000 \\ 0.000 & 0.000\end{array}$

24

14.996

IDEAL

QUTFUT SIG

MILLIVOLTS

59.984

8.3 .978

107.971

1.31 .965

155.958

179.952

203.946

227.939

251.9 .3

275.926

299.920

275.926

251.933

227.939

205.946

179.952

155.958

131.965

107.971

83.978

59.984

$\begin{array}{ccc}\text { CALIEFATION ACCUFACY } & \\ & \text { *LIMITED** } & \\ \text { MEASUFED } & \text { EFROR } \% & \text { OUT } \\ \text { OUTFUT SIG } & \text { FULL SCALE } & \text { OF } \\ \text { MILLIVOLTS } & & \text { TOLERANCE }\end{array}$

$0.00 \%$

$-0.06 \%$

$-0.08 \%$

$-0.08 \%$

$-0.07 \%$

$-0.07 \%$

$-0.06 \%$

$-0.05 \%$

$-0.03 \%$

$-0.01 \%$

$0.00 \%$

$-0.05 \%$

$-0.10 \%$

$-0.12 \%$

$-0.14 \%$

$-0.15 \%$

$-0.14 \%$

$-0.15 \%$

$-0.1 .3 \%$

$-0.08 \%$

$-0.01 \%$

\section{$\bar{z}=59.971$}


CURVEFIT OUTPUT FOR VTAB C2AP07 $\checkmark$

VOLTAGE $(V)$ OBS. PRESS. CALC. PRESS. DIFF. (PSIA) DIFFERENCE
DATE: 20-MAY -92

TIME: $16: 45: 25$
(PSIA)

$\begin{array}{rlllll}1 & 0.1899900 \mathrm{E}-04 & 0.0000000 \mathrm{E}+00 & 0.1616980 \mathrm{E}-01 & -0.1616980 \mathrm{E}-01 & 0.0000000 \mathrm{E}+00 \\ 2 & 0.2386400 \mathrm{E}-01 & 10.00000 & 10.00419 & -0.4191907 \mathrm{E}-02 & -0.4191907 \mathrm{E}-01 \\ 3 & 0.4781500 \mathrm{E}-01 & 20.00000 & 20.01887 & -0.1887076 \mathrm{E}-01 & -0.9435379 \mathrm{E}-01 \\ 4 & 0.7179800 \mathrm{E}-01 & 30.00000 & 30.03159 & -0.3158789 \mathrm{E}-01 & -0.1052930 \\ 5 & 0.9582200 \mathrm{E}-01 & 40.00000 & 40.04852 & -0.4852169 \mathrm{E}-01 & -0.1213042 \\ 6 & 0.1198220 & 50.00000 & 50.04505 & -0.4504749 \mathrm{E}-01 & -0.9009498 \mathrm{E}-01 \\ 7 & 0.1438320 & 60.00000 & 60.03783 & -0.3782853 \mathrm{E}-01 & -0.6304756 \mathrm{E}-01 \\ 8 & 0.1678940 & 70.00000 & 70.04682 & -0.4681656 \mathrm{E}-01 & -0.6688080 \mathrm{E}-01 \\ 9 & 0.1918900 & 80.00000 & 80.02543 & -0.2542685 \mathrm{E}-01 & -0.3178356 \mathrm{E}-01 \\ 10 & 0.2159280 & 90.00000 & 90.02107 & -0.2107370 \mathrm{E}-01 & -0.2341523 \mathrm{E}-01 \\ 1 & 0.2399390 & 100.0000 & 100.0076 & -0.7559560 \mathrm{E}-02 & -0.7559559 \mathrm{E}-02 \\ 2 & 0.2158260 & 90.00000 & 89.97866 & 0.2134324 \mathrm{E}-01 & 0.2371472 \mathrm{E}-01 \\ 3 & 0.1917200 & 80.00000 & 79.95474 & 0.4526231 \mathrm{E}-01 & 0.5657789 \mathrm{E}-01 \\ 14 & 0.1676770 & 70.00000 & 69.95657 & 0.4343131 \mathrm{E}-01 & 0.6204473 \mathrm{E}-01 \\ 5 & 0.1436360 & 60.00000 & 59.95628 & 0.4371986 \mathrm{E}-01 & 0.7286643 \mathrm{E}-01 \\ 6 & 0.1196280 & 50.00000 & 49.96428 & 0.3572271 \mathrm{E}-01 & 0.7144543 \mathrm{E}-01 \\ 17 & 0.9565400 \mathrm{E}-01 & 40.00000 & 39.97851 & 0.2148773 \mathrm{E}-01 & 0.5371932 \mathrm{E}-01 \\ 8 & 0.7164300 \mathrm{E}-01 & 30.00000 & 29.96692 & 0.3307942 \mathrm{E}-01 & 0.1102647 \\ 9 & 0.4767900 \mathrm{E}-01 & 20.00000 & 19.96205 & 0.3794962 \mathrm{E}-01 & 0.1897481 \\ & 0.2380300 \mathrm{E}-01 & 10.00000 & 9.978664 & 0.2133580 \mathrm{E}-01 & 0.2133580 \\ 2.1 & -0.1900100 \mathrm{E}-04 & 0.0000000 \mathrm{E}+00 & 0.2372668 \mathrm{E}-03 & -0.2372668 \mathrm{E}-03 & 0.0000000 \mathrm{E}+00\end{array}$

(PSIA)

EGREE OF POLYNOMIAL 3

OEFFICIENTS

$0.82039605 \mathrm{E}-02 \quad 0.41927728 \mathrm{E}+03-0.17652054 \mathrm{E}+02 \quad 0.30016025 \mathrm{E}+02$

$\pi S S=0.2182 \mathrm{E}-01 \quad \mathrm{SD}=0.3582 \mathrm{E}-01 \quad \mathrm{R} 2=0.999999 \mathrm{E}+00$ 
$-1$

RD-45A Rev. $11-18-91$

\begin{tabular}{|l|l|l|}
\hline \multicolumn{1}{|l|}{ INSTRUMENT STATUS } & RESE & IEST \\
\hline Certified & & \\
\hline Calibrated & & $\checkmark$ \\
\hline 3 For Ind. Only & $\checkmark$ & \\
\hline 4 Standardize & & \\
\hline 5 Certified c stand. & & \\
\hline 6 Calibrated a stand. & & \\
\hline 9 Other & & \\
\hline
\end{tabular}

INSTRUMENT CONDITION

AS RECEIVED (Certified Equipment only)

Meets Manufacturer's Tolerance $N$

operates within previously defined limits $N$ Deviates from Mfg or previously defined limits $Y$ Inoperative (Describe below) $X$

AS IEFT Limited (Describe below)

Meets Manufacturer's Tolerance other (Describe Below)

$$
\text { varof }
$$

Babcock \& Wilcox

- MCDermott company

B\&W Ser. No. 0880549

MANUFACTURER SENSOTEC

MODEL

ITEM

TJE/127810

MANUF . SER. NO. 201306

PROPERTY NO. 4427-01-111

ORDER NO.

SECTION

LOCATION

46

SCHEDULED $(Y-N)$

RECAL INT. (MOS.)

115

17- $Y$

12

TECH. PROCEDURE A0015

Babcock \& Wilcox RED Division certifies that the performance of this instrument was verified using standards whose accuracies are traceable to the National Institute of Standards and Technology. an accepted value of a natural physical constant a ratio type of calibration technique, or to a consensus standard.

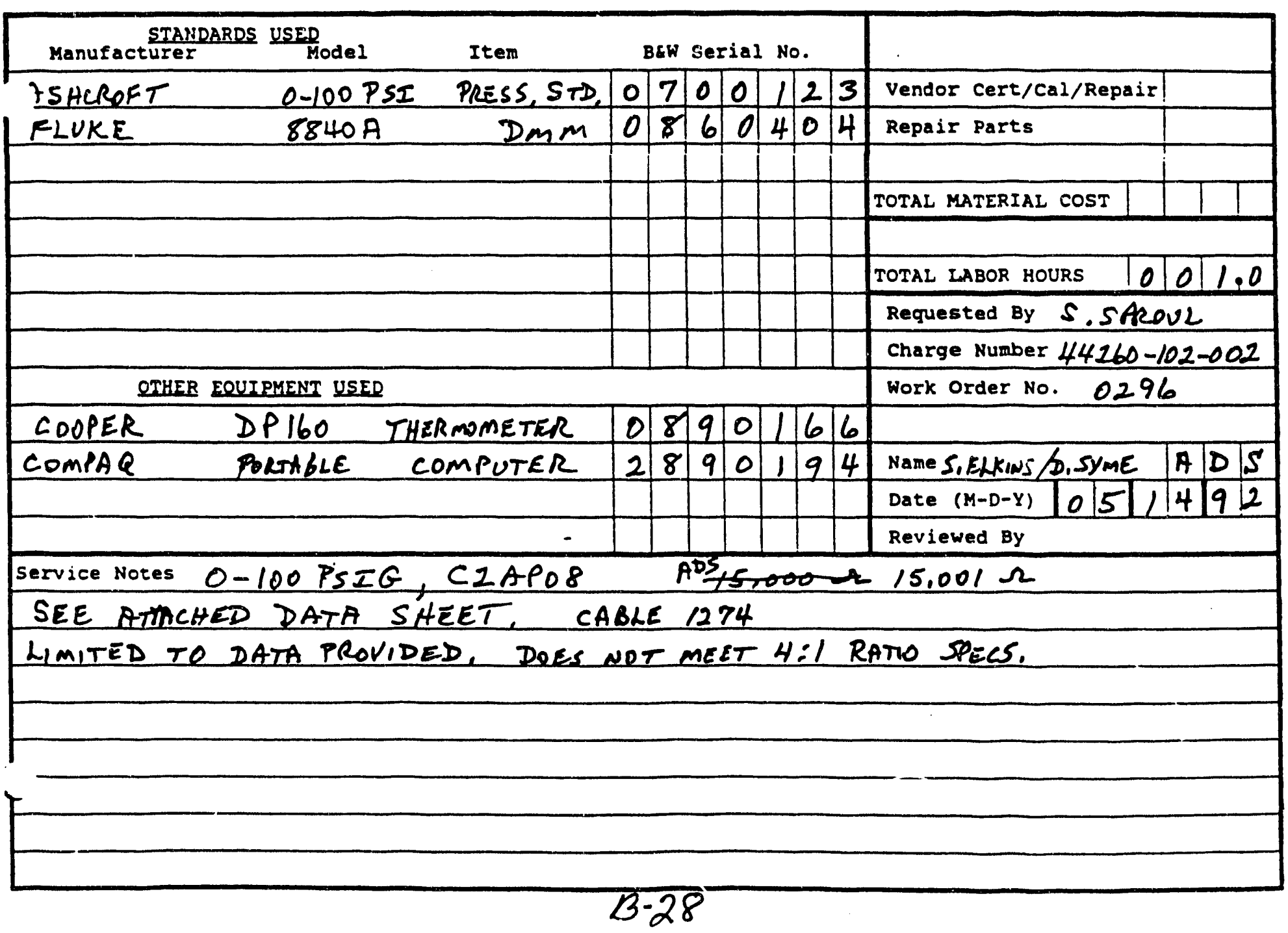


AS RECEIVED

riANSMITTEF:

RESS SOURCE

ASHCFOFT DIGIGAUGE
$0-100$ FSI
B\&W SER. No. 0700123

TOLEFANCE: $0.05 \%$

:ADOUT DEV:

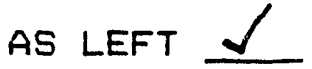

E\&WW S.N. O880549 $\because$

DATE: $14-M a y-92$

FFIOCEDURE : ARC-TF-015-08

F'FIOGFAM: TOO15_08.03A

NAME: S,ELK,NS Po. SYME

Ambient Temperature (F) 70
Facility I. D. Name C2AFOS

B\&W SER. NO. OB60404

TOLEFIANCE: $0.015 \%$

ILUE QF CUFRENT SENSING FESISTOF USED FOF TEST DATA ALUE OF CURRENT SENSING FESISTOF USED FOF CALIERATION FIESISTOF: MEASUFIING ACCURACY 24

IWEF SUFF'LY VOLTAGE

UTAL SEFIES FIESISTANCE

AFFLIED FFESSUFE .ANSMI TTEF:

F'SI

F'R'ESS SOURICE

FSI

$\begin{array}{rr}0.000 & 0.000 \\ 10.000 & 10.000 \\ 20.000 & 20.000 \\ 50.000 & 50.000 \\ 40.000 & 40.000 \\ 50.000 & 50.000 \\ 60.000 & 60.000 \\ 70.000 & 70.000 \\ 80.000 & 80.000 \\ 90.000 & 90.000 \\ 100.000 & 100.000 \\ 90.000 & 90.000 \\ 80.000 & 80.000 \\ 70.000 & 70.000 \\ 60.000 & 60.000 \\ 50.000 & 50.000 \\ 40.000 & 40.000 \\ 30.000 & 30.000 \\ 20.000 & 20.000 \\ 10.000 & 10.000 \\ 0.000 & 0.000\end{array}$

15.001

I DEAL

\section{OUTFUT SIG} MILLIVOLTS

60.004
84.006
108.007
132.009
156.010
180.012
204.014
228.015
252.017
276.018
300.020
276.018
252.017
228.015
204.014
180.012
156.010
132.009
108.007
84.006
60.004

CALIBRAATION ACCUFIACY

\section{**LIMITED**}

MEASUFED OUTFUT SIG MILLIVOLTS

EFF'OF' \% FULL SCALE
$0.17 \%$

15.001 OHMS

15.001 OHMS N.A.

$\begin{array}{rr}59.996 & 0.00 \% \\ 83.915 & -0.04 \% \\ 107.872 & -0.06 \% \\ 131.850 & -0.07 \% \\ 155.880 & -0.05 \% \\ 179.872 & -0.06 \% \\ 20.5689 & -0.06 \% \\ 227.925 & -0.04 \% \\ 251.936 & -0.03 \% \\ 275.967 & -0.02 \% \\ 299.996 & -0.01 \% \\ 275.958 & -0.03 \% \\ 251.848 & -0.07 \% \\ 227.791 & -0.09 \% \\ 203.715 & -0.13 \% \\ 179.699 & -0.13 \% \\ 155.720 & -0.12 \% \\ 131.710 & -0.12 \% \\ 107.759 & -0.10 \% \\ 83.854 & -0.06 \% \\ 59.996 & 0.00 \%\end{array}$

$$
\bar{z}=59.996
$$


CURVEFIT OUTPUT FOR VTAB C2AP08

VOLTAGE(V) OBS. PRESS. CALC. PRESS. DIFF. (PSIA) \& DIFFERENCE
DATE: $20-$ MAY -92

TIME: $16: 46: 25$
(PSIA)

$\begin{array}{rrr}1 & -0.1422405 \mathrm{E}-08 & 0.0000000 \mathrm{E}+00 \\ 2 & 0.2391900 \mathrm{E}-01 & 10.00000 \\ 3 & 0.4787600 \mathrm{E}-01 & 20.00000 \\ 4 & 0.7185400 \mathrm{E}-01 & 30.00000 \\ 5 & 0.9588400 \mathrm{E}-01 & 40.00000 \\ 6 & 0.1198760 & 50.00000 \\ 7 & 0.1438730 & 60.00000 \\ 8 & 0.1679270 & 70.00000 \\ 9 & 0.1919400 & 80.00000 \\ 10 & 0.2159710 & 90.00000 \\ 11 & 0.2400020 & 100.0000 \\ 12 & 0.2159420 & 90.00000 \\ 13 & 0.1918520 & 80.00000 \\ 14 & 0.1677950 & 70.00000 \\ 15 & 0.1437170 & 60.00000 \\ 16 & 0.1197030 & 50.00000 \\ 17 & 0.9572400 \mathrm{E}-01 & 40.00000 \\ 18 & 0.7171400 \mathrm{E}-01 & 30.00000 \\ 19 & 0.4776300 \mathrm{E}-01 & 20.00000 \\ 1 & 0.2385800 \mathrm{E}-01 & 10.00000 \\ 21 & -0.1422405 \mathrm{E}-08 & 0.0000000 \mathrm{E}+00\end{array}$

(PSIA)

$\begin{array}{lll}0.2329075 \mathrm{E}-02 & -0.2329075 \mathrm{E}-02 & 0.0000000 \mathrm{E}+00 \\ 10.01047 & -0.1046717 \mathrm{E}-01 & -0.1046717 \\ 20.02036 & -0.2035911 \mathrm{E}-01 & -0.1017956 \\ 30.02664 & -0.2663677 \mathrm{E}-01 & -0.8878925 \mathrm{E}-01 \\ 40.04398 & -0.4397677 \mathrm{E}-01 & -0.1099419 \\ 50.03665 & -0.3664684 \mathrm{E}-01 & -0.7329367 \mathrm{E}-01 \\ 60.02437 & -0.2436946 \mathrm{E}-01 & -0.4061577 \mathrm{E}-01 \\ 70.03056 & -0.3056211 \mathrm{E}-01 & -0.4366016 \mathrm{E}-01 \\ 80.01625 & -0.1625266 \mathrm{E}-01 & -0.2031583 \mathrm{E}-01 \\ 90.00778 & -0.7782277 \mathrm{E}-02 & -0.8646974 \mathrm{E}-02 \\ 99.99947 & 0.5334210 \mathrm{E}-03 & 0.5334210 \mathrm{E}-03 \\ 89.99573 & 0.4274978 \mathrm{E}-02 & 0.4749976 \mathrm{E}-02 \\ 79.97966 & 0.2033762 \mathrm{E}-01 & 0.2542202 \mathrm{E}-01 \\ 69.97566 & 0.2433732 \mathrm{E}-01 & 0.3476760 \mathrm{E}-01 \\ 59.95946 & 0.4054000 \mathrm{E}-01 & 0.6756666 \mathrm{E}-01 \\ 49.96462 & 0.3538037 \mathrm{E}-01 & 0.7076073 \mathrm{E}-01 \\ 39.97731 & 0.2269086 \mathrm{E}-01 & 0.5672715 \mathrm{E}-01 \\ 29.96825 & 0.3175421 \mathrm{E}-01 & 0.1058474 \\ 19.97318 & 0.2682494 \mathrm{E}-01 & 0.1341247 \\ 9.984962 & 0.1503761 \mathrm{E}-01 & 0.1503761 \\ 0.2329075 \mathrm{E}-02 & -0.2329075 \mathrm{E}-02 & 0.0000000 \mathrm{E}+00\end{array}$

DEGREE OF POLYNOMIAL

3

\section{COEFFICIENTS}

$0.23296709 E-02 \quad 0.41873770 E+03-0.13887114 E+02 \quad 0.21640320 E+02$
$E S S=0.1276 \mathrm{E}-01$
$\begin{aligned} S D & =0.2740 \mathrm{E}-01 \\ * S D & =0.5480 \mathrm{E}-01\end{aligned}$
$\mathbf{R 2}=$
$0.999999 E+00$
$2 * S D=0.5480 \mathrm{E}-01$ 
RESEARCH C DEVEIOPMENT DIVISION INSTRUMENT SERVICE IOG

D-45A Rev. $11-18-91$

\begin{tabular}{|c|c|c|}
\hline INSTRUMENT STATUS & 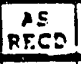 & $\begin{array}{c}A S \\
\text { ASTI } \\
\end{array}$ \\
\hline \multicolumn{3}{|l|}{ Certified } \\
\hline \multicolumn{3}{|l|}{ Calibrated } \\
\hline \multicolumn{3}{|l|}{ For Ind. Only } \\
\hline \multicolumn{3}{|l|}{ standardize } \\
\hline \multicolumn{3}{|l|}{ Certified \& stand. } \\
\hline \multicolumn{3}{|l|}{ Calibrated \& stand. } \\
\hline other & & \\
\hline
\end{tabular}

INSTRUMENT CONDITION

AS RECEIVED (Certified Equipment Only)

Meets Manufacturer's Tolerance $N$

operates within previously defined limits $N$ eviates from Mfg or previously defined limits $Y$ Inoperative (Describe below) $Y$

AS LEFT

Limited (Describe below)

Meets Manufacturer's Tolerance

other (Describe Below)
Babcock \& Wilcox

a McDermott company

B\&W Ser. No. 0880547

MANUFACTUPER

MODEL

ITEM

MANUF. SER. NO.

PROPERTY NO.

ORDER NO.

SENSOTEC

TJE/127810

TRANSMITR, PRES

195582

4427-01-109

$\begin{array}{lc}\text { SECTION } & 46 \\ \text { IOCATION } & 115 \\ \text { SCHEDULED (Y-N) } & 14 \\ \text { RECAL INT.(MOS.) } & 12\end{array}$

TECH. PROCEDURE A0015

Babcock \& Wilcox RED Division certifies that the performance of this instrument was verified using standards whose accuracies are traceable to the National Institute of Standards and Technology. an accepted value of a natural physical constant, a ratio type of calibration technique, or to a consensus standard.

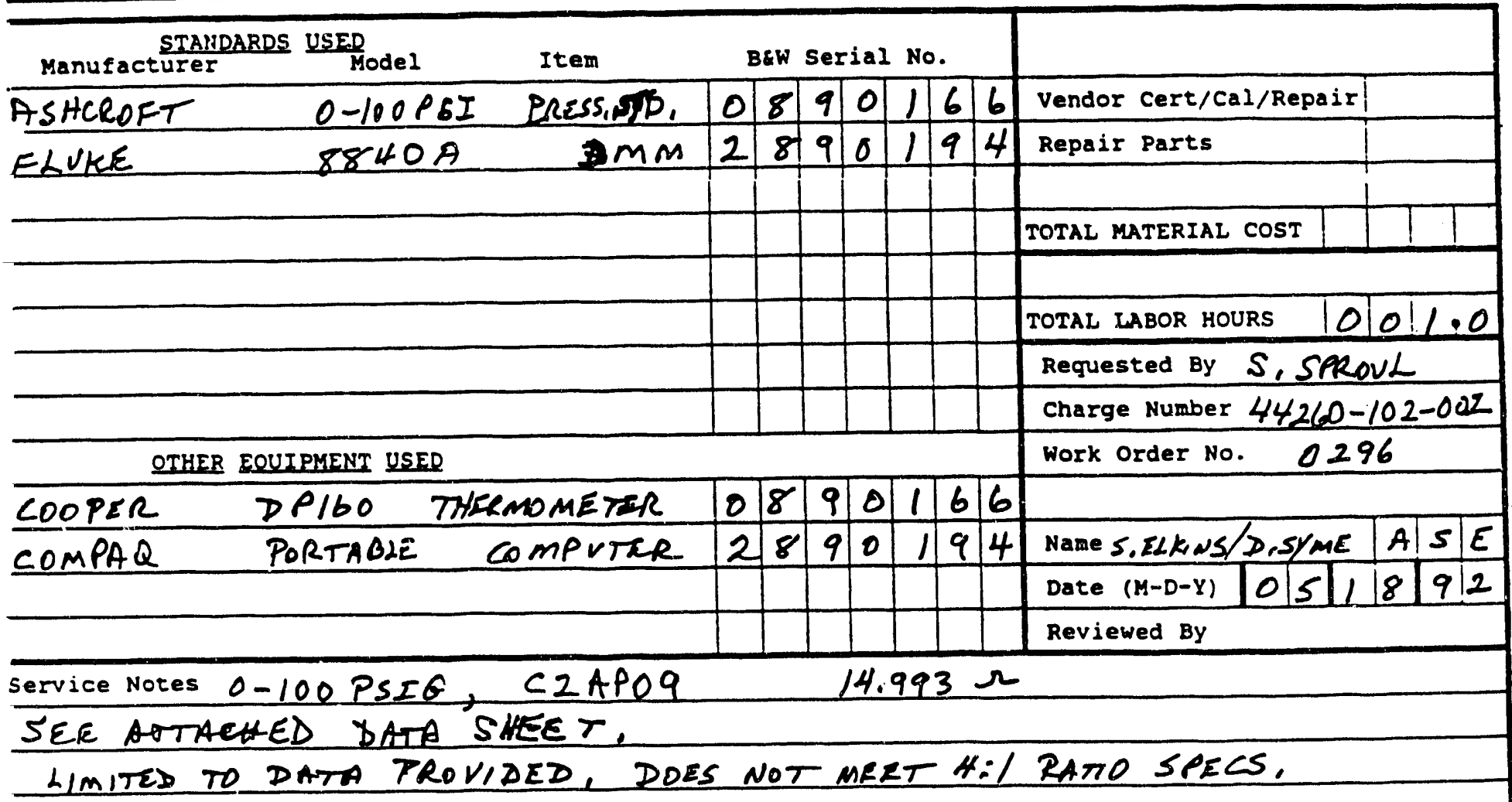


AS FIECEIVED

TRANSMITTEF:

SENSOTEC TJE 100 FSI

TOLEFANCE: $0.10 \%$

F'RESS SOUFICE

$$
\begin{array}{r}
\text { ASHCROFT DIGIGAUGE } \\
\text { O-100 FSI } \\
\text { E\&W SER. NO. } 0700123 \\
\text { TOLEFIANCE: } 0.05 \%
\end{array}
$$

READOUT DEV:

$$
\begin{aligned}
\text { FLUKE } 8840 \mathrm{~A} \\
\text { E\&W SER. NO: } 0860404 \\
\text { TOLEFANCE: } 0.015 \%
\end{aligned}
$$

AS LEFT

B\&W S.N. 0880547

DATE: $18-M a y-92$

FROCEDURE : AF'C-TF-O15-08

$=$

FROGRAM: T0015_08.03A

NAME: S.ELKINS/D.SYME

Ambient Temperature (F) 75

Facility I. D. Name CZAFí

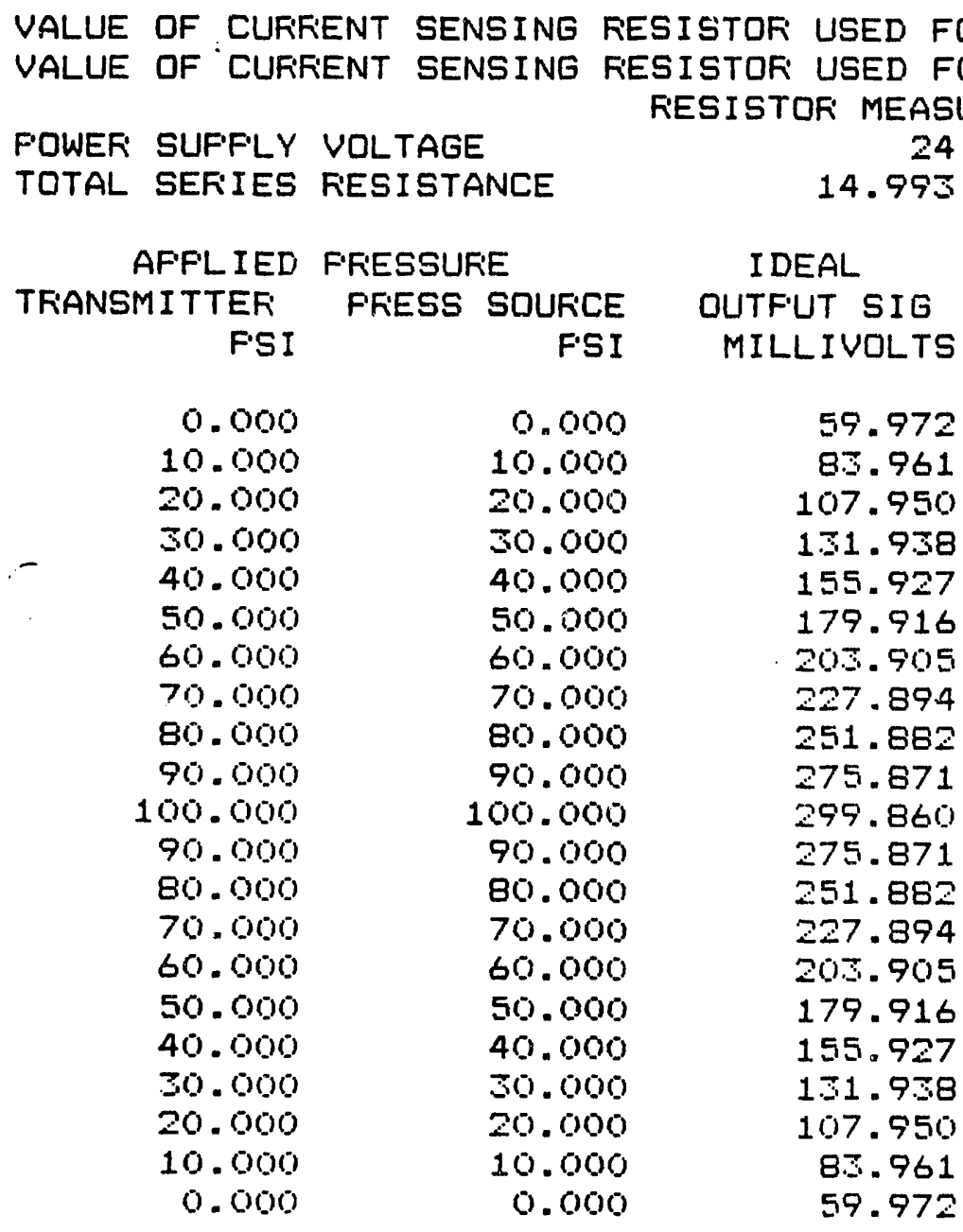

\author{
14.993 OHMS \\ 14.993 OHMS \\ N.A.
}

SURING ACCURIACY

$\begin{array}{ccc}\text { CALIERATION ACCUFIACY } & \\ & \text { **LIMITED** } & \\ \text { MEASUFED } & \text { EFFIOF } \% & \text { OUT } \\ \text { OUTFUT SIG } & \text { FULL SCALE } & \text { OF } \\ \text { MILLIVOLTS } & & \text { TOLEFANCE }\end{array}$

$\begin{array}{rr}59.954 & -0.01 \% \\ 83.840 & -0.05 \% \\ 107.805 & -0.06 \% \\ 131.786 & -0.06 \% \\ 155.812 & -0.05 \% \\ 179.781 & -0.06 \% \\ 203.793 & -0.05 \% \\ 227.864 & -0.01 \% \\ 251.858 & -0.01 \% \\ 275.886 & 0.01 \% \\ 299.892 & 0.01 \% \\ 275.818 & -0.02 \% \\ 251.749 & -0.06 \% \\ 227.739 & -0.06 \% \\ 203.709 & -0.08 \% \\ 179.706 & -0.09 \% \\ 155.714 & -0.09 \% \\ 151.713 & -0.09 \% \\ 107.736 & -0.09 \% \\ 8 . .831 & -0.05 \% \\ 59.940 & -0.01 \%\end{array}$

$\bar{z}=59.947$ 
CURVEFIT OUTPUT FOR VTAB C2AP09 $V$

DATE: $20-\mathrm{MAX}-92$

TIME: $16: 47: 23$

VOLTAGE (V) OBS. PRESS. CALC. PRESS. DIFF. (PSIA) O DIFFERENCE

(PSIA)

(PSIA)

$\begin{array}{llllll}1 & 0.7001046 \mathrm{E}-05 & 0.0000000 \mathrm{E}+00 & 0.7347121 \mathrm{E}-02 & -0.7347121 \mathrm{E}-02 & 0.0000000 \mathrm{E}+00 \\ 2 & 0.2389300 \mathrm{E}-01 & 10.00000 & 9.997588 & 0.2412280 \mathrm{E}-02 & 0.2412280 \mathrm{E}-01 \\ 3 & 0.4785800 \mathrm{E}-01 & 20.00000 & 20.00824 & -0.8236585 \mathrm{E}-02 & -0.4118292 \mathrm{E}-01 \\ 4 & 0.7183900 \mathrm{E}-01 & 30.00000 & 30.01469 & -0.1468530 \mathrm{E}-01 & -0.4895101 \mathrm{E}-01 \\ 5 & 0.9586500 \mathrm{E}-01 & 40.00000 & 40.03079 & -0.3078522 \mathrm{E}-01 & -0.7696304 \mathrm{E}-01 \\ 6 & 0.1198340 & 50.00000 & 50.01581 & -0.1580840 \mathrm{E}-01 & -0.3161681 \mathrm{E}-01 \\ 7 & 0.1438460 & 60.00000 & 60.01321 & -0.1321040 \mathrm{E}-01 & -0.2201733 \mathrm{E}-01 \\ 8 & 0.1679170 & 70.00000 & 70.03142 & -0.3141790 \mathrm{E}-01 & -0.4488271 \mathrm{E}-01 \\ 9 & 0.1919110 & 80.00000 & 80.01563 & -0.1562731 \mathrm{E}-01 & -0.1953414 \mathrm{E}-01 \\ 0 & 0.2159390 & 90.00000 & 90.01383 & -0.1382833 \mathrm{E}-01 & -0.1536481 \mathrm{E}-01 \\ 1 & 0.2399450 & 100.0000 & 100.0045 & -0.4511975 \mathrm{E}-02 & -0.4511976 \mathrm{E}-02 \\ 2 & 0.2158710 & 90.00000 & 89.98553 & 0.1446837 \mathrm{E}-01 & 0.1607596 \mathrm{E}-01 \\ 3 & 0.1918020 & 80.00000 & 79.97027 & 0.2972727 \mathrm{E}-01 & 0.3715909 \mathrm{E}-01 \\ 4 & 0.1677920 & 70.00000 & 69.97940 & 0.2059974 \mathrm{E}-01 & 0.2942820 \mathrm{E}-01 \\ 5 & 0.1437520 & 60.00000 & 59.97824 & 0.2175545 \mathrm{E}-01 & 0.3625908 \mathrm{E}-01 \\ 6 & 0.1197590 & 50.00000 & 49.98457 & 0.1542561 \mathrm{E}-01 & 0.3035122 \mathrm{E}-01 \\ 7 & 0.9576700 \mathrm{E}-01 & 40.00000 & 39.9895 & 0.1005347 \mathrm{E}-01 & 0.2513368 \mathrm{E}-01 \\ 18 & 0.7176600 \mathrm{E}-01 & 30.00000 & 29.98424 & 0.1576038 \mathrm{E}-01 & 0.5253459 \mathrm{E}-01 \\ 9 & 0.4778900 \mathrm{E}-01 & 20.00000 & 19.97943 & 0.2056960 \mathrm{E}-01 & 0.1028480 \\ & 0.2388400 \mathrm{E}-01 & 10.00000 & 9.993826 & 0.6174022 \mathrm{E}-02 & 0.6174022 \mathrm{E}-01 \\ 21 & -0.6998954 \mathrm{E}-05 & 0.0000000 \mathrm{E}+00 & 0.1487635 \mathrm{E}-02 & -0.1487635 \mathrm{E}-02 & 0.0000000 \mathrm{E}+00\end{array}$

EGREE OF POLYNOMIAL 3

OEFFICIENTS

$0.44169406 E-02 \quad 0.41853469 E+03 \quad-0.12565750 E+02 \quad 0.21589198 E+02$

SS $=0.6200 \mathrm{E}-02 \quad \begin{aligned} \mathrm{SD} & =0.1910 \mathrm{E}-01 \quad \mathrm{R} 2=0.100000 \mathrm{E}+01 \\ 2 * \mathrm{SD} & =0.3819 \mathrm{E}-01\end{aligned}$ 
RD-45A Rev. $11-18-91$

RESEARCH D DEVLOPMENT DIVISION INSTRUNENT SERVICE IOG

\begin{tabular}{|l|l|l|}
\hline \multicolumn{2}{|c|}{ INSTRUMENT STATUS } & $\begin{array}{c}\text { AS } \\
\text { CISE }\end{array}$ \\
\hline 1 Certified & & \\
\hline Calibrated & & \\
\hline 3 For Ind. Only & $\checkmark$ & \\
\hline 4 standardize & & \\
\hline 5 Certified c stand. & & \\
\hline 6 Calibrated a stand. & & \\
\hline 9 other & & \\
\hline
\end{tabular}

INSTRUMENT CONDITION

AS RECEIVED (Certified Equipmeñt only)

Meets Manufacturer's Tolerance $N$

Operates within previously defined limits $\mathrm{N}$

Deviates from $\mathrm{Mfg}$ or previously defined limits $Y$

Inoperative (Describe below) $\mathbf{Y}$

AS LEFT Limited (Describe below)

Meets Hanufacturer's Tolerance

other (Describe Below)
Babcock \& Wilcox

- McDermoti company

B\&W Ser. No. 0880548

MANUFACTURER

MODEL

ITEM

MANUF. SER. NO.

PROPERTY NO.

ORDER NO.

SECTION

IOCATION

SCHEDULED ( $Y-N$ )

RECAL INT. (MOS.)

\section{SENSOTEC}

TJE/127810

TRANSMITR, PRES

195586

4427-01-110

\section{6 \\ 115 \\ $+y$ \\ 12}

TECH. PROCEDURE AO015

Babcock \& Wilcox R\&D Division certifies that the performance of this instrument was verified using standards whose accuracies are traceable to the National Institute of standards and Technology, an accef ed value of a natural physical constant, - ratio :ype of calibration technique, or to a consensus standard.
STAUDARDS USED

Manufacturer

ASHCROFT

FLUKE
Model

Item

BeW Serial No.

0

\section{3}

1

Vendor Cert/Cal/Repair

anm

\section{OTHER EOUIPMENT USED}

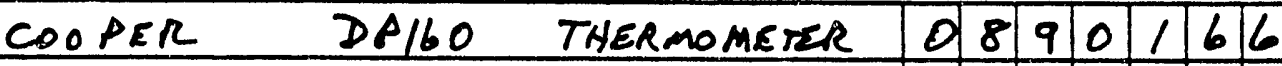

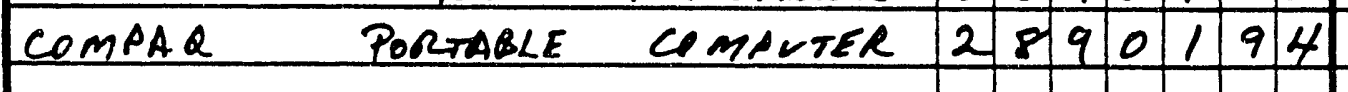

\footnotetext{
Service notes 0 -100 PSIF, C2AP10 15,002 $\Omega$

SEE ATTACHEP PATA SHEKT.
}

$$
\text { LIMITED TO PATA PROVIDED. DOES NOT MEET HEI RATD SPECS, }
$$

\section{TOTAL MATERIAL COST}

\begin{tabular}{|l|l|l|l|}
\hline TOTAL LABOR HOURS & 0 & 0 & 1.0 \\
\hline
\end{tabular} Requested By S, SPROVL Charge Number 44260-102-002 work order No. 0296 Name S,ELkins/D SYmE $A$ D D 5 \begin{tabular}{l|l|l|l|l|l|l} 
Date $(M-D-Y)$ & 0 & 5 & 1 & 8 & 9 & 2 \\
\hline
\end{tabular}

Reviewed By 
AS REECEIVED

\begin{tabular}{|c|c|c|}
\hline FANSMITTEF: : & $\begin{array}{r}\text { SENSOTEC } \\
100 \\
\text { TOLEFANCE: }\end{array}$ & $\begin{array}{l}\text { TIE } \\
\text { F.SI } \\
0.10 \%\end{array}$ \\
\hline 'FEESS SOURCE & $\begin{array}{r}\text { ASHCFIOFT } \\
0-100 \\
\text { B\&W SER. NO } \\
\text { TOLEFIANCE: }\end{array}$ & $\begin{array}{l}\text { DIGIGAUGE } \\
\text { FSI } \\
0700123 \\
0.05 \%\end{array}$ \\
\hline EADOUT DEV: & $\begin{array}{l}\text { FL UKE } \\
\text { B\&W Ser. No. } \\
\text { TOLEFIANCE: }\end{array}$ & $\begin{array}{l}8840 A \\
0860404 \\
0.015 \%\end{array}$ \\
\hline
\end{tabular}

AS

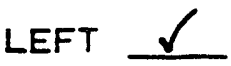

B\&W S.N. 0880548

DATE: $18-\operatorname{May}-92$

FFIOCEDUFIE : AFIC-TP-015-08

FROGRAM: T0015_08.03A

NAME: S, ELLINS/D, SYMiE

Ambient Temperature (F) 75

Facility I. D. Name C2AF10

ALUE OF CUFIENT SENSING FESISTOR USED FOF TEST DATA $\triangle A L U E$ OF CUFIRENT SENSING RESISTOF USED FOF CALIBFATION

OWEF SUFFLY VOLTAGE

OTAL SEFIES FIESISTANCE

AFFLIED FFESSURE FIANSMITTEF: FSI F'RESS SOURICE. F'S I

$\begin{array}{rr}0.000 & 0.000 \\ 10.000 & 10.000 \\ 20.000 & 20.000 \\ 30.000 & 30.000 \\ 40.000 & 40.000 \\ 50.000 & 50.000 \\ 60.000 & 60.000 \\ 70.000 & 70.000 \\ 80.000 & 80.000 \\ 50.000 & 90.000 \\ 100.000 & 100.000 \\ 90.000 & 90.000 \\ 80.000 & 80.000 \\ 70.000 & 70.000 \\ 60.000 & 60.000 \\ 50.000 & 50.000 \\ 40.000 & 40.000 \\ 30.000 & 30.000 \\ 20.000 & 20.000 \\ 10.000 & 10.000 \\ 0.000 & 0.000\end{array}$

FESISTOF MEASUFING ACCURACY 24 14.997

IDEAL. OUTFUTT SIG MILLIVOLTS

59.988
85.985
107.978
131.974
155.969
179.964
203.959
227.954
251.950
275.945
299.940
275.945
251.950
227.954
20.959
179.964
155.969
131.974
107.978
8.985
59.988

CALIEFATION ACCUFACY **LIMITED** MEASUFED EFFIOR \% DUTFUT SIG FULL SCALE MILLIVOLTS

$$
\begin{aligned}
& 14.997 \text { DHMS } \\
& 14.997 \text { OHMS } \\
& \text { N.A. }
\end{aligned}
$$

$\begin{array}{rr}59.962 & -0.01 \% \\ 85.920 & -0.03 \% \\ 107.870 & -0.05 \% \\ 131.854 & -0.05 \% \\ 155.872 & -0.04 \% \\ 179.866 & -0.04 \% \\ 205.856 & -0.04 \% \\ 227.909 & -0.02 \% \\ 251.908 & -0.02 \% \\ 275.951 & 0.00 \% \\ 299.949 & 0.00 \% \\ 275.853 & -0.04 \% \\ 251.794 & -0.06 \% \\ 227.792 & -0.07 \% \\ 203.728 & -0.10 \% \\ 179.738 & -0.09 \% \\ 155.812 & -0.07 \% \\ 131.830 & -0.06 \% \\ 107.858 & -0.05 \% \\ 85.956 & -0.01 \% \\ 59.958 & -0.02 \%\end{array}$

$$
\bar{z}=59.950
$$


CURVEFIT OUTPUT FOR VTAB C2AP10

DATE: 20-MAY -92

TIME: $16: 48: 34$

VOLTAGE (V) OBS. PRESS. CALC. PRESS. DIFF. (PSIA) \& DIFFERENCE

(PSIA)

(PSIA)

$\begin{array}{rlcccc}1 & 0.1199846 \mathrm{E}-04 & 0.0000000 \mathrm{E}+00 & -0.2134769 \mathrm{E}-02 & 0.2134769 \mathrm{E}-02 & 0.0000000 \mathrm{E}+00 \\ 2 & 0.2397000 \mathrm{E}-01 & 10.00000 & 10.00266 & -0.2660673 \mathrm{E}-02 & -0.2660673 \mathrm{E}-01 \\ 3 & 0.4792000 \mathrm{E}-01 & 20.00000 & 19.99936 & 0.6400015 \mathrm{E}-03 & 0.3200008 \mathrm{E}-02 \\ 4 & 0.7190400 \mathrm{E}-01 & 30.00000 & 30.00549 & -0.5486208 \mathrm{E}-02 & -0.1828736 \mathrm{E}-01 \\ 5 & 0.9592200 \mathrm{E}-01 & 40.00000 & 40.02102 & -0.2101902 \mathrm{E}-01 & -0.5254756 \mathrm{E}-01 \\ 6 & 0.1199160 & 50.00000 & 50.02177 & -0.2176939 \mathrm{E}-01 & -0.4353879 \mathrm{E}-01 \\ 7 & 0.1439060 & 60.00000 & 60.01608 & -0.1608172 \mathrm{E}-01 & -0.26802877 \mathrm{E}-01 \\ 8 & 0.1679590 & 70.00000 & 70.03185 & -0.3185081 \mathrm{E}-01 & -0.4550115 \mathrm{E}-01 \\ 9 & 0.1919580 & 80.00000 & 80.02035 & -0.2035468 \mathrm{E}-01 & -0.2544335 \mathrm{E}-01 \\ 10 & 0.2160010 & 90.00000 & 90.02238 & -0.2238442 \mathrm{E}-01 & -0.2487158 \mathrm{E}-01 \\ 11 & 0.2399990 & 100.0000 & 100.0009 & -0.9157941 \mathrm{E}-03 & -0.91579411 \mathrm{E}-03 \\ 12 & 0.2159030 & 90.00000 & 89.98163 & 0.183743 \mathrm{E}-01 & 0.2041604 \mathrm{E}-01 \\ 13 & 0.1918440 & 80.00000 & 79.97292 & 0.2708140 \mathrm{E}-01 & 0.3385175 \mathrm{E}-01 \\ 14 & 0.1678420 & 70.00000 & 69.98314 & 0.168568 \mathrm{E}-01 & 0.2408124 \mathrm{E}-01 \\ 15 & 0.1437780 & 60.00000 & 59.96277 & 0.3723084 \mathrm{E}-01 & 0.6205140 \mathrm{E}-01 \\ 15 & 0.1197880 & 50.00000 & 49.96843 & 0.3156862 \mathrm{E}-01 & 0.6313723 \mathrm{E}-01 \\ 17 & 0.9586200 \mathrm{E}-01 & 40.00000 & 39.99600 & 0.3995087 \mathrm{E}-02 & 0.9987717 \mathrm{E}-02 \\ 18 & 0.7188000 \mathrm{E}-01 & 30.00000 & 29.99548 & 0.4524210 \mathrm{E}-02 & 0.1508070 \mathrm{E}-01 \\ 19 & 0.4790800 \mathrm{E}-01 & 20.00000 & 19.99435 & 0.5647596 \mathrm{E}-02 & 0.2823798 \mathrm{E}-01 \\ 1 & 0.2400600 \mathrm{E}-01 & 10.00000 & 10.01769 & -0.1769059 \mathrm{E}-01 & -0.1769059 \\ 21 & -0.1200154 \mathrm{E}-04 & 0.0000000 \mathrm{E}+00 & -0.1215949 \mathrm{E}-01 & 0.1215949 \mathrm{E}-01 & 0.0000000 \mathrm{E}+00\end{array}$

DEGREE OF POLYNOMIAL 2

COEFFICIENTS

$-0.71464861 E-02 \quad 0.41769666 E+03-0.41444587 E+01$

ESS $=0.7414 \mathrm{E}-02 \quad \mathrm{SD}=0.2030 \mathrm{E}-01 \quad \mathrm{R2}=0.100000 \mathrm{E}+01$ 
CD-45A Rev. $12-18-91$

\begin{tabular}{l|l|l|}
\hline INSTRUMENT STATUS & RECE & AS \\
\hline Certified & \\
\hline Calibrated & & \\
\hline For Ind. only & & $\checkmark$ \\
\hline Standardize & & \\
\hline Certified stand. & & \\
\hline Calibrated s stand. & & \\
\hline Other & & \\
\hline
\end{tabular}

INSTRUMENT CONDITION

AS RECEIVED (Certified Equipment Only)

Meets Manufacturer's Tolerance $N$ Operates within previously defined limits $\mathrm{N}$ reviates from Mfg or previously defined limits $Y$ Inoperative (Describe below) $x$

AS IEFT Limited (Describe below) Meets Manufacturer's Tolerance other (Describe Below)
EARCH \& DEVIOPIENT DIVISION INSTRUYENT SERVICE IOG

\begin{tabular}{|l|l|l|}
\hline TYPE OF SERVICE \\
\hline Certification 1 & \\
\hline Calibration & 2 & \\
\hline Maintenance & 3 & \\
\hline Repair & 4 & \\
\hline Vendor Cert. & 5 & \\
\hline Vendor Calib. & 6 & \\
\hline Vendor Repair & 7 & \\
\hline Orher & 9 & \\
\hline
\end{tabular}

\section{MANUFACTURER}

MODEL

ITEM

MANUF. SER. NO.

PROPERTY NO.

ORDER NO.

SECTION

LOCATION

SCHEDULED $(\mathrm{Y}-\mathrm{N})$

RECAL INT. (MOS.)
Babcock \& Wilcox

a McDermoti company

B\&W Ser. No. 0880554

\section{SENSOTEC}

TJE/127810

TRANSMITR, PRES

201322

4427-01-116

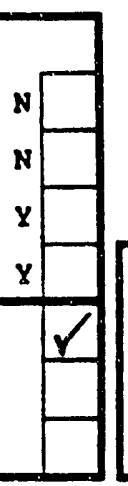

46

115

$-17-Y$

12

TECH. PROCEDURE A0015

Babcock Wilcox R\&D Division certifies that the performance of this instrument was verified using standards whose accuracies are traceable to the National Institute of standards and Technology, an accepted value of a natural physical constant, a ratio type of calibration technique, or to a consensus standard.

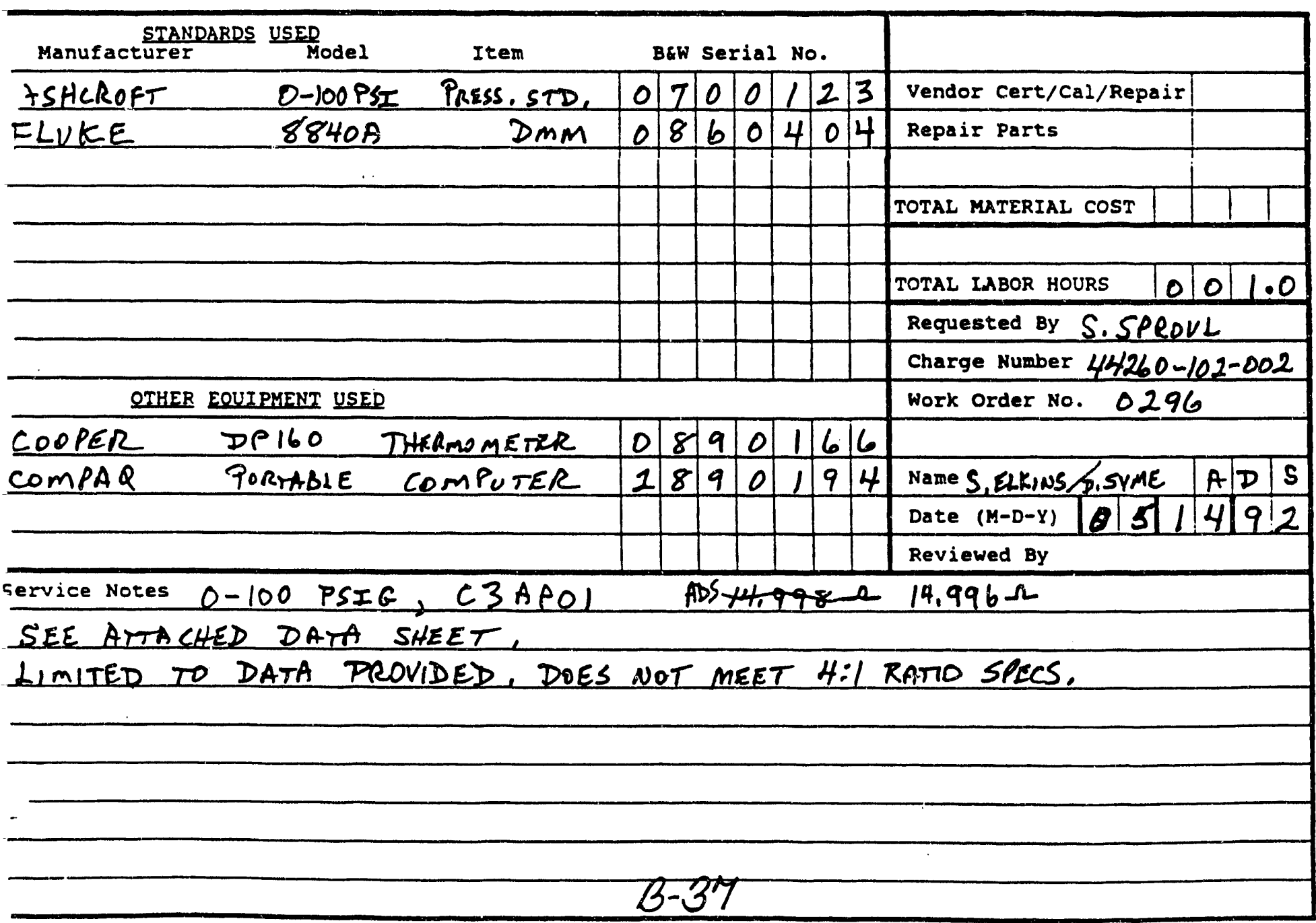


AS FIECEIVED

TRANSMITTER:

FRESS SOURCE

ASHCFIOFT DIGIGAUGE
O-10O FSI
B\&W SER. NO. 0700123
TOLEFIANCE: $0.05 \%$

FEADOUT DEV:
AS LEFT $\mathscr{\nearrow}$

B\&W S.N. 0880554 $\because$ DATE: 14-May-92 F'RIOCEDURE : AFIC-TF-015-08 PFIOGFIAM: T0015_08.03A

NAME: S.ELFINS/D. SYME

Ambient Temperature (F) 70

Facility I. D. Name CBAFOI

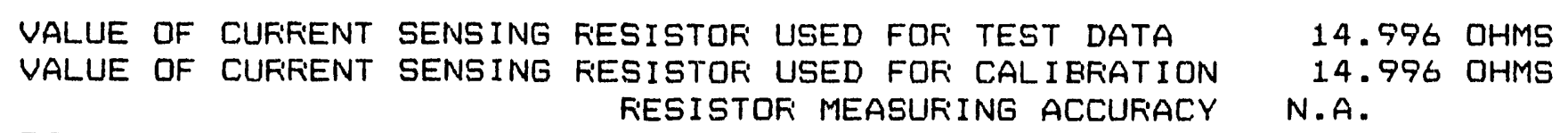

FOWEF SUFF'LY VOLTAGE

TOTAL SEFIIES FESISTANCE

AFF'LIED FFIESSURE
TRIANSMITTER FRESS SOURCE
FSI 24 14.996 I DEAL OUTFUT SIG MILLIVOLTS

59.984
$8 . .978$
107.971
131.965
155.958
179.952
205.946
227.939
251.93.
275.926
299.920
275.926
251.935
227.959
205.946
179.952
155.958
151.965
107.971
85.978
59.984

CALIBFATION
MEASUFED
OUTFUT SIG
MILLIVOLTS

ACCUFIACY

**LIMITED** ERROR \% FULL SCALE 0.000 10.000 20.000 30.000 40.000 50.000 60.000 70.000 80.000 90.000 100.000 90.000 80.000 70.000 60.000 50.000 40.000 30.000 20.000 10.000 0.000

59.980
85.879
107.801
131.780
155.826
1.79 .801
205.805
227.879
251.888
275.927
299.936
275.870
251.775
227.778
203.709
179.705
155.698
151.679
107.726
83.802
59.956

$0.17 \%$ OUT OF TOLEFANCE

$$
\bar{z}=59.968
$$


CURVEFIT OUTPUT FOR VTAB C3APOI

DATE: 20-MAY -92

TIME: $16: 49: 39$

VOLTAGE (V) OBS. PRESS. CALC. PRESS. DIFF. (PSIA) \& DIFFERENCE

(PSIA)

(PSIA)

$\begin{array}{rlllll}1 & 0.1200158 \mathrm{E}-04 & 0.0000000 \mathrm{E}+00 & 0.9188135 \mathrm{E}-02 & -0.9188135 \mathrm{E}-02 & 0.0000000 \mathrm{E}+00 \\ 2 & 0.2391100 \mathrm{E}-01 & 10.00000 & 10.01293 & -0.1292795 \mathrm{E}-01 & -0.1292795 \\ 3 & 0.4783300 \mathrm{E}-01 & 20.00000 & 20.00978 & -0.9784686 \mathrm{E}-02 & -0.4892343 \mathrm{E}-01 \\ 4 & 0.7181200 \mathrm{E}-01 & 30.00000 & 30.01638 & -0.1637691 \mathrm{E}-01 & -0.5458970 \mathrm{E}-01 \\ 5 & 0.9585800 \mathrm{E}-01 & 40.00000 & 40.03928 & -0.3927994 \mathrm{E}-01 & -0.9819985 \mathrm{E}-01 \\ 6 & 0.1198330 & 50.00000 & 50.02348 & -0.2348111 \mathrm{E}-01 & -0.4696221 \mathrm{E}-01 \\ 7 & 0.1438350 & 60.00000 & 60.01232 & -0.1232400 \mathrm{E}-01 & -0.2053999 \mathrm{E}-01 \\ 8 & 0.1679110 & 70.00000 & 70.02785 & -0.2784801 \mathrm{E}-01 & -0.3978287 \mathrm{E}-01 \\ 9 & 0.1919200 & 80.00000 & 80.01391 & -0.1391169 \mathrm{E}-01 & -0.1738961 \mathrm{E}-01 \\ 0 & 0.2159590 & 90.00000 & 90.01338 & -0.1338221 \mathrm{E}-01 & -0.1486913 \mathrm{E}-01 \\ 11 & 0.2399680 & 2 . j 0000 & 100.0038 & -0.3814291 \mathrm{E}-02 & -0.3814291 \mathrm{E}-02 \\ 2 & 0.2159020 & 90.00000 & 89.98967 & 0.1033108 \mathrm{E}-01 & 0.1147898 \mathrm{E}-01 \\ 3 & 0.1918070 & 80.00000 & 79.96691 & 0.3308860 \mathrm{E}-01 & 0.4136075 \mathrm{E}-01 \\ 14 & 0.1678100 & 70.00000 & 69.98584 & 0.1416251 \mathrm{E}-01 & 0.2023216 \mathrm{E}-01 \\ 1.5 & 0.1437410 & 60.00000 & 59.97321 & 0.2678608 \mathrm{E}-01 & 0.4464347 \mathrm{E}-01 \\ .6 & 0.1197370 & 50.00000 & 49.98352 & 0.1648253 \mathrm{E}-01 & 0.3296506 \mathrm{E}-01 \\ 77 & 0.9573000 \mathrm{E}-01 & 40.00000 & 39.98595 & 0.1404683 \mathrm{E}-01 & 0.3511708 \mathrm{E}-01 \\ 18 & 0.7171100 \mathrm{E}-01 & 30.00000 & 29.97426 & 0.2574496 \mathrm{E}-01 & 0.8581652 \mathrm{E}-01 \\ .9 & 0.4775800 \mathrm{E}-01 & 20.00000 & 19.97847 & 0.2153412 \mathrm{E}-01 & 0.1076706 \\ & 0.2383400 \mathrm{E}-01 & 10.00000 & 9.980725 & 0.1927522 \mathrm{E}-01 & 0.1927522 \\ 21 & -0.1199842 \mathrm{E}-04 & 0.0000000 \mathrm{E}+00 & -0.8669987 \mathrm{E}-03 & 0.8669987 \mathrm{E}-03 & 0.0000000 \mathrm{E}+00\end{array}$

IEGREE OF POLYNOMIAL 3

COEFFICIENTS

$0.41599066 \mathrm{E}-02 \quad 0.41896391 \mathrm{E}+03-0.16601765 \mathrm{E}+02 \quad 0.30229825 \mathrm{E}+02$

ESS $=0.8112 \mathrm{E}-02 \quad \mathrm{SD}=0.2184 \mathrm{E}-01 \quad \mathrm{R} 2=0.100000 \mathrm{E}+01$

$2 \star S D=0.4369 \mathrm{E}-01$ 
$x i=45 A^{\prime}$ Rev. $11-18-91$

RESEARCH \& DEVELOPMENT DIVISION INSTRUMENT SERVICE LOG

\begin{tabular}{|l|l|l|}
\hline \multicolumn{1}{|c|}{ INSTRUMENT STATUS } & R'́s & AS \\
\hline 1 Certified & & \\
\hline Calibrated & & \\
\hline 3 For Ind. Only & & \\
\hline 4 standardize & & \\
\hline 5 certified \& stand. & & \\
\hline 6 Calibrated \& stand. & & \\
\hline 9 other & & \\
\hline
\end{tabular}

\begin{tabular}{|r|r|}
\hline INSTRUMENT CONDITION & Other \\
\hline AS RECEIVED (Certified Equipment Only) & 9 \\
Operates within previously defined limits \\
Meets Manufacturer's Tolerance \\
Deviates from Mfg or previously defined limits \\
Inoperative (Describe below) \\
\hline AS LEFT I Meets Manufacturer's Tolerance \\
\hline \\
Other (Describe Below)
\end{tabular}

Babcock \& Wilcox

- McDermoticompany

B\&W Ser. No. 0890536

MANUFACTURER SENSOTEC

MODEL

ITEM

MANUF. SER. NO.

PROPERTY NO.

ORDER NO.

$\begin{array}{lc}\text { SECTION } & 46 \\ \text { LOCATION } & 115 \\ \text { SCHEDULED (Y-N) } & -14 Y \\ \text { RECAL INT.(MOS.) } & 12\end{array}$

TECH. PROCEDURE A0015

Babcock \& Wilcox R\&D Division certifies that the performance of this instrument was verified using standards whose accuracies are traceable to the National Institute of standards and Technology, an wcepted value of a natural physical constant, a rat 0 type of calibration technique, or to a consensus standard.

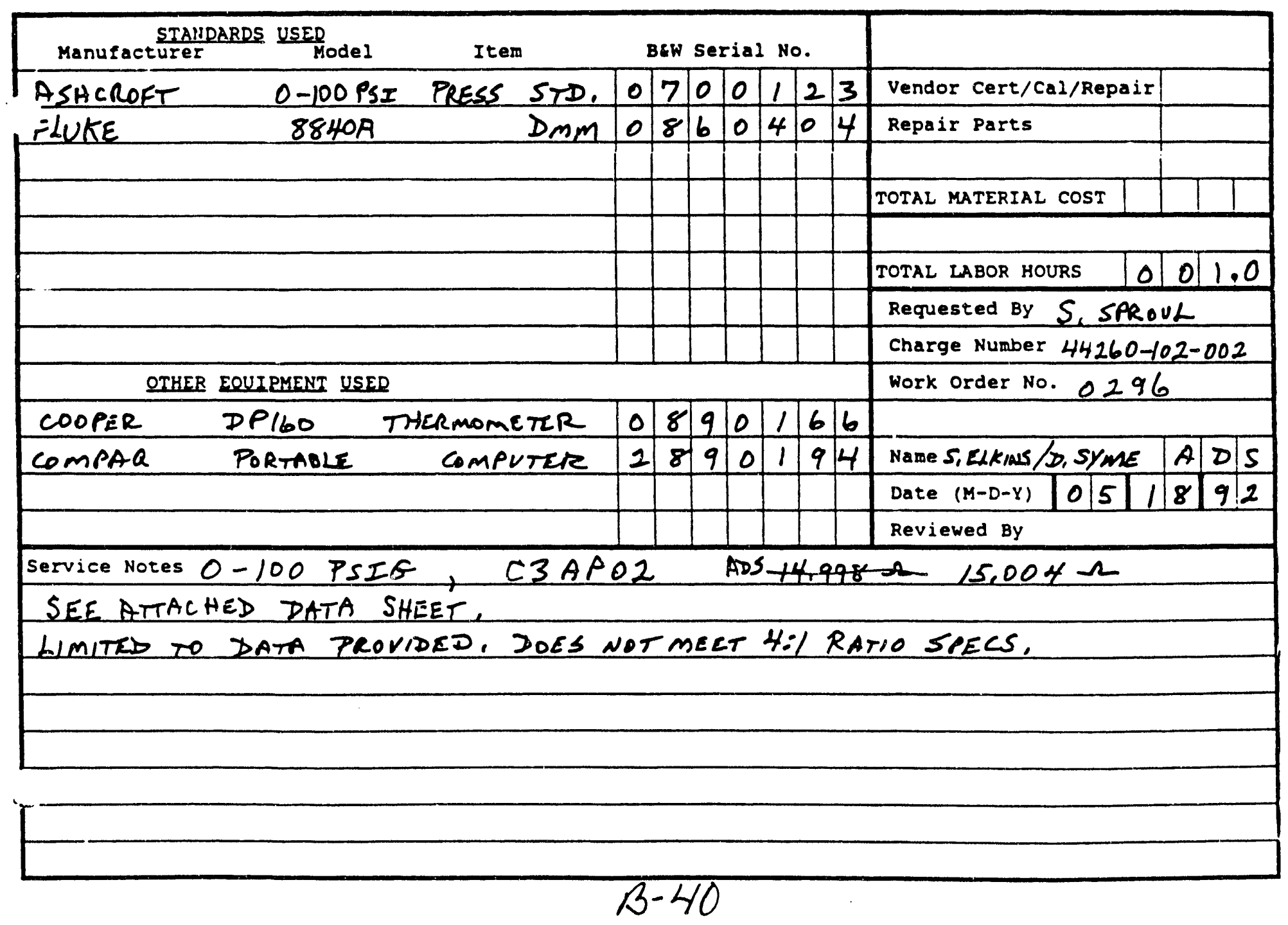


AS RIECE IVED

\section{'RANSMITTEF':}

SENSOTEC TJE

$100 \mathrm{~F} \cdot \mathrm{SI}$

TOLEFIANCE: $0.10 \%$

FESS SOURICE

\section{ASHCFIFT DIGIGAUGE \\ $0-100 \mathrm{~F} \cdot \mathrm{SI}$}

EREW Ser. No. 070012S

TOLEFIANCE: $0.05 \%$

:EADOUT DEV:
AS

LEFT

E\&WW S.N. 0890536 DATE: 18-MaY-92

FFIOCEDURE : ARC-TF-015-08

FFIOGRAM: TOO15_08.03A

NAME: S.EKKINS/D. SŸre

Ambient Temperature (F) 73

Facility I. D. Name C.SAFOI

E\&W SER. No. 0860404

TOLEFIANCE: $0.015 \%$

'ALUE OF CUFFENT SENSING FESISTOF' USED FOF' TEST DATA

ALUE OF: CURIFENT SENSING FESISTOF USED FOF CALIEFIATION

'OWEF' SUFFLY VOLTAGE

DTAL SEFIES FIESISTANCE

AF'FLIED F'FESSUFE RANSMITTER: F'SI

FFESS SDURICE
FSI

$\begin{array}{rr}0.000 & 0.000 \\ 10.000 & 10.000 \\ 20.000 & 20.000 \\ 30.000 & 50.000 \\ 40.000 & 40.000 \\ 50.000 & 50.000 \\ 60.000 & 60.000 \\ 70.000 & 70.000 \\ 80.000 & 80.000 \\ 50.000 & 90.000 \\ 100.000 & 100.000 \\ 50.000 & 90.000 \\ 80.000 & 80.000 \\ 70.000 & 70.000 \\ 60.000 & 60.000 \\ 50.000 & 50.000 \\ 40.000 & 40.000 \\ 30.000 & 50.000 \\ 20.000 & 20.000 \\ 10.000 & 10.000 \\ 0.000 & 0.000\end{array}$

\section{FA}

FFEESS SOURCE
FGI

$$
\begin{array}{r}
0.000 \\
10.000 \\
20.000 \\
30.000 \\
40.000 \\
50.000 \\
60.000 \\
70.000 \\
80.000 \\
90.000 \\
00.000 \\
90.000 \\
80.000 \\
70.000 \\
60.000 \\
50.000 \\
40.000 \\
30.000 \\
20.000 \\
10.000 \\
0.000
\end{array}
$$

\begin{tabular}{|c|c|c|c|c|}
\hline 14.998 & CAL I ERATION & $\begin{array}{l}1 \mathrm{ACC} \\
* * L I\end{array}$ & $\begin{array}{l}\text { UFAACY } \\
\text { MITED*** }\end{array}$ & 0.17 \\
\hline IDEAL & MEASUFED & EF:FID & Fi $\quad \%$ & QUT \\
\hline $\begin{array}{l}\text { OUTFUT SIG } \\
\text { MILLIVOLTS }\end{array}$ & $\begin{array}{l}\text { OUTFUT SIG } \\
\text { MILLIVDLTS }\end{array}$ & FULL & SCALE & $\begin{array}{l}\text { DF } \\
\text { TOLEFIANCE }\end{array}$ \\
\hline 59.992 & 59.983 & & $0.00 \%$ & \\
\hline 83.989 & 85.913 & & $-0.0 .5 \%$ & \\
\hline 107.986 & 107.892 & & $-0.04 \%$ & \\
\hline 131.982 & 131.901 & & $-0.03 \%$ & \\
\hline 155.979 & 155.936 & & $-0.02 \%$ & \\
\hline 179.976 & 179.934 & & $-0.02 \%$ & \\
\hline 203.973 & 205.909 & & $-0.03 \%$ & \\
\hline 227.970 & 227.96 .5 & & $0.00 \%$ & \\
\hline 251.966 & 251.965 & & $0.00 \%$ & \\
\hline 275.963 & 275.980 & & $0.01 \%$ & \\
\hline 299.960 & 299.943 & & $-0.01 \%$ & \\
\hline 275.963 & 275.910 & & $-0.02 \%$ & \\
\hline 251.966 & 251.860 & & $-0.04 \%$ & \\
\hline 227.970 & 227.830 & & $-0.06 \%$ & \\
\hline 203.973 & 205.779 & & $-0.08 \%$ & \\
\hline 179.976 & 179.796 & & $-0.08 \%$ & \\
\hline 155.979 & 155.850 & & $-0.06 \%$ & \\
\hline 131.982 & 131.840 & & $-0.06 \%$ & \\
\hline 107.966 & 107.847 & & $-0.06 \%$ & \\
\hline 8.989 & 8.8 .877 & & $-0.05 \%$ & \\
\hline 59.992 & 59.958 & & $-0.01 \%$ & \\
\hline
\end{tabular}

FIESISTOF MEASUFIING ACCURIACY
59.985

8.913

155.956

179.954

2013.909

227.965

251.965

275.980

299.94.

275.918

203.779

179.796

55.830

7.877

$2=59.471$
277.87
14.998 DHMS

14.998 OHMS

N.A. 
CURVEFIT OUTPUT FOR VTAB c3ap02 ${ }^{\circ}$

VOLTAGE (V) OBS. PRESS. CALC. PRESS. DIFF. (PSIA) DIFFERENCE
DATE: 20-MAY-92

TIME: $16: 50: 35$

(PSIA)

(PSIA)

$\begin{array}{rlcccc}1 & 0.1199900 \mathrm{E}-04 & 0.0000000 \mathrm{E}+00 & 0.9631241 \mathrm{E}-02 & -0.9631241 \mathrm{E}-02 & 0.0000000 \mathrm{E}+00 \\ 2 & 0.2394200 \mathrm{E}-01 & 10.00000 & 10.00062 & -0.6196465 \mathrm{E}-03 & -0.6196465 \mathrm{E}-02 \\ 3 & 0.4792100 \mathrm{E}-01 & 20.00000 & 20.00509 & -0.5092571 \mathrm{E}-02 & -0.2546286 \mathrm{E}-01 \\ 4 & 0.7193000 \mathrm{E}-01 & 30.00000 & 30.01605 & -0.1604761 \mathrm{E}-01 & -0.5349205 \mathrm{E}-01 \\ 5 & 0.9596500 \mathrm{E}-01 & 40.00000 & 40.03276 & -0.3275858 \mathrm{E}-01 & -0.8189645 \mathrm{E}-01 \\ 6 & 0.1199630 & 50.00000 & 50.02993 & -0.2993472 \mathrm{E}-01 & -0.5986943 \mathrm{E}-01 \\ 7 & 0.1439380 & 60.00000 & 60.01438 & -0.1438240 \mathrm{E}-01 & -0.2397066 \mathrm{E}-01 \\ 8 & 0.1679920 & 70.00000 & 70.02953 & -0.2953204 \mathrm{E}-01 & -0.4218863 \mathrm{E}-01 \\ 9 & 0.1919940 & 80.00000 & 80.02180 & -0.2179846 \mathrm{E}-01 & -0.2724808 \mathrm{E}-01 \\ 10 & 0.2160090 & 90.00000 & 90.01921 & -0.1920689 \mathrm{E}-01 & -0.2134099 \mathrm{E}-01 \\ 11 & 0.2399720 & 100.0000 & 99.99566 & 0.4343399 \mathrm{E}-02 & 0.4343399 \mathrm{E}-02 \\ 12 & 0.2159390 & 90.00000 & 89.99007 & 0.9934540 \mathrm{E}-02 & 0.1103838 \mathrm{E}-01 \\ 13 & 0.1918890 & 80.00000 & 79.97809 & 0.2191277 \mathrm{E}-01 & 0.2739096 \mathrm{E}-01 \\ 14 & 0.1678590 & 70.00000 & 69.97416 & 0.2583969 \mathrm{E}-01 & 0.3691385 \mathrm{E}-01 \\ 15 & 0.1438080 & 60.00000 & 59.96025 & 0.3974966 \mathrm{E}-01 & 0.6624943 \mathrm{E}-01 \\ 16 & 0.1198250 & 50.00000 & 49.97246 & 0.2754391 \mathrm{E}-01 & 0.5508782 \mathrm{E}-01 \\ 17 & 0.9585900 \mathrm{E}-01 & 40.00000 & 39.98859 & 0.1140771 \mathrm{E}-01 & 0.2851927 \mathrm{E}-01 \\ 18 & 0.7186900 \mathrm{E}-01 & 30.00000 & 29.99062 & 0.9380517 \mathrm{E}-02 & 0.3126839 \mathrm{E}-01 \\ \mathrm{C} & 0.4787600 \mathrm{E}-01 & 20.00000 & 19.98632 & 0.1367630 \mathrm{E}-01 & 0.6838151 \mathrm{E}-01 \\ \mathrm{~J} & 0.2390600 \mathrm{E}-01 & 10.00000 & 9.985595 & 0.1440521 \mathrm{E}-01 & 0.1440521 \\ 21 & -0.1300100 \mathrm{E}-04 & 0.0000000 \mathrm{E}+00 & -0.8104490 \mathrm{E}-03 & 0.8104490 \mathrm{E}-03 & 0.0000000 \mathrm{E}+00\end{array}$

DEGREE OF POLYNOMIAL 3

COEFFICIENTS

$0.46196485 \mathrm{E}-02 \quad 0.41766761 \mathrm{E}+03-0.69021855 \mathrm{E}+01 \quad 0.11576528 \mathrm{E}+02$

ESS $=0.8486 \mathrm{E}-02 \quad \mathrm{SD}=0.2234 \mathrm{E}-01 \quad \mathrm{R} 2=0.100000 \mathrm{E}+01$

$2 \star S D=0.4469 E-01$ 
:D-45A Rev. 12-18-91

RESEARCH \& DEVELOPMENT DIVISION INSTRURENT SERVICE IOG

\begin{tabular}{l|l|l|}
\hline INSTRUMENT STATUS & RÉCD & LES \\
\hline Certified & & \\
\hline Calibrated & & \\
\hline For Ind. Only & & \\
\hline Standardize & & \\
\hline Certified \& stand. & & \\
\hline Calibrated s stand. & & \\
\hline Other & & \\
\hline
\end{tabular}

INSTRUMENT CONDITION

AS RECEIVED (Certified Equipment Only)

Meets Manufacturer's Tolerance $N$ operates within previously defined limits $\mathrm{N}$ leviates from $\mathrm{Mfg}$ or previously defined limits $Y$ Inoperative (Describe below) $y$

Limited (Describe below)

Meets Manufacturer's Tolerance Other (Describe Below)
CSAtos

Babrock \& Wilcox

a MeDermort company

B\&W Ser. No. 0880552
MANUFACTURER

MODEL

ITEM

MANUF. SER. NO.

PROPERTY NO.

ORDER NO.

SECTION

LOCATION

SCHEDULED ( $Y-N)$

RECAL INT. (MOS.)
SENSOTEC

TJE/ 127810

TRANSMITR, PRES

201314

4427-01-114

\section{TECH. PROCEDURE A0015}

\author{
46 \\ 115 \\ *y \\ 12
}

Babcock \& Wilcox R\&D Division certifies that the performance of this instrument was verified using standards whose accuracies are traceable to the National Institute of standards and Technology, an accepted value of a natural physical constant. a ratio type of calibration technique, or to a consensus standard.
STALDARDS USED

Manufacturer

2SHCROFT

ELUKE
Model

Item

Bew Serial No.

O-100PSI PRESS, STD,

\begin{tabular}{l|l|l|l|l|l|l|l|}
0 & 7 & 0 & 0 & 1 & 2 & 3 & Vendor Cert/Cal/Repair
\end{tabular}

08

\begin{tabular}{l|l|l|l|l|l|l}
8 & 6 & 0 & 4 & 0 & 4 & Repair Parts \\
\hline
\end{tabular}

8840A DMM

\begin{tabular}{ll}
\hline \multicolumn{3}{c}{ OTHER EOUIPMENT USED } \\
\hline COOPER & DPILO THERMOMETER \\
COMPAR & PORTABLE S COMPVTER
\end{tabular}

\begin{tabular}{llllllll}
\hline & & & & & & \\
\hline & & & & & & \\
\hline & & & & & & \\
\hline & & & & & & \\
\hline & & & & & &
\end{tabular}

TOTAL MATERIAL COST

TOTAL LABOR HOURS 01011.0 Requested By S.SPROUL Charge Number 44260-102-002 Work Order No. 0296 COMPAR PORTABLE COMPUTER

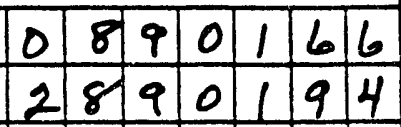

Name S. ELKWS/D. SYME A \begin{tabular}{l|l|l|l|l|l|l|} 
Date $(M-D-Y)$ & 0 & 5 & 1 & 8 & $9 ! 2$ \\
\hline
\end{tabular}

Reviewed By

ervice notes 0 -100 PSIG, C3APO3, 15,000 $\Omega$

SEE ATACHED DATA SHEET. LIMITED TO DATA PROVIDED, DOES NOT MEET H:I RATIO SPECS, 
AS F'ECEIVED

TRANSMITTEF:

PRESS SOURICE

$$
\begin{aligned}
\text { ASHCFOFT } & \text { DIGIGAUGE } \\
\text { O-100 } & \text { FSI } \\
\text { B\&W SER. NO. } & 0700123 \\
\text { TOLEFANCE: } & 0.05 \%
\end{aligned}
$$

READOUT DEV:
AS LEFT

E\&W S.N. OBBOSis2 DATE: 18-MaY-92

FFOCEDURE : ARC-TF-015-08 PFIOGFAM: TOO15_08.03A

NAME: S.ELKINS/P, Symir

Ambient Temperature

Facility I. D. Name
(F) 72

CSAFOS

B\&W SER. NO. OB60404

TOLERANCE: $0.015 \%$

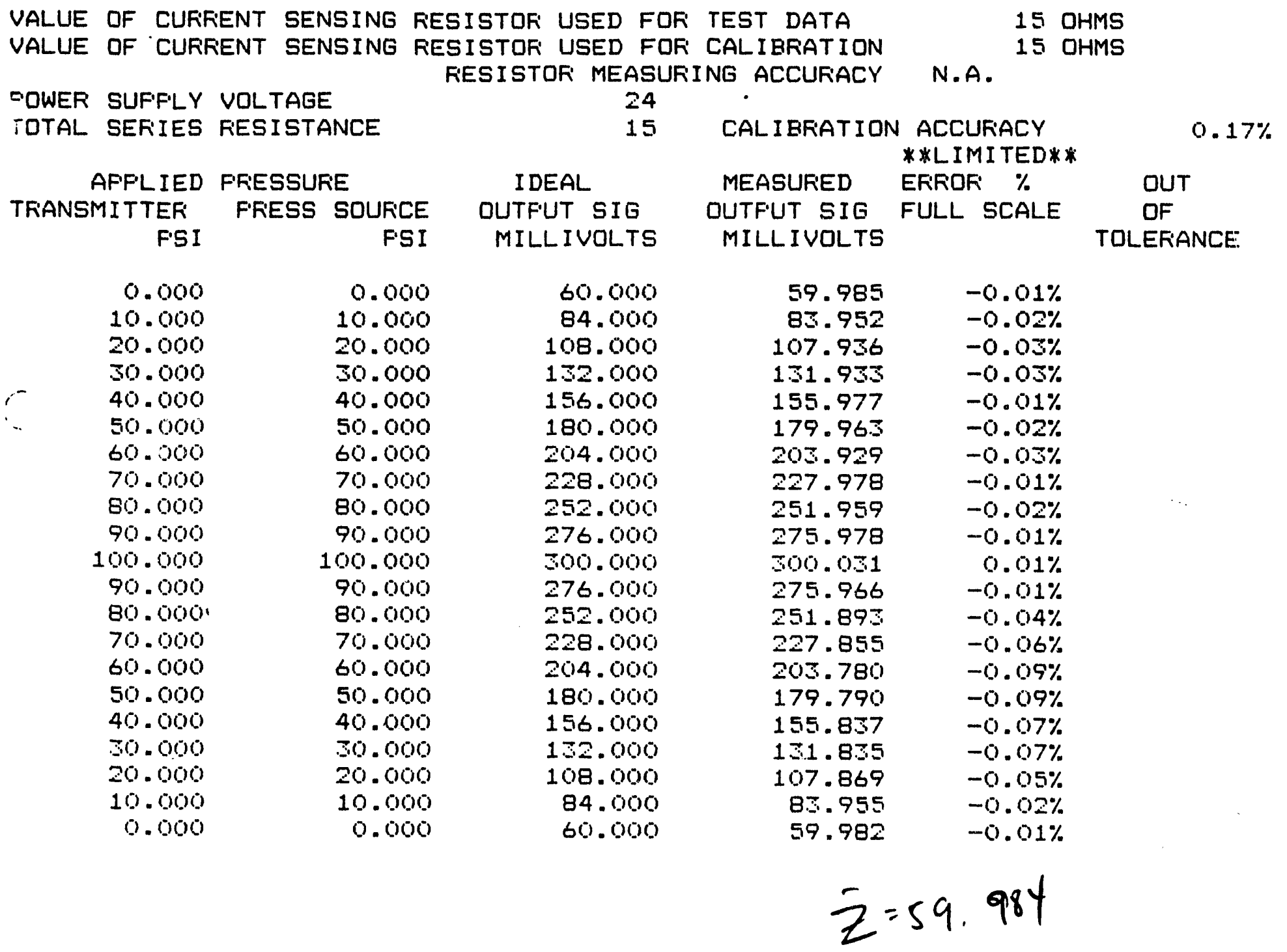


CURVEFIT OUTPUT FOR VTAB C3AP03;

VOLTAGE(V) OBS. PRESS.
CALC. PRESS. DIFF. (PSIA)
DATE: 20-MAY-92

TIME: 16:51:30

(PSIA)

(PSIA)

$\begin{array}{rl}1 & 0.1001463 \mathrm{E}-05 \\ 2 & 0.2396800 \mathrm{E}-01 \\ 3 & 0.4795200 \mathrm{E}-01 \\ 4 & 0.7194900 \mathrm{E}-01 \\ 5 & 0.9599300 \mathrm{E}-01 \\ 6 & 0.1199790 \\ 7 & 0.1439450 \\ 8 & 0.1679940 \\ 9 & 0.1919750 \\ 10 & 0.2159940 \\ 11 & 0.2400470 \\ 2 & 0.2159820 \\ 3 & 0.1919090 \\ 14 & 0.1678710 \\ 1.5 & 0.1437960 \\ 6 & 0.1198060 \\ 17 & 0.9585300 \mathrm{E}-01 \\ 18 & 0.7185100 \mathrm{E}-01 \\ 9 & 0.4788500 \mathrm{E}-01 \\ 1 & 0.2397100 \mathrm{E}-01 \\ 21 & -0.1998537 \mathrm{E}-05\end{array}$

$0.0000000 E+00$

10.00000

20.00000

30.00000

40.00000

50.00000

60.00000

70.00000

80.00000

90.00000

100.0000

90.00000

80.00000

70.00000

60.00000

50.00000

40.00000

30.00000

20.00000

10.00000

$0.0000000 E+00-0.2649402 E-02$

10.00288
20.00994
30.01809
40.04151
50.03642
60.01868
70.03118
80.01103
90.00237
100.0035
89.99738
79.98357
69.97998
59.95663
49.96435
39.98316
29.97723
19.98199
10.00414
0.2649402 E-02

10.00288

30.01809

40.04151

50.03642

60.01868

70.03118

90.00237

100.0035

89.99738

49.96435

39.98316

29.97723

19.98199
2

C.OEFFICIENTS

$0.18149949 E-02$

$0.41750899 E+03-0.37565059 E+01$

ESS $=0.9926 \mathrm{E}-02$

$$
\begin{aligned}
S D & =0.2348 \mathrm{E}-01 \\
2 * S D & =0.4697 \mathrm{E}-01
\end{aligned}
$$

$0.1396875 \mathrm{E}-02$

$-0.2883222 \mathrm{E}-02$

$-0.9939246 \mathrm{E}-02$

$-0.1809413 \mathrm{E}-01-0.6031378 \mathrm{E}-01$

$-0.4151164 E-01-0.1037791$

$-0.3642255 E-01-0.7284509 E-01$

$-0.1868246 \mathrm{E}-01-0.3113743 \mathrm{E}-01$

$-0.3117564 \mathrm{E}-01-0.4453652 \mathrm{E}-01$

$-0.1103112 \mathrm{E}-01-0.1378890 \mathrm{E}-01$

$-0.2369634 \mathrm{E}-02-0.2632927 \mathrm{E}-02$

$-0.3507793 \mathrm{E}-02-0.3507793 \mathrm{E}-02$

$0.2621001 E-02 \quad 0.2912224 E-02$

$0.1642930 E-01 \quad 0.2053663 E-01$

$0.2002278 \mathrm{E}-01 \quad 0.2860397 \mathrm{E}-01$

$0.4336533 E-01 \quad 0.7227555 E-01$

$0.3565068 \mathrm{E}-01 \quad 0.7130136 \mathrm{E}-01$

$0.1683872 \mathrm{E}-01 \quad 0.4209680 \mathrm{E}-01$

$0.2276881 \mathrm{E}-01 \quad 0.7589603 \mathrm{E}-01$

$0.1800974 \mathrm{E}-01 \quad 0.9004868 \mathrm{E}-01$

$-0.4135208 E-02-0.4135209 E-01$

$0.2649402 \mathrm{E}-02$

$0.0000000 \mathrm{E}+00$ 
1

RD-45A Rev. 11-18-91

\begin{tabular}{|c|c|c|}
\hline INSTRUMENT STATUS & S:5 & AS \\
\hline I certified & & \\
\hline Calibrated & & \\
\hline 3 For Ind. Only & & \\
\hline 4 standaroize & & \\
\hline 5 certilied a stand. & & \\
\hline 6 Calibrated c stand. & & \\
\hline 9 Other & & \\
\hline
\end{tabular}

INSTRUMENT CONDITION

AS RECEIVED (Certified Equipment only)

Meets Manufacturer's Tolerance $N$

Operates within previously defined limits $\mathrm{N}$ Deviates from $\mathrm{Mfg}$ or previously defined $11 \mathrm{mits} Y$ Inoperative (Describe below) $x$

AS LEFT

Meets Manufacturer's Tolerance

other (Describe Below)

\section{MAN U I \\ Babcock \& Wilcox \\ a McDermoti company}

B\&W Ser. NO. 0880539

MANUFACTURER

MODEL

ITEM

MANUF. SER. NO.

PROPERTY NO.

ORDER NO.

$\begin{array}{lc}\text { SECTION } & 46 \\ \text { LOCATION } & 115 \\ \text { SCHEDUIED (Y-N) } & \# Y \\ \text { RECAL INT.(MOS.) } & 12\end{array}$

SENSOTEC

TJE/ 127810

TRANSMITR, PRES

188757

4427-01-101

46

$\# Y$

12

\section{TECH. PROCEDURE A0015}

Babcock wilcox RLD Division certifies that the performance of this instrument was verified using standards whose accuracies are traceable to the National Institute of Standards and Technology, an accepted value of a natural physical constant. a ratio type of calibration technique, or to a consensus standard.

\section{STANDARDS USED}

Manufacturer Model Item BEW Serial No.

QSHCROFT O-100 PSI PRESS. STD,

(1)

FLUKE $8840 A$ DMm

\begin{tabular}{l|l|l|}
\hline & 0 & 7 \\
\hline & 0 & 8 \\
\hline
\end{tabular}

213 40.4
Vendor Cert/Cal/Repair

Repair Parts

TOTAL MATERIAL COST

TOTAL LABOR HOURS 101011.0 Requested By $S$, S PRovL Charge Number $44160-101-002$ Work Order No. 0296 OTHER EQUIPMENT USED

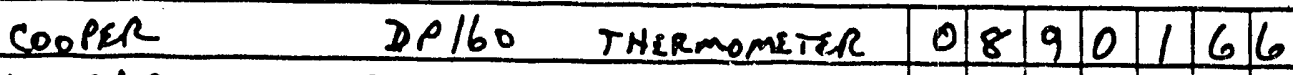

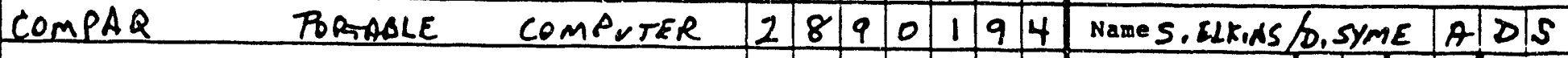
\begin{tabular}{l|l|l|l|l|l|l} 
Date $(M-D-Y)$ & 0 & 5 & 1 & 4 & $9 ! 2$ \\
\hline
\end{tabular} Reviewed By
Service Notes
O-100 PSIG,
C4APOI
$15,000 \Omega$

SEE ATACHED DATA SHEET. LIMITED TO DATA PROVIDES, DOES NET MEET H:I RATO SPECS. 
AS FECEIVED

\begin{tabular}{|c|c|c|}
\hline RAANSMITTEF': & $\begin{array}{r}\text { SENSOTEC } \\
100 \\
\text { TOLERANCE: }\end{array}$ & $\begin{array}{l}\text { TJE } \\
\text { FSI } \\
0.10 \%\end{array}$ \\
\hline 'RESS SLULIRCE & $\begin{array}{r}\text { ASHCFIOFT } \\
0-100 \\
\text { B\&W! SER. NO. } \\
\text { TOLEFIANCE: }\end{array}$ & $\begin{array}{l}\text { DIGIGAUGE } \\
\text { FSI } \\
0700125 \\
0.05 \%\end{array}$ \\
\hline EADOUT DEV: & $\begin{array}{l}\text { FLUHE } \\
\text { E\&W Ser. NO. } \\
\text { TOLEFIANCE: }\end{array}$ & $\begin{array}{l}8840 A \\
0860404 \\
0.015 \%\end{array}$ \\
\hline
\end{tabular}

AS

LEFT

E\&WW S.N. O8805.39... DATE: $14-$ May-92

FRIOCEDURE : AFIC-TF-015-08

FROGFIAM: TOO15_08.0BA

NAME: S,ELK,US/D. SYme

Ambient Temperature ( $F$ ) 70

Facility I. D. Name

ALUE OF CURFENT SENSING FESISTOF USED FOF TEST DATA 'ALLUE OF CUFFFENT SENSING FESISTOF' USED FOF CALIBRATION FEESISTOF MEASUFIING ACCURIACY

N.A.

- OWEF SUFF'YY VO!_TAGE

OTAL SEFIIES FIESISTANCE

AFF'LIED F'RESSURE FIANSMITTEF FSI FIFIESS SOUFICE

$$
\begin{array}{r}
0.000 \\
10.000 \\
20.000 \\
50.000 \\
40.000 \\
50.000 \\
60.000 \\
70.000 \\
80.000 \\
90.000 \\
100.000 \\
90.000 \\
80.000 \\
70.000 \\
60.000 \\
50.000 \\
40.000 \\
30.000 \\
20.000 \\
10.000 \\
0.000
\end{array}
$$
F'SI

\section{4}

15

IDEAL

OUTFUT SIG

MILLIVOLTS

$$
\begin{array}{r}
0.000 \\
10.000 \\
20.000 \\
50.000 \\
40.000 \\
50.000 \\
60.000 \\
70.000 \\
80.000 \\
90.000 \\
100.000 \\
90.000 \\
80.000 \\
70.000 \\
60.000 \\
50.000 \\
40.000 \\
30.000 \\
20.000 \\
10.000 \\
0.000
\end{array}
$$

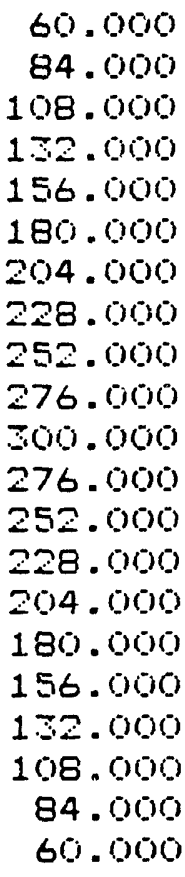

CALIEFIATION
MEASUFED
OUTFUT SIG
MILLIVOLTS

ACCUFIACY

**LIMITED** ERROF \% FULL SCALE MILLIVOLTS
15 OHMS

15 OHMS

59.989
83.885
107.840
1.1 .828
155.864
179.848
$20 . .848$
227.919
251.890
275.944
299.979
275.849
251.748
227.678
205.642
179.627
155.677
131.668
107.716
83.807
59.961$$
\begin{array}{r}
0.00 \% \\
-0.05 \% \\
-0.07 \% \\
-0.07 \% \\
-0.06 \% \\
-0.06 \% \\
-0.06 \% \\
-0.03 \% \\
-0.05 \% \\
-0.02 \% \\
-0.01 \% \\
-0.06 \% \\
-0.11 \% \\
-0.15 \% \\
-0.15 \% \\
-0.10 \% \\
-0.15 \% \\
-0.14 \% \\
-0.12 \% \\
-0.08 \% \\
-0.02 \%
\end{array}
$$$$
\bar{z}=59.975
$$

$0.17 \%$

OUT QF TOLEFIANCE 
CURVEFIT OUTPUT FOR VTAB C4APOIJ

DATE: 20-MAY-92

TIME: $16: 52: 35$

VOLTAGE $(V)$ OBS. PRESS. CALC. PRESS. DIFF. (PSIA) \& DIFFERENCE

(PSIA)

(PSIA)

$\begin{array}{rlcccc}1 & 0.1400176 \mathrm{E}-04 & 0.0000000 \mathrm{E}+00 & 0.1829549 \mathrm{E}-01 & -0.1829549 \mathrm{E}-01 & 0.0000000 \mathrm{E}+00 \\ 2 & 0.2391000 \mathrm{E}-01 & 10.00000 & 10.00777 & -0.7766683 \mathrm{E}-02 & -0.7766683 \mathrm{E}-01 \\ 3 & 0.4786500 \mathrm{E}-01 & 20.00000 & 20.01425 & -0.1424552 \mathrm{E}-01 & -0.7122760 \mathrm{E}-01 \\ 4 & 0.7185300 \mathrm{E}-01 & 30.00000 & 30.02683 & -0.2682714 \mathrm{E}-01 & -0.8942381 \mathrm{E}-01 \\ 5 & 0.9588900 \mathrm{E}-01 & 40.00000 & 40.05173 & -0.5173359 \mathrm{E}-01 & -0.1293340 \\ 6 & 0.1198730 & 50.00000 & 50.04726 & -0.4725892 \mathrm{E}-01 & -0.9451785 \mathrm{E}-01 \\ 7 & 0.1438730 & 60.00000 & 60.04176 & -0.4175999 \mathrm{E}-01 & -0.6959999 \mathrm{E}-01 \\ 8 & 0.1679440 & 70.00000 & 70.05810 & -0.5809900 \mathrm{E}-01 & -0.8299857 \mathrm{E}-01 \\ 9 & 0.1919150 & 80.00000 & 80.02513 & -0.2513398 \mathrm{E}-01 & -0.3141747 \mathrm{E}-01 \\ 10 & 0.2159690 & 90.00000 & 90.01896 & -0.1896370 \mathrm{E}-01 & -0.2107078 \mathrm{E}-01 \\ 11 & 0.2405040 & 100.0000 & 99.99718 & 0.2821052 \mathrm{E}-02 & 0.2821052 \mathrm{E}-02 \\ 12 & 0.2158740 & 90.00000 & 89.97951 & 0.2049119 \mathrm{E}-01 & 0.2276799 \mathrm{E}-01 \\ 13 & 0.1917730 & 80.00000 & 79.96611 & 0.3388639 \mathrm{E}-0 & 0.4235798 \mathrm{E}-01 \\ 14 & 0.1677030 & 70.00000 & 69.95785 & 0.4214670 \mathrm{E}-01 & 0.6020958 \mathrm{E}-01 \\ 15 & 0.1436670 & 60.00000 & 59.95601 & 0.4399340 \mathrm{E}-01 & 0.7332233 \mathrm{E}-01 \\ 16 & 0.1196520 & 50.00000 & 49.95519 & 0.4480953 \mathrm{E}-01 & 0.8961906 \mathrm{E}-01 \\ 17 & 0.9570200 \mathrm{E}-01 & 40.00000 & 39.97377 & 0.2623035 \mathrm{E}-01 & 0.6557588 \mathrm{E}-01 \\ 18 & 0.7169300 \mathrm{E}-01 & 30.00000 & 29.96007 & 0.3993133 \mathrm{E}-01 & 0.1331044 \\ 19 & 0.4774100 \mathrm{E}-01 & 20.00000 & 19.96247 & 0.3753200 \mathrm{E}-01 & 0.1876600 \\ 1 & 0.2383200 \mathrm{E}-01 & 10.00000 & 9.975172 & 0.2482799 \mathrm{E}-0 . & 0.2482799 \\ \angle 1 & -0.139984 \mathrm{E}-04 & 0.0000000 \mathrm{E}+00 & 0.6585912 \mathrm{E}-02 & -0.6585912 \mathrm{E}-02 & 0.0000000 \mathrm{E}+00\end{array}$

DEGREE OF POLYNOMIAL 2

COEFFICIENTS

$0.12439965 E-01 \quad 0.41819927 E+03-0.66796461 E+01$

ESS $=0.2398 E-01 \quad \begin{aligned} \text { SD } & =0.3650 E-01 \\ 2 * S D & =0.7301 E-01\end{aligned} \quad$ R2 $=0.999999 E+00$ 
RD-45A Rev. $11-18-91$

\begin{tabular}{|l|l|l|}
\hline INSTRUMENT STATUS & $\begin{array}{c}\text { AS } \\
\text { RECE }\end{array}$ & \begin{tabular}{c} 
AS \\
\hline Certified
\end{tabular} \\
\hline Calibrated & & \\
\hline 3 For Ind. Only & & $\checkmark$ \\
\hline 4 standardize & & \\
\hline 5 Certified c stand. & & \\
\hline 6 Calibrated S Stand. & & \\
\hline 9 other & & \\
\hline
\end{tabular}

INSTRUMENT CONDITION

AS RECEIVED (Certified Equipment only)

Meets Manufacturer's Tolerance N

Operates within previously defined limits $N$ Deviates from Mfg or previously defined limits $Y$ Inoperative (Describe below) $x$

AS LIFT Limited (Describe below)

Meets Manufacturer's Tolerance

other (Describe Below)
Babcock \& Wilcox

a MeDermotr company

B\&W Ser. No. 0880553
MANUFACTUKER

MODEL

ITEM

MANUF. SER. NO.

PROPERTY NO.

ORDER NO.

SECTION

LOCATION

SCHEDULED $(Y-N)$

RECAI INT.(MOS.)
SENSOTEC

TJE/127810

TRANSMITR, PRES :

201321

4427-01-115

46

115

$+5$

12

\section{TECH. PROCEDURE A0015}

Babcock C Wilcox RED Division certifies that the performance of this instrument was verified using standards whose accuracies are traceable to the National Institute of Standards and Technology, an accepted value of a natural physical constant, I a ratio type of calibration technigue, or to a consensus standard.

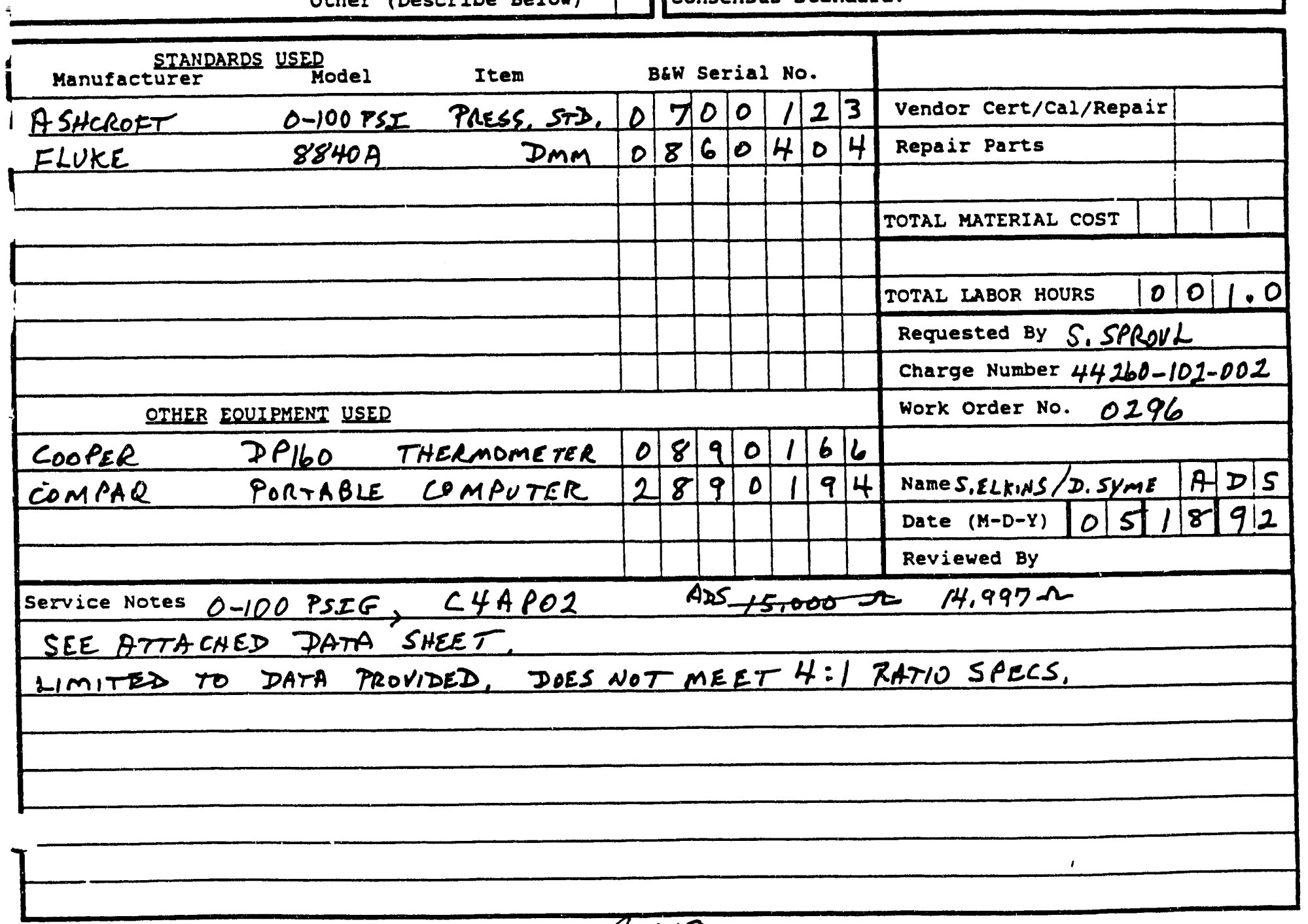


AS RECEIVED

TRIANSMITTEF:

SENSOTEC TJE

100 F'SI

TOLEFIANCE : $0.10 \%$

FFIESS SOUFICE

$$
\begin{aligned}
\text { ASHCROFT } & \text { DIGIGAUGE } \\
0-100 & \text { FSI } \\
\text { B\&W SER. NO. } & 0700123 \\
\text { TOLERANCE: } & 0.05 \%
\end{aligned}
$$

READOUT DEV:

FLUKKE 8840A

E\&W Ser. No. 0860404

TOLERIANCE : $0.015 \%$
AS LEFT

E\&W S.N. OB80553

DATE: $\quad 18-\operatorname{MaY}-92$

PROCEDURE: ARC-TF-015-OB

FFIOGFIM: TOO15_0B.0JA'

NAME: S.ELKINS/D, SŸ̈ME

Ambient Temperature (F) 75

Facility I. D. Name

\section{C4AFO2}

VALUE OF CURFENT SENSING FESISTOF USED FOF TEST DATA

VALUE OF "CUFIFENT SENSING FIESISTOF" USED FOF' CALIBFATION

FOWEF SUFFLY VOLTAGE

TOTAL SERIES FIESISTANCE

AFPLIED FRESSURE

TRIANSMITTEF: FSI

F'RESS SDURICE

F'SI

0.000
10.000
20.000
50.000
40.000
50.000
60.000
70.000
80.000
90.000
100.000
90.000
80.000
70.000
60.000
50.000
40.000
30.000
20.000
10.000
0.000
FESISTOF: MEASURING ACCUFIACY 24

14.997

IDEAL

QUTFUT SIG

MILLIVOLTS

59.988
83.983
107.978
131.974
155.969
179.964
205.959
227.954
251.950
275.945
299.940
275.945
251.950
227.954
203.959
179.964
155.969
131.974
107.978
85.983
59.988

14.997 DHMS 14.997 OHMS N.A.

$\begin{array}{clc}\text { CALIEFATION ACCURACY } & \\ & * * \text { IMITED** } & \\ \text { MEASURED } & \text { EFIROF \% } & \text { OUT } \\ \text { OUTFUT SIG } & \text { FULL SCALE } & \text { OF } \\ \text { MILLIVOLTS } & & \text { TOLEFIANCE }\end{array}$


CURVEFIT OUTPUT FOR VTAB C4APO2 $J$

DATE: 20-MAY -92

TIME: $16: 53: 30$

VOLTAGE(V) OBS. PRESS. CALC. PRESS. DIFF. (PSIA) \& DIFFERENCE

(PSIA)

(PSIA)

$\begin{array}{rrrrrr}1 & -0.7998896 \mathrm{E}-05 & 0.0000000 \mathrm{E}+00 & 0.3236739 \mathrm{E}-03 & -0.3236739 \mathrm{E}-03 & 0.0000000 \mathrm{E}+00 \\ 2 & 0.2389400 \mathrm{E}-01 & 10.00000 & 10.00058 & -0.5834413 \mathrm{E}-03 & -0.5834413 \mathrm{E}-02 \\ 3 & 0.4780900 \mathrm{E}-01 & 20.00000 & 19.99640 & 0.3599191 \mathrm{E}-02 & 0.1799596 \mathrm{E}-01 \\ 4 & 0.7177600 \mathrm{E}-01 & 30.00000 & 30.00404 & -0.4036394 \mathrm{E}-02 & -0.1345465 \mathrm{E}-01 \\ 5 & 0.9578300 \mathrm{E}-01 & 40.00000 & 40.01842 & -0.1842234 \mathrm{E}-01 & -0.4605585 \mathrm{E}-01 \\ 6 & 0.1198010 & 50.00000 & 50.02743 & -0.2742973 \mathrm{E}-01 & -0.5485946 \mathrm{E}-01 \\ 7 & 0.1437910 & 60.00000 & 60.01482 & -0.1481670 \mathrm{E}-01 & -0.2469449 \mathrm{E}-01 \\ 8 & 0.1678880 & 70.00000 & 70.03674 & -0.3673649 \mathrm{E}-01 & -0.5248069 \mathrm{E}-01 \\ 9 & 0.1919260 & 80.00000 & 80.02412 & -0.2411994 \mathrm{E}-01 & -0.3014993 \mathrm{E}-01 \\ 10 & 0.2160040 & 90.00000 & 90.01811 & -0.1811174 \mathrm{E}-01 & -0.2012416 \mathrm{E}-01 \\ 11 & 0.2400660 & 100.0000 & 99.99545 & 0.4546843 \mathrm{E}-02 & 0.4546843 \mathrm{E}-02 \\ 12 & 0.2159000 & 90.00000 & 89.97497 & 0.2503372 \mathrm{E}-01 & 0.2781524 \mathrm{E}-01 \\ 13 & 0.1918310 & 80.00000 & 79.98467 & 0.1533130 \mathrm{E}-01 & 0.1916413 \mathrm{E}-01 \\ 14 & 0.1677610 & 70.00000 & 69.98394 & 0.1605639 \mathrm{E}-01 & 0.2293770 \mathrm{E}-01 \\ 15 & 0.1436740 & 60.00000 & 59.96613 & 0.3386798 \mathrm{E}-01 & 0.5644663 \mathrm{E}-01 \\ 16 & 0.1196870 & 50.00000 & 49.97995 & 0.2005388 \mathrm{E}-01 & 0.4010776 \mathrm{E}-01 \\ 17 & 0.9570500 \mathrm{E}-01 & 40.00000 & 39.98590 & 0.1409880 \mathrm{E}-01 & 0.3524699 \mathrm{E}-01 \\ 18 & 0.7172400 \mathrm{E}-01 & 30.00000 & 29.98233 & 0.1766593 \mathrm{E}-01 & 0.5888642 \mathrm{E}-01 \\ 19 & 0.4781900 \mathrm{E}-01 & 20.00000 & 20.00058 & -0.5784688 \mathrm{E}-03 & -0.2892344 \mathrm{E}-02 \\ 1 & 0.2388900 \mathrm{E}-01 & 10.00000 & 9.998493 & 0.1507456 \mathrm{E}-02 & 0.1507456 \mathrm{E}-01 \\ \angle 1 & 0.7001104 \mathrm{E}-05 & 0.0000000 \mathrm{E}+00 & 0.6602559 \mathrm{E}-02 & -0.6602559 \mathrm{E}-02 & 0.0000000 \mathrm{E}+00\end{array}$

JEGREE OF POLYNOMIAL 2

ZOEFFICIENTS

$0.36719508 E-02 \quad 0.41859230 E+03-0.86410493 E+01$

ESS $=0.6847 \mathrm{E}-02 \quad \mathrm{SD}=0.1950 \mathrm{E}-01 \quad \mathrm{R} 2=0.100000 \mathrm{E}+01$

$2 \star S D=0.3901 E-01$ 
RD-45A Rev. 11-18-91

\begin{tabular}{|c|c|c|c|}
\hline & INSTRUMENT STATUS & ATED & LEST \\
\hline & certified & & \\
\hline & Caljbrated & & \\
\hline 31 & For Ind. Ondy & $\checkmark$ & \\
\hline 45 & Standardize & & \\
\hline & Certified stand. & & \\
\hline & Calibrated stand. & & \\
\hline & other & & \\
\hline
\end{tabular}

INSTRUMENT CONDITION

AS RECEIVED (Certified Equipment Ondy)

Meets Manufacturer's Tolerance $N$

Operates within previously defined limits $N$ Deviates from Mfg or previously defined linits $Y$ Inoperative (Describe below) $x$

AS IEET Limited (Describe below)

Meets Manufacturer's Tolerance

other (Describe Below) NSTRUMENT SERVICE IOC

\begin{tabular}{|c|c|}
\hline Certilication & 1 \\
\hline Calibration & 2 \\
\hline Maintenance & 3 \\
\hline Repair & 4 \\
\hline Vendor Cert. & 5 \\
\hline Vendor Calib. & 6 \\
\hline Vendor Repair & 7 \\
\hline other & 9 \\
\hline
\end{tabular}

MANUFACTURER

MODEL

ITEM

MANUF. SER. NO.

PROPERTY NO.

ORDER NO.

SECTION

LOCATION

SCHEDULED ( $Y-N)$

RECAL INT. (MOS.)
U4iru

Babcock \& Wilcox

a McDermott company

B\&W Ser. No. 0880538

SENSOTEC

TJE/ 127810

TRANSMITR, PRES

188753

4427-01-100

\section{6}

115

$+y$

12

\section{TECH. PROCEDURE A0015}

Babcock \& Wilcox RGD Division certifies that the performance of this instrument was verified using standards whose accuracies are traceable to the National Institute of standards and Technology an accepted value of a natural physical constant, a ratio type of calibration technique, or to a consensus standard.

\begin{tabular}{|c|c|c|c|c|c|c|}
\hline Manufacturer $\frac{\text { STAMDARDS }}{\text { USED }}$ & Item & & stw ser & erial & No. & \\
\hline ASHCROET & PRESS, STD, & 0 & \begin{tabular}{l|l|}
7 & 0 \\
\end{tabular} & 0 & 1123 & Vendor Cert/Cal/Repair \\
\hline ELUKE & DMM & 0 & \begin{tabular}{|l|l|}
8 & 6 \\
\end{tabular} & 01 & \begin{tabular}{l|l|l|}
4 & 0 & 4 \\
\end{tabular} & Repair Parts \\
\hline & & & & & & \\
\hline & & & & & & TOTAL MATERIAL COST \\
\hline & & & & & & \\
\hline & & & & & & TOTAL LABOR HOURS \\
\hline & & & & & & Requested By S, SPRoUL \\
\hline & & & & & & Charge Number $44260-102-002$ \\
\hline OTHER EOUIPMENT USED & & & & & & Work order No. 0296 \\
\hline COOPRR & THER MOMETER & 0 & 89 & 90 & $166 \mid 6$ & \\
\hline PORAABLE & COmPuTRR & 1 & 89 & 0 & 1994 & 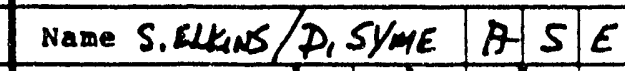 \\
\hline & & & & & & \begin{tabular}{|l|l|l|l|l|l|} 
Date $(M-D-Y)$ & 0 & 5 & 1 & 8 & 9 \\
\end{tabular} \\
\hline & & & & & & Reviewed By \\
\hline Service Notes $0-100$ PSIE & C4APOS & & & 14. & $994 \Omega$ & \\
\hline SEE ATTACHED DATA & SHEET & & & & & \\
\hline LIMITED TO DAM PR & DIDED, DOE & $\bar{N}$ & or $M$ & MEL & ET $4=1$ & RATE SPECS. \\
\hline
\end{tabular}


AS RECEIVED

FIANSMITTER:

P'FESS SOURCE

SENSOTEC TJE

100 FSI

TOLEFANCE: $0.10 \%$

$\begin{aligned} \text { ASHCFOFT } & \text { DIGIGAUGE } \\ 0-100 & \text { FSI } \\ \text { B\&W SER. NO: } & 0700123 \\ \text { TOLEFANCE: } & 0.05 \%\end{aligned}$
PRESS SOURCE

FLUKE 8840 A

E\&W Ser. No. 0860404 TOLEFANCE : $0.015 \%$
AS LEFT

B\&W S.N. OBBO538

DATE: 18-MaY-92

PFOCEDURE : ARC-TF-O15-OB

FRIOGRAM: TOO15_0B.03A

NAME: S.ELKINS/P.SŸinE

Ambient Temperature (F) 75

Facility I. D. Name C4AFOS
IALUE OF CUFFENT SENSING FIESISTOF' USED FOF TEST DATA
IALUE OF :CUFIRENT SENSING FESISTOFi USED FOF CALIERATION
$\begin{array}{ll}14.954 & \text { aHMS } \\ 14.994 & \text { OHMS }\end{array}$
FESISTOF: MEASUFIING ACCUFIACY N.A.

TOWEF SUFFLY VOLTAGE

-DTAL SEFIES REESISTANCE

AF'FLIED FFESSURE

FIANSMITTEF'

F'SI

F'RESS SOURICE

FSI

0.000

10.000

20.000

30.000

40.000

50.000

60.000

70.000

80.000

90.000

100.000

90.000

80.000

70.000

60.000

50.000

40.000

30.000

20.000

10.000

0.000
0.000
10.000
20.000
30.000
40.000
50.000
60.000
70.000
80.000
90.000
00.000
90.000
80.000
70.000
60.000
50.000
40.000
30.000
20.000
10.000
0.000

$$
24
$$

14.954

IDEAL

OUTFUT SIG

MILLIVOLTS

59.976

8.9666

107.957

131.947

155.938

179.928

203.918

227.909

251.879

275.890

299.880

275.890

251.899

227.909

205.918

179.928

155.938

131.947

107.957

83.966

59.976
CALIERIATION ACCURACY

**LIMITED**

MEASURED EFFIOF \%

OUTFUT SIG FULL SCALE

MILLIVOLTS

$\begin{array}{rr}57.885 & 0.00 \% \\ 83.876 & -0.04 \% \\ 107.819 & -0.06 \% \\ 131.792 & -0.06 \% \\ 155.800 & -0.06 \% \\ 179.764 & -0.07 \% \\ 205.770 & -0.06 \% \\ 227.821 & -0.04 \% \\ 251.816 & -0.03 \% \\ 275.875 & -0.01 \% \\ 279.874 & 0.00 \% \\ 275.704 & -0.08 \% \\ 251.648 & -0.10 \% \\ 227.654 & -0.11 \% \\ 203.599 & -0.13 \% \\ 179.593 & -0.14 \% \\ 155.604 & -0.14 \% \\ 151.625 & -0.15 \% \\ 107.692 & -0.11 \% \\ 83.787 & -0.07 \% \\ 59.915 & -0.03 \%\end{array}$

$$
\bar{z}=59.950
$$


CURVEFIT OUTPUT FOR VTAB C4AP03 $\sqrt{ }$

VOLTAGE(V) OBS. PRESS. CALC. PRESS. DIFF. (PSIA) \& DIFFERENCE
DATE: 20-MAY-92

TIME: $16: 54: 27$

(PSIA)

(PSIA)

$\begin{array}{rlllll}1 & 0.3499846 \mathrm{E}-04 & 0.0000000 \mathrm{E}+00 & 0.2363186 \mathrm{E}-01 & -0.2363186 \mathrm{E}-01 & 0.0000000 \mathrm{E}+00 \\ 2 & 0.2392600 \mathrm{E}-01 & 10.00000 & 10.01405 & -0.1405007 \mathrm{E}-01 & -0.1405007 \\ 3 & 0.4786900 \mathrm{E}-01 & 20.00000 & 20.01875 & -0.1875373 \mathrm{E}-01 & -0.9376866 \mathrm{E}-01 \\ 4 & 0.7184200 \mathrm{E}-01 & 30.00000 & 30.02851 & -0.2851170 \mathrm{E}-01 & -0.9503900 \mathrm{E}-01 \\ 5 & 0.9585000 \mathrm{E}-01 & 40.00000 & 40.04538 & -0.4538123 \mathrm{E}-01 & -0.1134531 \\ 6 & 0.1198140 & 50.00000 & 50.03641 & -0.3640533 \mathrm{E}-01 & -0.7281066 \mathrm{E}-01 \\ 7 & 0.1438200 & 60.00000 & 60.03744 & -0.3743990 \mathrm{E}-01 & -0.6239984 \mathrm{E}-01 \\ 8 & 0.1678710 & 70.00000 & 70.04969 & -0.4969394 \mathrm{E}-01 & -0.7099134 \mathrm{E}-01 \\ 9 & 0.1918660 & 80.00000 & 80.03113 & -0.3112706 \mathrm{E}-01 & -0.3890882 \mathrm{E}-01 \\ 10 & 0.2159230 & 90.00000 & 90.03082 & -0.3082205 \mathrm{E}-01 & -0.3424672 \mathrm{E}-01 \\ 11 & 0.2399240 & 100.0000 & 99.99973 & 0.2725967 \mathrm{E}-03 & 0.2725967 \mathrm{E}-03 \\ 12 & 0.2157540 & 90.00000 & 89.96060 & 0.3939934 \mathrm{E}-01 & 0.4377705 \mathrm{E}-01 \\ 13 & 0.1916980 & 80.00000 & 79.96127 & 0.3873146 \mathrm{E}-01 & 0.4841432 \mathrm{E}-01 \\ 14 & 0.1676840 & 70.00000 & 69.97188 & 0.2812371 \mathrm{E}-01 & 0.4017673 \mathrm{E}-01 \\ 15 & 0.1436490 & 60.00000 & 59.96623 & 0.3377311 \mathrm{E}-01 & 0.5628852 \mathrm{E}-01 \\ 16 & 0.1196430 & 50.00000 & 49.96514 & 0.3486115 \mathrm{E}-01 & 0.6972231 \mathrm{E}-01 \\ 17 & 0.9565400 \mathrm{E}-01 & 40.00000 & 39.96363 & 0.3636555 \mathrm{E}-01 & 0.9091388 \mathrm{E}-01 \\ 18 & 0.7167500 \mathrm{E}-01 & 30.00000 & 29.95881 & 0.4119209 \mathrm{E}-01 & 0.1373070 \\ 19 & 0.4774200 \mathrm{E}-01 & 20.00000 & 19.96571 & 0.3429416 \mathrm{E}-01 & 0.1714708 \\ 1 & 0.2383700 \mathrm{E}-01 & 10.00000 & 9.976847 & 0.2315296 \mathrm{E}-01 & 0.2315296 \\ 21 & -0.3500154 \mathrm{E}-04 & 0.0000000 \mathrm{E}+00 & -0.5650727 \mathrm{E}-02 & 0.5650727 \mathrm{E}-02 & 0.0000000 \mathrm{E}+00\end{array}$

COEFFICIENTS

$0.89912173 E-02 \quad 0.41832266 E+03-0.65129419 E+01$

ESS $=0.2206 \mathrm{E}-01 \quad \begin{aligned} \mathrm{SD} & =0.3501 \mathrm{E}-01 \\ 2 * \mathrm{SD} & =0.7001 \mathrm{E}-01\end{aligned} \quad \mathrm{R} 2=0.999999 \mathrm{E}+00$ 


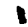

RD-45A Rev. $11-18-91$

\begin{tabular}{l|l|l|}
\hline INSTRUMENT STATUS & AS & AS \\
\hline Certified & & \\
\hline Calibrated & & \\
\hline For Ind. Only & $\checkmark$ & \\
\hline Standardize & & \\
\hline Certified stand. & & \\
\hline Calibrated stand. & & \\
\hline Other & & \\
\hline
\end{tabular}

INSTRUMENT CONDITION

AS RECEIVED (Certified Equipment only)

Meets Manufacturer's Tolerance $N$

Operates within previously defined limits $N$ jeviates from $\mathrm{Mfg}$ or previously defined limits $Y$ L. Inoperative (Describe below) $y$

AS LEFT

Limited (Describe below)

Meets Manufacturer's Tolerance

other (Describe Below)
Babcock \& Wilcox

- McDermoti company

B\&W Ser. NO. 0880536

MANUFACTURER SENSOTEC

MODEL - TJE/127810

ITEY

TRANSMITR, PRES:

MANUF . SER. NO. 179672

PROPERTY NO.

ORDER NO.

SECTION

46

LOCATION

SCHEDULED $(Y-N)$

RECAL INT. (MOS.)

115

if $Y$

12

TECH. PROCEDURE A0015

Babcock \& Wilcox RED Division certifies that the performance of this instrument was verified using standards whose accuracies are traceable to the National Institute of Standards and Technology, a ratio type of calibration technique, or to a consensus standard.

STAIDARDS USED

Manufacture

Model

Item

Bew Serial No.

A SHCROFT 0-100 PSI PRESS, STD,

$=1$ LKE $8840 \mathrm{~A}$ DMM

\begin{tabular}{l|l|l|l|l|l|l|}
0 & 7 & 0 & 0 & 1 & 2 & 3 \\
0 & 8 & 6 & 0 & 4 & 0 & 4
\end{tabular}

Vendor Cert/Cal/Repair Repair Parts

TOTAL MATERIAL COST

TOTAL IABOR HOURS 10.0 .1 .0 Requested BY S, SPROUL Charge Number 44260-102-002

OTHER EOUIPMENT USED Work order No. 0296

\begin{tabular}{|ll|l|l|l|l|l|l|l|}
\hline COOPER DP160 THERMO mERER & 0 & 8 & 9 & 0 & 1 & 6 & 6
\end{tabular} ComPAQ PIRTHBLR COMPUTER $\sim$

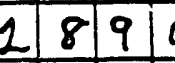
Name 5.ELKINS/D, SYME $A$ S

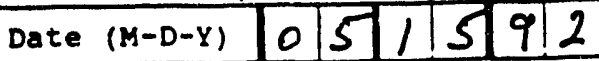

Reviewed By

Service notes $0-100$ PSIG, IPAPOL $15.004 \Omega$

SEE ATTACHED DATA SHEET. LIMITED TO DATA PROVIDED DOKS NOT MCET H:I RAPO SPECS, 
$?$

TRANSMITTER:

AS FECEIVED

SENSOTEC TJE

100 F'SI

TOLEFANCE: $0.10 \%$

FRESS SOURCE

\section{ASHCFIOFT DIGIGAUGE \\ 0-100 F.SI}

B\&W Ser. No. 0700123

TOLEFANCE : $0.05 \%$

FEADOUT DEV:

FLUKE 8840A
E\&W Ser. NO. 0860404

AS LEFT

E\&W S.N. 0880536.

DATE: 15-May-92

FROCEDUFE : ARC-TF-015-08

F'FOGFAM: T0015_08.03A

NAME: S,ELkWS /D, syme

Ambient Temperature (F) 70

Facility I. D. Name IFAFO1 TOLERANCE : $0.015 \%$

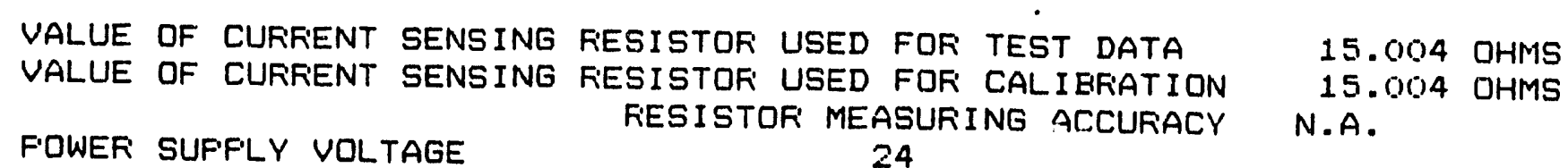

TOTAL SEFIES RESISTANCE

AFPLIED FRESSUFE

TFIANSMITTEF FRIESS SQURICE

FSI F'SI

0.000
10.000
20.000
30.000
40.000
50.000
60.000
70.000
80.000
90.000
100.000
90.000
80.000
70.000
60.000
50.000
40.000
30.000
20.000
10.000
0.000

0.000

10.000

20.000

30.000

40.000

50.000

60.000

70.000

80.000

90.000

100.000

90.000

80.000

70.000

60.000

50.000

40.000

30.000

20.000

10.000

0.000
24

15.004

I DEAL

OUTFUT SIG

MILLIVOLTS

60.016

84.022

108.029

1.2 .055

156.042

180.048

204.054

228.061

252.067

276.074

300.080

276.074

252.067

228.061

204.054

180.048

156.042

1.32 .035

108.029

84.022

60.016
CAL IEFATION ACCURACY

MEASURED

QUTFUT SIG

MILLIVOLTS

$\begin{array}{rr}59.969 & -0.02 \% \\ 83.941 & -0.03 \% \\ 107.981 & -0.02 \% \\ 132.020 & -0.01 \% \\ 156.112 & 0.03 \% \\ 180.082 & 0.01 \% \\ 204.096 & 0.02 \% \\ 228.137 & 0.03 \% \\ 252.096 & 0.01 \% \\ 276.080 & 0.00 \% \\ 300.060 & -0.01 \% \\ 276.060 & -0.01 \% \\ 252.088 & 0.01 \% \\ 228.122 & 0.03 \% \\ 204.058 & 0.00 \% \\ 180.047 & 0.00 \% \\ 156.063 & 0.01 \% \\ 131.988 & -0.02 \% \\ 108.003 & -0.01 \% \\ 83.964 & -0.02 \% \\ 59.947 & -0.03 \%\end{array}$

**L IMITED**

$0.17 \%$

EFFIOF \%

FULL SCALE

T

OUT
OF
TOLEFIANCE

立: 59.950 
CURVEFIT OUTPUT FOR VTAB ipap01 $\mathcal{J}$

VOLTAGE (V) OBS. PRESS. CALC. PRESS. DIFF. (PSIA)
DATE: 20-MAY-92

TIME: $16: 57: 21$
(PSIA)

$\begin{array}{llll}1 & 0.1100026 \mathrm{E}-04 & 0.0000000 \mathrm{E}+00 & 0.8575692 \mathrm{E}-02 \\ 2 & 0.2398300 \mathrm{E}-01 & 10.00000 & 9.989323 \\ 3 & 0.4802300 \mathrm{E}-01 & 20.00000 & 19.99551 \\ 4 & 0.7206200 \mathrm{E}-01 & 30.00000 & 29.99979 \\ 5 & 0.9615400 \mathrm{E}-01 & 40.00000 & 40.02602 \\ 6 & 0.1201240 & 50.00000 & 50.00276 \\ 7 & 0.1441380 & 60.00000 & 60.00046 \\ 8 & 0.1681790 & 70.00000 & 70.01345 \\ 9 & 0.1921380 & 80.00000 & 79.99769 \\ 0 & 0.2161220 & 90.00000 & 89.99912 \\ 1 & 0.2401020 & 100.0000 & 100.0070 \\ 2 & 0.2161020 & 90.00000 & 89.99078 \\ 3 & 0.1921300 & 80.00000 & 79.99435 \\ 4 & 0.1681640 & 70.00000 & 70.00720 \\ 5 & 0.1441000 & 60.00000 & 59.98464 \\ 6 & 0.1200890 & 50.00000 & 49.98819 \\ 7 & 0.9610500 \mathrm{E}-01 & 40.00000 & 40.00563 \\ 8 & 0.7203000 \mathrm{E}-01 & 30.00000 & 29.98647 \\ 9 & 0.4804500 \mathrm{E}-01 & 20.00000 & 20.00467 \\ 1 & 0.2400600 \mathrm{E}-01 & 10.00000 & 9.998898 \\ 1 & -0.1099974 \mathrm{E}-04 & 0.0000000 \mathrm{E}+00 & -0.5857472 \mathrm{E}-03\end{array}$

(PSIA)

\begin{tabular}{rr}
$-0.8575692 \mathrm{E}-02$ & \multicolumn{1}{l}{$0.0000000 \mathrm{E}+00$} \\
$0.1067663 \mathrm{E}-01$ & 0.1067663 \\
$0.4488489 \mathrm{E}-02$ & $0.2244245 \mathrm{E}-01$ \\
$0.2080978 \mathrm{E}-03$ & $0.6936595 \mathrm{E}-03$ \\
$-0.2602338 \mathrm{E}-01$ & $-0.6505846 \mathrm{E}-01$ \\
$-0.2757861 \mathrm{E}-02$ & $-0.5515722 \mathrm{E}-02$ \\
$-0.4612405 \mathrm{E}-03$ & $-0.7687341 \mathrm{E}-03$ \\
$-0.1345037 \mathrm{E}-01$ & $-0.1921481 \mathrm{E}-01$ \\
$0.2310596 \mathrm{E}-02$ & $0.2888246 \mathrm{E}-02$ \\
$0.8763262 \mathrm{E}-03$ & $0.9736958 \mathrm{E}-03$ \\
$-0.7043519 \mathrm{E}-02$ & $-0.7043519 \mathrm{E}-02$ \\
$0.9219621 \mathrm{E}-02$ & $0.1024402 \mathrm{E}-01$ \\
$0.5645424 \mathrm{E}-02$ & $0.7056780 \mathrm{E}-02$ \\
$-0.7201372 \mathrm{E}-02$ & $-0.1028768 \mathrm{E}-01$ \\
$0.1536205 \mathrm{E}-01$ & $0.2560342 \mathrm{E}-01$ \\
$0.1181135 \mathrm{E}-01$ & $0.2362270 \mathrm{E}-01$ \\
$-0.5630498 \mathrm{E}-02$ & $-0.1407625 \mathrm{E}-01$ \\
$0.1352512 \mathrm{E}-01$ & $0.4508373 \mathrm{E}-01$ \\
$-0.4667694 \mathrm{E}-02$ & $-0.2333847 \mathrm{E}-01$ \\
$0.1102171 \mathrm{E}-02$ & $0.1102171 \mathrm{E}-01$ \\
$0.5857472 \mathrm{E}-03$ & $0.0000000 \mathrm{E}+00$
\end{tabular}

EGREE OF POLYNOMIAL 3

OEFFICIENTS

$0.39948631 E-02 \quad 0.41642907 E+03-0.36843736 E+01 \quad 0.16616281 E+02$

$: S S=0.1912 \mathrm{E}-02 \quad \mathrm{SD}=0.1060 \mathrm{E}-01 \quad \mathrm{R2}=0.100000 \mathrm{E}+01$

$2 * S D=0.2121 \mathrm{E}-01$ 
RD-45A Rev. $11-18-91$

RESEARCH DEVELOPMENT DIVISION INSTRUMENT SERVICE IOG

\begin{tabular}{|c|c|c|}
\hline INSTRUMENT STATUS & Resco & $\begin{array}{c}\text { AS } \\
\text { LERT }\end{array}$ \\
\hline 2 Certified & & \\
\hline Calibrated & & \\
\hline 3 For Ind. Only & & \\
\hline 4 standardize & & \\
\hline 5 Certified \& stand. & & \\
\hline 6 calibrated stand. & & \\
\hline 9 other & & \\
\hline
\end{tabular}

INSTRUMENT CONDITION

AS RECEIVED (Certified Equipment only) Meets Manufacturer's Tolerance N Operates within previously defined limits $N$ Deviates from Mfg or previously defined limits $Y$ Inoperative (Describe below) $y$ AS LEFT Limited (Describe below) Meets Manufacturer's Tolerance other (Describe Below)

\begin{tabular}{|l|l|l|}
\hline \multicolumn{2}{|l|}{ TYPE OF SERVICE } \\
\hline Certification 2 & \\
\hline Calibration & 2 & \\
\hline Maintenance & 3 & \\
\hline Repair & 4 & \\
\hline Vendor Cert. & 5 & \\
\hline Vendor Calib. & 6 & \\
\hline Vendor Repair 7 & \\
\hline Other & 9 & \\
\hline
\end{tabular}

MANUFACTURER MODEL ITEM MANUF. SER. NO. PROPERTY NO. ORDER NO.

SECTION

IOCATION

SCHEDULED ( $Y-N)$

RECAL INT. (MOS.) consensus standard.
Babcock \& Wilcox

a McDermort company

B\&W Ser. No. 088055

SENSOTEC

TJE/127810

TRANSMITR, PRES

201695

4427-01-118

\section{TECH. PROCEDURE A0015}

Babcock \& Wilcox RED Division certifies that the performance of this instrument was verified using standards whose accuracies are traceable to the National Institute of standards and Technology. an accepted value of a natural physical constant. a ratio type of calibration technique, or to a

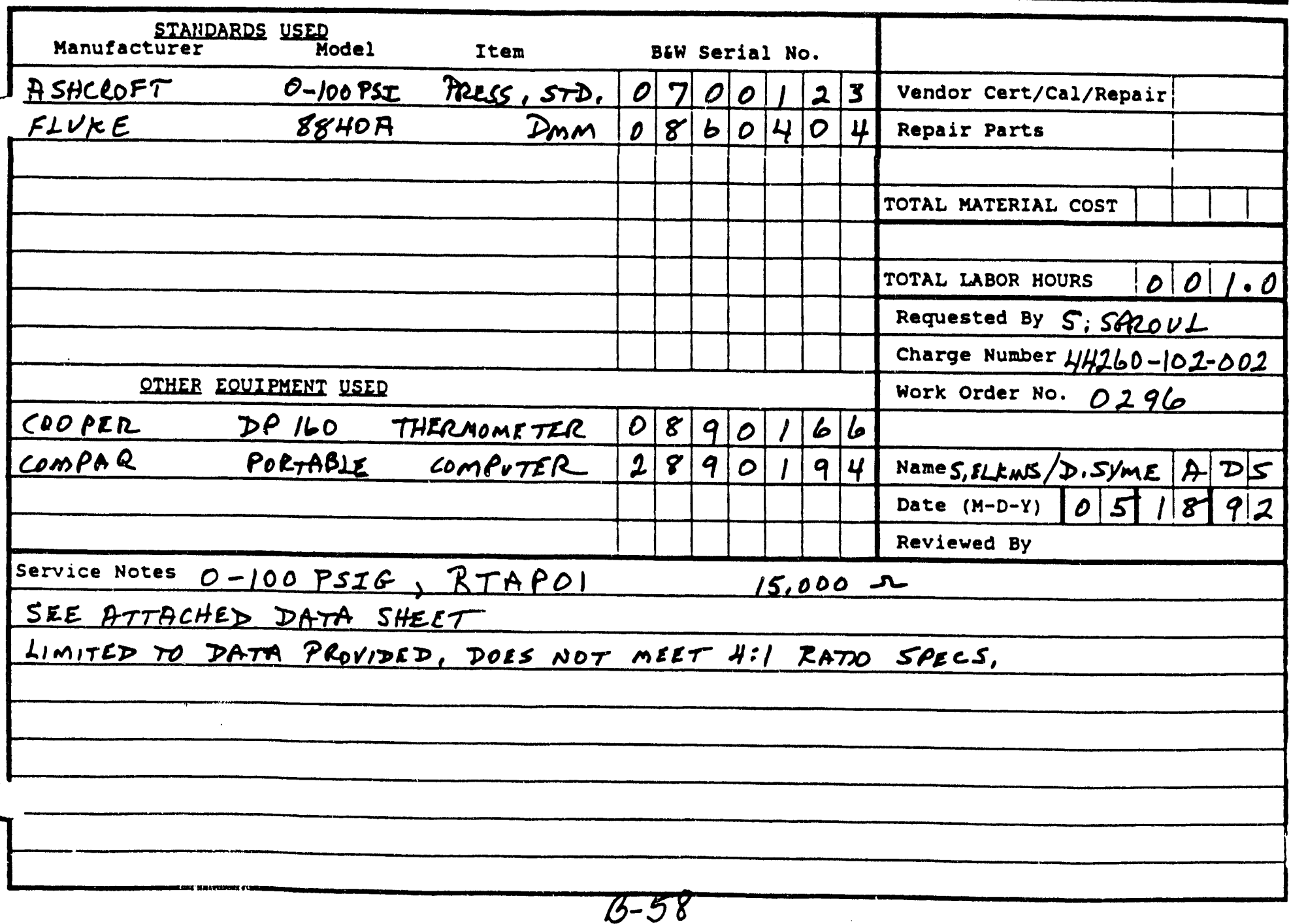



As

LEFT

AS FIECEIVED

'FANSMITTEF:

SENSOTEC TJE 100 PSI

TOLEFANCE: $0.10 \%$

FRESS SOUFICE

$$
\begin{gathered}
\text { ASHCFOFT DIGIGAUGE } \\
0-100
\end{gathered}
$$

E\&W Ser. NO. 0700125 TOLERANCE: $0.05 \%$

:EADOUT DEV:

\section{E\&:W Ser. NO. OB60404} TOLEFIANCE : $0.015 \%$

'ALUE OF CUFFENT SENSING RESISTOF' USED FOF' TEST DATA $\checkmark A L U E$ OF :CURRENT SENSING FESISTOF: USED FOF CALIBRATION 'OWEF' SUF'PLY VOLTAGE OTAL SEFIES FIESISTANCE FEESISTOF MEASURIING ACCUFIACY 24 15

$$
\text { AF'F'LIED F'REESSUFE }
$$
FIANSMITTEF' FSI F'FIESS SOUFICE F'SI
0.000

10.000

20.000

30.000

40.000

50.000

60.000

70.000

80.000

90.000

100.000

90.000

80.000

70.000

60.000

50.000

40.000

50.000

20.000

10.000

0.000
0.000

10.000

20.000

30.000

40.000

50.000

60.000

70.000

80.000

100.000

90.000

80.000

70.000

60.000

50.000

40.000

30.000

20.000

10.000

0.000
90.000
IDEAL OUTFUT SIG MILLIVOLTS

60.000

84.000

108.000

132.000

156.000

180.000

204.000

228.000

252.000

276.000

300.000

276.000

252.000

228.000

204.000

180.000

156.000

1.52 .000

108.000

84.000

60.000
CALIBFATION ACCUFACY

**LIMITED**

MEASUFED EFIFIOF \% DUTFUT SIG FULL SCALE MILLIVOLTS

15 OHMS

15 OHMS

N.A.
0880556

FRDGFIAM: T0015_08.03A

NAME: S.ELHas Lo. SYM̈E

(F) 69.4

FITAFO1 
CURVEFIT OUTPUT FOR VTAB rtap01 $\sqrt{ }$

VOLTAGE (V) OBS. PRESS. CALC. PRESS. DIFF. (PSIA)
DATE: 20-MAY-92

TIME: 16:59:03 (PSIA) (PSIA)

$\begin{array}{rlccrr}1 & -0.1499914 \mathrm{E}-04 & 0.0000000 \mathrm{E}+00 & -0.7821622 \mathrm{E}-02 & 0.7821622 \mathrm{E}-02 & 0.0000000 \mathrm{E}+00 \\ 2 & 0.2396000 \mathrm{E}-01 & 10.00000 & 9.999426 & 0.5737799 \mathrm{E}-03 & 0.5737799 \mathrm{E}-02 \\ 3 & 0.4794000 \mathrm{E}-01 & 20.00000 & 20.00403 & -0.4028742 \mathrm{E}-02 & -0.2014371 \mathrm{E}-01 \\ 4 & 0.7192600 \mathrm{E}-01 & 30.00000 & 30.00640 & -0.6399893 \mathrm{E}-02 & -0.2133298 \mathrm{E}-01 \\ 5 & 0.9595300 \mathrm{E}-01 & 40.00000 & 40.02112 & -0.2112105 \mathrm{E}-01 & -0.5280263 \mathrm{E}-01 \\ 6 & 0.1199520 & 50.00000 & 50.01943 & -0.1942840 \mathrm{E}-01 & -0.3885681 \mathrm{E}-01 \\ 7 & 0.1439440 & 60.00000 & 60.01008 & -0.1008120 \mathrm{E}-01 & -0.1680200 \mathrm{E}-01 \\ 8 & 0.1680150 & 70.00000 & 70.02887 & -0.2886986 \mathrm{E}-01 & -0.4124266 \mathrm{E}-01 \\ 9 & 0.1919990 & 80.00000 & 80.00670 & -0.6704547 \mathrm{E}-02 & -0.8380683 \mathrm{E}-02 \\ 10 & 0.2160470 & 90.00000 & 90.00641 & -0.6411162 \mathrm{E}-02 & -0.7123513 \mathrm{E}-02 \\ 11 & 0.2400780 & 100.0000 & 99.99429 & 0.5705875 \mathrm{E}-02 & 0.5705875 \mathrm{E}-02 \\ 12 & 0.2160190 & 90.00000 & 89.99477 & 0.5229109 \mathrm{E}-02 & 0.5810121 \mathrm{E}-02 \\ 13 & 0.1919850 & 80.00000 & 80.00088 & -0.8816412 \mathrm{E}-03 & -0.1102051 \mathrm{E}-02 \\ 14 & 0.1679130 & 70.00000 & 69.98643 & 0.1357433 \mathrm{E}-01 & 0.1939191 \mathrm{E}-01 \\ 15 & 0.1438440 & 60.00000 & 59.96845 & 0.3155057 \mathrm{E}-01 & 0.5258428 \mathrm{E}-01 \\ 16 & 0.1198490 & 50.00000 & 49.97653 & 0.2347266 \mathrm{E}-01 & 0.4694531 \mathrm{E}-01 \\ 17 & 0.9587400 \mathrm{E}-01 & 40.00000 & 39.98820 & 0.1179924 \mathrm{E}-01 & 0.2949810 \mathrm{E}-01 \\ 18 & 0.7188700 \mathrm{E}-01 & 30.00000 & 29.99014 & 0.9859604 \mathrm{E}-02 & 0.3286535 \mathrm{E}-01 \\ 19 & 0.4791000 \mathrm{E}-01 & 20.00000 & 19.99152 & 0.8484485 \mathrm{E}-02 & 0.4242242 \mathrm{E}-01 \\ 1 & 0.2398500 \mathrm{E}-01 & 10.00000 & 10.00986 & -0.9858837 \mathrm{E}-02 & -0.9858838 \mathrm{E}-01 \\ 21 & 0.1400086 \mathrm{E}-04 & 0.0000000 \mathrm{E}+00 & 0.4285936 \mathrm{E}-02 & -0.4285936 \mathrm{E}-02 & 0.0000000 \mathrm{E}+00\end{array}$

DEGREE OF POLYNOMIAL 2

COEFFICIENTS

$-0.15594505 E-02 \quad 0.41750199 E+03-0.41152162 E+01$

ESS $=0.4179 \mathrm{E}-02 \quad \mathrm{SD}=0.1524 \mathrm{E}-01 \quad \mathrm{R2}=0.100000 \mathrm{E}+01$ 
. D-45A Rev. $13-18-91$

RESEARCH \& DEVELOPAENT DIVISION INSTRURENT SERVICE LOG

\begin{tabular}{|l|l|l|}
\hline INSTRUMENT STATUS & RÉEA & AS \\
\hline - Certified & \\
\hline Caibrated & & \\
\hline For Ind. Only & & \\
\hline Standardize & & \\
\hline Certified s stand. & & \\
\hline Calibrated S Stand. & & \\
\hline Other & & \\
\hline
\end{tabular}

INSTRUMENT CONDITION

AS RECEIVED (Certified Equipment only)

Meets Manufacturer's Tolerance $N$

Operates within previously defined limits $N$ reviates from $\mathrm{Mfg}$ or previously defined limits $Y$ Inoperative (Describe below) $y$ AS IEFT Limited (Describe below) Meets Manufacturer's Tolerance other (Describe Below)
Babcóck \& Wilcox

a MeDermott company

B\&W Ser. No. 0880555
MANUFACTURER

MODEL

ITEM

MANUF. SER. NO.

PROPERTY NO.

ORDER NO.

\section{SECTION}

LOCATION

SCHEDULED ( $Y-N$ )

RECAL INT. (MOS.)
SENSOTEC

TJE/ 127810

TRANSMITR, PRES

201323

4427-01-117
TECH. PROCEDURE A0015

Babcock Wilcox RED Division certifies that the performance of this instrument was verified using standards whose accuracies are traceable to the National Institute of standards and Technology, an accepted value of a natural physical constant, , a ratio type of calibration technique, or to a consensus standard.

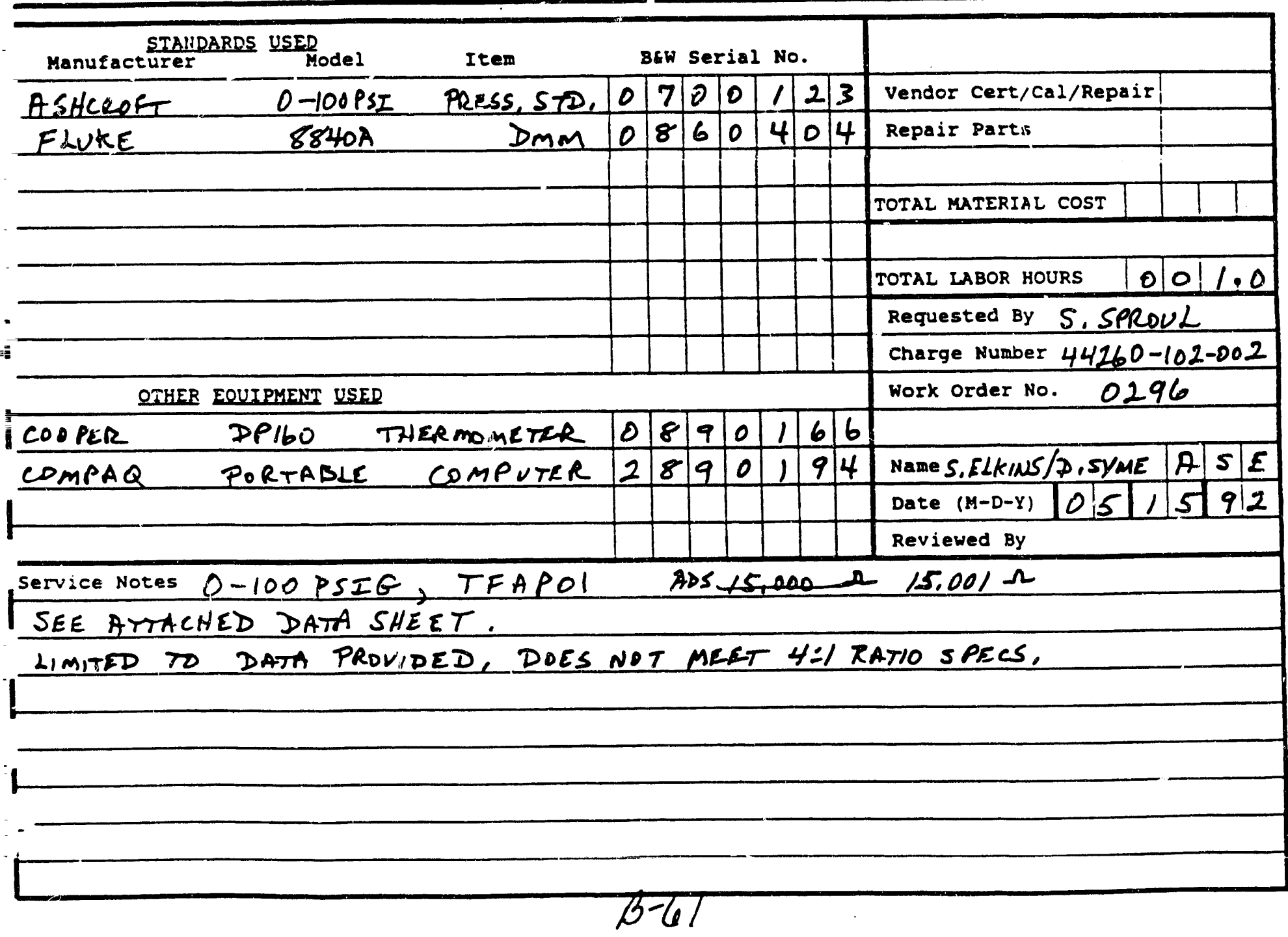


AS FIECEIVED

TRANSMITTER:

SENSOTEC TJE

100 F.SI

TOLERANCE: $0.10 \%$

PRESS SOURCE

\section{ASHCROFT DIGIGAUGE O-100 FSI}

B\&W Ser. No. 0700123

TOLEFANCE : $0.05 \%$

FEADOUT DEV:

E\&W Ser. NO. OB60404

AS LEFT

E\&W S.N. 0880555:

DATE: 15-Maÿ-92

FROCEDURE : ARC-TF-015-0B

FROGRAM: TOO15_OB.03A

NAME: S,ELKINS/D, SYME

Ambient Temperature (F) 70

Facility I. D. Name TOLEFIANCE: $0.015 \%$

VALUE OF CUFIFENT SENSING RESISTOR USED FOR TEST DATA

VALUE OF CURFENT SENSING FESISTOF USED FOF CALIERATION

POWEF SUFFLY VOLTAGE

TOTAL SERIES FESISTANCE

AFFLIED FFESSURE

TRANSMITTEF

F'SI

F'KEESS SOUF'CE

$$
\begin{array}{r}
0.000 \\
10.000 \\
20.000 \\
30.000 \\
40.000 \\
50.000 \\
60.000 \\
70.000 \\
80.000 \\
90.000 \\
100.000 \\
90.000 \\
80.000 \\
70.000 \\
60.000 \\
50.000 \\
40.000 \\
30.000 \\
20.000 \\
10.000 \\
0.000
\end{array}
$$

F'SI

FESISTOF MEASUFIING ACCUFIACY 24

$$
15.001
$$

IDEAL

OUTFUT SIG

MILLIVOLTS

$$
\begin{array}{r}
0.000 \\
10.000 \\
20.000 \\
30.000 \\
40.000 \\
50.000 \\
60.000 \\
70.000 \\
80.000 \\
90.000 \\
100.000 \\
90.000 \\
80.000 \\
70.000 \\
60.000 \\
50.000 \\
40.000 \\
30.000 \\
20.000 \\
10.000 \\
0.000
\end{array}
$$

15.001
IDEAL
OUTFUT SIG
MILLIVOLTS
60.004
84.006
108.007
132.009
156.010
180.012
204.014
228.015
252.017
276.018
300.020
276.018
252.017
228.015
204.014
180.012
156.010
132.009
108.007
84.006
60.004

15.001 OHMS 15.001 OHMS N.A.
$0.17 \%$

**LIMITED**

EFIFIOR \% OUTFUT SIG FULL SIAALE MILLIVOLTS

OUT
OF
TOLEFIANCE

$\begin{array}{rr}59.977 & -0.01 \% \\ 83.799 & -0.09 \% \\ 107.741 & -0.11 \% \\ 131.729 & -0.12 \% \\ 155.770 & -0.10 \% \\ 179.729 & -0.12 \% \\ 20.547 & -0.11 \% \\ 227.833 & -0.08 \% \\ 251.861 & -0.06 \% \\ 275.940 & -0.03 \% \\ 300.044 & 0.01 \% \\ 275.894 & -0.05 \% \\ 251.790 & -0.09 \% \\ 227.704 & -0.13 \% \\ 203.652 & -0.15 \% \\ 179.667 & -0.14 \% \\ 155.664 & -0.14 \% \\ 131.665 & -0.14 \% \\ 107.677 & -0.14 \% \\ 83.763 & -0.10 \% \\ 59.972 & -0.01 \%\end{array}$

$$
\xi=59.925
$$


VOLTAGE(V) OBS. PRESS. CALC. PRESS. DIFF. (PSIA) \& DIFFERENCE (PSIA)

(PSIA)

$\begin{array}{rl}1 & 0.2001764 \mathrm{E}-05 \\ 2 & 0.2382400 \mathrm{E}-01 \\ 3 & 0.4776600 \mathrm{E}-01 \\ 4 & 0.7175400 \mathrm{E}-01 \\ 5 & 0.9579500 \mathrm{E}-01 \\ 6 & 0.1197540 \\ 7 & 0.1437720 \\ 6 & 0.1678580 \\ 5 & 0.1918860 \\ 0 & 0.2159650 \\ 11 & 0.2400690 \\ 2 & 0.2159190 \\ 3 & 0.1918150 \\ 14 & 0.1677290 \\ 1.5 & 0.1436770 \\ .6 & 0.1196920 \\ 17 & 0.9568900 \mathrm{E}-01 \\ 18 & 0.7169000 \mathrm{E}-01 \\ .9 & 0.4770200 \mathrm{E}-01 \\ 1 & 0.2378800 \mathrm{E}-01 \\ 41 & -0.2998236 \mathrm{E}-05\end{array}$

$0.0000000 \mathrm{E}+00$
10.00000
20.00000
30.00000
40.00000
50.00000
60.00000
70.00000
80.00000
90.00000
100.0000
90.00000
80.00000
70.00000
60.00000
50.00000
40.00000
30.00000
20.00000
10.00000
$0.0000000 \mathrm{E}+00$

$0.1448882 \mathrm{E}-01$

9.990581

20.00103

30.01626

40.04062

50.01936

60.01275

70.02600

80.00825

90.00632

100.0109

89.98723

79.97877

69.97239

59.97324

49.99355

39.99644

29.98956

19.97430

9.975517

$0.1239318 \mathrm{E}-01$

\begin{abstract}
EGREE OF POLYNOMTAL
\end{abstract}
3

\title{
SOEFFICIENTS
}

$0.13649825 \mathrm{E}-01$

$0.41912847 E+03-0.15212809 E+02$

$0.18379541 \mathrm{E}+02$

$\overline{\mathrm{N} S S}=0.7324 \mathrm{E}-02$

\author{
$S D=0.2076 \mathrm{E}-01$ \\ $2 * S D=0.4151 \mathrm{E}-01$
}

$\mathbf{R 2}=$

$0.100000 E+01$ 
RD-45A Rev. 11-18-91

RESEARCH \& DEVELOPHENT DIVISION INSTRUNENT SERVICE LOG

\begin{tabular}{|l|l|l|}
\hline \multicolumn{1}{|c|}{ INSTRUMENT STATUS } & R'S & AS \\
\hline 1 Certified & LETS \\
\hline Calibrated & & \\
\hline 3 For Ind. Only & & $\checkmark$ \\
\hline 4 standardize & & \\
\hline 5 Certified stand. & & \\
\hline 6 Calibrated c stand. & & \\
\hline 9 other & & \\
\hline
\end{tabular}

INSTRUMENT CONDITION

AS RECEIVED (Certified Equipment only)

Meets Manufacturer's Tolerance $N$

Operates within previously defined limits $N$ Deviates from Mfg or previously defined limits $Y$ Inoperative (Describe below) Y

AS LEFT Limited (Describe below)

Meets Manufacturer's Tolerance

Other (Describe Below)
BSDYUI

Babcock \& Wilcox

acDermoti company

B\&W Ser. NO. 0880524

MANUFACTURER SENSOTEC

MODEL - 21309-22

ITEM TRANSMITR, D-P

MANUF. SER. NO. 168680

PROPERTY NO.

ORDER NO.

SECTION

LOCATION

SCHEDULED ( $\mathrm{Y}-\mathrm{N})$

RECAL INT. (MOS.)

4427-01-128

\section{6}

115

it Y

12

TECH. PROCEDURE A0015

Babcock \& Wilcox RGD Division certifies that the performance of this instrument was verified using standards whose accuracies are traceable to the National Institute of standards and Technology. an accepted value of a natural physical constant a ratio type of calibration technique, or to a consensus standard.

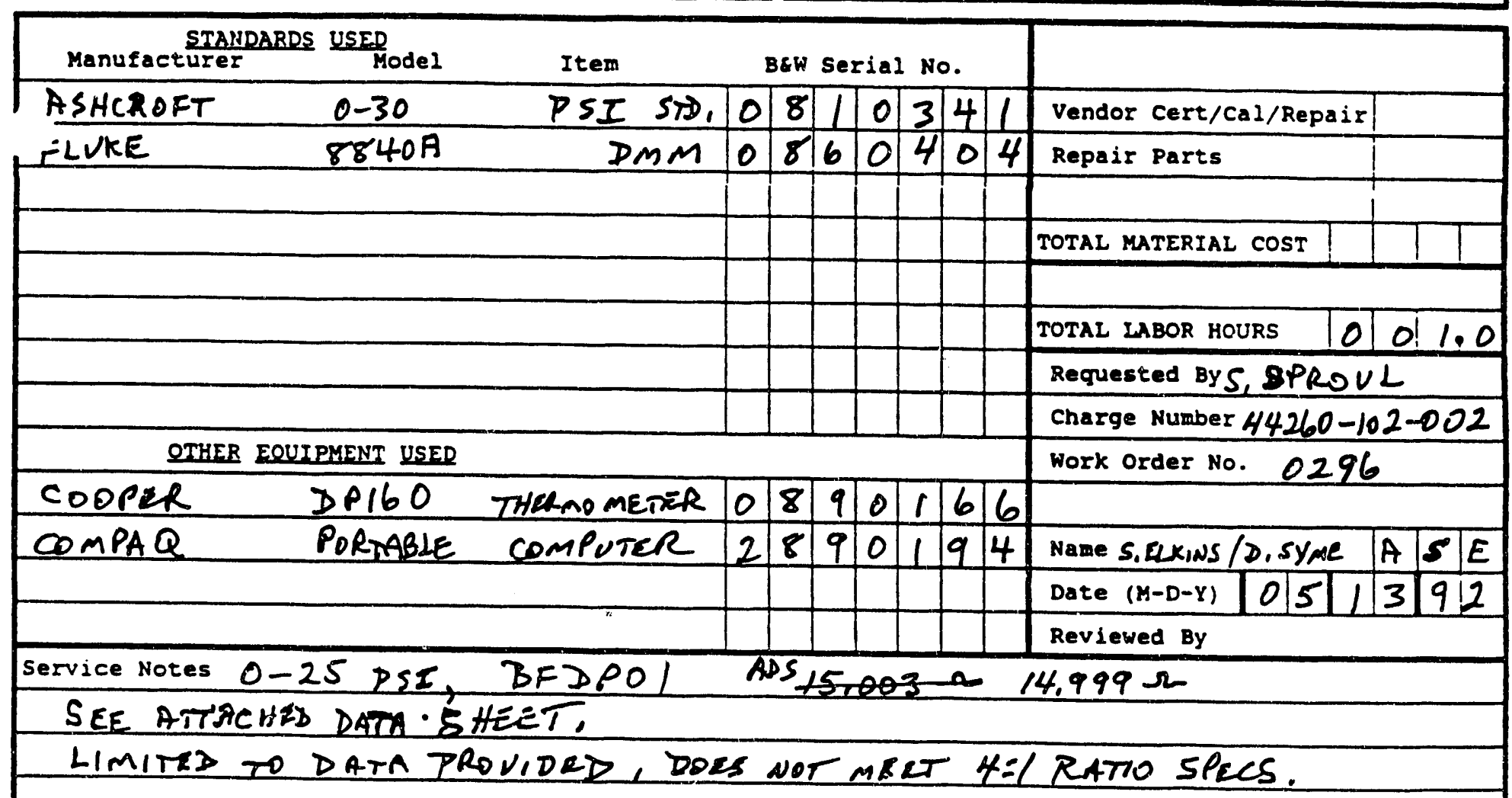


AS FIECEIVED

AS LEFT
BE.W S.N. 0880524.

DATE: 13-May-92

FROCEDUR' : AFIC-TF-O15-OB

FFIOGFIAM: T0015_08.0BA

NAME : S,ELKINS/D, SYmE

Ambient Temperature (F) 73

Facility I. D. Name EFDFO1

B\&W Ser. NO. 0860404

TOLEFIANCE: $0.015 \%$

'ALUE OF CURFENT SENSING FESISTOF' USED FOR TEST DATA JALUE OF CURFEENT SENSING FESISTOF USED FOF CALIBFATION 'OWEF' SUFF'LY VOLTAGE IOTAL SEFIIES FESISTANCE

AF'F'LIED F'FESSURE IFIANSMITTEF' F'FESS SOURICE F'SI F'SI

0.000

2.500

5.000

7.500

10.000

12.500

15.000

17.500

20.000

22.500

25.000

22.500

20.000

17.500

15.000

12.500

10.000

7.500

5.000

2.500

0.000
0.000

2.500

5.000

7.500

10.000

12.500

15.000

17.500

20.000

22.500

25.000

22.500

20.000

17.500

15.000

12.500

10.000

7.500

5.000

2.500

0.000
FIESISTOF: MEASUFINNG ACCUFACY 24

14.999

IDEAL

OUTFUT SIG

MILLIVOLTS

59.996

8.994

107.995

131.991

155.990

179.988

203.986

227.985

251.983

275.982

299.980

275.982

251.983

227.985

205.986

179.988

155.990

131.991

107.993

83.994

59.996

MEASURED

OUTFUT SIG

MILLIVOLTS
CALIBFA ATION ACCUFIACY

**LIMITED**

EFIRIOF \%

FULL SCALE

14.999 DHMS

14.999 OHMS

N.A.

$\begin{array}{rr}59.992 & 0.00 \% \\ 83.961 & -0.01 \% \\ 107.976 & -0.01 \% \\ 131.949 & -0.02 \% \\ 156.010 & 0.01 \% \\ 180.112 & 0.05 \% \\ 204.109 & 0.05 \% \\ 228.117 & 0.06 \% \\ 252.123 & 0.06 \% \\ 276.001 & 0.01 \% \\ 299.957 & -0.01 \% \\ 275.994 & 0.01 \% \\ 252.110 & 0.05 \% \\ 228.114 & 0.05 \% \\ 204.123 & 0.06 \% \\ 180.153 & 0.06 \% \\ 156.122 & 0.06 \% \\ 131.920 & -0.05 \% \\ 107.992 & 0.00 \% \\ 85.951 & -0.02 \% \\ 59.943 & -0.02 \%\end{array}$

$\bar{z}=59.968$ 
CURVEFIT OUTPUT FOR VTAB bfdP01

DATE: 1-JUN-92

TIME: 14:15:07

VOLTAGE (V) OBS. DP(PSI) CALC. DP(PSI) DIFF (PSIA) \& DIFFERENCE

$\begin{array}{rlccrr}1 & 0.2400158 \mathrm{E}-04 & 0.0000000 \mathrm{E}+00 & 0.8583078 \mathrm{E}-02 & -0.8583078 \mathrm{E}-02 & 0.0000000 \mathrm{E}+00 \\ 2 & 0.2399300 \mathrm{E}-01 & 2.500000 & 2.499657 & 0.3434070 \mathrm{E}-03 & 0.1373628 \mathrm{E}-01 \\ 3 & 0.4800800 \mathrm{E}-01 & 5.000000 & 4.996559 & 0.3440609 \mathrm{E}-02 & 0.6881218 \mathrm{E}-01 \\ 4 & 0.7198100 \mathrm{E}-01 & 7.500000 & 7.490142 & 0.9857857 \mathrm{E}-02 & 0.1314381 \\ 5 & 0.9604200 \mathrm{E}-01 & 10.00000 & 9.993930 & 0.6070017 \mathrm{E}-02 & 0.6070017 \mathrm{E}-01 \\ 6 & 0.1201440 & 12.50000 & 12.50304 & -0.3040558 \mathrm{E}-02 & -0.2432446 \mathrm{E}-01 \\ 7 & 0.1441410 & 15.00000 & 15.00227 & -0.2270506 \mathrm{E}-02 & -0.1513670 \mathrm{E}-01 \\ 8 & 0.1681490 & 17.50000 & 17.50369 & -0.3694781 \mathrm{E}-02 & -0.2111303 \mathrm{E}-01 \\ 9 & 0.1921550 & 20.00000 & 20.00596 & -0.5959487 \mathrm{E}-02 & -0.2979743 \mathrm{E}-01 \\ 10 & 0.2160330 & 22.50000 & 22.49592 & 0.4077488 \mathrm{E}-02 & 0.1812217 \mathrm{E}-01 \\ 11 & 0.2399890 & 25.00000 & 24.99506 & 0.4938031 \mathrm{E}-02 & 0.1975213 \mathrm{E}-01 \\ 12 & 0.2160260 & 22.50000 & 22.49519 & 0.4807590 \mathrm{E}-02 & 0.2136707 \mathrm{E}-01 \\ 13 & 0.1921420 & 20.00000 & 20.00460 & -0.4604148 \mathrm{E}-02 & -0.2302074 \mathrm{E}-01 \\ 14 & 0.1681460 & 17.50000 & 17.50338 & -0.3382142 \mathrm{E}-02 & -0.1932652 \mathrm{E}-01 \\ 15 & 0.1441550 & 15.00000 & 15.00373 & -0.3728878 \mathrm{E}-02 & -0.2485919 \mathrm{E}-01 \\ 16 & 0.1201650 & 12.50000 & 12.50523 & -0.5227199 \mathrm{E}-02 & -0.4181759 \mathrm{E}-01 \\ 17 & 0.9615400 \mathrm{E}-01 & 10.00000 & 10.00559 & -0.5587167 \mathrm{E}-02 & -0.5587167 \mathrm{E}-01 \\ 18 & 0.7195200 \mathrm{E}-01 & 7.500000 & 7.487125 & 0.1287496 \mathrm{E}-01 & 0.1716662 \\ 19 & 0.4802400 \mathrm{E}-01 & 5.000000 & 4.998223 & 0.1776697 \mathrm{E}-02 & 0.3553393 \mathrm{E}-01 \\ 20 & 0.2398300 \mathrm{E}-01 & 2.500000 & 2.498617 & 0.1382915 \mathrm{E}-02 & 0.5531659 \mathrm{E}-01 \\ 21 & -0.2499842 \mathrm{E}-04 & 0.0000000 \mathrm{E}+00 & 0.3491629 \mathrm{E}-02 & -0.3491629 \mathrm{E}-02 & 0.0000000 \mathrm{E}+00\end{array}$

( TGREE OF POLYNOMIAL 2

COEFFICIENTS
$0.60891419 \mathrm{E}-02 \quad 0.10390712 \mathrm{E}+03 \quad 0.90993490 \mathrm{E}+00$

ESS $=0.6354 \mathrm{E}-03 \quad \begin{aligned} \mathrm{SD} & =0.5942 \mathrm{E}-02 \\ 2 * \mathrm{SD} & =0.1188 \mathrm{E}-01\end{aligned} \quad \mathrm{R2}=0.999999 \mathrm{E}+00$ 
RD-45A Rev. $11-18-91$

\begin{tabular}{|l|l|l|}
\hline INSTRUMENT STATUS & RF́CD & AS \\
\hline 1 Certified & & \\
\hline$?$ Calibrated & & \\
\hline 3 For Ind. Only & $\checkmark$ & \\
\hline 4 standardize & & \\
\hline 5 Certified \& Stand. & & \\
\hline 6 Calibrated \& Stand. & & \\
\hline 9 other & & \\
\hline
\end{tabular}

INSTRUMENT CONDITION

AS RECEIVED (Certified Equipment only)

Meets Manufacturer's Tolerance $N$

Operates within previously defined limits $N$ Deviates from Mfg or previously defined limits $Y$

\begin{tabular}{|r|r|r|} 
& Inoperative (Describe below) & \\
\hline AS LEFT & Limited (Describe below) & $\checkmark$ \\
\hline Meets Manufacturer's Tolerance & \\
\cline { 2 - 3 } & Other (Describe Below) & \\
\hline
\end{tabular}

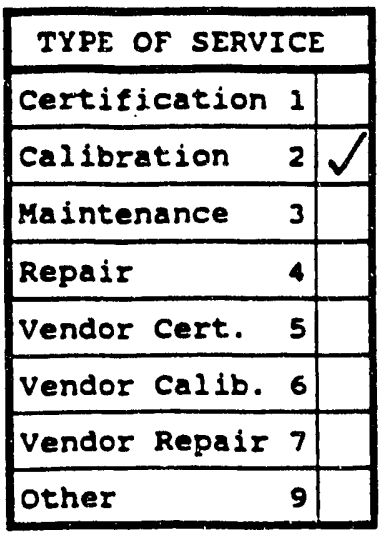

MANUFACTURER

MODEL

ITEM

MANUF. SER. NO.

PROPERTY NO.

ORDER NO.

SECTION

LOCATION

SCHEDULED ( $Y-N)$

RECAL INT. (MOS.) consensus standard.
Bấbcock \& Wilcox

a McDermoti company

B\&W Ser. No. 0880525

SENSOTEC

21309-22

TRANSMITR, D-P

190605

4427-01-129

46

115

$-17 y$

12

\section{TECH. PROCEDURE A0015}

Babcock Wilcox R\&D Division certifies that the performance of this instrument was verified using standards whose accuracies are traceable to the National Institute of Standards and Technology, an accepted value of a natural physical constant, a ratio type of calibration technique, or to a

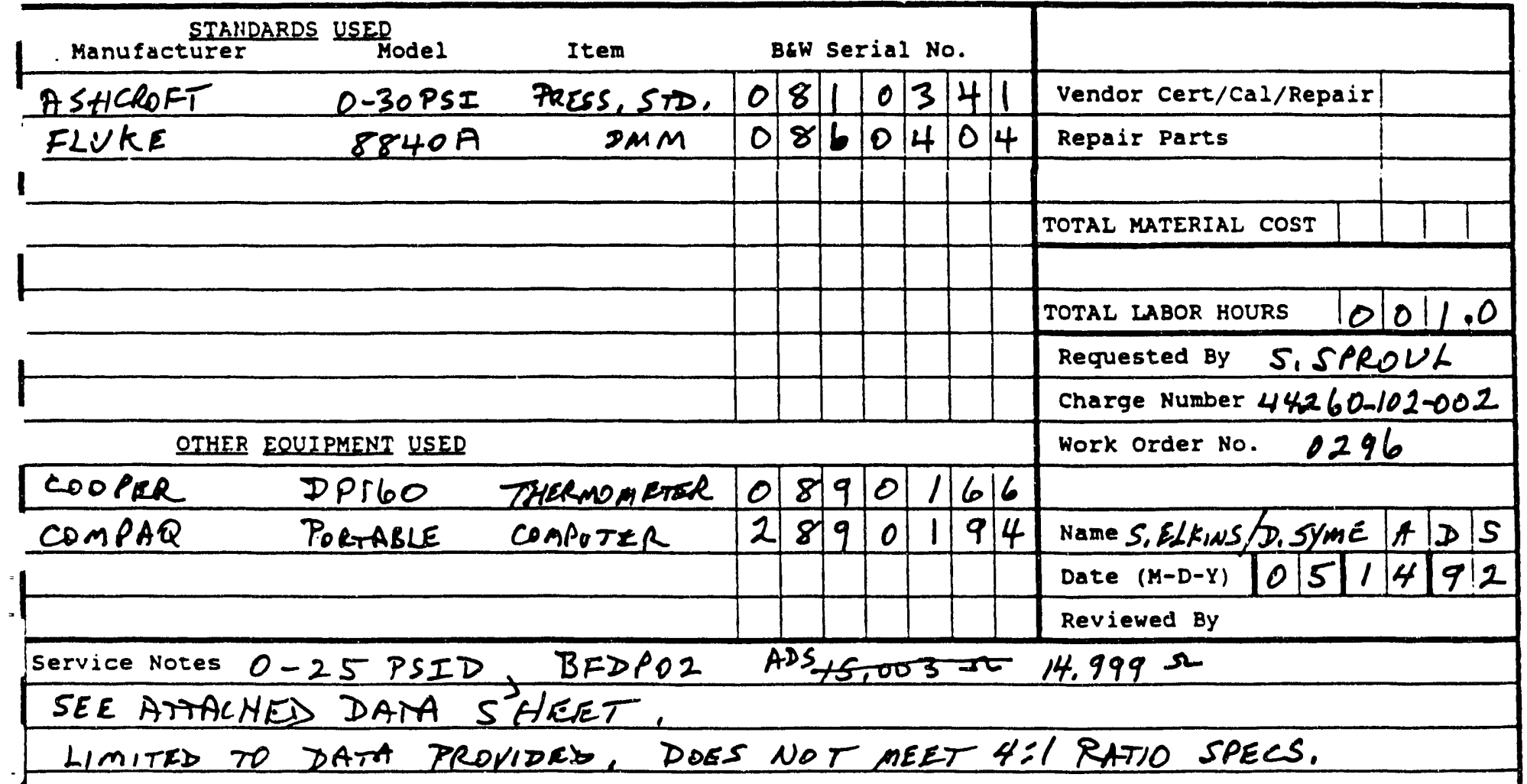


AS FECEIVED

TFANSMITTEF:

SENSOTEC $Z$

25 F'SI

TOLERANCE : $0.25 \%$

FRESS SOUFICE

$$
\begin{aligned}
\text { ASHCFOFT } & \text { DIGIGAUGE } \\
0-30 & \text { FSI } \\
\text { E\&W SER. NO. } & 0810341 \\
\text { TOLEFANCE: } & 0.06 \%
\end{aligned}
$$

FEADOUT DEV:

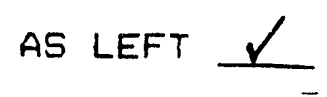

E\&W S.N. OB8O525 :

DATE: 14-May-92

FFIOCEDUFE : AFIC-TF-015-08

FFIOGFIAM: TOO15_08.03A

NAME: S,ELKINS/D.SYME

Ambient Temperature (F) 68

Facility I. D. Name EFDFO?

\section{E\&W Ser. No. 0860404 \\ TOLEFANCE : $0.015 \%$}

VALUE OF CUFRENT SENSING FIESISTOF USED FOF TEST DATA

VALUE OF CUFIFENT SENSING FESISTOF USED FOF CALI IBFIATION

FOWEF SUFF'Y VOLTAGE

TOTAL SEFIES RESISTANCE

AFF'LIED F'RESSURE

TFIANSMITTEF:

F'SI

F'FESS SOUF'CE

0.000

2.500

5.000

7.500

10.000

12.500

15.000

17.500

20.000

22.500

25.000

22.500

20.000

17.500

15.000

12.500

10.000

7.500

5.000

2.500

0.000
F'S I

FESISTOF: MEASUFIING ACCUFIACY 24

14.999

IDEAL

OUTFUT SIG

MILLIVOLTS

0.000

2.500

5.000

7.500

10.000

12.500

15.000

17.500

20.000

22.500

25.000

22.500

20.000

17.500

15.000

12.500

10.000

7.500

5.000

2.500

0.000
59.996

83.994

107.993

131.991

155.990

179.988

203.986

227.985

251.983

275.982

299.980

275.982

251.983

227.985

203.986

179.988

155.990

131.991

107.995

83.994

59.996

$\begin{array}{cl}\text { CALIEFIATION ACTUFAACY } \\ \text { **LIMITED** } \\ \text { MEASUFED } & \text { EFFIOF \% } \\ \text { OUTFUT SIG } & \text { FULL SCALE } \\ \text { MILLIVOLTS } & \end{array}$

14.999 OHMS

14.999 OHMS

N.A.

ACPUFIACY

EFFIOF \% MILLIVOLTS

$\begin{array}{rr}59.996 & 0.00 \% \\ 84.094 & 0.04 \% \\ 108.092 & 0.04 \% \\ 152.147 & 0.06 \% \\ 156.251 & 0.11 \% \\ 180.015 & 0.14 \% \\ 204.316 & 0.14 \% \\ 228.240 & 0.11 \% \\ 252.196 & 0.09 \% \\ 276.061 & 0.08 \% \\ 299.888 & -0.04 \% \\ 276.000 & 0.01 \% \\ 252.155 & 0.07 \% \\ 228.202 & 0.09 \% \\ 204.272 & 0.12 \% \\ 180.316 & 0.14 \% \\ 156.266 & 0.12 \% \\ 152.175 & 0.08 \% \\ 108.120 & 0.05 \% \\ 84.084 & 0.04 \% \\ 60.009 & 0.01 \%\end{array}$

$0.5 \%$

OUT

OF

$$
\bar{z}=60.00 \%
$$


CURVEFIT OUTPUT FOR VTAB bfdp02'

DATE : 20-MAY -92

TIME: $16: 32: 13$

VOLTAGE(V) OBS. DP(PSI) CALC. DP(PSI) DIFF (PSIA) \& DIFFERENCE

$\begin{array}{llllrr}1 & -0.7001243 \mathrm{E}-05 & 0.0000000 \mathrm{E}+00 & 0.3832620 \mathrm{E}-02 & -0.3832620 \mathrm{E}-02 & 0.0000000 \mathrm{E}+00 \\ 2 & 0.2409100 \mathrm{E}-01 & 2.500000 & 2.501942 & -0.1941508 \mathrm{E}-02 & -0.7766030 \mathrm{E}-01 \\ 3 & 0.4808900 \mathrm{E}-01 & 5.000000 & 4.992438 & 0.7561557 \mathrm{E}-02 & 0.1512311 \\ 4 & 0.7214400 \mathrm{E}-01 & 7.500000 & 7.491609 & 0.8390619 \mathrm{E}-02 & 0.1118749 \\ 5 & 0.9624800 \mathrm{E}-01 & 10.00000 & 9.998641 & 0.1358578 \mathrm{E}-02 & 0.1358578 \mathrm{E}-01 \\ 6 & 0.1203120 & 12.50000 & 12.50428 & -0.4279320 \mathrm{E}-02 & -0.3423456 \mathrm{E}-01 \\ 7 & 0.1443130 & 15.00000 & 15.00611 & -0.6110485 \mathrm{E}-02 & -0.4073657 \mathrm{E}-01 \\ 8 & 0.1682370 & 17.50000 & 17.50265 & -0.2651525 \mathrm{E}-02 & -0.1515157 \mathrm{E}-01 \\ 9 & 0.1921930 & 20.00000 & 20.00527 & -0.5269195 \mathrm{E}-02 & -0.2634598 \mathrm{E}-01 \\ 0 & 0.2160580 & 22.50000 & 22.50110 & -0.1103922 \mathrm{E}-02 & -0.4906321 \mathrm{E}-02 \\ 1 & 0.2398850 & 25.00000 & 24.99568 & 0.4323538 \mathrm{E}-02 & 0.1729415 \mathrm{E}-01 \\ 2 & 0.2159970 & 22.50000 & 22.49472 & 0.5279007 \mathrm{E}-02 & 0.2346225 \mathrm{E}-01 \\ 3 & 0.1921520 & 20.00000 & 20.00098 & -0.9836976 \mathrm{E}-03 & -0.4918488 \mathrm{E}-02 \\ 4 & 0.1681990 & 17.50000 & 17.49868 & 0.1316055 \mathrm{E}-02 & 0.7520317 \mathrm{E}-02 \\ 5 & 0.1442690 & 15.00000 & 15.00152 & -0.1521470 \mathrm{E}-02 & -0.1014313 \mathrm{E}-01 \\ 6 & 0.1203130 & 12.50000 & 12.50438 & -0.4383501 \mathrm{E}-02 & -0.3506801 \mathrm{E}-01 \\ 7 & 0.9626300 \mathrm{E}-01 & 10.00000 & 10.00020 & -0.2024197 \mathrm{E}-03 & -0.2024197 \mathrm{E}-02 \\ 18 & 0.7217200 \mathrm{E}-01 & 7.500000 & 7.494520 & 0.5479977 \mathrm{E}-02 & 0.7306636 \mathrm{E}-01 \\ 9 & 0.4811700 \mathrm{E}-01 & 5.000000 & 4.995346 & 0.4654130 \mathrm{E}-02 & 0.9308259 \mathrm{E}-01 \\ 20 & 0.2408100 \mathrm{E}-01 & 2.500000 & 2.500904 & -0.9042870 \mathrm{E}-03 & -0.3617148 \mathrm{E}-01 \\ 21 & 0.5998757 \mathrm{E}-05 & 0.0000000 \mathrm{E}+00 & 0.5179512 \mathrm{E}-02 & -0.5179512 \mathrm{E}-02 & 0.0000000 \mathrm{E}+00\end{array}$

GREE OF POLYNOMIAL 2

:OEFFICIENTS

$0.45579981 E-02 \quad 0.10360710 E+03 \quad 0.23864897 E+01$

TSS $=0.3897 \mathrm{E}-03 \quad \mathrm{SD}=0.4653 \mathrm{E}-02 \quad \mathrm{R2}=0.100000 \mathrm{E}+01$ 


\begin{tabular}{|l|l|l|}
\hline \multicolumn{1}{|c|}{ INSTRUMENT STATUS } & Ás & ASS \\
\hline 1 Certified & & \\
\hline Calibrated & & $\checkmark$ \\
\hline 3 For Ind. Only & $\checkmark$ & \\
\hline 4 standardize & & \\
\hline 5 certified s stand. & & \\
\hline 6 calibrated c stand. & & \\
\hline 9 other & & \\
\hline
\end{tabular}

INSTRUMENT CONDITION

AS RECEIVED (Certified Equipment only)

Meets Manufacturer's Tolerance $N$ Operates within previously defined limits $N$ Deviates from Mfg or previously defined limits $Y$

AS LEFT Limited (Describe below)

Meets Manufacturer's Tolerance

other (Describe Below)
Babcock \& Wilcox

a McDermost company

B\&W Ser. No. 0880531

MANUFACTURER SENSOTEC

MODEL

ITEM

PROPERTY NO.

ORDER NO.

4427-01-135

$\begin{array}{lc}\text { SECTION } & 46 \\ \text { LOCATION } & 115 \\ \text { SCHEDUIED (Y-N) } & -\$ 4 \\ \text { RECAL INT.(MOS.) } & 12\end{array}$

TECH. PROCEDURE A0015

Babcock o Wilcox RGD Division certifies that the performance of this instrument was verified using standards whose accuracies are traceable to the National Institute of standards and Technology. an accepted value of a natural physlcal constant, - ratio type of calibration technique, or to a consensus standard.

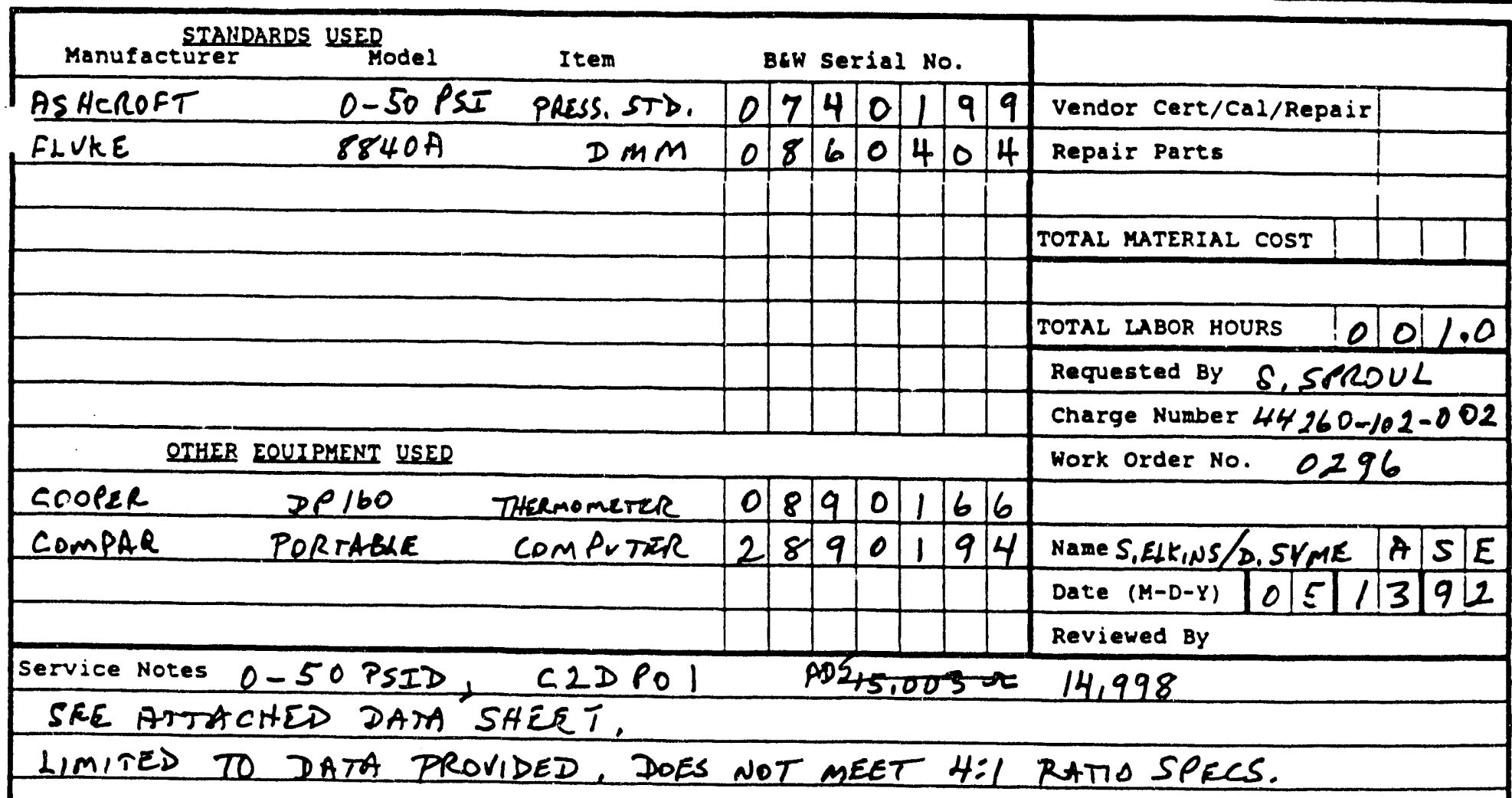


AS REECEIVED

IFIANSMITTER:

SENSOTEC $Z$

50 F'SI

TOL.EFANCE: $0.25 \%$

'RESS SOUFICE

$$
\begin{gathered}
\text { ASHCFOFT DIGIGAUGE } \\
\text { O-50 FSI }
\end{gathered}
$$$$
\text { B\&W Ser. NO. } 0740199
$$$$
\text { TOLEFANCE : } 0.05 \%
$$

\section{FLUKSE 8840A \\ B\&W Ser. No. 0860404 \\ TOLEFIANCE : $0.015 \%$ \\ IEADOUT DEV:}

AS LEFT $\mathscr{d}$

ERW S.N. O8BO5S1: " DATE: 15-May-192 FFOCEDURE : ARCC-TF-015-OB FFIOGFIM: TOO15_08.0IA

NAME: S, EXRLAS S. SYME

\author{
Ambient Temperature (F) 71 \\ Facility I. D. Name CaDFO1
}

IALUE OF CUFFENT SENSING FESISTOF USED FOF TEST DATA
JALUE OF CUFFENT SENSING FESISTOF USED FOR CALIEFATION JALUE OF CUFFENT SENSING FESISTOF USED FOR CALIBRATION
FESISTOR MEASUFING ACCUFACY

'OWEF' SUF'F'LY VOLTAGE IOTAL SEFIES FIESISTANCE

AF'F'LIED F'RESSURE IFANSMI TTEF: F'S I

$\begin{array}{rr}0.000 & 0.000 \\ 5.000 & 5.000 \\ 10.000 & 10.000 \\ 15.000 & 15.000 \\ 20.000 & 20.000 \\ 25.000 & 25.000 \\ 50.000 & 50.000 \\ 35.000 & 35.000 \\ 40.000 & 40.000 \\ 45.000 & 45.000 \\ 50.000 & 50.000 \\ 45.000 & 45.000 \\ 40.000 & 40.000 \\ 35.000 & 35.000 \\ 30.000 & 30.000 \\ 25.000 & 25.000 \\ 20.000 & 20.000 \\ 15.000 & 15.000 \\ 10.000 & 10.000 \\ 5.000 & 5.000 \\ 0.000 & 0.000\end{array}$
24

14.998

IDEAL OUTFUT SIG
MILLIVOLTS

59.992 8.989 107.986 151.982 155.979 179.976 203.975 227.970 251.966 275.963 299.960 275.96 .5 251.966 227.970 20.975 179.976 155.979 131.982 107.986 8.989 59.992

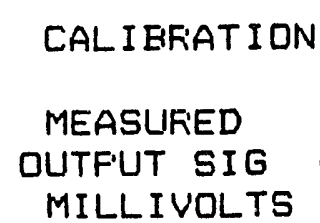

14.598 OHMS 14.998 OHMS N.A.

$\begin{array}{lc}\text { ACCUFACY } & 0.32 \% \\ \text { * *LIMITED** } & \\ \text { ERFIOR } \% & \text { OUT } \\ \text { FULL SCALE } & \text { OF } \\ & \text { TOLERIANCE }\end{array}$

$\begin{array}{rr}60.009 & 0.01 \% \\ 83.864 & -0.05 \% \\ 107.825 & -0.07 \% \\ 151.892 & -0.04 \% \\ 155.964 & -0.01 \% \\ 180.000 & 0.01 \% \\ 203.910 & -0.05 \% \\ 227.961 & 0.00 \% \\ 251.894 & -0.03 \% \\ 275.955 & 0.00 \% \\ 299.919 & -0.02 \% \\ 275.990 & 0.01 \% \\ 251.974 & 0.00 \% \\ 227.985 & 0.01 \% \\ 203.930 & -0.02 \% \\ 179.978 & 0.00 \% \\ 155.990 & 0.00 \% \\ 131.912 & -0.05 \% \\ 107.850 & -0.06 \% \\ 83.893 & -0.04 \% \\ 60.009 & 0.01 \%\end{array}$

$\bar{z}=60.009$ 
CURVEFIT OUTPUT FOR VTAB C2AP01/

DATE: 20-MAY-92

TIME: $16: 20: 07$

VOLTAGE (V) OBS. DP(PSI) CALC. DP(PSI) DIFF (PSIA) \& DIFFERENCE

$\begin{array}{rlcccc}1 & 0.1039743 \mathrm{E}-08 & 0.0000000 \mathrm{E}+00 & 0.1835668 \mathrm{E}-01 & -0.1835668 \mathrm{E}-01 & 0.0000000 \mathrm{E}+00 \\ 2 & 0.2385500 \mathrm{E}-01 & 5.000000 & 4.987400 & 0.1260039 \mathrm{E}-01 & 0.2520078 \\ 3 & 0.4781400 \mathrm{E}-01 & 10.00000 & 9.978106 & 0.2189406 \mathrm{E}-01 & 0.2189406 \\ 4 & 0.7188300 \mathrm{E}-01 & 15.00000 & 14.99173 & 0.8274508 \mathrm{E}-02 & 0.5516339 \mathrm{E}-01 \\ 5 & 0.9595500 \mathrm{E}-01 & 20.00000 & 20.00597 & -0.5969945 \mathrm{E}-02 & -0.2984972 \mathrm{E}-01 \\ 6 & 0.1199910 & 25.00000 & 25.01272 & -0.1271553 \mathrm{E}-01 & -0.5086211 \mathrm{E}-01 \\ 7 & 0.1439010 & 30.00000 & 29.99322 & 0.6784933 \mathrm{E}-02 & 0.2261644 \mathrm{E}-01 \\ 8 & 0.1679520 & 35.00000 & 35.00309 & -0.3085179 \mathrm{E}-02 & -0.8814798 \mathrm{E}-02 \\ 9 & 0.1918850 & 40.00000 & 39.98338 & 0.1162434 \mathrm{E}-01 & 0.2906084 \mathrm{E}-01 \\ 10 & 0.2159460 & 45.00000 & 45.00033 & -0.3287941 \mathrm{E}-03 & -0.7306535 \mathrm{E}-03 \\ 11 & 0.2399100 & 50.00000 & 49.99208 & 0.7923363 \mathrm{E}-02 & 0.1584673 \mathrm{E}-01 \\ 12 & 0.2159810 & 45.00000 & 45.00762 & -0.7619362 \mathrm{E}-02 & -0.1693192 \mathrm{E}-01 \\ 13 & 0.1919650 & 40.00000 & 40.00504 & -0.5039818 \mathrm{E}-02 & -0.1259954 \mathrm{E}-01 \\ 14 & 0.1679740 & 35.00000 & 35.00767 & -0.7667822 \mathrm{E}-02 & -0.2190806 \mathrm{E}-01 \\ 15 & 0.1439210 & 30.00000 & 29.99738 & 0.2618895 \mathrm{E}-02 & 0.8729649 \mathrm{E}-02 \\ 16 & 0.1199690 & 25.00000 & 25.00813 & -0.8132886 \mathrm{E}-02 & -0.3253154 \mathrm{E}-01 \\ 17 & 0.9598100 \mathrm{E}-01 & 20.00000 & 20.01139 & -0.1138580 \mathrm{E}-01 & -0.5692898 \mathrm{E}-01 \\ 18 & 0.7190300 \mathrm{E}-01 & 15.00000 & 14.99589 & 0.4108469 \mathrm{E}-02 & 0.2738980 \mathrm{E}-01 \\ 19 & 0.4784100 \mathrm{E}-01 & 10.00000 & 9.983730 & 0.1626990 \mathrm{E}-01 & 0.1626990 \\ 20 & 0.2388400 \mathrm{E}-01 & 5.000000 & 4.993440 & 0.6559633 \mathrm{E}-02 & 0.1311927 \\ 21 & 0.1039743 \mathrm{E}-08 & 0.0000000 \mathrm{E}+00 & 0.1835668 \mathrm{E}-01 & -0.1835668 \mathrm{E}-01 & 0.0000000 \mathrm{E}+00\end{array}$

( :GREE OF POLYNOMIAL 1

COEFFICIENTS

$0.18356463 E-01 \quad 0.20830195 E+03$

ESS $=0.2501 E-02 \quad$ SD $=0.1147 E-01 \quad R 2=0.999999 E+00$

$2 \star S D=0.2295 \mathrm{E}-01$ 
ID-45A Rev. $21-18-91$

RESEARCH \& DEVELOPMENT DIVISION INSTRUMENT SERVICE LOG

\begin{tabular}{|l|l|l|}
\hline INSTRUMENT STATUS & \multicolumn{1}{|c|}{ PÉCE } & AS \\
\hline Certified & & \\
\hline Calibrated & & \\
\hline For Ind. Only & & \\
\hline standardize & & \\
\hline Certified stand. & & \\
\hline Calibrated stand. & & \\
\hline Other & & \\
\hline
\end{tabular}

INSTRUMENT CONDITION

AS RECEIVED (Certified Equipment only)

Meets Manufacturer's Tolerance $N$

Operates within previously defined limits $N$ Deviates from $x$ g or previously defined limits $Y$ Inoperative (Describe below) $Y$

AS LEFT Limited (Describe below)
Meets Manufacturer's Tolerance

other (Describe Below)

Babcock \& Wilcox

- McDermoti company

B\&W Ser. No. 0880522

\section{MANUFACTURER \\ MODEL \\ ITEM \\ MANUF. SER. NO. \\ PROPERTY NO. \\ SENSOTEC \\ TRANSMITR, D-P \\ 195043 \\ 4427-01-126}

ORDER NO.

$\begin{array}{lc}\text { SECTION } & 46 \\ \text { LOCATION } & 115 \\ \text { SCHEDULED(Y-N) } & -14-Y \\ \text { RECAL INT.(MOS.) } & 12\end{array}$

TECH. PROCEDURE A0015

Babcock Wilcox RED Division certifies that the performance of this instrument was verified using standards whose accuracies are traceable to the National Institute of Standards and Technology, an accepted value of a natural physical constant, a ratio type of calibration technique, or to a consensus standard.

\begin{tabular}{|c|c|c|c|}
\hline Manufact $\frac{\text { STAIIDARDS }}{u r e r}$ & $\frac{\text { USED }}{\text { Model }}$ & Item & \\
\hline ASHCROFT & $0-10$ PSI & PRESS, STD, & 0 \\
\hline FLUKE & $8840 A$ & $D M M$ & 0 \\
\hline
\end{tabular}

BEW Serial No.

8110020

\begin{tabular}{|l|l|l|l|l|l|}
\hline 8 & 1 & 0 & 2 & 2 & 5 \\
\hline 8 & 5 & 0 & 4 & 5 & 3
\end{tabular}

Vendor Cert/Cal/Repair

\begin{tabular}{llllll|l|l|l} 
& & & & & & \\
\hline & & & & & & & & \\
\hline & & & & & & & \\
\hline & & & & & & & \\
\hline
\end{tabular}

Repair Parts

TOTAL MATERIAL COST

TOTAL LABOR HOURS

01012.5

Requested BY ScT SPROVL

Charge Number 44260-102-002

work order No. 0381

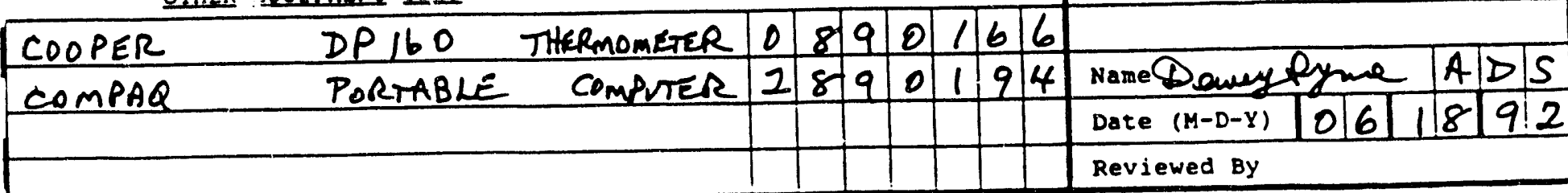

Service Notes 0-10 PSI C2DPO2, REPLACES 0880519, $14.999 \Omega$

SEE ATTACHED DATA SHEET. LIMITED DUE TO NOT MEETING

H:I RATTO SPECS. 
AS FIECEIVED

TFANSMITTEF: :

SENSOTEC $z$

10 F'SI

TOLEFANCE : $0.25 \%$

F'RESS SOUFICE

$$
\begin{aligned}
\text { ASHCFIOFT } & \text { DIGIGAUGE } \\
0-10 & \text { FSI } \\
\text { H\&W SER. NO. } & 0810225 \\
\text { TOLEFIANCE: } & 0.05 \%
\end{aligned}
$$

FEADOUT DEV:

FLUHE 8840A

E\&W Ser. No. 085045S

TOLEFIANCE: $0.015 \%$

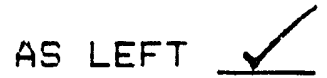

BEW S.N. O880522

DATE: $18-J u n-92$

F'FIOCEDUFE : AFIC-TF-015-08

FFIOGFAM: TOO15 08.03A

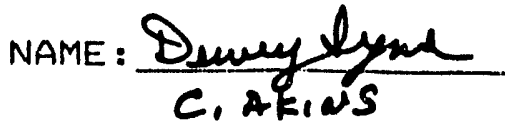

Ambient Temperature (F) 70

Facility I. D. Name CZDFOZ

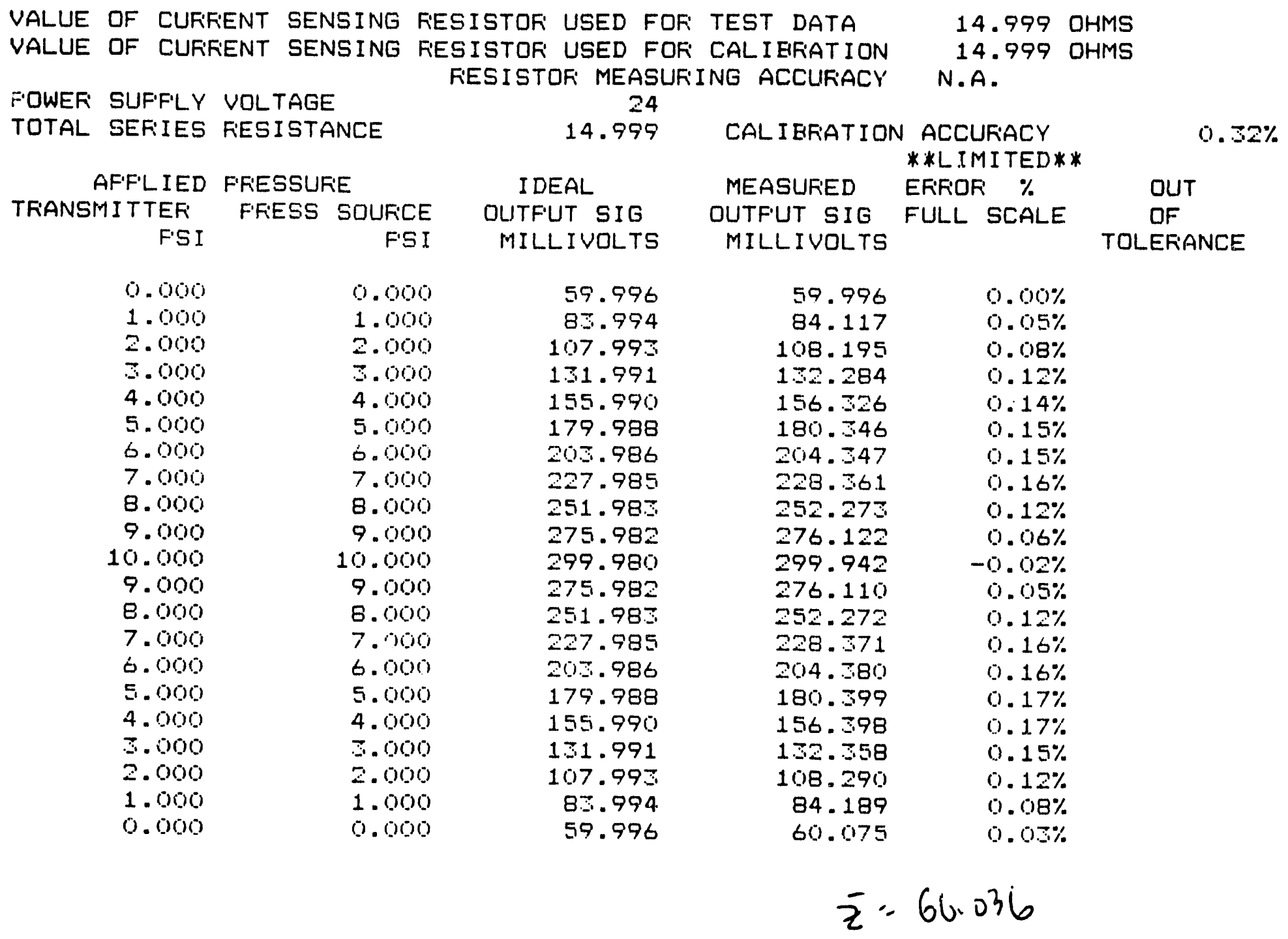


VOLTAGE(V) OBS. DP(PSI) CALC. DP(PSI) DIFF (PSIA) DIFFERENCE

$\begin{array}{rccccc}1 & -0.3999986 \mathrm{E}-04 & 0.0000000 \mathrm{E}+00 & -0.7997043 \mathrm{E}-03 & 0.7997043 \mathrm{E}-03 & 0.0000000 \mathrm{E}+00 \\ 2 & 0.2408100 \mathrm{E}-01 & 1.000000 & 0.9985279 & 0.1472057 \mathrm{E}-02 & 0.1472057 \\ 3 & 0.4815900 \mathrm{E}-01 & 2.000000 & 1.997373 & 0.2626740 \mathrm{E}-02 & 0.1313370 \\ 4 & 0.7224800 \mathrm{E}-01 & 3.000000 & 2.997974 & 0.2026220 \mathrm{E}-02 & 0.6754067 \mathrm{E}-01 \\ 5 & 0.9629000 \mathrm{E}-01 & 4.000000 & 3.997917 & 0.2082592 \mathrm{E}-02 & 0.5206481 \mathrm{E}-01 \\ 6 & 0.1203100 & 5.000000 & 4.998238 & 0.1761641 \mathrm{E}-02 & 0.3523282 \mathrm{E}-01 \\ 7 & 0.1443110 & 6.000000 & 5.999058 & 0.9417380 \mathrm{E}-03 & 0.1569563 \mathrm{E}-01 \\ 8 & 0.1683250 & 7.000000 & 7.001711 & -0.1711004 \mathrm{E}-02 & -0.2444292 \mathrm{E}-01 \\ 9 & 0.1922370 & 8.000000 & 8.001388 & -0.1387843 \mathrm{E}-02 & -0.1734804 \mathrm{E}-01 \\ 10 & 0.2160860 & 9.000000 & 8.999706 & 0.2940264 \mathrm{E}-03 & 0.3266960 \mathrm{E}-02 \\ 11 & 0.2399060 & 10.00000 & 9.998081 & 0.1918737 \mathrm{E}-02 & 0.1918737 \mathrm{E}-01 \\ .2 & 0.2160740 & 9.000000 & 8.999203 & 0.7966661 \mathrm{E}-03 & 0.8851846 \mathrm{E}-02 \\ 3 & 0.1922350 & 8.000000 & 8.001346 & -0.1346010 \mathrm{E}-02 & -0.1682512 \mathrm{E}-01 \\ 14 & 0.1683350 & 7.000000 & 7.002129 & -0.2128802 \mathrm{E}-02 & -0.3041145 \mathrm{E}-01 \\ 15 & 0.1443440 & 6.000000 & 6.000435 & -0.4352199 \mathrm{E}-03 & -0.7253665 \mathrm{E}-02 \\ 16 & 0.1203630 & 5.000000 & 5.000447 & -0.4469898 \mathrm{E}-03 & -0.8939796 \mathrm{E}-02 \\ 17 & 0.9636200 \mathrm{E}-01 & 4.000000 & 4.000914 & -0.9139415 \mathrm{E}-03 & -0.2284854 \mathrm{E}-01 \\ 26 & 0.7232200 \mathrm{E}-01 & 3.000000 & 3.001050 & -0.1049568 \mathrm{E}-02 & -0.3498559 \mathrm{E}-01 \\ 19 & 0.4825400 \mathrm{E}-01 & 2.000000 & 2.001317 & -0.1316786 \mathrm{E}-02 & -0.6583928 \mathrm{E}-01 \\ 20 & 0.2415300 \mathrm{E}-01 & 1.000000 & 1.001513 & -0.1512837 \mathrm{E}-02 & -0.1512837 \\ 21 & 0.3900014 \mathrm{E}-04 & 0.0000000 \mathrm{E}+00 & 0.2471122 \mathrm{E}-02 & -0.2471122 \mathrm{E}-02 & 0.0000000 \mathrm{E}+00\end{array}$

EGREE OF POLYNOMIAL 2

TOEFFICIENTS

$0.85640284 E-03 \quad 0.41402864 E+02 \quad 0.11194405 E+01$

ESS $=0.5019 \mathrm{E}-04 \quad \mathrm{SD}=0.1670 \mathrm{E}-02 \quad \mathrm{R} 2=0.100000 \mathrm{E}+01$

$2 * S D=0.3340 E-02$ 
RD-45A Rev. $12-18-91$

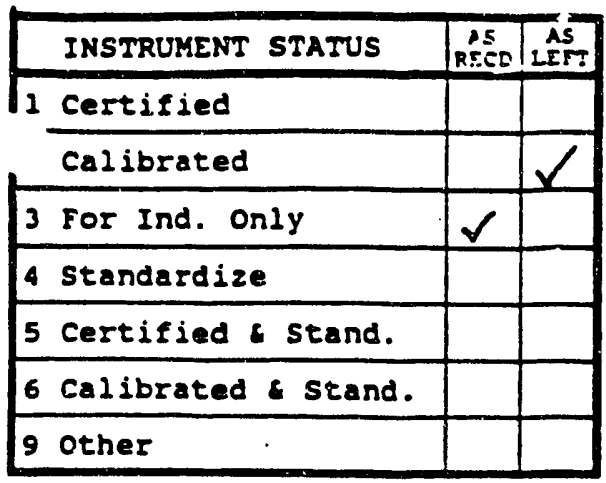

INSTRUMENT CONDITION

AS RECEIVED (Certified Equipment Only)

Meets Manusacturer's Tolerance $N$

Operates within previously defined limits $N$ Deviates from Mfg or previously defined limits $X$

\begin{tabular}{r|r|} 
Inoperative (Describe below) & \\
AS LEFT Limited (Describe below) \\
Meets Manufacturer's Tolerance \\
Other (Describe Below)
\end{tabular}

Babcock \& Wilcox

acDermote company

B\&W Ser. No. 0D80532

MANUFACIURER

MODEL

ITEY

MANUF, SER. NO.

PROPERTY NO.

ORDER NO.

SENSOIEC

21311-03

TRANSMITR, D-P

197074

4427-01-136

\section{SECTION}

IOCATION

SCHEDUIED ( $\mathrm{Y}-\mathrm{N})$

RECAL INT.(MOS.)

46

115

ti $y$

12

\section{TECH. PROCEDURE}

Babcock \& Wilcox RED Division certifies that the performance of this instrument was verified using standards whose accuracies are tracesble to the National Institute of standards and Technology. an accepted value of a natural physical constant, a ratio type of calibration technique, or to a consensus standard.

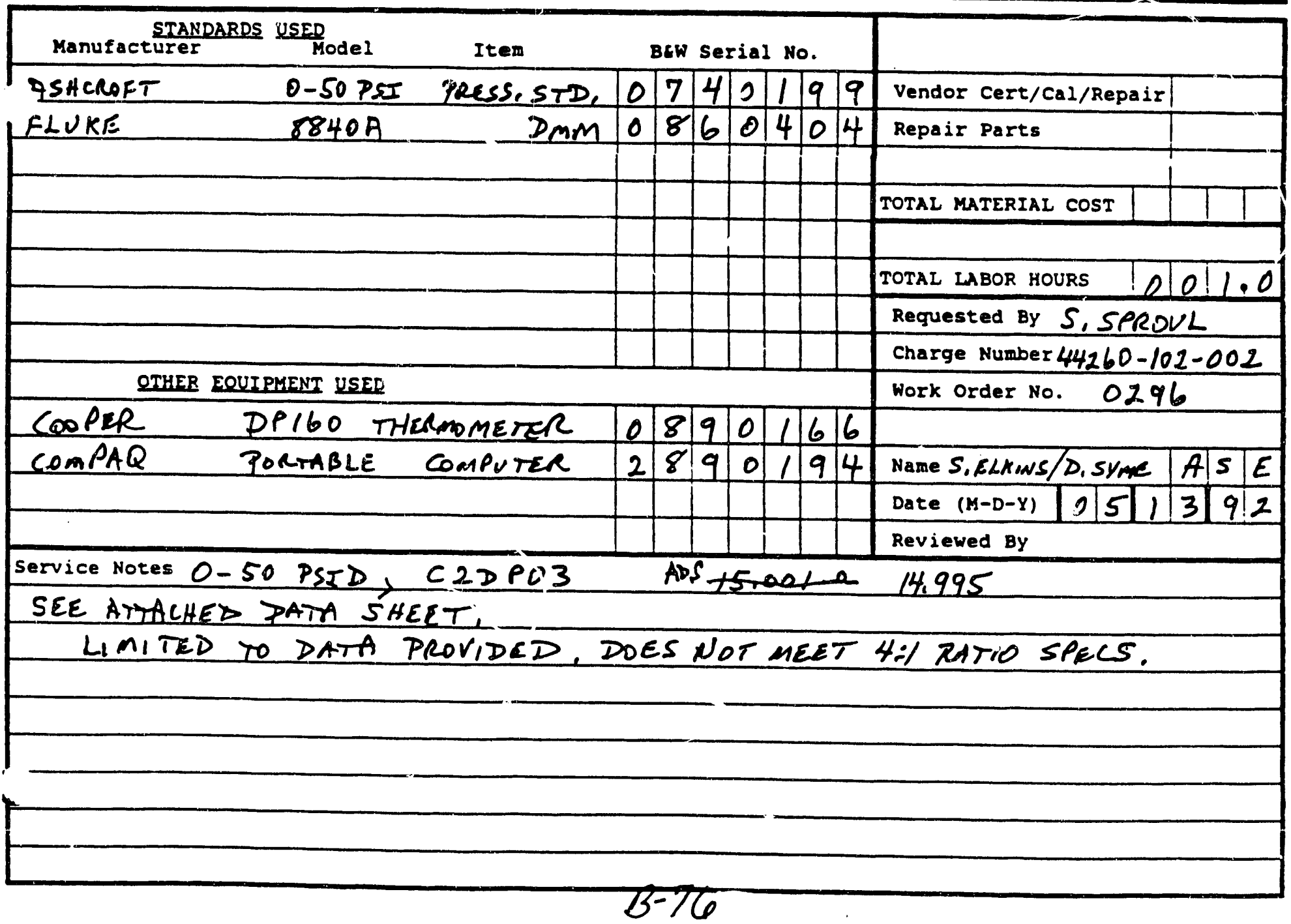


AS RECEIVED

$$
\text { SENSOTEC } Z
$$

$50 \mathrm{~F} S \mathrm{SI}$

TOLERANCE: $0.25 \%$

ASHCROFT DIGIGAUGE

O-50 F.SI

E\&W Ser. No. 0740199

TOLEFIANCE : $0.05 \%$

\section{FLUIKE $8840 \mathrm{~A}$}

B\&W Ser. NO. O860404

TOLEFIANCE: $0.015 \%$
AS LEFT

B\&W S.N. 0880532.

DATE: 15-May-92

FFIOCEDUFE : ARIC-TF-015-08

FFIDGRAM: T0015_08.03A

NAME: S.ELKINS \$. SYME

Ambient Temperature (F) 72

Facility I. D. Name C2DFOS
VALUE OF CURFENT SENSING FESISTOF USED FOFi TEST DATA ' $A$ ALUE OF CUFRENT SENSING FIESISTOF USED FOF CALIBFATION POWEF SUF'FLY VOLTAGE TOTAL SEFIIES RESISTANCE

AFFLIED FRESSURE TFIANSMITTEF: FSI FFESS SOURCE FSI

$\begin{array}{rr}0.000 & 0.000 \\ 5.000 & 5.000 \\ 10.000 & 10.000 \\ 15.000 & 15.000 \\ 20.000 & 20.000 \\ 25.000 & 25.000 \\ 30.000 & 30.000 \\ 55.000 & 35.000 \\ 40.000 & 40.000 \\ 45.000 & 45.000 \\ 50.000 & 50.000 \\ 45.000 & 45.000 \\ 40.000 & 40.000 \\ 55.000 & 35.000 \\ 50.000 & 30.000 \\ 25.000 & 25.000 \\ 20.000 & 20.000 \\ 15.000 & 15.000 \\ 10.000 & 10.000 \\ 5.000 & 5.000 \\ 0.000 & 0.000\end{array}$

FESISTOF MEASUFIING ACCUFACY

$$
\begin{array}{r}
24 \\
14.995
\end{array}
$$

I DEAL OUTFUT SIG MILLIVOLTS

59.980

83.972

107.964

131.956

155.948

179.940

203.932

227.924

251.916

275.908

275.908

227.924

203.932

179.940

155.948

131.956

107.964

83.972

59.980
299.900

251.916
14.995 OHMS 14.995 OHMS

N.A.

\section{CALIBFIATION}

MEASUFED OUTFUT SIE MILLIVOLTS

**LIMITED**

EFIFIOF \%

FULL SCALE

59.989

83.637

107.610

131.680

155.759

179.763

20.3 .771

227.779

251.841

275.829

299.841

275.851

251.780

227.820

203.801

179.800

155.791

131.723

107.663

8.707

60.006

$$
\begin{array}{r}
0.00 \% \\
-0.14 \% \\
-0.15 \% \\
-0.12 \% \\
-0.08 \% \\
-0.07 \% \\
-0.07 \% \\
-0.06 \% \\
-0.03 \% \\
-0.03 \% \\
-0.02 \% \\
-0.02 \% \\
-0.06 \% \\
-0.04 \% \\
-0.05 \% \\
-0.06 \% \\
-0.07 \% \\
-0.10 \% \\
-0.13 \% \\
-0.11 \% \\
0.01 \%
\end{array}
$$

$0.32 \%$

DUT

OF
TOLERANCE

\section{$\bar{z}=59.998$}


CURVEFIT OUTPUT FOR VTAB C2dp03 $J$

DATE: 20-MAY -92

TIME: $16: 23: 54$

VOLTAGE(V) OBS. DP(PSI) CALC. DP(PSI) DIFF (PSIA) - DIFFERENCE

$\begin{array}{rrrrrr}1 & 0.8998178 \mathrm{E}-05 & 0.0000000 \mathrm{E}+00 & 0.3175356 \mathrm{E}-01 & -0.3175356 \mathrm{E}-01 & 0.0000000 \mathrm{E}+00 \\ 2 & 0.2363900 \mathrm{E}-01 & 5.000000 & 4.967323 & 0.3267722 \mathrm{E}-01 & 0.6535445 \\ 3 & 0.4761200 \mathrm{E}-01 & 10.00000 & 9.968403 & 0.3159668 \mathrm{E}-01 & 0.3159668 \\ 4 & 0.7168200 \mathrm{E}-01 & 15.00000 & 14.98737 & 0.1263004 \mathrm{E}-01 & 0.8420025 \mathrm{E}-01 \\ 5 & 0.9576100 \mathrm{E}-01 & 20.00000 & 20.00586 & -0.5857808 \mathrm{E}-02 & -0.2928904 \mathrm{E}-01 \\ 6 & 0.1197650 & 25.00000 & 25.00637 & -0.6369452 \mathrm{E}-02 & -0.2547781 \mathrm{E}-01 \\ 7 & 0.1437730 & 30.00000 & 30.00537 & -0.5372568 \mathrm{E}-02 & -0.1790856 \mathrm{E}-01 \\ 8 & 0.1677810 & 35.00000 & 35.00203 & -0.2033682 \mathrm{E}-02 & -0.5810520 \mathrm{E}-02 \\ 9 & 0.1918430 & 40.00000 & 40.00758 & -0.7583628 \mathrm{E}-02 & -0.1895907 \mathrm{E}-01 \\ 10 & 0.2158310 & 45.00000 & 44.99540 & 0.4602146 \mathrm{E}-02 & 0.1022699 \mathrm{E}-01 \\ 11 & 0.2398430 & 50.00000 & 49.98586 & 0.1413922 \mathrm{E}-01 & 0.2827845 \mathrm{E}-01 \\ 12 & 0.2158530 & 45.00000 & 44.99997 & 0.2876938 \mathrm{E}-04 & 0.6393195 \mathrm{E}-04 \\ 13 & 0.1917820 & 40.00000 & 39.99490 & 0.5103056 \mathrm{E}-02 & 0.1275764 \mathrm{E}-01 \\ 14 & 0.1678220 & 35.00000 & 35.01056 & -0.1056480 \mathrm{E}-01 & -0.3018514 \mathrm{E}-01 \\ 15 & 0.1438030 & 30.00000 & 30.01162 & -0.1161777 \mathrm{E}-01 & -0.3872592 \mathrm{E}-01 \\ 16 & 0.1198020 & 25.00000 & 25.01408 & -0.1407548 \mathrm{E}-01 & -0.5630193 \mathrm{E}-01 \\ 17 & 0.9579300 \mathrm{E}-01 & 20.00000 & 20.01253 & -0.1252560 \mathrm{E}-01 & -0.6262802 \mathrm{E}-01 \\ 18 & 0.7172500 \mathrm{E}-01 & 15.00000 & 14.99633 & 0.3665980 \mathrm{E}-02 & 0.2443987 \mathrm{E}-01 \\ 19 & 0.4766500 \mathrm{E}-01 & 10.00000 & 9.979457 & 0.2054277 \mathrm{E}-01 & 0.2054277 \\ 20 & 0.2370900 \mathrm{E}-01 & 5.000000 & 4.981929 & 0.1807091 \mathrm{E}-01 & 0.3614182 \\ 21 & 0.8001822 \mathrm{E}-05 & 0.0000000 \mathrm{E}+00 & 0.3530244 \mathrm{E}-01 & -0.3530244 \mathrm{E}-01 & 0.0000000 \mathrm{E}+00\end{array}$

( GREE OF POİYNOMIAL 2

COEFFICIENTS

$0.33632002 E-01 \quad 0.20875780 E+03-0.20316335 E+01$

ESS $=0.6256 \mathrm{E}-02 \quad \mathrm{SD}=0.1864 \mathrm{E}-01 \quad \mathrm{R} 2=0.999999 \mathrm{E}+00$

$2 * S D=0.3729 \mathrm{E}-01$ 


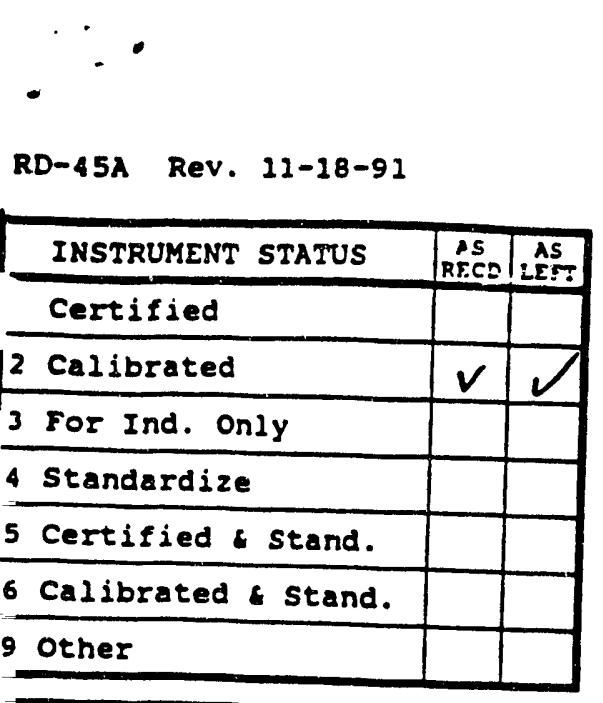

INSTRUMENT CONDITION

AS RECEIVED (Certified Equipment only)

Meets Manufacturer's Tolerance $N$

Operates within previously defined limits $\mathrm{N}$ eviates from Mfg or previously defined limits $Y$ Inoperative (Describe below) $Y$ AS LEFT Limited (Describe below) Meets Manufacturer's Tolerance Other (Describe Below)

\section{C3DPOI \\ $3 a b c c$ \\ acDermoti company} INCH DEVELOPAENT DIVISION

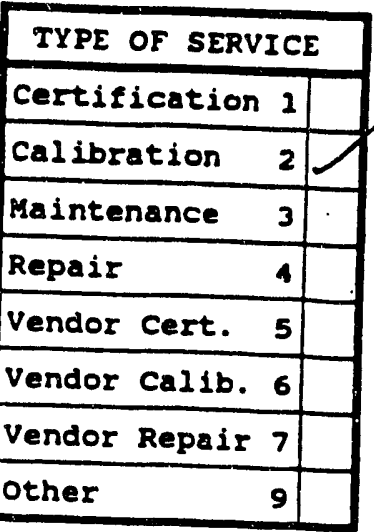

MANUFACTURER

MODEL

ITEM

MANUF . SER. NO.

PROPERTY NO.

ORDER NO.

B\&W Ser. NO. 0880533

SENSOTEC

21311-03

TRANSMITR, D-P

200013

4427-01-137

$\begin{array}{lr}\text { SECTION } & 46 \\ \text { LOCATION } & 115 \\ \text { SCHEDULED(Y-N) } & Y \\ \text { RECAL INT.(MOS.) } & 12\end{array}$

TECH. PROCEDURE

Babcock Wilcox RED Division certifies that the performance of this instrument was verif that the standards whose accuracies are was verified using National Institute of an accepted value of Standards and Technology, a ratio type of of a natural physical constant. consensus standard.

\section{MTAMDARDS USED}

Manufacturer Hodel

Model Item tsheveft Digigange Press ST0 07740.10 luke: Digigange Press. STD $8840 A$ D.M.M.

OTHER EOUIPMENT USED

ooper DP-160 ompaq Portable computer

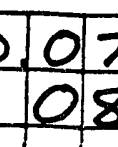

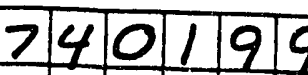
850454
Vendor Cert/Cal/Repair

Repair Parts

TOTAL MATERIAL COST

TOTAL LABOR HOURS $0.0 / 1.5$ Requested By Scot Sproul Charge Number $4 / 4260.102 .002$ Work order No. 0342

\section{6}

2890194 Name tewey yme ADDS

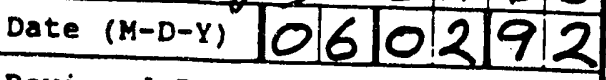
Reviewed By

rvice Notes $0-50$ PSI C3DPOI

See a ttached data sheet imited to data provided, does wat 
AS RECEIVED

TRANSMITTER:

FRESS SOURCE

$\begin{aligned} \text { ASHCFOFT } & \text { DIGIGAUGE } \\ 0-50 & \text { FSI } \\ \text { E\&WW SER. NO: } & 0740199 \\ \text { TOLEFANCE: } & 0.05 \%\end{aligned}$

FEADOUT DEV:

FLUK:E 8840A

E\&W SER. NO. 0850454

TOLEFIANCE : $0.015 \%$
AS LEFT $\sqrt{ }$

E\&W S.N. 0880533

DATE: $02-J 4 I n-92$

PROCEDURE : ARC-TF-015-08

FFIOGRAM: TOO15_08.03A

NAME: Pewey Pyue

Ambient Temperature (F) 75

Facility I. D. Name C.DPO1
VALUE OF CUFFENT SENSING FIESISTOF USED FOF TEST DATA

VALUE OF CUFFENT SENSING FESISTOF USED FOF CALIBFATION

FOWEF SUFFLY VOLTAGE

TOTAL SEFIIES RESISTANCE

AFF'LIED F'RESSURE :ANSMI TTEF:

F'SI

0.000

5.000

10.000

15.000

20.000

25.000

30.000

55.000

40.000

45.000

50.000

45.000

40.000

5.000

30.000

25.000

20.000

15.000

10.000

5.000

0.000
F'RESS SOURICE

F'SI

$$
0.000
$$

5.000

10.000

15.000

20.000

25.000

30.000

35.000

40.000

45.000

50.000

45.000

40.000

55.000

30.000

25.000

20.000

15.000

10.000

5.000

0.000
RIESISTOF: MEASUFIING ACCUFIACY 24

14.998

IDEAL

OUTFUT SIG

MILLIVOLTS

59.992
83.989
107.986
131.982
155.979
179.976
20.3 .973
227.970
251.966
275.963
299.960
275.963
251.966
227.970
203.973
179.976
155.979
131.982
107.986
83.989
59.992

CALIEFAATION ACCUFACY

**LIMITED**

MEASUFED

QUTFUT SIG

MILLIVOLTS

EFFIOF \%

FULL SCALE

60.003
83.873
107.812
1.1 .828
155.882
179.876
205.855
227.882
251.871
275.950
299.929
275.954
251.882
227.852
205.911
179.875
155.904
151.834
107.803
83.853
59.979
14.998 DHMS

14.998 OHMS

N.A. 
AS FIECEIVED

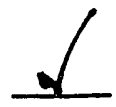

RANSMITTEF:

FRESS SOUFICE

EADOUT DEV:

\author{
SENSOTEC $Z$ \\ 50 F'SI \\ TOLEFANCE : $0.25 \%$ \\ ASHCFOFT DIGIGAUGE \\ $0-50$ F.SI \\ B\&.W Ser. Na. 0740199 \\ TOLEFANCE: $0.05 \%$ \\ FLUKEE 8840A \\ E\&W Ser. No. 0850454 \\ TOLEFIANCE: $0.015 \%$
}

AS LEFT

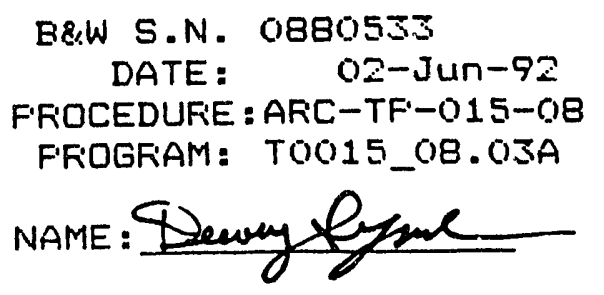

Ambient Temperature (F) 75

Facility I. D. Name CBDFO1

\begin{abstract}
IALUE OF CUFFENT SENSING FESISTOF USED FOF TEST DATA JALUE OF CUFFENT SENSING FESISTOF USED FOF: CALIEFATION

FIESISTOF: MEASUF: ING ACCUFIACY

$$
14.998 \text { OHMS }
$$$$
14.998 \text { OHMS }
$$

N.A.
\end{abstract}

'OWEF' SLFFLY VOLTAGE

TOTAL SEFIES FESISTANCE

AFF'L-IED FFESSUFE TFIANSMI TTEF<smiles>CC[As]</smiles>

0.000

5.000

10.000

15.000

20.000

25.000

30.000

5.000

40.000

45.000

50.000

45.000

40.000

55.000

30.000

25.000

20.000

15.000

10.000

5.000

0.000
F'FIESS SOUFICE

F'SI

0.000

5.000

10.000

15.000

20.000

25.000

30.000

35.000

40.000

45.000

50.000

45.000

40.000

35.000

30.000

25.000

20.000

15.000

10.000

5.000

0.000

$$
24
$$

14.998

I DEAL

OUTFUT SIG
MILLIVOLTS

59.992
85.989
107.986
1.1 .982
155.979
179.976
205.975
227.970
251.966
275.963
299.960
275.965
251.966
227.970
203.973
179.976
155.979
131.982
107.986
83.989
59.992

CALIBFATION ACCUFACY **LIMITED**

MEASUFED DUTFUT SIG MILLIVOLTS

EFFIOR \% FULL SCALE

$$
\begin{array}{r}
59.886 \\
85.766 \\
107.699 \\
151.747 \\
155.804 \\
179.799 \\
205.853 \\
227.794 \\
251.806 \\
275.842 \\
299.835 \\
275.852 \\
251.786 \\
227.826 \\
203.805 \\
179.760 \\
155.785 \\
131.723 \\
107.687 \\
83.737 \\
59.833
\end{array}
$$


$r$ VOLTAGE(V) OBS. DP(PSI) CALC. DP(PSI) DIFF (PSIA) DIFFERENCE

$\begin{array}{rlllll}1 & 0.1200164 \mathrm{E}-04 & 0.0000000 \mathrm{E}+00 & 0.1526318 \mathrm{E}-01 & -0.1526318 \mathrm{E}-01 & 0.0000000 \mathrm{E}+00 \\ 2 & 0.2388200 \mathrm{E}-01 & 5.000000 & 4.994711 & 0.5288517 \mathrm{E}-02 & 0.1057703 \\ 3 & 0.4782100 \mathrm{E}-01 & 10.00000 & 9.986931 & 0.1306854 \mathrm{E}-01 & 0.1306854 \\ 4 & 0.7183700 \mathrm{E}-01 & 15.00000 & 14.99358 & 0.6423436 \mathrm{E}-02 & 0.4282291 \mathrm{E}-01 \\ 5 & 0.9589100 \mathrm{E}-01 & 20.00000 & 20.00650 & -0.6504673 \mathrm{E}-02 & -0.3252336 \mathrm{E}-01 \\ 6 & 0.1198850 & 25.00000 & 25.00529 & -0.5294533 \mathrm{E}-02 & -0.2117813 \mathrm{E}-01 \\ 7 & 0.1438640 & 30.00000 & 29.99933 & 0.6711251 \mathrm{E}-03 & 0.2237084 \mathrm{E}-02 \\ 8 & 0.1678910 & 35.00000 & 35.00173 & -0.1725153 \mathrm{E}-02 & -0.4929008 \mathrm{E}-02 \\ 9 & 0.1918800 & 40.00000 & 39.99458 & 0.5422764 \mathrm{E}-02 & 0.1355691 \mathrm{E}-01 \\ 10 & 0.2159590 & 45.00000 & 45.00452 & -0.4520565 \mathrm{E}-02 & -0.1004570 \mathrm{E}-01 \\ 11 & 0.2399380 & 50.00000 & 49.99202 & 0.7975764 \mathrm{E}-02 & 0.1595153 \mathrm{E}-01 \\ 12 & 0.2159630 & 45.00000 & 45.00535 & -0.5352679 \mathrm{E}-02 & -0.1189484 \mathrm{E}-01 \\ 13 & 0.1918910 & 40.00000 & 39.99687 & 0.3133699 \mathrm{E}-02 & 0.7834246 \mathrm{E}-02 \\ 14 & 0.1678610 & 35.00000 & 34.99548 & 0.4519795 \mathrm{E}-02 & 0.1291370 \mathrm{E}-01 \\ 15 & 0.1439200 & 30.00000 & 30.01099 & -0.1098992 \mathrm{E}-01 & -0.3663306 \mathrm{E}-01 \\ 16 & 0.1198840 & 25.00000 & 25.00509 & -0.5086232 \mathrm{E}-02 & -0.2034493 \mathrm{E}-01 \\ 17 & 0.9591300 \mathrm{E}-01 & 20.00000 & 20.01109 & -0.1108879 \mathrm{E}-01 & -0.5544395 \mathrm{E}-01 \\ 18 & 0.7184300 \mathrm{E}-01 & 15.00000 & 14.99483 & 0.5172813 \mathrm{E}-02 & 0.3448542 \mathrm{E}-01 \\ 19 & 0.4781200 \mathrm{E}-01 & 10.00000 & 9.985055 & 0.1494509 \mathrm{E}-01 & 0.1494509 \\ 20 & 0.2386200 \mathrm{E}-01 & 5.000000 & 4.990540 & 0.9459980 \mathrm{E}-02 & 0.1891996 \\ 21 & -0.1199836 \mathrm{E}-04 & 0.0000000 \mathrm{E}+00 & 0.1025580 \mathrm{E}-01 & -0.1025580 \mathrm{E}-01 & 0.0000000 \mathrm{E}+00\end{array}$

DEGREE OF POLYNOMIAL 2

CeFficients

$0.12759145 E-01 \quad 0.20864083 E+03-0.14173950 E+01$
F.SS $=$
$0.1433 \mathrm{E}-02$
$\begin{aligned} S D & =0.8924 \mathrm{E}-02 \\ 2 \star S D & =0.1785 \mathrm{E}-01\end{aligned}$
$\mathbf{R 2}=$
$0.100000 E+01$ 


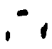

RD-45A

\begin{tabular}{|c|c|c|}
\hline INSTRUMENT STATUS & $\begin{array}{ll}A 5 \\
\text { A.s.co }\end{array}$ & $\begin{array}{l}\text { AS } \\
E \pi \\
\end{array}$ \\
\hline 1 Certified & & \\
\hline Calibrated & & \\
\hline For Ind. only & 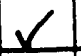 & \\
\hline 4 standardize & & \\
\hline Certified \& stand. & & \\
\hline 6 Calibrated Stand. & & \\
\hline other & & \\
\hline
\end{tabular}

RESEARCH \& DEVELOPHENT DIVISION INSTRUIENT SERVICE LOG

\begin{tabular}{|c|c|}
\hline \multicolumn{2}{|c|}{ TYPE OF SERVICE } \\
\hline Certification 1 & \\
\hline Calibration & \\
\hline Maintenance & \\
\hline Repair & \\
\hline Vendor cert. 5 & \\
\hline Vendor calib. 6 & \\
\hline Vendor Repair 7 & \\
\hline other & 9 \\
\hline
\end{tabular}

AS RECEIVED (Certified Equipment only)

: Meets Manufacturer's Tolerance $N$ Operates within previously defined limits $\mathrm{N}$ jeviates from Mfg or previously defined limits $Y$ 1 Inoperative (Describe below) $y$ AS LEFT Limited (Describe below) Meets Manufacturer's Tnlerance other (Describe Below)
Babcock \& Wilcox

acDermott company

B\&W Ser. No. 0880520

MANUFACTURER

SENSOTEC

MODEL

ITEM

23.309-15

TRANSMITR, D-P

187402

MANUF. SER. NO.

4427-01-124

ORDER NO.

SECTION

LOCATION

46

SCHEDULED $(\mathrm{Y}-\mathrm{N})$

115

RECAL INT.(MOS.)

$+y$

12
TECH. PROCEDURE A0015

Babcock Wilcox RED Division certifies that the performance of this instrument was verified using standards whose accuracies are traceable to the standards whose accuracies are trace Technology. an accepted value of a natural physical constant. * a ratio type of calibration technique, or to a consensus standard.

\section{STAUDARPS USED}

Manufacturer

ASHCROFT TLUKE
Model

Item

F $0-10$ PSE PRESS, STD. Bew Serial No. $8840 \mathrm{~A}$ PMM \begin{tabular}{l|l|l|l|l|l|l}
0 & 8 & 1 & 0 & 2 & 2 & 5 \\
0 & 8 & 6 & 0 & 4 & 0 & 4
\end{tabular}

Vendor Cert/Cal/Repair Repair Parts TOTAL MATERIAL COST TOTAL IABOR HOURS Requested By S.SPRoul Charge Number $44160-102-002$ work order No. 0296

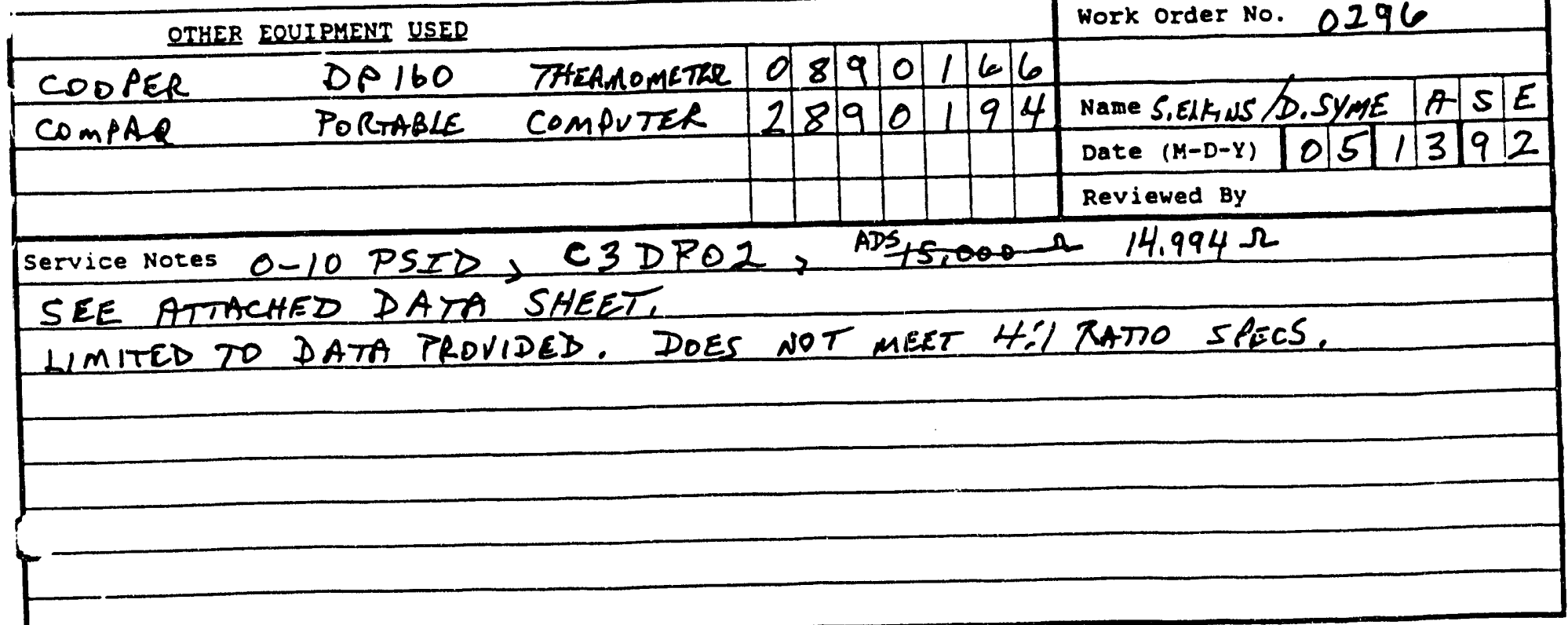


AS RECEIVED

TRANSMITTER :

SENSOTEC $Z$

$10 \mathrm{FSI}$

TOLERANCE : $0.25 \%$

FRESS SOUFICE

$$
\begin{gathered}
\text { ASHCFOFT DIGIGAUGE } \\
0-10 \text { FSI }
\end{gathered}
$$

B\&wW Ser. No. 0810225

TOLEFAANCE: $0.05 \%$

REAंDOUT DEV:

FLUKE $8840 A$

E\&W Ser. NO. 0860404

TOLEFANCE: $0.015 \%$
AS

LEFT

E\&W S.N. 0880520

DATE: $13-M a Y-92$

FFIOCEDUFE : ARC-TF-015-08

FFIOGFIAM: TOO15_0B.03A

NAME: S. ELKINS b, SYME

Ambient Temperature ( $F$ ) 71

Facility I. D. Name CBDFOZ
VALUE OF CUFFENT SENSING RESISTOF USED FOF TEST DATA VALUE OF CUFFENT SENSING FESISTOF USED FOF CALIEFIATION FOWER SUFFLY VOLTAGE TOTAL SEFIES FESISTANCE

AF'FLIED F'RESSURE

TRANSMI TTEF: F.SI F'FIESS SOURICE FSI

$\begin{array}{rr}0.000 & 0.000 \\ 1.000 & 1.000 \\ 2.000 & 2.000 \\ 5.000 & 5.000 \\ 4.000 & 4.000 \\ 5.000 & 5.000 \\ 6.000 & 6.000 \\ 7.000 & 7.000 \\ 8.000 & 8.000 \\ 9.000 & 9.000 \\ 10.000 & 10.000 \\ 9.000 & 9.000 \\ 8.000 & 8.000 \\ 7.000 & 7.000 \\ 6.000 & 6.000 \\ 5.000 & 5.000 \\ 4.000 & 4.000 \\ 3.000 & 5.000 \\ 2.000 & 2.000 \\ 1.000 & 1.000 \\ 0.000 & 0.000\end{array}$

FESISTOF: MEASUFIING ACCUFIACY

\section{4}

14.994

IDEAL

OUTFUT SIG

MILLIVOLTS

59.976

83.966

107.957

131.947

155.938

179.928

203.918

227.909

251.899

275.890

299.880

275.890

251.899

227.909

20.918

179.928

155.938

131.947

107.957

83.966

59.976
CALIEFIATION ACCUFIACY

**L IMITED**

MEASUFIED

OUTFUT SIG

MILLIVOLTS

EFROF \%

FULL SCALE

59.976

84.054

108.065

132.021

156.051

180.062

204.013

228.024

251.971

275.877

299.734

275.817

251.904

227.901

205.909

179.938

155.955

151.959

107.958

8. .973

59.914

$$
\begin{array}{r}
0.00 \% \\
0.04 \% \\
0.04 \% \\
0.03 \% \\
0.05 \% \\
0.06 \% \\
0.04 \% \\
0.05 \% \\
0.03 \% \\
-0.01 \% \\
-0.06 \% \\
-0.03 \% \\
0.00 \% \\
0.00 \% \\
0.00 \% \\
0.00 \% \\
0.01 \% \\
0.00 \% \\
0.00 \% \\
0.00 \% \\
-0.03 \%
\end{array}
$$

$0.32 \%$

DUT OF TOLERANCE

$$
\bar{z}=59.945
$$


CURVEFIT OUTPUT FOR VTAB C3APO2 $/$

DATE: $20-\mathrm{MAY}-92$

TIME: $16: 27: 54$

VOLTAGE(V) OBS. DP(PSI) CALC. DP(PSI) DIFF (PSIA) \& DIFFERENCE

$\begin{array}{llllll}1 & 0.3100153 \mathrm{E}-04 & 0.0000000 \mathrm{E}+00 & 0.9125592 \mathrm{E}-03 & -0.9125592 \mathrm{E}-03 & 0.0000000 \mathrm{E}+00 \\ 2 & 0.2410900 \mathrm{E}-01 & 1.000000 & 1.002852 & -0.2852121 \mathrm{E}-02 & -0.2852121 \\ 3 & 0.4812000 \mathrm{E}-01 & 2.000000 & 2.002458 & -0.2457680 \mathrm{E}-02 & -0.1228840 \\ 4 & 0.7207600 \mathrm{E}-01 & 3.000000 & 3.000225 & -0.2253522 \mathrm{E}-03 & -0.7511741 \mathrm{E}-02 \\ 5 & 0.9610600 \mathrm{E}-01 & 4.000000 & 4.001529 & -0.1528528 \mathrm{E}-02 & -0.3821320 \mathrm{E}-01 \\ 6 & 0.1201170 & 5.000000 & 5.002494 & -0.2493558 \mathrm{E}-02 & -0.4987117 \mathrm{E}-01 \\ 7 & 0.1440680 & 6.000000 & 6.001409 & -0.1409008 \mathrm{E}-02 & -0.2348347 \mathrm{E}-01 \\ 8 & 0.1680790 & 7.000000 & 7.003280 & -0.3279673 \mathrm{E}-02 & -0.4685248 \mathrm{E}-01 \\ 9 & 0.1920260 & 8.000000 & 8.002931 & -0.2931480 \mathrm{E}-02 & -0.3664350 \mathrm{E}-01 \\ 10 & 0.2159320 & 9.000000 & 9.001322 & -0.1321582 \mathrm{E}-02 & -0.1468424 \mathrm{E}-01 \\ 11 & 0.2397890 & 10.00000 & 9.998113 & 0.1886666 \mathrm{E}-02 & 0.1886666 \mathrm{E}-01 \\ 12 & 0.2158720 & 9.000000 & 8.998815 & 0.1184771 \mathrm{E}-02 & 0.1316412 \mathrm{E}-01 \\ 13 & 0.1919590 & 8.000000 & 8.000134 & -0.1339801 \mathrm{E}-03 & -0.1674751 \mathrm{E}-02 \\ 14 & 0.1679560 & 7.000000 & 6.998146 & 0.1853717 \mathrm{E}-02 & 0.2648167 \mathrm{E}-01 \\ 15 & 0.1439640 & 6.000000 & 5.997071 & 0.2929456 \mathrm{E}-02 & 0.4882427 \mathrm{E}-01 \\ 16 & 0.1199930 & 5.000000 & 4.997323 & 0.2676890 \mathrm{E}-02 & 0.5353780 \mathrm{E}-01 \\ 17 & 0.9601000 \mathrm{E}-01 & 4.000000 & 3.997527 & 0.2472588 \mathrm{E}-02 & 0.6181470 \mathrm{E}-01 \\ 18 & 0.7201400 \mathrm{E}-01 & 3.000000 & 2.997642 & 0.2357531 \mathrm{E}-02 & 0.7858437 \mathrm{E}-01 \\ 19 & 0.4801300 \mathrm{E}-01 & 2.000000 & 1.998002 & 0.1997859 \mathrm{E}-02 & 0.9989295 \mathrm{E}-01 \\ 20 & 0.2402800 \mathrm{E}-01 & 1.000000 & 0.9994808 & 0.5192345 \mathrm{E}-03 & 0.5192345 \mathrm{E}-01 \\ 21 & -0.3099847 \mathrm{E}-04 & 0.0000000 \mathrm{E}+00 & -0.1666811 \mathrm{E}-02 & 0.1666811 \mathrm{E}-02 & 0.0000000 \mathrm{E}+00\end{array}$

( GREE OF POLYNOMIAL 2

COEFFICIENTS

$-0.37718993 E-03 \quad 0.41602750 E+02 \quad 0.39320188 E+00$

ESS $=0.8957 \mathrm{E}-04 \quad \mathrm{SD}=0.2231 \mathrm{E}-02 \quad \mathrm{R2}=0.100000 \mathrm{E}+01$

$2 * S D=0.4461 \mathrm{E}-02$ 
RESEARCH \& DEVELOPMENT DIVISION INSTRUMENT SERVICE IOG

RD-45A Rev. $11-18-91$

\begin{tabular}{|c|c|c|}
\hline INSTRUMENT STATUS & $\begin{array}{ll}\text { As } \\
\text { RT.Co }\end{array}$ & 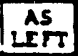 \\
\hline 1 Certified & & \\
\hline calibrated & & \\
\hline 3 For Ind. Only & $\checkmark$ & \\
\hline 4 Standardize & & \\
\hline 5 certlfied stand. & & \\
\hline 6 Calibrated stand. & & \\
\hline 9 Other & & \\
\hline
\end{tabular}

INSTRUMENT CONDITION

AS RECEIVED (Certified Equipment only)

Meets Manulacturer's Tolerance $N$ operates within previously defined limits $N$ Deviates from Mfg or previously defined limits $Y$ Inoperative (Describe below) $Y$

AS LEFT
TYPE OF SERVICE

Certification 1

\begin{tabular}{|lr|l|}
\hline Calibration & 2 & $ل$ \\
\hline Maintenance & 3 & \\
\hline Repair & 4 & \\
\hline Vendor Cert. & 5 & \\
\hline Vendor Calib. & 6 & \\
\hline Vendor Repair & 7 & \\
\hline Other & 9 & \\
\hline
\end{tabular}

MANUFACTURER

MODEL

ITEM

MANUF. SER. NO.

PROPERTY NO.

ORDER NO.

SECTION

LOCATION

SCHEDULED ( $Y-N)$

RECAL INT. (MOS.)
Babcock \& Wilcox

- McDermoti company

B\&W Ser. No. 0880534

SENSOTEC

21311-03

TRANSMITR, D-P

200017

4427-01-138

TECH. PROCEDURE AO015

Babcock \& Wilcox RCD Division certifies that the performance of this instrument was verified using standards whose accuracles are tracaable to the National Institute of standards and Technology, an accepted value of a natural physical constant, a ratio type of calibration technique, or to a consensus standard.

other (Describe Below)

Mecte Mamuecturer's Tolerance

Manufacturer

Model

Item

Bew Serial No.

ASHCROFT O-50 PSI PRESS, STP FLUKE $8840 A$ DMM

\begin{tabular}{l|l|l|l|}
0 & 7 & 4 & 0 \\
\hline 0 & 8 & 6 & 0 \\
\hline
\end{tabular}

\begin{tabular}{l|l|l}
0 & 1 \\
\hline 0 & 4 & 0 \\
\hline & & 0 \\
\hline
\end{tabular}

\begin{tabular}{l|l|l}
9 & 9 \\
\hline 0 & 4 & \\
\hline
\end{tabular}

Vendor Cert/Cal/Repair

Repair Parts

TOTAL MATERIAL COST

TOTAL LABOR HOURS

01011.0 Requested By $S$, SPROUL Charge Number $44260-101-002$ Work Order No. 0296

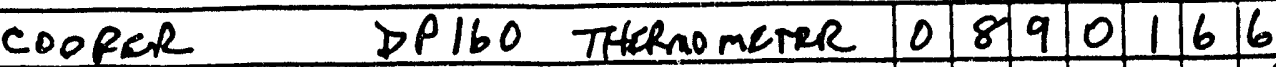
COMPAQ PORABLE COMPUTER

\begin{tabular}{|c|c|c|c|c|c|c|c|c|c|}
\hline & 89 & 0 & 1 & 9 & 4 & Name S, ELkiNS & ID sYMe & $A$ & $D[5$ \\
\hline & & & & & & Date $(M-D-Y)$ & 0.51 & 3 & 912 \\
\hline & & & & & & Reviewed By & & & \\
\hline
\end{tabular}

- Service Notes O-50 PSID,

(4DPO) $14.994 \Omega$

SEE AYTACHED DATA SHEET,

LIMITES TO PATA PROVIDED, DOES NOT MEET HII RATIO SPECS, 
AS FECEIVED

IRANSMITTEF:

'REESS SOUFICE

$$
\begin{aligned}
\text { ASHCFOFT } & \text { DIGIGAUGE } \\
\text { O-5O FSI } & \text { FS } \\
\text { E\&W SER. NO. } & 0740199 \\
\text { TOLERANCE: } & 0.05 \%
\end{aligned}
$$

:EADOUT DEV:
AS LEFT $\mathscr{\swarrow}$

B\&W S.N. 0880334 .. DATE: $\quad 13-M a y-92$

PFIOCEDURE : ARC-TF-015-08

FFIOGFIM: TOO15_08.03A

NAME : s, ILKINS/S. SYME

Ambient Temperature (F) 72

Facility I. D. Name C4DFO1

\section{E\&W Ser. No. 0860404 \\ TOLERANCE: $0.015 \%$}

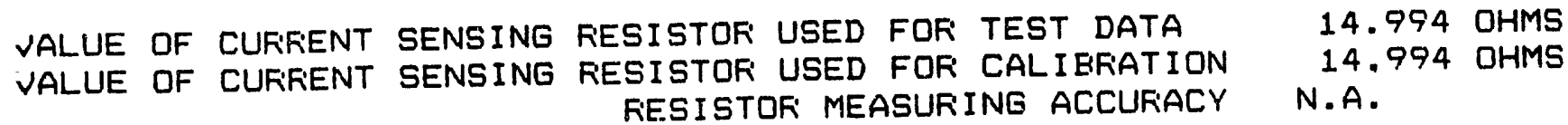

$\because O W E F$ SUFFLY VOLTAGE TOTAL SEFIES FIESISTANCE

AFFLIED FRESSURE
TFANSMITTEF FFIESS SOUFICE
F'SI

$\begin{array}{rr}0.000 & 0.000 \\ 5.000 & 5.000 \\ 10.000 & 10.000 \\ 15.000 & 15.000 \\ 20.000 & 20.000 \\ 25.000 & 25.000 \\ 30.000 & 30.000 \\ 35.000 & 35.000 \\ 40.000 & 40.000 \\ 45.000 & 45.000 \\ 50.000 & 50.000 \\ 45.000 & 45.000 \\ 40.000 & 40.000 \\ 35.000 & 35.000 \\ 30.000 & 50.000 \\ 25.000 & 25.000 \\ 20.000 & 20.000 \\ 15.000 & 15.000 \\ 10.000 & 10.000 \\ 5.000 & 5.000 \\ 0.000 & 0.000\end{array}$

$$
24
$$

14.994

IDEAL

OUTFUT SIG

MILLIVOLTS

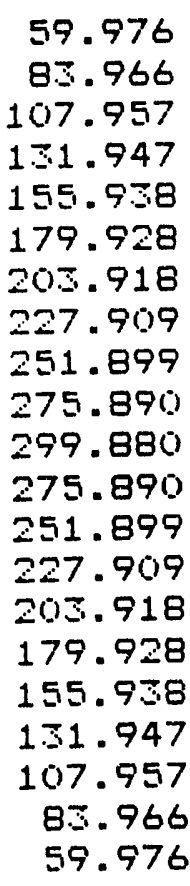

CALIBFATION ACCURACY

**LIMITED**

MEASURED ERFIOR \% OUTFUT SIG FULL SCALE MILLIVOLTS

$\begin{array}{rr}59.997 & 0.01 \% \\ 83.561 & -0.17 \% \\ 107.488 & -0.20 \% \\ 131.532 & -0.17 \% \\ 155.610 & -0.14 \% \\ 179.617 & -0.13 \% \\ 205.650 & -0.11 \% \\ 227.703 & -0.09 \% \\ 251.780 & -0.05 \% \\ 275.828 & -0.03 \% \\ 299.839 & -0.02 \% \\ 275.945 & 0.02 \% \\ 251.929 & 0.01 \% \\ 227.977 & 0.03 \% \\ 20.917 & 0.00 \% \\ 179.948 & 0.01 \% \\ 15 \% .904 & -0.01 \% \\ 151.803 & -0.06 \% \\ 107.768 & -0.08 \% \\ 83.765 & -0.08 \% \\ 60.021 & 0.02 \%\end{array}$

$0.32 \%$

OUT

DF TOLEFANCE

$$
z=-60.009
$$


CURVEFIT OUTPUT FOR VTAB C4dPOIJ

DATE: $20-$ KAY -92

TIME: $16: 28: 51$

VOLTAGE(V) OBS. DP(PSI) CALC. DP(PSI) DIFF (PSIA) DIFFERENCE

$\begin{array}{llllll}1 & -0.1199896 \mathrm{E}-04 & 0.0000000 \mathrm{E}+00 & 0.3438477 \mathrm{E}-01 & -0.3438477 \mathrm{E}-01 & 0.0000000 \mathrm{E}+00 \\ 2 & 0.2355200 \mathrm{E}-01 & 5.000000 & 4.953110 & 0.4689038 \mathrm{E}-01 & 0.9378077 \\ 3 & 0.4747900 \mathrm{E}-01 & 10.00000 & 9.944888 & 0.5511197 \mathrm{E}-01 & 0.5511197 \\ 4 & 0.7152300 \mathrm{E}-01 & 15.00000 & 14.95832 & 0.4168396 \mathrm{E}-01 & 0.2778930 \\ 5 & 0.9560100 \mathrm{E}-01 & 20.00000 & 19.97606 & 0.2393876 \mathrm{E}-01 & 0.1196938 \\ 6 & 0.1196080 & 25.00000 & 24.97625 & 0.2375151 \mathrm{E}-01 & 0.9500605 \mathrm{E}-01 \\ 7 & 0.1436410 & 30.00000 & 29.97909 & 0.2091125 \mathrm{E}-01 & 0.6970418 \mathrm{E}-01 \\ 8 & 0.1676940 & 35.00000 & 34.98333 & 0.1667491 \mathrm{E}-01 & 0.4764260 \mathrm{E}-01 \\ 9 & 0.1917710 & 40.00000 & 39.98978 & 0.1021787 \mathrm{E}-01 & 0.2554469 \mathrm{E}-01 \\ 10 & 0.2158190 & 45.00000 & 44.98744 & 0.1255985 \mathrm{E}-01 & 0.2791079 \mathrm{E}-01 \\ 11 & 0.2398300 & 50.00000 & 49.97465 & 0.2535202 \mathrm{E}-01 & 0.5070404 \mathrm{E}-01 \\ 12 & 0.2159360 & 45.00000 & 45.01175 & -0.1174833 \mathrm{E}-01 & -0.2610741 \mathrm{E}-01 \\ 13 & 0.1919200 & 40.00000 & 40.02076 & -0.2075584 \mathrm{E}-01 & -0.5188960 \mathrm{E}-01 \\ 14 & 0.1679680 & 35.00000 & 35.04031 & -0.4031495 \mathrm{E}-01 & -0.1251856 \\ 15 & 0.1439080 & 30.00000 & 30.03465 & -0.3465340 \mathrm{E}-01 & -0.1155113 \\ 16 & 0.1199390 & 25.00000 & 25.04517 & -0.4517002 \mathrm{E}-01 & -0.1806801 \\ 17 & 0.9589500 \mathrm{E}-01 & 20.00000 & 20.03731 & -0.3731235 \mathrm{E}-01 & -0.1865618 \\ 18 & 0.7179400 \mathrm{E}-01 & 15.00000 & 15.01481 & -0.1480664 \mathrm{E}-01 & -0.9871095 \mathrm{E}-01 \\ 19 & 0.4775900 \mathrm{E}-01 & 10.00000 & 10.00329 & -0.3286912 \mathrm{E}-02 & -0.3286912 \mathrm{E}-01 \\ 20 & 0.2375400 \mathrm{E}-01 & 5.000000 & 4.995263 & 0.4736601 \mathrm{E}-02 & 0.9473201 \mathrm{E}-01 \\ 21 & 0.1200104 \mathrm{E}-04 & 0.0000000 \mathrm{E}+00 & 0.3939586 \mathrm{E}-01 & -0.3939586 \mathrm{E}-01 & 0.0000000 \mathrm{E}+00\end{array}$

( 'GREE OF POLYNOMIAL 2

COEFFICIENTS

$0.36890099 E-01 \quad 0.20879528 E+03-0.23925242 E+01$

ESS $=0.1955 E-01 \quad S D=0.3295 E-01 \quad R 2=0.999996 E+00$

$2 \star S D=0.6590 \mathrm{E}-01$ 
D-45A Rev. $11-28-91$

RESEARCH \& DEVELOPAENT DIVISION INSTRUMENT SERVICE IOG

\begin{tabular}{|c|c|c|c|}
\hline INSTRUMENT STATUS & $\begin{array}{c}\text { AS } \\
\text { RE.CE } \\
\end{array}$ & $\begin{array}{c}\text { AS } \\
\text { LETT }\end{array}$ & TYPE OF SERVICI \\
\hline Certified & & & Certification 1 \\
\hline Calibrared & $\checkmark$ & & Calibration \\
\hline For Ind. only & & & Maintenance \\
\hline standardize & & & Repair \\
\hline 5 certified st stand. & & & Vendor cert. 5 \\
\hline 5 Calibrated a stand. & & & Vendor Calib. 6 \\
\hline 9 Other & & & Vendor Repair 7 \\
\hline & & & Other \\
\hline
\end{tabular}

INSTRUMENT CONDITION

AS RECEIVED (Certified Equipment only)

Meers Manufacturer's Tolerance $N$

operates within previously defined limits $\mathrm{N}$

Deviates from Mfg or previously defined liwits $X$ Inoperative (Describe below) $y$

AS LEFT

Limited (Describe below)

Meets Manufacturer's Tolerance

other (Desc:-ibe Below)

ORDER NO.
Babcock \& Wilcox

a MCDermost company

B\&W Ser. No. 0880521

MANUFACTURER SENSOTEC

MODEL

ITEM

21309-15

TRANSMITR, D-P

MANUF . SER. NO. 187436

PROPERTY NO. 4427-01-125

SECTION

46

LOCATION

115

SCHEDDULED ( $Y-N)$

$\mathbf{Y}$

RECIL INT. (MOS.)

12

TECH. PROCEDURE

Babcock Wilcox RED Division certifies that the performance of this instrument was verified using pertormance or eccuracies are traceable to the standard institute of standards and Technology, National Institute of standards and ical constant, a ratio type of calibration technique, or to a consensus standard.

Manufacturer Model

Item

Bew serial No.

\begin{tabular}{ccc}
\hline Asheroft & 2780 & Press. STP. \\
Flukel & 88404 & D. U.M.
\end{tabular}
\begin{tabular}{l|l|l|l|l|l|l}
0 & 8 & 1 & 0 & 2 & 2 & 5 \\
\hline 0 & 8 & 5 & 0 & 4 & 5 & 4
\end{tabular} Vendor Cert/Cal/Repair Repair Parts TOTAL MATERIAL COST Eluke

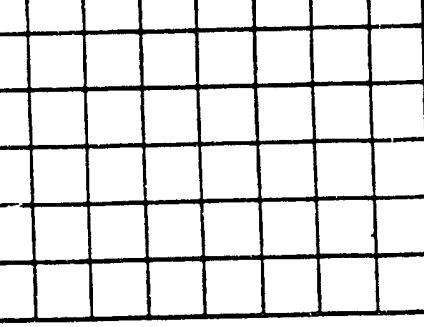

OTHER EOUIPMENT USED

TOtal Labor hours b O $/ / .5$ Requested by Scotf Spron 1 Charge Number $44260 \cdot 102.002$ work order No. 0342
Cooper DP 160 Themameter 00899001166 Compaq Portable Computer 28.9001494 Name Dewey bre $A|D| S$

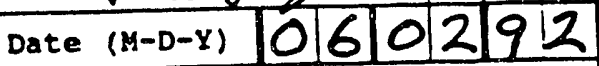
Reviewed By

service notes O-10 PSI C4DPOZ

Calibratien limited to data provided see attached data sheet HAD ZERO SHIFT FIOM BACK FILING. RECALIBRATED 
AS RECEIVED

TRANSMITTER:

FRESS SOUFICE

ASHCFOFT DIGIGAUGE
$0-10$ PSI
B\&W SER. NO. O810225

TOLEFIANCE : $0.05 \%$

FEADQUT DEV:

SENSOTEC $Z$

$10 \mathrm{FSI}$

TOLEFIANCE: $0.25 \%$

FLUKE 8840A

B\&W SET. NO. O850454

TOLEFIANCE: $0.015 \%$
AS LEFT

B\&W S.N. OB80521

DATE: $01-J 4 I n-92$

FROCEDIJRE : ARIC-TF-015-08

FFIOGRAM: TO015_09.03A

NAME: Aoming Lerome

Ambient Temperature (F) 75

Facility I. D. Name C4DFO2
VALUE OF CUFFENT SENSING FESISTOF USED FOF TEST DATA VALUE OF CUFFENT SENSING FESISTOF USED FOF CALIBFATION OOWER SUF'FLY VOLTAGE TOTAL SEFIIES RESISTANCE

AFFLIED FIFESSUFIE TFANSMITTEF' F'S I FFIESS SOUFICE F'SI

$\begin{array}{rr}0.000 & 0.000 \\ 1.000 & 1.000 \\ 2.000 & 2.000 \\ 3.000 & 5.000 \\ 4.000 & 4.000 \\ 5.000 & 5.000 \\ 6.000 & 6.000 \\ 7.000 & 7.000 \\ 8.000 & 8.000 \\ 9.000 & 9.000 \\ 10.000 & 10.000 \\ 9.000 & 9.000 \\ 8.000 & 8.000 \\ 7.000 & 7.000 \\ 6.000 & 6.000 \\ 5.000 & 5.000 \\ 4.000 & 4.000 \\ 5.000 & 3.000 \\ 2.000 & 2.000 \\ 1.000 & 1.000 \\ 0.000 & 0.000\end{array}$

FESISTOF: MEASUIFING ACCUFIACY

$$
24
$$

14.993

IDEAL
OUTFUT SIG
MILLIVILTS

59.972
83.961
107.950
131.938
155.927
179.916
203.905
227.894
251.882
275.871
299.860
275.871
251.882
227.894
20.905
179.916
155.927
151.938
107.950
8.961
59.972
CALIEFIATION ACCURACY

**LIMITED**

MEASURED EFIFIOF \% OUTFUT SIG FULL SCALE MILLIVOLTS

59.961
84.029
108.071
132.073
156.045
180.053
204.037
228.033
251.993
275.894
299.777
275.893
251.990
228.030
204.041
180.058
156.094
132.088
108.091
84.033
60.006

$0.00 \%$

$0.03 \%$

$0.05 \%$

$0.06 \%$

$0.05 \%$

$0.06 \%$

$0.06 \%$

$0.06 \%$

$0.05 \%$

$0.01 \%$

$-0.03 \%$

$0.01 \%$

$0.04 \%$

$0.06 \%$

$0.06 \%$

$0.06 \%$

$0.07 \%$

$0.06 \%$

$0.06 \%$

$0.0 .3 \%$

$0.01 \%$
$0.32 \%$

OUT

OF

TOLEFIANCE

$$
\bar{z}=59.984
$$


AS

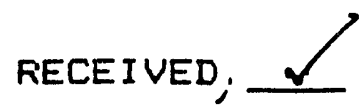

$\therefore$ :ANSMI TTEF:

SENSOTEC $Z$

10 F'SI

TOLEFANCE: $0.25 \%$

FiESS SOURCE

$\begin{aligned} \text { ASHCROFT } & \text { DIGIGAUGE } \\ \text { O-10 FSI } & \text { F. } \\ \text { B\&W SER. NO: } & 0810225 \\ \text { TOLEFIANCE: } & 0.05 \%\end{aligned}$

¿ADJUT DEV:

E\&W Ser. No. 0850454

AS LEFT

B\&W S.N. O880521

DATE: $01-J u n-92$

FFIOCEDURE : ARC-TF-015-08

FFIOGFIM: TO015_08.03A

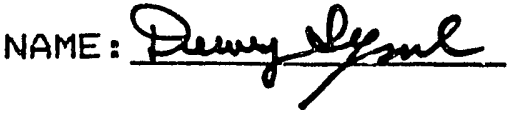

Ambient Temperature (F) 75

Facility I. D. Name C4DPOZ TOLEFIANCE: $0.015 \%$
ILUE OF CUFFEN
SENSING
FIESISTOF USED FOF TEST DATA
ALUE OF CURFENT SENSING RESISTOF USED FOF CALIBFIATION
FIESISTOR MEASUFING ACCUFIACY

$$
\begin{aligned}
& 14.993 \text { OHMS } \\
& 14.99 .3 \text { aHMS }
\end{aligned}
$$$$
\text { N.A. }
$$

JWEF SUF'FLY VOLTAGE

JTAL SEFIIES FESISTANCE

AFF'LIED FRESSUFE RANSMITTEF F'SI F'RESS SOUFICE F'SI

$\begin{array}{rr}0.000 & 0.000 \\ 1.000 & 1.000 \\ 2.000 & 2.000 \\ 3.000 & 3.000 \\ 4.000 & 4.000 \\ 5.000 & 5.000 \\ 6.000 & 6.000 \\ 7.000 & 7.000 \\ 8.000 & 8.000 \\ 9.000 & 9.000 \\ 10.000 & 10.000 \\ 9.000 & 9.000 \\ 8.000 & 8.000 \\ 7.000 & 7.000 \\ 6.000 & 6.000 \\ 5.000 & 5.000 \\ 4.000 & 4.000 \\ 5.000 & 5.000 \\ 2.000 & 2.000 \\ 1.000 & 1.000 \\ 0.000 & 0.000\end{array}$
24

14.993

IDEAL DUTFUT SIG MILLIVOLTS

59.972

83.961 107.950

131.938

155.927

179.916

203.905

227.894

251.882

275.871

299.860

275.871

251.882

227.894

205.905

179.916

155.927

131.938

107.950

83.961

59.972
CAL I BFAATION ACCLFACY

**LIMITED**

MEASURED EFIRTI \% DUTFUT SIG FULL SCALE MILLIVOLTS

62.033
86.036
110.044
134.026
157.963
181.948
205.857
229.809
253.708
277.577
301.414
277.560
253.697
229.787
205.846
181.912
157.960
134.020
110.060
86.047
62.052

$0.86 \%$

$0.87 \%$

$0.87 \%$

$0.87 \%$

$0.85 \%$

$0.85 \%$

$0.81 \%$

$0.80 \%$

$0.76 \%$

$0.71 \%$

$0.65 \%$

$0.70 \%$

$0.79 \%$

$0.81 \%$

$0.8 .5 \%$

$0.85 \%$

$0.87 \%$

$0.88 \%$

$0.87 \%$

$0.87 \%$
$0.76 \%$
$0.32 \%$

OUT

OF

TOLEFIANCE

YES

YES

YES

YES

YES

YES

YES

YES

YES

YES

YES

YES

YES

YES

YES

YES

YES

YES

YES

YES

YES 
DATE: 2-JUN-92

TIME: 11:00:54

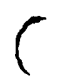

VOLTAGE(V) OBS. DP(PSI) CALC. DP(PSI)

$\begin{array}{llcc}1 & -0.2299854 \mathrm{E}-04 & 0.0000000 \mathrm{E}+00 & -0.9825019 \mathrm{E}-03 \\ 2 & 0.2404500 \mathrm{E}-01 & 1.000000 & 0.9999127 \\ 3 & 0.4808700 \mathrm{E}-01 & 2.000000 & 2.000318 \\ 4 & 0.7208900 \mathrm{E}-01 & 3.000000 & 2.999648 \\ 5 & 0.9606100 \mathrm{E}-01 & 4.000000 & 3.998317 \\ 6 & 0.1200690 & 5.000000 & 4.999074 \\ 7 & 0.1440530 & 6.000000 & 5.999420 \\ 8 & 0.1680490 & 7.000000 & 7.000854 \\ 9 & 0.1920090 & 8.000000 & 8.001373 \\ 10 & 0.2159100 & 9.000000 & 9.000014 \\ 11 & 0.2397930 & 10.00000 & 9.998485 \\ 12 & 0.2159090 & 9.000000 & 8.999972 \\ 13 & 0.1920060 & 8.000000 & 8.001248 \\ 14 & 0.1680460 & 7.000000 & 7.000729 \\ 15 & 0.1440570 & 6.000000 & 5.999587 \\ 16 & 0.1200740 & 5.000000 & 4.999283 \\ 17 & 0.9611000 \mathrm{E}-01 & 4.000000 & 4.000359 \\ 18 & 0.7210400 \mathrm{E}-01 & 3.000000 & 3.000273 \\ 19 & 0.4810700 \mathrm{E}-01 & 2.000000 & 2.001150 \\ 20 & 0.2404900 \mathrm{E}-01 & 1.000000 & 1.000079 \\ 21 & 0.2200146 \mathrm{E}-04 & 0.0000000 \mathrm{E}+00 & 0.8883218 \mathrm{E}-03\end{array}$

DIFF (PSIA)

\% DIFFERENCE

$0.9825019 \mathrm{E}-03 \quad 0.0000000 \mathrm{E}+00$

$0.8732024 E-04$

$0.8732024 E-02$

$-0.3178341 \mathrm{E}-03$

$-0.1589170 \mathrm{E}-01$

$0.3520218 \mathrm{E}-03$

$0.1683112 \mathrm{E}-02$

$0.9256676 \mathrm{E}-03$

$0.5803080 \mathrm{E}-03$

$-0.8540446 \mathrm{E}-03$

$-0.1373305 E-02$

$-0.1355261 \mathrm{E}-04$

$0.1514960 \mathrm{E}-02$

$0.2824197 \mathrm{E}-04$

$-0.1247995 \mathrm{E}-02$

$-0.7288076 \mathrm{E}-03$

$0.4134235 \mathrm{E}-03$

$0.7171846 \mathrm{E}-03$

$-0.3588205 E-03$

$-0.2726917 \mathrm{E}-03$

$-0.1150295 \mathrm{E}-02$

$-0.7907353 \mathrm{E}-04$

$0.1173406 \mathrm{E}-01$

$0.4207779 E-01$

$0.1851335 \mathrm{E}-01$

$0.9671800 \mathrm{E}-02$

$-0.1220064 E-01$

$-0.1716631 \mathrm{E}-01$

$-0.1505845 \mathrm{E}-03$

$0.1514960 E-01$

$0.3137997 E-03$

$-0.1559993 E-01$

$-0.1041154 E-01$
$0.6890392 E-02$

$0.1434369 \mathrm{E}-01$

$-0.8970513 E-02$

$-0.9089722 E-02$

$-0.5751473 \mathrm{E}-01$

$-0.8883218 \mathrm{E}-03$

$-0.7907353 E-02$

$0.0000000 E+00$

- DEGREE OF POLYNOMIAL 2

C JEFFICIENTS

$-0.26364184 E-04 \quad 0.41573860 E+02 \quad 0.51113723 E+00$

ESS $=0.1523 \mathrm{E}-04 \quad \begin{aligned} \mathrm{SD} & =0.9199 \mathrm{E}-03 \\ 2 * \mathrm{SD} & =0.1840 \mathrm{E}-02\end{aligned} \quad \mathrm{R2}=0.100000 \mathrm{E}+01$ 
RESEARCH DEVELOPIENT DIVISION INSTRUVENT SERVICE LOG

RD-45A ReV. $12-18-91$

\begin{tabular}{|l|l|l|}
\hline INSTRUMENT STATUS & $\begin{array}{c}\text { AS } \\
\text { RICE }\end{array}$ & IET \\
\hline Certified & & \\
\hline Calibrated. & & \\
\hline For Ind. Only & & \\
\hline Standardize & & \\
\hline 5 certified stand. & & \\
\hline 6 calibrated stand. & & \\
\hline 9 other & & \\
\hline
\end{tabular}

INSTRUMENT CONDITION

AS RECEIVED (Certified Equipment only)

Meets Manufacturer's Tolerance $N$

Operates within previously defined limits $\mathrm{N}$ Deviates from $\mathrm{Mfg}$ or previously defíned limits $y$ Inoperative (Describe below) $Y$ AS LEFT Iimited (Describe below) Meets Manufacturer's Tolerance other (Describe Below)
Iroroy

Babcock \& Wilcox

a MCDermost company

B\&W Ser. No. 0880526

MANUFACTURER SENSOTEC

MODEI - 21309-22

ITEM TRANSMITR, D-P

MANUF . SER. NO. 195498

PROPERTY NO. 4427-01-130

ORDER NO.

SECTION 46

IOCATION 115

SCHEDULED $(Y-N) \quad Y$

RECAI INT • (MOS.) 12

TECH. PROCEDURE

Babcock Wilcox RfD Division certifies that the Babcock a Wilcox Ris instrument was verified using performance of this instes are traceable to the standards whose accuracies are traceable to thology. National Institute of standards and rechnology, an accepted value of a natural physical constant a ratio type of calibration technique, or to consensus standart.

\section{STHIJDARDS USED}

Manufacturer

ASHCROFT

FLVKE

0 -30PSI $8840 A$
Item

Bew Serial No.

Bew serial No. \begin{tabular}{r|l|l|l|l|l|l|l|l} 
PRESS, ST2. & 0 & 8 & 1 & 0 & 3 & 4 & 1 & Vendor Cert/Cal/Repair \\
\hline DMM & 0 & 8 & 5 & 0 & 4 & 5 & 4 & Repair Parts \\
\hline
\end{tabular} \begin{tabular}{llllll|l}
0 & 8 & 5 & 0 & 4 & 5
\end{tabular}

TOTAL MATERIAL COST

TOTAL IABOR HOURS 01011.5 Requested BY SCOTT SPROUL Charge Number 44260-102-002 work order No. 0341

\section{OTHER EOUIPMENT USED}

COOPER

DP160 THER MOMETER

\begin{tabular}{|l|l|l|l|l|l|l|}
\hline 0 & 8 & 9 & 0 & 1 & 6 & 6 \\
\hline 2 & 8 & 9 & 0 & 1 & 9 & 4 \\
\hline
\end{tabular}

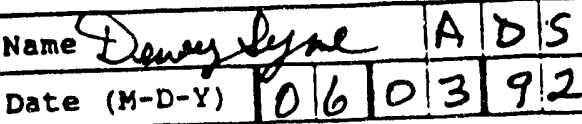
COMPAQ PORTABLE COMPUTER

Reviewed BY

Service Notes RESET ZERe of RECALIBRATED. HAD ZERO SNJET IPDP O4 $0-25$ PSI SEE ATTACHED DATA SHEET. LIMITED TO DATA PROVIDED, DOES NOT MEET H:I RATIO SPESS. 
AS RECEIVED

TRANSMITTEF:

SENSOTEC $Z$

25 F'SI

TOLEFANCE: $0.25 \%$

FRESS SOURICE

$$
\begin{aligned}
& \text { ASHCFOFT } \text { DIGIGAUGE } \\
& 0-30 \text { FSI } \\
& \text { BE.W SER. NO: } 0810341 \\
& \text { TOLEFIANCE: } 0.06 \%
\end{aligned}
$$

READOUT DEV:

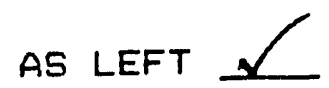

E\&W S.N. 0880526 DATE: $\quad 03-J U \Pi-92$

FFIOCEDURE : ARC-TF-015-08

FRIOGRIAM: T0015_0B.03A

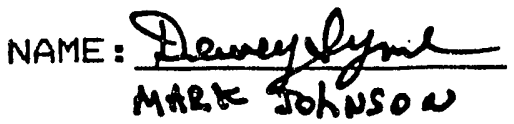

Ambient Temperature (F) 75

Facility I. D. Name

IFDFO4

E\&W Ser. NO. 0850454

TOLERANCE: $0.015 \%$

$$
\begin{aligned}
& 14.996 \text { OHMS } \\
& 14.996 \text { OHMS } \\
& \text { N.A. }
\end{aligned}
$$
FESISTOF MEASUFING ACCURACY 24

FOWEF' SUFFLY VOLTAGE

TOTAL SERIES FESISTANCE

AFFLIED FRESSURE TFIANSMITTEF

F'S I

0.000

2.500

5.000

7.500

10.000

12.500

15.000

17.500

20.000

22.500

25.000

22.500

20.000

17.500

15.000

12.500

10.000

7.500

5.000

2.500

0.000
FRESS SDUFICE

F'SI

0.000

2.500

5.000

7.500

10.000

12.500

15.000

17.500

20.000

22.500

25.000

22.500

20.000

17.500

15.000

12.500

10.000

7.500

5.000

2.500

0.000
14.996

IDEAL OUTFUT SIG MILLIVOLTS

59.984

8.978

107.971

131.965

155.958

179.952

203.946

227.939

251.953

275.926

299.920

275.926

251.935

227.9 .39

205.946

179.952

155.958

131.965

107.971

8. .978

59.984

\section{CAL I BRATION}

MEASUFED OUTFUT SIG MILLIVOLTS

* *LIMITED**

ERROR \%

FULL SCALE

59.942
84.004

108.020

132.049

156.127

180.234

204.239

228.197

252.254

276.132

300.013

276.107

252.256

228.228

204.232

180.254

156.199

1.32 .044

108.076

83.974

59.960

$$
\begin{array}{r}
-0.02 \% \\
0.01 \% \\
0.02 \% \\
0.03 \% \\
0.07 \% \\
0.12 \% \\
0.12 \% \\
0.11 \% \\
0.13 \% \\
0.09 \% \\
0.04 \% \\
0.08 \% \\
0.13 \% \\
0.12 \% \\
0.12 \% \\
0.12 \% \\
0.10 \% \\
0.03 \% \\
0.04 \% \\
0.00 \% \\
-0.01 \%
\end{array}
$$

$0.3 .3 \%$

QUT

OF

TOLEFIANCE 


\begin{tabular}{|c|c|c|c|c|c|}
\hline & $E(V)$ & OBS. DP(PSI) & iI) & IFF & \\
\hline $\begin{array}{l}11 \\
12 \\
12 \\
13 \\
14 \\
15 \\
16 \\
17 \\
18 \\
19 \\
10\end{array}$ & $\begin{array}{l}-0.8999916 \mathrm{E}-05 \\
0.2405300 \mathrm{E}-01 \\
0.4806900 \mathrm{E}-01 \\
0.7209800 \mathrm{E}-01 \\
0.9617600 \mathrm{E}-01 \\
0.1202830 \\
0.1442880 \\
0.1682460 \\
0.1922830 \\
0.2161810 \\
0.2400620 \\
0.2161560 \\
0.1922850 \\
0.1682770 \\
0.1442810 \\
0.1202830 \\
0.9624800 \mathrm{E}-01 \\
0.7209300 \mathrm{E}-01 \\
0.4812500 \mathrm{E}-01 \\
0.2402300 \mathrm{E}-01 \\
0.9000084 \mathrm{E}-05\end{array}$ & $\begin{array}{l}2.500000 \\
5.000000 \\
7.500000 \\
10.00000 \\
12.50000 \\
15.00000 \\
17.50000 \\
20.00000 \\
22.50000 \\
25.00000 \\
22.50000 \\
20.00000 \\
17.50000 \\
15.00000 \\
12.50000 \\
10.00000 \\
7.500000 \\
5.000000 \\
2.500000 \\
0.0000000 \mathrm{E}+00\end{array}$ & $\begin{array}{l}0.4157776 \mathrm{E}-02 \\
2.500937 \\
4.994678 \\
7.491502 \\
9.995156 \\
12.50357 \\
15.00311 \\
17.49947 \\
20.00580 \\
22.49936 \\
24.99286 \\
22.49675 \\
20.00601 \\
17.50270 \\
15.00238 \\
12.50357 \\
10.00265 \\
7.490982 \\
5.000495 \\
2.497823 \\
0.6024886 \mathrm{E}-02\end{array}$ & $\begin{array}{r}-0.4157776 \mathrm{E}-02 \\
-0.9373786 \mathrm{E}-03 \\
0.5322285 \mathrm{E}-02 \\
0.8498427 \mathrm{E}-02 \\
0.4843617 \mathrm{E}-02 \\
-0.3570983 \mathrm{E}-02 \\
-0.3106469 \mathrm{E}-02 \\
0.5263774 \mathrm{E}-03 \\
-0.5804808 \mathrm{E}-02 \\
0.6372504 \mathrm{E}-03 \\
0.7139690 \mathrm{E}-02 \\
0.3246689 \mathrm{E}-02 \\
-0.6013420 \mathrm{E}-02 \\
-0.2704866 \mathrm{E}-02 \\
-0.2377337 \mathrm{E}-02 \\
-0.3570983 \mathrm{E}-02 \\
-0.2645627 \mathrm{E}-02 \\
0.99018151 \mathrm{E}-02 \\
-0.4945902 \mathrm{E}-03 \\
0.2176637 \mathrm{E}-02 \\
-0.6024886 \mathrm{E}-02\end{array}$ & $\begin{array}{r}0.0000000 E+00 \\
-0.3749514 \mathrm{E}-01 \\
0.1064457 \\
0.1133124 \\
0.4843617 \mathrm{E}-01 \\
-0.2856787 \mathrm{E}-01 \\
-0.2070979 \mathrm{E}-01 \\
0.3007871 \mathrm{E}-02 \\
-0.2902404 \mathrm{E}-01 \\
0.283224 \mathrm{E}-02 \\
0.2855876 \mathrm{E}-01 \\
0.1442973 \mathrm{E}-01 \\
-0.3006710 \mathrm{E}-01 \\
-0.1545638 \mathrm{E}-01 \\
-0.1584891 \mathrm{E}-01 \\
-0.2856787 \mathrm{E}-01 \\
-0.2645627 \mathrm{E}-01 \\
0.1202420 \\
-0.9891805 \mathrm{E}-02 \\
0.8706547 \mathrm{E}-01 \\
0.0000000 \mathrm{E}+00\end{array}$ \\
\hline
\end{tabular}

DEGREE OF POLYNOMIAL 2

JEFFICIENTS

$0.50913221 E-02$

$0.10372832 E+03$

$0.15016764 \mathrm{E}+01$

ESS $=0.4520 E-03 \quad \begin{aligned} S D & =0.5011 E-02 \\ 2 * S D & =0.1002 E-01\end{aligned} \quad$ R2 $=0.100000 E+01$ 
RESEARCH C DEVELOPKENT DIVISION INSTRUMENT SERVICE LOG
Babcock \& Wilcox

- McDermort company

\begin{tabular}{|l|l|l|}
\hline INSTRUMENT STATUS & RÉEg & iś \\
\hline 1 Certified & \\
\hline Calibrated & & \\
\hline 3 For Ind. Only & $\checkmark$ & \\
\hline 4 standardize & & \\
\hline 5 Certified \& stand. & & \\
\hline 6 Calibrated a stand. & & \\
\hline 9 other & & \\
\hline
\end{tabular}

INSTRUMENT CONDITION

\begin{tabular}{|c|c|}
\hline Certification & \\
\hline Calibration & \\
\hline Maintenance & \\
\hline Repair & \\
\hline Vendor Cert. & \\
\hline Vendor Calib. & \\
\hline Vendor Repair & \\
\hline other & \\
\hline
\end{tabular}

AS RECEIVED (Certified Equipment only)

Meets Manufacturer's Tolerance $N$

operates within previously defined limits $N$ Deviates from Mfg or previously defined limits $X$ Inoperative (Describe below) $Y$

AS LEFT

\section{Limited (Describe below)}

Meets Manufacturer's Tolerance other (Describe Below)

\section{MANUFACTURER \\ MODEL \\ ITEM}

MANUF. SER. NO.

PROPERTY NO.

ORDER NO.

SECIION

LOCATION

SCHEDUIED ( $Y-N$ )

RECAI INT. (MOS.)
B\&W Ser. No. 0880527

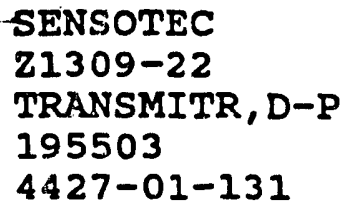

SENSOTEC

Z1309-22

TRANSMITR, D-P

195503

$4427-01-131$

46

115

$-1+y$

12

\section{TECH. PROCEDURE A0015}

Babcock Wilcox RED Division certifies that the performance of this instrument was verified using standards whose accuracies are traceable to the National Institute of standards and Technology, an accepted value of a natural physical constant, a ratio type of calibration technique, or to a consensus standard.

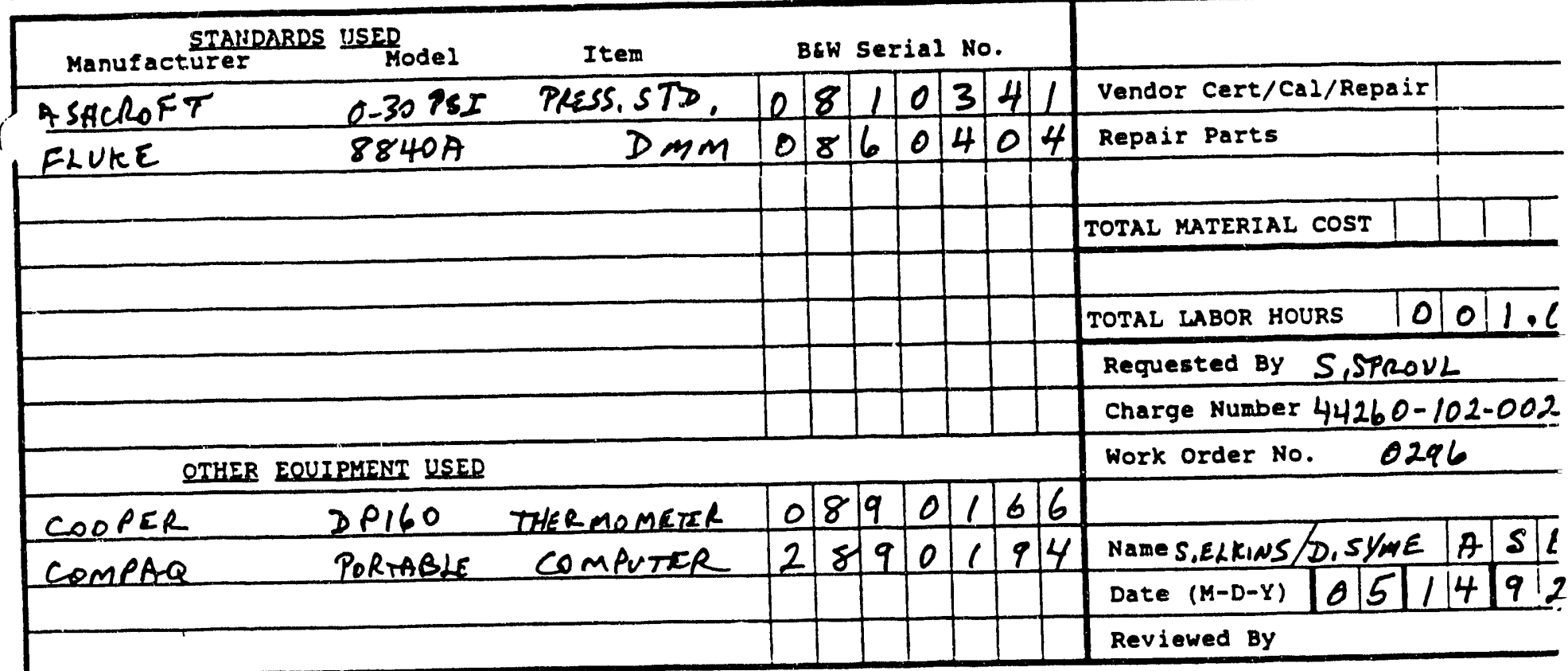

Service Notes $0-25$ PSID, IPDPO5 $14.999 \Omega$

SEE ATACHED DATA SHEET,

LIMITED TO DATA PROVIDED. DOES NOT MEET 4:I RATIO SPES. 
AS RECEIVED

SENSOTEC $Z$

25 F'SI

TOLEFANCE: $0.25 \%$

FRESS SOURECE

$0-30$ F'SI
E\&W Ser. NO. 0810341

ASHCFIOFT DIGIGAUGE

TOLEFIANCE: $0.06 \%$

:EADOUT DEV:

FLUKE 8840A

E\&W SER. NO. 0860404

TOLEFIANCE: $0.015 \%$
AS

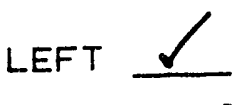

E\&W S.N. OB80527"

DATE: 14-May-92

FROCEDURE : AFIC-TF-015-08

FFIOGRAM: TOO15_08.03A

NAME: S,ELKINS/D, SYME

Ambient Temperature (F) 70

Facility I. D. Name IFDFOS
VALUE OF CUFFENT SENSING FIESISTOF USED FOF TEST DATA

'/ALUE OF CUFIFENT SENSING FESISTOF USED FOF CALIEFIATION FESISTOF MEASUFING ACCURIACY
FOWEF: SUFFLY VOLTAGE

TOTAL SEFIES FESISTANCE

\section{AF'FILIED FFESSURE}

TFIANSMITTEF: F'SI

$\begin{array}{rrr}0.000 & 0.000 & 59.972 \\ 2.500 & 2.500 & 83.961 \\ 5.000 & 5.000 & 107.950 \\ 7.500 & 7.500 & 131.938 \\ 10.000 & 10.000 & 155.927 \\ 12.500 & 12.500 & 179.916 \\ 15.000 & 15.000 & 205.905 \\ 17.500 & 17.500 & 227.894 \\ 20.000 & 20.000 & 251.882 \\ 22.500 & 22.500 & 275.871 \\ 25.000 & 25.000 & 299.860 \\ 22.500 & 22.500 & 275.871 \\ 20.000 & 20.000 & 251.882 \\ 17.500 & 17.500 & 227.894 \\ 15.000 & 15.000 & 20.505 \\ 12.500 & 12.500 & 179.916 \\ 10.000 & 10.000 & 155.927 \\ 7.500 & 7.500 & 151.958 \\ 5.000 & 5.000 & 107.950 \\ 2.500 & 2.500 & 83.961 \\ 0.000 & 0.000 & 59.972\end{array}$

24
14.993

IDEAL OUTFUTT SIG MILLIVOLTS
CAL IEFIATION

MEASUFED OUTFUT SIG MILLIVOLTS

$$
\begin{aligned}
& 14.993 \text { OHMS } \\
& 14.993 \text { OHMS } \\
& \text { N.A. }
\end{aligned}
$$

$\begin{array}{rr}59.975 & 0.00 \% \\ 84.035 & 0.03 \% \\ 108.136 & 0.08 \% \\ 132.211 & 0.11 \% \\ 156.284 & 0.15 \% \\ 180.386 & 0.20 \% \\ 204.356 & 0.19 \% \\ 228.268 & 0.16 \% \\ 252.187 & 0.13 \% \\ 275.986 & 0.05 \% \\ 299.753 & -0.04 \% \\ 275.906 & 0.01 \% \\ 252.080 & 0.08 \% \\ 228.165 & 0.11 \% \\ 204.245 & 0.14 \% \\ 180.288 & 0.16 \% \\ 156.278 & 0.15 \% \\ 132.159 & 0.09 \% \\ 108.115 & 0.07 \% \\ 84.027 & 0.03 \% \\ 59.570 & 0.00 \%\end{array}$

$\bar{z}=57.973$ 
DATE: 20-MAY -92 TIME: $16: 36: 00$

$-$

\begin{tabular}{|c|c|c|c|c|c|}
\hline & VOLTAGE(V) & BS. DP (PSI) & CALC. DP (PSI) & DIFF (PSIA) & \& DIFFERENCE \\
\hline $\begin{array}{l}1 \\
2 \\
3 \\
4 \\
5 \\
6 \\
7 \\
8\end{array}$ & $\begin{array}{l}0.1998520 \mathrm{E}-05 \\
0.2406000 \mathrm{E}-01 \\
0.4816300 \mathrm{E}-01 \\
0.7223800 \mathrm{E}-01 \\
0.9631100 \mathrm{E}-01 \\
0.1204130 \\
0.1443830 \\
0.1682950 \\
0.1922140 \\
0.2160130 \\
0.2397800 \\
0.2159330 \\
0.1921070 \\
0.1681920 \\
0.1442700 \\
0.1203150 \\
0.9630500 \mathrm{E}-01 \\
0.7218600 \mathrm{E}-01 \\
0.4814000 \mathrm{E}-01 \\
0.2405400 \mathrm{E}-01 \\
-0.3001480 \mathrm{E}-05\end{array}$ & $\begin{array}{l}00 \\
00 \\
00 \\
00 \\
00 \\
00 \\
00 \\
00 \\
00 \\
00 \\
00 \\
00 \\
00 \\
00 \\
00 \\
00 \\
00 \\
00 \\
00 \\
0 O E+00\end{array}$ & $\begin{array}{l}1 E-02 \\
4 \\
7 \\
2 \\
4 \\
6 \\
8 \\
2 \\
6 \\
8 \\
8 \\
0 \\
6 \\
6 \\
9 \\
5 \\
9 \\
9 \\
0 \\
2 \\
4 E-02\end{array}$ & $\begin{array}{r}-0.70386 \\
0.24160 \\
0.34932 \\
0.37575 \\
0.51613 \\
-0.94606 \\
-0.93825 \\
-0.69234 \\
-0.88607 \\
-0.18842\end{array}$ & $\begin{array}{l}0000 E+00 \\
4034 E-01 \\
6468 E-01 \\
0000 E-01 \\
1397 E-02 \\
8516 E-01 \\
5030 E-01 \\
6275 E-01 \\
0396 E-01 \\
4621 E-02 \\
9017 E-01 \\
9801 E-01 \\
9808 E-01 \\
5677 E-01 \\
7526 E-01 \\
0712 E-02 \\
0515 E-01 \\
1430 \\
5915 \\
5042 \\
0000 E+00\end{array}$ \\
\hline
\end{tabular}

- DEGREE OF POLYNOMIAL 2

(TEFFICIENTS

$0.68319136 \mathrm{E}-02 \quad 0.10344546 \mathrm{E}+03 \quad 0.32036882 \mathrm{E}+01$

ESS $=0.6532 \mathrm{E}-03 \quad \begin{aligned} S D & =0.6024 \mathrm{E}-02 \\ 2 * S D & =0.1205 \mathrm{E}-01\end{aligned} \quad \mathrm{R2}=0.999999 \mathrm{E}+00$


$\cdot$

RD-45A Rev. $11-18-91$

\begin{tabular}{|l|l|l|}
\hline INSTRUMENT STATUS & RISE & AS \\
\hline Certified & & \\
\hline Calibrated & & \\
\hline For Ind. Only & & \\
\hline Standardize & & \\
\hline Certified i stand. & & \\
\hline Calibrated c stand. & & \\
\hline Other & & \\
\hline
\end{tabular}

INSTRUMENT CONDITION
RESERRCH \& DEVELOPAENT DIVISION INSTRUTENT SERVICE IOG

\section{IDPPO 6 \\ Babcock \& Wilcox \\ - McDermott company}

B\&W Ser. No. 0880528

MANUFACTURER SENSOTEC

MODEL

ITEM

21309-22

TRANSMITR, D-P

MANUP. NO. 201803

PROPERTY NO.

4427-01-132

ORDER NO.

SECTION 46

LOCATION 115

SCHEDULED $(Y-N) \quad Y$

RECAI INT. (MOS.) 12

TECH. PROCEDURE

Babcock \&ilcox RED Division certifies that the performance of this instrument was verified using standards whose accuracies are traceable to the
National Institute of standards and Technology. an accepted value of a natural physical constant a ratio type of calibration technique, or to a consensus standard.

Meets Manufacturer's Tolerance N

Operates within previously defined limits $N$ eviates from Mfg or previously defined limits $Y$ Inoperative (Describe below) $x$ Meets Manufacturer's rolerance other (Describe Below)

\section{Limited (Describe below)}

.

Item BCW Serial No.

STAIIDARDS USED

Manufacturer Model

ASHCROFT $0-30$ PSI PeES, STD. $8840 A$

\begin{tabular}{|l|l|l|l|l|l|l|}
\hline 0 & 8 & 1 & 0 & 3 & 4 & 1 \\
0 & 8 & 5 & 0 & 4 & 5 & 4 \\
\hline
\end{tabular}

Vendor Cert/Cal/Repair

Repair Parts

TOTAL MATERIAL COST

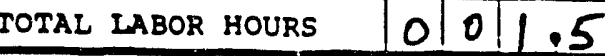

Requested BY SLOTT SPROVL

Charge Number 44260-102-002

Work Order No. 0341

OTHER EQUIPMENT USED

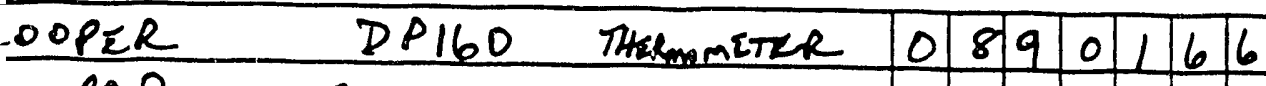

IMPAQ PORTABLE COMPUTRR

\begin{tabular}{ll|l|l|l|l}
1 & 8 & 9 & 0 & 1 & 9
\end{tabular}

Name Deweyefyre $A \cdot D \mid S$

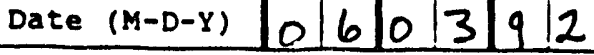
Reviewed By

Ivice Notes RESET ZFRO \& RELALIBRATED, HAD ZRRO SHIFT, IPDPO6 -25 PSI SEE ATACHED DATA SHEET, 1 MITUS TR PATA PROVIDED, DOES NOT MEET 4:1 RATTO SPECS, 
AS RECEIVED

SENSOTEC $Z$

$25 \mathrm{~F} \cdot \mathrm{SI}$

TOLEFIANCE: $0.25 \%$

FRESS SQUFICE

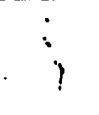

READOUT' DEV:

\author{
ASHCFIFT DIGIGAUGE \\ 0-30 FSI \\ B\&WW Ser. No. 0810:41 \\ TOLEFANCE: $0.06 \%$
}

FLUKE $8840 A$
B\&W SER. NO. 0850454
TOLEFANCE: $0.015 \%$
AS LEFT

E\&W S.N. 0880528

DATE: $\quad 03-J 4 I-92$

FROCEDURE : ARC-TF-015-08

FFIOGFAM: T0015_08.03A

NAME: Douref Byme

Ambient Temperature (F) 75
Facility I. D. Name

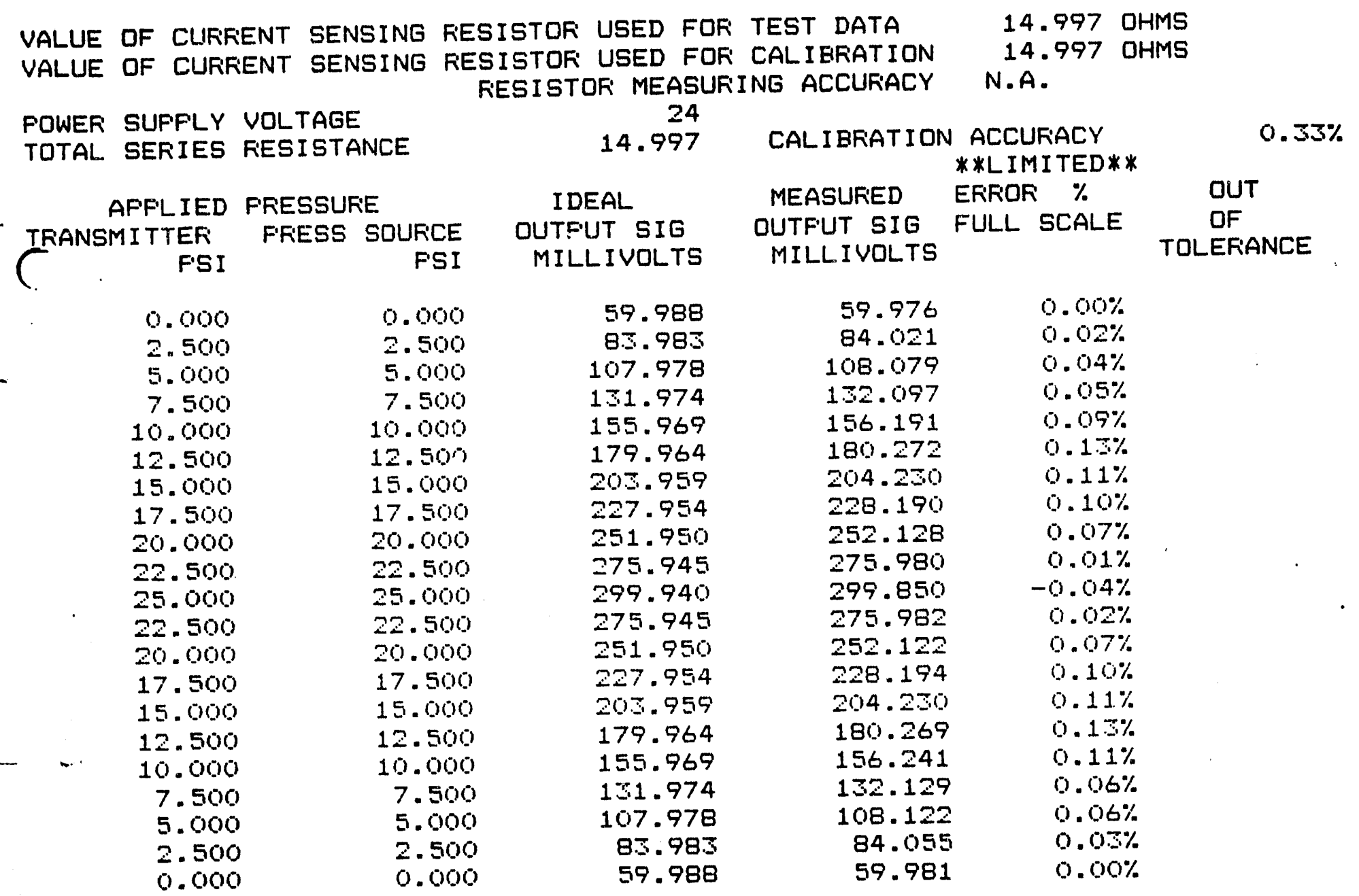




\begin{tabular}{|c|c|c|c|c|c|}
\hline & VOLTAGE (V) & OBS. DP (PSI) & CALC. DP (PSI) & DIFF (PSIA) & \& DIFFERENCE \\
\hline $\begin{array}{c}1 \\
2 \\
3 \\
4 \\
5 \\
6 \\
7 \\
8 \\
9 \\
10 \\
11 \\
12 \\
13 \\
14 \\
15 \\
16 \\
17 \\
18 \\
19 \\
20\end{array}$ & $\begin{array}{l}-0.2999197 \mathrm{E}-05 \\
0.2404200 \mathrm{E}-01 \\
0.4810000 \mathrm{E}-01 \\
0.7211800 \mathrm{E}-01 \\
0.9621200 \mathrm{E}-01 \\
0.1202930 \\
0.1442510 \\
0.1682110 \\
0.1921490 \\
0.2160010 \\
0.2398710 \\
0.2160030 \\
0.1921430 \\
0.1682150 \\
0.1442510 \\
0.1202900 \\
0.9626200 \mathrm{E}-01 \\
0.7215000 \mathrm{E}-01 \\
0.4814300 \mathrm{E}-01 \\
0.2407600 \mathrm{E}-01 \\
0.2000803 \mathrm{E}-05\end{array}$ & $\begin{array}{l}0.0000000 \mathrm{E}+00 \\
2.500000 \\
5.000000 \\
7.500000 \\
10.00000 \\
12.50000 \\
15.00000 \\
17.50000 \\
20.00000 \\
22.50000 \\
25.00000 \\
22.50000 \\
20.00000 \\
17.50000 \\
15.00000 \\
12.50000 \\
10.00000 \\
7.500000 \\
5.000000 \\
2.500000 \\
0.0000000 \mathrm{E}+00\end{array}$ & $\begin{array}{l}0.4433861 \mathrm{E}-02 \\
2.497615 \\
4.994821 \\
7.490546 \\
9.996850 \\
12.50449 \\
15.00197 \\
17.50233 \\
20.00304 \\
22.49740 \\
24.99628 \\
22.49761 \\
20.00241 \\
17.50275 \\
15.00197 \\
12.50417 \\
10.00205 \\
7.493873 \\
4.999287 \\
2.501142 \\
0.4952024 \mathrm{E}-02\end{array}$ & $\begin{array}{r}-0.4433861 \mathrm{E}-02 \\
0.2384979 \mathrm{E}-02 \\
0.5178856 \mathrm{E}-02 \\
0.9453641 \mathrm{E}-02 \\
0.3149694 \mathrm{E}-02 \\
-0.4485556 \mathrm{E}-02 \\
-0.1974749 \mathrm{E}-02 \\
-0.2328290 \mathrm{E}-02 \\
-0.3038327 \mathrm{E}-02 \\
0.2598895 \mathrm{E}-02 \\
0.3718677 \mathrm{E}-02 \\
0.2389632 \mathrm{E}-02 \\
-0.2411199 \mathrm{E}-02 \\
-0.2745933 \mathrm{E}-02 \\
-0.1974749 \mathrm{E}-02 \\
-0.4172989 \mathrm{E}-02 \\
-0.2054194 \mathrm{E}-02 \\
0.6126721 \mathrm{E}-02 \\
0.7130842 \mathrm{E}-03 \\
-0.1142311 \mathrm{E}-02 \\
-0.4952024 \mathrm{E}-02\end{array}$ & $\begin{array}{r}0.0000000 E+00 \\
0.9539918 \mathrm{E}-01 \\
0.1035771 \\
0.1260486 \\
0.3149694 \mathrm{E}-01 \\
-0.3588445 \mathrm{E}-01 \\
-0.1316499 \mathrm{E}-01 \\
-0.1330451 \mathrm{E}-01 \\
-0.1519164 \mathrm{E}-01 \\
0.1155065 \mathrm{E}-01 \\
0.1487471 \mathrm{E}-01 \\
0.1062058 \mathrm{E}-01 \\
-0.1205599 \mathrm{E}-01 \\
-0.1569104 \mathrm{E}-01 \\
-0.1316499 \mathrm{E}-01 \\
-0.3338391 \mathrm{E}-01 \\
-0.2054194 \mathrm{E}-01 \\
0.8168962 \mathrm{E}-01 \\
0.1426168 \mathrm{E}-01 \\
-0.4569244 \mathrm{E}-01 \\
0.0000000 \mathrm{E}+00\end{array}$ \\
\hline
\end{tabular}

\section{DEGREE OF POLYNOMIAL 2}

JEFFICIENTS

$0.47446754 \mathrm{E}-02 \quad 0.10363253 \mathrm{E}+03 \quad 0.23132344 \mathrm{E}+01$
ESS $=0.3192 \mathrm{E}-03$
$S D=0.4211 \mathrm{E}-02$
$R 2=0.100000 E+01$ 
RD-45A Rev. $11-18-91$

\begin{tabular}{|l|l|l|}
\hline \multicolumn{1}{|c|}{ INSTRUMENT STATUS } & Rr.en & AS \\
\hline 1 Certified \\
\hline Calibrated & & \\
\hline 3 For Ind. Only & & $\checkmark$ \\
\hline 4 standardize & & \\
\hline 5 certified stand. & & \\
\hline 6 calibrated stand. & & \\
\hline 9 other & & \\
\hline
\end{tabular}

INSTRUMENT CONDITION

AS RECEIVED (Certified Equiprent only)

Meets Manufacturer's Tolerance $N$

Operates within previously defined IImits $N$ Deviates from Mfg or previously defined limits $Y$ Inoperative (Describe below) $x$ AS LEFI Limited (Describe below)

Meets Manufacturer's Tolerance other (Describe Below) $|r|$ Hri $\mid$

Babcock \&Wilcox

- McDermotl company

B\&W Ser. No. 0880529

MANUFACTURER

SENSOTEC

MODEL

21309-22

ITEM

TRANSMITR, D-P

KANUF. SER. NO. 201804
PROPERTY NO.

ORDER NO.

SECTION

LOCATION

SCHEDULED ( $Y-N$ )

RECAL INT. (MOS.)

$4427-01-133$

\section{6 \\ 115 \\ $-1+Y$ \\ 12}

\section{TECH. PROCEDURE A0015}

Babcock W Wilcox RGD Division certifies that the performance of this instrument was verified using standards whose accuracies are traceable to the National Institute of standards and Technology, an accopted value of a natural physical constant, a ratio type of calibration technique, or to a consensus standard.
STAIDARDS USED

Manufacturer

ISACROFT

ELVKE
Item BCW serial No.

PRES, STP. $0-30$ PSI $8840 \mathrm{~A}$ $D M M$

\begin{tabular}{|l|l|l|l|l|l|l|l|}
\hline 0 & 8 & 1 & 0 & 3 & 4 & 1 & Vendor Cert/Cal/Repair \\
\hline 0 & 8 & 6 & 0 & 4 & 0 & 4 & Repair Parts \\
\hline
\end{tabular}

TOTAL MATERIAL COST

TOTAL LABOR HOURS $\quad 0$ O 011.0 Re quested By $S$, SProl/ Charge Number 44/260-102-002 Work order No. 0296

OTHER EOUTPHENT USED

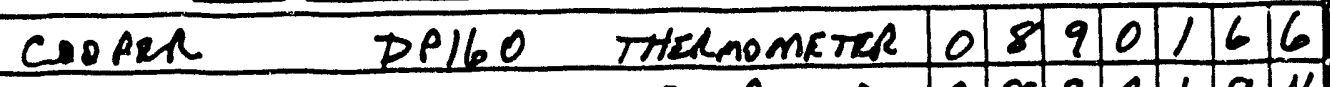
compaQ rortable compvTER

service Notes $0-25$ PSID, TFD POI ADS $4999 \Omega 14.995 \Omega$ SEE ATTACHED DATA SHELT, LIMITED TO DATA PROVIDED, DOES NOT MEET $4: 1$ RATIO SPECS. 
AS FECEIVED

FANSMITTEF:

ESS SQUFICE

EADOUT DEV:
AS

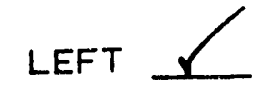

B\&W S.N. 0880529 DATE: 14-Maÿ-92 FROCEDURE : ARC-TF-015-08 FFIOGFIAM: TOO15_08.03A

NAME : S,ELK,NS/S, STME

Ambient Temperature (F) 70

Facility I. D. Name

B\&W SET. NO. 0860404

TOLERANCE: $0.015 \%$

JALLIE OF CUFFENT SENSING FESISTOF USED FOF TEST DATA

ALUE OF CUFRENT SENSING FESISTOF USED FOF CALIBFATION

FOWEF: SUFF'LY VOLTAGE

TOTAL SEFIIES FESISTANCE

AFFLIED F'FESSURE TFANSMITTEF F'FESS SOUFICE FSI

$$
\begin{array}{r}
0.000 \\
2.500 \\
5.000 \\
7.500 \\
10.000 \\
12.500 \\
15.000 \\
17.500 \\
20.000 \\
22.500 \\
25.000 \\
22.500 \\
20.000 \\
17.500 \\
15.000 \\
12.500 \\
10.000 \\
7.500 \\
5.000 \\
2.500 \\
0.000
\end{array}
$$
FESISTOF MEASURING ACCUFACY
24

14.995

IDEAL OUTFUT SIG MILLIVOLTS

59.980
83.972
107.964
131.956
155.948
179.940
205.932
227.924
251.916
275.908
299.900
275.908
251.916
227.924
203.932
179.790
155.748
1.1 .956
107.964
85.972
59.980
DUTFUT SIG
CALIBFIATION ACCUFIACY **LIMITED** MEASUFED MILLIVOLTS

$$
0.33
$$

OUT

DF TOLEFANCE

$\begin{array}{rr}59.996 & 0.01 \% \\ 84.071 & 0.04 \% \\ 108.055 & 0.04 \% \\ 132.099 & 0.06 \% \\ 156.109 & 0.07 \% \\ 180.203 & 0.11 \% \\ 204.141 & 0.09 \% \\ 228.115 & 0.08 \% \\ 252.038 & 0.05 \% \\ 275.939 & 0.01 \% \\ 299.796 & -0.04 \% \\ 275.757 & -0.06 \% \\ 251.949 & 0.01 \% \\ 227.999 & 0.03 \% \\ 204.022 & 0.04 \% \\ 180.071 & 0.05 \% \\ 150.069 & 0.05 \% \\ 151.996 & 0.02 \% \\ 108.009 & 0.02 \% \\ 83.961 & 0.00 \% \\ 59.975 & 0.00 \%\end{array}$

$\bar{z}=59.986$ 
CURVEFIT OUTPUT FOR VTAB tfAPOI

DATE: 20-MAY-92

TIME: $16: 33: 18$

\begin{tabular}{|c|c|c|c|c|c|}
\hline & VOLI & DP (PSI) & $P$ (PSI) & SIA) & IFFERENCE \\
\hline $\begin{array}{r}1 \\
2 \\
3 \\
4 \\
5 \\
6 \\
7 \\
8 \\
9 \\
10 \\
11 \\
12 \\
13 \\
14 \\
15 \\
16 \\
17 \\
18 \\
19\end{array}$ & $\begin{array}{l}0.1000098 \mathrm{E}-04 \\
0.2408500 \mathrm{E}-01 \\
0.4806900 \mathrm{E}-01 \\
0.7211300 \mathrm{E}-01 \\
0.9612300 \mathrm{E}-01 \\
0.1202170 \\
0.1441550 \\
0.1681290 \\
0.1920520 \\
0.2159530 \\
0.2398100 \\
0.2157710 \\
0.1919630 \\
0.1680130 \\
0.1440360 \\
0.1200850 \\
0.9608300 \mathrm{E}-01 \\
0.7201000 \mathrm{E}-01 \\
0.4802300 \mathrm{E}-01 \\
0.2397500 \mathrm{E}-01 \\
-0.1099902 \mathrm{E}-04\end{array}$ & $\begin{array}{l}0.0000000 \mathrm{E}+00 \\
2.500000 \\
5.000000 \\
7.500000 \\
10.00000 \\
12.50000 \\
15.00000 \\
17.50000 \\
20.00000 \\
22.50000 \\
25.00000 \\
22.50000 \\
20.00000 \\
17.50000 \\
15.00000 \\
12.50000 \\
10.00000 \\
7.500000 \\
5.000000 \\
2.500000\end{array}$ & $\begin{array}{l}0.4584974 \mathrm{E}-02 \\
2.505641 \\
4.999105 \\
7.500673 \\
10.00057 \\
12.51108 \\
15.00720 \\
17.50892 \\
20.00718 \\
22.50498 \\
25.00003 \\
22.48595 \\
19.99788 \\
17.49681 \\
14.99478 \\
12.49732 \\
9.996401 \\
7.489953 \\
4.994321 \\
2.494209\end{array}$ & $\begin{array}{r}-0.4584974 \mathrm{E}-02 \\
-0.5640680 \mathrm{E}-02 \\
0.8949766 \mathrm{E}-03 \\
-0.6729063 \mathrm{E}-03 \\
-0.5674768 \mathrm{E}-03 \\
-0.1108059 \mathrm{E}-01 \\
-0.7196686 \mathrm{E}-02 \\
-0.7196820 \\
-0.892446 \mathrm{E}-02 \\
-0.7177541 \mathrm{E}-02 \\
-0.4981944 \mathrm{E}-02 \\
-0.2887305 \mathrm{E}-04 \\
0.1404517 \mathrm{E}-01 \\
0.2120068 \mathrm{E}-02 \\
0.3186813 \mathrm{E}-02 \\
0.5216525 \mathrm{E}-02 \\
0.2678471 \mathrm{E}-02 \\
0.3598828 \mathrm{E}-02 \\
0.1004733 \mathrm{E}-01 \\
0.5679084 \mathrm{E}-02 \\
0.5791041 \mathrm{E}-02 \\
-0.2404185 \mathrm{E}-02\end{array}$ & $\begin{array}{l}0.0000000 \mathrm{E}+00 \\
-0.2256272 \\
0.1789953 \mathrm{E}-01 \\
-0.8972084 \mathrm{E}-02 \\
-0.5674768 \mathrm{E}-02 \\
-0.8864474 \mathrm{E}-01 \\
-0.4797791 \mathrm{E}-01 \\
-0.5098541 \mathrm{E}-01 \\
-0.3588770 \mathrm{E}-01 \\
-0.2214197 \mathrm{E}-01 \\
-0.1154922 \mathrm{E}-03 \\
0.6242299 \mathrm{E}-01 \\
0.1060034 \mathrm{E}-01 \\
0.1821036 \mathrm{E}-01 \\
0.3477683 \mathrm{E}-01 \\
0.2442776 \mathrm{E}-01 \\
0.3598828 \mathrm{E}-01 \\
0.1339644 \\
0.1135817 \\
0.2316416 \\
0.0000000 \mathrm{E}+00\end{array}$ \\
\hline
\end{tabular}

DEGREE OF POLYNOMIAL 2

(OEFFICIENTS

J.35464012E-02 $0.10384708 E+03 \quad 0.16156422 E+01$

$\begin{aligned} \text { ESS }=0.8167 E-03 \quad R D & =0.6736 E-02 \\ 2 * S D & =0.1347 \mathrm{E}-01\end{aligned} \quad$ R2 $=0.999999 \mathrm{E}+00$ 
. 1

RD-45A Rev. $11-18-91$

\begin{tabular}{|c|c|}
\hline INSTRUMENT STATUS & 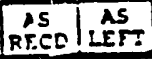 \\
\hline 1 Certified & \\
\hline Cadibrated & \\
\hline 3 For Ind. Only & $\sqrt{ }$ \\
\hline 4 Standardize & \\
\hline 5 certified stand. & \\
\hline 6 Calibrated stand. & \\
\hline 9 other & \\
\hline
\end{tabular}

INSTRUMENT CONDITION

AS RECEIVED (Certified Equipment only)

Meets Manufacturer's Tolerance $N$

operates within previously defined limits $N$ (Deviates from Mfg or previously defined limits $Y$ Inoperative (Describe below) $Y$

AS IEFT limited (Describe below)

Meets Manufacturer's Tolerance
Babcock \& Wilcox

- MCDermoti company

B\&W Ser. No. 0880535

MANUFACTURER

MODEL

ITEM

MANUF. SER. NO.

PROPERTY NO.

ORDER NO.

SECTION

IOCATION

SCHEDULED ( $Y-N)$

RECAI INT. (MOS.)
SENSOTEC

21311-03

TRANSMITR, D-P

200018

4427-01-139

\section{6 \\ $-4 y$ \\ 12}

TECH. PROCEDURE A0015

Babcock Wilcox RED Division certifies that the babcock wance of this instrument was verified using performance of this insies are traceable to the standards whose accuracies are traceable to the National Institute of standards and Technology, an accepted value of a natural physical constan a ratio type of calibration technique, or to consensus standard.

other (Describe Below)

STANDARDS USED

Manufacturer

Hodel Item BtW serial No.

ASHCROFT

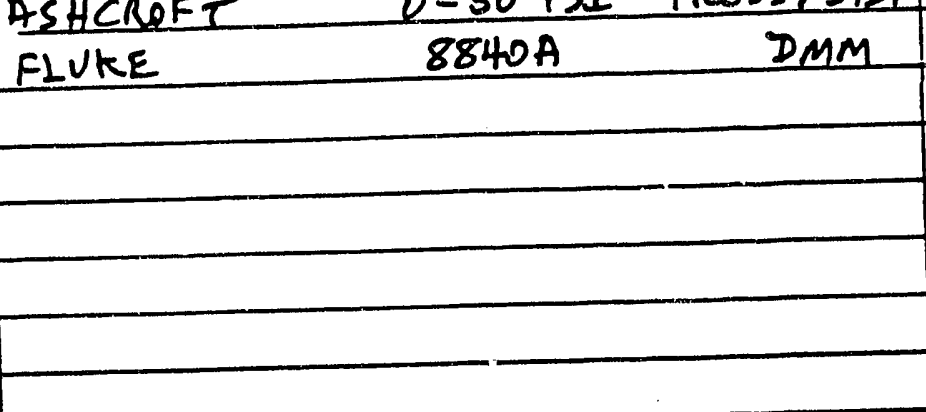

$\frac{0-50 \text { PSI }}{8840 \mathrm{~A}}$

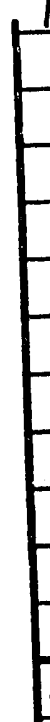

OTHER EOUIPYENT USED

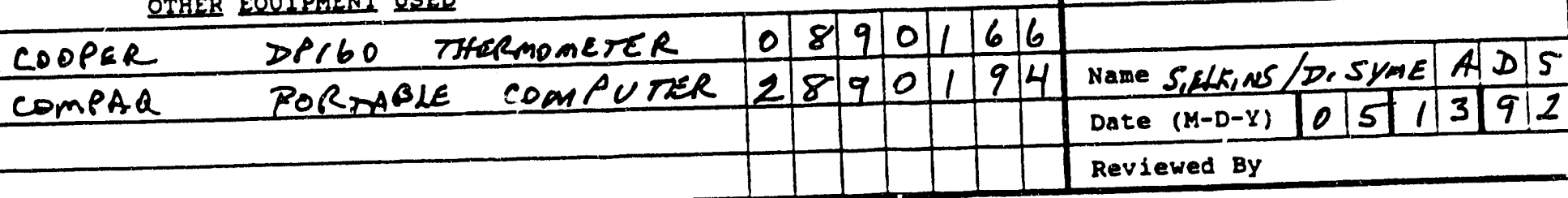

Service notes $0-50$ PSID, TFPPO2 $15.003 \Omega$

SEE DTTACHEP PATA SHEET.

L.IMITED TO DATA PROVIDED, DOES NoT MEA 4:1 RATO SPECS. 
AS RECEIVED

SENSOTEC $Z$

50 FSI

TOLEFANCE : $0.25 \%$

PRESS SOURCE

$\begin{aligned} \text { ASHCFIFT } & \text { DIGIGAUGE } \\ \text { O-5O } & \text { FSI } \\ \text { B\&W SER. NO. } & 0740199\end{aligned}$

TOLEFIANCE: $0.05 \%$

READOUT DEV:

FLUKEE 8840A

E\&WW SER. NO. O860404

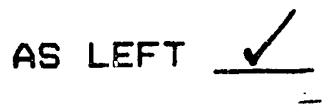

B\&W S.N. OB80535

DATE: 13-May-92

PROCEDURE : ARC-TP-O15-OB

PRIOGFIAM: T0015_08.03A

NAME: S,ELk,NS/D, SYME

Ambient Temperature

Facility I. D. Name

(F) 72

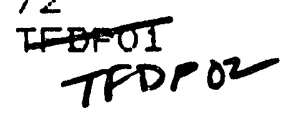

VALUE OF CURFENT SENSING FIESISTOF USED FOR TEST DATA VALUE OF CUFFENT SENSING RESISTOF USED FOF CALIERATION FOWEF SUFFLY VOLTAGE

TOTAL SEFIES RESISTANCE

$$
\text { AF'FLIED F'FESSURE }
$$

TFANSMITTEF: F'SI

F'RESS SOUFICE FSI

$\begin{array}{rr}0.000 & 0.000 \\ 5.000 & 5.000 \\ 10.000 & 10.000 \\ 15.000 & 15.000 \\ 20.000 & 20.000 \\ 25.000 & 25.000 \\ 30.000 & 30.000 \\ 35.000 & 35.000 \\ 40.000 & 40.000 \\ 45.000 & 45.000 \\ 50.000 & 50.000 \\ 45.000 & 45.000 \\ 40.000 & 40.000 \\ 35.000 & 35.000 \\ 30.000 & 30.000 \\ 25.000 & 25.000 \\ 20.000 & 20.000 \\ 15.000 & 15.000 \\ 10.000 & 10.000 \\ 5.000 & 5.000 \\ 0.000 & 0.000\end{array}$

FESISTOF: MEASURING ACCURACY 24 15.003

IDEAL QUTFUT SIG MILLIVOLTS
60.012
84.017
108.022
1.2 .026
156.031
180.036
204.041
228.046
252.050
276.055
300.060
276.055
252.050
228.046
204.041
180.036
156.031
132.026
108.022
84.017
60.012

$15.00 \mathrm{~S}$ OHMS 15.003 OHMS N.A.

$\begin{array}{ccc}\text { CALIEFATION ACCUFACY } & \\ & \text { **LIMITED** } & \\ \text { MEASURED } & \text { ERROR \% } & 0.32 \% \\ \text { OUTFUT SIG } & \text { FULL SCALE } & \text { OUT } \\ \text { MILLIVOLTS } & & \text { TOLERANCE }\end{array}$

$$
\bar{z}=60.027
$$




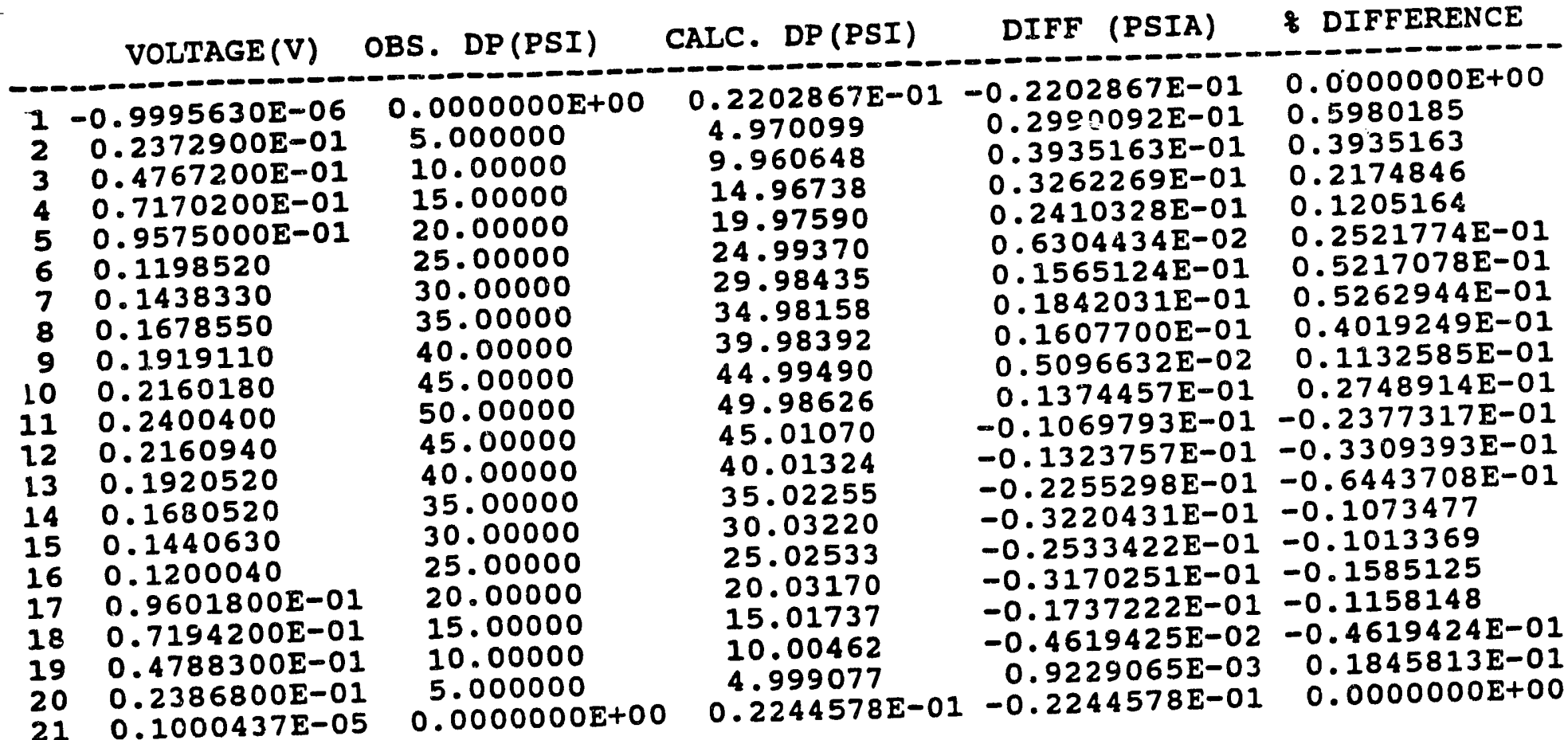

$10.1000437 \mathrm{E}-05$

DEGREE OF POLYNOMIAL 2

$\left(\begin{array}{l}\text { OEFFICIENTS } \\ \text { J.22237132E-01 } \\ 0.20855562 \mathrm{E}+03-0.16951483 \mathrm{E}+01\end{array}\right.$
ESS $=0.9981 \mathrm{E}-02 \quad \begin{aligned} \text { SD } & =0.2355 \mathrm{E}-01 \\ 2 \star S D & =0.4709 \mathrm{E}-01\end{aligned} \quad \mathrm{R} 2=0.999998 \mathrm{E}+00$




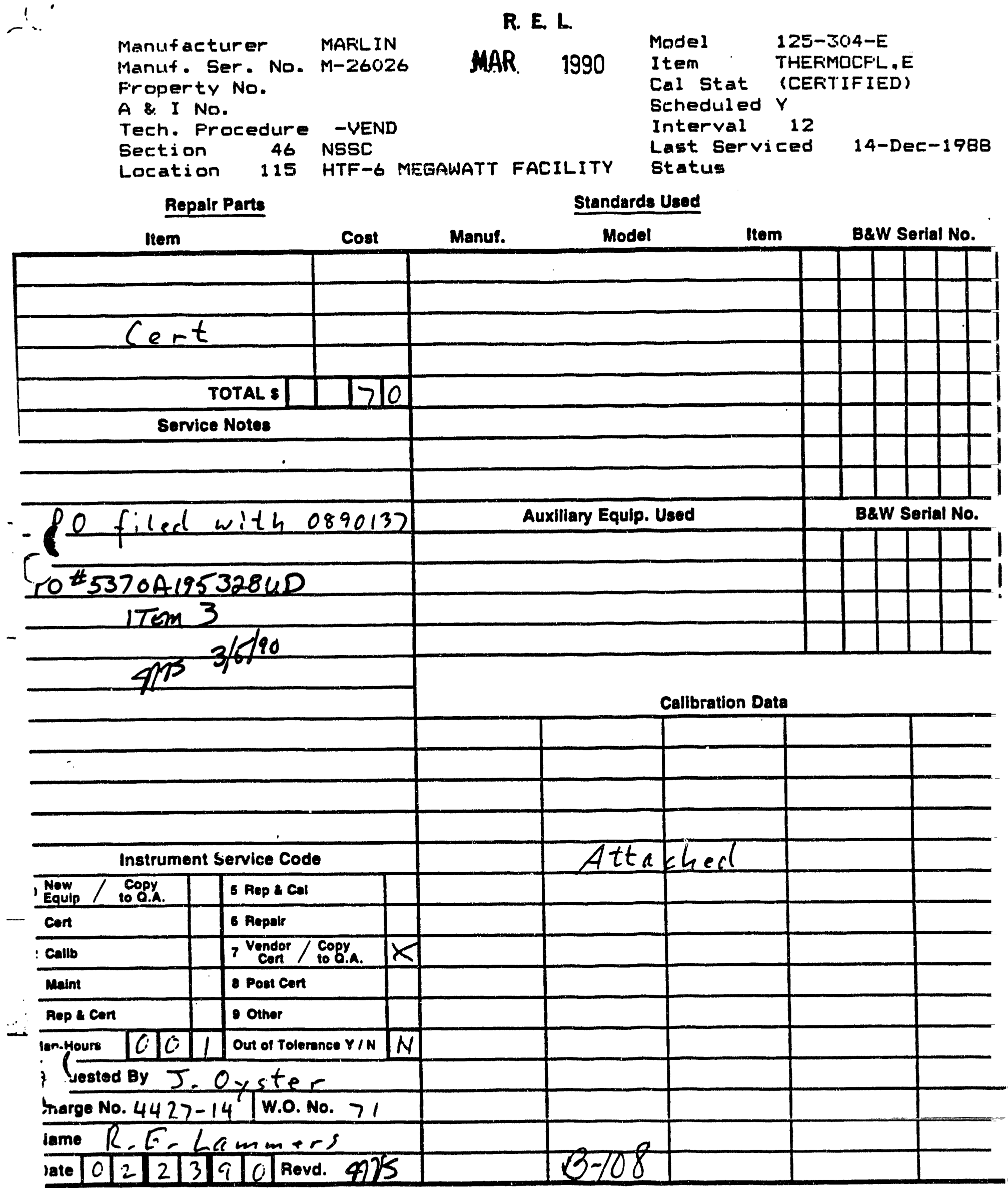


MARLIN JOB NO.

oNBS - Draceable

CERTIFICATION No.

853400

Certification

$M-33321$

BAECOCK \& WILCOX COMPANY

P.0.5370A195328

Cat. No. 125-304-E-1U-0-T (48')-2FF-15'

Thermocouple

REFERENCE M-26026

TEMP $M-33321$ Change firem $M-26026$

-F DEV, F E (volts)

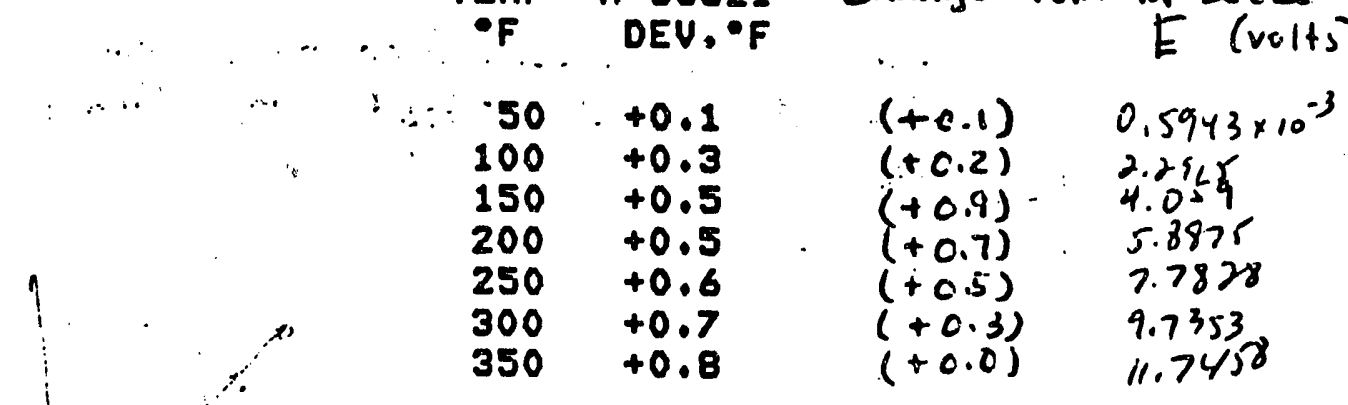

STANDARD: TYPE N N.I.S.T. 244669A

DATED: $09 / 19 / 89$

FFOCEDURE: MCF 103

CONDITIONS: AMBIENT 75 F $(+/-2)$

REV. 1: $08 / 25 / 87$

CONFORMS TO: MIL-STD-45662

R.H. $<50 \%$

Rev. 08/01/88

$\frac{-1}{\operatorname{ses}}$ 
CURVEFIT OUTPUT FOR VTAB iptC01

DATE: 26-MAY-92

TIME: $14: 38: 23$

\begin{tabular}{|c|c|c|c|c|c|}
\hline & VOLI & OBS. & AI & $\mathbf{E}$ & FFE \\
\hline & $\begin{array}{l}0.5943000 \mathrm{E}-03 \\
0.2291500 \mathrm{E}-02 \\
0.4059000 \mathrm{E}-02 \\
0.5887500 \mathrm{E}-02 \\
0.7782800 \mathrm{E}-02 \\
0.9735300 \mathrm{O}-02 \\
0.1174580 \mathrm{D}-01\end{array}$ & $\begin{array}{l}150.0000 \\
200.0000 \\
250.0000 \\
300.0000 \\
350.0000\end{array}$ & $\begin{array}{l}50.00411 \\
99.98105 \\
150.0351 \\
199.9677 \\
250.0147 \\
299.9975 \\
349.9999\end{array}$ & $\begin{array}{r}-0.4106501 \mathrm{E}-02 \\
0.1895481 \mathrm{E}-01 \\
-0.3505160 \mathrm{E}-01 \\
0.3227923 \mathrm{E}-01 \\
-0.1468064 \mathrm{E}-01 \\
0.2537544 \mathrm{E}-02 \\
0.6714703 \mathrm{E}-04\end{array}$ & $\begin{array}{r}-0.8213002 \mathrm{E}-02 \\
0.1895481 \mathrm{E}-01 \\
-0.2336773 \mathrm{E}-01 \\
0.1613962 \mathrm{E}-01 \\
-0.5872255 \mathrm{E}-02 \\
0.8458481 \mathrm{E}-03 \\
0.1918487 \mathrm{E}-04\end{array}$ \\
\hline
\end{tabular}

DEGREE OF POLYNOMIAL 4

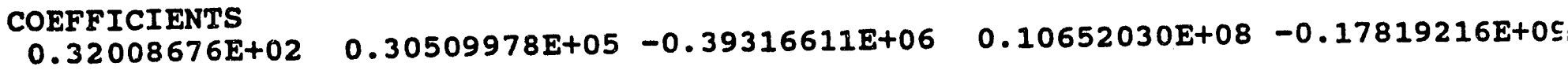

ESS $=0.2869 \mathrm{E}-02 \quad \begin{aligned} \mathrm{SD} & =0.3787 \mathrm{E}-01 \\ 2 \star \mathrm{SD} & =0.7575 \mathrm{E}-01\end{aligned} \quad$ R2 $=0.100000 \mathrm{E}+0.1$

TEMPERATURES (F) FOR TYPE J TC

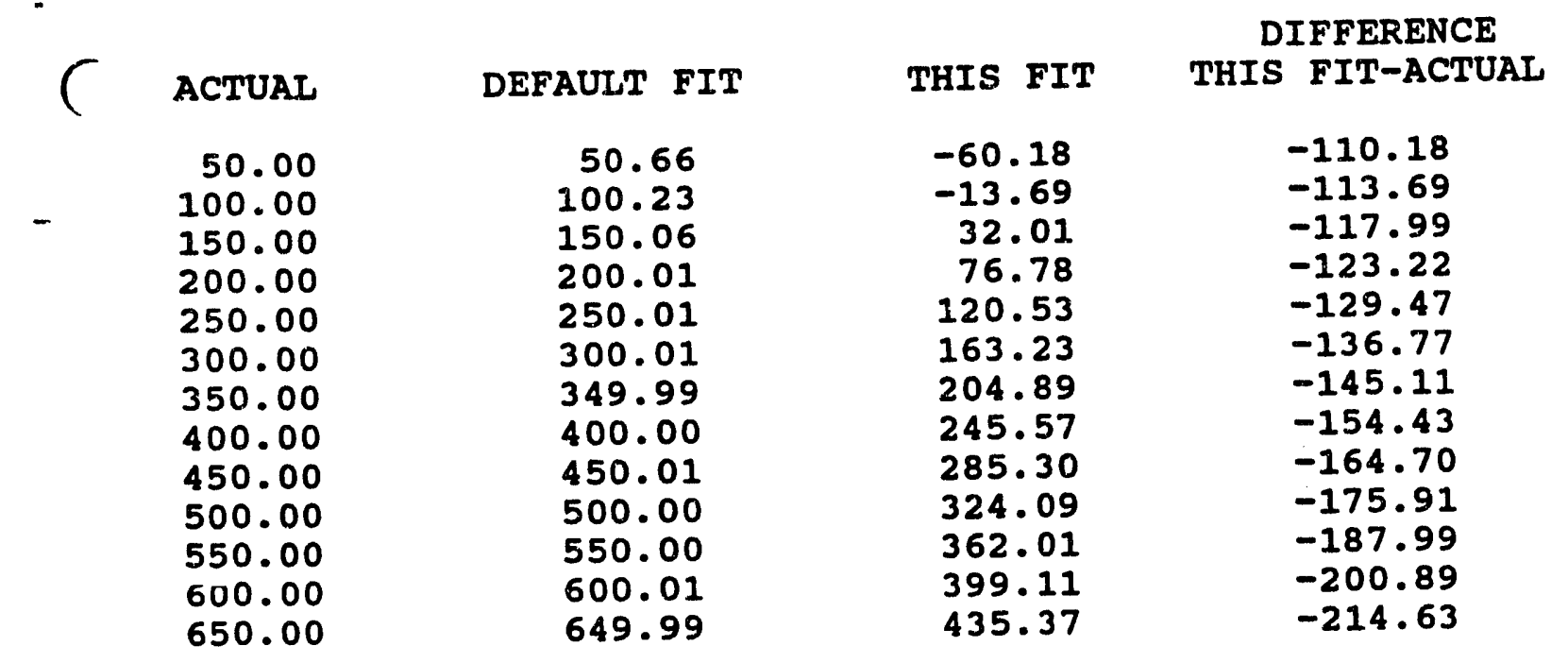


\%absock \& Wilcox

\}. McDermott company
RESEARCH \& DEVELOPMENT DIVISION

INSTRUMENT SERVICE LOG
IPTCO2 ADA5A

$3-3 c-90$

MEV. DATE 7.2098

E\&W Ser. No. OEBOSE

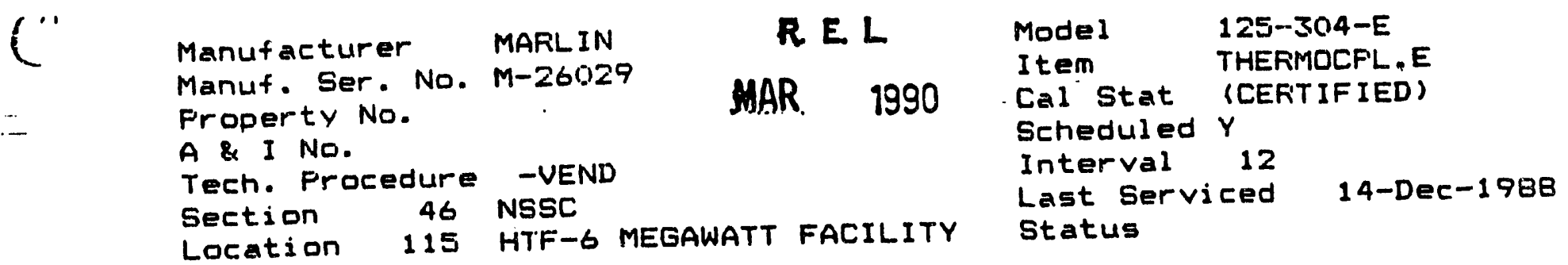

Repair Parts

Standards Used

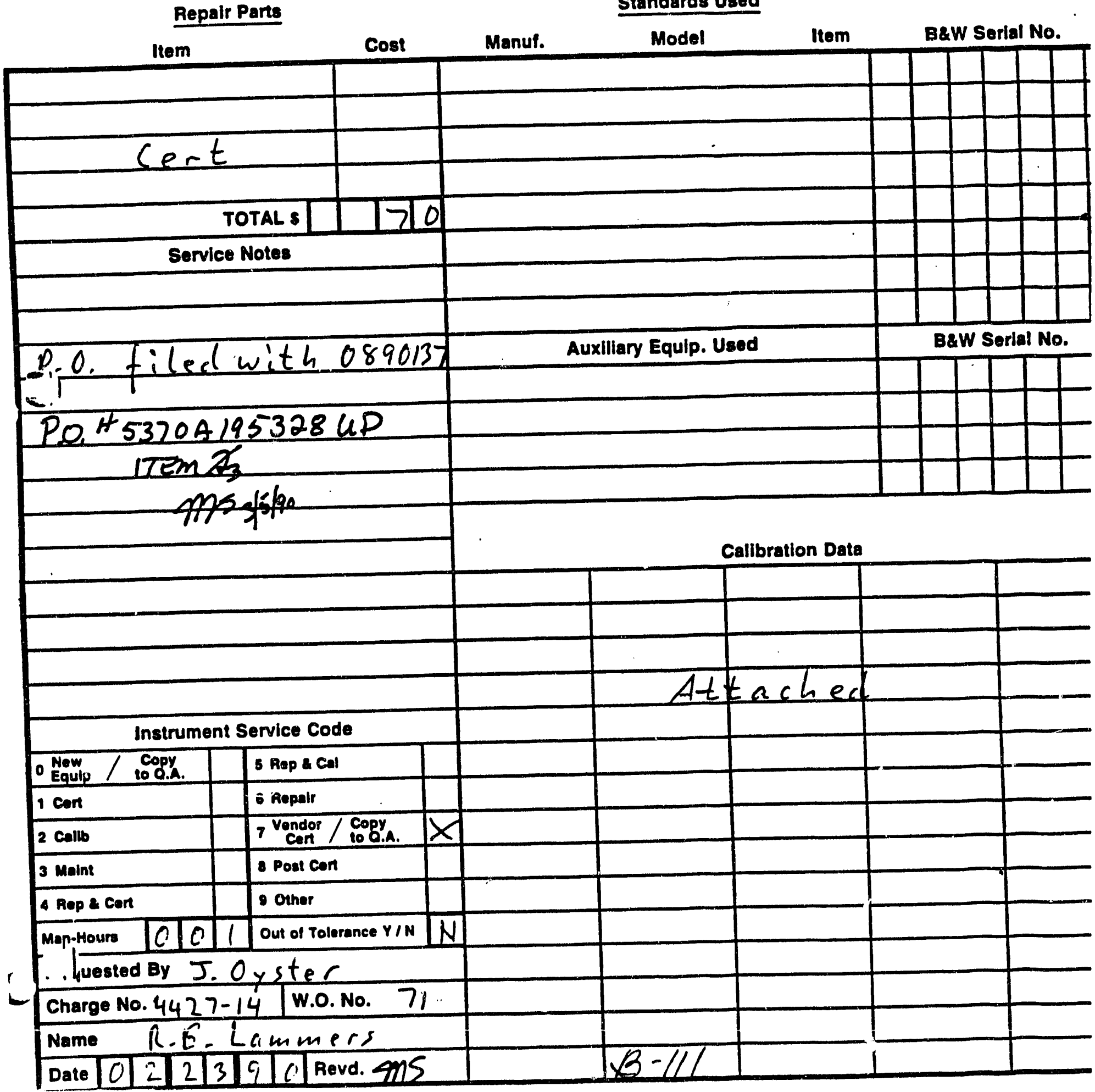




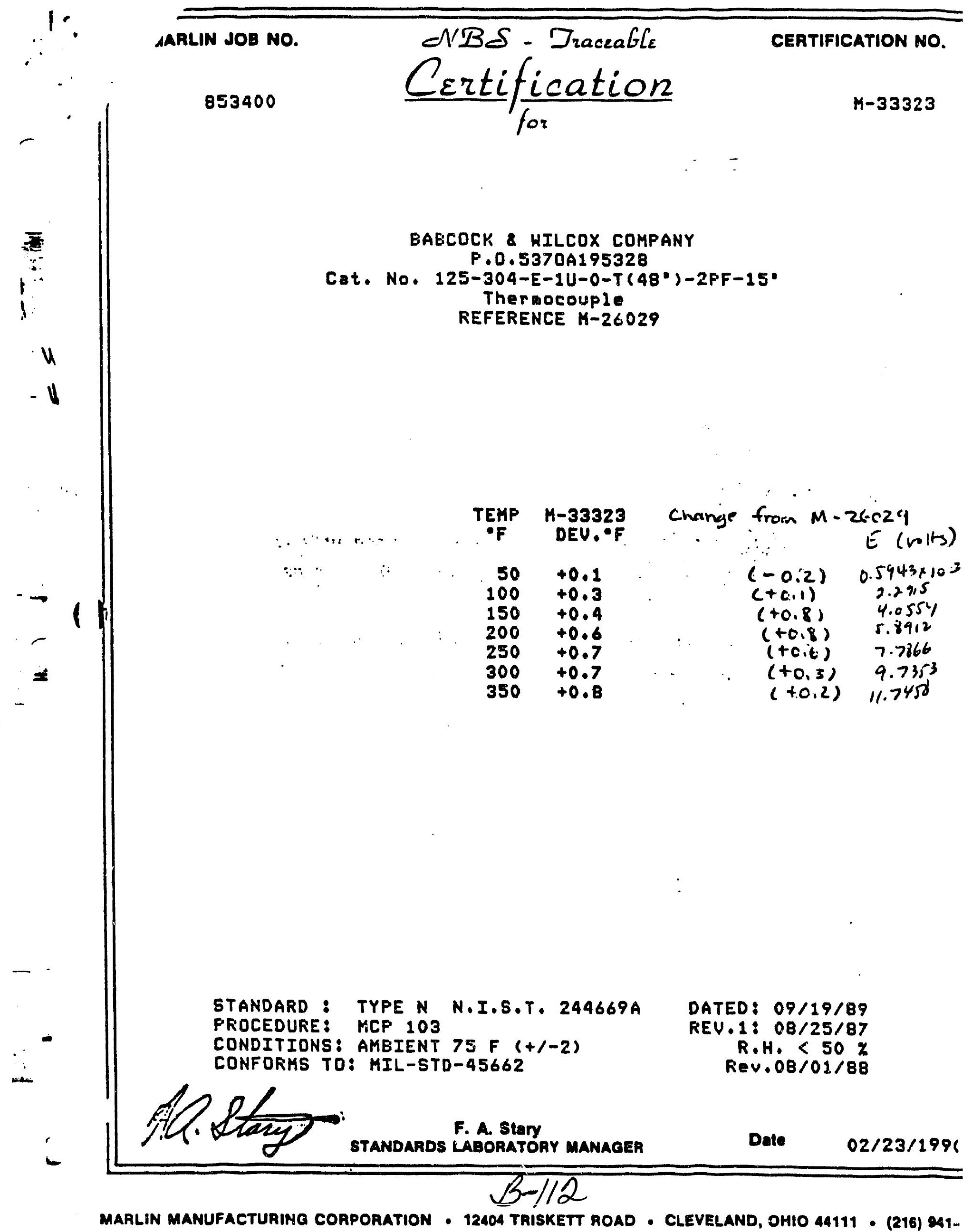


CURVEFIT OUTPUT FOR VTAB IPTC02

DATE: 26-MAY-92

TIME: $14: 47: 34$

VOLTAGE $(V)$ OBS. TEMP(F) CALC. TEMP(F) DIFFERENCE \& DIFFERENCE

\begin{tabular}{|c|c|c|c|c|c|}
\hline 3 & $\begin{array}{l}0.5943000 E-03 \\
0.2291500 E-02 \\
0.4055400 E-02 \\
0.5891200 E-02 \\
0.7786600 E-02 \\
0.9735300 E-02 \\
0.1174580 E-01\end{array}$ & $\begin{array}{l}50.00000 \\
100.0000 \\
150.0000 \\
200.0000 \\
250.0000 \\
300.0000 \\
350.0000\end{array}$ & $\begin{array}{l}49.98833 \\
100.0442 \\
149.9458 \\
200.0070 \\
250.0376 \\
299.9699 \\
350.0072\end{array}$ & $\begin{array}{r}0.1166553 E-01 \\
-0.4419972 E-01 \\
0.5423580 E-01 \\
-0.7009688 E-02 \\
-0.3756695 E-01 \\
0.3007622 E-01 \\
-0.7201196 E-02\end{array}$ & $\begin{array}{r}0.2333106 \mathrm{E}-01 \\
-0.4419972 \mathrm{E}-01 \\
0.3615720 \mathrm{E}-01 \\
-0.3504844 \mathrm{E}-02 \\
-0.1502678 \mathrm{E}-01 \\
0.1002541 \mathrm{E}-01 \\
-0.2057484 \mathrm{E}-02\end{array}$ \\
\hline
\end{tabular}

DEGREE OF POLYNOMIAL 4

COEFFICIENTS

$\begin{array}{llllll}0.31906911 E+02 & 0.30688495 E+05 & -0.45405448 E+06 & 0.17495795 E+08 & -0.42394605 E+09\end{array}$

ESS $=0.7448 \mathrm{E}-02 \quad \begin{aligned} \mathrm{SD} & =0.6102 \mathrm{E}-01 \\ 2 * \mathrm{SD} & =0.1220 \mathrm{E}+00\end{aligned} \quad$ R2 $=0.100000 \mathrm{E}+01$

TEMPERATURES(F) FOR TYPE J TC

$\begin{array}{rcrc}\text { (ACTUAL } & \text { DEFAULT FIT } & \text { THIS FIT } & \begin{array}{c}\text { DIFFERENCE } \\ \text { THIS FIT-ACTUAL }\end{array} \\ 50.00 & 50.66 & -61.50 & -111.50 \\ 100.00 & 100.23 & -14.21 & -114.21 \\ 150.00 & 150.06 & 31.91 & -118.09 \\ 200.00 & 200.01 & 76.83 & -123.17 \\ 250.00 & 250.01 & 120.58 & -129.42 \\ 300.00 & 300.01 & 163.22 & -136.78 \\ 350.00 & 349.99 & 204.82 & -145.18 \\ 400.00 & 400.00 & 245.49 & -154.51 \\ 450.00 & 450.01 & 285.25 & -164.75 \\ 500.00 & 500.00 & 324.09 & -175.91 \\ 550.00 & 550.00 & 362.00 & -188.00 \\ 600.00 & 600.01 & 398.95 & -201.05 \\ 650.00 & 649.99 & 434.79 & -215.21\end{array}$


- Manufacturer MAFLIN

- Manuf. Ser. No. M-26030

Froperty No.

A \& I No.

Tech. Frocedure -VEND

R.EL

Mode 1

125-3014-E

MAR. 1990

Section

46 NSSC

Location

115 HTF-6 MEGAWATT FACILITY

Item

THERMOCF'L, E

Cal stat (CERTIFIED)

Scheduled $Y$

Interval 12

Last Serviced

Status

14-Dec-1988

Repair Parts

Standards Used

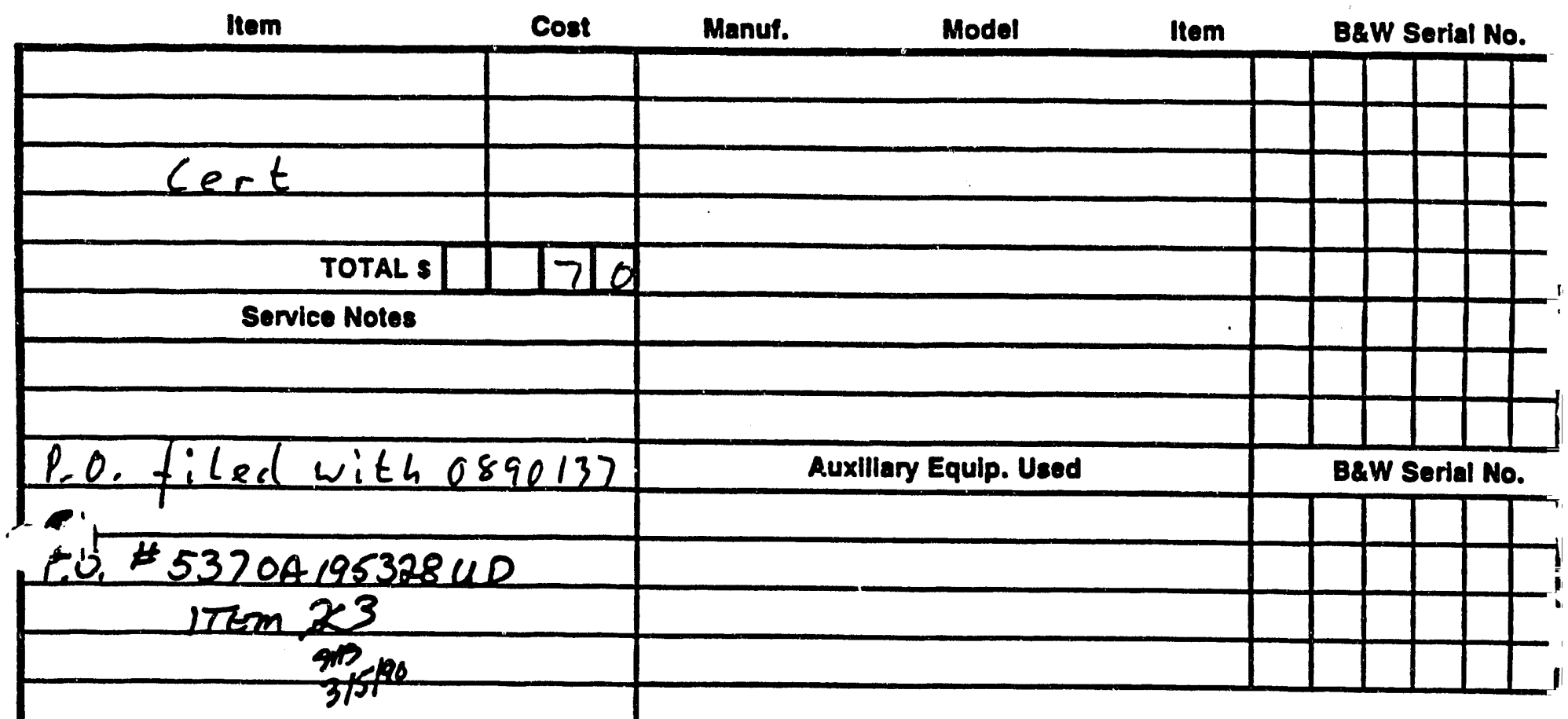

Calibration Data

Instrument Service Code

\begin{tabular}{|c|c|}
\hline 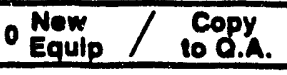 & 5 Rop \& Cal \\
\hline $1 \mathrm{Con}$ & 6 Aepalr \\
\hline 2 callb & $\begin{array}{l}7 \text { Vendor / Cogy } \\
\text { Cent / to o.A. }\end{array}$ \\
\hline 3 Malnt & - Poat Cort \\
\hline 4 Rap a Con & 0 Other \\
\hline Man-Hours & Out of Tolerance Y I N \\
\hline Re $\int_{\text {. urge No. } 4 \mathrm{~L}}$ & ter \\
\hline Name & mers \\
\hline Date & Revd. \\
\hline
\end{tabular}


\RLIN JOB NO.

853400

\section{-NBS- Jraccable \\ Certification}

\author{
BAECOCK \& HILCOX COMPANY \\ P. $0.5370 A 195328$ \\ Cat. No. 125-304-E-1U-0-T(48') -2PF-15० \\ Thermocouple \\ REFERENCE M-26030
}

TEMP $M-33322$ Cnange from M 26030

-F DEV. $F$... E E

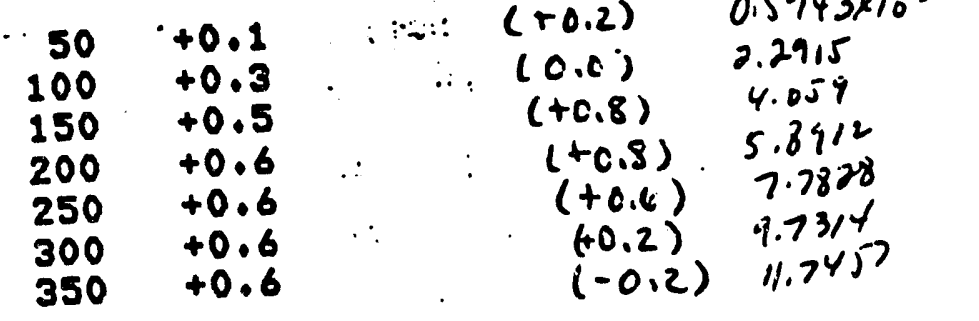

STANDARD: TYPE N N.I.S.T. 244669A

DATED: $09 / 19 / 89$ PFOCEDURE: MCF 103 CONDITIONS: AMEIENT $75 F(+1-2)$ CONFORMS TO: MIL-STD-45662 
CURVEFIT OUTPUT FOR VTAB iPtC03

DATE: 26-MAY-92

TIME: 14:50:01

\begin{tabular}{|c|c|c|c|c|c|}
\hline & VOLTAGE (V) & OBS. $\operatorname{TEMP}(F)$ & CALC. TEMP (F) & DIFFERENCE & DIFFERENCE \\
\hline $\begin{array}{l}1 \\
2 \\
3 \\
4 \\
5 \\
6 \\
7\end{array}$ & $\begin{array}{l}0.5943000 \mathrm{E}-03 \\
0.2291500 \mathrm{E}-02 \\
0.4059000 \mathrm{E}-02 \\
0.5891200 \mathrm{E}-02 \\
0.7782800 \mathrm{E}-02 \\
0.9731400 \mathrm{O}-02 \\
0.1174570 \mathrm{E}-01\end{array}$ & $\begin{array}{l}50.00000 \\
100.0000 \\
150.0000 \\
200.0000 \\
250.0000 \\
300.0000 \\
350.0000\end{array}$ & $\begin{array}{l}49.99809 \\
100.0067 \\
149.9935 \\
199.9967 \\
250.0103 \\
299.9931 \\
350.0015\end{array}$ & $\begin{array}{r}0.1907110 E-02 \\
-0.6713872 E-02 \\
0.6507279 E-02 \\
0.3287547 E-02 \\
-0.1032342 E-01 \\
0.6876622 E-02 \\
-0.1541266 E-02\end{array}$ & $\begin{array}{r}0.3814219 E-02 \\
-0.6713872 \mathrm{E}-02 \\
0.4338186 \mathrm{E}-02 \\
0.1643774 \mathrm{E}-02 \\
-0.4129367 \mathrm{E}-02 \\
0.2292207 \mathrm{E}-02 \\
-0.4403618 \mathrm{E}-03\end{array}$ \\
\hline
\end{tabular}

DEGREE OF POLYNOMIAL 4

COEFFICIENTS

$0.31932508 E+02 \quad 0.30665102 E+05-0.46103999 E+06$

$0.20123609 E+08-0.58411532 E+C$

ESS -

$0.2581 E-03$

$\begin{aligned} S D & =0.1136 \mathrm{E}-01 \\ 2 * S D & =0.2272 \mathrm{E}-01\end{aligned}$

$R 2=0.100000 E+01$

TEMPERATURES(F) FOR TYPE J TC

ACTUAL

50.00
100.00
150.00
200.00
250.00
300.00
350.00
400.00
450.00
500.00
550.00
600.00
650.00
DEFAULT FIT

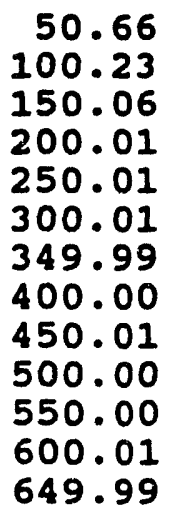

THIS FIT

-61.54
-14.18
31.93
76.81
120.53
163.17
204.82
245.56
285.37
324.19
361.92
398.43
433.45

DIFFERENCE THIS FIT-ACTUAL

-111.54
-114.18
-118.07
-123.19
-129.47
-136.83
-145.18
-154.44
-164.63
-175.81
-188.08
-201.57
-216.55 


$$
\begin{aligned}
& \text { Matis. res } \\
& \text { पे }-1,--71
\end{aligned}
$$

7W * $1 \quad 0.90000000000 D+01 \quad-0.10000000000 D-05 \quad 0.63897000000 D-07$

$\begin{array}{lllll}\text { ROW * } 2 & -0.10000000000 \mathrm{D}-05 & 0.63897000000 \mathrm{D}-07 & -0.51091000000 \mathrm{D}-13 \\ \text { ROW * } 3 & 0.63897000000 \mathrm{D}-07 & -0.51091000000 \mathrm{D}-13 & 0.80265664500 \mathrm{D}-15 \\ \text { ROW * } 4 & 0.28800000000 \mathrm{D}+03 & -0.19900000000 \mathrm{D}-02 & 0.20457480000 \mathrm{D}-05\end{array}$

REVERSED CURVEEIT AROUND $32 F$

\begin{tabular}{llrrrr} 
& OBSERVED & CALCULATED & \multicolumn{1}{c}{ DIFFERENCE } & PERCENT & X(I) \\
1 & 28.00000 & 28.00437 & $-0.4373553 E-02$ & $-0.1561983 E-01$ & $0.1300000 E-03$ \\
2 & 29.00000 & 28.99133 & $0.8671130 E-02$ & $0.2990045 E-01$ & $0.9800000 E-04$ \\
3 & 30.00000 & 30.00726 & $-0.7257809 E-02$ & $-0.2419270 E-01$ & $0.6500000 E-04$ \\
4 & 31.00000 & 30.99059 & $0.9411080 E-02$ & $0.3035832 E-01$ & $0.3300000 E-04$ \\
5 & 32.00000 & 32.00278 & $-0.2780397 E-02$ & $-0.8688740 E-02$ & $0.0000000+00$ \\
6 & 33.00000 & 33.01307 & $-0.1307439 E-01$ & $-0.3961937 E-01$ & $-0.3300000 E-04$ \\
7 & 34.00000 & 33.99094 & $0.9058685-02$ & $0.2664319 E-01$ & $-0.6500000 E-04$ \\
8 & 35.00000 & 34.99750 & $0.2502153 E-02$ & $0.7149009 E-02$ & $-0.98000000-04$ \\
9 & 36.00000 & 36.00216 & $-0.2156898 E-02$ & $-0.5991384 E-02$ & $-0.1310000 E-03$
\end{tabular}

DEGREE OF POIYNOMIAL 2

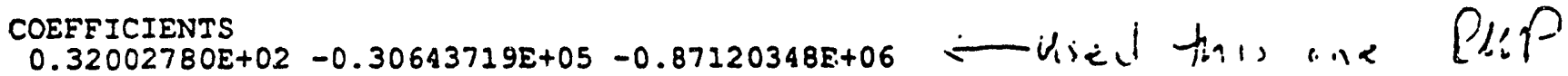
$\dot{y-i ;--i \mid}$ 
Bábock \& Wilcox

- MeDormott company
RESIEARCH \& DEVELOPMENT DIVISION INSTRUMENT SERVICE LOG
NSTCOI RDA5A $3-3 c-9 c$ hEV. DATE T2OAN

E\&W Ser. No. OBBOE:

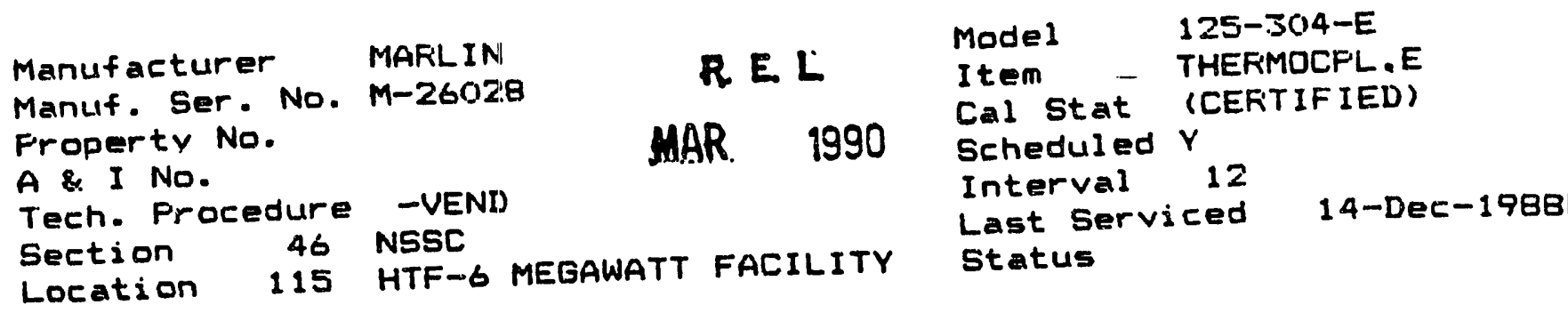

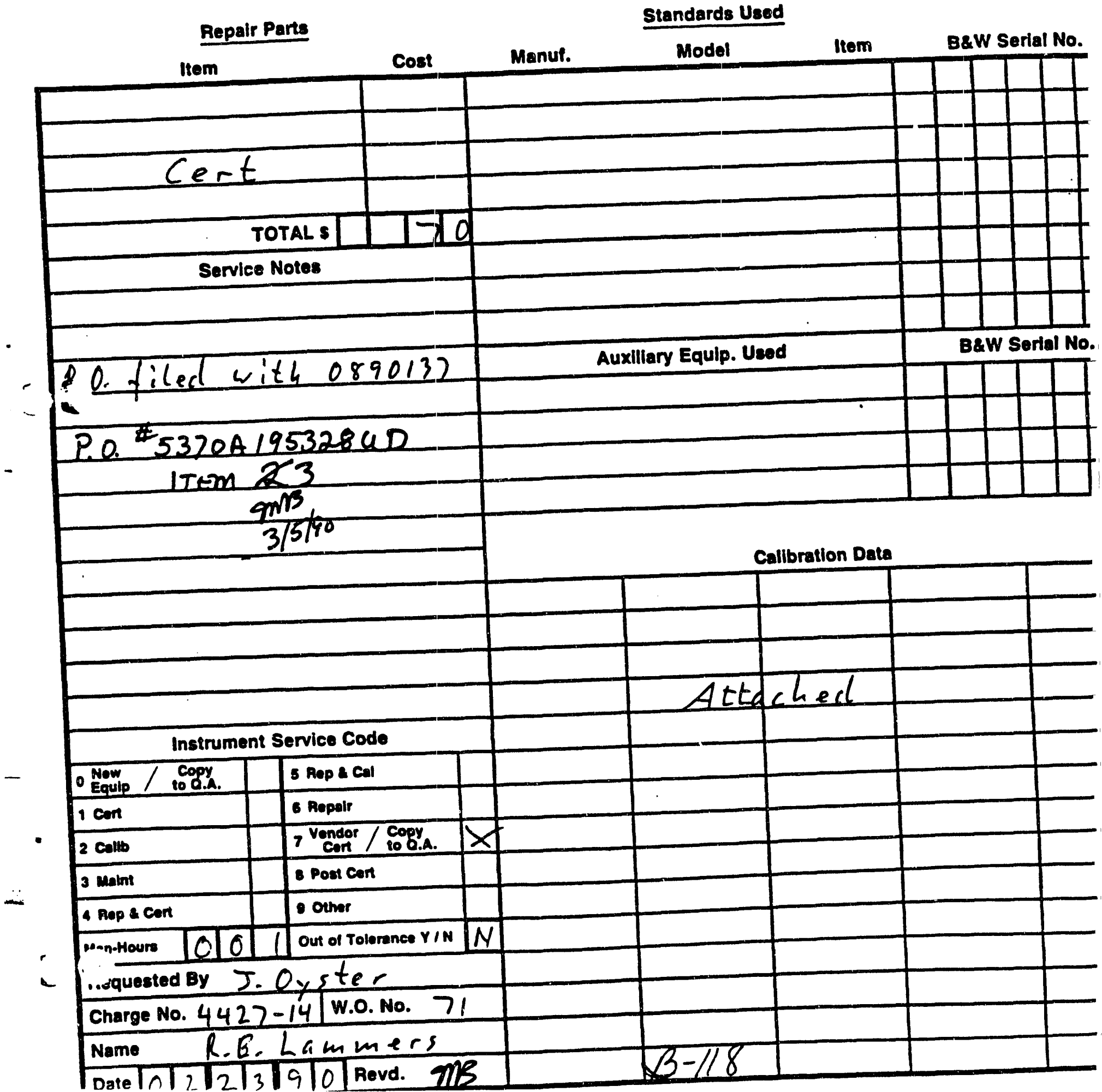




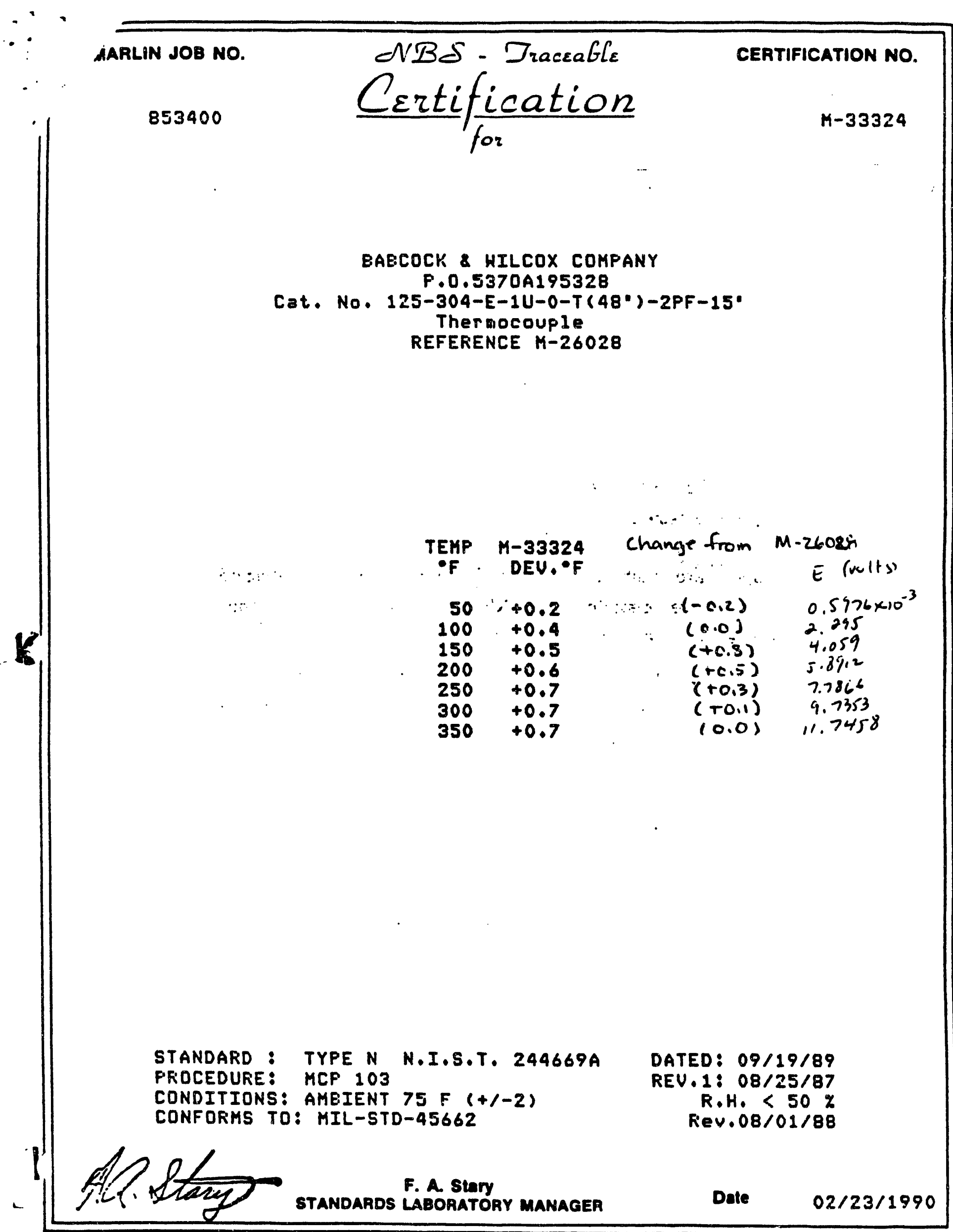

STANDARD : TYPE N N.I.S.T. 244669A

DATED: $09 / 19 / 89$

CONDITIONS: AMEIENT $75 \mathrm{~F}(+/-2)$

CONFORMS TO: MIL-STD-45662

R.H. $<50 \%$

Rev.08/01/88

F. A. stary

STANDARDS LABOAATORY MANAGER 


\begin{tabular}{lcccrr} 
& VOLTAGE $(V)$ & OBS. TEMP (F) & CALC. TEMP(F) & DIF IERENCE & P DIFFERENCE \\
\hline 1 & $0.5976000 E-03$ & 50.00000 & 49.99189 & $0.8105829 E-02$ & $0.1621166 E-01$ \\
2 & $0.2295000 E-02$ & 100.0000 & 100.0286 & $-0.2859184 \mathrm{E}-01$ & $-0.2859184 \mathrm{E}-01$ \\
3 & $0.4059000 \mathrm{E}-02$ & 150.0000 & 149.9722 & $0.2778905 \mathrm{E}-01$ & $0.1852603 \mathrm{E}-01$ \\
4 & $0.5891200 \mathrm{E}-02$ & 200.0000 & 199.9863 & $0.1371056 \mathrm{E}-01$ & $0.6855278 \mathrm{E}-02$ \\
5 & $0.7786600 \mathrm{E}-02$ & 250.0000 & 250.0436 & $-0.4359120 \mathrm{E}-01$ & $-0.1743648 \mathrm{E}-01$ \\
6 & $0.9735300 \mathrm{E}-02$ & 300.0000 & 299.9709 & $0.2912607 \mathrm{E}-01$ & $0.9708689 \mathrm{E}-02$ \\
7 & $0.1174580 \mathrm{E}-01$ & 350.0000 & 350.0065 & $-0.6548458 \mathrm{E}-02$ & $-0.1870988 \mathrm{E}-02$
\end{tabular}

DEGREE OF POLYNOMTAL 4

COEFFICIENTS

$0.31846369 E+02 \quad 0.30612890 E+05-0.42488239 E+06$

$0.14232950 E+08-0.30780354 E+C$

ESS $=0.4635 \mathrm{E}-02 \quad \begin{aligned} \mathrm{SD} & =0.4814 \mathrm{E}-01 \\ 2 * \mathrm{SD} & =0.9628 \mathrm{E}-01\end{aligned} \quad \mathrm{R} 2=0.100000 \mathrm{E}+01$

TEMPERATURES(F) FOR TYPE J TC

$\begin{array}{rr}\text { ACTUAL } & \text { DEFAULT FIT } \\ 50.00 & 50.66 \\ 100.00 & 100.23 \\ 150.00 & 150.06 \\ 200.00 & 200.01 \\ 250.00 & 250.01 \\ 300.00 & 300.01 \\ 350.00 & 349.99 \\ 400.00 & 400.00 \\ 450.00 & 450.01 \\ 500.00 & 500.00 \\ 550.00 & 550.00 \\ 600.00 & 600.01 \\ 650.00 & 649.99\end{array}$

THIS FIT

$-61.01$

$-14.09$

31.85

76.71

120.48

163.16

204.80

245.50

285.26

324.09

362.01

399.04

435.08
DIFFERENCE

THIS FIT-ACTUAL

$-111.01$

$-114.09$

$-118.15$

$-123.29$

$-129.52$

$-136.84$

$-145.20$

$-154.50$

$-164.74$

$-175.91$

$-187.99$

$-200.96$

$-214.92$ 


$1, n$
RD-45A Rev. $11-28-91$
\begin{tabular}{|l|l|l|}
\hline INSTRUMENT STATUS & RECD & AS \\
\hline 1 Certified & $V$ & $\checkmark$ \\
\hline 2 Calibrated & & \\
\hline 3 For Ind. Only & & \\
\hline 4 Standardize & & \\
\hline 5 Certified $~$ stand. & & \\
\hline 6 Calibrated s stand. & & \\
\hline 9 Other & & \\
\hline
\end{tabular}

INSTRUMENT CONDITION

RESEARCH \& DEVELOPMENT DIVIS A INSTRUMENT SERVICE IOG

\begin{tabular}{r} 
AS RECEIVED (Certified Equipment Only) \\
Meets Manufacturer's Tolerance $\mathrm{N}$ \\
Operates within previously defined limits $\mathrm{N}$ \\
Deviates from Mfg or previously defined limits $\mathrm{Y}$ \\
\hline AS LEFT \\
Inoperative (Describe below)
\end{tabular}

\section{Babcock \& Wilcox}

a MeDermott company

B\&W Ser. No. 0880504

MANUFACTURER MARIIN

MODEL PRT1-MC-48

ITEM SENSOR, TMP, RES

MANOF. SER. NO. M-25373

PROPERTY NO. 4427-01-062

ORDER NO.

SECTION 46

LOCATION 125

SCHEDULED ( $\mathrm{Y}-\mathrm{N})$

RECAL INT.(MOS.) 12 MAR 021992

\section{TECH. PROCEDURE VEND}

Babcock \& Wilcox RCD Division certifies that the performance of this instrument was verified using standards whose accuracies are traceable to the National Institute of standards and Technology, an accepted value of a natural physical constant, a ratio type of calibration technique, or to a consensus standard.

\begin{tabular}{|lll}
\hline STANDARDS USED & Model Item & BGW Serial No.
\end{tabular}

OTHER EOUIPMENT USED

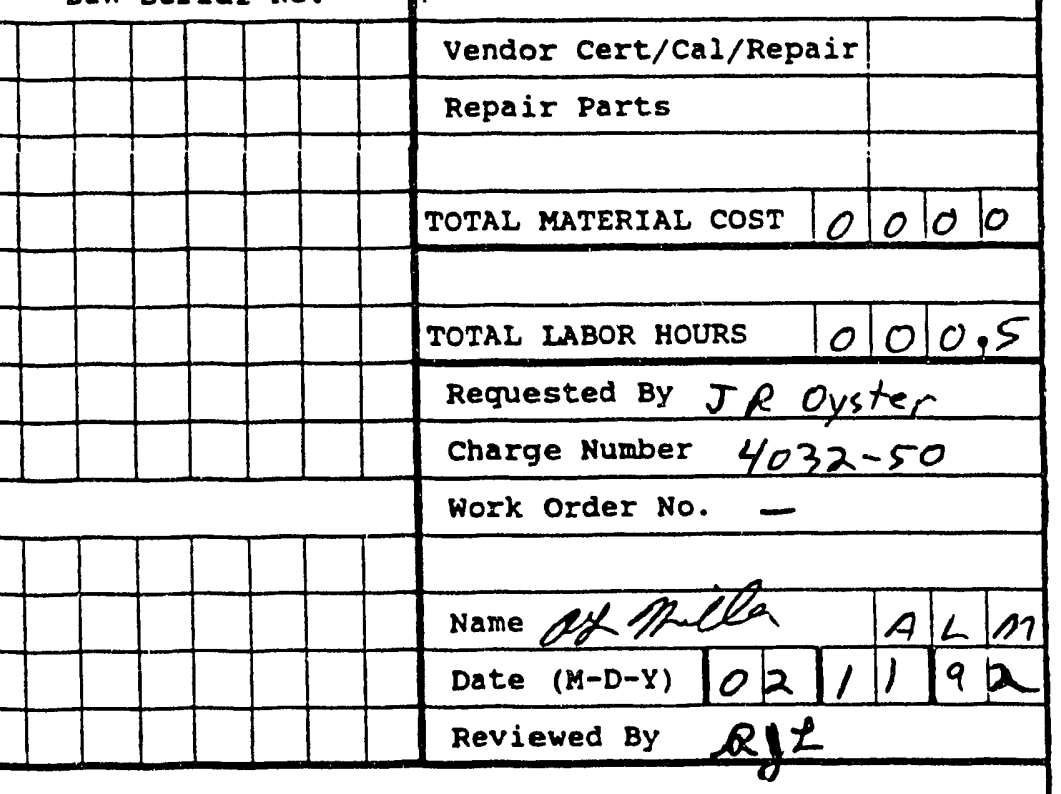

Service Notes
Previous Hours $=12.5$

Cument Hours $=00.0$

Remaining Hours $=7987.5$ 
1: THE BABCOCK \& WILCOX CO JANY - ALLIANCE RESEARCH CENTER LONG HAND MEMORANDUM

C To R.E. Lammers, Instrument DePt.

\begin{tabular}{l|l}
\hline From J.R. OYSTER, N.S.S.\&C. SECTION & \\
\hline Subj. SRP TEMPERATURE SENSOR RE-GERTIFICATION & $\begin{array}{l}\text { Filo No. } \\
\text { or Rot. SRP }\end{array}$ \\
\hline
\end{tabular}

Please extend the certification on the RTD unit

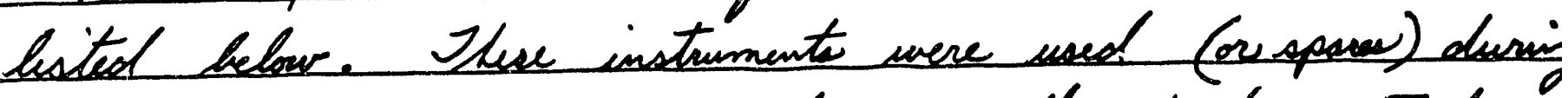
Save. River Flow Excursion Testing on the HTF/NPR Tent facility. No additional hours should be added, as they. have not been used since the last reporting period.

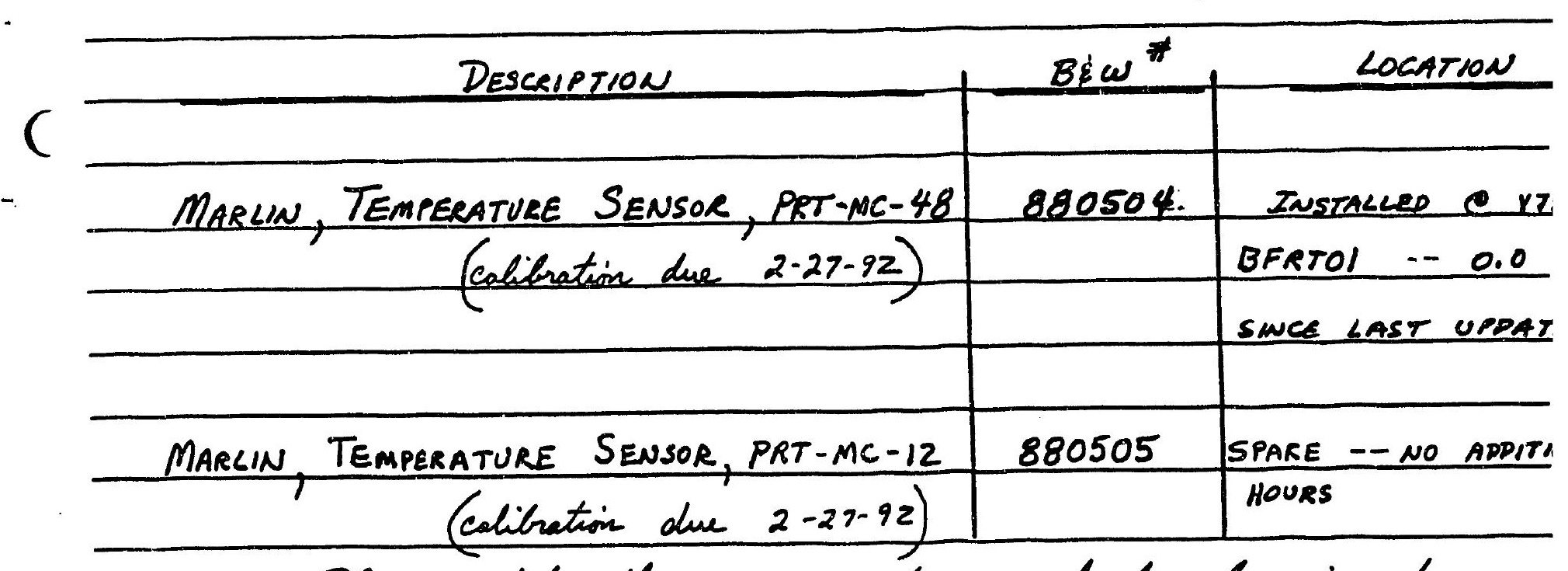

Please update thieve service logs and forward copies to me e There unit could be used on un coming test schedule te start this year in the Upflow Tent Loop. The charge *\$032-50 - for the update.

CC: DiP. BIRMINGHAM

Thank yous,

Q.e.colyotor

( RM. PRIVATE

KIT. REYNOLDS

Ko. SCHULZE

$3-122$ 


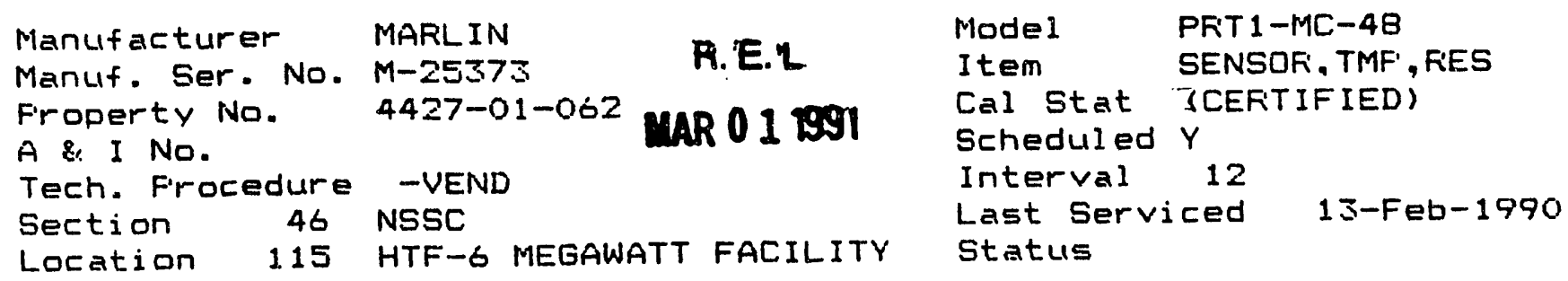

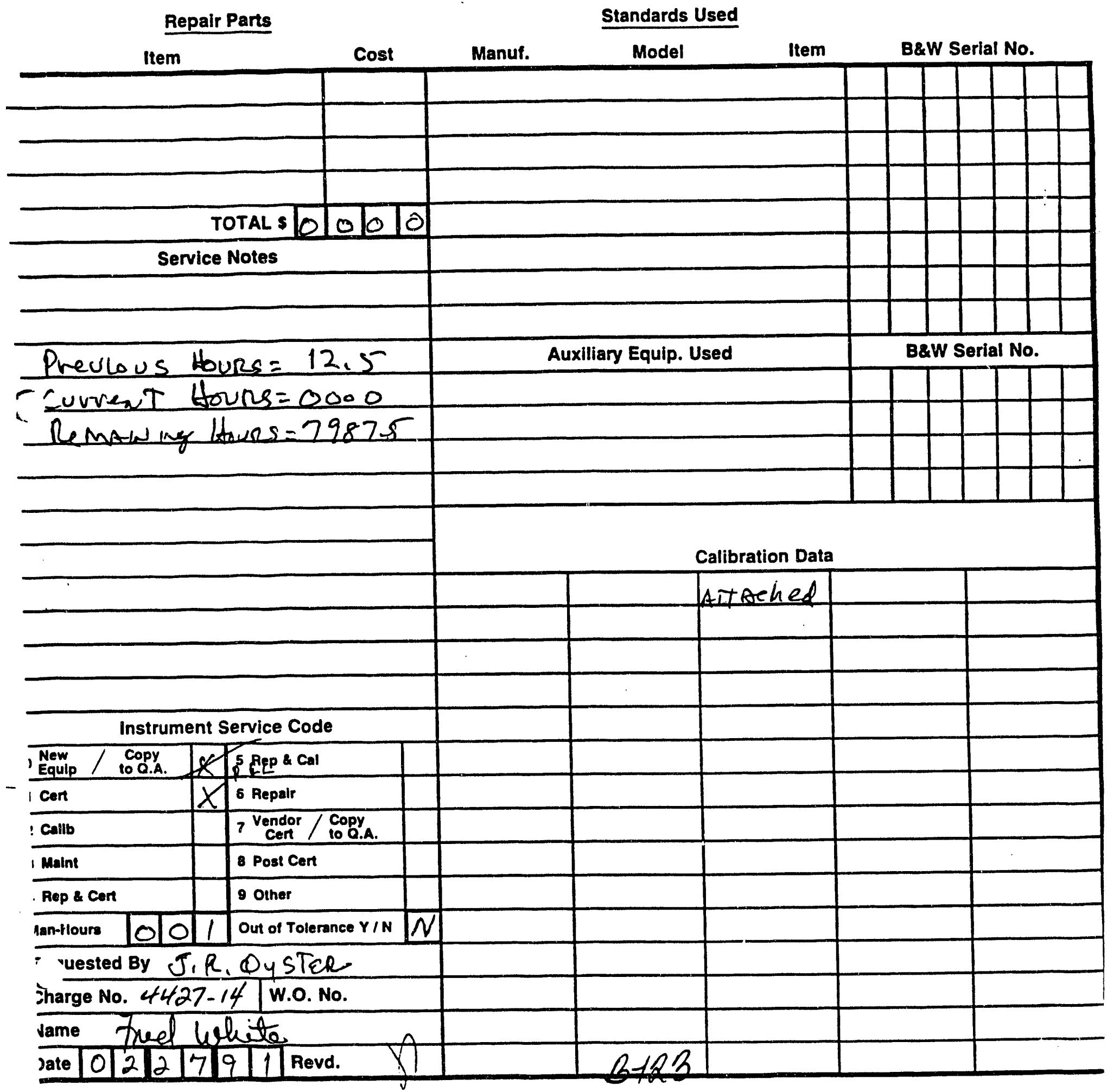




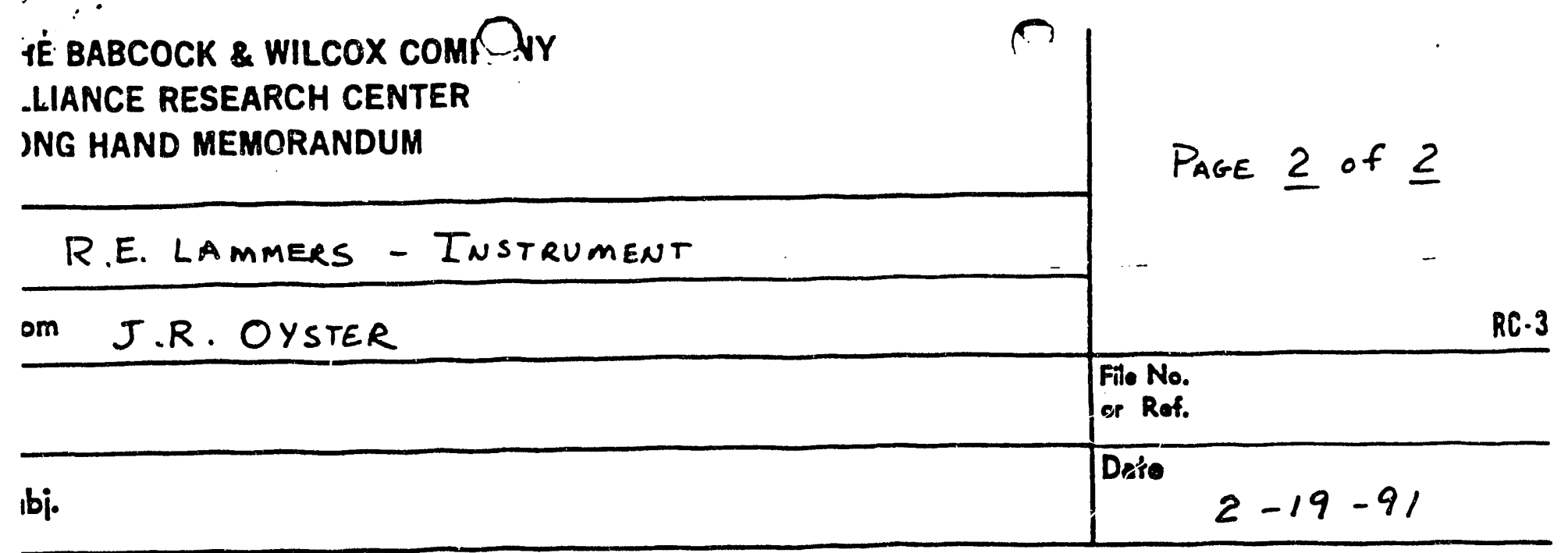

Also please re-certify the following three RTO units for another 12 month period. The location and hours of usage are as noted:

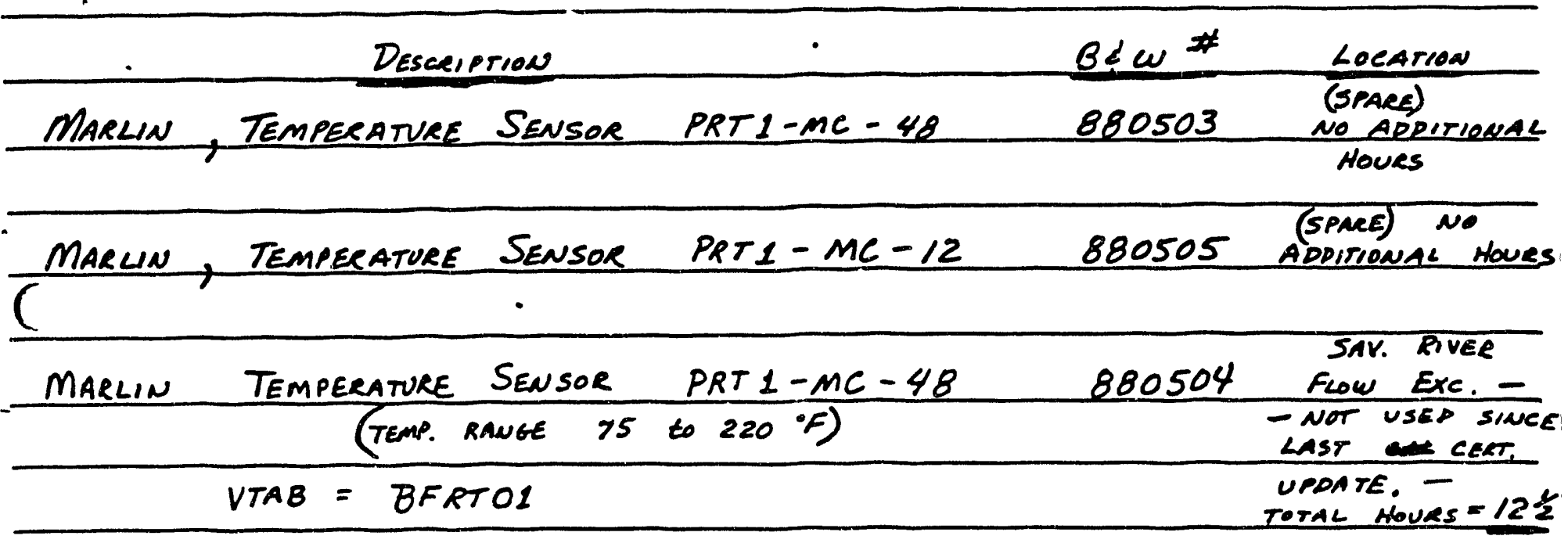

Please update the above instruments and change their instrument service logs to reflect as noted. Use this charge number for these updates ("4427-14).

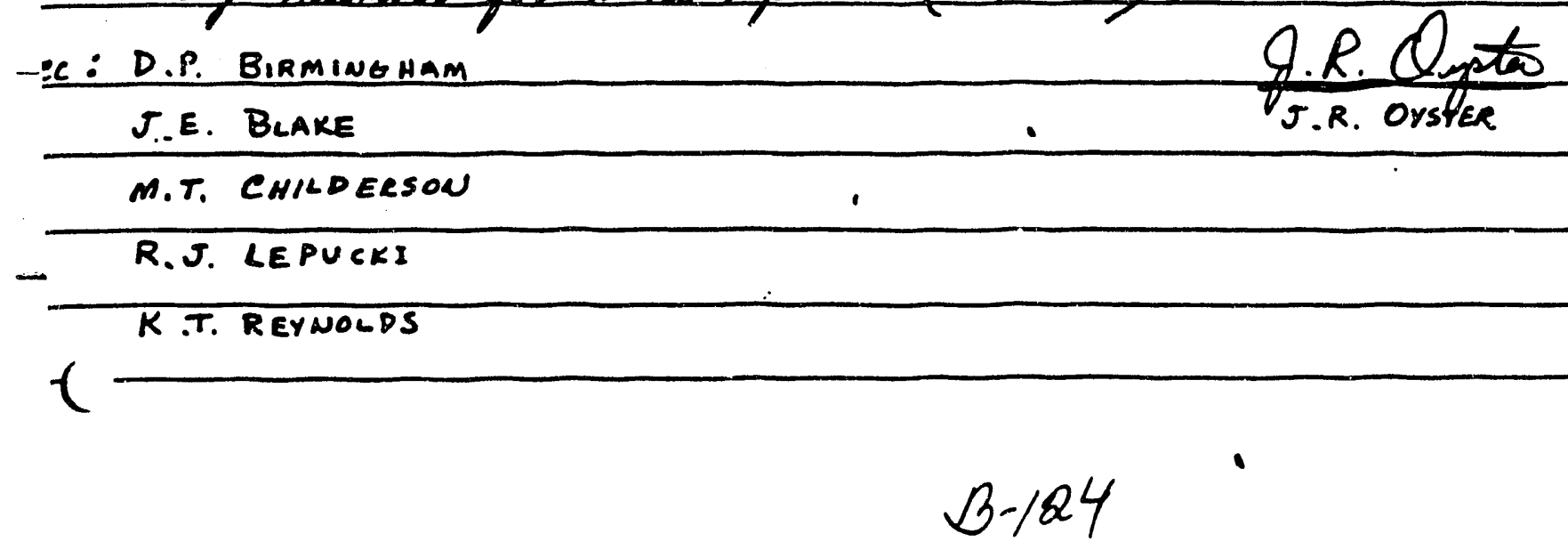




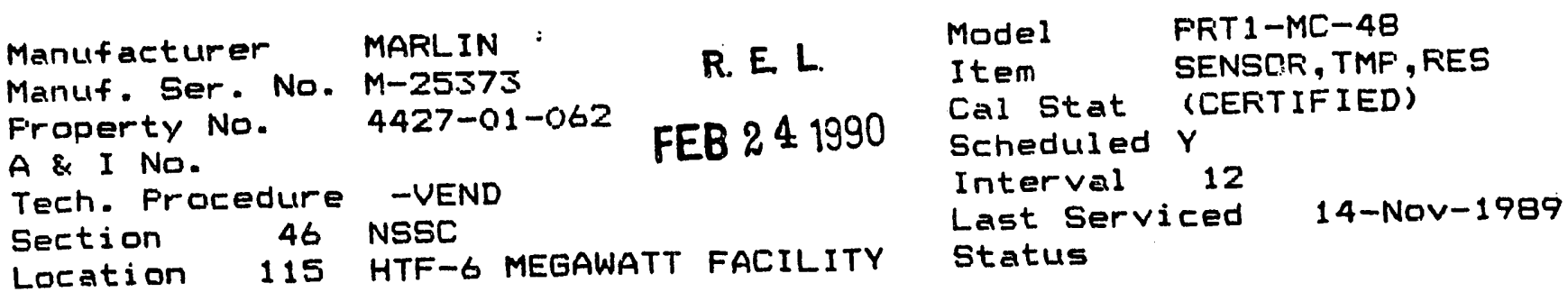

\section{Repair Parts}

Standards Used

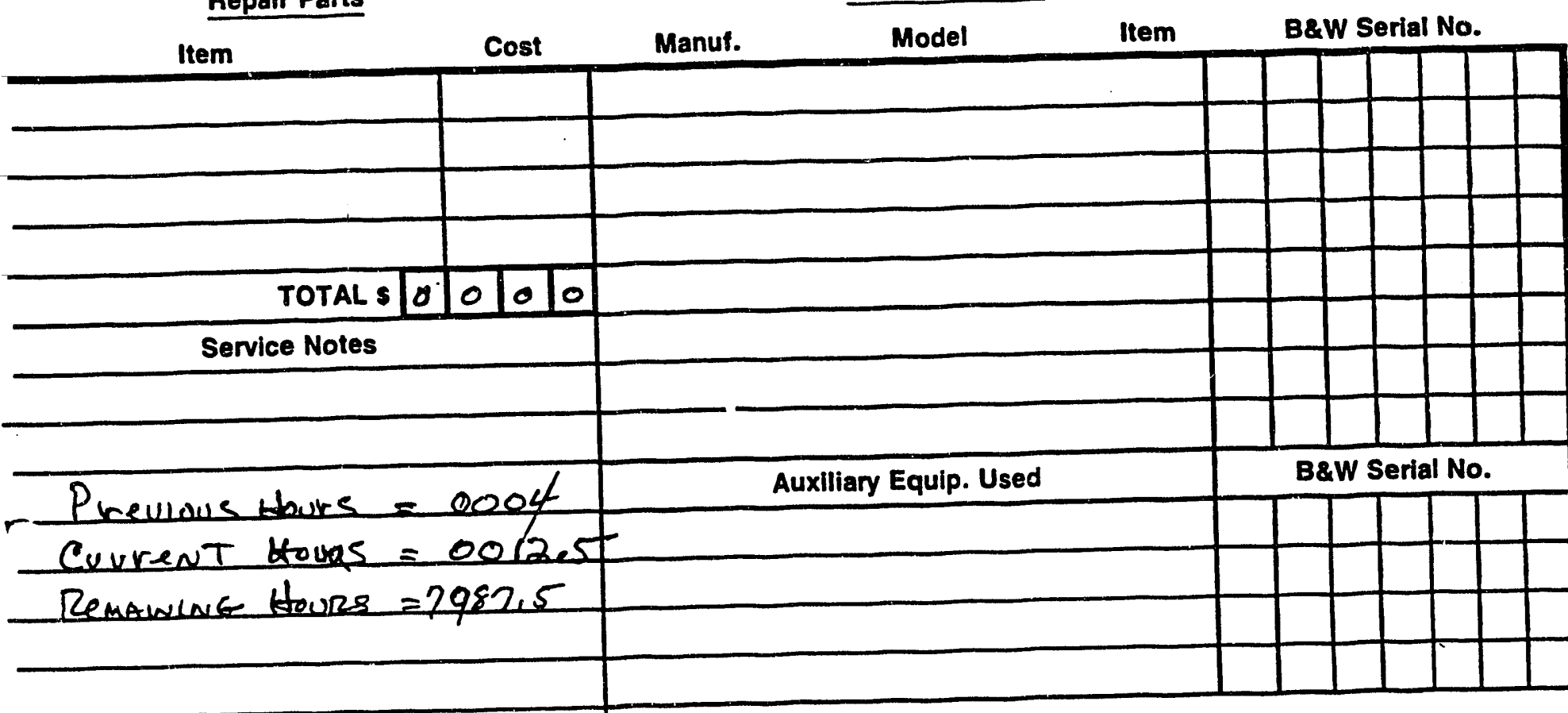

Calibration Data

Instrument Service Code

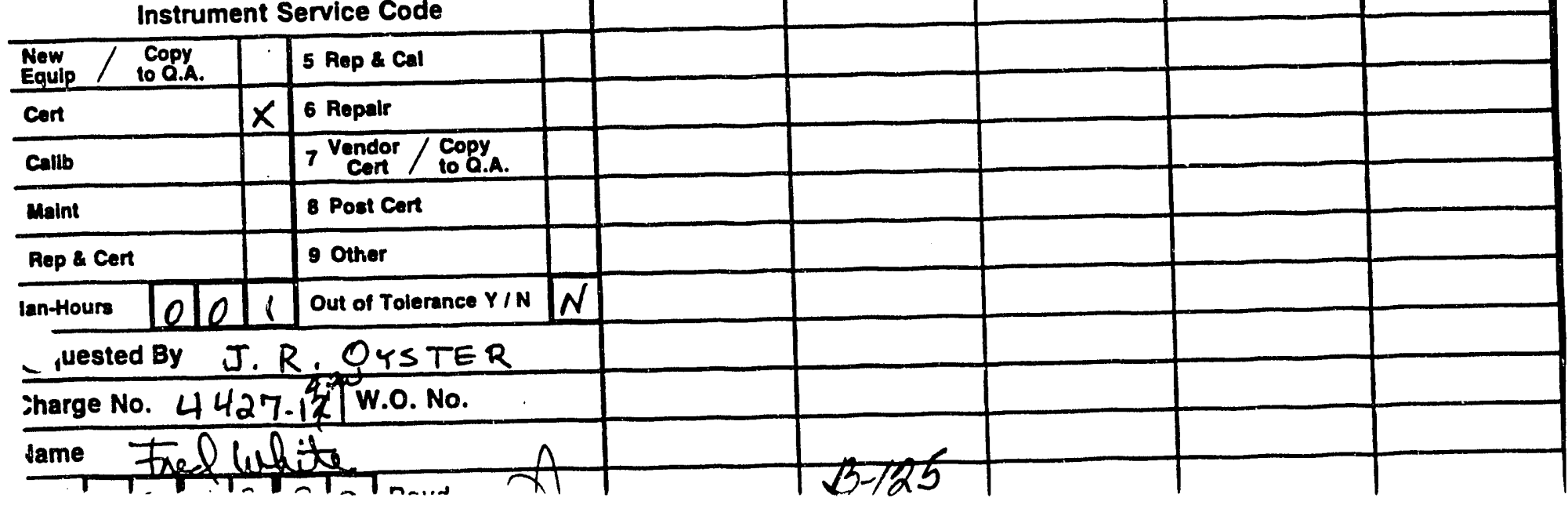




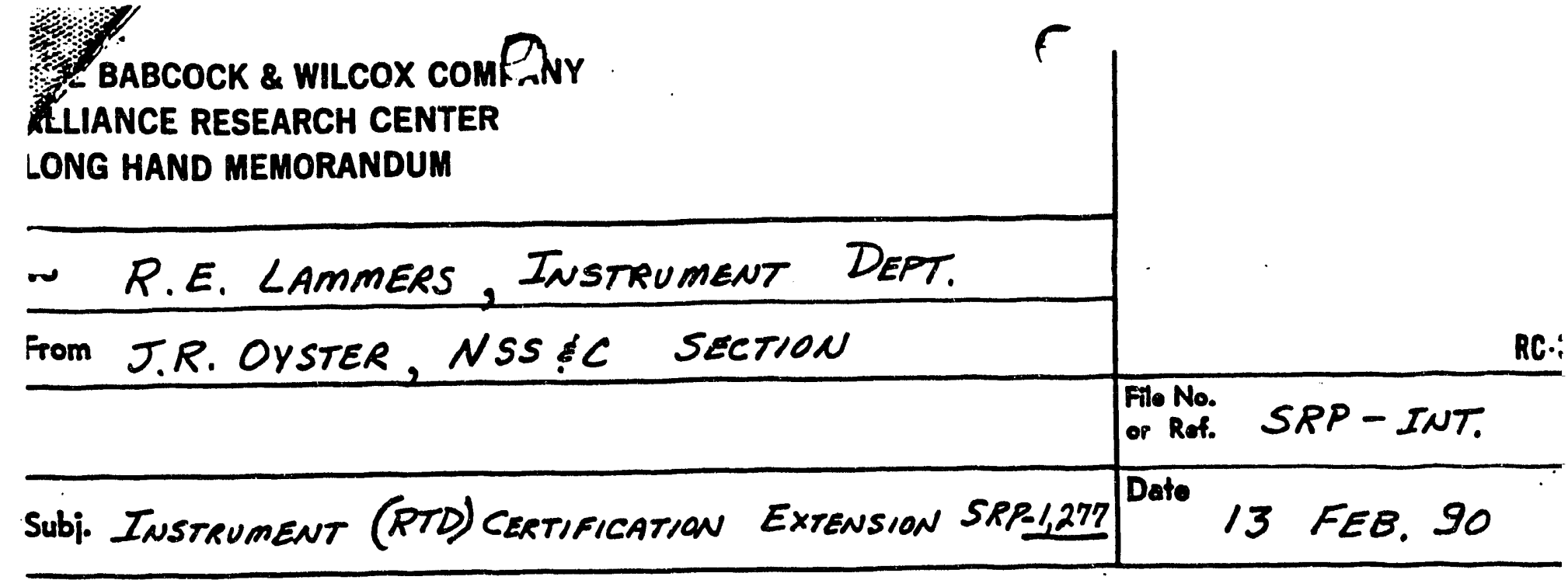

Please extend the recently due certification on the following instrument, bated in the SRP flow excursion, outlet pleven.

SP IDENTIFICATION - - BFRTO1

Bíw No - 880504

Manuf. - MARLIN

INST. - R TD, UNGRD. $\frac{1}{16}$ INCH SS. SHEATH

This instrument is silver soldered into position and is not easily removed. It has approximately $12 \frac{1}{2}$ hours at a tempenotiere range of 75 t 220 degree $F$.

Please update the isistumint service loges to reflect the hours of operation and send copier to G.R. Dots.

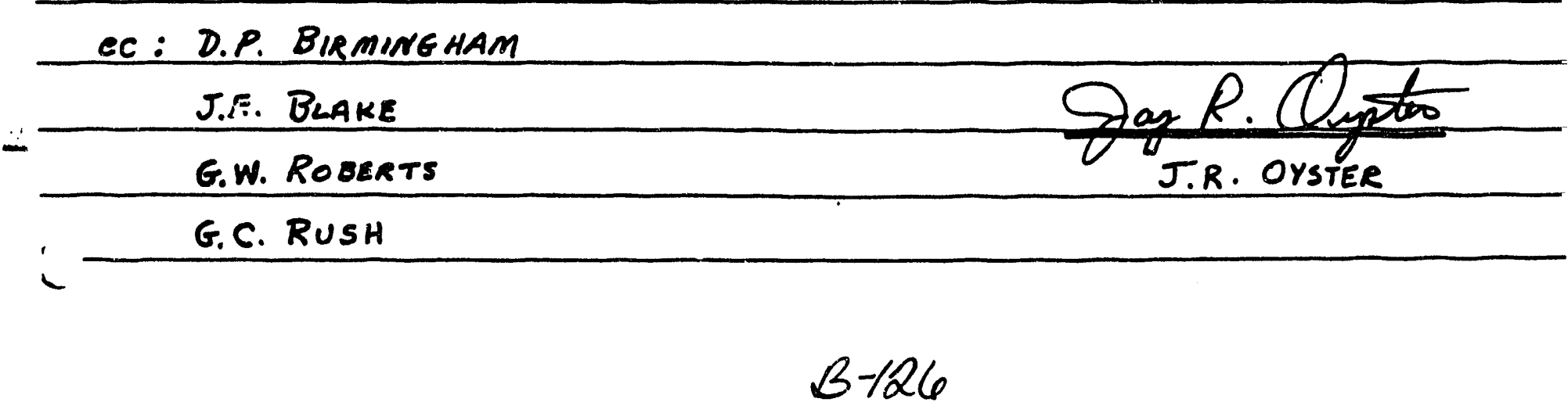




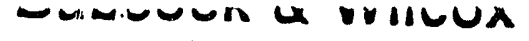

a McDermott company

-

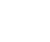

Manuf acturer

MARLIN

Manuf. Ser. No. M-25373

Property No. 4427-01-062

$A \&$ \& $\mathrm{No}$.

Tech. Procedure - VEND

Section

Location

HT TR\&FL MECH

HTF-6 MEGAWATT FACILITY
$\operatorname{Pin} 11 / 7 / 8 \varepsilon$

ROA5A

REV. DATE 7.2018

B\&W Ser. No. 088056

Model PRT1-MC -48

Item SENSOR, TMP, RES

Cal stat (HOT EnttBRATED)

Scheduded-AYY CERTIfICS

Interval 12

Last Seruiced

Status
Repair Parts

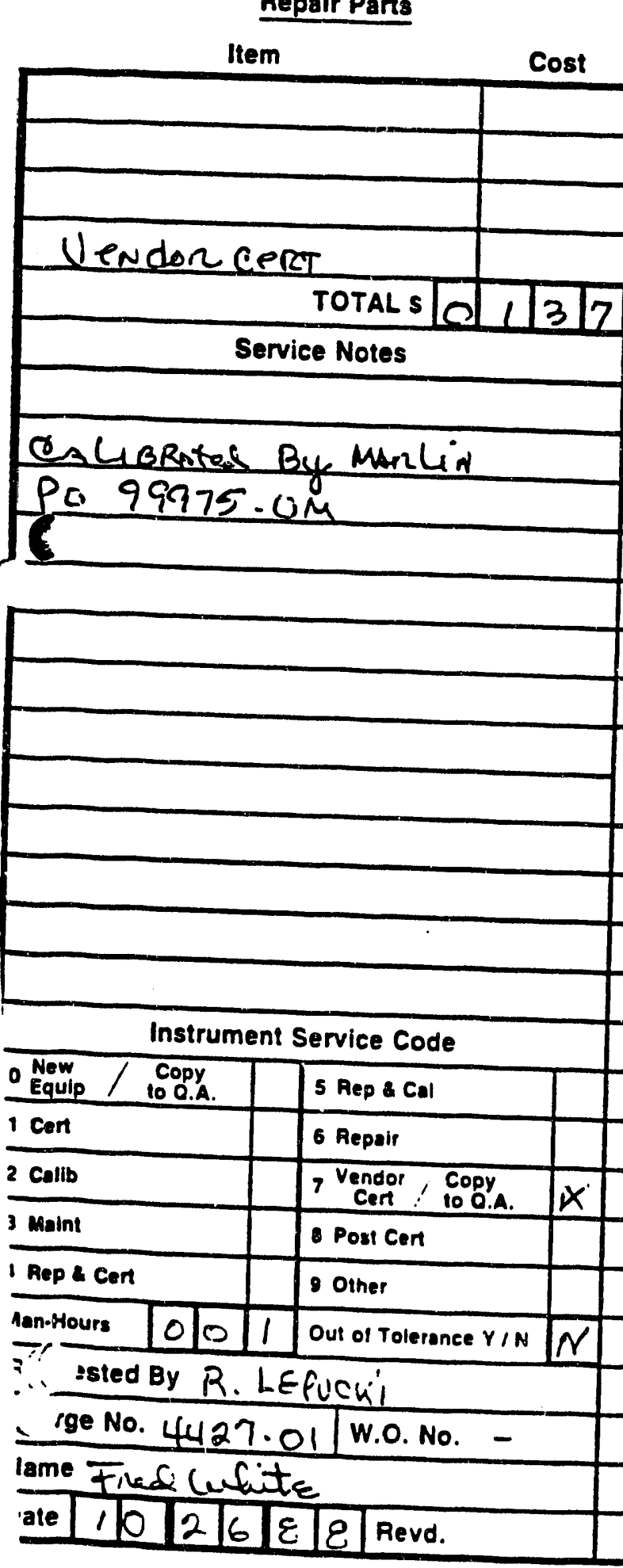

\section{Standards Used}

Manuf. Model
Item

B\&W Serial No.

Auxiliary Equip. Used

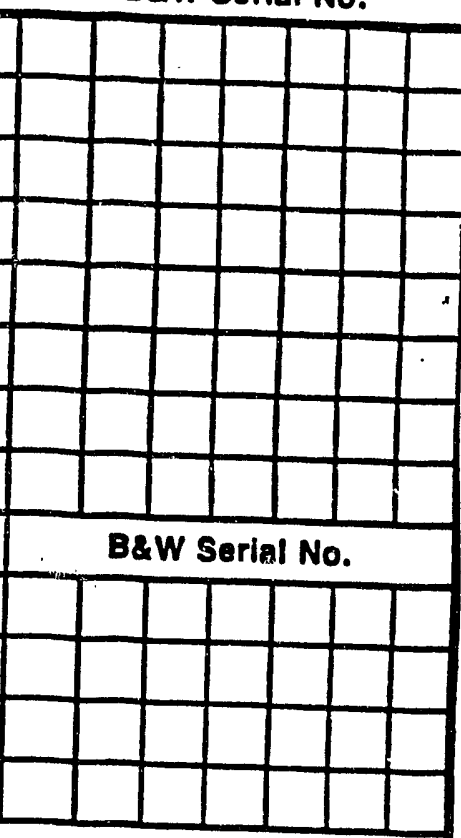

Calibration Data

A TIAched 
- Málin jos no.

880300

\title{
oNBS - Jraczalle \\ Aertification
}

$M-25373$

\author{
REPQRI OE CALIBRAIIDN \\ FOR
}

PLAIINUM RESISIANCE IHERMOMEIER

The above platinum resistance thermometer was calibrated on 10/20/8 Ear use with a continuous current of $1.0 \mathrm{milliamps}$ through the thermometer.

The Eallowing velues wera found for constants in the Formulas given in the International Practical Temperature Scale of 1968 (IPIS-6日):

$$
\begin{array}{ll}
\text { Alphe }=.003848543 & \text { Delta }-1.495581 \\
A=3.906101 E-03 & \text { B }=-5.755807 E-07
\end{array}
$$

The pertinent formulas are given in the discussion on the Eollowing pages.

The resistance at zero degrees Celsius was found to be 100.090 ohms.

All observations were made by comparisun with platinum intervals by the National Bureau of Standards. The most recent calibration is deted 07/21/85 N.B.S. No. 237502. 
Babcock \& Wilcox ${ }^{2}$

a McDermott company
RESEARCH \& DEVELOPMENT DIVISION

INSTRUMENT SERVICE LOG nite ill oles

NEV. DATE 7.20A

B\&W Ser. No. 0880504
Manufacturer MARLIN

Manuf. Ser. No. M-25373

Property No. 4427-01-062

$A \&$ \& No.

Tech. Procedure - Vend

$\begin{array}{lcc}\text { Section } & 4 \mathrm{G} & \text { HT REFL MECH } \\ \text { Location } & 115\end{array}$
BEAT 1

Model PRT1-MC-48

$1 \mathrm{tem}$ SENSOR, IMP, RES

Cal stat (HOF EATBATEO)

Scheduled-tYY CERTIfIES

Interval 12

Last Serviced
Repair Parts

Item
Cost
Manuf.

Standards Used

Model
Item
B\&W Serial No.

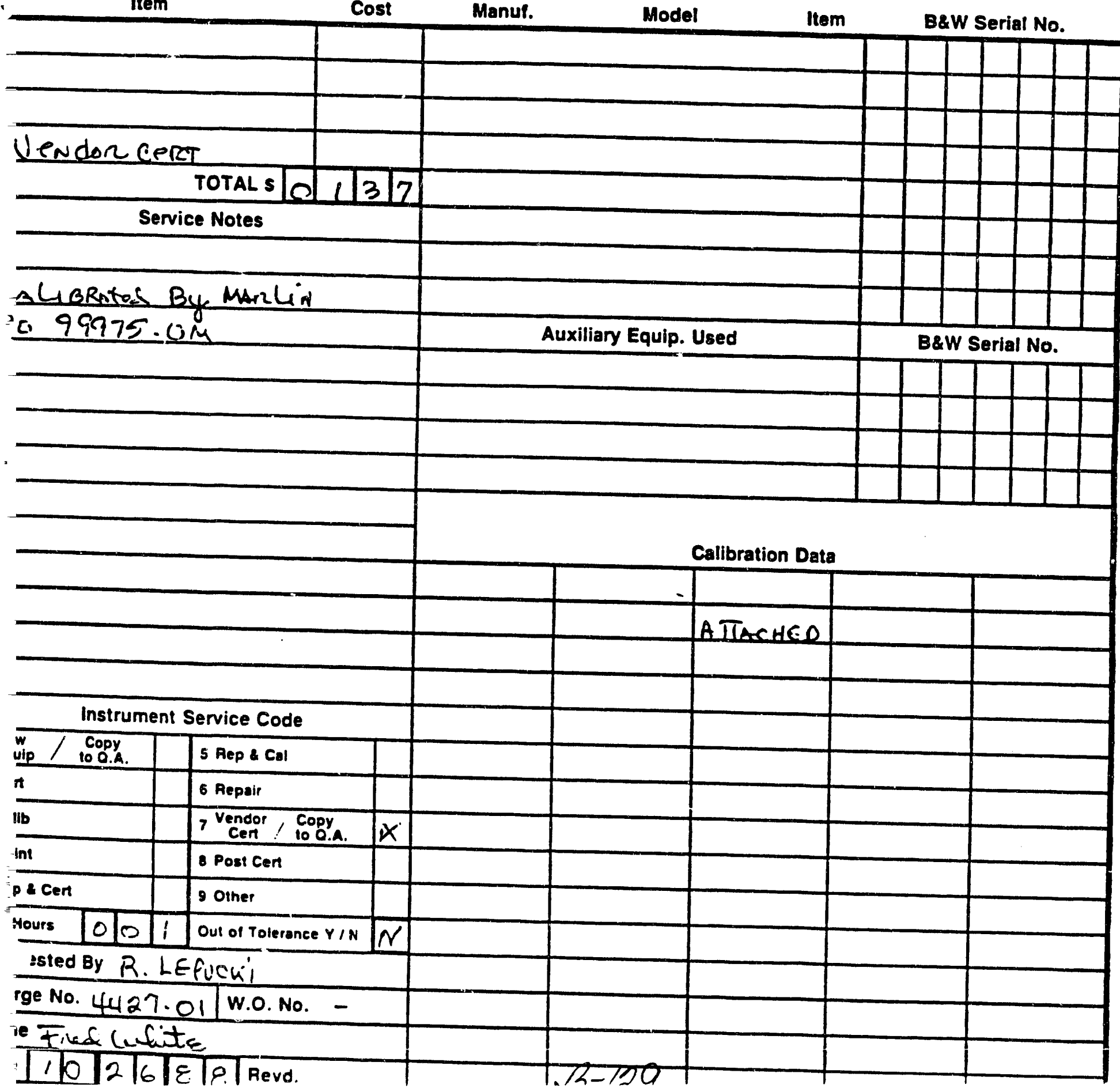


MARLIN JOB NO.

$$
\begin{aligned}
& \text { onBS - Jraceable } \\
& \text {-ertification }
\end{aligned}
$$

CERTIFICATION NO.

880300

$M-25373$

\author{
REPORI OF CALIBRAIION
}

FDR

PLATINUM RESISIANCE IHERMOMETER

The above platinum resistance thermometer was alibrated on 10/20/B日 for use with a continuous current of $1.0 \mathrm{mdlli}$ amps through the thermometar.

The following velues were found $F$ ar nonstants in the formules given in the International Precticel Iemperature scale of 1968 (IPIS-6日):

$$
\begin{array}{ll}
\text { Alpha }=.003848543 & \text { Delta }-1.495581 \\
A=3.906101 E-03 & \text { B }=-5.755807 E-07
\end{array}
$$

The pertinent formules are given in the discusision on the following pages.

The resistance at zero degrees Celsius was found to be 100.090 ohms.

All observations were made by comparison with platinum resistance thermometer standards calibrated at regular intervals by the National Bureau of Standards. The most recent calibration is deted 07/21/86 N.B.S. No. 237502 . 
- MARLIN JOB NO.

$$
\begin{aligned}
& \text { NBS - Dracealle } \\
& \text { Rertification } \\
& \text { for } \\
& \text { REPORT OF CALIBRATION } \\
& \text { PLAIINUM RESISTANCE IHERMDMEIER }
\end{aligned}
$$$$
\text { CERTIFICATION No. }
$$$$
880300
$$

The above platinum resistance thermometer was calibrated on 10/20/8B for use with a continuous current of 1.0 milliamps through the thermometer.

The following values were found for constants in the formules given in the International Practicel Iemperature Scale of 1958 (IPIS-EB):

$$
\text { Alphe - .003848543 Delte - } 1.495581
$$$$
A=3.905101 E-03 \quad B=-5.755807 E-07
$$

The pertinent formulas are given in the discussion on the following pages.

The resistance at zero degrees Celsius was found to be 100.090 ohms. The resistance at 100.042 degrees Celsius was 138.626 ohms and the resistance at 200.258 degrees Celsius was found to be 176.051 ohms.

All observations were made by comparison with platinum resistance thermometer standards calibrated at regular intervals by the National Bureau of Standards. The most recent celibration is dated $07 / 21 / 86$ N.B.S. No. 237502.

\author{
PROCEDURE: Comparison NBS Monograph-126
}




\section{9 \\ N.B.S. CERTIFIED LALURATIIFY STANIJARDS}

INSTRUMENTS:

VOLTAGE STANDARD:

RESISTANCE THERMOMETER:

RESISTANCE STANDARD:

THERMOCOUPLES:

RIBEON FILAMENT LAMP:

SHUNT:

FIXED POIITS :
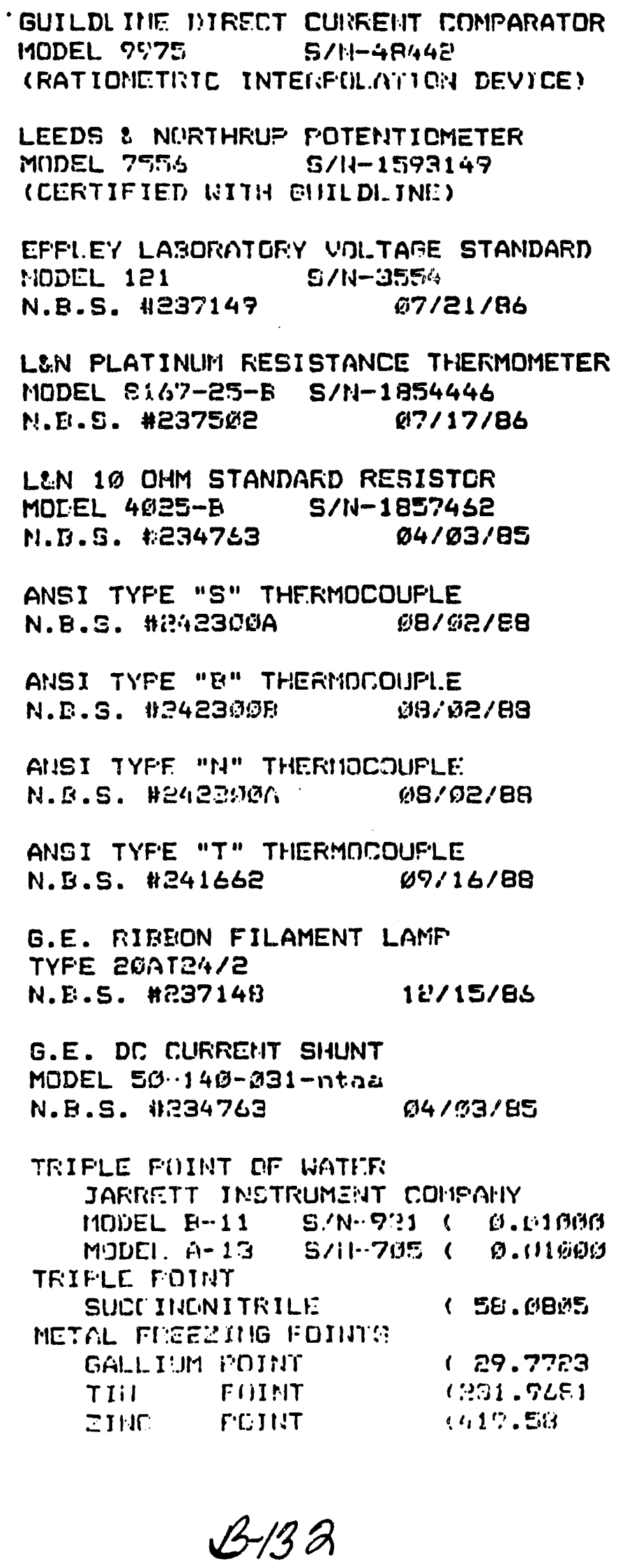


\begin{tabular}{|c|c|c|c|c|c|c|c|c|c|c|c|}
\hline$\vdots$ & c & $\vdots$ & $?$ & 3 & 4 & 5 & 6 & 7 & $\varepsilon$ & 9 & : 0 \\
\hline 管 & 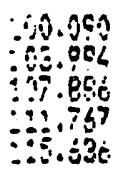 & 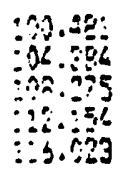 & 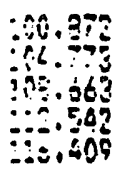 & 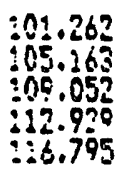 & 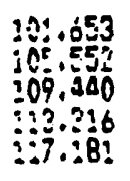 & 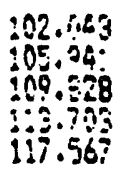 & $\begin{array}{l}: 02.434 \\
\vdots 06.39: \\
\vdots 10.206 \\
\vdots 4.096 \\
\vdots 7.052\end{array}$ & 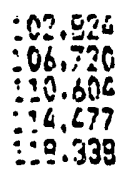 & 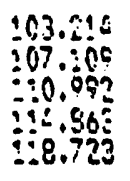 & 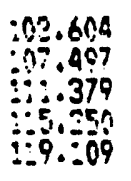 & 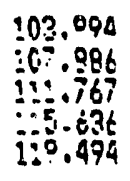 \\
\hline 9 & 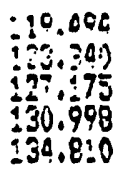 & 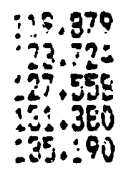 & 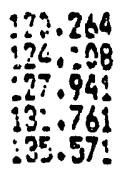 & $\begin{array}{l}120.649 \\
224.492 \\
258.373 \\
132.943 \\
135.951 \\
.35 .951\end{array}$ & $\begin{array}{r}779.028 \\
729.876 \\
728.706 \\
332.524 \\
136.331\end{array}$ & 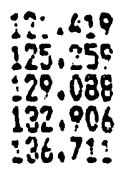 & 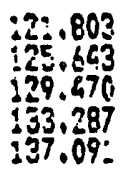 & 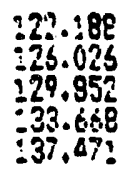 & $\begin{array}{l}122.579 \\
125.409 \\
130.235 \\
136.048 \\
137.85:\end{array}$ & $\begin{array}{l}56 \\
92 \\
16 \\
29 \\
3:\end{array}$ & $\begin{array}{r}130 \\
130 \\
? \\
3\end{array}$ \\
\hline & 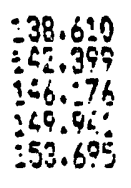 & 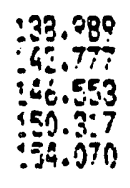 & 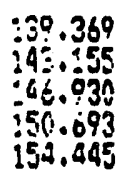 & $\begin{array}{r}139.748 \\
149.533 \\
147.307 \\
150 \\
150.069 \\
154.819\end{array}$ & $\begin{array}{l}100.197 \\
\vdots 43.919 \\
107.683 \\
151.046 \\
155 .: 994\end{array}$ & $\begin{array}{r}140.506 \\
44.264 \\
34.060 \\
55.060 \\
155.568\end{array}$ & 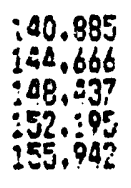 & 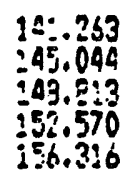 & 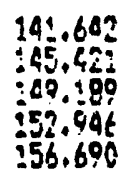 & $\begin{array}{l}142 \\
145 \\
149 \\
553 \\
15 ?\end{array}$ & $\begin{array}{l}342 \\
\vdots 46 \\
\vdots 49 \\
\vdots 50 \\
\vdots 5.5\end{array}$ \\
\hline 58 & 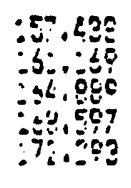 & 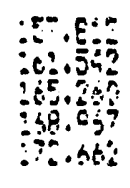 & 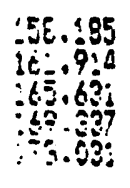 & $\begin{array}{r}158.559 \\
36 . .256 \\
360.009 \\
660.907 \\
172.400\end{array}$ & 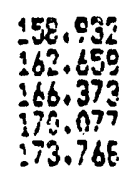 & 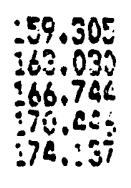 & $\begin{array}{r}55.670 \\
359.402 \\
369.915 \\
370.05 \\
39.505\end{array}$ & $\begin{array}{r}160.051 \\
\vdots 6.776 \\
667.485 \\
79.95 \\
374.974 \\
9.974\end{array}$ & $\begin{array}{l}160.428 \\
160.146 \\
109.05 t \\
7955 \\
375.242\end{array}$ & 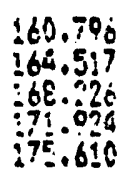 & 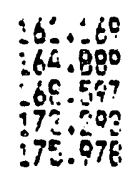 \\
\hline$\because$ & 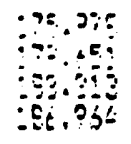 & ל & 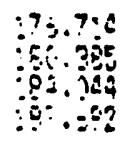 & $\begin{array}{l}77.090 \\
789.75 \\
38.40 \\
: 88.0566\end{array}$ & 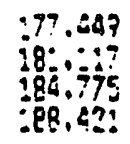 & 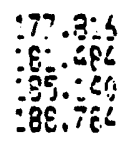 & 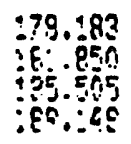 & 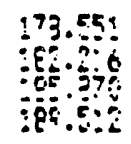 & 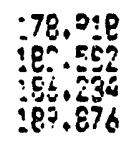 & 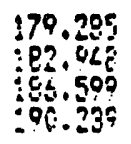 & \\
\hline & & & & ? & 4 & & & & 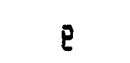 & & $\because$ \\
\hline
\end{tabular}




\section{TEMPERATURE RANGE: $0^{\circ}$ TO $630.74^{\circ} \mathrm{C}$}

Temperatures between $0^{\circ} \mathrm{C}$ and $630.74^{\circ} \mathrm{C}$ on the new International Practical Temperature Scale of 1968 (IPTS-58) are defined by the indications (resistance values) of standard platinum resistance thermometers and the following expressions:

$$
\begin{gathered}
t=t^{\prime}+M\left(t^{\prime}\right) \\
t^{\prime}=\frac{1}{a}\left(\frac{R_{t}}{R_{0}}-1\right)+6\left(\frac{t^{\prime}}{100}-1\right) \frac{t^{\prime}}{100} \\
M\left(t^{\prime}\right)=.045\left(\frac{t^{\prime}}{100}\right)\left(\frac{t^{\prime}}{100}-1\right)\left(\frac{t^{\prime}}{419.59}-1\right)\left(\frac{t^{\prime}}{630.74}-1\right) .
\end{gathered}
$$

where $t$ is the temperature, at the outside of the tube protecting the platirum resistor, in ' $C$ on the International Practical Temperature Seale of 1958 , and $R_{t}$ and $R_{0}$ ore the resistances of the platinum resistor at $t^{\circ}$ ar.d $0^{\circ} C$ respectively, messured with a continuous current through the flatinum resistor. The value of this current and the values of the constants a and 8 found for this thermometer are given on the previous paige. The value 'of $M\left(t^{\prime}\right)$, given by expression (3), is the same for all thermomezers and $i_{3}$ a function only of the quantity $t^{\prime}$. The addition of the small value represented by (3) serves to make the IPTS-63 conform more closely to the thermodynamic scale than can be done with only the simple quadratic or expression (2).

in alternate form which is completely equivalent to expression. (2) is

$$
R_{t}=P_{0}\left(1+A t^{\prime}+B t^{\prime 2}\right)
$$

in seme instances expression (4) is less dificicult :0 callulate than (2). it.e constants $A$ and $B$ used in $(4)$ are related directly to $a$ and 5 .

$$
\begin{aligned}
& A=a(1-5 / 100) \\
& B=-0 j / 10^{4}
\end{aligned}
$$

EAUTION: THE VALUE: SF $A, B$, ANO 3 ?II THE NEN 1953 SCALE A2E DIS-

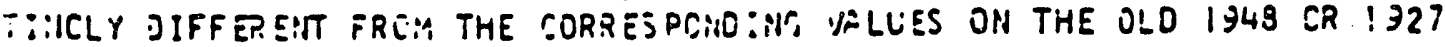

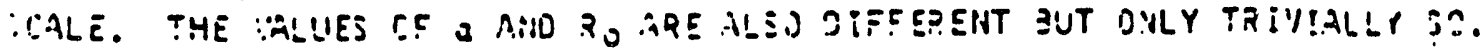




\section{Babcock \& Wilcox}

acDermott company
RESEARCH \& DEVELOPMENT DIVISIOR'

INSTRUMENT SERVICE LOG
RD-45A

MEV. DATE T.20.9
IPRTOI

$$
2-22-90 g R l \text {. }
$$

E\&W Ser: No. OBBi5:5

$\begin{array}{ll}\text { Manufacturer } & \text { MAfiLIN } \\ \text { Manuf. Ser. No. M-25374 } \\ \text { Froperty No. } & 4427-01-0 \dot{ }\end{array}$

A \& I No.

Tech. Procedure -VEND

Section 46 NSSC

Location

\section{Model}

Item

Cal stat

Scheduled $Y$

Interval 12

Last Serviced

Status
FRT 1-MC-12

SENSOF, TMF', FEES

(CEFT IF IED)

14-Nov-1989

\section{Repair Parts}

HTF-6 MEGAWATT FACILITY

Standards Used

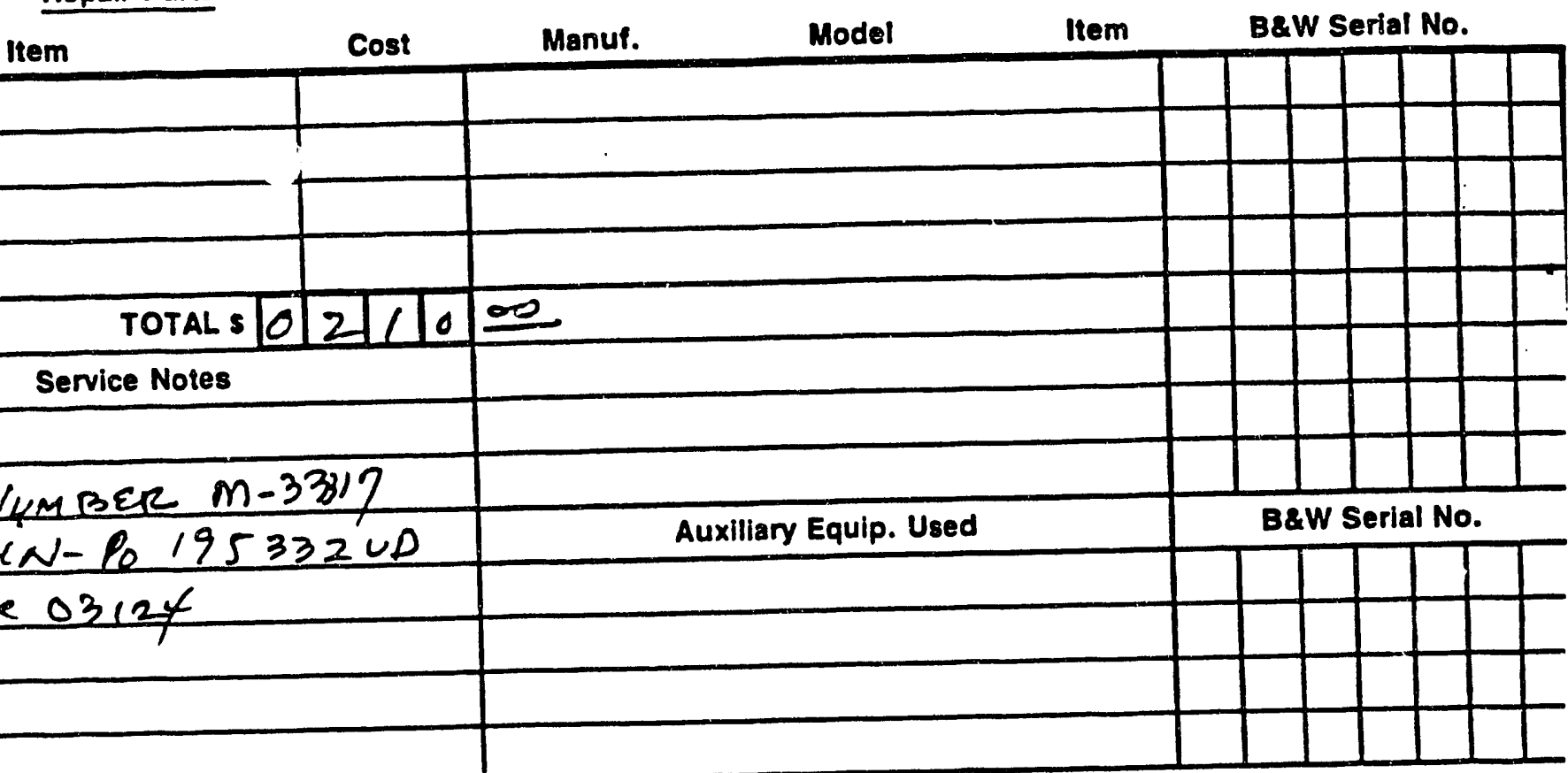

Test NUMBER $m-33317$

MARlin-Po 195332 UD

$=$ code 03124
Calibration Data

Instrument Service Code

\begin{tabular}{|c|c|}
\hline 0 New $/ \begin{array}{c}\text { Copy } \\
\text { to } 0 . A .\end{array}$ & 5 Rep a Cal \\
\hline 1 Cont & 6 Repair \\
\hline 2 Callb & $7 \begin{array}{c}\text { Vendor } \\
\text { Cent } / \text { Copy } \\
\text { to O.A. }\end{array}$ \\
\hline 3 Maint & 8 Post Cen \\
\hline 4 Rep a Cert & 9 Other \\
\hline "an-Hours & Out of Tolerance $Y / N$ \\
\hline
\end{tabular}

- equested By Jary CystER

\begin{tabular}{ll|l|l}
\hline Charge No. 4427.14 & W.o. No.
\end{tabular}

Name 17 al calde

Attached




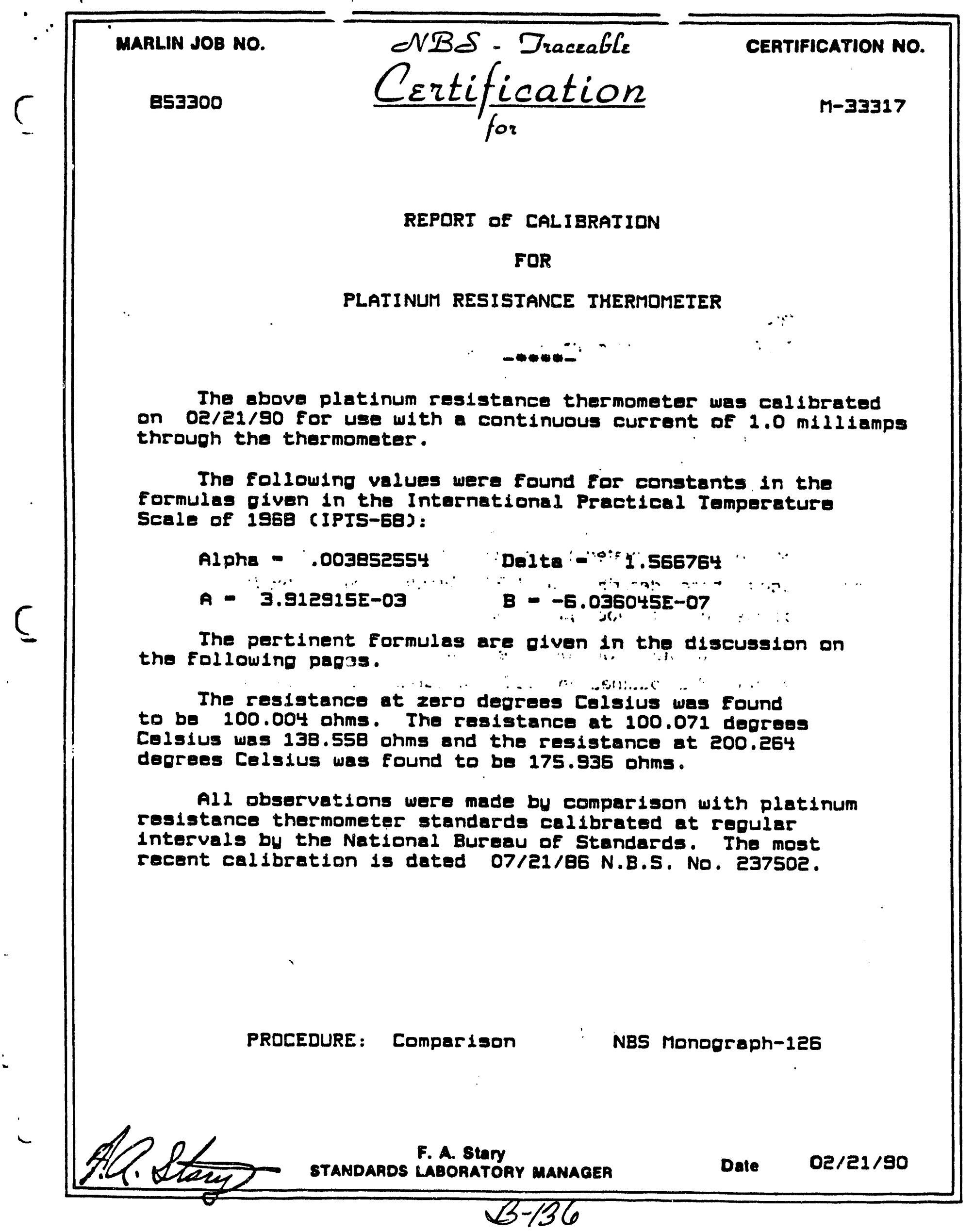




\begin{tabular}{|c|c|c|c|c|c|c|c|c|c|c|c|c|}
\hline 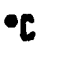 & 0 & 1 & 2 & 3 & 4 & 5 & 6 & 7 & 8 & 9 & 10 & \\
\hline $\begin{array}{l}8 \\
10 \\
20 \\
30 \\
40\end{array}$ & $\begin{array}{l}100.004 \\
103.911 \\
107.806 \\
111.689 \\
115.560\end{array}$ & $\begin{array}{l}100.395 \\
104.301 \\
108.195 \\
112.077 \\
115.946\end{array}$ & $\begin{array}{l}100.786 \\
104.691 \\
108.584 \\
112.464 \\
116.332\end{array}$ & $\begin{array}{l}101.17 \\
105.081 \\
108.972 \\
112.851 \\
116.719\end{array}$ & $\begin{array}{l}101.568 \\
105.470 \\
109.361 \\
113.239 \\
117.105\end{array}$ & $\begin{array}{l}101.959 \\
105.860 \\
109.749 \\
113.626 \\
117.491\end{array}$ & $\begin{array}{l}102.350 \\
106.249 \\
110.137 \\
114.013 \\
117.876\end{array}$ & $\begin{array}{l}102.740 \\
106.639 \\
110.525 \\
114.400 \\
118.262\end{array}$ & & $\begin{array}{l}21 \\
17 \\
01 \\
73 \\
33\end{array}$ & $\begin{array}{l}11 \\
69 \\
39 \\
60\end{array}$ & \\
\hline $\begin{array}{l}30 \\
60 \\
70 \\
80 \\
90\end{array}$ & $\begin{array}{l}119.418 \\
123.265 \\
127.100 \\
130.922 \\
134.733\end{array}$ & $\begin{array}{l}119.804 \\
123.649 \\
127.483 \\
131.304 \\
135.113\end{array}$ & $\begin{array}{l}120.189 \\
124.033 \\
127.865 \\
131.685 \\
135.493\end{array}$ & $\begin{array}{l}120.574 \\
124.417 \\
128.248 \\
132.067 \\
135.873\end{array}$ & $\begin{array}{l}120.959 \\
124.800 \\
128.630 \\
132.448 \\
136.254\end{array}$ & $\begin{array}{l}121.343 \\
125.184 \\
129.012 \\
132.829 \\
136.633\end{array}$ & $\begin{array}{l}121.728 \\
125.567 \\
129.395 \\
133.210 \\
137.013\end{array}$ & $\begin{array}{l}122.112 \\
125.951 \\
129.777 \\
133.591 \\
137.393\end{array}$ & $\begin{array}{l}77 \\
34 \\
2 \\
2\end{array}$ & $\begin{array}{l}122.881 \\
126.717 \\
130.541 \\
134.352 \\
138.152\end{array}$ & 5 & \\
\hline & $\begin{array}{l}138.531 \\
142.317 \\
146.092 \\
149.854 \\
153.604\end{array}$ & $\begin{array}{l}138.910 \\
142.695 \\
146.468 \\
150.229 \\
153.978\end{array}$ & $\begin{array}{l}139.289 \\
143.073 \\
146.845 \\
150.605 \\
154.352\end{array}$ & $\begin{array}{l}139.668 \\
143.451 \\
147.222 \\
150.980 \\
154.727\end{array}$ & $\begin{array}{l}140.047 \\
143.829 \\
147.598 \\
151.355\end{array}$ & $\begin{array}{l}140.426 \\
144.206 \\
147.974 \\
151.730 \\
155.474\end{array}$ & $\begin{array}{l}140.804 \\
144.583 \\
148.350 \\
152.105 \\
155.848\end{array}$ & $\begin{array}{l}141 \\
144 \\
148 \\
152 \\
156\end{array}$ & $\begin{array}{l}141.561 \\
145.338 \\
149.102 \\
152.855\end{array}$ & $\begin{array}{l}39 \\
15 \\
78 \\
29\end{array}$ & 14 & \\
\hline 17 & $\begin{array}{l}157.342 \\
161.068 \\
164.782 \\
168.484 \\
172.173\end{array}$ & $\begin{array}{l}157.715 \\
161.440 \\
165.152 \\
168.853 \\
172.542\end{array}$ & $\begin{array}{l}158.088 \\
161.812 \\
165.523 \\
169.222 \\
172.910\end{array}$ & $\begin{array}{l}158.461 \\
162.183 \\
165.899 \\
169.592 \\
173.278\end{array}$ & $\begin{array}{l}158 \\
162 \\
166 \\
169 \\
173\end{array}$ & $\begin{array}{l}159.206 \\
162.926 \\
166.634 \\
170.330 \\
174.014\end{array}$ & $\begin{array}{l}79 \\
98 \\
99 \\
99\end{array}$ & $\begin{array}{l}159 \\
163 \\
167 \\
171 \\
174\end{array}$ & & $\begin{array}{l}96 \\
11 \\
14 \\
05 \\
84\end{array}$ & $\begin{array}{l}16 \\
16 \\
17 \\
17\end{array}$ & \\
\hline 22 & $\begin{array}{l}175.851 \\
179.516 \\
183.170 \\
186.811\end{array}$ & $\begin{array}{l}176.218 \\
179.818 \\
183.535\end{array}$ & $\begin{array}{l}176.585 \\
180.248 \\
183.899 \\
187.538\end{array}$ & $\begin{array}{l}176.952 \\
180.614 \\
184.264 \\
187.902\end{array}$ & $\begin{array}{l}177.319 \\
180.979 \\
184.628 \\
188.265\end{array}$ & $\begin{array}{l}177.685 \\
181.345 \\
184.992 \\
188.628\end{array}$ & $\begin{array}{l}052 \\
10 \\
356 \\
991\end{array}$ & $\begin{array}{l}178.418 \\
182.075 \\
185.72 \\
189.35\end{array}$ & & & & \\
\hline & 0 & 4 & 2 & 2 & & 5 & & 7 & 8 & & 10 & \\
\hline
\end{tabular}




\section{9 \\ NATIONAL INSTITUTE OF STANDARDS AND TECHNOLOGY CERTIFIED LAEORATORY STANDAFDS}

INSTRUMENTS:

VOLTAGE' STANDARD:

\section{RESISTANCE THERMOMETER:}

\section{RESISTANCE STANDARD:}

THERMOCOUPLES:

C

FIBBON FILAMENT LAMF:

SHUNT:

FIXED POINTS:

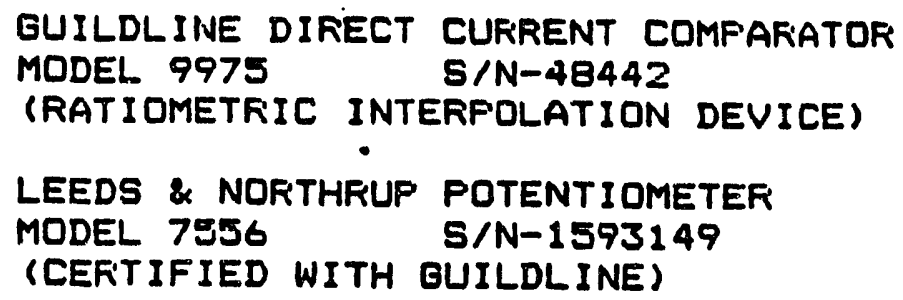

EPFLEY LABOFATORY VOLTAGE STANDARD MODEL 121

N.I.S.T. \#242636 . 12/14/88

L\&N FLATINUM RESISTANCE. THERMOMETER MODEL 8167-25-E S/N-1854446

N.I.S.T. \#237502 07/17/86

LEN 10 OHM STANDARD RESISTOR

MODEL 4O2S-B . S/N-1857462

N.I.S.T. \#244720 $10 / 19 / 89$

ANSI TYFE "S" THERMOCOUPLE

N.I.S.T. \#244669A 09/20/89

AN5I TYFE "B" THERMOCOUPLE

N.I.S.T. \#244669B 09/20/89

ANSI TYFE "N" THERMOCOUPLE

N.I.S.T. \#244669A $12 / 01189$

ANSI TYFE "T" THERMOCOUFLE

N.I.S.T. \#241662 $09 / 16 / 88$

G.E. RIEBON FILAMENT LAMF

TYFE 2OAT24/2

N.I.S.T. \#237148 12/15/86

G.E. DC CURFENT SHUNT

MODEL 50-140-031-ntaa

N.I.S.T. \#234763 04/03/85

TRIIFLE POINT OF WATER JAFRETT INSTFIUMENT COMFANY

MODEL E-11 S/N-921 ( $0.01000 \mathrm{C})$ MODEL A-13 5/N-705 ( 0.01000 C)

TRIIFLE FOINT SUCCINONITRILE $\quad(58.1805$ C)

METAL FREEZING FOINTS GALLIUM FOINT TIN FOINT ZINC FOINT

$(29.7723$ C)

$(231.9681 \quad C)$

1419.58

C) 


\section{TEMPERATURE RANGE: $0^{\circ}$ TO $630.74^{\circ} \mathrm{C}$}

Temperatures between $0^{\circ} \mathrm{C}$ and $630.74^{\circ} \mathrm{C}$ on the new International Practical Temperature Scale of 1968 (IPTS-68) are defined by the indications (resistance values) of standard platinum resistance thermometers and the following expressions:

$$
\begin{aligned}
& t=t^{\prime}+M\left(t^{\prime}\right) \\
& t^{\prime}=\frac{1}{a}\left(\frac{R_{t}}{R_{0}}-1\right)+8\left(\frac{t^{\prime}}{100}-1\right) \frac{t^{\prime}}{100} \\
& M\left(t^{\prime}\right)=.045\left(\frac{t^{\prime}}{100}\right)\left(\frac{t^{\prime}}{100}-1\right)\left(\frac{t^{\prime}}{419.58}-1\right)\left(\frac{t^{\prime}}{630.74}-1\right)
\end{aligned}
$$

where $t$ is the temperature, at the outside of the tube protecting the platinum resistor, in $C$ on the International Practical Temperature Scale of 1968, and $R_{t}$ and $R_{0}$ are the resistances of the platinum resistor at $t^{\circ}$ and $0^{\circ} \mathrm{C}$ respectively, measured with a continuous current through the platinum resistor. The value of this current and the values of she constants a and $\delta$ found for this thermometer are given on the previous page. The value of $M\left(t^{\prime}\right)$, given by expression (3), is the same for all thermomezers and is a function only of the quantity $t^{\prime}$. The addition of the small value represented by (3) serves to make the IPTS-68 conform more closely to the thermodynamic scale than can be done with only the simple quadratic of expression (2).

in alternate foim which is completely equivalent to expression (2) is

$$
R_{t}=P_{0}\left(1+A_{t}{ }^{\prime}+B_{t} t^{2}\right)
$$

in some instances expression (4) is less dificult to caleulate than (2). the constanis $A$ and $B$ used in (4) are related directly to a and $\delta$.

$$
\begin{aligned}
& A=a(1+s / 100) \\
& B=-a j / 10^{4}
\end{aligned}
$$

CAUTION: THE VALUES SF 2, B, AHO : DH THE NIEN 1953 jCALE ARE D ISTTHCLY DIFFER ENT FRLY THE CORRESPOND?NG JALUES ON THE OLD 1949 CR 1927 ICALE. THE IALUES R.F \& AND RO ARE ALSO S:FEE?ENT ZUT JIVLY TRIVIALLY SO. 
RD-15A Rev. $11-28-91$

\begin{tabular}{|l|l|l|}
\hline INSTRUMENT STATUS & RECD & IEST \\
\hline Certified & & \\
\hline Calibrated & & \\
\hline 3 For Ind. Only & & \\
\hline 4 Standardize & & \\
\hline 5 Certified s stand. & & \\
\hline 6 Calibrated s stand. & & \\
\hline 9 other & & \\
\hline
\end{tabular}

INSTRUMENT CONDITION

AS RECEIVED (Certified Equipment only)

Meets Manufacturer's Tolerance $N$

Operates within previously defined limits $N$

Deviates from $M f g$ or previously defined limits $Y$

Inoperative (Describe below) $Y$

AS LEFI

Limited (Describe below)

Meets Manufacturer's Tolerance

other (Describe Below)
Post Cert of Ret. Re

Babcock \& Wilce

- MeDermo:t company

B\&W Ser. NO.' 0760 :

MANUFACTURER

MODEL

ITEM

MANUF. SER. NO.

PROPERTY NO.

ORDER NO.

SECTION

IOCATION

SCHEDULED $(Y-N)$

L\&N

4030-B

RESISTR, STNDRD

1838925

RECAL INT.(MOS.)

46

181

$Y$

12

\section{TECH. PROCEDURE A0456-00}

Babcock Wilcox RCD Division certifies that performance of this instrument was verified $u$ : standards whose accuracies are traceable to ti National Institute: of Standards and Technolog: an accepted value of a natural physical const: a rat lo type of calibration technique, or to consensus standard.
STANDARDS USED

Manufacturer Model

ESI SP3632/242D RESISMEAS SYSTIEA ERTCO ASTM-17C THERMOMETER
Bew Serial No.

\begin{tabular}{l|l|l|l|l|l|l|l|}
\hline 0 & 8 & 0 & 0 & 2 & 9 & 6 & Vendor Cert/Cal/Repair
\end{tabular}

\begin{tabular}{|c|c|c|c|c|c|c|c|}
\hline$a$ & 8 & 2 & 0 & 3 & 1 & 3 & Repair Parts \\
\hline
\end{tabular}

TOTAL"MATERIAL COST

TOTAL IABOR HOURS

Requested BY JOYSTER

Charge Number $4032-50$

work order No. $T R / S$.

OTHER EOUTPMENT USTD

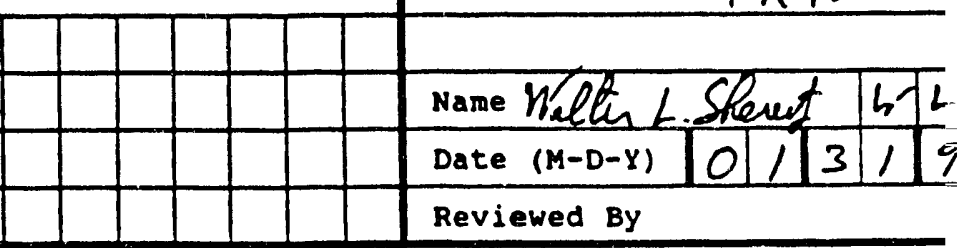

Service Notes

SEE ATTHCHED SHEET 


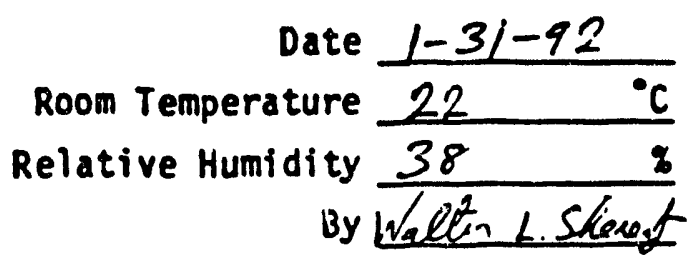

STANDARD RESISTOR CALIBRATION

OF

$L \$ N^{\prime} C A T, 4030-B$

BEW OT60179, MFE S.N.1838425

THIS RESISTOR HAS A VALUE AT $25.0^{\circ} \mathrm{C}$ OF:

99,9998

This value is obtained from the measured value at $22,4^{\circ} \mathrm{c}, \mathrm{R}_{t}$ below, plus corrections for deviation from $25.0^{\circ} \mathrm{C}$ from a Temperature Correction Table furnished by the manufacturer or a calibration laboratory, and/or the formula:

$$
R_{t}=R_{25}\left[1+\alpha(t-25)+B(t-25)^{2}\right]
$$

where: $R_{t}=$ Resistance at $t^{\circ} \mathrm{C}$

$R_{25}=$ Resistance at $25^{\circ} \mathrm{C}$

$\alpha$ and $B=$ Resistor constants furnished by manufacturer or calibration laboratory.

The $\alpha$ and $B$ values from latest temperature coefficient data are:

$$
\begin{aligned}
& \alpha=0,000003609 \\
& B=-0,000000522
\end{aligned}
$$


1 RD-45A ReV $11-18-91$

\begin{tabular}{|l|l|l|}
\hline \multicolumn{1}{|c|}{ INSTRUMENT STATUS } & ASECO & AS \\
\hline 1 Certified & & \\
\hline Calibrated & & \\
\hline 3 For Ind. Only & & \\
\hline 4 standardize & & \\
\hline 5 Certified s stand. & & \\
\hline 6 Calibrated s stand. & & \\
\hline 9 other & & \\
\hline
\end{tabular}

INSTRUMENT CONDITION

AS RECEIVED (Certified Equiprent only)

Meets Marufacturer's Tolerance $N$

operates within previously defined limits $N$ Deviates from MEg or previously defined linits $y$ Inoperative (Describe below) Y

AS LEFT

Iimited (Describe below)

Meets Manufacturer's Tolerance other (Describe Below)
WSTMU I

Babcock \& Wilcox

- MCDermott company

B\&W Ser. NO. 0880506
MANUFACTURER

MODEL

ITEM

MANUF. SER. NO.

PROPERTY NO.

ORDER NO.

SECTION

IOCATION

SCHEDULED ( $\mathrm{Y}-\mathrm{N})$

RECAI INT. (MOS.)
FLOW TECH

FT-64CISW

METER, TURBINE

640363

4427-03-095

46

115

ArY

12

\section{TECH. PROCEDURE VEND}

Babcock Wilcox RED Division certifies that the performance of this instrument was verified using standards whose accuracies are traceable to the National Institute of standards and Technology. an uccepted value of a natural physical constant, a ratio type of calibration technique, or to a consensus standard.

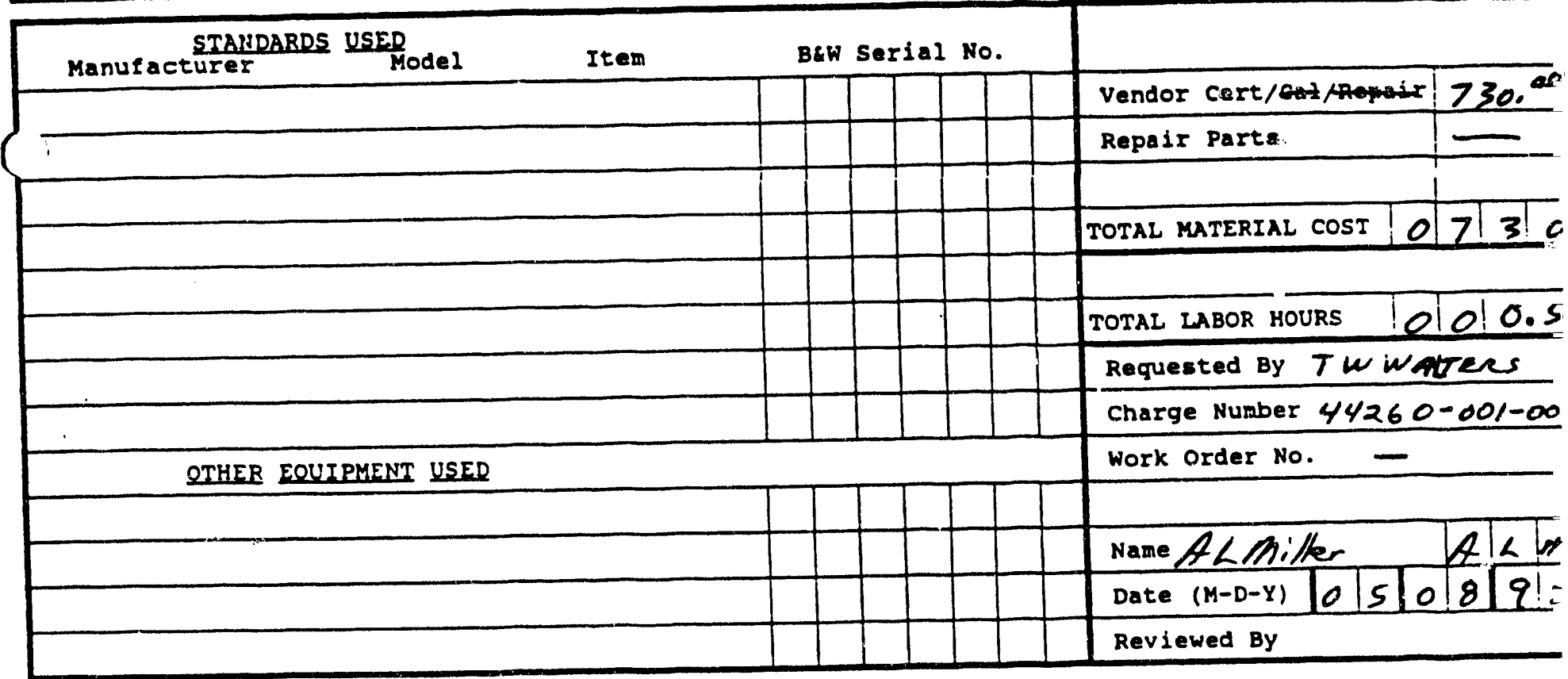

Service Notes

$$
\text { Po } 01685 \quad 29006
$$

Deviation report written because not all data points were within $\infty$ PO specified range + Acceptad"as is" by Tw walters. 


\section{Babcock \& Wilcox \\ a MCDermott company \\ Ð. ALLIANCE RESEARCH CENTER \\ 1562 BEESON STREET - ALLIANCE, OHIO 44601 \\ ATTN $2<12$. \\ LYNCHBURG RESEARCH CENTER \\ MT. ATHOS - LYNCHBURG, VIRGINIA 24505 \\ ATTN:}

\section{$\triangle$ PURCHASE ORDER DEVIATION REPORF}

NITIATOR ALC $M I L L E R$

Vendor EISCARR PokTEP
SECTION 184

P.O. NO. $0 / 685$
DATE $5-20-92$

PACK SLIP NO. 1119366

J shortage $\square$ overage $\square$ wrong matl $\square$ defectivematl $\square$ certification 区other Pata Print

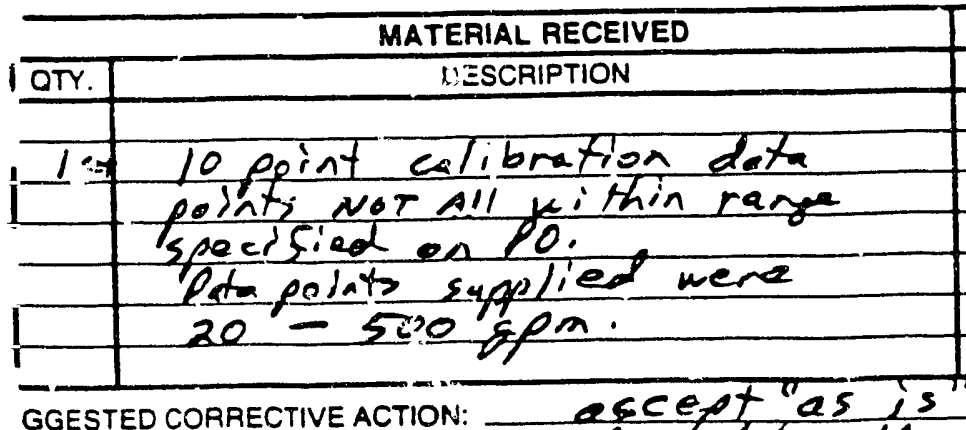
GGESTED CORRECTIVEACTION: eccept as is

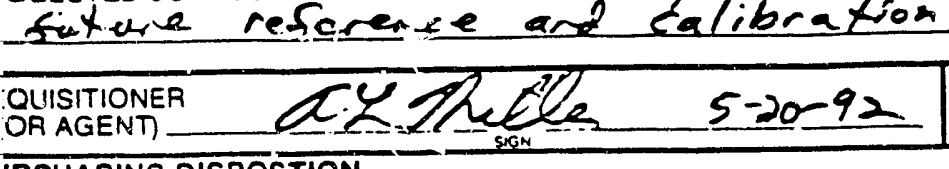
URCHASING DISPOSTION

ACCEPTI $\square$ CREUIT $\square$ YENDOR $\square$ RETURN $\square$ CHANGE $\square$ OTHER aPproval I

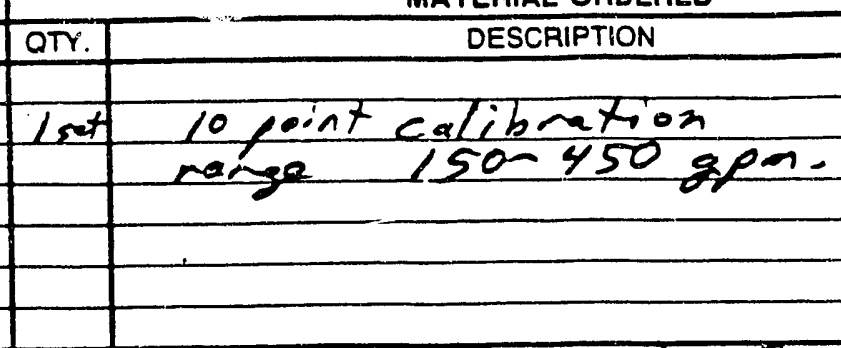
PAY INVOICE COMING TO REPLACE MATERIAL NOTICE

per Tw walte.s o nete sor.

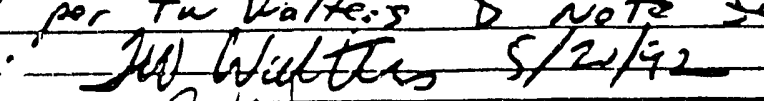

r

NITIATOR

] SHIPMENT TO VENDOR (REQUIRES PURCHASING APPROVAL)

-IATERIAL QTYIDESCRIPTION:

IEASON FOR SHIPMENT: $\square$ REPAIRS $\square$ CALIBRATION $\square$ P.O. DEVIATION (SEE ABOVE) $\square$ OTHER XPLAIN:
SPECIAL INSTRUCTIONS: $\square$ HAZARDOUS MATERLALS $\square$ OTHER

COMPANY:

STREET:

CITYISTATE:

ATTN:
APPROVAL

SECTION

DATE

$\square$ OTHER (SHIPMENT TU CUSTOMER, CONTRACTS, ETC)

\section{ATTN:} ZIP:

- TERIALS HANDLING USE ONLY \begin{tabular}{l|l|l|}
\hline NO. CTMS. & WEIGHT & \\
\hline & &
\end{tabular} FREIGHT BILL NO.
P.O. NO.: CHARGE NO:

SHIP VIA:

VALUE:

COPY TO: 


\section{PISCHERTER}

Babcock \& Wilcox Company Alliance Research Center 1562 Beeson Street NE Alliance, OH 44601-2165
Date: 11 May 1992

Subject: CERTIFICATION

Ref: $92 W 340593$

Customer I.D.\#: 0880506

\section{TURBINE FLOMMETER CALIBRATION ADCURACY CERTITFICATE}

A. METER IDENTIFICATION:

(1) Customer Purchase Order Number: 01685

(2) F\&P Serial Number: 92W340593

(3) Sales Order Number: RA39953FW.001

(4) Model Number: Flow Tech

B. CALIBRATION SPECIFICATIONS:

(1) Date of Calibration: 8 May 1992

(2) Metering Fluid: Water

(3) Flow Range: 500 to 22 Units: GPM

C. ACOURACY: \pm 1 \& of Rate flow using $\mathrm{mV}$ data

D. CALTBRATION FACIIITIIES:

The Measurement and Test Equipment and associated procedures used in the calibration of this meter are in accordance with MIL-STD 45662 , and are traceable to NIST (formerly NBS).

The basis of this traceability is through one or more of the following NIST Test Numbers:

MASS

DC Volt

AC Volt

RESISTANCE

AC RATIO

TEMPERATURE

LENGIH

PRESSURE

FREQUENCY

SONIC NOZZLES

Calibration procedure

QE-FTP-344 Rev. 0
TEST NLIBIAR

39761/737-228509

$245417 / 245243 / 100081$

$243344 / 24516 / 100082$

$242461 / 244478 / 100083$

246699

238455

$731 / 244239-89$

738-229929/738-232442/737-067044

$737-067043 / 523 / 236557$

WWVB

$731-241460-88 / 9103031004 / 38126 / 240257$

$238455 / 10379 / 738-232727 / 731-243669$

Very truly yours,

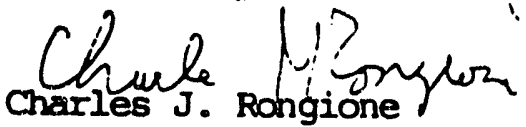

Sr. Flow Standards Engineer

CJR:bpl 
FISCHER : PORTER CO. WARMINSTER, PA

\author{
Serial No: 92 W340593 \\ Model No : Flow-TECH \\ Max Flow: 560 GPM(US) \\ Cust. ID:0880506
}

Date : 8 MAY 92

SIZE - : 4 "

SP GR $(6 \theta / 60): 1$

MODEL:FT-641SW-LED-5
RIG:4L

VISCOSITY: 1.0751 CST

RIG TEMP : $63.14^{\circ} \mathrm{F}$

CALIBRATION DATA

CALIBRATION FLUID: H2O

FLUID DEN. : .99872

\section{TEST DATA RESULTS}

\begin{tabular}{cc} 
Run & Rig Std. \\
\hline 01 & $\frac{R}{3-T 2-C 17}$ \\
02 & $3-T 2-C 17$ \\
03 & $3-T 2-C 17$ \\
04 & $3-T 2-C 17$ \\
05 & $3-T 2-C 17$ \\
06 & $3-T 2-C 17$ \\
07 & $2-T 2-C 20$ \\
08 & $2-T 2-C 20$ \\
09 & $2-T 2-C 20$ \\
10 & $2-T 2-C 20$ \\
--1 & - \\
11 & $3-T 2-C 17$ \\
12 & $3-T 2-C 17$
\end{tabular}

Hertz

$4 \overline{35.840000}$ 390.390000

343.380000

298.590000

255.820800

208.250000

365.068000

267.160000

168.820008

43.268000 $i$

253.580000
438.560000

\begin{tabular}{cr}
$K-$ cyc/Gal & $\frac{M V}{300.1000}$ \\
\hline 52.745 & 274.6000 \\
52.747 & 249.1000 \\
52.749 & 224.5000 \\
52.750 & 175.1000 \\
52.751 & 148.6000 \\
52.750 & 124.9000 \\
119.777 & 100.9000 \\
119.825 & 70.5000 \\
120.859 &
\end{tabular}

52.751

52.745
GPM(US)

495.7858

444.8680

390.5821

339.6278

298.8664

236.8700

182.8691

133.7755

84.3687

21.5736

288.4285

498.8811
$\begin{array}{ll}\Delta E= & K=\frac{\Delta E}{Q} \\ 240.1 & 0.4843 \\ 214.6 & 0.4833 \\ 289.1 & 0.4841 \\ 1645 & 0.4844 \\ 140.7 & 0.4851 \\ 11.5 .1 & 0.4854 \\ 88.6 & 0.4845 \\ 64.9 & 0.4851 \\ 40.9 & 0.4848 \\ 10.5 & 0.4867\end{array}$

139.7

241.4
0.4850
$0.48 ?$

CALIBRATED BY : NL

@ZERO FLOW $=60.0 \mathrm{~mJ}$

TEST EQUIFMENT :

QC \# Description

E-158/ RATE/RATIO

T-5B THERMOMETER

E-234 DVM

VAPORT 1,RM 200292 


$\begin{array}{rc}\text { ROW } & 1 \\ 3 & 0.4841000 \\ 4 & 0.4844000 \\ 5 & 0.4851000 \\ 6 & 0.4859000 \\ 7 & 0.4845000 \\ 8 & 0.4851000 \\ 9 & 0.4848000 \\ 10 & 0.4867000 \\ 11 & 0.4850000 \\ 12 & 0.4839000\end{array}$

DEGREE OF POLYNOMIAL
$0.12000000000 D+02 \quad 0.16503000000 D+000$
0.4842939
$-0.19$
$39229 \mathrm{E}-03$
$-0.4005844 \mathrm{E}-01$
0.1891000
$-0.1154352 \mathrm{E}-03-0.2383055 \mathrm{E}-01$
0.1645000
0.4847297
$0.3702562 E-03$
$0.7632574 E-01$
0.1407000
$0.9397393 E-03$
0.1934018
$-0.6988817 \mathrm{E}-03$
$-0.1442480$
$-0.3122898 \mathrm{E}-03$
$-0.6437638 E-01$
$-0.8283994 \mathrm{E}-03$
$-0.1708745$
$0.7978618 \mathrm{E}-03$
0.1639330
$0.2630525 \mathrm{E}-03$
$0.7701582 \mathrm{E}-04$
$0.5423763 E-01$
$0.1591565 \mathrm{E}-01$
0.1151000
$0.8860000 \mathrm{E}-1$
$0.6490000 \mathrm{E}-1$
$0.4090000 \mathrm{E}-1$
$0.1050000 \mathrm{E}-\mathrm{I}$
0.1399000
0.2414000

\begin{tabular}{|c|c|c|c|c|c|}
\hline ESS $=$ & 0.3 & $56 \mathrm{E}-05$ & $S D=0.6209 \mathrm{E}-03$ & $=0.574853 \mathrm{E}+00$ & \\
\hline ROW & $\#$ & 1 & $0.12000000000 D+02$ & $0.16503000000 D+01$ & $0.29125573000 D+0$ \\
\hline ROW & $\neq$ & 2 & $0.16503000000 D+01$ & $0.29125573000 D+00$ & $0.57091809129 D-0.1$ \\
\hline ROW & $\#$ & 3 & $0.29125573000 D+00$ & $0.57091809129 D-01$ & $0.11883638349 D-0.1$ \\
\hline ROW & $\#$ & 4 & $0.58171000000 D+01$ & $0.79941770000 \mathrm{D}+00$ & $0.14103811658 D+0 C$ \\
\hline
\end{tabular}

พSTMO1

$\begin{array}{rr} & \text { OBSERVED } \\ 1 & 0.4843000 \\ 2 & 0.4833000 \\ 3 & 0.4841000 \\ 4 & 0.4844000 \\ 5 & 0.4851000 \\ 6 & 0.4859000 \\ 7 & 0.4845000 \\ 8 & 0.4851000 \\ 9 & 0.4848000 \\ 10 & 0.4867000 \\ 11 & 0.4850000 \\ 12 & 0.4839000\end{array}$

CALCULATED
0.4838942
0.4840774
0.4842730
0.4844736
0.4846787
0.4849115
0.4851657
0.4854045
0.4856572
0.4859933
0.4846858
0.4838852

DIFFERENCE

$0.4057875 \mathrm{E}-03$

$-0.7773636 E-03$

$-0.1730044 E-03$

$-0.7357662 E-04$

$0.4213110 \mathrm{E}-03$

$0.9885405 \mathrm{E}-03$

$-0.6656728 \mathrm{E}-03$

$-0.3044520 \mathrm{E}-03$

$-0.8572481 E-03$

$0.7066611 E-03$

$0.3142275 \mathrm{E}-03$

$0.1478997 \mathrm{E}-04$
PERCENT

$0.8378845 E-01$

$-0.1608449$

$-0.3573733 \mathrm{E}-01$

$-0.1518923 E-01$

$0.8685034 \mathrm{E}-01$

0.2034453

$-0.1373938$

$-0.6276066 \mathrm{E}-01$

$-0.1768251$

0.1451944

$0.6478917 \mathrm{E}-01$

$0.3056411 E-02$

\begin{tabular}{l}
\multicolumn{1}{c}{$X(I)$} \\
0.2401000 \\
0.2146000 \\
0.1891000 \\
0.1645000 \\
0.1407000 \\
0.1151000 \\
$0.8860000 \mathrm{E}-0$ \\
$0.6490000 \mathrm{E}-0$ \\
$0.4090000 \mathrm{E}-0$ \\
$0.1050000 \mathrm{E}-0$ \\
0.1399000 \\
0.2414000
\end{tabular}

DEGREE OF POLYNOMIAL 2

\section{COEFFICIENTS}

$0.48611355 E+00-0.11549258 E-01 \quad 0.96038351 E-02$

$L^{-s S}=0.3828 \mathrm{E}-05$

ROW 1

$0.57091809129 D-01$

$$
S D=0.6522 E-03 \quad R 2=0.577907 E+00
$$

$0.12000000000 D+02 \quad 0.16503000000 D+01$

$0.29125573000 D+00$ 


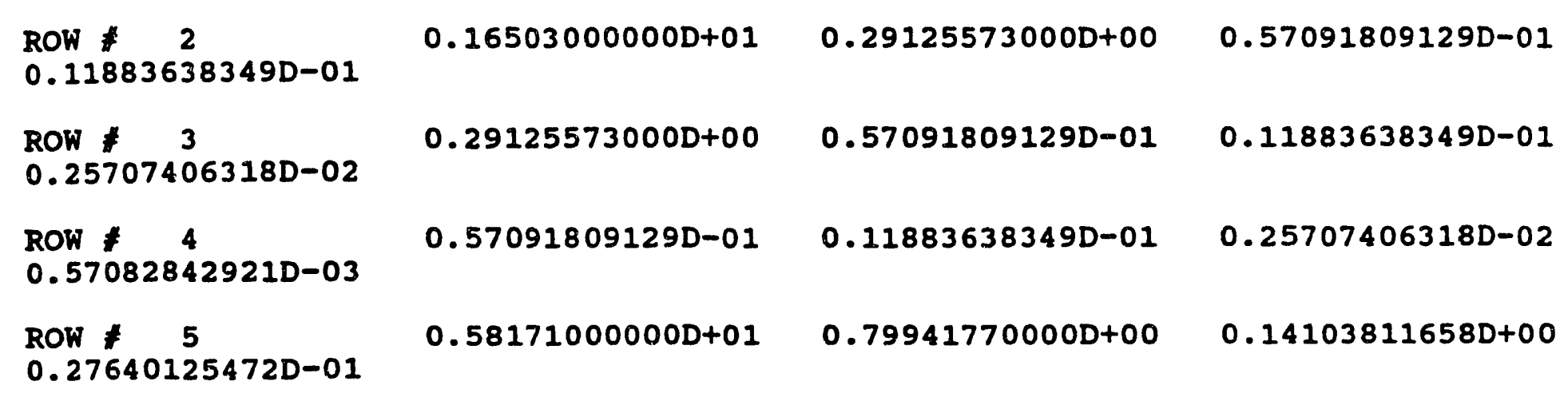

WSTM01

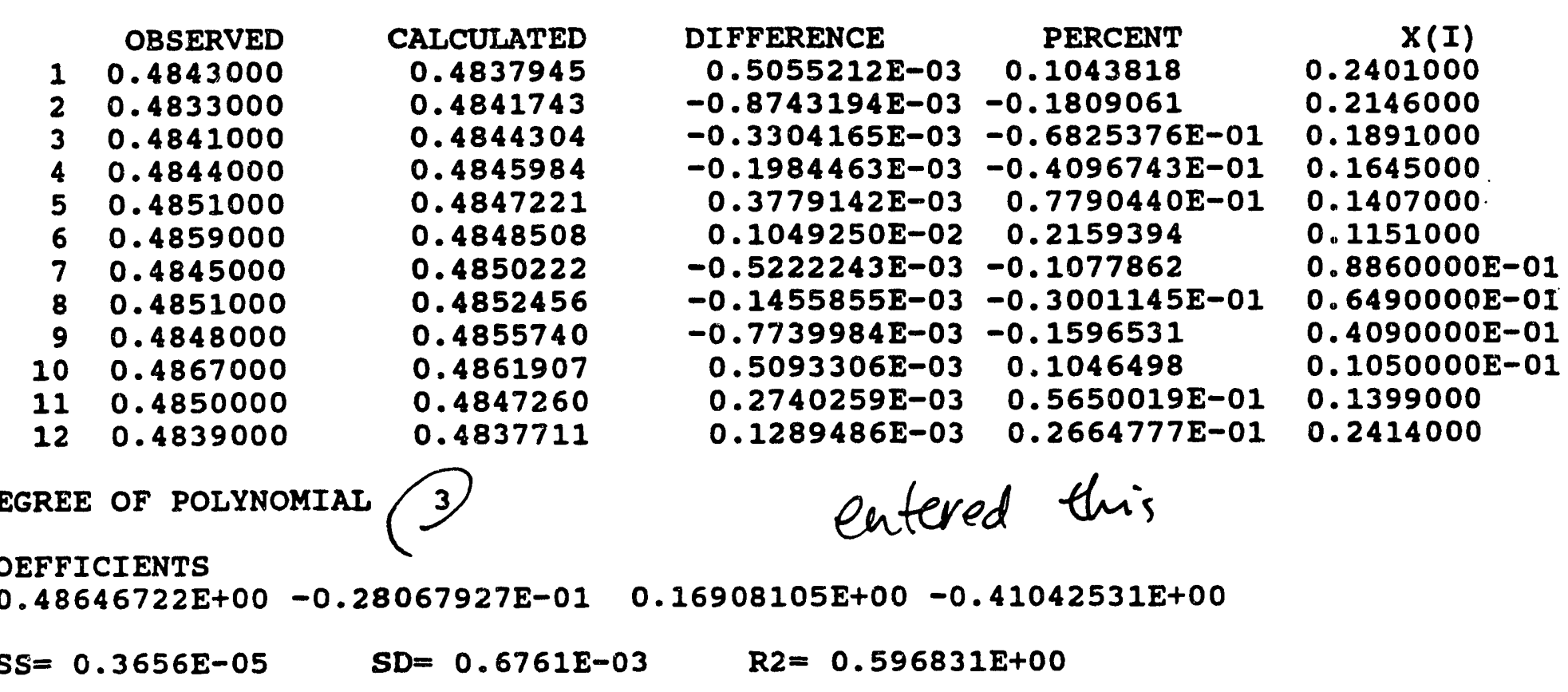

$$
K=\frac{\Delta E(m v)}{Q(g p m)}
$$

$B-1449$ 


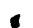

Nothing toriteport APS

RD-45A Rev. 11-18-91

\begin{tabular}{|l|l|l|}
\hline INSTRUMENT STATUS & RECD & AET \\
\hline Certified & \\
\hline 2 Calibrated & & \\
\hline 3 For Ind. Only & & \\
\hline 4 Standardize & & \\
\hline 5 Certified \& stand. & & \\
\hline 6 Calibrated \& stand. & & \\
\hline 9 Other & & \\
\hline
\end{tabular}

INSTRUMENT CONDITION

AS RECEIVED (Certified Equipment only)

Meets Manufacturer's Tolerance $N$

Operates within previously defined limits $N$

Deviates from Mfg or previously defined limits $Y$

AS LEFT

Limited (Describe below)

Meets Manufacturer's Tolerance

other (Describe Below)

\section{CZDPUZ \\ OUD $6 / 22 / a_{2} j$
Babcock \& Wilco \\ a MeDermotl company}

B\&W Ser. No.

088051

MANUFACTURER SENSOTEC

MODEL

ITEM

21309-15

MANUF SER NO TRANSMITR,D-P

No. 187394

ORDER NO.

4427-01-123

\section{SECTION}

IOCATION

46

SCHEDULED $(\mathrm{Y}-\mathrm{N})$

RECAL INT.(MOS.)

115

$Y$

12

\section{TECH. PROCEDURE}

Babcock \& W W cox RED Division certifies that the performance of this instrument was verified using National Institute of an accepted value of Standards and Technology. a ratio type of cal a natural physical constant. consensus standard.

\section{STANDARDS USED}

$\int$ Manufacturer Model Item BeW serial No.

\begin{tabular}{|ccc|c|c|}
\hline ASHCROFT & $0-10$ PSI & PRESS, STD & 0 & 8 \\
\hline FLUKE & $8840 A$ & DMM & 0 & 8 \\
\hline
\end{tabular}

\begin{tabular}{|c|c|}
\hline FLUKE $8840 \mathrm{~A}$ & \\
\hline & \\
\hline & \\
\hline & \\
\hline & \\
\hline & \\
\hline
\end{tabular}

\begin{tabular}{llllllll|l|l|}
\hline & & & & & & \\
\hline & & & & & & & \\
\hline & & & & & & & & \\
\hline & & & & & & \\
\hline
\end{tabular}

TOTAL MATERIAL COST

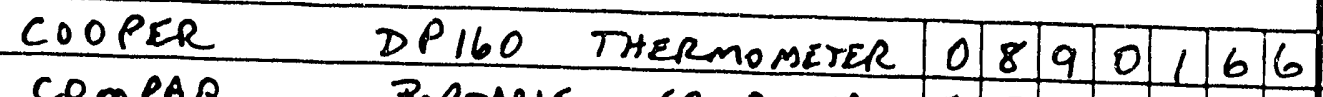
\begin{tabular}{l} 
COMPAQ PORTABLE COMPUTER \\
\hline
\end{tabular}

\begin{tabular}{l|l|l|l} 
TOTAL LABOR HOURS & 0 & 0 & 2,0 \\
\hline
\end{tabular} Requested BY SCOTT SRROLL Charge Number $44260-102-002$ Work Order No. 0342 $0-10$ PSI SEE ATTACHED DATA SHEET. LIMITED TO DATA PROVIDED. POES NOT MEET H:I RATIO SAECS. 
AS FECEIVED

TRANSMI TTEF:

SENSOTEC $Z$

10 F'SI

TOLEFIANCE : $0.25 \%$

'RESS SOURICE

\section{ASHCROFT DIGIGAUGE $0-10$ F.SI}

BR:W Ser. No. 0810225

TOLEFIANCE : $0.05 \%$

IEADOUT DEV:
AS LEFT

B\&W S.N. O880519

$$
\text { DATE: } \quad 03-J 4 n-92
$$

FROCEDUFE : AFIC-TF-015-08

FFIOGFIM: TOO15_08.03A

NAME: Dowey fype

Ambient Temperature (F) 75

Facility I. D. Name C2DFO2
FLUKE 8840A

E\&W Ser. No. OB50454

TOLEFIANCE: $0.015 \%$
'ALUE OF CUFIFENT SENSING FIESISTOF' USED FOF' TEST DATA

'ALUE OF CURFENT SENSING FIESISTOF' USED FOF' CALIEFATION
'OWEF' SUFFLY VOLTAGE

DTAL SEFIIES FESISTANCE

AF'F'LIED F'FIESSURE

FIANSMITTEF

FSI

FRESS SOURICE

F.SI

$\begin{array}{lr}0.000 & 0.000 \\ 1.000 & 1.000 \\ 2.000 & 2.000 \\ 3.000 & 3.000 \\ 4.000 & 4.000 \\ 5.000 & 5.000 \\ 6.000 & 6.000 \\ 7.000 & 7.000 \\ 8.000 & 8.000 \\ 9.000 & 9.000 \\ 10.000 & 10.000 \\ 9.000 & 9.000 \\ 8.000 & 8.000 \\ 7.000 & 7.000 \\ 6.000 & 6.000 \\ 5.000 & 5.000 \\ 4.000 & 4.000 \\ 3.000 & 3.000 \\ 2.000 & 2.000 \\ 1.000 & 1.000 \\ 0.000 & 0.000\end{array}$

FESISTOF MEASUFIING ACCURACY 24

14.999

IDEAL

OUTFUT SIG

MILLIVOLTS

59.996

8.5. 994

107.993

131.991

155.990

179.988

203.986

227.985

251.985

275.982

299.980

275.982

251.983

227.985

203.986

179.988

155.990

131.991

107.993

83.994

59.996 $\begin{array}{ll}14.999 & \text { OHMS } \\ 14.999 & \text { OHMS }\end{array}$

N.A.

$\begin{array}{clc}\text { CALIEFIATION ACCUFACY } & \\ \text { MELIMITED** } & \\ \text { MEASURED } & \text { EFIRIOF } \% & \text { OUT } \\ \text { QUTFUT SIG } & \text { FULL SCALE } & \text { OF } \\ \text { MILLIVOLTS } & & \text { TOLEFIANCE }\end{array}$


DATE: $\quad 3-J U N-92$

TIME: $11: 31: 43$

(

VOLTAGE(V) OBS. DP(PSI) CALC. DP(PSI)

DIFF (PSIA)

\& DIFFERENCE

$1-0.3400101 \mathrm{E}-04$

$0.0000000 E+00$

-0.4470691
0.9997427
2.000442

1.000000

2.000000

$0.4810200 \mathrm{E}-01$

3.000000

$0.7215400 E-01$

4.000000

5.000000

3.001110

$0.9616000 \mathrm{E}-01$

6.000000

0.1441760

7.000000

0.1681760

8.000000

9.000000

10.00000

9.000000

0.2399350

8.000000

7.000000

6.000000

4.000455

5.000848

6.001081

7.001945

8.002397

9.000263

0.2160180

9.998003

0.1921160

8.998843

0.1681470

8.000894

7.000735

0.1441070

5.000000

5.998204

4.000000

4.998973

0.9609500 E-01

3.000000

3.997748

2.997989

2.000000

1.998903

$0.4806500 \mathrm{E}-01$

1.000000

0.9995349

$0.0000000 \mathrm{E}+00$

$0.2336300 \mathrm{E}-02$

$0.4470691 \mathrm{E}-03$

$0.2573097 \mathrm{E}-03$

$-0.4422066 \mathrm{E}-03$

$-0.1110315 \mathrm{E}-02$

$-0.4550294 \mathrm{E}-03$

$-0.8479087 \mathrm{E}-03$

$-0.1080743 E-02$

$-0.1944788 \mathrm{E}-02$

$-0.2396633 \mathrm{E}-02$

$-0.2627812 \mathrm{E}-03$

$0.1996830 \mathrm{E}-02$

$0.1157193 E-02$

$-0.8940116 \mathrm{E}-03$

$-0.7350553 \mathrm{E}-03$

$0.1795892 \mathrm{E}-02$

$0.1027052 E-02$

$0.2251649 \mathrm{E}-02$

$0.2010932 \mathrm{E}-02$

$0.1096699 \mathrm{E}-02$

$0.4651468 \mathrm{E}-03$

$0.3299899 \mathrm{E}-04$

DEGREE OF POLYNOMIAL 2

C JEFFICIENTS

$0.96542800 E-03 \quad 0.41542820 E+02 \quad 0.51175891 E+00$

ESS $=0.4032 \mathrm{E}-04 \quad \begin{aligned} \mathrm{SD} & =0.1497 \mathrm{E}-02 \\ 2 * \mathrm{SD} & =0.2993 \mathrm{E}-02\end{aligned} \quad \mathrm{R} 2=0.100000 \mathrm{E}+01$ 


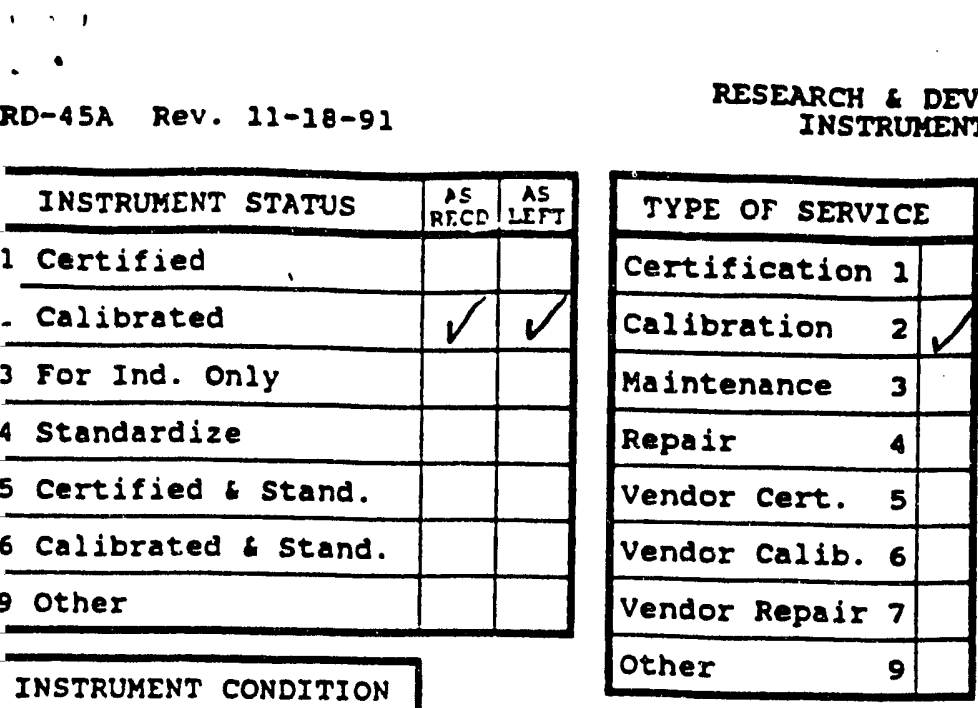

AS RECEIVED (Certified Equipment only)

Meets Manufacturer's Tolerance $N$

operates within previously defined limits $N$ Deviates from $\mathrm{Mfg}$ or previously defined limits $Y$ Inoperative (Describe below) $Y$ AS LEFT Limited (Describe below) Meets Manufacturer's Tolerance other (Describe Below)

\section{CRDPO2 $02 \mathrm{D} / 3 / 92$ Babcock \& Wilcox \\ a McDermoti company}

B\&W Ser. No. 0880519

MANUFACTURER

MODEL

ITEM

MANUF. SER. NO TRANSMITR, D-P

PROPERTY

ORDER NO.

SECTION

LOCATION

SCHEDULED $(Y-N)$

RECAL INT.(MOS.)

4427-01-123

46
115
$Y$
12

TECH. PROCEDURE

Babcock \& Wilcox RCD Division certifies that the performance of this instrument was verified using standards whose accuracies are traceable to the National Institute of standards and Technology, an accepted value of a natural physical constant. a ratio type of cal ibration technisu constant consensus standard.

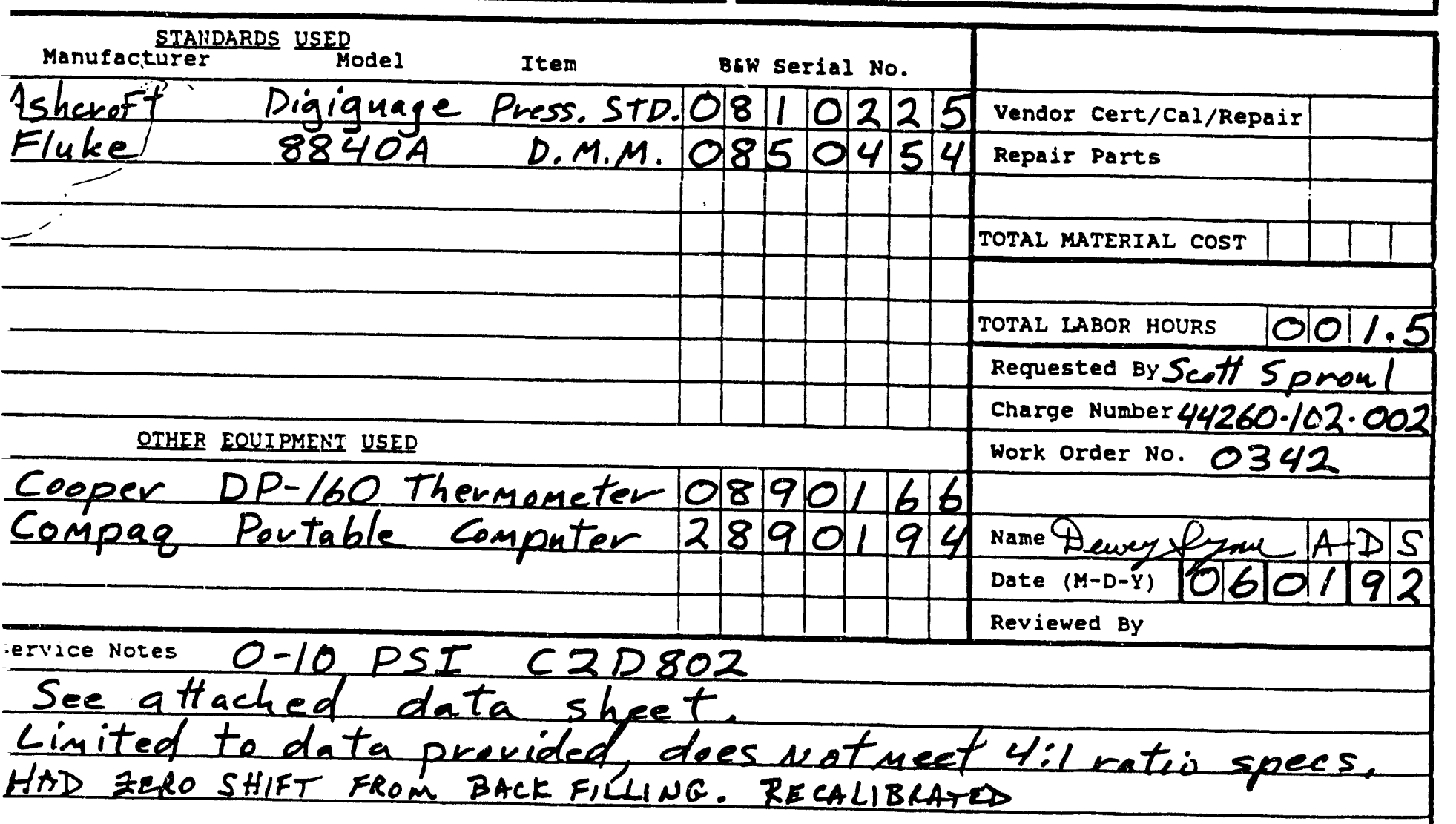


AS RECEIVED

IANSMITTEF:

SENSOTEC $Z$

$10 \mathrm{~F} S \mathrm{SI}$

TOLEFANCE : $0.25 \%$

PRESS SOURCE

$$
\begin{aligned}
& \text { ASHCFOFT } \text { DIGIGAUGE } \\
& \text { O-10 FSI } \text { FI } \\
& \text { B\&W SEF. NO. } 0810225 \\
& \text { TOLEFIANCE: } 0.05 \%
\end{aligned}
$$

FEADOUT DEV:

B\&W SER. NO. 0850454

AS LEFT

E\&W S.N. 0880519. DATE: 01-Jun-92

FROCEDURE : ARC-TF-O15-OE

FFIOGFAM: TOO15_0B.03A

NAME:

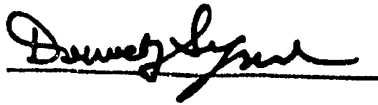

Ambient Temperature (F) 75

Facility I. D. Name CZDFOZ

VALUE OF CURFENT SENSING FEESISTOR USED FOF TEST DATA

VALUE OF CUFFENT SENSING FIESISTOR USED FOF CALIEFIATION

F'OWEF' SUFF'Y VOLTAGE

TOTAL SEFIES RESISTANCE

AFF'LIED F'FESSURE

TRANSMITTER

FSI

FRESS SOUF:CE

F'S I

0.000

$1: 000$

2.000

5.000

4.000

5.000

6.000

7.000

8.000

9.000

10.000

9.000

8.000

7.000

6.000

5.000

4.000

5.000

2.000

1.000

0.000
0.000

1.000

2.000

3.000

4.000

5.000

6.000

7.000

8.000

9.000

10.000

9.000

8.000

7.000

6.000

5.000

4.000

3.000

2.000

1.000

0.000
RESISTOF: MEASUFIING ACCUFACY 24

14.999

IDEAL

$$
\begin{aligned}
& \text { OUTFUT SIG } \\
& \text { MILLIVOLTS }
\end{aligned}
$$

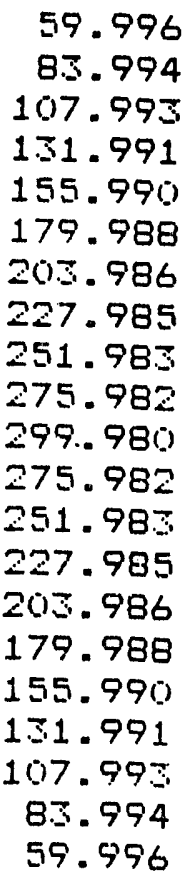

$\begin{array}{cl}\text { CALIBFATION ACCUFIACY } \\ & \text { **LIMITED** } \\ \text { MEASUFED } & \text { EFRIOF \% } \\ \text { OUTFUT SIG } & \text { FULL SCALE } \\ \text { MILLIVOLTS } & \end{array}$

14.999 OHMS

14.999 OHMS

N.A.

\section{**LIMITED** \\ $0.32 \%$

OUT
QF
TOLEFIANCE

$\begin{array}{rr}59.993 & 0.00 \% \\ 84.032 & 0.02 \% \\ 108.065 & 0.03 \% \\ 132.130 & 0.06 \% \\ 156.123 & 0.06 \% \\ 180.145 & 0.07 \% \\ 204.160 & 0.07 \% \\ 228.172 & 0.08 \% \\ 252.141 & 0.07 \% \\ 276.055 & 0.03 \% \\ 299.921 & -0.02 \% \\ 275.977 & 0.00 \% \\ 252.047 & 0.03 \% \\ 228.070 & 0.04 \% \\ 204.046 & 0.02 \% \\ 180.048 & 0.03 \% \\ 156.057 & 0.03 \% \\ 132.021 & 0.01 \% \\ 108.002 & 0.00 \% \\ 83.971 & -0.01 \% \\ 60.009 & 0.01 \%\end{array}$

$$
\bar{z}=60.001
$$


VOLTAGE (V) OBS. DP(PSI) CALC. DP(PSI)

DIFF (PSIA)

* DIFFERENCE

$\begin{array}{rl} & -0.8000762 \mathrm{E}-05 \\ 2 & 0.2403100 \mathrm{E}-01 \\ 3 & 0.4806400 \mathrm{E}-01 \\ 4 & 0.7212900 \mathrm{E}-01 \\ 5 & 0.9612200 \mathrm{E}-01 \\ 6 & 0.1201440 \\ 7 & 0.1441590 \\ 8 & 0.1681710 \\ 9 & 0.1921400 \\ 10 & 0.2160540 \\ 11 & 0.2399200 \\ 12 & 0.2159760 \\ 13 & 0.1920460 \\ 14 & 0.1680690 \\ 15 & 0.1440450 \\ 16 & 0.1200470 \\ 17 & 0.9605600 \mathrm{E}-01 \\ 18 & 0.7202000 \mathrm{E}-01 \\ 19 & 0.4800100 \mathrm{E}-01 \\ 20 & 0.2397000 \mathrm{E}-01 \\ 21 & 0.7999238 \mathrm{E}-05\end{array}$

$$
\begin{array}{ll}
0.0000000 E+00 & 0.1063804 E-02 \\
1.000000 & 1.000615
\end{array}
$$

2.000000

3.000000

4.000000

5.000000

6.000000

7.000000

8.000000

9.000000

10.00000

9.000000

7.000000

6.000000

5.000000

4.000000

3.000000

2.000000

1.000000

$0.0000000 \mathrm{E}+00$
2. 000371

3.001915

4.000915

5.001578

6.002402

7.003556

8.003369

9.001339

9.997755

8.998083

7.999447

6.999302

5.997650

4.997536

3.998167

2.997377

1.997750

0.9980780

$0.1728939 \mathrm{E}-02$
8.000000
$-0.1063804 \mathrm{E}-02$

$-0.6150028 \mathrm{E}-03$

$-0.3714892 \mathrm{E}-03$

$-0.1914775 \mathrm{E}-02$

$-0.9154280 \mathrm{E}-03$

$-0.1577581 \mathrm{E}-02$

$-0.2402240 \mathrm{E}-02$

$-0.3555821 \mathrm{E}-02$

$-0.1339246 \mathrm{E}-02$

$0.2245025 \mathrm{E}-02$

$0.1916553 \mathrm{E}-02$

$0.5526187 \mathrm{E}-03$

$0.6979151 \mathrm{E}-03$

$0.2349780 \mathrm{E}-02$

$0.2463971 \mathrm{E}-02$

$0.1833247 \mathrm{E}-02$

$0.2622642 E-02$

$0.2249862 \mathrm{E}-02$

$0.1921977 \mathrm{E}-02$

$-0.1728939 E-02$
$-0.3369265 E-02$
$0.0000000 E+00$

$-0.6150028 E-01$

$-0.1857446 E-01$

$-0.6382585 \mathrm{E}-01$

$-0.2288570 E-01$

$-0.3155163 \mathrm{E}-01$

$-0.4003734 \mathrm{E}-01$

$-0.5079745 E-01$

$-0.4211581 E-01$

$-0.1488051 \mathrm{E}-01$

$0.2245025 E-01$

$0.2129503 E-01$

$0.6907734 \mathrm{E}-02$

$0.9970216 E-02$

$0.3916300 \mathrm{E}-01$

$0.4927943 \mathrm{E}-01$

$0.4583117 E-01$

$0.8742140 E-01$

0.1124931

0.1921 .977

$0.0000000 E+00$

JEGREE OF POLYNOMIAL 2

JEFFICIENTS

$0.13964029 E-02 \quad 0.41570942 E+02 \quad 0.39363291 E+00$

$0.8328 E-04$

$S D=0.2151 \mathrm{E}-02$

$2 * S D=0.4302 \mathrm{E}-02$

R2 $=$

$0.100000 E+01$ 
RD-45A ReV. $11-18-91$

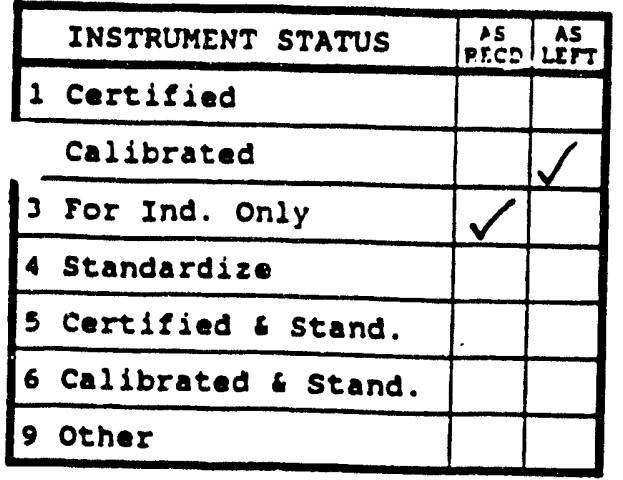

INSTRUMENT CONDITION

AS RECEIVED (Certified Eguipment Only)

Heets Manufacturer's Tolerance $N$

Operates within previously defined limits $\mathrm{N}$ Deviates from Mfg or previously defined limits $Y$ Inoperative (Describe below) $Y$

AS LEFT Limited (Describe below) Meets Manufacturer's Tolerance other (Describe Below)
ARCH \& DEVELOPHENT DIVISION INSTRUMENT SERVICE LOG

\begin{tabular}{|l|l|l|}
\hline \multicolumn{2}{|l|}{ TYPE OF SERVICE } \\
\hline Certification 1 & \\
\hline Calibration & 2 & \\
\hline Maintenance & 3 & \\
\hline Repair & 4 & \\
\hline Vendor Cert. & 5 & \\
\hline Vendor Ca.11b. & 6 & \\
\hline Vendor Repair & 7 & \\
\hline Other & 9 & \\
\hline
\end{tabular}

\section{SECTION}

\section{LOCATION}

SCHEDULED ( $Y-N)$

RECAL INT. (MOS.)
CZDPOZ

Babcock \& Wilcox

acDermolt company

B\&W Ser. No. 088051

SENSOTEC

21309-15

TRANSMITR, D-P

187394

4427-01-123

46

115

$+y$

12

\section{TECH. PROCEDURE AO015}

Babcock Wilcox RED Division certifies that the performance of this instrument was ver if that the standards whose accuracicsent was verifled using National Inst tute of cles are traceable to the National Institute of standards and Technology. a ratio type of cal a natural physical constant. a ratio type of calibration technique, or to consensus standard.

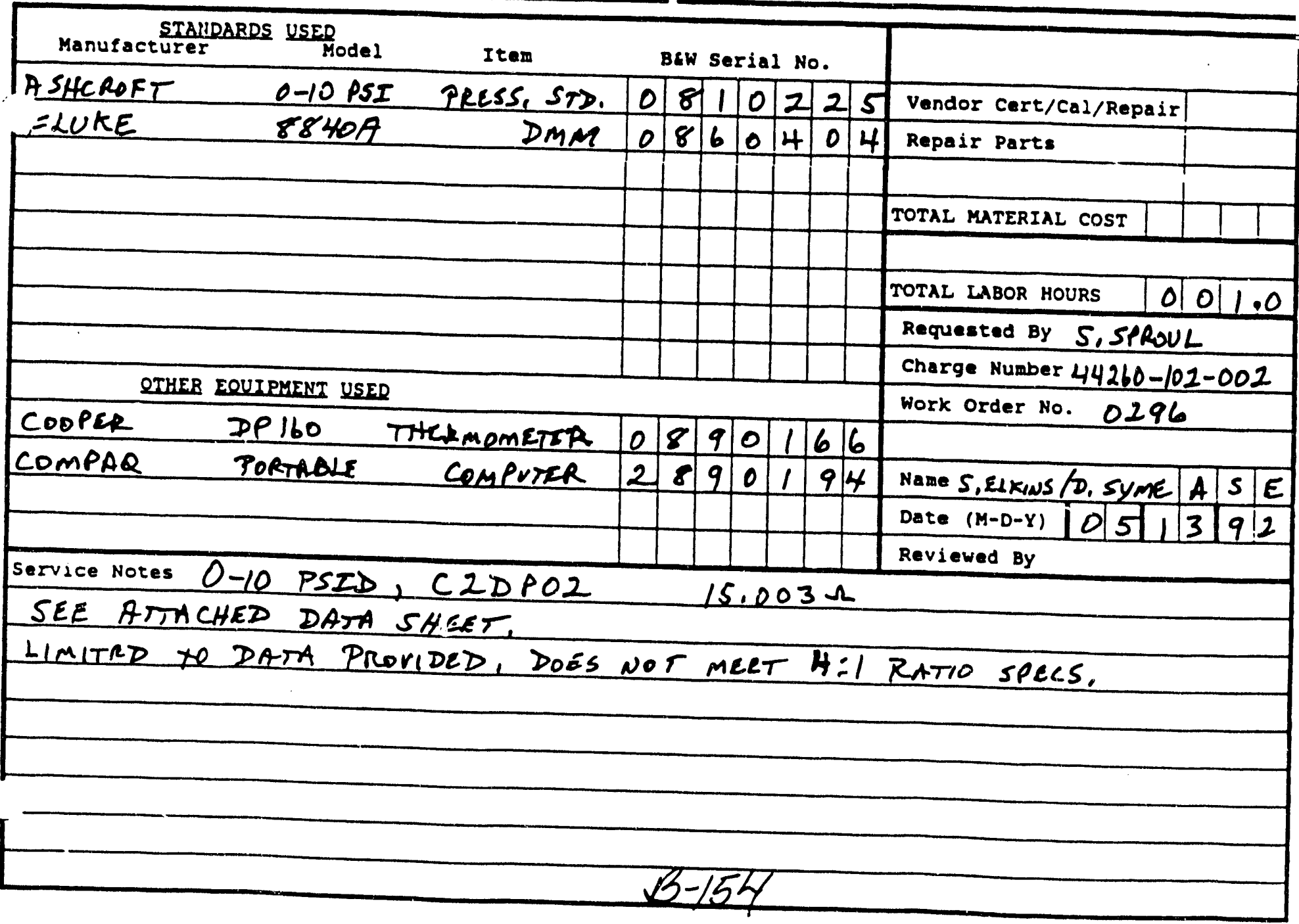


AS FECEIVED

\begin{tabular}{|c|c|c|}
\hline \multirow[b]{2}{*}{ FANSMITTER : } & AS FIECEIVED & \\
\hline & $\begin{array}{r}\text { SENSOTEC } \\
10 \\
\text { TOLERANCE: }\end{array}$ & $\begin{array}{l}\text { Z } \\
\text { F'SI } \\
0.25 \%\end{array}$ \\
\hline 'RESS SOUFICE & $\begin{array}{r}\text { ASHCFIOFT } \\
0-10\end{array}$ & $\begin{array}{l}\text { DIGIGAUGE } \\
\text { FSI }\end{array}$ \\
\hline & $\begin{array}{l}\text { B\&W Ser. NO. } \\
\text { TOLEFANCE: }\end{array}$ & $\begin{array}{l}0810225 \\
0.05 \%\end{array}$ \\
\hline EADOUT DEV: & $\begin{array}{l}\text { FLUKE } \\
\text { B\&W SER. No. } \\
\text { TOLERANCE: }\end{array}$ & $\begin{array}{l}8840 A \\
0860404 \\
0.015 \%\end{array}$ \\
\hline
\end{tabular}

AS LEFT

E\&W S.N. 0880519 DATE: $13-M a y-92$

FROCEDURE : ARC-TF-015-08

FROGFIAM: TO015_08.03A

NAME: S.ELKINS S. SyME

Ambient Temperature (F) 71

Facility I. D. Name c2dpoz

ALUE OF CURFENT SENSING RESISTOF USED FOFi TEST DATA

'ALUE OF CUFFENT SENSING FESISTOF' USED FOF' CALIBRATION

OWEF SUFF'LY VOLTAGE

OTAL SEFIES FIESISTANCE

AF'FLIED F'RESSUFE

'FANSMITTEF' F'RESS SOURCE

F'SI

$\begin{array}{lr}0.000 & 0.000 \\ 1.000 & 1.000 \\ 2.000 & 2.000 \\ 3.000 & 5.000 \\ 4.000 & 4.000 \\ 5.000 & 5.000 \\ 6.000 & 6.000 \\ 7.000 & 7.000 \\ 8.000 & 8.000 \\ 9.000 & 9.000 \\ 10.000 & 10.000 \\ 9.000 & 9.000 \\ 8.000 & 8.000 \\ 7.000 & 7.000 \\ 6.000 & 6.000 \\ 5.000 & 5.000 \\ 4.000 & 4.000 \\ 5.000 & 3.000 \\ 2.000 & 2.000 \\ 1.000 & 1.000 \\ 0.000 & 0.000\end{array}$

FEESISTOF: MEASUFING ACCUFACY 24

14.999

I DEAL

DUTFUT SIG

MILLIVOLTS

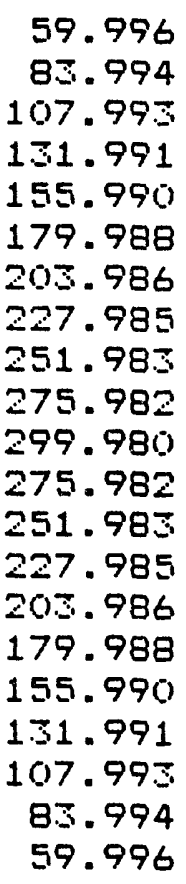

$\begin{array}{cl}\text { CAL IEFIATION ACCUFIACY } \\ \text { **LIMITED** } \\ \text { MEASUFIED } & \text { EFFIOF \% } \\ \text { OUTFUT SIG } & \text { FULL SCALE } \\ \text { MILLIVOLTS } & \end{array}$

14.999 OHMS

14.999 OHMS

N.A.

\section{ACCUFIACY $\quad 0.32 \%$ \\ OUT \\ OF \\ TOLERANCE}

$\bar{z}=60.020$ 
CURVEFIT OUTPUT FOR VTAB C2dp02 $\checkmark$

DATE: 20-MAY-92

TIME: $16: 22: 29$

VOLTAGE (V) OBS. DP(PSI) CALC. DP(PSI) DIFF (PSIA) \& DIFFERENCE

\begin{tabular}{rlcccc}
\hline 1 & $-0.5099974 \mathrm{E}-04$ & $0.0000000 \mathrm{E}+00$ & $-0.6090893 \mathrm{E}-03$ & $0.6090893 \mathrm{E}-03$ & $0.0000000 \mathrm{E}+00$ \\
2 & $0.2397400 \mathrm{E}-01$ & 1.000000 & 0.9982745 & $0.1725493 \mathrm{E}-02$ & 0.1725493 \\
3 & $0.4797300 \mathrm{E}-01$ & 2.000000 & 1.996554 & $0.3445981 \mathrm{E}-02$ & 0.1722990 \\
4 & $0.7197100 \mathrm{E}-01$ & 3.000000 & 2.995269 & $0.4731440 \mathrm{E}-02$ & 0.1577147 \\
5 & $0.9597000 \mathrm{E}-01$ & 4.000000 & 3.994501 & $0.5498637 \mathrm{E}-02$ & 0.1374659 \\
6 & 0.1201990 & 5.000000 & 5.003794 & $-0.3794076 \mathrm{E}-02$ & $-0.7588152 \mathrm{E}-01$ \\
7 & 0.1441320 & 6.000000 & 6.001233 & $-0.1233465 \mathrm{E}-02$ & $-0.2055776 \mathrm{E}-01$ \\
8 & 0.1681780 & 7.000000 & 7.003860 & $-0.3859679 \mathrm{E}-02$ & $-0.5513827 \mathrm{E}-01$ \\
9 & 0.1921270 & 8.000000 & 8.002917 & $-0.2916996 \mathrm{E}-02$ & $-0.3646245 \mathrm{E}-01$ \\
10 & 0.2160730 & 9.000000 & 9.002324 & $-0.2323747 \mathrm{E}-02$ & $-0.2581941 \mathrm{E}-01$ \\
11 & 0.2399150 & 10.00000 & 9.997861 & $0.2138565 \mathrm{E}-02$ & $0.2138565 \mathrm{E}-01$ \\
12 & 0.2159210 & 9.000000 & 8.995978 & $0.4021599 \mathrm{E}-02$ & $0.4468444 \mathrm{E}-01$ \\
13 & 0.1920480 & 8.000000 & 7.999621 & $0.3793510 \mathrm{E}-03$ & $0.4741888 \mathrm{E}-02$ \\
14 & 0.1680890 & 7.000000 & 7.000148 & $-0.1478371 \mathrm{E}-03$ & $-0.2111958 \mathrm{E}-02$ \\
15 & 0.1440800 & 6.000000 & 5.999066 & $0.9342171 \mathrm{E}-03$ & $0.1557028 \mathrm{E}-01$ \\
16 & 0.1201110 & 5.000000 & 5.000127 & $-0.1274345 \mathrm{E}-03$ & $-0.2548691 \mathrm{E}-02$ \\
17 & $0.9610400 \mathrm{E}-01$ & 4.000000 & 4.000082 & $-0.8198392 \mathrm{E}-04$ & $-0.2049598 \mathrm{E}-02$ \\
18 & $0.7213100 \mathrm{E}-01$ & 3.000000 & 3.001929 & $-0.1928812 \mathrm{E}-02$ & $-0.6429372 \mathrm{E}-01$ \\
19 & $0.4810400 \mathrm{E}-01$ & 2.000000 & 2.002004 & $-0.2004497 \mathrm{E}-02$ & -0.1002248 \\
20 & $0.2405000 \mathrm{E}-01$ & 1.000000 & 1.001435 & $-0.1435105 \mathrm{E}-02$ & -0.1435105 \\
21 & $0.5100026 \mathrm{E}-04$ & $0.0000000 \mathrm{E}+00$ & $0.3630739 \mathrm{E}-02$ & $-0.3630739 \mathrm{E}-02$ & $0.0000000 \mathrm{E}+00$
\end{tabular}

( GREE OF POLYNOMIAL 2

COEFFICIENTS

$0.15108130 E-02 \quad 0.41566941 E+02 \quad 0.41379784 E+00$

ESS $=0.1574 E-03 \quad$ SD $=0.2957 E-02 \quad R 2=0.999999 E+00$

$2 \star S D=0.5913 \mathrm{E}-02$

$\mathcal{B}-156$ 
RD-45A Rev. $12-18-91$

\begin{tabular}{|l|l|l|}
\hline INSTRUMENT STATUS & RISEn & AS \\
\hline 1 Certified & & \\
\hline 2 Calibrated & & $\checkmark$ \\
\hline 3 For Ind. Only & & \\
\hline 4 standardize & & \\
\hline 5 Certified stand. & & \\
\hline 6 Calibrated stand. & & \\
\hline 9 other & & \\
\hline
\end{tabular}

INSTRUMENT CONDITION

AS RECEIVED (Certified Equipment only)

Meets Manufacturer's Tolerance

Operares within previously defined limits $\mathrm{N}$ Deviates from $\mathrm{Mfg}$ or previously defined limits $\mathrm{Y}$ Inoperative (Describe below) $Y$

AS LEFT Limited (Describe below)

Heets Manufacturer's Tolerance

other (Describe Below)
(30PO)

Babcock \& Wilcox

a MeDermott company

B\&W Ser. NO. 0880533

MANUFACTURER

MODEL

ITEM

MANUF. SER. NO.

PROPERTY NO.

ORDER NO.

SECTION

LOCATION

SCHEDULED ( $\mathrm{Y}-\mathrm{N}$ )

RECAL INT. (MOS.)

\section{SENSOTEC}

21311-03

TRANSMITR, D-P

200013

4427-01-137

\section{6 \\ 115 \\ in $y$ \\ 12}

TECH. PROCEDURE A0015

Babcock \& Wilcox RED Division certifies that the performance of this instrument was verified using standards whose accuracies are traceable to the National Institute of Standards and Technology. an accepted value of a natural physical constant. a ratio type of calibration technique, or to a consensus standard.

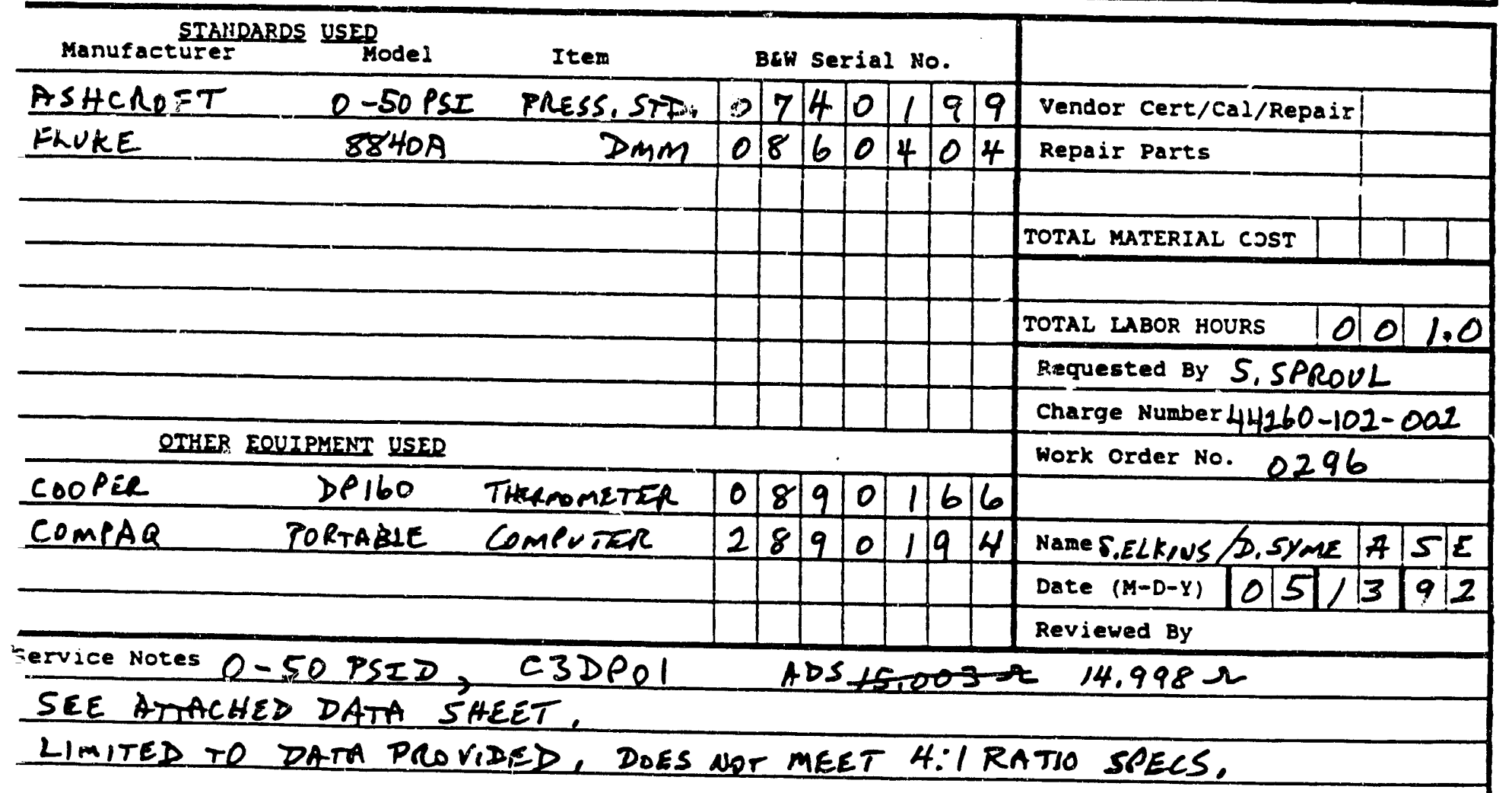


! TRANSMITTER:

AS RECEIVED

SENSOTEC $Z$

50 FSI

TOLERANCE : $0.25 \%$

FRESS SOURICE

$$
\begin{aligned}
\text { ASHCFOFT } & \text { DIGIGAUGE } \\
\text { O-5O FSI } & \text { F' } \\
\text { B\&W SER. NO. } & 0740199 \\
\text { TOLEFIANCE: } & 0.05 \%
\end{aligned}
$$

READOUT DEV:

$$
\begin{aligned}
& \text { FLUKE } 8840 \mathrm{~A} \\
& \text { B\&W SER. NO. } 0860404 \\
& \text { TOLEFIANCE: } 0.015 \%
\end{aligned}
$$

AS LEFT

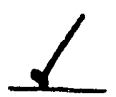

Ambient Temperature (F) 71

Facility I. D. Name
B\&W S.N. O880533. DATE: 13-May-92 FROCEDURE : ARC-TP-015-08

FRIOGRAM: TOO15_OB.03A NAME: S, ELLWWS/D, syme
VALUE OF CURFENT SENSING FESISTOR USED FOF TEST DATA VALUE OF CURRENT SENSING FIESISTOF USED FOF CALIBFATION POWEF SUFFLY VOLTAGE TOTAL SEFIES RESISTANCE

AFFLIED PRESSURE

TRANSMITTER FSI

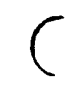

$$
\begin{array}{r}
0.000 \\
5.000 \\
10.000 \\
15.000 \\
20.000 \\
25.000 \\
30.000 \\
35.000 \\
40.000 \\
45.000 \\
50.000 \\
45.000 \\
40.000 \\
35.000 \\
30.000 \\
25.000 \\
20.000 \\
15.000 \\
10.000 \\
5.000 \\
0.000
\end{array}
$$

FIESISTOF MEASUFIING ACCURACY 24

14.998

IDEAL

$$
\begin{aligned}
& \text { OUTFUT SIG } \\
& \text { MILLIVOLTS }
\end{aligned}
$$

F'SI

$$
\begin{array}{r}
0.000 \\
5.000 \\
10.000 \\
15.000 \\
20.000 \\
25.000 \\
30.000 \\
35.000 \\
40.000 \\
45.000 \\
50.000 \\
45.000 \\
40.000 \\
35.000 \\
30.000 \\
25.000 \\
20.000 \\
15.000 \\
10.000 \\
5.000 \\
0.000
\end{array}
$$

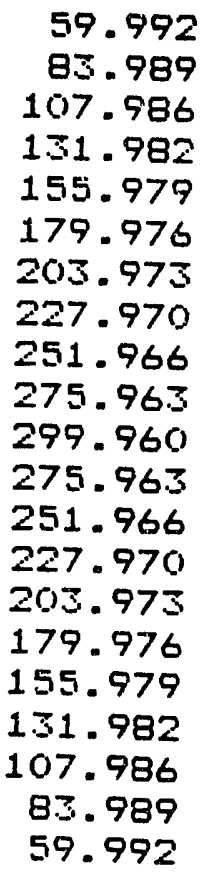

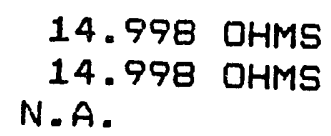

14.998 OHMS 14.998 OHMS N.A.

\section{ACCURACY \\ **LIMITED** \\ EFiRIOR \% \\ FULL SCALE}

MILLIVOLTS

$\begin{array}{rr}59.946 & -0.02 \% \\ 83.821 & -0.07 \% \\ 107.788 & -0.08 \% \\ 131.815 & -0.07 \% \\ 155.878 & -0.04 \% \\ 179.862 & -0.05 \% \\ 203.846 & -0.05 \% \\ 227.891 & -0.03 \% \\ 251.886 & -0.03 \% \\ 275.919 & -0.02 \% \\ 299.761 & -0.08 \% \\ 275.813 & -0.06 \% \\ 251.755 & -0.09 \% \\ 227.811 & -0.07 \% \\ 203.770 & -0.08 \% \\ 179.835 & -0.06 \% \\ 155.851 & -0.05 \% \\ 131.812 & -0.07 \% \\ 107.783 & -0.08 \% \\ 83.854 & -0.06 \% \\ 59.971 & -0.01 \%\end{array}$

$$
\bar{z}=59.959
$$


CURVEFIT OUTPUT FOR VTAB C3dP01 $V$

DATE: 20-MAY-92

TIME: $16: 26: 55$

VOLTAGE (V) OBS. DP(PSI) CALC. DP(PSI) DIFF (PSIA) \& DIFFERENCE

$\begin{array}{llllll}1 & -0.1300184 \mathrm{E}-04 & 0.0000000 \mathrm{E}+00 & 0.1119659 \mathrm{E}-01 & -0.1119659 \mathrm{E}-01 & 0.0000000 \mathrm{E}+00 \\ 2 & 0.2386200 \mathrm{E}-01 & 5.000000 & 4.989437 & 0.1056311 \mathrm{E}-01 & 0.0112621 \\ 3 & 0.4782900 \mathrm{E}-01 & 10.00000 & 9.986136 & 0.1386391 \mathrm{E}-01 & 0.1386391 \\ 4 & 0.7185600 \mathrm{E}-01 & 15.00000 & 14.99462 & 0.5384156 \mathrm{E}-02 & 0.3589438 \mathrm{E}-01 \\ 5 & 0.9591900 \mathrm{E}-01 & 20.00000 & 20.00987 & -0.9868934 \mathrm{E}-02 & -0.4934467 \mathrm{E}-01 \\ 6 & 0.1199030 & 25.00000 & 25.00793 & -0.7928814 \mathrm{E}-02 & -0.3171526 \mathrm{E}-01 \\ 7 & 0.1438870 & 30.00000 & 30.00526 & -0.5262001 \mathrm{E}-02 & -0.1754000 \mathrm{E}-01 \\ 8 & 0.1679320 & 35.00000 & 35.01458 & -0.1457575 \mathrm{E}-01 & -0.4164500 \mathrm{E}-01 \\ 9 & 0.1919270 & 40.00000 & 40.01274 & -0.1274484 \mathrm{E}-01 & -0.3186210 \mathrm{E}-01 \\ 0 & 0.2159600 & 45.00000 & 45.01810 & -0.1810026 \mathrm{E}-01 & -0.4022280 \mathrm{E}-01 \\ 1 & 0.2398020 & 50.00000 & 49.98296 & 0.1704490 \mathrm{E}-01 & 0.3408980 \mathrm{E}-01 \\ 2 & 0.2158540 & 45.00000 & 44.99603 & 0.3974770 \mathrm{E}-02 & 0.8832823 \mathrm{E}-02 \\ 3 & 0.1917960 & 40.00000 & 39.98546 & 0.1454054 \mathrm{E}-01 & 0.3635136 \mathrm{E}-01 \\ 4 & 0.1678520 & 35.00000 & 34.99791 & 0.2089503 \mathrm{E}-02 & 0.5970009 \mathrm{E}-02 \\ 5 & 0.1438110 & 30.00000 & 29.98943 & 0.1057230 \mathrm{E}-01 & 0.3524099 \mathrm{E}-01 \\ 6 & 0.1198760 & 25.00000 & 25.00230 & -0.2302654 \mathrm{E}-02 & -0.9210616 \mathrm{E}-02 \\ 7 & 0.9589200 \mathrm{E}-01 & 20.00000 & 20.00424 & -0.4241956 \mathrm{E}-02 & -0.2120978 \mathrm{E}-01 \\ 8 & 0.7185300 \mathrm{E}-01 & 15.00000 & 14.99399 & 0.6009467 \mathrm{E}-02 & 0.4006311 \mathrm{E}-01 \\ 9 & 0.4782400 \mathrm{E}-01 & 10.00000 & 9.985094 & 0.1490625 \mathrm{E}-01 & 0.1490625 \\ 0 & 0.2389500 \mathrm{E}-01 & 5.000000 & 4.996317 & 0.3682686 \mathrm{E}-02 & 0.7365372 \mathrm{E}-01 \\ 1 & 0.1199816 \mathrm{E}-04 & 0.0000000 \mathrm{E}+00 & 0.1640979 \mathrm{E}-01 & -0.1640979 \mathrm{E}-01 & 0.0000000 \mathrm{E}+00\end{array}$

IGREE OF POLYNOMIAL 2

OEFFICIENTS

$0.13907838 \mathrm{E}-01 \quad 0.20852775 \mathrm{E}+03-0.63165188 \mathrm{E}+00$

SS $=0.2547 E-02 \quad \begin{aligned} S D & =0.1190 E-01 \\ 2 * S D & =0.2379 E-01\end{aligned} \quad R 2=0.999999 E+00$ 
RESEARCH \& DEVELOPIENT DIVISION INSTRURENT SERVICE LOG

\begin{tabular}{|c|c|c|}
\hline INSTRUMENT STATUS & RISE & $\begin{array}{l}\text { AS } \\
\text { LERT }\end{array}$ \\
\hline 1 Certified & & \\
\hline Calibrated & & \\
\hline 3 For Ind. only & $\checkmark$ & \\
\hline 4 Standardize & & \\
\hline 5 certified stand. & & \\
\hline 6 calibrated a stand. & & \\
\hline 9 Other & & \\
\hline
\end{tabular}

INSTRUMENT CONDITION

AS RECEIVED (Certified Equipment Only)

Meets Manufacturer's Tolerance $N$ Operates within previously defined limits $N$ Deviates from Mig or previously defined limitz $Y$ Inoperative (Describe below) $y$

AS LEFT

Limited (Describe below)

Meets Manufacturar's Tolerance other (Describe Below)
- McDermoticompany

B\&W Ser. No. 088052

IANUFACTURER

MODEL

ITEM

SENSOTEC

Z1309-15

TRANSMITR, D-P

MANUF. SER. NO. 187436

PROPERTY NO.

ORDER NO.

4427-01-125

$\begin{array}{lc}\text { SECTION } & 46 \\ \text { LOCATION } & 115 \\ \text { SCHEDULED (Y-N) } & \mathbb{4} Y \\ \text { RECAL INT.(MOS.) } & 12\end{array}$

TECH. PROCEDURE AO015

Babcock wilcox RED Division certifies that the performance of this instrument was verified using standards whose accuracies are traceable to the National Institute of standards and Technology, an accepted value of a natural physical constant. a ratio type of calibration technique, or to consensus standard.

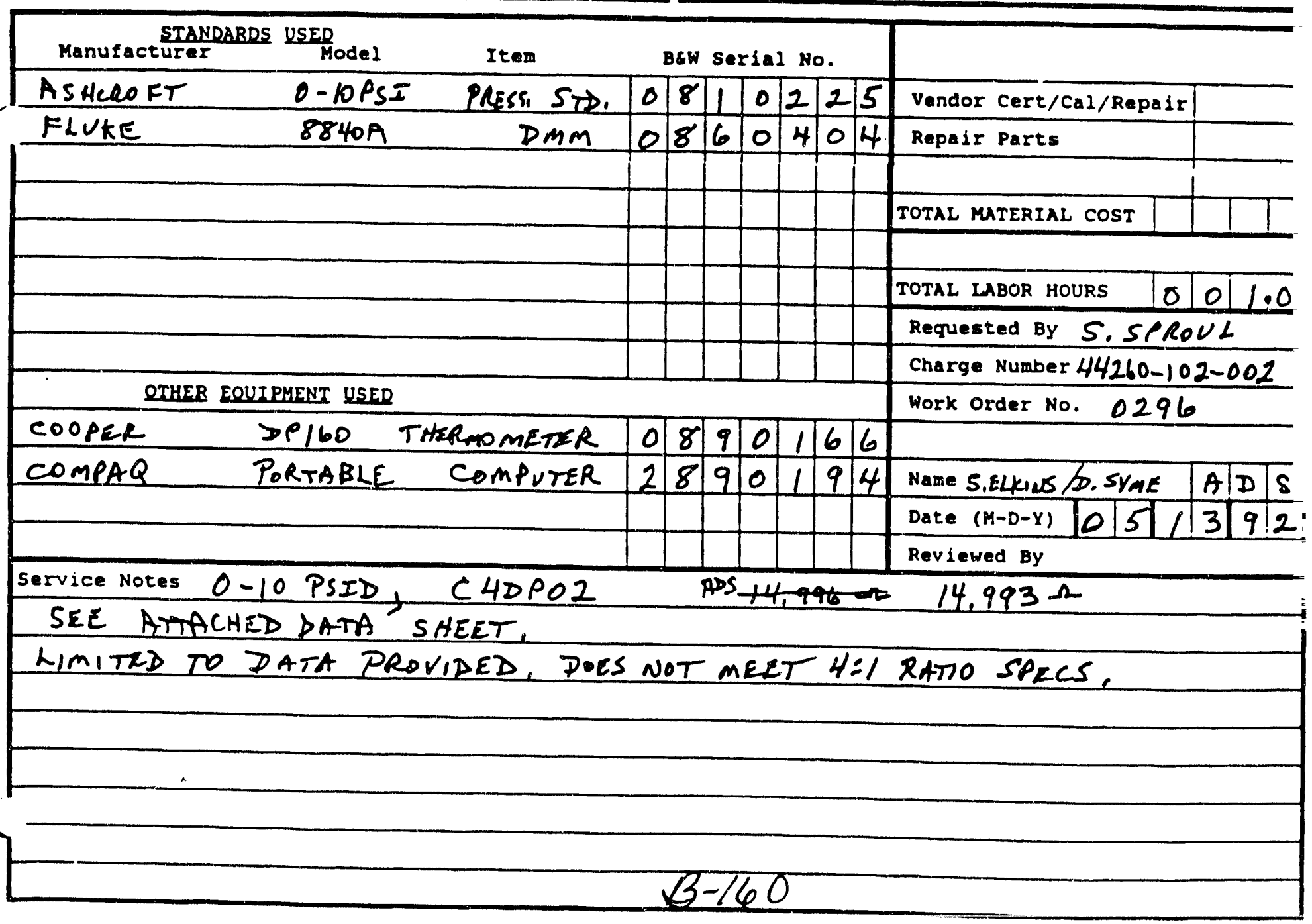


AS FIECEIVED

\section{FANSMITTEF:}

\section{SENSOTEC $Z$}

10 FSI

TOLERANCE : $0.25 \%$

RESS SOURCE

$\begin{aligned} \text { ASHCFOFT } & \text { DIGIGAUGE } \\ \text { O-10 FSI } & \text { FS } \\ \text { B\&W SER. NO: } & 0810225 \\ \text { TOLEFIANCE: } & 0.05 \%\end{aligned}$

EADOUT DEV:
AS LEFT

BEW S.N. 0880521 . DATE: 13-May-92

FROCEDURE : ARC-TF-015-08

FFIOGFIAM: T0015_08.03A

NAME: S,LKINS/D, SYME

Ambient Temperature (F) 71

Facility I. D. Name

FLUTE 8840A
B\&W SER. NO. 0860404

TOLEFAANCE : $0.015 \%$
ALUE OF CUFFENT SENSING FESISTOF USED FOR TEST DATA
ALUE OF CUFRENT SENSING FESISTOF USED FOR CALIBFIATION
FEESISTOF: MEASUFIING ACCUFACY
14.995 OHMS
14.993 OHMS
N.A.

OWEF SUFF'LY VOLTAGE

OTAL SEFIES FESISTANCE

AF'F'LIED FRESSUFE

RANSMITTEF

F'SI

F'RESS SOUFICE

F'S I

$\begin{array}{rr}0.000 & 0.000 \\ 1.000 & 1.000 \\ 2.000 & 2.000 \\ 3.000 & 3.000 \\ 4.000 & 4.000 \\ 5.000 & 5.000 \\ 6.000 & 6.000 \\ 7.000 & 7.000 \\ 8.000 & 8.000 \\ 9.000 & 9.000 \\ 10.000 & 10.000 \\ 9.000 & 9.000 \\ 8.000 & 8.000 \\ 7.000 & 7.000 \\ 6.000 & 6.000 \\ 5.000 & 5.000 \\ 4.000 & 4.000 \\ 3.000 & 3.000 \\ 2.000 & 2.000 \\ 1.000 & 1.000 \\ 0.000 & 0.000\end{array}$

14.993

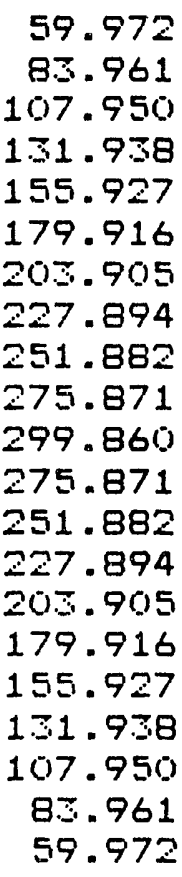
24

IDEAL

OUTFUT SIG

MILLIVOLTS

\begin{tabular}{|c|c|c|c|}
\hline $\begin{array}{r}24 \\
14.993\end{array}$ & CALIEFATION & $\begin{array}{l}\text { ACCUFACY } \\
\text { * *LIMITED** }\end{array}$ & \\
\hline $\begin{array}{l}\text { IDEAL } \\
\text { UTFUT SIG } \\
\text { MILLIVOLTS }\end{array}$ & $\begin{array}{l}\text { MEASURED } \\
\text { DUTFUT SIG } \\
\text { MILLIVOLTS }\end{array}$ & $\begin{array}{l}\text { EFFIOR } \% \\
\text { FULL SCALE }\end{array}$ & $\begin{array}{l}\text { OUT } \\
\text { OF } \\
\text { TQLERANCE }\end{array}$ \\
\hline
\end{tabular}

$$
\bar{z}=59.926
$$


CURVEFIT OUTPUT FOR VTAB C4dPOZ

DATE: $20-$ MAY -92

TIME: $16: 30: 25$

VOLTAGE (V) OBS. DP(PSI) CALC. DP(PSI) DIFF (PSIA) *-DIFFERENCE

$\begin{array}{rllllrr}1 & -0.1199949 \mathrm{E}-04 & 0.0000000 \mathrm{E}+00 & -0.4799287 \mathrm{E}-03 & 0.4799287 \mathrm{E}-03 & 0.0000000 \mathrm{E}+0 \mathrm{O} \\ 2 & 0.2406900 \mathrm{E}-01 & 1.000000 & 1.000578 & -0.5784023 \mathrm{E}-03 & -0.5784024 \mathrm{E}-01 \\ 3 & 0.4808900 \mathrm{E}-01 & 2.000000 & 1.999772 & 0.2282403 \mathrm{E}-03 & 0.1141202 \mathrm{E}-01 \\ 4 & 0.7207500 \mathrm{E}-01 & 3.000000 & 2.998219 & 0.1780676 \mathrm{E}-02 & 0.593587 \mathrm{E}-01 \\ 5 & 0.9609700 \mathrm{E}-01 & 4.000000 & 3.998835 & 0.1164983 \mathrm{E}-02 & 0.2912458 \mathrm{E}-01 \\ 6 & 0.1201020 & 5.000000 & 4.999412 & 0.5880364 \mathrm{E}-03 & 0.1176073 \mathrm{E}-01 \\ 7 & 0.1440620 & 6.000000 & 5.998780 & 0.1219524 \mathrm{E}-02 & 0.2032539 \mathrm{E}-01 \\ 8 & 0.1680790 & 7.000000 & 7.001195 & -0.1195464 \mathrm{E}-02 & -0.1707805 \mathrm{E}-01 \\ 9 & 0.1920420 & 8.000000 & 8.002024 & -0.2024163 \mathrm{E}-02 & -0.2530203 \mathrm{E}-01 \\ 10 & 0.2159050 & 9.000000 & 8.999339 & 0.6610667 \mathrm{E}-03 & 0.7345186 \mathrm{E}-02 \\ 11 & 0.2397980 & 10.00000 & 9.998570 & 0.1430004 \mathrm{E}-02 & 0.1430004 \mathrm{E}-01 \\ 12 & 0.2159050 & 9.000000 & 8.999339 & 0.6610667 \mathrm{E}-03 & 0.7345186 \mathrm{E}-02 \\ 13 & 0.1920240 & 8.000000 & 8.001272 & -0.1272132 \mathrm{E}-02 & -0.1590165 \mathrm{E}-01 \\ 14 & 0.1680870 & 7.000000 & 7.001529 & -0.1529477 \mathrm{E}-02 & -0.2184967 \mathrm{E}-01 \\ 15 & 0.1440850 & 6.000000 & 5.999740 & 0.2598763 \mathrm{E}-03 & 0.4331272 \mathrm{E}-02 \\ 16 & 0.1201260 & 5.000000 & 5.000413 & -0.4126669 \mathrm{E}-03 & -0.8253339 \mathrm{E}-02 \\ 17 & 0.9612800 \mathrm{E}-01 & 4.000000 & 4.000127 & -0.1267279 \mathrm{E}-03 & -0.3168197 \mathrm{E}-02 \\ 18 & 0.7209300 \mathrm{E}-01 & 3.000000 & 2.998969 & 0.1031153 \mathrm{E}-02 & 0.3437175 \mathrm{E}-01 \\ 19 & 0.4812000 \mathrm{E}-01 & 2.000000 & 2.001062 & -0.1061743 \mathrm{E}-02 & -0.5308713 \mathrm{E}-01 \\ 20 & 0.2407500 \mathrm{E}-01 & 1.000000 & 1.000828 & -0.8279090 \mathrm{E}-03 & -0.8279090 \mathrm{E}-01 \\ 21 & 0.1100051 \mathrm{E}-04 & 0.0000000 \mathrm{E}+00 & 0.4758707 \mathrm{E}-03 & -0.4758707 \mathrm{E}-03 & 0.0000000 \mathrm{E}+00\end{array}$

( IGREE OF POLYNOMIAL 2

COEFFICIENTS

$0.18728069 E-04 \quad 0.41556496 E+02 \quad 0.58060646 E+00$

ESS $=0.2274 \mathrm{E}-04 \quad \begin{aligned} \mathrm{SD} & =0.1124 \mathrm{E}-02 \\ 2 * \mathrm{SD} & =0.2248 \mathrm{E}-02\end{aligned} \quad$ R2 $=0.100000 \mathrm{E}+01$ 
AS FECEIVED

TFIANSMI TTEF:

SENSOTEC $Z$

25 F.SI

TOLEFANCE : $0.25 \%$

FRESS SOUFICE

$$
\begin{aligned}
\text { ASHCROFT } & \text { DIGIGAUGE } \\
\text { O-3O FSI } & \text { FSI } \\
\text { B\&W SER. NO. } & 0810341 \\
\text { TOLERANCE: } & 0.06 \%
\end{aligned}
$$

FEADOUT DEV:

\section{E\&W SER. NO. 0860404}

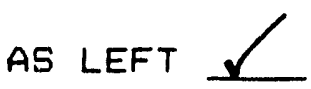

TOLEFANCE : $0.015 \%$

VALUE OF CUFFENT SENSING RESISTOF USED FOF TEST DATA

VALUE OF CUFFENT SENSING FESISTOF USED FOR CALIBFATION

FOWEF SUFF'LY VOLTAGE

TOTAL, SEFIES FESISTANCE

AFFLIED F'RESSURE

TFANSMITTEF

F'S I

$\begin{array}{rr}0.000 & 0.000 \\ 2.500 & 2.500 \\ 5.000 & 5.000 \\ 7.500 & 7.500 \\ 10.000 & 10.000 \\ 12.500 & 12.500 \\ 15.000 & 15.000 \\ 17.500 & 17.500 \\ 20.000 & 20.000 \\ 22.500 & 22.500 \\ 25.000 & 25.000 \\ 22.500 & 22.500 \\ 20.000 & 20.000 \\ 17.500 & 17.500 \\ 15.000 & 15.000 \\ 12.500 & 12.500 \\ 10.000 & 10.000 \\ 7.500 & 7.500 \\ 5.000 & 5.000 \\ 2.500 & 2.500 \\ 0.000 & 0.000\end{array}$

FESISTOF MEASUFIING ACCUFACY

$$
\begin{array}{r}
24 \\
14.997 \\
\text { IDEAL } \\
\text { QUTFUT SIG } \\
\text { MILLIVOLTS }
\end{array}
$$

QUTFUT SIG

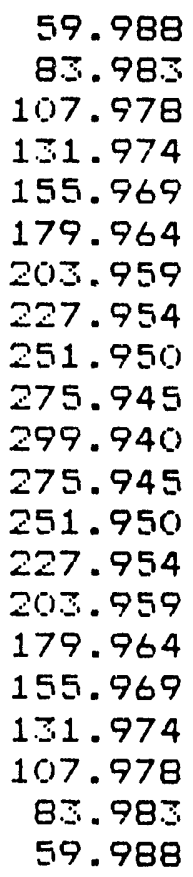

E\&W S.N. 0880528

DATE: 14-May-92

FFIOCEDUFE : ARC-TP-O15-OB

FFIOGFIAM: T0015_0B.03A

NAME: S,ELK,NS/D, SYME

Ambient Temperature (F) 70

Facility I. D. Name
IF'DF'O6

CALIBFITION
MEASURED
OUTFUT SIG
MILLIVOLTS

ACCUFIACY

* *L IMI TED**

ERFILR \%

FULL SCALE MILLIVOLTS

$\begin{array}{rr}59.953 & -0.01 \% \\ 84.019 & 0.02 \% \\ 108.068 & 0.04 \% \\ 132.123 & 0.06 \% \\ 156.203 & 0.10 \% \\ 180.295 & 0.14 \% \\ 204.233 & 0.11 \% \\ 228.218 & 0.11 \% \\ 252.149 & 0.08 \% \\ 276.061 & 0.05 \% \\ 299.880 & -0.03 \% \\ 275.966 & 0.01 \% \\ 252.113 & 0.07 \% \\ 228.180 & 0.09 \% \\ 204.210 & 0.10 \% \\ 180.239 & 0.11 \% \\ 156.182 & 0.09 \% \\ 132.088 & 0.05 \% \\ 108.053 & 0.03 \% \\ 84.005 & 0.01 \% \\ 59.932 & -0.02 \% \\ & \\ 2759.943 & \end{array}$

\section{$0.35 \%$}

OUT

OF

TOLEFIANCE 
VOLTAGE(V) OBS. DP(PSI) CALC. DP(PSI) DIFF (PSIA; \& DIFFERENCE

$\begin{array}{rlllll}1 & 0.9998283 E-05 & 0.0000000 E+00 & 0.5626411 \mathrm{E}-02 & -0.5626411 \mathrm{E}-02 & 0.0000000 \mathrm{E}+00 \\ 2 & 0.2407600 \mathrm{E}-01 & 2.500000 & 2.500650 & -0.6500618 \mathrm{E}-03 & -0.2600247 \mathrm{E}-01 \\ 3 & 0.4812500 \mathrm{E}-01 & 5.000000 & 4.996502 & 0.3497669 \mathrm{E}-02 & 0.6995337 \mathrm{E}-01 \\ 4 & 0.7218000 \mathrm{E}-01 & 7.500000 & 7.495568 & 0.4431573 \mathrm{E}-02 & 0.5908764 \mathrm{E}-01 \\ 5 & 0.9626000 \mathrm{E}-01 & 10.00000 & 9.999827 & 0.1727368 \mathrm{E}-03 & 0.1727368 \mathrm{E}-02 \\ 6 & 0.1203520 & 12.50000 & 12.50793 & -0.7932857 \mathrm{E}-02 & -0.6346286 \mathrm{E}-01 \\ 7 & 0.1442900 & 15.00000 & 15.00258 & -0.2580795 \mathrm{E}-02 & -0.1720530 \mathrm{E}-01 \\ 8 & 0.1682750 & 17.50000 & 17.50470 & -0.4700612 \mathrm{E}-02 & -0.2686064 \mathrm{E}-01 \\ 9 & 0.1922060 & 20.00000 & 20.00375 & -0.3754841 \mathrm{E}-02 & -0.1877421 \mathrm{E}-01 \\ 10 & 0.2161180 & 22.50000 & 22.50339 & -0.3386703 \mathrm{E}-02 & -0.1505201 \mathrm{E}-01 \\ 11 & 0.2399370 & 25.00000 & 24.99584 & 0.4157336 \mathrm{E}-02 & 0.1662934 \mathrm{E}-01 \\ 12 & 0.2160230 & 22.50000 & 22.49345 & 0.6549152 \mathrm{E}-02 & 0.2910734 \mathrm{E}-01 \\ 13 & 0.1921700 & 20.00000 & 19.99999 & 0.6474794 \mathrm{E}-05 & 0.3237397 \mathrm{E}-04 \\ 14 & 0.1682370 & 17.50000 & 17.50073 & -0.7344070 \mathrm{E}-03 & -0.4196611 \mathrm{E}-02 \\ 15 & 0.1442670 & 15.00000 & 15.00018 & -0.1826675 \mathrm{E}-03 & -0.1217784 \mathrm{E}-02 \\ 16 & 0.1202960 & 12.50000 & 12.50210 & -0.2099945 \mathrm{E}-02 & -0.1679956 \mathrm{E}-01 \\ 17 & 0.9623900 \mathrm{E}-01 & 10.00000 & 9.997642 & 0.2357815 \mathrm{E}-02 & 0.2357815 \mathrm{E}-01 \\ 18 & 0.7214500 \mathrm{E}-01 & 7.500000 & 7.491930 & 0.8069594 \mathrm{E}-02 & 0.1075946 \\ 19 & 0.4811000 \mathrm{E}-01 & 5.000000 & 4.994945 & 0.5055205 \mathrm{E}-02 & 0.1011041 \\ 20 & 0.2406200 \mathrm{E}-01 & 2.500000 & 2.499198 & 0.8021312 \mathrm{E}-03 & 0.3208525 \mathrm{E}-01 \\ 21 & -0.1100172 \mathrm{E}-04 & 0.0000000 \mathrm{E}+00 & 0.3450385 \mathrm{E}-02 & -0.3450385 \mathrm{E}-02 & 0.0000000 \mathrm{E}+00\end{array}$

DEGREE OF POLYNOMIAL 2

- OEFFICIENTS

J.45903860E-02 $0.10362028 \mathrm{E}+03 \quad 0.22392541 \mathrm{E}+01$

ESS $=0.3552 \mathrm{E}-03 \quad \begin{aligned} \mathrm{SD} & =0.4442 \mathrm{E}-02 \\ 2 \star S D & =0.8884 \mathrm{E}-02\end{aligned} \quad \mathrm{R2}=0.100000 \mathrm{E}+01$ 
- RD-45A Rev. $11-18-91$

\begin{tabular}{|l|l|l|}
\hline \multicolumn{1}{|c|}{ INSTRUMENT Status } & pífo & iś \\
\hline 1 Certified & & \\
\hline Calibrated & & \\
\hline 3 For Ind. Only & & \\
\hline 4 standardize & & \\
\hline 5 Certified s stand. & & \\
\hline 6 calibrated i stand. & & \\
\hline 9 other & & \\
\hline
\end{tabular}

INSTRUMENT CONDITION

AS RECEIVED (Certified Equipment only)

$\therefore$ Meets Manufacturer's Tolerance N Operates within previously defined limits $N$ Deviates from Mfg or previously defined limits $y$ Inoperative (Describe below) $y$ Meets Manufacturer's Tolerance other (Describe Below)
I DEVELOPMENT DIVISION INSTRUAENT SERVICE LOG

\begin{tabular}{|c|}
\hline Certification 2 \\
\hline Callbration \\
\hline Maintenance \\
\hline Repair \\
\hline Vendor cert. 5 \\
\hline Vendor calib. 6 \\
\hline Vendor Repair 7 \\
\hline other \\
\hline
\end{tabular}

\begin{tabular}{lll}
\hline & \\
\hline & \\
\hline & \\
\hline & \\
\hline & \\
\hline & \\
\hline & \\
\hline
\end{tabular}

MANUFACTURER

MODEL

ITEM

MANUF. SER. NO.

PROPERTY NO.

ORDER NO.

\section{SECTION \\ LOCATION \\ SCHEDULED ( $Y-N$ )}

RECAL INT.(MOS.)
Babcock \&'Wilcox

acDermoticompany

B\&W Ser. NO. 088052

SENSOTEC

Z1309-22

TRANSMITR, D-P .

195498

4427-01-130

46

115

it $y$

12

TECH. PROCEDURE A0015

Babcock \& Wilcox RED Division certifies that the standards whose this instrument was verified using National whose accuracies are traceable to the an accepted value of Standards and Technology. a ratio type of calibration technisical constant consensus standard.

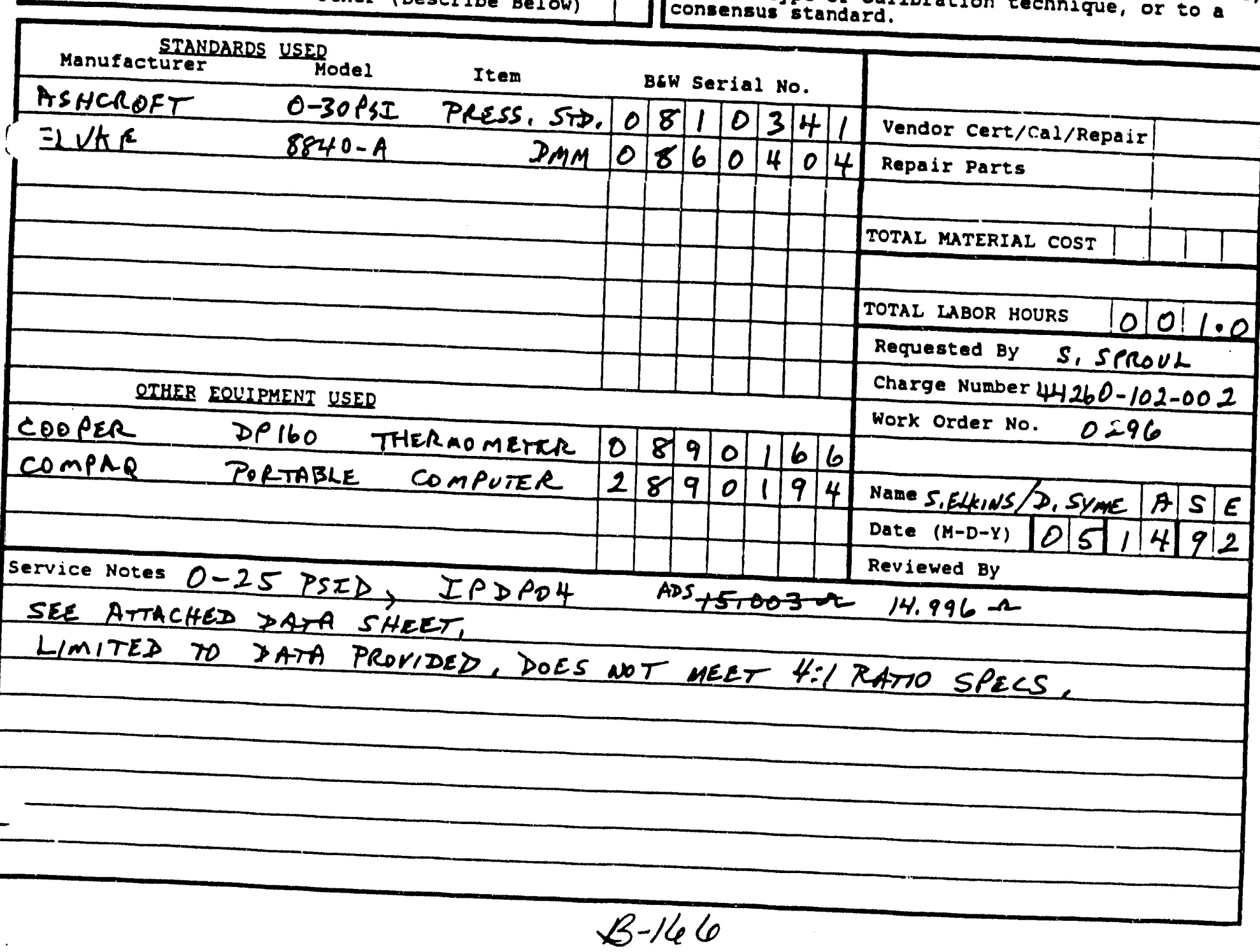




\author{
AS RECEIVED \\ SENSOTEC $Z$ \\ 25 F.SI \\ TOLEFANCE : $0.25 \%$ \\ :ANSMITTEF: \\ ASHCFOFT DIGIGAUGE \\ O- 30 FSI \\ B\&W Ser. No. 0810341 \\ TOLEFANCE : $0.06 \%$ \\ ADOUT DEV: \\ FLUJEE 8840A \\ B\&W Ser. No. 0860404 \\ TOLEFIANCE : $0.015 \%$
}

AS LEFT

E\&W S.N. 0880526

DATE: 14-Mä̈-92

FROCEDUFE : AFIC-TF-015-08

FFIOGFIAM: TO015_0B.03A

NAME: S.ELK INS S S. SYME

Ambient Temperature ( $F$ ) 70

Facility I. D. Name
ILUE OF CURFENT SENSING RESISTOF USED FOF TEST DATA

ILUE OF CURFENT SENSING RESISTOFi USED FOF CALIBRATION

TWER SUFFLY VOLTAGE

ITAL SEFIIES RESISTANCE

AFFLIED FRESSURE IANSMITTEF: F'SI

F'FESS SOUR'CE

0.000

2.500

5.000

7.500

10.000

12.500

15.000

17.500

20.000

22.500

25.000

22.500

20.000

17.500

15.000

12.500

10.000

7.500

5.000

2.500

0.000 F.SI

RESISTOF MEASUIFIING ACCURIACY 24

14.996

I DEAL

OUTFUT SIG

MILLIVOLTS

0.000

2.500

5.000

7.500

10.000

12.500

15.000

17.500

20.000

22.500

25.000

22.500

20.000

17.500

15.000

12.500

10.000

7.500

5.000

2.500

0.000
59.984

83.978

107.971

131.965

155.958

179.952

205.946

227.939

251.935

275.926

299.920

275.926

251.935

227.939

203.946

179.952

155.958

131.965

107.971

8.978

59.984

CALIERATION ACCUFACY
**LIMITED**
MEASURED
ERFIOR \%
OUTFUT SIG
MILLIVOLTS

14.956 OHMS

14.996 OHMS

N.A.

$\begin{array}{rr}59.996 & 0.00 \% \\ 83.982 & 0.00 \% \\ 107.988 & 0.01 \% \\ 131.995 & 0.01 \% \\ 156.044 & 0.04 \% \\ 180.115 & 0.07 \% \\ 204.106 & 0.07 \% \\ 228.065 & 0.05 \% \\ 252.046 & 0.05 \% \\ 275.966 & 0.02 \% \\ 299.757 & -0.07 \% \\ 275.930 & 0.00 \% \\ 252.030 & 0.04 \% \\ 227.999 & 0.02 \% \\ 204.032 & 0.04 \% \\ 180.048 & 0.04 \% \\ 156.039 & 0.03 \% \\ 131.938 & -0.01 \% \\ 107.931 & -0.02 \% \\ 85.906 & -0.03 \% \\ 59.931 & -0.02 \%\end{array}$

$\bar{z}=59.964$ 
CURVEFIT OUTPUT FOR VTAB IPdPO4V

DATE: 20-MAY-92

TIME: $16: 35: 01$

VOLTAGE(V) OBS. DP(PSI) CALC. DP(PSI) DIFF (PSIA) \& DIFFERENCE

$\begin{array}{rlclll}1 & 0.3199882 \mathrm{E}-04 & 0.0000000 \mathrm{E}+00 & 0.9852841 \mathrm{E}-02 & -0.9852841 \mathrm{E}-02 & 0.0000000 \mathrm{E}+00 \\ 2 & 0.2401800 \mathrm{E}-01 & 2.500000 & 2.502404 & -0.2403655 \mathrm{E}-02 & -0.9614619 \mathrm{E}-01 \\ 3 & 0.4802400 \mathrm{E}-01 & 5.000000 & 4.998437 & 0.1562877 \mathrm{E}-02 & 0.3125755 \mathrm{E}-01 \\ 4 & 0.7203100 \mathrm{E}-01 & 7.500000 & 7.495980 & 0.4020445 \mathrm{E}-02 & 0.5360594 \mathrm{E}-01 \\ 5 & 0.9608000 \mathrm{E}-01 & 10.00000 & 9.999300 & 0.6998811 \mathrm{E}-03 & 0.6998810 \mathrm{E}-02 \\ 6 & 0.1201510 & 12.50000 & 12.50632 & -0.6322592 \mathrm{E}-02 & -0.5058074 \mathrm{E}-01 \\ 7 & 0.1441420 & 15.00000 & 15.00642 & -0.6418458 \mathrm{E}-02 & -0.4278972 \mathrm{E}-01 \\ 8 & 0.1680990 & 17.50000 & 17.50437 & -0.4371357 \mathrm{E}-02 & -0.2497918 \mathrm{E}-01 \\ 9 & 0.1920820 & 20.00000 & 20.00644 & -0.6436679 \mathrm{E}-02 & -0.3218340 \mathrm{E}-01 \\ 10 & 0.2160020 & 22.50000 & 22.50333 & -0.3326114 \mathrm{E}-02 & -0.1478273 \mathrm{E}-01 \\ 11 & 0.2397930 & 25.00000 & 24.98813 & 0.1186653 \mathrm{E}-01 & 0.4746611 \mathrm{E}-01 \\ 12 & 0.2159660 & 22.50000 & 22.49957 & 0.4327942 \mathrm{E}-03 & 0.1923530 \mathrm{E}-02 \\ 13 & 0.1920660 & 20.00000 & 20.00477 & -0.4766986 \mathrm{E}-02 & -0.2383493 \mathrm{E}-01 \\ 14 & 0.1680350 & 17.50000 & 17.49770 & 0.2303671 \mathrm{E}-02 & 0.1316383 \mathrm{E}-01 \\ 15 & 0.1440680 & 15.00000 & 14.99870 & 0.1295220 \mathrm{E}-02 & 0.8634802 \mathrm{E}-02 \\ 16 & 0.1200840 & 12.50000 & 12.49934 & 0.6574958 \mathrm{E}-03 & 0.5259966 \mathrm{E}-02 \\ 17 & 0.9607500 \mathrm{E}-01 & 10.00000 & 9.998780 & 0.1220490 \mathrm{E}-02 & 0.1220490 \mathrm{E}-01 \\ 18 & 0.7197400 \mathrm{E}-01 & 7.500000 & 7.490048 & 0.9952043 \mathrm{E}-02 & 0.1326939 \\ 19 & 0.4796700 \mathrm{E}-01 & 5.000000 & 4.992509 & 0.7491139 \mathrm{E}-02 & 0.1498228 \\ 20 & 0.2394200 \mathrm{E}-01 & 2.500000 & 2.494504 & 0.5496245 \mathrm{E}-02 & 0.2198498 \\ 21 & -0.3300118 \mathrm{E}-04 & 0.0000000 \mathrm{E}+00 & 0.3100148 \mathrm{E}-02 & -0.3100148 \mathrm{E}-02 & 0.0000000 \mathrm{E}+00\end{array}$

( TGREE OF POLYNOMIAL 2

COEFFICIENTS

$0.65285591 E-02 \quad 0.10388759 E+03 \quad 0.12189226 E+01$

ESS $=0.6423 \mathrm{E}-03 \quad \mathrm{SD}=0.5974 \mathrm{E}-02 \quad \mathrm{R2}=0.999999 \mathrm{E}+00$

$2 * S D=0.1195 \mathrm{E}-01$ 

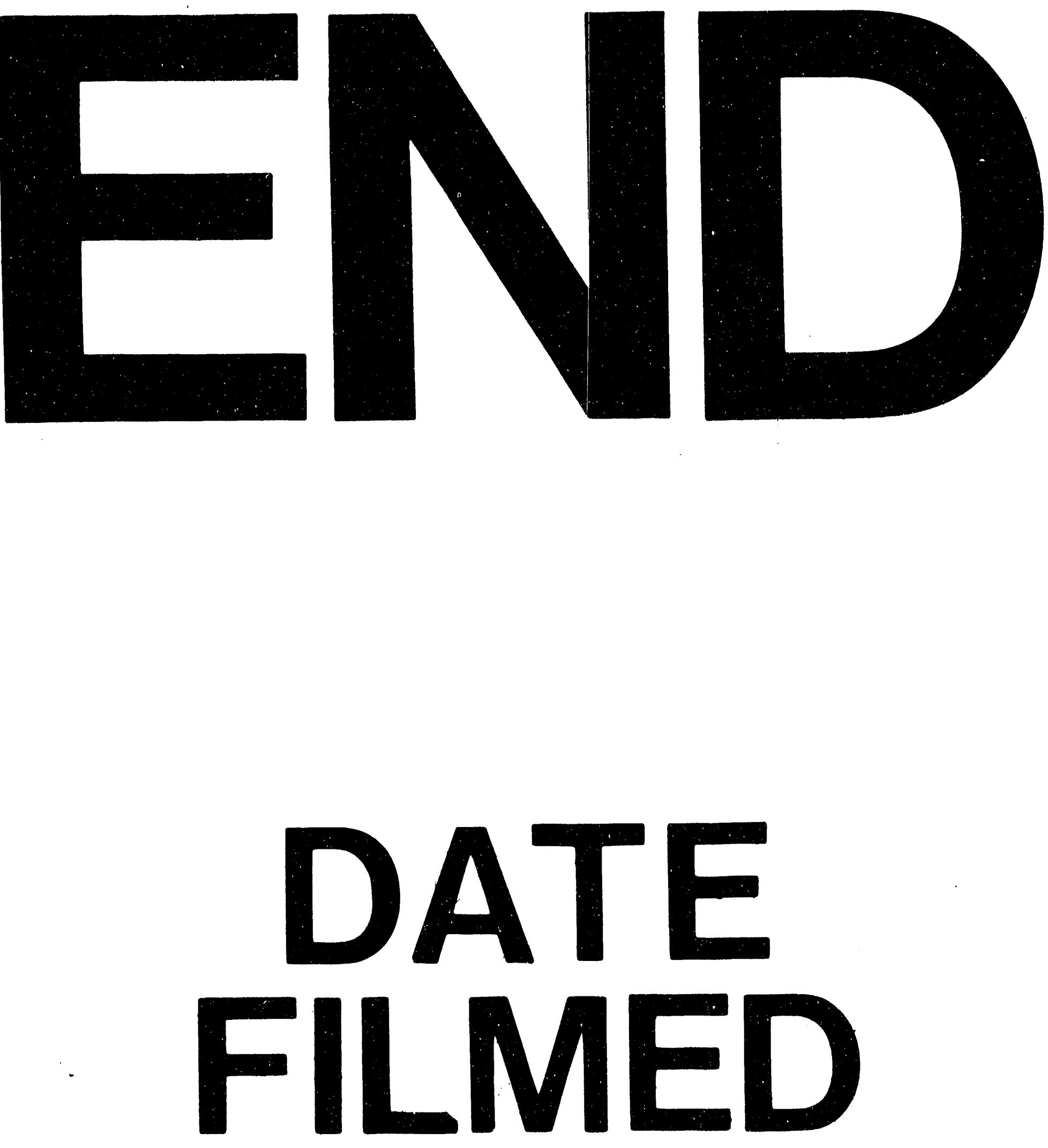

1

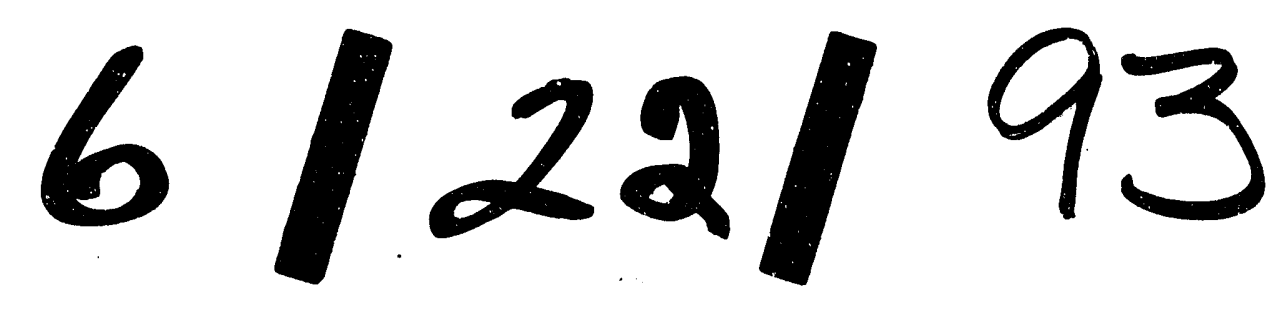


